

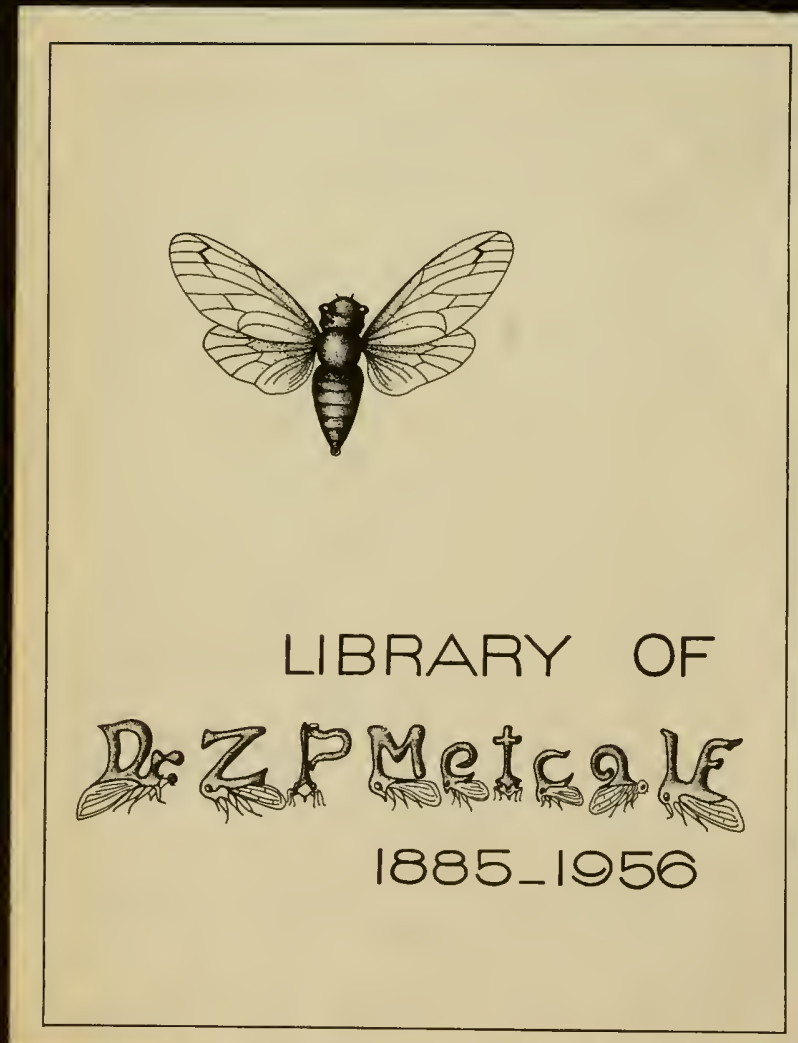





\section{INDEX}

\section{FAUNÆ NOVÆ ZEALANDIÆ}

EDITED BY

Captain F. W. HUTtON, F.R.S.

Published for the

Philosophical Institute of Canterbury,

Nerw Zealand.

LONDON :

DULAU \& CO., 37 SOHO SQUARE. I 904. 



\section{P REFACE.}

GINCE the publication of Dieffenbach's Travels in Neze $\infty$ Zealand, no list has been published of the animals inhabiting New Zealand and the neighbouring seas, although during the last sixty years our knowledge of them has increased very much. Under these circumstances, most of the zoologists in New Zealand have come to the conclusion that the time has arrived for making such a list-to be called Index Faunce Nove Zealandice-and last year they requested me to undertake the editorship. This I willingly did, as I was already assured of the help of all New Zealand zoologists. The Board of Governors of the New Zealand Institute was asked to publish a small edition of the work; but as it declined to do so, the Philosophical Institute of Canterbury, being generously supported by the Otago Institute, undertook the responsibility.

Mr. H. Suter, of Auckland, has supplied the Mollusca; Capt. T. Broun, of Auckland, the Coleoptera. Mr. P. Cameron, of England, has sent a list of the Hymenoptera. Prof. C. Chilton, of Christchurch, and Mr. G. M. Thomson, of Dunedin, have supplied the Crustacea. Professor Benham, of Dunedin, has furnished the Annulata and other Worms, except the terrestrial Turbellarians, which was drawn up by Professor Dendy, of Christchurch, who also supplied a list of the Holothurians. Mr. H. Farquhar, of Wellington, has revised the Echinoidea, Star-fishes, and Hydrozoa. Mr. F. W. Hilgendorf, of Lincoln, has given me a list of the Rotifera; and Prof. H. B. Kirk, of Wellington, has supplied the Porifera. 
The list-as its name implies-is an index only. It does not attempt to give complete references, but merely refers to one or more publications in which lists of synonyms, or descriptions, may be found. Also it is only a record, not a revision; and the only changes made in nomenclature are a few generic names, in the Diptera and Coleoptera, which have been substituted for others which are preoccupied. No doubt a complete list of references would have been very useful to the users of the book, but the expense would have been too great; and we hope that the Government may, some day, bring out a more perfect edition.

The area included in the Index is from the Kermadec Islands, in the north, to Macquarie Island, in the south; and it takes in Stations 165 to 17 I of the Challenger Expedition.

No species introduced by human agency, either intentionally or unintentionally, are admitted; but a list of them is given as an appendix. No doubt this list is very imperfect, and must be looked upon as a first attempt only. For help in it I have especially to mention Mr. Suter, for the Land Mollusca; Captain Broun, Mr. J. J. Walker, R.N., and Dr. Swale, for the Coleoptera ; and Professor Benham for the Earth-worms. Mr. Hilgendorf has supplied a general list, including the Aphidæ. It has been a labour of love for us all, and I wish heartily to thank all those who have helped me, and without whose assistance the Index could not have been completed. But especially I wish to thank Prof. Chilton for the trouble he has taken in helping me to correct the proofs.

\section{F. W. HUTTON.}

Canterbury Museum, Christchurch, N.Z. December 1903 . 


\section{LIST OF ABBREVIATIONS.}

A.M.N.H.-Annals and Magazine of Natural History.

C.B.M.-Catalogue of the British Museum.

C.M.M.N.Z.-Catalogue of the Marine Mollusca of N.Z., Wellington, 1873 .

J.L.S.-Journal of the Linnæan Society of London. Zoology.

MAN. CONCH.-Tryon and Pilsbry's Manual of Conchology.

M.N.Z.Col.-Manual of the New Zealand Coleoptera, Wellington, I880-93.

N.Z.J.S.-New Zealand Journal of Science, Dunedin.

P.L.S.N.S.W.-Proceedings of the Linnæan Society of New South Wales.

P.Z.S.-Proceedings of the Zoological Society of London.

T.E.S.-Transactions of the Entomological Society of London.

T.N.Z.I.--Transactions of the New Zealand Institute.

Q.J.M.S.-Quarterly Journal of the Microscopical Society of London. 


\section{CORRECTIONS.}

Page 28, for Mahua read Mohua.

$" 4 \mathrm{I}$, omit Lirus porosus.

"48, for Duymræia read Duymæra.

, 8o, for Aclia read Aclis.

, 94, for Lithophago read Lithophaga.

" IOI, for Amblytetes read Amblytelus.

" I02, for Rhyssus read Rhyssa.

" I I 5, for Protenia read Proternia.

"123, for Dolichermis read Dolichernis. 


\section{CONTENTS.}

\begin{tabular}{|c|c|c|c|c|c|c|c|c|}
\hline \multicolumn{3}{|c|}{ INTRODUCTION- } & \multicolumn{2}{|r|}{ PAGE } & \multicolumn{4}{|c|}{ ARTHROPODA $($ cont. $)-$} \\
\hline HISTORICAL & . & - & • & I & ORTHOPTERA & - & • & \\
\hline Elements of & THE & FAUN & & 4 & APTERA - & . & . & \\
\hline N.Z. NOT AN & OCEA & NIC I & & & MYRIAPODA & - & . & . \\
\hline LAND & - & . & - & I I & ONYCHOPHORA & - & . & - \\
\hline Outside Evin & ENCE & . & . & 12 & ARACHNIDA & . & . & \\
\hline Date of Isol & ATION & & - & 13 & MaLACOSTRACA & . & . & \\
\hline Geological E & EVIDE & NCE & . & 16 & ENTOMOSTRACA & . & - & \\
\hline OUTLYING IsL & ANDS & . & . & 19 & & & & \\
\hline BIBLIOGRAPHY & . & - & . & 21 & ANNULATA- & & & \\
\hline HORDATA- & & & & & $\begin{array}{l}\text { POLYCH.ETA } \\
\text { ECHIUROIDEA }\end{array}$ & & $\dot{\cdot}$ & $\cdot$ \\
\hline MAMMALIA & . & . & - & 25 & OLIGOCHATA & - & . & . \\
\hline Aves. & . & - & - & 27 & MYZostomIDA & . & . & \\
\hline Reptilia . & . & . & . & 39 & Sipunculacea & - & - & \\
\hline АMPHIBIA . & . & . & . & 40 & HIRUDINEA & . & 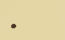 & 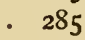 \\
\hline Pisces & - & - & & 40 & & & & \\
\hline Cyclostomat & A. & . & • & 55 & ECHINODERM & $A$ & A- & \\
\hline LEPTOCARDII & . & - & - & 55 & HOLOTHUROIDE & & - & \\
\hline TUNicata & & - & - & 55 & ECHINOIDEA & . & . & \\
\hline HEMICHORDA & . & - & - & $5^{6}$ & OPHI UROIDEA & . & • & \\
\hline OLLUSCA - & & & & & CRINOIDEA & & - & \\
\hline CEPHALOPODA & . & . & & & CEROIDEA & - & - & \\
\hline Gaster & . & • & • & 58 & MOLLUSCOIDA & $4-$ & & \\
\hline SCAPHOPODA & - & - & & 85 & BRACHIOPODA & & . & • \\
\hline AMPHINEURA & . & - & & 86 & PolyzoA . & & - & 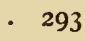 \\
\hline Pelecypoda & - & 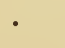 & - & 87 & & & & \\
\hline RTHROPOD & $A-$ & & & & $\mathrm{R}$ & & & \\
\hline HYN & . & . & & 9 & & & & \\
\hline LEPIDOPTERA & . & $0^{\circ}$ & & 103 & NEMATELMIN & $\mathrm{T}$ & $\mathrm{ES}-$ & \\
\hline Diptera . & . & - & - & I 26 & Nematoidea & - & - & \\
\hline Coleoptera & . & ${ }^{-}$ & - & 138 & GORDIACEA & - & . & \\
\hline HeMiptera & . & - & 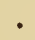 & 221 & ACANTHOCEPHA & & - & • \\
\hline NEURoptera & . & 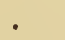 & - & 228 & СHATOGNATHA & & . & 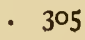 \\
\hline
\end{tabular}




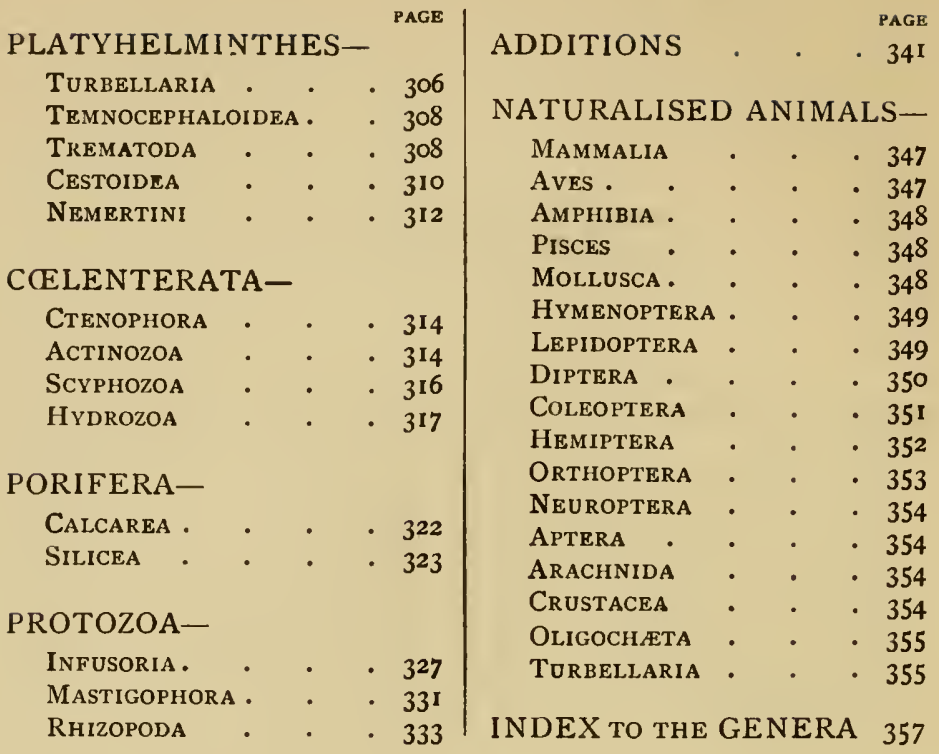




\section{N T R O D U C T I O N.}

\section{HISTORICAL.}

THE foundation of our knowledge of the fauna of New Zealand was laid by the naturalists who accompanied Captain Cook in his first and second voyages round the world. In the first voyage (r 769-70) Sir Joseph Banks and Dr. Solander were the naturalists and Mr. S. Parkinson was the artist. In the second voyage $\left(\mathrm{I}_{773-4)}\right)$ Dr. J. R. Forster was the naturalist and his son George was the artist. The information thus obtained at so much labour was nearly lost to the world by the Government declining to publish the results. The original drawings of $\mathrm{Mr}$. S. Parkinson and Mr. G. Forster, as well as the manuscript notes of Dr. Solander, are still in the British Museum.

The birds were described, from Parkinson's and Forster's drawings, by Latham in $1781-92$, in his General Synopsis of Birds; and these descriptions were incorporated by Gmelin in his edition of the Systema Natura Linnei, r788, when scientific names were given to the species. The manuscript of Dr. Forster's. description of the fishes was published by Schneider in his edition of Bloch's Systema Ichthyologie, generally with Forster's own names, but sometimes with new ones, and ultimately Forster's. descriptions of both birds and fishes were published in Berlin in 1844, under the name of Descriptiones Animalium qua in itinere ad maris australis terras per annos $1772, \mathrm{I} 773$ et $\mathrm{I} 774$ suscepto collegit, observavit et delineavit Joannes Reinhold Forster.

The few insects brought home by Sir Joseph Banks were described in $1774-5$ by Fabricius, and some of the types are retained in the British Museum. Dr. Solander described a few 
shells in 1784 ; and others, collected by the Forsters, were described by Chemnitz in $178 \mathrm{I}-8$, and Martyn in 1784 .

All this has complicated the nomenclature a good deal. Speaking of the Fishes Dr. Gill says: 'It is to be remarked that some of the species described by Forster have each received two names. Schneider, with the propensity common in his time to change a name because it did not seem to be as good a one as could be given, substituting for Forster's one of his own which suited him better. Nevertheless, Forster's, in every case but one, was also given. Forster's names were therefore actually published at the same time as Schneider's and in connection with Forster's own descriptions. Whose names, then, shall be adopted -Forster's or Schneider's? Opinion has differed on this point, some accepting Forster's, others Schneider's.*

The next contribution to our knowledge was made when the French corvette Coquille visited the Bay of Islands in 1822 , when the naturalists on board, MM. Lesson and Garnot, added three new species of birds to our fauna.

In 1827 the same ship, but now named the Astrolabe, examined all our outlying islands, and remained for some time in Queen Charlotte's Sound. The naturalists, MM. Quoy and Gaimard, were assiduous collectors, especially of Mollusks, and their results were published by the French Government in I 833 .

In I 840 Dr. E. Dieffenbach travelled through the North Island of New Zealand and the Chatham Islands for the New Zealand Company, and the collections he brought back with him were described by Dr. J. Gray and Dr. Richardson in the appendix to Dieffenbach's travels. In this book also Dr. J. Gray, Director of the British Museum, gave a list of the then known fauna of New Zealand.

In the same year the United States Exploring Expedition, under Captain Wilkes, visited Akaroa and the Bay of Islands, and the birds collected were described by Peale in 1848 ; the shells by Aug. Gould in $185_{2}$; the Crustacea by J. D. Dana in 1855 .

In $184 \mathrm{I}$ the French ships Astrolabe and Zelée visited the Auckland Islands, Port Chalmers, Akaroa, and the Bay of Islands. The records of this voyage, called Voyage au pole Sud, were pub-

* Mennoirs of Nat. Acad. Sciences, Washington, vi. p. 95. 
lished between $184 \mathrm{I}$ and 1846 , the natural history portion being by Hombron and Jacquinot. The New Zealand collections consisted of birds, shells, crabs, and beetles.

In the same year, I $\$ 4 \mathrm{I}$, H.M.S. Erebus and Terror, under the command of Sir James Ross, visited Campbell Island, the Auckland Islands, and the Bay of Islands, and the zoological results of the voyage were partly published in 1846 . This included the Mammals by Dr. J. Gray, the Birds by Mr. G. Gray, the Fishes by Sir John Richardson, and part of the Insects by Mr. A. White The publication was then interrupted for want of funds, but was completed in $1874, \mathrm{Mr}$. A. G. Butler taking the remainder of the Insects, Mr. E. J. Miers the Crustacea, and Mr. E. A. Smith the Mollusca.

By this time the colony had been established in the north, and some of the residents of Auckland collected and sent their specimens to the British Museum. The principal of these were Dr. Greenwood, who collected land shells, and Colonel Bolton, who collected insects. The insects were described by Mr. F. Walker, in his catalogues of the insects in the British Museum; and the shells partly by Dr. J. Gray and partly by M. Pfeiffer, in the Proceedings of the Zoological Society and the Malakozoologische Blätter.

During the years $1847-9$ H.M.S. Acheron was engaged surveying the coast of the islands, and the surgeon, Dr. Lyall, made a collection of Polyzoa, which were described by Mr. G. Busk in his catalogue of the Polyzoa in the British Museum.

In 1859 the Austrian frigate Novara visited Auckland, and descriptions of the zoological collections were published in Vienna in 1864-9. Unfortunately sufficient care was not taken to keep the New Zealand collections separate from those made in Australia and other parts of the world, and this has led to much confusion

H.M.S. Challenger visited. Wellington in 1874 , and the collections, chiefly of deep-sea animals, were described in the wellknown series of 'Challenger' Reports.

In the same year, 1874 , the French Transit of Venus Expedition established a station on Campbell Island, and the naturalist of the expedition, Dr. H. Filhol, collected and examined the animals. Subsequently he made a collecting tour in several parts of New Zealand, and the results were published in 1885 in the Mission de l'ile Campbell. The anatomy of the penguins, and a 
catalogue of the New Zealand Crustacea are the most important papers.

The German expedition for the same object visited Auckland Island, when Dr. Krone collected a few Diptera and land Mollusca.

In 1879 Mr. E. Meyrick visited New Zealand, and for several years collected and described the Lepidoptera.

Meantime local naturalists had been very active. The New Zealand Institute was established in 1868 , and its annual volume has always contained valuable contributions to the zoology of New Zealand.

Several descriptive catalogues, by different authors, were published between 187 I and I880. The first edition of Sir W. Buller's Birds of Nere Zealand appeared in 1873 , the second edition in I888. Mr. G. V. Hudson's Nere Zealand Moths and Butterfies in 1898 . Other papers, in various publications, will be found mentioned in the index.

\section{ELEMENTS OF THE NEIV ZEALAND FAUNA.}

Omitting a few stragglers among the birds, fishes, and butterflies, which have failed to establish themselves, the New Zealand fauna consists of two parts, one migratory, the other stationary.

The migratory portion, which is very small, includes two cuckoos and three or four species of Limicola, which reach us every October from the north through $\mathrm{New}$ Caledonia and I.ord Howe Island, and leave in or about March."

Among the list of fishes several names will be found of forms which are properly inhabitants of warmer seas than those of New Zealand, and are, no doubt, wanderers from the north. Such are Lampris, Brama, Sphyrana, Murana, and Ophisaurus. The Mackerel (S. australasicus) and the Sprat (C. antipodum) are irregular visitors to our coasts; while two species, the Kingfish (Seriola lalandii), and the Trevally (Caranx georgianus), appear to perform a regular migration, leaving us in the winter for the north. But very little is as yet known of their movements.

Of our fresh-water fishes, Retropinna richardsoni, Galaxias attenuatus, and the Eels (Anguilla) migrate to the sea to spawn, and the young fish ascend the rivers in the spring. The Lamprey

* Trans. N. Z. Inst. vol. xxxii. p. 25 I. 
(Geotria) breeds in the fresh water, but the young go down to the sea and reascend the rivers, while immature, in the spring, remaining there to breed. Prototroctes breeds in fresh water, and probably never goes to sea. The other species of Galaxias and Eleotris remain in fresh water all their lives.

The stationary portion forms the bulk of the fauna; and it may be divided into a small aboriginal element, including species which have no near relatives elsewhere, and larger Malayan, Australian, and Antarctic elements, as well as several other smaller ones. In many cases, however, it is hard to say in which group a species should be placed, for its relations are so widely scattered. Taking a broad view of the whole fauna, however, it may be said that the terrestrial portion is chiefly of Malayan origin, but with rather strong Palæarctic and Neo-tropical connections. 'The fresh-water fishes and crustacea are chiefly Australian and South Anterican, while the relations of the fresh-water mollusca are not known to me. The marine fauna is chiefly Australian and Antarctic.

I will give a short account of each of these different elements.

The Aboriginal Element. - Includes the Short-tailed Bat (Mystacops), the Tuatara (Sphenodon), and the Kiwi (Apteryx). To which may be added the following birds: Glaucopis, Heteralocha, Turnagra, Xenicus, Stringops, and Nestor. Also the land mollusk Carthea; and the following among the insects: Oniscigaster, Orectognathes, Paradetis, Notoreas, Palcomicra, Mneoarchac, Arctoneura, Casa, Paradoxa, Opifex, Tinemyia, Fraudator, Ostenia, Exsul, Brounia, Camiarus, Epistranus, Cyphotelus, Alema, Peneticus, Aphilon, Lorelus, Astetholes, Gastrosarus, and others. It is not necessary to assume that all these aboriginals date their arrival in New Zealand at the same time, for some may have become modified more rapidly than others. But most of them appear to belong to old forms, and probably they were among the first comers.

The Malayan and Melanesian Element.-This embraces nearly the whole of our land fauna, and a considerable portion of the marine fauna as well. I should define it as including all those species which have their nearest relatives in New Caledonia, New Guinea, or the Malay Archipelago; and whose ancestors, presumably, came to New Zealand from the north. It includes all the land birds-except those just mentioned, and Zosterops-all 
lizards, ${ }^{*}$ some fishes like Erythricthys, Parapercis, and Eleotris. Nearly the whole of the land Mollusca, including Endodonta, Placostylus, Rhytida, Athoracophorus, also many marine and freshwater shells, Monilea, Xenophora, Unio, and a very large number of insects, including the whole of the Phasmidce and Stenopelmatida. The Katipo Spider (Latrodectes) must also be included in this group, for it is found not only in Australia, but in the Malay Archipelago and India. There is also a closely allied form in Chili. Scyphax, a terrestrial isopod, occurs only in New Zealand and New Caledonia. Armadillo also is distinctly Melanesian.

The Ethiopian Element. - This is small, but remarkable. Most noticeable is the relation between the extinct Dinornithida and the also extinct Epiornithide of Madagascar. Our Fernbird (Sphenceacus) belongs to a genus which is found only in New Zealand and South Africa, but it has a near ally in Madagascar. There is a curious and easily recognised beetle, called Sternaula.t, which is also only found in New Zealand and Madagascar. And perhaps we should include the marine shell Littorina mauritiana, although it is also found in Australia.

The Oriental Element.-This includes species which have nearer relatives in the Oriental region than in the Malay Archipelago. Our tree lizards belong to a genus, Hoplodactylus, found only in New Zealand, Queensland, and India ; but there are some related forms in New Caledonia and the Malay Archipelago. Our frog, Leiopelma, has its nearest relative in China, and there are no known representatives of the family to which it belongs either in Australia or in the Malay Archipelago. The black butterfly, Percnodaimon, is a north temperate alpine form, and is said to have its nearest relative in the Himalaya. Also the May fly, Ichthybotus, is close to Ephemera of the northern temperate regions and India. The genus of marine shells, Ethalia, is not found nearer to New Zealand than Borneo.

Probably both the Ethiopian and Oriental elements ought to be placed with the Malayan element, the connecting links in the Malayan Archipelago having been exterminated or not yet found.

The Australian Element. - This I take to mean those species

* Gehyra oceanica may have been brought to New Zealand by the Maoris in their canoes, among the food stores. For undoubtedly it is by this means that it has become distributed through the islands of the Pacific Ocean. 
which have nearer relations in Australia or Tasmania than in any other part of the world, and which we may suppose came to New Zealand from the west. The Australian element in New Zealand does not include any species connected with the ancient typical Australian fauna, but is entirely made up of forms belonging to the Malayan element in the Australian fauna which overran Australia and Polynesia at a comparatively late date.

We have, therefore, two possible explanations of our Australian connection. Either (I) the ancestors of our animals crossed the Tasman sea and so came to us from the west, or (2) the relationship may be due to two branches of northern immigrants from New Guinea, one taking the line to Australia, the other to New Zealand. The first group forms the true Australian element, while the second group ought properly to be placed with the Malayan elemert; but it is not always easy to say from which direction the ancestors of our animals came. For example, the marine spider, Uliodon maritima, was first found in New Zealand and then in New South Wales, and was of course considered as belonging to the Australian element, but it has lately been found in New Caledonia.

I should select as examples of the Australian element the bat, Chalinolobus morio; and among birds, Zosterops carulescens, Hcamatopus unicolor, and Spatula rhynchotis; several marine fishes, e.g., Arripis and Latris; the land-shell, Laoma; some marine mollusca, like Megatebenus; some crabs, such as Paramithrax, Nectocarcinus, and cizius truncatus. The fresh-water isopod, Phreatoicus, and two or three land isopods; beetles like Pyronota, Adelium, Chrosis, and others; several moths, and perhaps the Land Planarians. Diporochceta, an earthworm from the Chatham lslands, may perhaps be added, although it is far more probable that these worms came to us by land from the north. There are also several species of Myriapods common to New Zealand and Australia or Tasmania, but these are easily conveyed in fruit, and may be late introductions.

When we consider that Tasmania and the South Island of New Zealand are in the region of strong westerly winds, and that gales are constantly traversing the T'asman Sea, we should expect to find the Australian element much stronger than it really is.

The Antarctic Element. - These are the animals whose ancestors we suppose came to New Zealand from the south or east, as we find that their relations are confined to southern latitudes. It 
contains chiefly marine forms, such as seals, penguins, cormorants, and the black-backed gull, as well, perhaps, as the black oyster-catcher. There are also several fishes, both fresh-water and marine. The former comprise Cheimarichthys, Prototroctes, Galaxias, and Geotria, but as a close relation to Galaxiascalled Cromeria - has lately been found in the Upper Nile, and a Galaxias in South Africa, we must reconsider our opinions about that genus. Galaxias attenuatus and the Lamprey are migratory, spending some parts of their lives in the sea. Of the marine Antarctic fishes we have Notothenia, Bovichthys, Congiopodus, Genjpterus, Gonorhynchus and Callorhynchus. There are also several Antarctic crustaceans, such as Cyclograpsus, Halicarcinus planatus, Rhynchocinetes typus, Leander affinis and Sphceroma gigas.

Of marine mollusca there are Ranella argus, Struthiolaria, Plaxiphora, Callochiton, Venus stutchburyi, Solenella australis, Mytilus magellanicus, and others. Also our Brachiopods are more closely related to those of the Straits of Magellan than they are to the Brachiopods of Tasmania. The family of earthworms called Acanthodrilide is abundant in New Zealand, Patagonia, South Africa, and Kergueien Land, while it is almost absent from Australia and Tasmania. It occurs, however, in New Caledonia, India, and the West Indian Islands, and so can hardly be considered as of Antarctic origin.

There may also be a few land invertebrates belonging to the Antarctic element, for the spider, Tetragnatha gulosa, is said to be found in the Chatham Islands, as well as in the island of St. Paul, but it may have been brought by a whaler.

I know of no insects that have a true Antarctic distribution.

The Neo-tropical Element.-The Merganser of the Auckland Islands has its nearest relative in Brazil. Our Blue Duck, Hymenolamus, forms, with Salvadorina of Waigou and Merganetta of the Andes, a distinct sub-family. And if Mr. Pycraft is right in his opinion that the Dinornithida are more closely allied to the Rhea than to the Cassowary or Emu, ${ }^{*}$ then this evidence must be added to the rest connecting New Zealand with South America, and perhaps Madagascar.

The Neo-tropical element is tolerably abundant in our insects. The Crane-fly, Tanyderus, is found in Amboina and Chili, while 
Macromastix is Australian as well as Chilian. Our large Dragonfly, Uropetala, is related to Petalura of Australia, and Phenes of Chili. In the Coleoptera there are the genera Tropopterus and Lagrioda. In the Lepidoptera we have the butterfly Argyrophenga, and the moths Gonophylla, Drepanodes, and other of the Notodontina, which are more nearly related to Chilian species than to those of Australia, as also are the species of Diptychophora and Siculodes in the PJralidina. Our fresh-water Crayfish, Paranephrops, is also closely related to those of Australia and South America.

The earthworms of the genus Microsolex are chiefly South American, but they are found in California and Madeira as well. The distribution of the little fresh-water, external parasitic worms called Temnocephala is very instructive. They occur not only in New Zealand and Australia but also in Mexico, Brazil, and Chili, as well as in the Malay Archipelago, the Philippine Islands, and Madagascar. They usually live on fresh-water crayfish; but in the Malay Archipelago they live upon fresh-water crabs; and in Brazil on fresh-water Chelonians and on Ampullaria. In both these places there are no fresh-water crayfish.

The Nearctic Element.-We find a small Nearctic element in the May-flies, Coloburiscus and Ameletus, found only in New Zealand and North America. Also in the fresh-water shell Latia, which is living in New Zealand and fossil in North America. It is closely related to Gundlachia, which inhabits Tasmania and North America. The little leach Microbdella Nor'e Zealandice is also congeneric with a species from North Carolina.

The Palceartic Element.-We have also some animals which show a close connection with species of Europe and Northern Asia, but which have no nearer relatives in the tropics. These includes the so-called bipolar forms, most of which are marine.

Among the whales we have the species of Bulanoptera and Ziphius cavirostris, and perhaps all the southern baleen whales should be included. The Black 'Teal (Fuligula Nov' Zealandia) must also be looked upon as a bipolar form; and it has a companion in the extinct Anas (?) finschi, which, according to Van Beneden, is closely allied to Clangula.

Among the fishes we have some deep-sea forms like Halagyreus johnstoni, Alepisaurus ferox, Argentina, and Lepidopus caudatus. But others do not inhabit the deep sea, e.g., Zeus faber, Cyttus, Polyprion, Conger vulgaris, Scymnus lichia, Echinorhinus 
spinosus, and Acanthias vulgaris. There are also a few crustacea and mollusca, such as Saxicava artica and Mytilus edulis, as well as many polyzoa, of which the southern forms are considered to be identical with those inhabiting the northern seas.

But bipolar species are not exclusively marine. Retropinna belongs to the northern Salmonidce. The fresh-water spider Cambridgea is almost identical with Argyroneta of Europe. Lycosa piratica is also found in New Zealand under conditions which make its importation by man very improbable. Brachaspis, a flightless grasshopper, is related to Pezotettix; and among the beetles there are Cillenum, Trogophlaus, Pycnomerus, Philothermus, Dendrophagus, Telmatophilus, Betarmon, and others.

Attempts have been made to account for the Scandinavian element in the New Zealand flora by the supposition of a general glacial epoch all over the world, during which the Arctic plants passed from mountain top to mountain top through the tropics. But it is now considered very doubtful if there ever was a general glacial epoch, and even if there was one it could not explain the occurrence in New Zealand of northern beetles, grasshoppers, and spiders. It seems more probable that these bipolar forms at some former time inhabited the tropics; but that owing to variation and change being more rapid in warm than in cold climates-a statement not yet proved-the older forms were exterminated in the warmer regions, being replaced by their more highly developed descendants, the present tropical fauna.

The Atlantic Island Element.-Lastly we see in some of the beetles a remarkable connection between the islands of the Atlantic and those of the Pacific, in which New Zealand shares. This is shown by the genera Rhynchogonus, Acalles, Pentarthrum, and Aglycyderes, all belonging to the Rhynchophora; and Xenoscelis, belonging to the Nitulida. It is most probable that these, also, are relics of a former more universal distribution; the islands serving as harbours of refuge, while their continental relations were exterminated.

Of Uncertain Origin.-The distribution of Peripatus is difficult to explain, as it is found in the West Indies, Central and South America, and South Africa, as well as in the Malay Peninsula, New britain, Australia, and New Zealand. It is supposed to have originated in Central America, and to have migrated from thence. If this is correct, it could not have reached Chili before the Miocene period, for before that time 
South America was isolated from the North. At any rate it cannot be considered as an Antarctic form, and I an inclined to place it in the Neo-tropical element.

\section{NEW ZEALAND NOT AN OCEANIC ISLAND.}

An examination of the pages of this Index will show that the New Zealand fauna is not altogether an insular one-that is, one that has crossed over an oceanic barrier-but is largely derived through a former connection with the mainland.

(I) In the first place we have a number of animals which it is impossible to believe crossed over the sea :-

The Tuatara and lizards, as well as the frog;

Our large land mollusca and slugs;

The fresh-water shells, and especially Unio;

The fresh-water cray-fish;

Many insects, such as the large Curculionida; the wingless grasshoppers; Stenopelmatide, and Phasmide ;

Many of the Neuroptera, which pass most of their lives in fresh water ;

The earth-worms and land planarians.

Perhaps the Kiwi and Moas should be mentioned, but it is possible that their ancestors may have flown across a moderately broad strait.

(2) In the second place, the relations of the fauna are not what we should expect if they had been over-sea wanderers; for the Tasmanian and Australian element is weak in comparison with the Malayan element.

(3) Thirdly, the low type of a great part of the fauna, which, however, shows a great variety of forms, especially in the insects. The fauna is essentially an old one, and many of the higher types are absent.

(4) Fourthly, the occurrence of migratory birds which could never have found their way here if they had not originally followed a land connection.

(5) Fifthly, the number of gymnosperms and orchids in the flora-plants which are not found on oceanic islands-confirms the conclusion arrived at from the animals.

To explain the connection between New Zealand and some ancient land it is necessary to assume the existence of a former continent, either in the Antarctic regions or in the Pacific, of which Patagonia, Australia, New Guinea, and New Zealand formed 
parts. But in either case the land communication must have been broken up a very long time ago, because the Indo-Pacific marine fauna differs so much from that of South America.

The land mollusca of Polynesia furnish the best evidence for a former Pacific continent. For, as Mr. H. Pilsbry has pointed out, it is not a collection of waifs and strays, but a harmonious group of a very early type. These land shells-belonging to the genus Endodonta-occur all through the Polynesian Islands, New Zealand, Eastern Australia, New Guinea, the Philippine Islands, and have an outlier in Ceylon. This outlier furnishes good evidence that the ancient type at one time inhabited India, Burma, and the Malay Peninsula, and that its migration has been from the north and not from the south. But as this genus is not known to extend to South America, the evidence fails to explain the South American connection.

The best zoological evidence for a former Antarctic continent is furnished by the earth-worms belonging to the family Acanthodrilida, which do not occur anywhere in the Northern Hemisphere, and are very scarce in Australia, but common in New Zealand and New Caledonia. However, there is an outlier in Madagascar which seems to imply a northern origin for the family. The floras of the Antarctic Islands are also considered as evidence of a former Antarctic continent, or group of Antarctic islands.

\section{OUTSIDE EVIDENCE.}

We cannot study the geographical relations of the New Zealand fauna with any prospect of success without taking into consideration the faunas of neighbouring countries, and there are two subjects of special importance to which I must draw attention.

(1) The first is the fact that Australia shows a connection with South America in which New Zealand does not share. We see this in the Mammalia, the family of fresh-water tortoises called Chelydida, the extinct tortoise Miolania, and in the snakes belonging to the families Typlopidae and Ilysiida, as well as three sub-families of the Colubrida; also the frogs and the fresh-water fishes Osteoglossum and Ceratodus, the latter having been found fossil in Patagonia. In addition there are a large number of insects, such as Gnostida, Buprestida, and Longicornia, among the beetles, and Castniidce among the moths. And in this con- 
nection we must remember the outliers of the Iguanida found in Fiji and in Madagascar.

The Australian Neo-tropical element is therefore much more considerable than that of New Zealand, and is chiefly subtropical ; while that of New Zealand is temperate. Also it should be noticed that the Chilian and Patagonia fauna consists of two parts- $(a)$ an older indigenous fauna, and $(b)$ a later immigrant fauna from the north. The Australian and New Zealand connections are entirely with the older fauna.

(2) The second important fact is the poverty of the present Antarctic terrestrial fauna and flora. Only a small beetle, a dipterous insect, and two or three species belonging to the Collembola, as well as a few kinds of mosses and lichens, have been found there : a striking contrast to the fauna and flora of similar climates in the Arctic regions.

Now, it is evident that the insects could not have been blown or carried to where the mosses happened to be. Their ancestors must have travelled to their present positions by land; and, this being so, we have two possible explanations to account for the difference between Arctic and Antarctic floras and faunas :

I. The mosses and insects may be survivals from a time when no angiospermous plants were in existence, the land on which they live having been isolated from other lands ever since. This supposes them to be relics of the Jurassic period.

2. The present fauna and flora may be the remains of a more extensive flora and fauna which once inhabited the southern regions, but which has been destroyed by a glacial epoch. In this case it is necessary to explain how it is that we find such remarkable floras on southern islands like Kerguelen, Macquarie, and Hermite, not very far north of the desolate regions of the South Shetlands and Victoria Land. It is to be hoped that the Antarctic explorations now going on will throw some light on this question.

\section{DATE OF ISOLATION.}

In estimating the time or times when New Zealand was connected with the mainland, we must first take into consideration the great differences between its fauna and those of Australia, South America, and South Africa. This shows decisively that the period of connection was a very ancient one, for New Zealand must have been isolated before the spread of 
many groups of animals, and must have remained isolated ever since.

Professor Gill, writing about the fishes, says that 'the number of species apparently peculiar to the province, and, therefore, modified from other or earlier representatives, indicates a long period of isolation in accordance with its distance from the nearest continents and the depth of the intervening ocean.' He thinks it probable 'that there existed some terrestria! passage-way between the several [Antarctic] regions at a time as late as the close of the Mesozoic. The separation of the several areas must, however, have occurred little later than the earlier Tertiary, inasmuch as the salt-water fishes of corresponding isotherms found along the coasts of the now widely-separated lands are to such a large extent specifically different.' 'In general,' he says, 'change seems to take place more rapidly among marine animals than fresh-water representatives of the same class.' And thus he explains the closer relations in Galaxias and Geotria than in the marine fishes.

Mr. H A. Pilsbry thinks that the Epiphallngonous land-snails invaded New Guinea from the north before the Eocene period, and yet they were unable to reach New Zealand.

It must be remembered that the flora of New Zealand shows the same elements as the fauna, and. as the Antarctic plants are far more numerous than the undoubted Antarctic land animals, I tried a good many years ago to use them as a means of ascertaining whether the migration from the north occurred before or alter the east and west migration And, judging by the extent of modification the two elements among the plants have undergone in New Zealand, I arrived at the conclusion that the tropical migration, taken as a whole, was anterior to the Antarctic migration.

A study of the fauna confirms this opinion. For while in the Malayan element we find a large number of endemic genera, the Antarctic element consists largely of species which are found elsewhere; or, like the shags, are only slightly modified. This shows that their spread has been later.

This conclusion, however, does not prevent us from supposing that some Antarctic immigrants may have been in New Zealand as long or evell longer than some Malayan immigrants. For there may have been several periods of migration.

Indeed, the Malayan element in the fauna shows considerable 
differences in modification. In all the orders of insects we can recognise that certain families are probably late comers, for they are made up of fragmentary groups, and the genera are rarely endemic. For example, we have in the Lepidoptera, the Papilionina, the Sphingida and the Arctiada; in the Diptera we have the Muscide; in the Hymenoptera, the Apidie and Pompilida; in the Coleoptera, the Cicindelida, Buprestida, and the Lamellicornia; in the Hemiptera, the Pentatomida and Cicadida: in the Orthoptera we have the Gryllida, Locustida, and Blattida; in the Neuroptera, the Odonata.

On the other hand, there are groups which are comparatively well represented, and we recognise them as ancient inhabitants because a large proportion of the genera are endemic. Thus in the Lepidoptera we have the Scopariida and the Tineina; in the Diptera, the Tipulide; in the Hymenoptera, the Formicidce; in the Coleoptera, the Pselaphida, Colydiida, Dascyllidee, and the Curculionida; in the Hemiptera, the Fulgorida; in the Orthoptera, the Stenopelmatida and Phasmida; in the Neuroptera we have the Trichoptera.

The Mud-fish (Neochanna) must have been a long time in New Zealand, because it has become so much modified; and if it is, as Dr. Gunther has suggested, a degraded form of Galaxias, then Galaxias must have been here for a still longer time. But Galaxias attemuatus is found in Patagonia and Tasmania ; and it is more closely related to $G$. maculatus of Tierra del Fuego and Patagonia than to any of the New Zealand or Australian species. From which it is evident that it must either have spread at a comparatively late period from New Zealand to Patagonia, or that Galaxias has been introduced twice into New Zealand.

Again, in the Antarctic element, the earth-worms point to a period of distribution long anterior to that of the marine fishes, Crustacea and Mollusca, which form the greater part of the group; so that here, also, we seem to have two different periods of migration.

The connection between New Zealand and South America shown by those species the geographical relations of which are also Malayan, and which cannot be considered as Antarctic, appears to have been at a very remote period No species are identical, and only a few genera, chiefly in insects. In birds such as Hymenolcemus and the Struthious birds, the relations are between members of the same family only. It is the same with the 
Australian-South American connection, as shown by the frogs and the parrots. The latter are a group of birds which probably originated in the Malay Archipelago or Australia and spread to South America. According to Professor W. K. Parker, the Australian Magpies (Gymnorliza), and the Wood-swallows (Artamus), and even some of the Fly-catchers (Petroica) are more closely related to South American birds than to any others.* And Professor Huxley says that there is a close relation between the Megapodide and the Cracida of South America. $\dagger$ But all these relations are distant, and imply a very ancient connection.

\section{GEOLOGICAL EVIDENCE.}

We will now see what light the geology of New Zealand throws on these questions.

In the Triassic period New Zealand appears to have formed part of the eastern coast line of a continent which probably included Australia and Tasmania. In the middle of the Jurassic period-the date is not very certain, for the fossils have not yet been properly examined-this state of things changed, and the range of mountains running from Stewart Island to the East Cape was formed, and with it the branch range from Taupo to the North Cape. From that time forward the connection with Australia has been slight, as proved by the difference in the fossils, so that we may assume that the Tasman Sea was formed at the same time as the New Zealand Alps, and that it has been in existence ever since. Any subsequent direct land communication between New Zealand on the one hand and Tasmania or Australia on the other, is highly improbable.

This mountain chain is of the Alpine type. and has a granitic core, consequently when it was first formed it probably extended further on each side than it does now; indeed, we see that the north-western portion has been largely removed by denudation. Since its formation it has been twice partially submerged, New Zealand in each case being reduced to a cluster of islands. The first of these depressions was in the Upper Cretaceous, the second in the Oligocene and Miocene periods. We also find evidence that the land stood lower than it does now in the

* Trans. Zool. Soc. x. p. 25I, \&c.

+ Pro. Zool. Soc. 1868, p. 296. 
Pleistocene and perhaps in the newer Pliocene. The evidence for the third depression consists of raised beaches and marine fossiliferous beds in the southern part of the North Island. We also know that Cook's Strait was in existence in the Pleistocene period, because the species of Moas found in the peat-bogs and alluvial deposits of the South Island are specifically different from those found in the North Island.

So we have the older Cretaceous, the Eocene, and the older Pliocene as periods during which New Zealand may have been of much larger dimensions than it is now.

The Cretaceous fossils have not yet been described except the plants. These differ much from those of Australia, but as Baron von Ettingshausen, who described them, had a very limited acquaintance with the existing flora of New Zealand, the names he has given to the fossils cannot always be relied upon as accurate. It is, however, evident that we have here the basis of our present Phanerogamous flora; and, if this is the case, it is a proof that the New Zealand mountains have not been totally submerged since the older part of the Cretaceous period.

A Unio has been found at the Malvern Hills, which may be taken as a proof of land communication between New Zealand and some other country in the lower Cretaceous period, for Unio is not known in any oceanic island.

With the plants and the fresh-water Unio no doubt there came a number of land animals, although we have not found any of their remains. Probably they were land mollusca, insects of various orders, myriapods and worms, but no vertebrates except Sphenodon.

The known Oligocene and Miocene faunas consist chiefly of marine mollusks, which differ very much from the contemporaneous mollusca of Australia. One remarkable peculiarity is that we have in the Miocene sixteen species of shallow-water invertebrates, which are also found in the Miocene of Patagonia, implying a shallow-water communication between the two countries. Many of the Mollusca are very large, much larger than those inhabiting the New Zealand coasts at the present day, so that we must suppose that the sea in the Miocene period was warmer than it is now.

Pectunculus laticostatus and Dosinia grayi, which are Eocene only in Australia, first appeared in New Zealand in the Mioccne, and still live there. Typhis is Eocene in Australia, Miocene in 
New Zealand, where it became extinct before the Pliocene. Murex and Trophon, both of which are Eocene in Australia, did not reach New Zealand until the Pliocene period. This appearance of shells in Australia before they are known in New Zealand shows that a large part of our present marine fauna reached us in Miocene and Pliocene times from Australia and not from an Antarctic continent.

In the coal-measures of Moreley Creek, Southland-probably of Eocene age-a Unio (U. inflatus) has been found which may have been derived from the Cretaceous Unio already mentioned. This species, however, appears to have become extinct without leaving any descendants. Probably it was killed off by the submergence in the Oligocene and Miocene periods.

In the lignite beds of the Dunstan in Otago-probably of older Pliocene age-another Unio has been found which appears to belong to our recent species, Diplodon aucklandicus, which is only a variety of $D$. menziesii. This species has its nearest allies in New Guinea, so that its existence in New Zealand in the older Pliocene is evidence that during that period New Zealand was in direct communication with New Guinea.

Under a lava stream at Timaru a few bones of a small moa (Anomalopteryx antiqua) and of an Apteryx have been found, which are either upper Miocene or lower Pliocene in age, and other Moa bones have been found in Pliocene beds near Napier, and footprints of Pliocene or Pleistocene moas have been found near Gisborne, but no trace of them has hitherto been found in either the Miocene or Eocene coal-measures.

The question whether or not a glacial epoch occurred in the southern hemisphere similar to that of the north, has an important bearing on the distribution of animals and plants. So far as New Zealand is concerned, there can be no doubt but that the glaciers of the South Island were formerly of much greater dimensions than they are now. But there is no evidence that they reached the sea, although some of the old moraines are now below the sea level, and there is no palæontological evidence of a colder climate in the Pliocene or Pleistocene periods than exists now. On the contrary, the evidence points to a gradual cooling ever since the Miocene period. On the whole it seems probable that the former extension of the glaciers was due to the land standing 3000 or 4000 feet higher in the older Pliocene times than it does now. The very distinct flora of the Antarctic 
Islands is also evidence that no glacial epoch has taken place in the Southern Hemisphere.

The large proportion of shallow-water Miocene fossils common to Patagonia, New Zealand, and Australia proves that either in the Eocene or Oligocene, shallow water, warmer than it is at present, extended through the South Pacific; but as none of the Miocene mammals nor land birds of Patagonia passed to New Zealand, there could not have been a continuous land connection. Probably it was a series of coral islands. This will account for the spread of the penguins, which are Oligocene in New Zealand and Miocene in Patagonia, but it is doubtful whether it will account for the insects. Certainly it cannot explain the connection between the Vertebrata of Australia and those of South America.

\section{OUTLYING ISLANDS.}

Of the outlying islands the Snares and Bounty Islands are composed entirely of granite, and no doubt at one time they formed part of New Zealand. The Kermadecs, Antipodes, and Auckland Islands are entirely volcanic. The Chatham Islands are volcanic, with some contemporaneous fossiliferous limestones and tuffs of early Miocene age, resting on a low platform of ancient schistose rocks, which form the northern portion of the largest island. Consequently these islands were formed in the Miocene period.

Campbell Island is also volcanic, on a basis of chalky limestone and tuffaceous beds, from which a few fossils have been obtained. The evidence is not sufficient to form any decided opinion, but the fossiliferous beds appear to be Oligocene in age.

Antipodes Island is formed largely of stratified volcanic tuffs, and the island appears to be a submarine volcano which has been subsequently elevated.

The geological structure of Macquarie Island is unknown to me.

The faunas of the Kermadecs, Antipodes, Snares, and Macquarie Island are very imperfectly known, except the birds. The only land animal on Bounty Islands is Ischyroplectron isolatum, an orthopterous insect, which was found by Captain Fairchild when turning over large stones to build the provision depot. The Bounty Islands have no vegetation except sea-weeds.

A Shining Cuckoo (Chalcococcyx), in an exhausted state, was 
once seen on Macquarie Island; * and formerly it was inhabited by rails and parrakeets. The IVood-hen (Ocydromus) now found on it was introduced by the Maoris for food.

The Chatham and Auckland Islands have fresh-water speries of Galaxias, which do not appear to go down to the sea, and are similar to those of New Zealand. Pitt Island, one of the Chatham group, has a lizard closely related to Lygosoma moko of New Zealand. Both the Chatham and Auckland Islands have small forest birds, as well as slugs and beetles, none of which could cross over a sea barrier. IWe may therefore suppose that they were once connected with New Zealand, and derived the main part of their faunas overland.

Much the same may be said for the Snares; but the case of Campbell Island is different. Here we have a Galaxias and a small land shell, but no resident land birds nor beetles. Three Godwit were shot in 1894 by Mr. Borchgrevink's exploring party; and the little Zosterops has made its way there, but there are no parrakeets nor larks. If, however, the presence of earth-worms is a proof of a former land connection with some larger mass, then at one time New Zealand must have extended through Campbell Island to Macquarie Island.

The number of endemic species, and even genera, among the land animals of these islands proves that they have been long separated, and altogether precludes the idea of a comparatively recent Antarctic continent connected with New Zealand. They also preclude the idea of a very cold climate having existed in the southern hemisphere since the islands were separated from New Zealand, that is since the Pliocene period. For this would have killed off most of the inhabitants.

* Campbell's Nests and Eggs of Australian Birds, p. 580. 


\section{BIBIIOGRAPHY.}

I 5 53. Sir ЈоSерн Ноокеr. Flora Nove Zealandice (Introduction).

I 857 . L. Ruetineyer. Über die Herkunft unserer Thieratelt.

¿870. T. H. Huxley. Presidential Address to the Geological Society of London. Q.J.G.S., vol. xxvi.

I 873 . F. IV. Hutton. 'On the Geographical Relations of the New Zealand Fauna.' Trans. N. Z. I., v. p. 227 ; and Ann. Mag. Nat. Hist., ser. 4, vol. xiii. p. 25.

I874. A. Milne-Ėdwards. 'Recherches sur la Faune Ancienne des Iles Mascareignes.' Ann. Sci. Nat., ser. 5, vol. xix. p. I.

I 375. T. Gill. 'On the Geographical Distribution of Fishes.' Ann. Mag. Nat. Hist., ser. 4, vol. xv. p. 25 I.

I876. A. R. IVAllace. The Geographical Distribution of Animals, vol. 1 .

H. N. Mosely. ' Botany of the Antarctic Islands.' Jour. Linn. Soc. Botany, vol. xv. p. 485 .

I880. A. R. Wallace. Island Life, p. 455.

I882. E. Blanchard. 'Proofs of the Subsidence of a Southern Continent during recent Geological Epochs.' N.Z. Jour. Sci, vol. i. p. 25 I (abstract from the Comptes Rendus).

H. F1Lhol. 'Geological and Zoological Relations of Campbell Island with the neighbouring Southern Lands.' N. Z. Jour. Sci., vol. i. p. 259.

I $88_{4}$. F. IV. HutTon. 'On the Origin of the Fauna and Flora of New Zealand.' Part I. N. Z. Jour. Sci., vol. ii. p. I ; and Ann. Mag. Nat. Hist., ser. 5, vol xiii. p. 425 .

I885. F. W. Hutton 'On the Origin of the Fauna and Flora of New Zealand.' Part II. N. Z. Jour. Sci., vol. ii. p. 249 ; and Ann. Mag. Nat. Hist., ser. 5, vol. xv. p. 77.

I890. W. T. BLANFord. Presidential Address to the Geological Society of Lordon. "Q. J G. S., vol. xlvi. p. 99.

1892. C. Hedley. 'The Range of Placostylus.' Pro. Linn. Soc. $N$. S. Wales, ser. 2, vol. xii. p. 335. 
I 892. H. v. JHERING. 'On the Ancient Relations between New Zealand and South America.' Trans. N. Z. I., vol. xxiv. p. 43 I.

I893. T. Gill. 'A Comparison of Antipodal Faunas.' Mem. Nat. Acad. of Sci. Washington, vol. vi. p. 91.

H. O. Forbes. 'The Chatham Islands.' Supp. Papers, Roy. Geographical Soc., vol. iii. p. 607.

H. O. Forbes. 'Antarctica.' Natural Science, vol iii. p. 54; and Fortnightly Review for May 1893.

C. Hedley. 'On the Relation of the Fauna and Flora of Australia to those of New Zealand.' Natural Science, vol. iii. p. 187 .

1895. C. Hedley. 'Considerations on the Surviving Refugees in Austral Lands of Ancient Antarctic Life.' Pro. Roy. Soc. N. S. Wales, vol. xxix. p. 278.

I 896. R. LydeKKer. Geographical Distribution of Mammals.

F. W. Hutton. 'Theoretical Explanations of the Distribution of Southern Faunas.' Pro. Linn. Soc. N. S. Wales, vol. xxi. p. 36 .

H. Deane. 'President's Address to the Linn. Soc. N. S. Wales,' vol. xxi. p. 82 I.

I897. A. Heilprin. 'Les Régions Antarctiques.' Rev. Scient. vol. vii. p. 329 .

I898. D'Arcy W. Thomson. 'On Supposed Resemblances between Arctic and Antarctic Faunas.' Pro. Roy. Soc. Edin., vol. xxii. p. 3 I 1 .

I 899. G. Pfeffer. 'Ueber die gegenseitigen Beziehungen der arktischen und antarktischen Fauna.' Verh. Deutsch. Zool. Ges., vol. ix. p. 266. See also Ann. Mag. Nat. Hist., ser. 7, vol. vii. p. 301 , 'On the Mutual Relations of the Arctic and Antarctic Faunas.'

A. E. Ortmann. 'G. Pfeffer und die Bipolaritat.' Zool. Anz., vol. xxii. p. $2 \mathrm{I} 4$.

A. E. Ortmann. 'On some New Facts lately presented in Opposition to the Hypothesis of Bipolarity of Marine Faunas. Amer. Naturalist, vol. xxxiii. p. $5^{8} 3$.

C. Hedley, 'A Zoo-geographical Scheme for the MidPacific.' Pro. Linn. Soc. N. S. Wales, vol. xxiv. p. 39 I. 
1900. H. F. Osborn. 'The Geological and Faunal Relations of Europe and America during the Tertiary Period.' Science, n. s. vol. xi. p. 561 .

H. A. Pilsbry. 'Genesis of Mid-Pacific Faunas.' Pro. Acad. Nat. Sci. Phil., 1900, p. 568.

I90I. A. E. Ortmann. "The Theories of the Origin of the Antarctic Faunas and Floras.' Amer. Nat., vol. xxxv. p. I39.

Miss E. Pratt. 'Bipolar Theory.' Manchester Memoirs, vol. xlv. no. 14.

1902. A. E. ORtmann. Report on the Tertiary Invertebrates of Patagonia, p. I 10.

A. Dendy. 'The Chatham Islands.' Manchester Memoirs, vol, xlvi. no. I 2.

IV. B. Benнaм. 'Earth-worms and Palæogeography.' Report Aust. Ass. Adv. Science, Hobart, vol. ix.

E. A. Smith. 'On the Supposed Similarity between the Mollusca of the Arctic and Antarctic Regions.' Presidential Address to the Malacological Society of London.

C. F. ANCEY. 'Considérations sur les faunes macalogiques des parties australes du globe.' Jour. de Conch., xlix. 12.

H. Suter. 'Observations on M. Ancey's Paper.' Jour. de Conch., xlix. 3 I6. 



\section{PHYLUM, CHORDATA}

CLASS, MAMMALIA.

Sub-class Eutheria.

Order Chiroptera.

Family VeSPERTILIONIDE.

Chalinolobus, Peters, 1866.

morio, Gray. Dobson, A.M.N.H. 6, iv. 62. C. tuberculatus,

Cat. Chi. B.M. 248 ; Tomes, P.Z.S. I $857,1.35$.

Family Embai.lonuride.

Mystacops, Flower, I891.

tuberculatus, Gray. Dobson, Cat. Chi. B.M. $44^{2}$ (Mystacina); Tomes, P.Z.S. 1857,138 .

Order CaRnivora.

Sub-order Pinnipedia.

Family OTARIIDE.

Eumetopias, Gill, 1866.

hookeri, Gray. Clark, P.Z.S. 1873,765 . Challenger Reports, xxvi. 78 .

Arctocephalus, F. Cuvier, 1824.

forsteri, Lesson. Clark, P.Z.S. 1875,650 , and $1884,196$.

Challenger Reports, xxvi. 87. A. cinereus, Hector, T.N.Z.I. iv. 196.

Family РносіDж.

Ogmorhinus, Peters, i 875 .

leptonyx, Blainville. Challenger Reports, xxvi. 64 .

Macrorhinus, F. Cuvier, 1824.

leoninus, Linnæus. Flower, P.Z.S. IS81, 146. Challenger Reports, xxvi. 69.

Order CETACFa.

Sub-order Mystacoceti.

Family BaLÆNIDE.

Balæna, Linnæus, r 766.

australis, Gray. Hector, T.N.Z.I. x. 3.35: Challenger

Reports, I. 32 ; P.Z.S. I 90 I, P. 44. 
Neobalæna, Gray, i8 7 I.

marginata, Gray. Hector, T.N.Z.I. x. 331; Beddard, T.Z.S. xvi. 87 .

Megaptera, Gray, I 846 .

longimana, Rudolphi. Challenger Reports, i. 3o. M. lalandii, Hector, T.N.Z.I. x. 335 .

Balænoptera, Lacépède, 1804.

musculus, Linnæus. B. sibbaldi, Hutton, T.N.Z.I. vii. 266. physalus, Linnæus. $\quad P$. australis, Hector, T.N.Z.I. x. 336 ; B. musculus, Parker, T.N.Z.I. xvii. 3 .

rostrata, Fabricius. Haast, T.N.Z.I. xiii. 169; Benham, P.Z.S. I 901,$280 ;$ B. huttoni, Hector, T.N.Z.I. x. 337 ; Benham, T.N.Z.I. xxxiv. I 51 .

Sub-order ODONTOCETI.

Family PhYSETERIDE.

Sub-family PhYSETERINÆ.

Physeter, Linnæus, I 766. macrocephalus, Linnæus. Hector, T.N.Z.I. x. 337 .

Cogia, Gray, I 846 .

breviceps, Blainville. Hector, T.N.Z.I. x. 337 ; Benham, T.N.Z.I. xxxiv. I 55 ; and P.Z.S. 1902, 54.

Sub-family ZipHIIN\%.。

Ziphius, F. Cuvier, 1823 .

cavirostris, Cuvier. Hector, T.N.Z.I. x. 342 ; Challenger Reports, i. 27 ; Parker, P.Z.S. $1887,342$.

Mesoplodon. Gervais, 1850.

layardi, Gray. Hector, T.N.Z.I. x. 341 ; Haast, P.Z.S. I 876,478 ; C. guntheri, Gray, A.M.N.H. 4 , vii. 368 .

hectori, Gray. Hector, T.N.Z.I. x. 339.

grayi, Haast. P.Z.S. 1876,7 and 457 ; T.Z.N.I. ix. $45^{\circ}$. australis, Flower. T.Z.S. x. 4 I9 $; B$. hectori, Hector,T.Z.N.I. vii. 262 .

haasti, Flower. T.Z.S. x. 4 I 9.

Berardius, Duvernoy, $185 \mathrm{I}$ arnouxi, Duvernoy. Hector, T.N.Z.I. x. $33^{8}$.

Delphinapterus, Lacépède, 1804.

Family DELPHINIDE. leucas, Pallas. Hector, T.N.Z.I. xvii. 209.

Cephalorhynchus, Gray, $185^{\circ}$.

hectori, Beneden. Hector, T.N.Z.I. xvii. 209 ; Flower, P.Z.S. 1883,476 . E. clancula, Hector, T.N.Z.I. ix. 350. 
Orca, Gray, 1846.

gladiator, Gray. Hector, T.N.Z.I. xvii. 208.

Pseudorca, Reinhart, I862.

crassidens, Owen. Hector, T.N.Z.I. xvii. 209.

Globicephalus, Lesson, 1842 .

melas, Traill. Hector, T.N.Z.I. xvii. 209.

Grampus, Gray, I 846 .

griseus, Lesson. G. richardsoni, Hector, T.N.Z.I. xvii. 209.

Delphinus, Linnæus, I 766 .

delphis, Linnæus Hector, T.N.Z.I. xvii. 2 10; Flower, T.Z.S. xi. I ; D. novce zealandice, Hutton, T.N.Z.I. ix. 349 .

Tursiops, Gervais, 1855 .

tursio, Buonaterre. Hector, T.N.Z.I. xvii. 210 ; Flower, T.Z.S. xi. I ; and P.Z.S. I 883. $478 ; T$. metis, Hutton, A.M.N.H. 4, xvi. 357 .

Prodelphinus, Gervais, 1880

obscurus, Gray. Flower, P.Z.S. I 883, 495; Hutton. T.N.Z.I. ix. 349; Hector, T.N.Z.I. xvii. 2 I I.

euphrosyne, Gray. Flower, P.Z.S. 1883, 497 ; Hector, T.N.Z.I. xvii. 2 II.

Glaucopis, Gmelin, I 788 .

CLASS, AVES.

Sub-class Carinate.

Order Passeres.

Sub-order ACROMYODI.

Family CORviDE.

cinerea, Latham. C.B.M. iii. 142 ; Buller, 'Birds of New Zealand,' i. 5 .

wilsoni, Bonaparte. C.B.M. iii. I43; Buller, i. I.

Family STURNide.

Heteralocha, Cabanis, I 851 .

acutirostris, Gould. C.B.M. iii. 143 ; Buller, i. 7 .

Creadion, Vieillot, i 8 I 6 .

carunculatus, Gmelin. C.B. M. iii. I 44 ; Buller, i. 18 and 2 I.

Turnagra, Lesson, $183 \mathrm{I}$.

Family TURDIDE.

crassirostris, Gmelin. C.B.M. vii. 4 ; Buller, i. 3 I.

tanagra, Schlegel. C.B.M. vii. 5 ; Buller, i. 26. 


\section{Family Sylviade:}

Sphenœacus, Strickland, I84I.

punctatus, Quoy and Gaimard. C.B.M. vii. 97 ; Buller,i. 60.

fulvus, Gray. C.B.M. vii. 98 ; Buller, i. $6 \mathbf{1}$.

rufescens, Buller. C.B.M. vii. 98 ; Buller, i. 62 .

Family MUSCICAPIDE.

Petroica, Swainson, I 832 .

macrocephala, Gmelin. C.B.M. iv. I 76 ; Buller, i. 42.

toitoi, Garnot. C.B.M. iv. I 74; Buller, i. 39.

Miro, Lesson, I831.

albifrons, Gmelin. C.B.M. iv. 235 ; Buller, i. 36.

australis, Sparrmann. C.B.M. iv 235 ; Buller, i. 33.

traversi, Buller. C. B.M. iv. 236 ; Buller, i. 38 .

dannefordi, Rothschild. Nov. Zool. i. 688 (1894).

Pseudogerygone, Sharpe, 1878.

igata, Quoy and Gaim. C.B.M. iv. 2 I 8 ; Buller, i. 44 and 50. albofrontata, Gray. C.B.M. iv. 222 ; Buller, i. 49.

Rhipıdura, Vigors and Horsfield, i $82 \%$.

flabellifera, Gmelin. C.B.M. iv. 308 ; Buller, i 69 .

fuliginosa, Sparmann. C. B. M. iv. 316 ; Buller, i. 72.

Family PARIDE.

Certhiparus, De Lafresnaye, I 842.

albicillus, Lesson. C.B.M. viii. 75 ; Buller, i. 53.

novæ-zealandiæ. Gmelin. C.B.M. viii. 76 ; Buller, i. 5 I.

Mahua, Lesson, i 837 .

ochrocephala, Gmelin. C.B.MI. viii. 76 ; Buller, i. $5^{6 .}$

Pogonornis, Gray, I 846 .

Family Meliphagidæ.

cincta, Dubus. C.B.M. ix. 25 I ; Buller, i. IоI.

Anthornis, Gray, I $84 \mathrm{I}$.

melanura, Sparrmann. C.B.M. ix. 255 ; Buller, i. 85 .

melanocephala, Gray. C. B. M. ix. 256 ; Buller, i. 92.

Prosthemadera, Gray, i 840.

novæ-zealandiæ, Gmelin. C.B.M. ix: 257 ; Buller, i. 94.

Family Zosteropide.

Zosterops, Vigors and Horsfield, 1827 .

cærulescens, Latham. C.B.M. ix. $\mathbf{5}_{52}^{2}$; Buller, i. 77 .

Anthus, Bechstein, I 807 .

Family MotaCilluU:.

novæ-zealandiæ, Gmelin. C.B.M. x. 6r6; Buller, i. 64 .

aucklandicus, Gray. C.B.M. x. 6 r 7 .

steindachneri, Reischek. T.N.Z.I. xxi. 388 . 
Sub-order MEsomyodi.

Family Xenicide.

Acanthidositta, De Lafresnaye, 1842.

chloris, Sparrmann. C.B.M. xiv. 45 I ; Buller, i. I I3.

Xenicus, Gray, I 855 .

longipes, Gmelin. C.B.M. xiv. $45^{2}$; Buller, i. 108.

gilviventris, Pelzeln. C.B.M. xiv. 453 ; Buller, i. I I r.

Traversia, Rothschild, i $\$ 94$.

lyalli. Rothschild. Bull. Brit. Ornith. Club, iv. no. xxii. p. x. ;

X. insularis, Buller, Ibis, I 895,236 ; T.N.Z.I. xxviii. $34 \mathrm{I}$.

\section{Order Picarie.}

Halcyon, Swainson, I82 I.

\section{Family ALCEDINIDE.}

vagans. Lesson. C.B.M. xvii. 27 I ; Buller, i. 122.

Family Cuculidæ.

Chalcococcyx, Cabanis, I 863 .
lucidus, Gmelin.
C.B.M. xix. 295 ; Buller, i. I 32.

Urodynamis, Salvadori, I880.

taitensis, Sparrmann. C.B.M. xix. 314 ; Buller, i. 27.

Order Striges.

Family BuBONIDÆ.

Ninox, Hodgson, 1837 .

novæ-zealandiæ, Gmelin. C B.M. ii. I73; Buller, i. 192.

Sceloglaux, Kaup, i 848 .

albifacies, Gray. C.B.M. ii. 187 ; Buller, i. 198.

Order Psittaci.

Family Nestoride.

Nestor, Wagler, 1832.

notabilis, Gould. C.B.M. xx. 4 ; Buller, i. I66.

meridionalis, Gmelin. C.B.M. xx. 5 ; Buller, i. 150.

Family Psittacide.

Sub-family Platycercine.

Cyanorhamphus, Bonaparte, I 854 .

unicolor, Vigors. C.B.M. xx. 581 ; Buller, i. $\mathbf{1} 48$.

novæ-zealandiæ, Sparmann. C.B.M. xx. 58I ; Buller, i.

I 37 .

aucklandicus, Bonaparte. C.B.M. xx. $5^{84}$.

erythrotis. Wagler. C.B.M. xx. $5^{86}$.

cyanurus, Salvadori. C.B.M. xx. 587 .

auriceps, Kuhl. C.B.M. xx 587 ; Buller, i. 142.

malherbei, Souancé. C.B.M. xx. 590; Buller, i. 146 (alpinus). 
Family STRINGOPIDE.

Stringops, Gray, I 845 .

habroptilus, Gray. C.B.M. xx. 599 ; Buller, i. 176.

Order ACcipitres.

Family FALCONIDE.

Sub-family FaLCONinÆ.

Nesierax, Oberholsen, i 899.

novæ-zealandiæ, Gmelin. C.B.M. i. 372 ; Buller, i. 2 I 3. australis, Hombron and Jacquinot. C.B.M. i. 373 ; Buller, i. 220 .

Sub-family ACCIPITRINÆ.

Circus, Lacépède, r 806.

gouldi, Bonaparte. C.B.M. i. 72 ; Buller, i. 206.

\section{Order Gallinæ.}

Family Phasianide.

Coturnix, Moehring, $175^{2}$.

novæ-zealandiæ, Gmelin. C.B.M. xxii. 245 ; Buller, i. 225.

Order COLumbe.

Family Treronide.

Sub-family CARPOPHAGINE.

Hemiphaga, Bonaparte, I 854.

novæ-zealandiæ, Gmelin. C.B.M. xxi. 236 ; Buller, i. 229. chathamensis, Rothschild. P.Z.S. I89r, p. 312 ; C.B.M.

xxi. 239.

Order Gralle.

Family RAI.LID E.

Hypotænidia, Reichenbach, I 852.

philippensis, Linnæus. C.B.M. xxiii. 39 ; Buller, ii. 95. macquariensis, Hutton, Ibis, 1879 ; C.B.M. xxiii. 43 . muelleri, Rothschild. Bull. Brit. Orn. Club, I893; T.N.Z.I. xxvi. I80; C.B.M. xxiii. 330 ; $R$. brachypus, Buller, ii. 100.

Nesolimnas, Andrews, 1896 .

dieffenbachii, Gray. C.B.M. xxiii. 47 ; Buller, ii. 12 r.

Cabalus, Hutton, 1873 .

modestus, Hutton, Ibis, 1872 ; C.B.M. xxiii. 331 , plate 6.

Ocydromus, Wagler, I 830 .

earli, Gray. Buller, ii. I 5 ; O. greyi, Buller, ii. I05.

australis, Sparrmann. C.B.M. xxiii. 64 (part); Buller, ii. I 16.

hectori, Hutton, T.N.Z.I. vi. I Iо; O. brachypterus, Buller, ii. I 19 .

brachypterus, Lafresnaye. C.B.M. xxiii. $67 ; 0$. fuscus, Buller, ii. I I 2. 
Porzana, Vieillot, 18 r 6 .

affinis, Gray. C.B.M. xxiii. 106 : Buller, ii. 103.

tabuensis, Gmelin. C.B.M. xxiii. I I I Buller, ii. ror.

Porphyrio, Brisson, 1760 .

melanonotus, Temminck. C.B.M. xxiii. 205 ; Buller, ii. 79. chathamensis, Sharpe, Ibis, I893; C.B.M. xxiii. 202.

Notornis, Owen, I 848 .

hochstetteri, Meyer. Benham, T.N.Z.I. xxxi. 146; $N$. mantelli, C.B.M. xxiii. 208 ; Buller, ii. 85 .

Order Herodiones.

Herodias, Boie, 1822 .

$$
\text { Family ARDEIDE. }
$$

timoriensis, Cuvier. C.B.M. xxvi. $98 ;$ A. egretta, Buller, ii. 124.

Notophoyx, Sharpe, r 898 .

novæ-hollandiæ, Latham. C.B.M. xxvi. 109 ; Buller, ii. I 34 .

Demiegretta, Blyth, I846.

sacra, Gmelin. C.B.M. xxvi. I 37 ; Buller, ii. I 28.

Nycticorax, Rafinesque, 1815 .

caledonicus, Gmelin. C.B.M. xxvi. 158 ; Buller, ii. I 39.

Ardetta, Grey, 1842.

pusilla, Vieillot. C.B.M. xxvi. 234; A. masulata, Buller, ii. I 36 .

Botaurus, Brisson, 1760 .

pœciloptilus, Wagler. C.B.M. xxvi. 258 ; Buller, ii. 142.

Order LimicolÆ

Family CHARADRIIDÆ.

Sub-family ARENARIINE.

Arenaria, Brisson, i 760 .

interpres, Linnæus. C.B.M. xxiv. 92 ; Buller, ii. I4.

Sub-family HæMATOPODINÆ.

Hæmatopus, Linnæus, I 766.

longirostris, Vieillot. C.B.M. xxiv. I r2; Buller, ii. 16 . unicolor, Wagler. C.B.M. xxiv. I 8 ; Buller, ii. 18.

Sub-family CharadriIN

Charadrius, Linnæus, i 766.

dominicus, Müller. C.B.M. xxiv. 196 ; C. fulvus, Buller, ii. 6 . 
Ochthodromus, Reichenbach, I 852. obscurus, Gmelin. C.B.M. xxiv. 2 I I B Buller, ii. I. bicinctus, Jardine and Selby. C.B.M. xxiv. 2 I 2 ; Buller, ii. 3.

Thinornis, Gray, 1846.

novæ.zealandiæ, Gmelin. C.B.M. xxiv. 304 ; Buller, ii. I I. rossi, Gray. C.B.M. xxiv. 305.

Anarhynchus, Quoy and Gaimard, 1830.

frontalis, Quoy and Gaimard. C.B.M. xxiv. 306 ; Buller, ii. 9 .

Sub-family Himantopodinæ.

Himantopus, Brisson, 1760.

picatus, Ellman. C.B.M. xxiv. 319: H. leucocephalus, Buller, ii. $2 \mathbf{I}$, and $H$. albicollis, Buller, ii. 27.

melas, Hombron and Jacquinot. C.B.M.xxiv. $323 ; H$. novezealandia, Buller, ii. 24.

Recurvirostra, Linnæus, I 766 .

novæ-hollandiæ, Vieillot. C.B.M. xxiv. 333; Buller, ii. 20.

Sub-family TOTANide.

Limosa, Brisson, I 760 .

novæ-zealandiæ, Gray. C.B.M. xxiv. 377 ; Buller, ii. 40.

Sub-family Scolopacinæ.

Limonites, Kaup, I 829 . ruficollis, Pallas. C.B.M. xxiv. 545 .

Heteropygia, Coues, I86I.

acuminata, Horsfield. C.B.M. xxiv. 566 ; Buller, ii. 37.

Tringa, Linnæus, I 766 .

canutus, Linnæus. C.B.M. xxiv. 593 ; Buller, ii. 35.

Gallinago, Leach, i 8 I 6 .

aucklandica, Gray. C.B.M. xxiv. 66I ; Buller, ii. 32. huegeli, Tristram. C.B.M. xxiv. 663 .

pusilla, Buller. C.B.M. xxiv. 663 ; Buller, ii. 33 .

Order Gaviæ.

Family LARIDE.

Sub-family STERnine.

Hydroprogne, Kaup, I829. caspia, Pallas. C.B.M. xxv. 32 ; Buller, ii. 73.

Sterna, Linnæus, I 766.

albistriata, Gray, C.B.M. xxv. 48; Buller, ii. 70. vittata, Gmelin. C.B.M. xxv. 5 I and 456.

frontalis, Gray. C.B.M. xxv. 97 ; Buller, ii. 68. fuliginosa, Gmelin. C.B.M. xxv. 106 and 457. nereis, Gould. C.B.M. xxv. II 2 ; Buller, ii. 75 . 
Procelsterna, Lafresnaye, I 842 .

cinerea, Gould. C.B.M. xxv. I 35 ; Buller, ii. 78 .

Micranous, Saunders, 1895 .

leucocapillus, Gould. C.B.M. xxv. 145; A. melanogenys, Cheeseman; T.N.Z.I. xxiii. 22 I.

Gygis, Wagler, I 832 .

candida, Gmelin. C. B.M. xxv. I49.

Larus, Linnæus, 766.

Sub-family LARINÆ.

bulleri, Hutton. C.B.M. xxv. 233 ; Buller, ii. 58 .

scopulinus, Gray. C.B.M. xxv. $23^{8}$; Buller, ii. 56 .

dominicanus, Lichtenstein. C.B.M. xxv. 245 ; Buller, ii. 47 .

Family StercorariIDE.

Megalestris, Bonaparte, 1856.

antarctica, Lesson. C.B.M. xxv. 3 I9; Buller, ii. 63 .

Stercorarius, Brisson, I 760.

crepidatus, Gmelin, C.B.M. xxv. 327 ; Buller, ii. 66 .

Order Steganopodes.

Family Phä̈thontide.

Phaëthon, Linnæus, I 766.

rubricauda, Bodd. C.B.M. xxvi. 45 I ; Buller, ii. 186.

Family FreGatide.

Fregata, Brisson, 1760 .

aquila, Linnæus. C.B.M. xxvi. 443 : Buller, ii. I82.

Sula, Brisson, 1760.

Family SULıDÆ.

serrator, Gray. C.B.M. xxvi. 428 ; Buller, ii. I 77.

cyanops, Sundervall C.B.M. xxvi. 430; Cheeseman,

T.N.Z.I. xxii. 223.

Family Phalacrocoracide.

Phalacrocorax, Brisson, i 760.

carbo, Linnæus. C.B.M. xxvi. $340 ; P$. nove hollandia, Buller, ii. I 45,

varius, Gmelin. C.B.M xxvi. 394 ; Buller, ii. I 49. sulcirostris, Brandt. C.B M. xxvi. 376 .

melanoleucus, Vieillot. C.B.M. xxvi. 398 ; Buller, ii. 173. brevirostris, Gould. C.B.M. xxvi. 400 ; Buller, ii. I68. punctatus, Sparrmann. C.B.M. xxvi. 354 ; Buller, ii. I64. featherstoni, Buller. C B.M. xxvi. 356 ; Buller, ii. I 66 . chalconotus, Gray C.B.M. xxvi. 369 ; Buller, ii. 162 , and P. glaucus, $\mathrm{x}_{3} 3$. 
Phalacrocorax, campbelli, Filhol. C.B.M.xxvi. ${ }^{\delta} \delta_{7} ;$ P.nycthemerus, Buller, ii. 167.

colensoi, Buller. C.B.M. xxvi. 386 ; Buller, ii. 16 r.

stewarti, Ogilvie-Grant. C.B.M. xxvi. 385 .

ranfurlyi, Ogilvie-Grant. Bull. B. O. Club, xi. (r901).

carunculatus, Gmelin. C.B.M. xxvi. 384 ; Buller, ii. 155 ; $P$. huttoni, Buller, ii. I 74 .

onslowi, Forbes. C.B.M. xxvi. $385 ; P$. imperialis, Buller, ii. 153 .

traversi, Rothschild. Bull. Brit. Ornith. Club, viii. (1898).

Order Tubinares.

Family Procellarilde.

Sub-family OCEANiTinÆ.

Oceanites, Keyserling and Blasius, 1840 . oceanicus, Kuhl. C.B.M. xxv. $35^{8}$; Buller, ii. $25^{\circ}$.

Garrodia, Forbes, I 88 I.

nereis, Gould. C.B.M. xxv. $36 \mathrm{I}$; Buller, ii. 247 .

Pelagodroma, Reichenbach, I 852 .

marina, Latham. C.B.M. xxv. 362 ; Buller, ii. 248.

Cymodroma, Ridgway, 1884.

melanogaster, Gould. C.B.M. xxv. 364 ; Buller, ii. 249.

Family PUfFinide.

Puffinus, Brisson, 1760 .

\section{Sub family Puffininte.}

bulleri, Salvin. C.B.M. xxv. 371 ; Buller, ii 240.

chlororhynchus, Lesson. C.B.M. xxv. 372 ; Buller, ii. 235. gavia, Forster. C.B.M. xxv. 381 ; Buller, ii. 236.

assimilis, Gould. C.B.M. xxv. 384 ; Buller, ii. 239. carneipes, Gould. C.B.M. xxv. 385 ; Buller, ii. 234. griseus, Gmelin. C.B.M. xxv. 386 ; Buller, ii. 232. tenuirostris, Temminck. C.B.M. xxv. 388 ; Buller, ii. 230.

Priofinus, Hombron and Jacquinot, $\mathrm{r} 844$. cinereus, Gmelin. C.B.M. xxv. 390 ; Buller, ii. 241 .

Priocella, Hombron and Jacquinot, i 844 . glacialoides, Smith. C.B.M. xxv. 393 ; Buller, ii. 228 .

Majaqueus, Reichenbach, r 864. æquinoctialis, Linnæus. C.B.M. xxv. 395 .

parkinsoni, Gray. C.B.M. xxv. 397 ; Buller, ii. 242.

Estrelata, Bonaparte, I 855 macroptera, Smith. C.B.M. xxv. 399; Buller, ii. 245. lessoni, Garnot. C.B.M. xxv. 401 ; Buller ii. 2 I 9 . nigripennis, Rothschild. C.B.M. xxv. 409. 
CEstrelata cervicalis, Salvin. C.B.M. xxv. 4 I I.

neglecta, Schlegel. C.B.M. xxv. 412 ; Buller, ii. 224.

gularis, Peale. C.B.M. xxv. 414; C. affinis, Buller, ii. 223. axillaris, Salvin. C.B.M. xxv. 4 I 8 .

cooki, Gray. C.B.M. xxv. 4I 7 ; Buller, ii. 217.

Sub-family Fulmarine.

Ossifraga, Hombron and Jacquinot, 1844.

gigantea, Gmelin. C.B.M. xxv. 422 ; Buller, ii. 225.

Daption, Stephens, 1826.

capensis, Linnæus. C.B.M. xxv. 428 ; Buller, ii. 2 r5.

Halobæna, Geoff. St. Hilaire, 1856 .

cærulea, Gmelin. C.B.M. xxv. 431 ; Buller, ii. 2 I 4.

Prion, Lacépède, I 80 I.

vittatus, Gmelin. C.B.M. xxv. 432 ; Buller, ii. 2 I I.

banksi, Gould. C.B.M. xxv. 434 ; Buller, ii. 2 I 0.

desolatus, Gmelin. C.B.M. xxv. 434 ; P. turtur, Buller, ii. 209.

ariel, Gould. C.B.M. xxv. 436 ; Buller, ii. 212.

Pelecanoides, Lacépède, I 801 .

Family Pelecanoididæ.

urinatrix, Gmelin. C.B.M. xxv. 437 ; Buller, ii. 207.

exsul, Salvin. C.B.M. xxv. 43 I.

Diomedea, Linnæus, I 766 .

Family Diomedeide.

regia, Buller. C.B.M. xxv. 443 .

exulans, Linnæus. C.B.M. xxv. 44 I ; Buller, ii. I 89. melanophrys, Boie. C.B.M. xxv. 447 ; Buller, ii. 198.

bulleri, Rothschild. C.B.M. xxv. 448 .

Thalassogeron, Ridgway; I 884 .

salvini, Rothschild. C.B.M. xxv. $45^{\circ} ;$ D. cauta, Buller, ii. 203.

culminatus, Gould. C B.M. xxv. $45 \mathrm{I}$; Buller, ii. 20 r.

chlororhynchus, Gmelin. C.B.M. xxv. 45I; Buller, ii. 202.

Phœbetria, Reichenbach, 1852.

fuliginosa, Gmelin. C.B.M. xxv. 453 ; Buller, ii. 205.

Order IMPENNES.

Family SPHeniscide.

Aptenodytes, Forster, I 78 I.

patagonica, Forster. C.B.M. xxvi. 627 ; A. longirostris, Buller, ii. 306 . 
Pygoscelis, Wagler, 1832.

papua, Forster. C.B.M. xxvi. 63I ; P.taniatus, Buller, ii. 304 .

Catarrhactes, Brisson, I 760 .

chrysocome, Forster. C.B.M. xxvi. 635 ; Buller, ii. 290. pachyrhynchus, Gray. C.B.M. xxvi. 638 ; Buller, ij. 287 . sclateri, Buller. C.B.M. xxvi. 640 ; Buller, ii. 289 .

schlegeli, Finsch. C.B.M. xxvi. 643; E. chrysolophus, Buller, ii. 297 and 298.

Megadyptes, Milne-Edwards, I880.

antipodum, Homb. and Jacq. C.B.M. xxvi. 644 ; Buller, ii. 294.

Eudyptula, Bonaparte, 1856 .

minor, Forster. C.B.M. xxvi. 646 ; Buller, ii. 300 and 302 . albosignata, Finsch. C.B.M. xxvi. 648 .

Order Pygopodes.

Family POD1C1PEDIDE.

Podicipes, Latham, 1787 .

cristatus, Linnæus. C.B.M. xxvi. 544 ; Buller, ii. 283.

rufipectus, Gray. C.B.M. xxvi. 523 ; Buller, ii. 280 .

Order ANSERES.

Family ANATIDE.

Sub-family Anatine.

Casarca, Bonaparte, 1838 .

variegata, Gmelin. C.B.M. xxvii. 183 ; Buller, ii. 264.

Anas, Linnæus, I 766 .

superciliosa, Gmelin. C.B.M. xxvii. 206 ; Buller, ii. 25 I.

Nettion, Kaup, 1829.

castaneum, Eyton. C.B.M. xxvii. 252.

gibberifrons, S. Müller. C.B.M. xxvii. 254 ; Buller, ii. 26 I.

Elasmonetta, Salvadori, I 895 .

chlorotis, Gray. C.B.M. xxvii. 287 ; Buller, ii. 257.

Nesonetta, Gray, I 844 .

aucklandica, Gray. C.B.M. xxvii. 289 ; Buller, ii. 263.

Spatula, Boie, 1822 .

rhynchotis, Latham. C.B.M. xxvii. 314; Rhynchaspis variegata, Buller, ii. 269 .

Sub-family Fuligulinæ.

Nyroca, Fleming, I 822 .

australis, Eyton. C.B.M. xxvii. 350 ; Buller, ii. 275 . 
Fuligula, Stephens, 1824 .

novæ-zealandiæ, Gmelin. C.B.M. xxvii. ; 368 ; Buller, ii. 273.

Sub-family MerganetTinæ.

Hymenolæmus, Gray, I 843 .

malacorhynchus, Gmelin. C.B.M. xxvii 455 ; Buller, ii. 276.

Sub-family MERGINÆ.

Merganser, Brisson, 1760.

australis, Hombron and Jacquinot. C.B.M. xxvii. 484 ; Buller, ii. 279.

Sub-class RatiTæ.

Order APTERyges.

Family APTERYGIDE.

Apteryx, Shaw, i8r3.

australis, Shaw. C.B.M. xxvii. 604 ; Buller, ii. 322 ; Parker,

Trans. Phil. Soc. I $891, \mathrm{~B}, 25$. A. lawryi, Rothschild;

C.B.M. xxvii. 606 .

mantelli, Bartlett. C.B.M. xxvii. 607. A. bulleri, Buller, ii. 308 .

oweni, Gould. C.B.M. xxvii. 609 ; Buller, ii. 327.

occidentalis, Rothschild. C.B.M. xxvii. 6ro; Novitates

Zoologicæ, vi. 384 , pl. 12.

haasti, Potts. C.B.M. xxvii. 6 I i ; Buller, ii. 330.

\section{APPENDIX TO AVES.}

List of Wanderers which have been found in New Zealand.

Order Passeres.

Family CAM PEPHAGIDE.

Graucalus melanops, Latham. C.B.M. iv. 30 ; Buller, i. 66.

Family Meliphagidæ.

Acanthochæra carunculata, Latham. C.B.M. ix. 263; Buller, i. Iс6.

Family HiRundinide.

Petrochelida nigricans, Vieillot. C.B.M. x. r90; Buller, i. 76 .

\section{Order Picarie.}

Family CYPSELIDE.

Micropus pacificus, Blyth. C.B.M.xvi. 448 ; Buller, i. I 7 .

Family Coracilde:

Eurystomus australis, Swainson. C.B.M. xvii. 36. $E$. pacificus, Buller, i. I 8 . 


\section{Order ACCIPITRES}

Family FaLCONIDE.

Cerchneis cenchroides, Vigors and Horsfield. C.B.M. i. $43^{1}$; Buller, T.N.Z.I. xxviii. 359 .

Order Herodiones.

Family Plataleide.

Platalea regia, Gould. C.B.M. xxvi. 47 ; Buller, ii. 144.

Plegadis, Kaup, I829.

Family IBIDE.

falcinellus, Linnæus. C. B.M. xxvi. 29.

Order LIMICOLE.

Family CHARADRIIDE.

Sub-family LobivanelLiNe.

Lobivanellus lobatus, Latham. C.B.M. xxiv. I 39; Buller, ii. 13 .

Sub-family CHARADRIINE.

Egiatilis ruficapilla, Temminck. C.B.M. xxiv. 286; Buller, ii. 5 .

Sub-family Totaninæ.

Limosa hudsonica, Lath. C.B.M. xxiv. 388 .

Numenius cyanopus, Vieillot. C.B.M. xxiv. $35^{\circ}$; Buller, ii. 45 .

Mesoscolopax minutus, Gould. C.B.M. xxiv. 371 ; Hutton, T.N.Z.I. xxxv.

Heteractitis incanus, Gmelin. C.B.M. xxiv. 453; Buller, ii. $3^{8}$.

Glottis nebularius, Gunner. C.B.M. xxiv. 48I ; Hutton, T.N.Z.I. xxxiv. 253.

Gallinago australis, Latham. C.B.M.xxiv. 653 ; Cheeseman, T.N.Z.I. xxxi. I05.

Sub-family Phalaropinæ.

Crymophilus fulicarius, Linnæus. C.B.M. xxiv. 693; Buller, ii. 30.

Order Gaviæ.

Family LARIDE.

Sub-family STERnine.

Hydrochelidon leucoptera, Meisner and Schinz. C.B.M. xxv. 6 ; Buller, ii. 77 .

Order Steganopodes.

Family Fregatide.

Fregata ariel, Gould. C.B.M. xxvi. 447. T. minor, Buller, ii. 185 . 
Family SULide.

Sula sula, Linnæus. C.B.M. xxvi. 436 . S. fusca, Hamilton, T.N.Z.I. xxi. I 28.

Family Phalacrocoracide.

Sub-family Plotine.

Plotus novæ-hollandiæ, Gould. C.B.M. xxvi. 4r 7 ; Buller, ii. 175 .

Order ANSEREs.

Family ANatidæ.

Sub-family ANatine.

Dendrocygna eytoni, Gould. C.B.M. xxvii. 165 ; Buller, ii. 268.

Gehyra, Gray, 1834 .

\author{
CLASS, REPTILIA. \\ Order Squamata. \\ Sub-order LACERTILIA. \\ Family Geckonide.
}

oceanica, Lesson. Boulenger Cat. Lizards in B.M. i. ${ }^{2} 2$.

A specimen from Moko-hinou is in the British Museum.

Naultinus, Gray, 1840 .

grayi, Bell. Lucas and Frost, T.N.Z.I. xxix. 267 (in part); Colenso, T.N.Z.I. xix 147. Variety sulphureus, Buller, T.N.Z.I. iii. 8.

elegans, Gray. Lucas and Frost, T.N.Z.I. xxix. 267 (in part. $\quad N$. pentagonalis, Colenso, T.N.Z.I. xxii. 262.

rudis, Fischer. Lucas and Frost, T.N.Z.I. xxix. 270.

Hoplodactylus, Fitzinger, I 843

maculatus, Gray. Lucas and Frost, T.N.Z.I. xxix. 27 I. pacificus, Gray. Lucas and Frost, T.N.Z.I. xxix. 272. granulatus, Gray. Lucas and Frost, T.N.Z.I. xxix. 273.

Lygosoma, Gray, 1828 .

Family SCINCIDE.

grande, Gray. Lucas and Frost, T.N.Z.I. xxix. 275.

moco, Dumeril and Bibron. Lucas and Frost, T.N.Z.I. xxix. 276.

dendyi, Boulenger. A.M.N.H. 7, ix. 196.

lineo-ocellatum, Dumeril. Lucas and Frost, T.N.Z.I.

xxix. 277.

smithii, Gray. Lucas and Frost, T.N.Z.I. xxix. 277.

æneum, Girard. Lucas and Frost, T.N.Z.I. xxix. 278.

ornatum, Gray. Lucas and Frost, T.N.Z.I. xxix. 279. 
Order RHyNCHOCEPHALIA.

Family HatTERIIDE.

Sphenodon, Gray, i83i.

punctatus, Gray. Cat. Chelonians B.M. 2; Dendy,

Q.J.M.S. xlii. I ; Howes, T.Z.S. xvi. I ; Harrison, Q.J.M.S. xliv. I6r.

\section{APPENDIX TO REPTILIA.}

List of Wanderers which have been found in New Zealand.

Order Squamata.

Sub-order Ophidia.

Family COLUBRIDE.

Platurus colubrinus, Schneider. C.B.M. iii. 308.

Hydrus platurus, Linnæus. C.B.M. iii. 267.

Order Chelonia.

Family SPHARGIDE.

Dermochelys coriacea, Linnæus. C.B.M. Io; Cheeseman, T.N.Z.I. xxv. 108.

CLASS, AMPHIBIA.

Order ANURA.

Sub-order Phaneroglossa.

Family DiscoglossidÆ.

Liopelma, Fitzinger, I86 I.

hochstetteri, Fitzinger. C.B.M. 448 ; T N.Z.I. xii. 250.

CLASS, PISCES.*

Sub-class Teleostoni.

Order Teleostei.

Sub-order ACANTHOPTERI.

Polyprion, Cuvier, I8I 7 .

Family SERRANIDE.

prognathus, Foster. Hutton, T.N Z.I. xxii. 276 ; No. 26.

Gilbertia, Jordan, I89o.

semicincta, Cuvier. C.B.M. and ed. i. 307. Plectropoma huntii, Hector, T.N.Z.I. vii. 240 ; Hutton, T.N.Z.I. xxviii. 3I4.

* For a Bibliography see Hamilton, T.N.Z.I. xxxiv. 539. 
Cæcioperca, Castelnau, I 872 .

lepidoptera, Forster. C.B.M. 2nd ed. i. 3 I 2 ; T.N.Z.I. xxii. 276 ; No. 23 (Anthias).

Caprodon, Temminck, 1844 .

longimanus, Gunther. C.B.M. 2nd ed. i. 3 I 5 ; T.N.Z.I. xxii. 276 ; No. 24 (Anthias).

Lirus, Lowe, I 836 .

$$
\text { Family LUTJANIDE. }
$$

porosus, Richardson (Diagramma), Challenger Reports, xxxi. 1 I.

Plagiogenion, Forbes, I 890.

rubiginosus, Hutton. T.N.Z.I. xxii. 277, No. 28.

Family Pentacerotide.

Pseudopentaceros, Gill, I 893 (?).

richardsoni, Smith. T.N.Z.I. xxii. 277 , No. 27 (Pentaceros).

Arripis, Jennings, I 840 .

Family ARRIPIDIDE.

trutta, Cuvier. A. salar, Hutton, Cat. Fishes of N.Z. 2.

Emmelichthys, Richardson, I 846 .

nitidus, Richardson. Cat. Fishes N.Z. 3 (Erythrichthys).

Family Pinelepteride.

Incisidens, Gill, r 893 (?).

simplex, Richardson. T.N.Z.I. xxii. 277 , No. 33 (Girella). multilineata, Clarke. T.N.Z.I. xxxi. 98 (Girella).

Atypichthys, Gunther, I862.

strigatus, Gunther. Kirk, T.N.Z.I. xii. 308 (Atypus).

Scorpis, Cuvier, I83I.

Family SQUamipinnes.

violaceus, Hutton. T.N.Z.I. xxii. 278 (Neprotichthys); Regan, A.M.N.H. 7, x. I 16.

Family MULLIDE.

Upeneichthys, Bleeker, I $85^{\circ}$.

vlamingii, Cuvier. Hector, T.N.Z.I. ix. 465.

Pagrosomus, Gill, 1893 (?).

Family SPARIDE.

auratus, Forster. Pagrus unicolor, Cat. Fishes N.Z. 6 ;

T.N.Z.I. ix. 353 .

Family HaPLODACTYLide.

Haplodactylus, Gunther, 1859 .

meandratus, Solander. T.N.Z.I. xxii. 277 , No. 34 . 
Chironemus, Cuvier, 1829 .

fergusoni, Hector. 'T.N.Z.I. vii. 243, and ix. 467 (Haplodactylus).

spectabilis, Hutton. T.N.Z.I. xxii. 277 , No. 35 (Chilodactylus).

Chilodactylus, Lacépède, I 803.

macropterus, Forster. Cat. Fishes N.Z. 8.

douglasi, Hector. T.N.Z.I. vii. 244 .

Mendosoma, Guichen, 1847 .

lineata, Gray. T.N.Z.I. xxii. 277 , No. $4 \mathrm{I}$.

Latris, Richardson, 1840.

ciliaris, Forster. Cat. Fishes N.Z. 9.

lineata, Forster. Cat Fishes N.Z. 8.

aerosa, Hutton. T.N.Z.I. ix. 353 .

Family SCORPÆNIDE.

Sebastapistes, Streets, I 877 .

percoides, Solander. T.N.Z.I. v. 26I, and ix. 353 (Sebastes).

barathri, Hector. T.N.Z.I. vii. 245 (Sebastes).

Scorpæna, Cuvier, 1829 .

cruenta, Solander. Cat. Fishes N.Z. Io; T.N.Z.I. v. 26 I.

byssoensis, Richardson. Hector, T.N.Z.I. xvi. 323.

Cottapistus, Bleeker, i $\delta_{7} 6$

cottoides, Linnæus. Cat. Fishes N.Z. i I (Prosopodasys).

Family Congiopodide.

Congiopodus, Perry, 1871 .

leucopœecilus, Richardson. T.N.Z.I. xxii. 277 , No. 49

(Agriopus) ; Stenhouse, T.N.Z.I. xxvi. II I.

peruvianus, Cuvier. Hutton, T.N.Z.I. xxviii. 3 I 4.

Family CoTtıdÆ.

Neophrynichthys, Gunther, 1876.

latus, Hutton. T.N.Z.I. xxii. 280 , No. 100 ; Gill, Pro. U.S.

National Mus. xi 327.

Family Trachinide.

Leptoscopus, Gill, I 859 . macropygius, Richardson. T.N.Z.I. xxii. 279 , No. 88.

Crapatalus, Gunther, I86r.

novæ-zealandiæ, Gunther. T.N.Z.I. xxii. 279 , No. 89.

Genyagnus, Gill, I86r.

maculatus, Forster. $K$. monopterygium, Bloch; Cat. Fishes

N.Z. 23. 
Kathesostoma, Gunther, 1860.

lævis, Bloch. Cat. Fishes N.Z. 23; K. giganteum, Haast, T.N.Z.I. v. 274 .

fluviatilis, Hutton. Cat. Fishes N.Z. 24 ; 'T. N.Z.I. xxviii. 315.

Bovichthys, Cuvier, 1831 .

variegatus, Richardson. T.N.Z.I. xxii. 279 , No. 92.

Notothenia, Richardson, 1844 .

maoriensis, Haast. 'T.N.Z.I. v. 276.

angustata, Hutton. T.N.Z l. viii. 2 I 3 .

cornucola, Richardson. 'T.N.Z.I. v. 262.

arguta, Hutton. T.N.Z.I. xi. 339.

microlepidota, Hutton. T N.Z.I. viii. 2 I 3.

parva, Hutton. T.N.Z.I. x1. 339.

Cheimarrichthys, Haast, 1874 .

forsteri, Haast. T.N.Z.I. vi. 103.

Parapercis, Steindachner, I884.

colias, Forster. Cat. Fishes N.Z. 25 (Percis).

gilliesii, Hutton. A.M.N H. 5 , iii. 53 .

Family BERYCIDÆ.

Beryx, Cuvier, I829.

affinis, Gunther. T.N.Z.I. xxii. 278 , No. 54.

Trachyichthys, Shaw, I $8-(?)$.

trailii, Hutton. T.N.Z.I. xvii. I62; Challenger Reports, xxii. 23.

elongatus, Gunther. Cat. Fishes N.Z. I 2.

intermedius, Hector. T.N.Z.I. vii. 245.

Xiphias, Linnæus, 1748 .

Family XIPHIDE.

gladius, Linnæus. T.N.Z.I. xxii. 278 , No. 55.

Histiophorus, Cuvier, $\mathrm{I}_{3} \mathrm{I}$.

herschelii, Gray. T.N.Z.I xxii. 278 , No. 56.

Lepidopus, Gouan, 1770 .

Family LEPIDOPODIDE.

caudatus, Euphrasen. T.N.Z.I. xxii. 278 , No. 57.

elongatus, Clarke T.N.Z.I. xi. 294.

Thyrsites, Cuvier, I 83 I.

Family Genpylide.

atun, Euphrasen. Cat. Fishes N.Z. 13.

Promethichthys, Gill, i 893 .

prometheus, Webb and Berthel. Hector, T.N.Z.I. xvi. 322. 
Caranx, Lacépède, 1800.

Family Carangida,

georgianus, Cuvier. Cat. Fishes N.Z. I6.

koheru, Hector. T.N.Z.I. vii. 247.

Trachurus, Rafinesque, I8 10.

trachurus, Linnæus. Cat. Fishes N.Z. э6.

Seriola, Cuvier, 1829.

lalandii, Cuvier. Cat. Fishes N.Z. I 7.

Naucrates, Rafinesque, r 8 Io.

ductor, Linnæus. Cat. Fishes N.Z. I8.

Evistius, Gill, I 893 .

huttoni, Gunther. T.N.Z.I. xxii. 278 , No. 70 (Platystethus).

Cubiceps, Lowe, I 843 .

Family Stromatide.

gracilis, Lowe (?). T.N.Z.I. xxviii. $3^{15}$

Seriolella, Guichen, I 847 .

porosa, Guichen (=Neptomenus dolbula, Gunther), Regan;

A M.N.H. 7, x. 128 ; T.N.Z.I. viii. 2 I 1.

bilineata, Hutton. T.N.Z.I. xxviii. 3 I4; Regan; A.M.N.H. $7, \mathrm{x} .128$.

brama, Gunther. Cat. Fishes N.Z. 2 I (Neptomenus).

Centrolophus, Lacépède, 1802.

maoricus, Ogilby. Rec. Aust. Mus. ii. 64, r 893.

Lirus, Lowe, 1833 .

porosus, Richardson (Diagramma). Regan, A.M.N.H. 7, x. 202.

Family CORYPHÆNIDE.

Lampris, Betzius, I 799.

guttatus, Gill, Mem. Nat. Acad. Sci. Phil. vi. I 15 ; L. lima, Hector, T.N.Z.I. xvi. 322.

Pteraclis, Cuvier, I833.

velifer, Pallas. C.B.M. I, ii. 4 I I ; Ogilby; Records Aust.

Mus. ii. 65.

Brama, Schneider, 180 I.

squamosa, Hutton. T.N.Z.I. viii. 2 ro (Toxotes); T.N.Z.I. ix. 465 .

Scomber, Linnæus, 1748 .

Family SCOMBRIDæ.

australiasicus, Cuvier. Cat. Fishes N.Z. 2 I.

Thynnus, Cuvier, I 83 I.

pelamys, Linnæus. Specimen in Canterbury Museum. 
Sarda, Cuvier, is 89.

chilensis, Cuvier. Cat. Fishes N.Z. 22 (Pelamys).

Lepidothynnus, Gunther, 1889 .

huttoni, Gunther. Challenger Reports, xxxi. I5; Regan, A.M.N.H. 7 , x. 120.

Cybium, Cuvier, I83 I.

guttatum, Bleeker. T.N.Z I. xxviii. 3 I 5 .

Gasterochisma, Richardson, I 845 .

melampus, Richardson. T.N.Z.I.vi. I04; Regan, A.M.N.H.

$7, \mathrm{X} . \mathrm{I} 2 \mathrm{O}$.

Family ECHENEIDIDE.

Remora, Willoughby, 686.

brachyptera, Lowe. T.N.Z.I. viii. 2 I 7 (Echeneis).

Family CaPROIDE.

Capromimus, Gill, I 893 .

abbreviatus, Hector. T.N.Z.I. vii. 247 (Platystethus).

Zeus, Linnæus, 1748 .

faber, Linnæus. Cat. Fishes N.Z. I8.

Cyttus, Gunther, 1860.

australis, Richardson. Cat. Fishes N.Z. 19.

novæ-zealandiæ, Arthur. T.N.Z.I. xvii. I63 (Zeus).

Rhombocyttus, Gill, I 893 .

traversi, Hutton. T.N.Z.I. v. 261.

Diretmus, Johnston, I 863.

aureus, Campbell. T.N.Z.I. xi. 297 (Discus); Challenger

Reports, xxii. 45 .

Family Ceratidie.

Egœonichthys, Clarke, I 878 .

appellii, Clarke T.N.Z.I. x. 245 .

Family Triglide.

Trigla, Linnæus, I 748.

kumu, Lesson. Cat. Fishes N.Z. 28.

Lepidotrigla, Gunther, 1860.

vanessa, Richardson. T.N.Z.I. xxii. $53^{\circ}$.

brachyptera, Hutton. T.N.Z.I. v. 263 .

Family GoBIDæ.

Gobiomorphus, Gill, 1893 (?).

gobioides, Cuvier. T.N.Z.I. v. 263 (Eleotris).

Eleotris, Gronovius, ${ }_{7} 6_{3}$.

radiata, Quoy and Gaimard. T.N.Z.I. v. 263 .

huttoni, Ogilby. P.L.S.N.S.W. 1894, ix. 369. 
Gobius, Linnæus, 1748 .

lentiginosus, Richardson. Cat. Fishes N.Z. 29; Gunther, 'Study of Fishes,' 486, fig. 220.

Family Heneroccetide.

Hemerocœtes, Valenciennes, I837. acanthorhynchus, Forster. Cat. Fishes N.Z. 37.

Family BLENNidÆ.

Ericentrus, Gill, 1893. rubrus, Hutton. T.N.Z.I. v. 264 (Clinus).

Cologrammus, Gill, 1893 .

flavescens, Hutton. T.N.Z.I. v. 264 (Clinus).

Cristiceps, Cuvier, $18{ }_{3} 6$.

australis, Cuvier. T.N.Z.I. v. 264 .

Tripterygion, Risso, 1826.

tripinne, Forster. T. forsteri, Cuvier, T.N.Z.I. ix. 354.

decemdigitatum, Clarke. T.N.Z.I. xi. 292.

medium, Gunther. T.N.Z.I. xxii. 280, No. 14.

dorsale, Clarke T.N.Z.I. xi. 29 I.

varium, Forster. Cat. Fishes N.Z. 33.

nigripenne, Cuvier. T.N.Z.I. v. 263.

robustum, Clarke. T.N.Z.I. xxii. 28 , No. I I 7 .

Auchenopterus, Gunther, I86I.

aysoni, Hector. T N.Z.I. xxxiv. 240.

Family Acanthoclinide.

Acanthoclinus, Jennings, I 842 .

littoreus, Forster. Cat. Fishes N.Z. 34 .

taumaka, Clarke. T.N.Z.I. xi. 393.

Family ATHERINIDE.

Atherina, Linnæus, 1766 .

pingius, Lacépède. Kirk, T.N.Z.I. xii. 309.

Family SpHYrenide.

Sphyræna, Artedi, $173^{8 .}$

obtusata, Cuvier. Kirk, T.N.Z.I. xii. 3 Iо.

Mugil, Linnæus, I 748 .

Family MUgILIDæ.

cephalotus, Cuvier. T.N.Z.I. xxii. $28 \mathrm{I}$, No. 123.

Agonostomus, Telfaire, I830.

forsteri, Bloch. Cat. Fishes N.Z. 37.

Family Macrorhamphoside.

Centiscops, Gill, I862.

humerosus, Richardson. Cat. Fishes N.Z. $3^{8}$ (Centriscus). 
Diplocrepis, Gunther, I86I.

Family Gobiesocide.

puniceus, Richardson. T.N.Z.I. xxii. 281 , No. 126.

Trachelochismus, Brisson, I 846 .

pinnulatus, Forster. T.N.Z.I. xxii. $28 \mathrm{I}$, No. 27.

littoreus, Forster (Cyclopterus). T. guttulatus, Hutton, Cat.

Fishes N.Z. 4 I.

Crepidogaster, Gunther, r86r.

hectoris, Gunther. T.N.Z.I. xxii. 28I, No. I 2 I.

simus, Hutton. T.N.Z.I. xxviii. 3 I 6.

Family Trachypteride.

Trachypterus, Gouan, I 770.

altivelis, Kner. T.N.Z.I. xxii. 28 I, No. 132.

arawata, Clarke. T.N.Z.I. xiii. I95.

Lophotes, Giorna, 1803 .

Family LopHOTIDE.

fiskei, Gunther. T.N.Z.I. xxvi. 223 , and xxix. 25 I.

Regalicus, Nardo, I 827.

Family Regalicide.

pacificus, Haast. T.N.Z.I. x. 247 , and xi. 269.

argenteus, Parker. T.N.Z.I. xvi. 284 , and xx. 20.

grillii, Lindroth. T.N.Z.I. xxiv. 192.

Family Notacanthide.

Notacanthus, Lacépède. 1803 .

sexspinis, Richardson. Cat. Fishes N.Z. 39.

Sub-order Pharyngognathi.

Family ODACıde.

Odax, Cuvier, 1839.

vittatus, Solander. T.N.Z.I. xxii. 282 , No. 144.

Coridodax, Gunther, r 862.

pullus, Forster. T.N.Z.1. xxii. 282 , No. 145.

Family LABRIDE.

Pseudolabrus, Bleeker, I $86 \mathrm{r}$.

fucicola, Richardson. T.N.Z.I. xxii. 28 I, No. 137.

roseipunctatus, Hutton. T.N.Z.I. xii. 455.

bothryocosmus, Richardson. T.N.Z.I. v. 265 .

miles, Gill. L. coccinea. T.N.Z.I. xxii. 282 , No. 139.

celidotus, Forster. Cat. Fishes N.Z. $4^{2}$; Gunther, A.M.N.H.

4, xvii. 398.

cinctus, Hutton. T.N.Z.I. ix. 354 . 
Pictilabrus, Gill, I 892.

laticlavius, Richardson. Hector, T.N.Z.I. xvi. 323.

Cymolutes, Gunther, I86r.

sandeyeri, Hector. T.N.Z.I. xvi. 323.

Lepidoplois, Gill, 1863 .

unimaculatus, Gunther. Hector, T.N.Z.I. xvi. 323.

Duymræia, Bleeker, I856.

flagillifera, Cuvier. Hector, T.N.Z.I. xxii. 527 ; Gunther, C.F.B.M. iv. I 2 I.

Sub-order ANacanthin.

Family Pleuronectide.

Caulopsetta, Gill, I893.

scapha, Forster. Cat. Fishes N.Z. 51. (Pseudorhombus.) boops, Hector. T.N.Z.I. vii. 249.

hectoris, Gunther. Challenger Reports, xxii. I63.

Brachypleura, Gunther, I 862.

novæ-zealandiæ, Gunther. Hutton, Cat. Fishes N.Z. 5 ०.

Ammotretis, Gunther, 1862.

rostratus, Gunther. Hutton, T.N.Z.I. viii. 215.

guntheri, Hutton. T.N.Z.I. v. 267.

Rhombosolea, Gunther, 1862.

plebeia, Richardson. $R$. monopus. Hutton, T.N.Z.I. xxii. 283 , No. 170.

flesoides, Gunther. Hutton, T.N.Z.I. xxii. 283 . No. I 7 I. tapirina, Gunther. Hutton, T.N.Z.I. xxii. 283 , No. 172. retiaria, Hutton. T.N.Z.I. xxii. 283 , No. 173 .

Apsetta, Kyle, I90I.

thompsoni, Kyle. P Z.S. 1900, p. 986.

Peltorhamphus, Gunther, I862.

novæ-zealandiæ, Gunther. Hutton, Cat. Fishes N.Z. 52.

Family GADIDÆ.

Physiculus, Kaup, 1858 . bacchus, Forster. Hutton, Cat. Fishes N.Z. 46 (Lotella);

Beattie, T.N.Z.I. xxiii. 7 I.

rhacinus, Forster. Hutton, T.N.Z.I. vi. plate 18 , fig. 74 .

Pseudophycis, Gunther, I862.

breviusculus, Richardson. Hutton, Cat. Fishes N.Z. 47 .

Halargyreus, Gunther, I 862.

johnsonii, Gunther. Hutton, Cat. Fishes N.Z. 45.

Onos, Risso, i 826 .

novæ-zealandiæ, Hector. T.N.Z.I. vi. Io7 (Motella). 
Bathygadus, Gunther, 1878 .

cottoides, Gunther. Hutton, T.N.Z.I. xxii. 282 , No. 147 .

Merluccius, Rafinesque, i8 ro.

gayi, Guichen. Hutton, T.N.Z.I. xxii. 282, No. 148.

Auchenoceros, Gunther, I889.

punctatus, Hutton. T.N.Z.I. xxii. 282 , No. I 54.

Family OpHIDIDE.

Hypolycodes, Hector, I $88 \mathrm{I}$.

haastii, Hector. T.N.Z.I. xiii. 194.

Dinematichthys, Bleeker, 1854 .

consobrinus, Hutton. Hector, T.N.Z.I. ix. 466.

Genypterus, Philippi, I 855 .

blacodes, Forster. Hutton, Cat. Fishes N.Z. 48.

Family MACRURIDÆ.

Cœlorhynchus, Agassiz, I 843 .

parallelus, Gunther. Challenger Reports, xxii. I 25.

australis, Richardson. Hutton, Cat. Fishes N.Z. 78.

Macrurus, Bloch, 1787 .

rudis, Gunther. Challenger Reports, xxii. I 3 I.

serrulatus, Gunther. Challenger Reports, xxii. I33.

Cetonurus, Gunther, I 887.

crassiceps, Gunther. Challenger Reports, xxii. 143.

Chalinura, Goode and Bean, 1886.

murrayi, Gunther. Challenger Reports, xxii. I46.

Optonurus, Gunther, 1887 .

denticulatus, Richardson. Hutton, Cat. Fishes N.Z. 49.

Nematonurus, Gunther, 1887.

armatus, Hector. T.N.Z.I. vii. 249; Challenger Reports, xxii. I 50 .

Trachyrhynchus, Bonaparte, I8- (?).

longirostris, Gunther. Challenger Reports, xxii. 153.

Macrurorus, Gunther, 1873.

novæ-zealandiæ, Hector. T.N.Z.I. iii. I 36 (Coryphanoides).

Sub-order Physostomi.

Family Exoccetrde.

Exocœtus, Linnæus, I 748.

micropterus, Cuvier. Hutton, Cat. Fishes N.Z. 54.

speculiger, Cuvier. Hutton, Cat. Fishes N.Z. 55.

ilma, Clarke. T.N.Z.I. xxxi. 94. 
Hemirhamphus, Cuvier, 18 I 7 . intermedius, Cuvier. Hutton, Cat. Fishes N.Z. 53.

Scombresox, Lacépède, I803. forsteri, Cuvier. Hutton, Cat. Fishes N.Z. 53.

Family Sternoptychide.

Argyropelecus, Cocco, 1829. intermedius, Clarke. T.N.Z.I. x. 244.

Sternoptyx, Herman, I $7-$ (?).

diaphana, Herman. Gunther, Challenger Reports, xxii. 169.

Phosichthys, Hutton, 1872.

argenteus, Hutton. T.N.Z.I. v. 269. Challenger Reports, xxii. 178.

Maurolicus, Cocco, I 829. australis, Hector. T.N.Z.I. vii. 250.

Gonostoma, Rafinesque, i 8 io. microdon, Gunther. Challenger Reports, xxii. I 75 .

Family SCOPELIDE.

Lampadena, Goode and Bean, 1895. parvimana, Gunther. Hutton, T.N.Z.I. v. 269 (Scopelus).

Myctophum, Rafinesque, r8ro. hectoris, Gunther. T.N.Z.I. ix. 47 I. boops, Richardson. Hutton, T.N.Z.I. v. 269.

Rhinoscopelus, lütken, I 892. coruscans, Richardson. Hutton, T.N.Z.I. v. 270.

Neoscopelus, Johnson, 1863. macrolepidotus, Johnson. Gunther, Challenger Reports, xxii. 196.

Alepisaurus, Lowe, 1833. ferox, Lowe. Hutton, T.N.Z.I. xxxiv. 197.

Bathysaurus, Gunther, 1878 .

ferox, Gunther. Hutton, T.N.Z.I. xxii. 283 , No. 179.

Chlorophthalmus, Bonaparte, I84I. gracilis, Gunther. Hutton, T.N.Z.I. xxii. 283 , No. I80.

Bathypterois, Gunther, I 878 . longifilis, Gunther. Challenger Reports, xxii. I 85.

Family HaPLOChITONIDE.

Prototroctes, Gunther, I 864. oxyrhynchus, Gunther. Hutton, Cat. Fishes N.Z. 57. 
Family ARgentinide.

Argentina, Linnæus, 1748.

sphyræna, Linnæus. Challenger Reports, xxii. 2 I 8 ; $A$. decagon, Clarke, T.N.Z.I. xi. 296.

elongata, Hutton. A.M.N.H. 5, iii. 53; Challenger Reports, xxii. 218.

Retropinna, Gill, I862.

richardsoni, Gill. Hutton, T.N.Z.I. xxii. 284 , No. 194.

Galaxias, Cuvier, I 8 I 7 .

Family GaLaxide.

alepidotus, Forster. Hutton, Cat. Fishes N.Z. $5^{8}$.

var. brocchus, Richardson. Voy. Erebus \& Terror, Fishes, 76 .

kokopu, Clarke. T.N.Z.I. xxxi. 88

fasciatus, Gray. Hutton, Cat. Fishes N.Z. 59.

bollonsi, Hutton. T.N.Z.I. xxxiv, I 92.

postvectis, Clarke. T.N.Z.I. xxxi. 88.

robinsoni, Clarke. T.N.Z.I. xxxi. 89.

brevipinnis, Gunther. Hutton, Cat. Fishes N.Z. 59.

var. grandis, Haast. T.N.Z.I. v. 278.

lynx, Hutton. T.N.Z.I. xxviii. 317.

attenuatus, Jennings. Hutton, Cat. Fishes N.Z. 60.

Neochanna, Gunther, I867.

apoda, Gunther. Hutton, Cat. Fishes N.Z. 6r.

Family GoNORHYNCHIDE.

Gonorhynchus, Gronovius, 1763 .

greyi, Richardson. Hutton, Cat. Fishes N.Z. 62.

Chanos, Lacépède, 1803 .

Family Clupeide.

salmoneus, Forster. Hutton, Cat. Fishes N.Z. 64 .

Clupea, Linnæus, r 748.

sagax, Jenyns. Hutton, T.N.Z.I. xxii. 284 , No. 199.

antipoda, Hector. Hutton, Cat. Fishes N.Z. I33; T.N.Z.I. xv. 203.

Stolephorus, Lacépède, $x 803$.

encrasicholus, Linnæus. Hutton, Cat. Fishes N.Z. 99.

Muræna, Linnæus, $\mathbf{1} 766$.

Fumily Murenide.

krullii, Hector, T.N.Z.I. ix. 468.

Ophisurus, Lacépède, I 800.

novæ-zealandiæ, Hector. T.N.Z.I. ii. 34. 
Anguilla, Thunberg, 1790 . aucklandii, Richardson. Hutton, Cat. Fishes N.Z. 64. australis, Richardson. Hutton, Cat. Fishes N.Z. 65.

Congermuræna, Kaup, I866.

habentata, Richardson. Hutton, Cat. Fishes N.Z. 66.

Leptocephalus, Gronovius, 1754 .

conger, Willoughby. Hutton, Cat. Fishes N.Z. 66.

\section{Sub-order LOPHOBRANCH11.}

Family HIPPOCAMPIDE.

Hippocampus, Leach, I 8 I 4 abdominalis, Lesson. Hutton, Cat. Fishes N.Z. 70.

Solegnathus, Swainson, I 839 .

spinossissimus, Gunther. Hutton, Cat. Fishes N.Z. 69 ; T.N.Z.I. xxii. 285 , No. 214 .

Family Sygnathıde.

Stigmatophora, Kaup, I 853 . longirostris, Hutton. Cat. Fishes N.Z. 69 ; T.N.Z.I. xxii. 285 , No. 2 I 3.

Ichthyocampus, Kaup, 1853 .

filum, Gunther. Hutton, Cat. Fishes N.Z. 68.

Doryichthys, Kaup, 1853 .

elevatus, Hutton. Cat. Fishes N.Z. 68.

Siphostoma, Rafinesque, i 8 го.

pelagica, Linnæus. Hutton, Cat. Fishes N.Z. 67.

blainvilliana, Eydoux and Soul. Gill, Nat. Acad. of Sci.

Washington, vi. I 22.

Sub-order Plectognathi.

Family Balistidæ.

Monacanthus, Cuvier, I 8 I 7 .

scaber, Forster. T.N.Z.I. xxii. 285 , No. 2 I 6.

Family Tetraodontide.

Amblyrhynchotus, Bibron, 1855 .

richei, Fremiville. Cat. Fishes N.Z. 72.

Crayracion, Klein, 1743 .

gillbanksii, Clarke. T.N.Z.I. xxix. 245.

Gastrophysus, Müller, $184^{2}$.

cheesemanni, Clarke. T.N.Z.I. xxix. 248.

Family DIODONTIDÆ.

Dicotylichthys, Kaup, I 85. jaculiferus, Cuvier. Hutton, T.N.Z.I. r. 27 I. 
Mola, Nardo, 1827 .

Family MOLIDE.

mola, Linnæus, Gill. Mem. Acad. Nat. Sci. Phil. vi. I2 2.

\section{Sub-class HoLOCEPHALI. \\ Family CHIMÆRIDÆ.}

Callorhynchus, Gronovius, 1754 .

antarcticus, Lacépède. Hutton, T.N.Z.I. xxii. 276 , Nos. 21 and 22 ; Hector, T.N.Z.I. xxxiv. pl. xiv.

Chimæra, Linnæus, 1766 .

monstrosa, var. australis, Hector. T.N.Z.I. xxxiv. 239.

Sub-class Elasmobranchil.

Order SELACHII.

Sub-order HYPo'RemI.

Aëtobatus, Blainville, r 828 .

Family AётоватIDÆ.

tenuicaudatus, Hector. T.N.Z.I. ix. 286 (Myliobatis).

Family Dasybatide.

Dasybatis, Bonaparte, I $84 \mathrm{I}$.

brevicaudatus, Hutton. T.N.Z.I. viii. 216 (Trygon).

Torpedo, Dumeril, 1806 .

Family TORPEDINIDE.

fairchildi, Hutton. Cat. Fishes N.Z. 83.

fusca, Parker. T.N.Z.I. xvi. 28I.

Family NARCOBATIDE.

Astrape, Muller and Henle, 1837.

aysoni, Hamilton. T.N.Z.I. xxxiv. 225.

Raia, Linnæus, 1748.

Family RAIIDE.

nasuta, Solander. Hutton, T.N.Z.I. xxii. 276 , No. 18.

Sub-order ASTEROSPONDYLI.

Family SPHYRNIDÆ.

Sphyrna, Rafinesque, i 8 ro.

zygæna, Linnæus. Z. malleus, Hutton, T.N.Z.I. xxii. 275, No. 3 .

Family GaLEIDE.

Carcharias, Rafinesque, I 8 Io.

lamia, Risso. Challenger Reports, xxxi. 5.

Carcharinus, Blainville, I 828 .

brachyurus, Gunther. Hutton, Cat. Fishes N.Z. 75. 
Galeorhinus, Blainville, 1828 .

australis, Macleay. Hutton, T.N.Z.I. xxii. 275, No. 2 (Galeus).

Galeus, Cuvier, I 8 I 7 .

antarcticus, Gunther. Hutton, T.N.Z.I. xxii. 275 , No. 4 (Mustelus). Parker, T.N.Z.I. xxii. 33I.

Family Alopirdæ.

Alopias, Rafinesque, 1810.

vulpes, Gmelin. Hutton, Cat. Fishes N.Z. 78.

Family Cetorninidæ.

Cetorhinus, Blainville, I 8 I 6.

maximus, Gunn. Cheeseman, T.N.Z.I. xxiii. I26 (Selache).

Family ISURIDÆ.

Carcharodon, A. Smith, I837.

carcharias, Müller. C. rondeletii, Hutton, Cat. Fishes N.Z. 78.

Isurus, Rafinesque, I8ro.

cornubicus, Gmelin. Haast, T.N.Z.I. vii. 237.

Family SCYLlioRHINIDE.

Cephaloscyllium, Gill, I86 I.

laticeps, Dumeril. Hutton, T.N.Z.I. xxii. 276 , No. 9.

Sub-order Cyclospondyl.

Family OXYNOTIDE.

Oxynotus, Rafinesque, i 8 Io.

bruniensis, Ogilby. Records Aust. Mus. ii. 62; C. salviana, T.N.Z.I. xxii. 276 , No. Io.

Family SQUAL1D瓜.

Squalus, Linnæus, I 748 .

acanthias, Linnæus. A. vulgaris, Hutton, Cat. Fishes N.Z. 76.

Family DaLATIIDE.

Dalatias, Rafinesque, 1810.

lichia, Cuvier. Parker, 'I.N.Z.I. xv. 223 (Scymus).

Family ECHINORHINIDÆ.

Echinorhinus, Blainville, I 828 .

spinosus, Cuvier. Parker, T.N.Z.I. xvi. 280.

Sub-order OPISTHARTHRI.

Family HEXaNCHIDE

Notorhynchus, Ayres, 1856

indicus, Cuvier. Hutton, Cat. Fishes N.Z. 79 (Notidanus). 


\section{CLASS, CYCLOSTOMATA.}

Geotria, Gray, 1851 .

Family Pteromyzontide.

australis, Gray. Hutton, T.N.Z.I. xxii. 285 , Nos. $22 \mathrm{I}$ and 222 ; Dendy, T.N.Z.I. xxxiv. I 47.

Family Heptatremide.

Heptatrema, Duméril, i 8 - (?).

cirrata, Forster. Hutton, Cat. Fishes N.Z. 87 (Bdellostoma).

CLASS, LEPTOCARDII.

Heteropleuron, Kirkaldy, i 894.

Family BRACHIOSTOMIDE.

hectori, Benham. Q.J.M.S. xliv. 273 (1901).

\section{SUB-PHYLUM, UROCHORDA.}

\section{CLASS, TUNICATA.}

Order AscidiACEe.

Sub-order Ascidie-COMPOSITE.

Family BOTRYLLIDE.

Botryllus,, Lamarck, I 80 I.

racemosus, Quoy and Gaimard. Voy. Astrolabe, Zool. iii. 620 (Gen. dub.).

Family Distomide.

Cystodytes, Drasche, I 883 .

aucklandicus, Nott. T.N.Z.I. xxiv. 323 .

perspicuus, Nott. T N.Z.I. xxiv. 326 .

Family DIDEMNIDE.

Leptoclinum, Milne-Edwards, $184 \mathrm{I}$.

niveum, Nott. T.N.Z.I. xxiv. 308 .

densum, Nott. T.N.Z.I. xxiv. 3 I I.

tuberatum, Nott. T.N.Z I. xxiv. 3 I 4 .

maculatum, Nott. T.N.Z.I. xxiv. 316.

Polysyncraton, Nott, 1892.

Family - (?). paradoxum, Nott. T.N.Z.I. xxiv. 3 I8. fuscum, Nott. T.N.Z.I. xxiv. $32 \mathrm{I}$. 
Sub-order Ascidiæ-SIMPLICES.

Boltenia, Savigny, 18 I 6 .

Family BOLTENIDE.

pachydermatina, Herdman. Challenger Reports, vi. 89 ;

Watt, T.N.Z.I. xxiv. 334 .

Culeolus, Herdman, i882.

wyville-thomsoni, Herdman. Challenger Reports, vi. ז०3.

Polycarpa, Heller, 1877.

Family Cynthide.

elata, Heller. Sitz. Akad. Wien, 1878 (Loc. dub.).

nebulosa, Heller. Sitz. Akad. Wien, 1878 (Loc. dub.).

Ascidia, Linnæus, I 758.

Family AscIDIDÆ.

erythrostoma, Quoy and Gaimard. Voy. Astrolabe, Zool. iii. 609 (Gen. dub.).

janthinoctoma, Quoy and Gaimard. Voy. Astrolabe, Zool. iii. 6ro (Gen. dub.).

cærulea, Quoy and Gaimard. Voy. Astrolabe, Zool. iii. 6 I I (Gen, dub.).

\section{Order Thaliacea.}

Family Pyrosomatıde.

Pyrosoma,, Péron, I804.

sp. innom. Herdman. Challenger Reports, xxvii. pp. I3 and 35 .

elegans, Lamarck (?). Challenger Reports, xxvii. p. 35.

Salpa, Forskal, I 775 .

Family SALP1DÆ.

costata-tilesii, Quoy. Voy. Astrolabe, Zool. iii. 570;

Chall. Rep. xxvii. 6o.

infundibuliformis, Quoy. Voy. Astrolabe, Zool. iii. 587.

cordiformis-zonaria, Quoy. Challenger Reports, xxvii. 7 o. democratica-mucronata, Forster. Challenger Reports, xxvii. 79.

Family Doliolide.

Doliolum, Quoy and Gaimard, I 835 .

denticulatum, Quoy. Challenger Reports, xxvii. 44. ehrenbergii, Krohn. Challenger Reports, xxvii. 46.

\section{CLASS, HEMICHORDA.}

Family Harrimancide.

Dolichoglossus, Spengel, 1893 .

otagoensis, Benham. T.N.Z.I. xxxii. 9 (Balanoglossus). 


\section{PHYLUM, MOLLUSCA.}

\section{CLASS, CEPHALOPODA.}

Order Octopoda.

Family OCTOPODIDE.

Octopus, Lamarck, I 799.

maorum, Hutton. Man. N.Z. Moll. I.

communis, Park. T.N.Z.I. xvii. I 98.

Pinnoctopus, d'Orbigny, $\mathrm{I} 845$.

cordiformis, Quoy and Gaimard. Man. N.Z. Moll. 2;

Man. Conch. I, i. I 28.

Eledone, Leach, 1817 .

verrucosa, Verrill. 'Blake' Report, I05.

Family Argonautide.

Argonauta, Linnæus, 1758.

argo, Linnæus. Austral. Mus. Sydney, Cat. xv. I ; A. bulleri, T. W. Kirk.

nodosa, Solander. Austral. Mus. Sydney, Cat. xv. $2: A$. gracilis, T. W. Kirk.

Family AMPHITRETIDж.

Amphitretus, Hoyle, 1885 .

pelagicus. Hoyle. Challenger Reports, xvi. 67.

Family CirRoteUthide.

Cirroteuthis, Eschricht, 1836 .

meangensis, Hoyle. Challenger Reports, xvi. 63 .

Order Decapoda.

Taonius, Steenstrup, 186 I.

\section{Family Cranchinde.}

suhmii (Lankester), Hoyle. Challenger Reports, xvi. 45, I92.

Calliteuthis, Verrill, I880.

Family ChIRoteUthIDÆ.

? reversa, Verrill. Ceph. N.E. America, 295. 
Family ONıCHOTEUTHIDÆ.

Onichoteuthis, Lichtenstein, I818.

banksii, Leach. O. bartlingii, Lesueur. Man. Conch. I, i. 168 .

Family OMMASTREPHIDE.

Todarodes, Steenstrup, I880.

sloanii, Gray. Man. N.Z. Moll. 3 ; Man. Conch. I, i. 180.

Architeuthis, Steenstrup, 1856.

verrillii, T. W. Kirk. T.N.Z.I. xiv. 284 .

kirkii, Robson. T.N.Z.I. xix. I55.

longimanus, T. W. Kirk. T.N.Z.I. xx. 34 .

Steenstrupia, T. W. Kirk, I882.

stockii, T. W. Kirk. T.N.Z.I. xiv. 286.

Family LoLıGinide.

Sepioteuthis, Blainville, I 825 .

lessoniana, Férussac. Austral. Mus. Sydney, Cat. xv. I4; Man. Conch. I, i. I 52.

bilineata, Quoy and Gaimard. Austral. Mus. Sydney, Cat. xv. $15 ;$ Man. Conch. I, i. I54.

Sepiola, Leach, 1817 .

$$
\text { Family SEPIOLIDE. }
$$

pacifica, T. W. Kirk. T.N.Z.I. xiv. 283 .

Sepia, Linnæus, $175^{8}$.

$$
\text { Family SePIIDE. }
$$

apama, Gray. Austral. Mus. Sydney, Cat. xv. I1; Man. Conch. I, i. I 94.

Family SPIRULidæ.

Spirula, Lamarck, I 799 .

peroni, Lamarck. Austral. Mus Sydney, Cat. xv. Io; Man.

Conch. I, i. 205.

\section{CLASS, GASTEROPODA.}

Sub-class Euthyneura.

Order Pulmonata.

Sub-order STYLOMMATOPHORA.

Family ONCIDIDE.

Onchidella, Gray, I850. patelloides, Quoy and Gaimard. Man. N.Z. Moll..28. nigricans, Quoy and Gaimard. Man. N.Z. Moll. 28. irrorata, Gould. Man. N.Z. Moll. 28. campbelli, Filhol. Mission, Ile Campbell, 52 I. obscura, Plate. Zool. Jahrb. Anat. vii. 207. 
Family ATHORACOPHORIDE.

Athoracophorus, Gould, $1852=$ Janella, Gray, 1850 .

bitentaculatus, Quoy and Gaimard. P.L.S.N.S.W. 2, vii. 66 I ; P.Mal.S. ii. 247.

antipodum, Gray. P.Mal.S. ii. 248.

papillatus, Hutton. P.Mal.S. ii. 25 I.

marmoreus, Hutton. P.Mal.S. ii. 255.

marmoratus, v. Martens. Nova Acta, etc. liv. 7 I.

verrucosus var. nigricans, v. Martens. Nova Acta, etc.

liv. 77 .

verrucosus var. fasciata, v. Martens. Nova Acta, etc.

liv. 79 .

dubius, Cockerell. P.Mal.S. ii. 249.

simrothi, Suter. P.Mal.S. ii 34 .

dendyi, Suter. P.Mal.S. ii. 253 .

schauinslandi, Plate. Zool. Jahrb. Anat. xi. 193-269.

Family TORNATELLINIDE.

Tornatellina, Beck, 1837 .

novoseelandica, Pfeiffer. P.L.S.N.S.W. 2, vii. 660.

\section{Family Bulimulide.}

Placostylus, Beck, I 837 .

hongii, Lesson. P.L.S.N.S.W. 2, vii. 632 ; Man. Conch. 2, xiii. 22.

var. novoseelandica, Pfeiffer. P.L.S.N.S.W. 2, vii. 632 ; Man. Conch. 2, xiii. 24.

var. candida, Crosse. P.L.S.N.S.W. 2, vii. 632; Man. Conch, 2, xiii. 25.

\section{Family Patulide.}

Genus Endodonta, Albers, i 850.

Charopa, Albers, 1860.

coma, Gray. P.L.S.N.S.W. 2, vii. 653; Man. Conch. 2, iii. 22.

var. globosa, Suter. P.L S.N.S.W. 2, vii. 654 .

, multicostata, Murdoch. P.Mal.S. ii. I6r.

egesta, Gray. P.L.S.N.S.IV. 2, vii. 659; Man. Conch. 2, iii. 23 .

buccinella, Reeve. P.L.S.N.S.W. 2, viii. 497; Man.

Conch. 2, viii. $98 ; E$. sylvia. Hutton, T.N.Z.I. xvi. I 75 .

var. serpentinula, Suter. P.L.S.N.S.W. 2, vii. 657.

anguiculus, Reeve. P.L.S.N.S.W. 2, vii. 655; Man.

Conch. 2. iii. 23.

var. montivaga, Suter. P.L.S.N.S.W. 2, viii. 498.

„ fuscosa, Suter. T.N.Z.I. xxvi. I 35.

corniculum, Reeve. P.L.S.N.S.IV. 2, vii. 655; Man.

Conch. 2, iii. 24 . 
Charopa var. maculata, Suter. T.N.Z.I. xxiii. 89; Man. Conch. 2, viii. 96.

infecta, Reeve. P.L.S.N.S.W. 2, vii. 657 ; Man. Conch. 2, iii. 23.

var. irregularis, Suter. T.N.Z.I. xxii. 224 ; Man. Conch. 2 , viii. 98 .

var. alpestris, Suter. T.N.Z.I. xxiii. 88 ; Man. Conch. 2, viii. 99 .

caput-spinulæ, Reeve. P.L.S.N.S.W. 2, vii. 659; Man. Conch. 2, iii. 102.

biconcava, Pfeiffer. P.L.S.N.S.W. 2, vii. 658; Man. Conch. 2, i. I30.

modicella, Férussac, var. vicinalis, Mousson. Journ. de Conch. xxi. I I 2 ; Man. Conch. 2, iii. 39.

bianca, Hutton. P.L.S.N.S.IV. 2, vii. 655 ; Man. Conch. 2, viii. 97 .

var. montana, Suter. T.N.Z.I. xxiii. 88 ; Man. Conch. 2, viii. 97 .

tapirina, Hutton. P.L.S.N.S.W. 2, vii. $65^{6}$; Man. Conch. 2 , iii. 23 .

colensoi, Suter. P.L.S.N.S.IV. 2, vii. 657 ; Man. Conch. 2, viii. 99 .

variecostata, Suter. T.N.Z.I. xxii. 225; Man. Conch. 2, viii. 100.

subantialba, Suter. T.N.Z.I. xxii. 226 ; Man. Conch. 2, viii. I04.

huttoni, Suter. T.N.Z.I. xxii. 226 ; Man. Conch. 2, viii. r 04. moussoni, Suter. T.N.Z.I xxii. 227 ; Man. Conch. 2, viii. I05. sterkiana, Suter. T.N.Z.I. xxiii. 85 ; Man. Conch. 2, viii. Ior. var. reeftonensis, Suter. T.N.Z.I. xxiv. 294 ; Man. Conch. 2, viii. IO2.

var. major, Suter. T.N.Z.I. xxiv. 295.

brouni, Suter. T.N.Z.I. xxiii. 86; Man. Conch. 2 viii. 102. eremita, suter. T.N.Z.I. xxiii. 87 ; Man. Conch. 2, viii. 103. mutabilis, Suter. T.N.Z.I. xxiii. 84 ; Man. Conch. 2, viii. Iо r. pseudocoma, Suter. P.L.S.N.S.W. 2, viii. 495.

segregata, Suter. P.L.S.N.S.IV. 2, viii. 496.

prestoni, Sykes. P.Mal.S. i. 218.

roseveari, Suter. P.Mal.S. ii. 34 .

titirangiensis, Suter. P.Mal.S. ii. 35 .

vortex, Murdoch P.Mal.S. ii. I60. otagoensis, Suter. P.Mal.S. iii. 286.

subinfecta, Suter. P.Mal.S. iii. 286.

Eschrodomus, Pilsbry, I892.

stipulata, Reeve. P.L.S.N S.IV. 2, vii. 659 ; Man. Conch. 2, iii. 94 .

barbatula, Reeve. P.L.S.N.S.IV. 2, vii. 66o; Man. Conch. 2 , iii. 95 . 
Phenacharopa, Pilsbry, I893.

novoseelandica, Pfeiffer. P.L.S.N.S.W. 2, vii. 660.

Thaumatodon, Pilsbry, I893.

varicosa, Pfeiffer. P.L.S.N.S.W. 2, viii. 493 ; Man. Conch.

2, iii. 23 ; T.N.Z.I. xxvi. I 28.

tau, Pfeiffer. P.Mal.S. iii. 32 I ; Man. Conch. 2. viii. 84 .

jessica, Hutton. P.L.S.N.S.W. 2, vii. 65I ; Man. Conch. 2 , viii. 85 .

cryptobidens, Suter. T.N.Z.I. xxiii. 89 ; Man. Conch. 2, viii. 85 .

Ptychodon, Ancey, I 888.

leiodon, Hutton. T.N.Z.I. xv. I 35 ; Man. Conch. 2 , viii. 87 . pseudoleiodon, Suter. T.N.Z.I. xxii. 221 ; Man. Conch. 2 , viii. 88.

wairarapa, Suter. T.N.Z.I. xxii. 222 ; Man. Conch. 2, viii. 88. hectori, Suter. P.L.S.N.S.W. 2, vii. $65_{2}$; Man. Conch, 2, viii. 89 .

microundulata, Suter. T.N.Z.I. xxii. 223; Man. Conch. 2 , viii. 89 .

aorangi, Suter. T.N.Z.I. xxii. 223; Man. Conch. 2, viii. 90. hunuaensis, Suter. P.L.S.N.S.W. 2, viii. 494.

ureweraensis, Suter. Journ. Malac. vii. 53 .

Laoma, Gray, 1850.

Family LaOMide.

leimonias, Gray. P.L.S.N.S.W. 2, vii. 646; Man. Conch. 2 , iii. 68 .

pœcilosticta, Pfeiffer. P.L.S.N.S.W. 2, vii. 646; Man. Conch. 2, iii. 68.

marina, Hutton. P.L.S N.S.W. 2, vii. 646 ; Man. Conch. 2 , viii. 57 .

pirongiaensis, Suter. P.L.S.N.S.W. 2 , viii. $49 \mathrm{I}$.

ciliata, Suter. T.N.Z.I. xxvi. I 36.

elegans, Suter. P.Mal.S. ii. 35 .

Phrixgnathus, Hutton, I 883.

mariæ, Gray. P.L.S.N.S.W. 2, vii. 647; Man. Conch. 2 , iii. 37. erigone, Gray. P.L.S.N.S.W. 2, vii. 648 : Man. Conch. 2, iii. 37 .

glabriuscula, Pfeiffer. P.L.S.N.S.W. 2, vii. 650; Man.

Conch. 2, iii. 37.

regularis, Pfeiffer. P.L.S.N.S.W. 2, vii. 648 ; Man. Conch. 2 , iii. 37 .

sciadium, Pfeiffer. P.L.S.N.S.W. 2, vii. 650.

fatua, Pfeiffer. P.L.S.N.S.IV. 2, vii. 650.

conella, Pfeiffer. P.L.S.N.S.W. 2, vii. 647 ; Man. Conch.

2 , viii. 58 . 
Phrixgnathus campbellica, Filhol. P.L.S.N.S.W. 2 , vii. 649. ariel, Hutton. P.L.S.N.S.W. 2, vii. 647 ; Man. Conch, 2, viii. 59.

marginata, Hutton. P.L.S.N.S.W. 2, vii. 647; Man. Conch. 2, viii. 60.

celia. Hutton. P.L.S.N.S.W. 2, vii. 648; Man. Conch. 2, viii. 60 .

pumila, Hutton. P.L.S.N.S.W. 2, vii. 648 ; Man. Conch. 2 , viii. 63 .

phrynia, Hutton. P.L.S.N.S.W. 2, vii. 649: Man. Conch. 2, viii. 61 ; $L$. acanthinulopsis, Suter, P.L.S.N.S.W. 2, vii. 650 . var. major, Suter. P.Mal.S. ii. 259.

titania, Hutton. P.L.S.N.S.W. 2, vii. 650; Man. Conch. 2 , viii. 62 .

haasti, Hutton. P.L.S.N.S.W. 2, vii. $65^{\circ}$; Man. Conch. 2, viii. 62.

microreticulata, Suter. T.N.Z.I. xxii. 227 ; Man. Conch. 2 , viii. 63 .

raricostata, Suter. T.N.Z.I. xxii. 2 26; Man.Conch. 2, viii. I 00. allochroida, Suter. T.N.Z,I. xxii. 228 ; Man. Conch. 2, viii. 63. var. sericata, Suter. T.N.Z.I. xxii. 228 ; Man. Conch. 2, viii. 64 .

var. lateumbilicata, Suter. T.N Z.I. xxii. 228 ; Man. Conch. 2, viii. 64 .

transitans, Suter. T.N.Z.I. xxiv. 297; Man. Conch, 2, viii. 59. murdochi, Suter. T.N.Z.I. xxvi. I 36 .

cheesemani, Suter. T.N.Z.I. xxvi. I37.

lucida, Suter. P.Mal.S. ii. 36.

var. elevata, Suter. P.Mal.S. ii. 36.

spiralis, Suter. P.Mal.S. ii. 36.

moellendorffi, Suter. P.Mal.S. ii. 36 .

sublucida, Suter. P.Mal.S. ii. 37.

hamiltoni, Suter. P.Mal.S. ii. 37 .

Family Phenacohelicide.

Flammulina, v. Martens, I 873 .

zebra, Le Guillon. P.L.S.N.S.W. 2, vii. 643 ; Man. Conch. 2, i. I 28.

chiron, Gray. P.L.S.N.S.W. 2, vii. 644; Man. Conch. 2 , i. I 29. compressivoluta, Reeve. P.L.S.N.S.W. 2, vii. 643; Man.

Conch. 2, i. 128.

crebriflammis, Pfeiffer. P.L.S.N.S.W. 2, vii 644; Man. Conch. 2, i. I30 (not infundibulum, H. and J.).

alpina, Suter. Cornen-fulva, Suter, not of Pfeiffer, which is Hyalinia cellaria, Mueller, an introduced species. P.L.S. N.S.W. 2, vii. 644 ; Man. Conch. 2, viii. 76 .

cornea, Hutton. P.L.S.N.S.W. 2, vii. 643; Man. Conch.

2, viii. 75 . 
Flammulina jacquenetta, Hutton. P.L.S.N.S.W. 2, vii. 643 ; Man. Conch. 2, viii. 76.

perdita, Hutton. P.L.S.N.S.W. 2, vii. 644; Man. Conch. 2, viii. 76 .

costulata, Hutton. P.L.S.N.S.W. 2, vii. 645 ; Man. Conch. 2, viii. 73 .

feredayi, Suter. P.L.S.N.S.W. 2, vii. 645 ; Man. Conch. 2, viii. 74 .

var. glacialis, Suter. T.N.Z.I. xxiii. 92.

olivacea, Suter. P.L.S.N.S.W. 2, vii. 645 ; Man. Conch. 2 , viii. 75 .

pilsbryi, Suter. T.N.Z.I. xxvi. I33.

Suteria, Pilsbry, 1892.

ide, Gray. P.L.S.N.S.W. 2, vii. 642 ; Man. Conch. 2, ii. 2 10.

Phenacohelix, Suter, 1892.

pilula, Reeve. P.Mal.S. ii. 284 ; Man. Conch. 2, ii 2 I 2.

chordata, Pfeiffer. P.L.S.N.S.W. 2, vii. 642.

lucetta, Hutton. P.L.S.N.S.IV. 2, vii. 654 ; P. Mal. S. iii.

323.

perplexa, Murdoch. P.Mal.S. ii. I6 I.

ponsonbyi, Suter. P.Mal.S. ii. 285.

var. unicolor, Suter. T.N.Z.I. xxvi. I34.

Therasia, Hutton, $188_{3}$.

thaisa, Hutton. P.L.S.N.S.W. 2, vii. 64I ; Man. Conch. 2, viii. 70 .

celinde, Gray. P.L.S.N.S.W. 2, vii. 640; Man. Conch. 2, ii. $2 \mathrm{I}$.

ophelia, Pfeiffer. P.L.S.N.S.IV. 2, vii. 640; Man. Conch. 2, ii. 2 I .

decidua, Pfeiffer. P.L.S.N.S.W. 2, vii. 64I ; Man. Conch. 2 , viii. $7 \mathrm{I}$.

valeria, Hutton. P.L.S.N.S.W. 2, vii. 640; Man. Conch. 2, viii. 69 .

tamora, Hutton. P.L.S.N.S.W. 2, vii. 64I ; Man. Conch. 2 , viii. 70 .

traversi, E. A. Smith. P.L.S.N.S.W. 2, vii. 64I ; Man. Conch. 2, ii. 2 I 4.

Pyrrha, Hutton, I884.

cressida, Hutton. P.L.S.N.S.W. 2, vii. 640; Man. Conch. 2 , viii. 72 .

subincarnata, Suter. T.N.Z.I. xxvi. I33.

virescens, Suter. P.Mal.S. iii. 287.

Allodiscus, Pilsbry, 1892 .

dimorpha, Pfeiffer. P.L.S.N.S.IV. 2, vii. 638; Man. Conch. 2, ii. 2 I I. 
Allodiscus tullia, Gray. P.L.S.N.S.W. 2, vii, 638.; Man. Conch. 2, ii. 211.

granum, Pfeiffer. P.L.S.N.S.W. 2, vii. 43 ; miranda, Hutton, T.N.Z.I. xvi. 180; Man. Conch. 2, ii. 212 ; viii. 68. venulata, Pfeiffer. P.L.S.N.S.W. 2, vii. 638 . cassandra, Hutton. P.L.S.N.S.W. 2, vii. 638 ; Man. Conch. 2, viii. 66 .

planulata, Hutton. P.L.S.N.S.W. 2, vii. 638 ; Man. Conch. 2 , viii. 67 .

adriana, Hutton. P.L.S.N S.W. 2, vii. 639; Man. Conch. 2 , viii. 67 .

godeti, Suter. P.L.S.N.S.W. 2, vii. 639; Man. Conch. 2, viii. 68 .

wairoaensis, Suter. P.L.S.N.S.IV. 2, viii. 488.

urquharti, Suter. P.L.S.N.S.W. 2, viii. 489 .

smithi, Suter. T.N.Z.I. xxvi. I 34 .

rustica, Suter. T.N.Z.I. xxvi. I35.

chion, Sykes. P.Mal.S. ii. Io7.

mossi, Murdoch. P.Mal.S. ii. I62.

Gerontia, Hutton, 1883 .

pantherina, Hutton. P.L.S.N.S W. 2, vii. 637; Man.

Conch. 2 , viii. 65 .

cordelia, Hutton. P.L.S.N.S.W. 2, vii. 637 ; Man. Conch. 2, viii. 66.

Carthæa, Hutton, I 884 .

kivi, Gray. P.L.S.N.S.W. 2, vii. 633; Man. Conch. 2, iii. 37 .

Thalassohelix, Pilsby, 1892 .

zealandiæ, Gray. P.L.S.N.S.W. 2, vii. 637 ; Man. Conch.

2 , ii. 2 I 4 .

var antipoda, Hombron and Jacquinot. P.L.S.N.S.W. 2, vii. 637 : Man. Conch. 2, ii. 214.

aucklandica, Le Guillon. P.L.S.N.S.W. 2, vii. 637 .

ziczac, Gould. P.L.S.N.S.W. 2, vii. 635; Man. Conch. 2, i. 252 ; viii. 69 .

igniflua, Reeve. P.L.S.N.S.W. 2, vii. 636; Man. Conch. 2 , i. 129.

var. obnubila, Reeve. P.L.S.N.S.W. 2, vii. 636 .

propinqua, Hutton. P.L.S.N.S.W. 2, vii. 636 ; Man. Conch. 2 , viii. 72 .

Phacussa, Hutton, 1883 .

helmsi, Hutton. T.N.Z.I. xv. $137,138$.

var. maculata, Hutton. T.N.Z.I. xvi. 205.

hypopolia, Pfeiffer. P.L.S.N.S.IV. 2, vii. 635 ; Man. Conch.

2 , ii. I 8 I, 2 I 2

fulminata, Hutton. T.N.Z.I. xv. I 38 . 
Phacussa fulminata, var. costata, Suter. P.Mal.S. iii. 288. henryi, Suter. P.Mal.S. iii. 289.

Family LiMACıDE.

Helicarion, Férussac fils, 1822.

kermadecensis, E. A. Smith. P.L.S.N.S.W. 2, vii. 633;

Man. Conch. 2, i. 158.

Otoconcha, Hutton, i 884.

dimidiata, Pfeiffer. P.L.S.N.S.W. 2, vii. 634; Man. Conch. 2, i. 181 .

Xesta, Albers, 1850.

novaræ, Pfeiffer. P.L.S.N.S.IV. 2, vii. 644; T.N.Z.I. xxvi. I30.

Macrochlamys, Benson, 1832 (s.str.).

kermadeci, Pfeiffer. P.L.S.N.S.I. 2, vii. 634 .

Medyla, Albers, 1860 (Coneuplecta).

exposita, Mousson. P.L.S.N.S.IV. 2, vii. 634 ; Man. Conch. 2 , ii. 47 .

Rhytida, Albers, I 860.

Family RHYTID1DÆ.

greenwoodi, Gray. P.L.S.N.S.W. 2, vii. 630; Man. Conch. 2, i. 126 ; P.Mal.S. iv. 166.

dunniæ, Gray. P.L.S.N.S.W. 2, vii. 630; Man. Conch. 2, i. 126 .

var. beta, Pfeiffer. P.L.S.N.S.IV. 2, vii. 630; Man. Conch. 2, i. 126.

patula, Hutton. P.L.S.N.S.W. 2, vii. 630 .

citrina, Hutton. P.S.L.N.S.W. 2, vii. 631.

australis, Hutton. P.L.S.N.S.IV. 2 , vii. 63 I.

meesoni, Suter. P.L.S.N.S.W. 2, vii. 631 ; P.Mal.S. iv. 168.

Rhenea, Hutton, 1893 .

coresia, Gray. P.L.S.N.S.IW. 2, vii. 63I; Man. Conch. 2, i. I 30 ; P.Mal.S. iv. I 68.

jeffreysiana, Pfeiffer. P.L.S.N.S.IW. 2, vii. 63I ; Man.

Conch. 2, i. 129.

Paryphanta, Albers, 1850 .

busbyi, Gray. P.L.S.N.S.W. 2, vii. 629; Man. Conch. 2,

i. 127.

urnula, Pfeiffer. P.L.S.N.S.W. 2, vii. 630; Man. Conch. 2, i. 128.

hochstetteri, Pfeiffer. P.L.S.N.S.W. 2, vii. 629; Man.

Conch. 2, i. I 27 ; P.Mal.S. i. 5, iv. I7 r ; A.M.N.H. 7, vii. 68 ; Zool. Jahrb. xiv. 369.

gilliesi, E. A. Smith. P.L.S.N.S.W. 2, vii. 629; Man.

Conch. 2, i. I 27.

lignaria, Hutton. T.N.Z.I. xx. 43 ; xxxii. 22.

edwardi, Suter. P.Mal.S. iii. 290 ; A.M.N.H. 7 , vii. 70. 
Schizoglossa, Hedley, I893.

novoseelandica, Pfeiffer. P.L.S.N.S.IV. 2, vii. 628 ; P.Mal.S. iv. 169 ; A.M.N.H. 7 , vii. 71 .

\section{Sub-order Basommatophora.}

Family L1MNÆIDÆ.

Sub-family PlanORBINÆ.

Planorbis, (Guettard) Geoffroy, I 767.

corinna, Gray. P.L.S.N.S.W. 2, vii. 626.

Isidora, Ehrenberg, I831.

variabilis, Gray. P.L.S.N.S.IV. 2, vii. 627 ; gibbosa, Hutton;

T.N.Z.I. xiv. I 55, not of Gould; noveseelandice, Clessin,

Conch. Cab. 2, i. pt. 17, 372.

tabulata, Gould. P.L.S.N.S.W. 2, vii. 627 ; coromandelicus,

Dunker, Mal.Blätt. ix. I 50 ; hochstetteri, Dunker, l.c. I 50. antipodea, Sowerby. P.L.S.N.S.IW. 2, vii. 627 .

Sub-family LimNÆinÆ.

Limnæa, Lamarck, i80 I.

tomentosa, Pfeiffer. P.L.S.N.S.W. 2, vii. 625 .

leptosoma, Hutton. T.N.Z.I. xvii. 55 .

tenella, Hutton. T.N.Z.I. xvii. 55, $5^{6}$.

pusilla, Hutton. T.N.Z.I. xxii. 56.

tenisoni, Clessin, Conch. Cab. 2, i. pt. I 7,37 r.

var. alfredi, Suter. T.N.Z.I. xxii. 229 ; xxiv. 300.

Amphipeplea, Nilsson, I822.

arguta, Hutton. T.N.Z.I. xvii. 54.

ampulla, Hutton. T.N.Z.I. xvii. 55 .

var. globosa, Suter. T.N.Z.I. xxiii. 93.

Sub-family Ancylinæ.

Gundlachia, Pfeiffer, I 847.

- sp. T.N.Z.I. xxvi. 122.

Latia, Gray. $185^{\circ}$.

neritoides, Gray. P.L.S.N.S.W. 2, vii. 624 .

var. lateralis, Gould. P.L.S.N.S.IV. 2, vii. 624 .

var. petitiana, Fischer. Jour. de Conch. v. 84 .

var. gassiesiana, Fischer. Jour. de Conch. v. 167.

Family AMPHIBOLIDÆ.

Amphibola, Schumacher, I8I 7 .

crenata, Martyn, Man. N.Z. Moll. 35 ; A.M.N.H. 5 , iii. I8I ; avellana, Bruguière, $\mathrm{I} 789$.

Family AURICULID瓜.

Tralia, Gray, i 840 (Ophicardelus).

australis, Quoy and Gaimard. Man. N.Z. Moll. 34. 
Marinula, King, I8 32 .

filholi, Hutton. Man. N.Z. Moll. 34.

Leuconopsis, Hutton, I 884 .

obsoleta, Hutton. Man. N.Z. Moll. 34 ; P.L.S.N.S.W. xxv.

pl. xlviii. fig. I 6 .

Order OpISTHOBRANCHIA.

Sub-order Nudibranchia.

Archidoris, Bergh. 1892 .

Family DORIDIDE.

tuberculata, Cuvier. Man. N.Z. Moll, I25.

Doris, Linnæus, $175^{8}$.

punctata, Quoy and Gaimard. Man. N.Z. Moll. I25.

granulosa, Abraham. Man. N.Z. Moll. 126.

longula, Abraham. Man. N.Z. Moll. I 26.

muscula, Abraham. Man. N.Z. Moll. I 26.

lanuginata, Abraham. Man. N.Z. Moll. I 7 .

wellingtonensis, Abraham. Man. N.Z. Moll. I27.

rubicunda, Cheeseman. T.N.Z.I. xiii. 222.

flabellifera, Cheeseman. T.N.Z.I. xiii. 222.

luctuosa, Cheeseman. T.N.Z.I. xiv. 2 I 3.

Chromodoris, Alder and Hancock, 1855.

aureo-marginata, Cheeseman. T.N.Z.I. xiii, 223.

amœna, Cheeseman. T.N.Z.I. xviii. I 37.

Doriopsis, Pease, r 860.

Family DORIOPSIDE.

mammosa, Abraham. P.Z.S. 1877,266 ; T.N.Z.I. xiv. 214.

citrina, Cheeseman. T.N.Z.I. xiii. 223.

Family GONIODORIDE.

Acanthodoris, Gray, I850.

mollicella, Abraham. Man. N.Z. Moll. i28.

globosa, Abraham. Man. N.Z. Moll. I28.

Atagena, Gray, 1850 .

carinata, Quoy and Gaimard. Man. N.Z. Moll. I 28.

Family POLYCERIDe.

Euplocamus, Philippi, I 836 .

pacificus, Bergh. Challenger Reports, x. 57 .

Family ÆOLIDIDE.

Eolis, Cuvier, I 798.

gracilis, T. W. Kirk. T.N.Z.I. xv. 2 I 7.

leptosoma, Hutton. T.N.Z.I. xvi. 2 I 3. 
Fiona, Hancock and Embleton, 1853.

marına, Forskål. EEolis plicata. Hutton, T.N.Z.I. xiv. 166; Bergh, Opisthobranches Campagnes Hirondelle, 6-8, Pl. i. figs. 7-1 6 .

Eolidiella, Bergh, I892.

drusilla, Bergh. Erg. Reise Pacific, 233.

faustina, Bergh. L.c. 235 .

Facelina, Alder and Hancock, 1855 .

longicauda, Quoy and Gaimard. Man. N.Z. Moll. 129.

Cratena, Bergh. 1864.

corfei, Hutton. T.N Z.I. xiii. 203 ; xiv. 166.

Family Hermeide.

Stiliger, Ehrenberg, I 83 I. Calliopcea, d'Orbigny, 1837 .

felina, Hutton. T.N.Z.I. xv. I $18,133$.

Sub-order Tectibranchia.

Family SIPHONARIIDE.

Siphonaria, Sowerby, 1824 .

obliquata, Sowerby. T.N.Z.I. xv. I4I.

australis, Quoy and Gaimard. T.N.Z.I. xv. 142 ; A.M.N.H. 5, ix. $34 \mathrm{I}$.

zelandica, Quoy and Gaimard. T.N.Z.I. xv. 143 .

tristensis (Leach), Sowerby. S. redimiculum, Reeve.

T.N.Z.I. Xv. 143 .

Gadinia, Gray, 1824 .

nivea, Hutton T.N.Z.I. xv. I 44.

Family Pleurobranchide.

Pleurobranchus, Cuvier, 1804 .

ornatus, Cheeseman. P.Z.S. 1878,275 ; T.N.Z.I. xi. 378 ;

Man. Conch. I, xvi. 206.

aurantiacus, Risso. Zool. Jahrb. Syst. xiii. 2 ro; Man.

Conch. I, xvi. I 95 .

Pleurobranchæa, Meckel, i 8 I 3 .

maculata, Quoy and Gaimard. Vny. Astrol. ii. 301 ; Man.

Conch. I, xvi. 227.

novæ-zealandiæ, Cheeseman. P.Z.S. 1878,276 ; T.N.Z I.

xi. 378 ; Man. Conch. 1, xvi. 227.

var. granulosa, Bergh. Zool. Jahrb. Syst. xiii. 209.

Tethys, Linnæus, I $75^{8}$.

Family APLysIIDE.

brunnea, Hutton. T.N.Z.I. vii. 279 ; Man. Conch. I. xvi.

97.

venosa, Hutton. T.N.Z.I. vii. 279; Man. Conch, r, xvi. 98. 
Tethys tryoni, Meinertzhagen. T.N.Z.I. xii. 270 ; Man. Conch. I, xvi. 16, 98 .

hamiltoni, 'T. W. Kirk. T.N.Z.I. xiv. 283 ; Man. Conch. I, xvi. 98 .

Notarchus, Cuvier, I 8 I 7 .

glaucus, Cheeseman. P.Z.S. 1878,277 ; T.N.Z.I. xi. 379; Man. Conch. I, xvi. 146

Aglaja, Renier, I804.

$$
\text { Family AGLAJ1De. }
$$

cylindrica, Cheeseman. T.N.Z.I. xiii. 224 ; Man. Conch. I, xvi. 49.

Philine, Ascanius, 1772.

Family PhiLinida.

aperta, Linnæus. P. angasi, Crosse and Fischer. Man. N.Z. Moll. I 23 ; Man. Conch. I, xvi. 8.

Family AKERATIDÆ.

Akera, O. F. Mueller, 1776.

tumida, A. Adams. Man. N.Z. Moll. 122 ; Man. Conch. r, xv. 379 .

Haminea, Leach, I 847 .

zelandiæ, Gray. Man. N.Z. Moll. I 2 I; Man. Conch. I, xv. 373 .

cuticulifera, E. A. Smith. Man. N.Z. Moll. 122 ; Man. Conch. $1, x v \cdot 372$.

? castanea, A. Adams. Sow. Thes. ii. 584 ; Man. Conch. I, Xv. 374 .

Bulla, Linnæus, $175^{8}$. Family BULLIDE.

australis (Gray), Quoy and Gaimard. P.L.S.N.S.W. I, x. 89 ; Man. Conch. I, xv. 346.

quoyi, Gray. Man. N.Z. Moll. 2 I ; Man. Conch. I, Xv. 348.

Cylichna, Lovén, 1846 .

Family SCAPHANDRID

arachis, Quoy and Gaimard. Voy. Astrol. ii. 361 ; Man. Conch. I, Xv. 318.

striata, Hutton. Man. N.Z. Moll. I20; Man. Conch. I, xv. 319.

Actæon, Montfort, i 8 ro.

Family ACTÆONIDÆ.

kirki, Hutton. Man. N.Z. Moll. I I9; Man. Conch. I. xv. 146.

Solidula, Fischer de Waldheim, r 807 .

alba, Hutton. Man. N.Z. Moll. I 9 ; Man. Conch. I xv. 146. 
Solidula gracilis, T. W. Kirk. T.N.Z.I. xiv. 268 ; Man. Conch. I, Xv. 146.

huttoni, T. W. Kirk. T.N.Z.I. xiv. 268 ; Man. Conch. I, xv. 147 .

Leucotina, A. Adams, I 860.

pura, A. Adams. Man. Conch. I, xv. I 72.

Bullinula, Beck, 1840. Bullina, Adams, non Férussac.

scabra, Gmelin. Man. N.Z. Moll. I 20 ; Man. Conch. r, xv. I $76 ;$ B. lineato, Wood.

Family TORNATINID瓜.

Tornatina, A. Adams, I $85^{\circ}$.

pachys, Watson. Challenger Reports, xv. 660; Man. Conch. I, xv. I 98 .

Family CaVolinid «.

Cavolinia, Abildgaard, r 79 r.

tridentata, Forskål. Austral. Mus. Sydney, Cat. xv. 38 .

Sub-class STTREPTONEURA.

Sub-order HETEROPODA.

Family PTEROTRACH位E.

Carinaria, Lamarck, I80 I.

australis, Quoy and Gaimard. Voy. Astrol. ii. 394.

Sub-order Platypoda.

a. Toxoglossa.

Family TEREBRIDÆ.

Terebra, Bruguière, I 789 .

tristis, Deshayes. P.L.S.N.S.IV. I, X. I 18 ; Man. Conch. I, vii. 36 .

Family Conide.

Sub-family PLeURotominÆ.

Pleurotoma, Lamarck, 1822.

ischna, Watson. T.N.Z.I. xxxi. 65 .

Genotia, H. and A. Adams, 1853 .

engonia, Watson. T.N.Z.I. xxxi. 65 .

Drillia, Gray, 1838 .

lævis, Hutton. T.N.Z.I. xxxi. 66.

amœna, E. A. Smith. 'T.N.Z.I. xxxi. 66.

Spirotropis, Sars, 1878 .

bulbacea, Watson. T.N.Z.I. xxxi. 67 .

Sub-family Clavatuline.

Surcula, H. and A. Adams, r 853 .

novæ-zelandiæ, Reeve. T.N.Z.I. xxxi. 67 ; Man. Conch.

I, vi. I 84 . 
Surcula trailli, Hutton. T.N.Z.I. xxxi. 68; Man. Conch. I, vi. 206,208 . albula, Hutton. T.N.Z.I. xxxi. 68. cheesemani, Hutton. T.N.Z.I. xxxi. 69. gypsata, Watson. T.N.Z.I. xxxi. 69. varians, Hutton. 'T.N.Z.I. xxxi. 69. verrucosa, Suter. T.N.Z.I. xxxi. 70.

Mangilia (Leach), Risso, I 826.

Sub-family MangILIINe. ula, Watson. T.N.Z.I. xxxi. 7 I. goodingi, E. A. Smith. T.N.Z.I. xxxi. 71 . dictyota, Hutton. T.N.Z.I. xxxi. 72 . subaustralis, Suter. T.N.Z.I. xxxi. 72. flexicostata, Suter. T.N.Z.I. xxxi. 73 .

Clathurella, Carpenter, 1857 .

sinclairi, E. A. Smith. T.N.Z.I. xxxi. 73; Man. Conch. I, vi. $28_{3}$.

subabnormis, Suter. T.N.Z.I. xxxi. 74. nodicincta, Suter. T.N.Z.I. xxxi. 74 .

Daphnella, Hinds, I844.

lymneiformis, Kiener. T.N.Z.I. xxxi. 75 ; Man. Conch. I, vi. 300 .

lacunosa, Hutton. T.N.Z.I. xxxi. 75 . substriata, Suter. T.N.Z.I. xxxi. 76 . protensa, Hutton. T.N.Z.I. xxxi. 76; Man. Conch. I, vi. 208. membranacea, Watson. T.N Z.I. xxxi. 77.

xanthias, Watson. T.N.Z.I. xxxi. 77 .

Cancellaria, Lamarck, I822.

Family Cancellarilde.

trailli, Hutton. Man. N.Z. Moll 46.

b. RaChigLOSSA.

Family MURICIDæ.

Murex, Linnæus, I 758 .

zelandicus, Quoy and Gaimard. T.N.Z.I. xvi. 218 ; Man. Conch. I, ii. 108.

octogonus, Quoy and Gaimard. T.N.Z.I. xvi. 2 I 8 ; Man. Conch. I, ii. I IO.

var. espinosa, Hutton. T.N.Z.I. xviii. 333 .

var. umbilicata, J. Woods. Journ. Malac. viii. 6I.

angasi, Crosse. T.N.Z.I. xvi. 2 I9; Man. Conch. I, ii. 88 eos, Hutton. Cat. Mar. Moll. N.Z. 8.

Purpura, Bruguière, 1789 .

succincta, Martyn. T.N.Z.I. xvi. $22 \mathrm{I}$; Man. Conch. I, ii. I 70 ; P. textiliosa, Lamarck. T.N.Z.I. xvi. 22 I. 
Purpura striata, Martyn. T.N.Z.I. xvi. 22 I. var. squamata, Hutton. Man. N.Z. Moll. 56. scobina, Quoy and Gaimard. T.N.Z.I. xvi. 222; Man. Conch. I, ii. I 70 .

var. albomarginata, Deshayes. T.N.Z.I. xvi. 222. var. rutila, Suter. Journ. Malac. vii. 55 . haustrum, Martyn. T.N.Z.I. xvi. 222 ; Man. Conch. $r_{\text {, }}$ ii. 160 .

tritoniformis, Blainville. P. Roy. S. Vict. n.s. x. 275 ; Man. Conch. I, ii. 156.

Trophon, Montfort, 18 io.

stangeri, Gray. T.N.Z.I. xvi. 2 19; Man. Conch. I, ii. 147. duodecimus, Gray. T.N.Z.I. xvi. 220 ; Man. Conch. I, ii. 147 .

ambiguus, Philippi. T.N.Z.I.xvi. 2 I 9 ; Man. Conch. I, ii. I33. var. cretacea, Reeve. Conch. Icon. Fusus, sp. 48.

var. pumila, Suter. Journ. Malac. vii. 55 . patens, Hombron and Jacquinot. Man. N.Z. Moll. 57 ; Man. Conch. I, ii. I 77. paivæ, Crosse. Man. N.Z. Moll. 49; Man. Conch. I, ii. r55. plebejus, Hutton. T.N.Z.I. xvi. 220 ; Man. Conch. I, ii. r45. inferus, Hutton. T.N.Z.I. xvi. $22 \mathrm{I}$; Man. Conch. r, ii. I45. cheesemani, Hutton. T.N.Z.I. xvi. 220.

\section{Family Columbellide.}

Columbella, Lamarck, I 799.

choava, Reeve. T.N.Z.I. xvi. 223. Man. Conch. I, v. I37. huttoni, Suter. For sulcata, Hutton, Man. N.Z. Moll. 45, preoccupied.

$$
\text { Family NASSID E. }
$$

Nassa, Lamarck, I 799.

glans, Linnæus. P. Roy. S. Vict. n.s. x. 280 ; Man. Conch. I, iv. 27.

ephamilla, Watson. Challenger Reports, xv. I 87 .

dissimilis, Watson. Challenger Reports, xv. I 75.

Family Buccinides.

Siphonalia, A. Adams, I 863.

nodosa, Martyns. Cat. Tert. Moll. B.M. i. $15^{8}$; Man. Conch. 1, iii. 136 .

mandarina, Duclos. Cat. Tert. Moll. B.M. i. 157; Man.

Conch. I, iii. I 38 .

var. caudata, Quoy and Gaimard. Voy. Astrol. ii. 503 .

var. valedicta, Watson. Challenger Reports, xv. 20 I.

- dilatata, Quoy and Gaimard. P. Roy. S. Vict. n.s. x. 272 ;

Man. Conch. I, iii. I 35 . 
Cominella, Gray, 1847 .

maculata, Martyn. T.N.Z.I. xvi. 231; Man. Conch. I, iii. 204 .

maculosa, Martyn. T.N.Z.I. xvi. 231; Man. Conch. I, iii. 204 .

testudinea, Chemnit\%. 'I.N.Z.I. xvi. 231; Man. Conch. I, iii. 203.

lineolata, Lamarck. P. Roy. S. Vict. n.s. x. 275 ; Man. Conch. I, iii. 204.

nassoides, Reeve. T.N.Z.I. xvi. 232; Man. Conch. I, iii. 206; C. veneris, Filhol, Mission Ile Campbell, 525.

lurida, Philippi. T.N.Z.I. xvi. 233 ; Man. Conch. I, iii. 206. virgata, H. and $\Lambda$. $\Lambda$ dams. 'T.N.Z.I. xvi. $23^{2}$; Man. Conch. I, iii. 204 .

huttoni, Kobelt. T.N.Z.I. xvi. 232 ; Man. Conch. 1, iii. 205. campbelli, Filhol. Mission Ile Campbell, 524.

Euthria, Gray, 1850.

lineata, Martyn. T.N.Z.I. xvi. 229 ; Man. Conch. I, iii. 5 x. var. traversi, Hutton. T.N.Z.I. xvi. 229 ; Man. Conch. r, iii. 136.

var. pertinax, v. Martens. 'T.N.Z.I.xvi. 229 ; Man. Conch. I, iii. I 5 I.

vittata, Quoy and Gaimard. 'T.N.L.I. xvi. 230; Man. Conch. I, iii. $5^{2}$.

antarctica, Reeve. T.N.Z.I. xxvi. 231 ; Man. Conch, r, iii. 150 .

littorinoides, Reeve. T.N.Z.I. xvi. 230; Man. Conch. i, iii. $15 \mathrm{I}$.

striata, Hutton. T.N.Z.I. xvi. 230.

martensiana, Hutton. T.N.Z.I. xvi. 230 ; Man. Conch. ז, iii. $15 \mathrm{I}$.

flavescens, Hutton. T.N.Z.I. xvi. 230.

Pisania, Bivona, $18 \mathbf{3 2}^{2}$.

reticulata, A. Mdams. P.Z.S. 1854,$138 ;$ Man. Conch. I, iii. 147 .

Fusus, Lamarck, 1799.

Family Fasciolaridi.

spiralis, A. Adams. T.N.Z.I. xvi. 227.

Taron, Hutton, I 883 .

dubius, Hutton. T.N.Z.I. xvi. 227 ; Man. Conch. I, ii. $x 56$.

Mitra, Lamarck, 1799.

Family Mitride.

melaniana, Lamarck. P. Roy. S. Vict. n.s. x. 186; Man.

Conch. I, iv. 127.

obscura, Hutton. P.Mal.S. ii. 201.

albopicta, E. A. Smith. P.Mal.S. iii. 21. 
Vulpecula, Blainville, 1824 . Turricula, Klein. 1753. rubiginosa, Hutton. T.N.Z.I. xvi. 226. planata, Hutton. T.N.Z.I. xrii. 315.

Family VolUTID

Scaphella, Swainson, I $\$_{32}$. gracilis, Swainson. Cat. Tert. Moll. B.... i. II 4 ; Man. Conch. I, iv. 94. pacifica, Lamarck. Cat. Tert. Moll. B.M. i. I 3 ; Man.

Conch. 1, iv. 94 . var. elongata, Swainson. T.N.Z.I. xvi. 225. lutea, Watson. Challenger Reports, xv. 254

Family Marginellide.

Marginella, Lamarck, I80I. muscaria, Lamarck. P. Roy. S. Vict. n.s. X. $1 \$_{9}$; Man. Conch. I, r. 23. turbinata, Sowerby: Cat. Tert. Moll. B.M. i. $8_{7}$; Man. Conch. I, v. 23.

pygmæa, Sowerby. P. Roy. S. Vict. 11.s. xi. I91; Man. Conch. I, v: 26, 93. infans, Reere. T.N.Z.I. xvi. 224. mustelina, Angas. P.Z.S. I87 1 , I4.

Ancilla, Lamarck, 799.

$$
\text { Family OLIvid.玉. }
$$

australis, Sowerby. Cat. Tert. Moll. B.M. i. 76 ; Man. Conch. I, v. 94 . mucronata, Sowerby. Reeve, Conch. Icon. sp. Io; $A$. albisulcata, Quoy and Gaimard, V. Astrol. iii. 19. pyramidalis, Reeve. Conch. Icon. sp. II. bicolor, Gray. A. noz'e-zelandia, Sowerby: A. nana, Watson, Challenger Reports, xv. 230.

\section{c. GYinoglossa.}

Family Pyramidellid.

Pyramidella, Lamarck, i 799. rosea, Hutton. Man. N.Z. Moll. 72.

Eulimella, Forbes, is 46 . deplexa, Hutton. T.N.Z.I. xvii. $3 \mathbf{I} 8$.

Odontostoma, Fleming, 1828 . angasi, Tryon. Man. Conch. I, viii. 362 . impolita, Hutton. Man. N.Z. Moll. 8I. hyphala, Watson. Challenger Reports, xv. $4 \delta_{3}$.

Turbonilla, Leach, 1826 . zealandica, Hutton. Man. N.Z. Moll. 72. 


\section{d. TENIOGLOSSA.}

Lotorium, Montfort, I 8 ro.

Family LOTORIIDE.

olearium, Linnæus. P.L.S.N.S.W. I, ix. 932 ; Man. Conch. I, iii. II.

tritonis, Linnæus. P.L.S.N.S.IV. I, ix. 932; Man. Conch. I, iii. 9 .

spengleri, Chemnitz. P.L.S.N.S.W. I, ix. 933; Man. Conch. I, iii. I6.

cornutum, Perry=exaratum, Reeve. P. Roy. S. Vict. n.s. x. 265 ; Man. Conch. I, iii. 22.

rubicundum, Perry = nodiferum, Lamarck. P.L.S.N.S.W. I, ix. 932 ; Man. Conch. r, iii. ro.

Apollo, Montfort, 1810 .

argus, Gmelin. P. Roy. S. Vict. n.s. x. 267 ; Man. Conch. I, iii. 44 .

australasia, Perry. A. leucostomus, Lamarck. P. Roy. S. Vict. n.s. 268 ; Man. Conch. I. iii. 42.

Family CAssıdıD.E.

Semicassis, Mörch, I $8_{52}$.

labiata, Perry=achatina, Lamarck. P. Roy. S. Vict. n.s. xii. 189 ; Man. Conch. I. vii. 278.

pyrum, Lamarck. P. Roy. S. Vict. n.s. xii. I 89 ; Man. Conch. I, vii. $27 \mathrm{~S}$.

\section{Family Dolinde.}

Dolium, Lamarck, i $80 \mathrm{r}$.

variegatum, Lamarck. Man. N.Z. Moll. 65 ; Man. Conch. I, vii. 262 .

costatum, Menke. Man. Conch. I, vii. 263 .

Trivia, Gray, $183 z$.

Family CYPRÆIDÆ.

europæa, Montagu. P.L.S.N.S.W. r, ix. 935 ; Man. Conch. I, vii. 205.

australis, Lamarck. P. Roy. S. Vict. n.s. xii. 187: Man. Conch. I, vii. 206.

Family STRUTHIOLARIID،.

Struthiolaria, Lamarck, I $8 \mathbf{1} 6$.

papulosa, Martyn. Cat. Tert. Moll. B.M. i. 2 I9; Man. Conch. I, vii. I 33 . vermis, Martyn. Cat. Tert. Moll. B.M. i. 219; Man. Conch. i. vii. 133 .

tricarinata, Lesson. Man. N.Z. Moll. 68. 


\section{Family VERMICULARIDE.}

Tenagodes, Guettard, 1770.

cumingi, Mörch. P.Z.S. I860, 403 ; Man. Conch. I. viii. I 90.

weldii, J. Woods. P. Roy. S. Vict. n.s. xii. 205; Man. Conch. 1, viii. I 9 I.

australis, Quoy and Gaimard. P. Roy. S. Vict. n.s. xii. 204 ; Man. Conch. I, viii. I89.

Vermicularia, Lamarck, I 799; Vermetus, Adamson, 1757.

lamellosa, Hutton. Man. N.Z. Moll. 85 ; Man. Conch. I, viii. 184 .

zelandica, Quoy and Gaimard. P.Z.S. 1862, 82; Man. Conch. I, viii. 182 .

novæ-hollandiæ, Rousseau. P. Roy. S. Vict. n.s. xii. 204 ; Man. Conch. I, viii. I79; $V$. monilifera, Hutton, Plioc. Moll. N.Z. 66.

rosea, Quoy and Gaimard. Man. N.Z. Moll. 85; Man. Conch. I, viii. 185 .

\section{Family TURRITELLIDÆ.}

Turritella, Lamarck, I 799.

rosea, Quoy and Gaimard. Cat. Tert. Moll. B.M. i. 240; Man. Conch. I, viii. 199.

pagoda, Reeve. Conch. Icon. sp. 60; Man. Conch. 1, viii. 204.

sinuata, Reeve. Conch. Icon. sp. 62 ; Man. Conch. 1, viii. 200.

vittata, Hutton. Man. N.Z. Moll. 84; Man. Conch. I, viii. 208; T. carlottae, Watson, Challenger Reports, xv. 478.

fulminata, Hutton. Man. N.Z. Moll. 84 ; Man. Conch. I, viii. 208.

kanieriensis, Harris. Cat. Tert. Moll. B.M. i. $24 \mathrm{I} ; T$. tricincta, Hutton.

Family Melaninde.

Melanopsis, Férussac père, 1807. trifasciata, Gray. P.L.S.N.S.W. 2, vii. 6 r8.

\section{Family CerithHide.}

Planaxis, Lamarck, 1822.

mollis, Sowerby. P.L.S.N.S.W. 2, ii. 993; Man. Conch. I, ix. 279.

Triforis, Deshayes, ${ }^{8}{ }_{24}$.

gemmulatus, Adams and Reeve. Voy. Samarang, 46;

Man. Conch. I, ix. I8I.

angasi, Crosse. Man. N.Z. Moll. 75; Man. Conch. r, ix. 179. 
Cerithiopsis, Forbes and Hanley, $\mathbf{1} 849$.

cerea, Sowerby. Thes. Conch. ii. 880; Man. Conch. I, ix. I 77 .

terebelloides, v. Martens. Man. N.Z. Moll. 74; Man. Conch. I, ix. 158 .

Potamides, Brongniart, i8 8 o.

bicarinatus, Gray. Man. N.Z. Moll. 74 ; Man. Conch, 1, ix. 163 .

subcarinatus, Sowerby. P.L.S.N.S.W. I, ix. 937 ; Man. Conch. 1, ix. 163 .

alternatus, Hutton. Man. N.Z. Moll. 74 ; Man. Conch. 1, ix. 165 .

tricarinatus, Hutton. P.L.S.N.S.W. I, ix. 937 ; Man. Conch. I, ix. 165 .

Bittium (Leach), Gray, 1847 .

lawleyanum, Crosse. Journ. de Conch. 1863, 87; Man. Conch. I, ix. I 54 .

exile, Hutton. T.N.Z.I. xvi. 2 I 4 ; Man. Conch. I, ix. 158.

cylindricum, Watson. Challenger Reports, xv. 550.

Family Assınineidæ.

Assiminea, Leach, 1828 .

antipodum, Filhol. Mission Ile Campbell, 523.

Family HydrobıDÆ.

Potamopyrgus, Stimpson, I 865.

corolla, Gould. P.L.S.N.S.IV. 2, vii. 6 I 9.

antipodum, Gray. P.L.S.N.S.W. 2, vii. 6rg.

cumingiana, Fischer. P.L.S.N.S.W. 2, vii. 6 rg.

spelæa, Frauenfeld. Man. N.Z. Moll. 82.

pupoides, Hutton. T.N.Z.I. xiv. I 46.

Family Rissonde.

Rissoia, Fréminville, I 813.

huttoni, Suter, P.Mal.s. iii. 2.

hamiltoni, Suter. P.Mal.S. iii. 2.

incidata, Frauenfeld. P.Mal.S. iii. 3 ; Man. Conch. 1, ix. 339. annulata, Hutton. P.Mal.S. iii. 3 . var. minor, Suter. P.Mal.S. iii. 3 .

subfusca, Hutton. P.Mal.S. iii. 4 ; Man. Conch. r, ix. 344 . var. micronema, Suter. P.Mal.S. iii. 4.

tenisoni, Tate. $R$. australis, T. Woods, non Sow., P.Mal.S. iii. 4 ; P.L.S.N.S.W. xxv. pl. xxv. fig. 4 .

fumata, Suter. P.Mal.S. iii. 5 .

foveauxiana, Suter. P.Mal.S. iii. 5 .

lubrica, Suter. P.Mal.S. iii. 5. 
Rissoia cheilostoma, T. Woods. R. plicata, Hutton, non Desh., P.Mal.S. iii. 6; Man. Conch. I, ix. 86, 366. var. lyalliana, Suter. P.Mal.S. iii. 6 .

Rissoina, d'Orbigny, I 840. rugulosa, Hutton. P.Mal.S. iii. 6. fasciata, A. Adams. P.Mal.S. iii. 7 ; Man. Conch. I, ix. 380. olivacea, Hutton. P.Mal.S. iii. 7 ; Man. Conch. I, ix. 397.

Barleeia, Clark, I853. rosea, Hutton. P.Mal.S. iii. 8; Man. Conch. I, ix. 393. neozelanica, Suter. P.Mal.S. iii. 8.

Realia, Gray, $185^{\circ}$.

Family Realide.

egea, Gray. P.L.S.N.S.W. 2, vii. 622. var. albina, Suter. T.N.Z.I. xxiv. 275 . turriculata, Pfeiffer. P.L.S.N.S.W. 2, vii. 622. hochstetteri, Pfeiffer. P.L.S.N.S.IV. 2, vii. 623 . carinella, Pfeiffer. P.L.S.N.S.IV. 2, vii. 623.

Omphalotropis, Pfeiffer, I 854.

vestita, Pfeiffer. P.L.S.N.S.W. 2, vii. 622.

Family Diplommatinide.

Palaina, Semper, 1865 . chordata, Pfeiffer. P.L.S.N.S.W. 2, vii. 620.

Paxillus, H. and A. Adams, I85 I. peregrinus, Gould. P.L.S.N.S.W. 2, vii. 620.

\section{Family CyClOPHORIDE.}

Lagochilus, Blanford, 1864.

cytora, Gray. P.L.S.N.S.W. 2, vii. 621 . lignarius, Pfeiffer. P.L.S.N.S.W. 2, vii. 621 . pannosus, Hutton. P.L.S.N.S.W. 2, vii. 621 . calvus, Hutton. P.L.S.N.S.W. 2, vii. 621 . pallidus, Hutton. P.L.S.N.S.W. 2 , vii. 621 . hedleyi, Suter. P.L.S.N.S.W. 2, viii. 484 . torquillus, Suter. P.L.S.N.S.W. 2, viii. 485 . fasciatus, Suter. T.N.Z.I. xxvi. I 32. chiltoni, Suter. P.Mal.S. ii. 33 . studeri, Suter. P.Mal.S. ii. 33 .

Family LITORINIDE.

Litorina, Férussac, 1821 . mauritiana, Lamarck. P.L.S.N.S.W. I, ix. 942; Man. Conch. I, ix. 247. cincta, Quoy and Gaimard. P.L.S.N.S.W. I, ix. 94 I. 
Lævilitorina, Pfeiffer, I 886.

caliginosa, Gould, U.S. Expl. Exp. 198 ; Man. Conch. 1 , ix. 254 .

?hamiltoni, E. A. Snith. P.Mal.S. iii. 22. (Paludestrina.)

Risellopsis, Kesteven, I902.

varia, Hutton. Man. N.Z. Moll. 79; Man. Conch. r, ix. 255;

Kesteven, Rec. Australian Mus. iv. 320.

Risella, Gray, I 840 .

melanostoma, Gmelin. Man. N.Z. Moll. 79; Man. Conch. I, ix. 262.

Family SOLARIIDE.

Solarium, Lamarck, I 799 .

luteum, Lamarck. P. Roy. S. Vict. n.s. xiii. I 39; Man.Conch. I, ix. I6.

reevei, Hanley. modesta, Cooper, P.Z.S. r862, 204; Man.

Conch. I, ix. I 2.

Family HiPPONYCidæ.

Hipponyx, Defrance, I8I9.

? australis, Lamarck. P. Roy. S. Vict. n.s. xii. I98; Man.

Conch. I. viii. I 36 .

Family CaPulide.

Calyptræa, Lamarck, 1799 .

maculata, Quoy and Gaimard. V. Astrol. iii. 422 ; C.nova zealandice, Lesson, V. Coq. ii. 395; Man. N.Z. Moll. 86.

scutum, Lesson. Man. N.Z. Moll. 86; Man. Conch. 1, viii. 122.

Crepidula, Lamarck, 799.

aculeata, Gmelin. Cat.'Tert.Moll.B.M. i. 247 ; Man. Conch. I, viii. I 29.

monoxyla, Lesson. Cat.Tert. Moll.B.M. i. 246; Man. Conch. I, viii. $\mathbf{2} 28$.

crepidula, Linnæus. C. unguiformis, Lamarck. Cat. Tert. Moll. B.M. i. 248 ; Man. Conch. I, viii. 130.

Family XENOPHORIDE.

Xenophora, Fischer de Waldheim, i807. pallidula, Reeve. P.Z.S. I 842, I62; Man. Conch. 1, viii. I6o.

Family Trichotropide.

Trichotropis, Broderip and Sowerby, 1829. inornata, Hutton. T. clathrata, Sowerby. Cat. Tert. Moll.

B.M. i. 233 ; Man. Conch. I, ix. 43.

Lippistes, Montfort, I 810.

benhami, Suter. Jour. Malac. ix. 65 . 
Family MaRSENIADE.

Marsenia, Leach. I819.

ophione, Gray. P.Z.S. 1849, I69; Man. Conch. I, viii. 63. cerebroides, Hutton. T.N.Z.I. xv. I 22.

Natica, Scopoli, 1777 .

\section{Family NaTiCide.}

zelandica, Quoy and Gaimard. Cat. Tert. Moll. B.M. i. 259 ;

Man. Conch. 1, viii. 22.

australis, Hutton. Man.N.Z.Moll. 72 ; Man.Conch. I, viii. 54. vitrea, Hutton. P.L.S.N.S.W. I, ix. 934 ; Man. Conch. 1, viii. 54 .

Amaura, Möller, I 842.

apora, Watson. Chall. Rep. xv. 454 .

\section{e. Ptenoglossa.}

Scalaria, Lamarck, i 801 .

\section{Family ScalariIDa.}

tenella, Hutton. P.L.S.N.S.W. I, ix. 943; Man. Conch. I, ix. 84 ; S. noroseeletndice, Clessin, Mart. and Chemn. 57.

philippinarum, Sowerby. P. Roy. S. Vict. n.s. xiii. I 44 ; Man. Conch. I, ix. 66.

jukesiana, Forbes. P. Roy. S. Vict. n.s. xiii. 143; Man. Conch. I, ix. 66 ; S. wellingtonensis, T. W. Kirk, T.N.Z.I. xii. 307 .

australis, Lamarck. P. Roy. S. Vict. n.s. xiii. I42; Man. Conch. 1 , ix. 76 .

zelebori, Frauenfeld. Man. N.Z. Moll. 69; Man. Conch. I, ix. $78 ;$ S. reevei, Clessin, Mart. and Chemn. 63.

Aclia, Lovén, i 846 .

hyalina, Hutton. P.L.S.N.S.W. I, ix. 935 ; Man. Conch. I, ix. 90.

Janthina, Lamarck, I 799.

Family JANTHINIDE.

fragilis, Lamarck. Reeve, Icon. sp. 6; Man. Conch. I, ix. 36. var. planospirata, Adams and Reeve; Man. Conch. I, ix. 36 : Z. balteata, Reeve, Conch. Icon. sp. II.

globosa, Swainson. Man. Conch. I, ix. 37.

exigua, I amarck. P. Roy. S. Vict. n.s. xiii. I 40 ; Man. Conch. I, ix. 37 .

Order Aspidobranchia.

Sub-order RHIPIDOGLOSSA.

Family HydROCENIDE.

Hydrocena, Parreyss, 1843.

purchasi, Pfeiffer. P.L.S.N.S.IV. 2, vii. 623; Man.N.Z.

Moll. 40. 
Family NERITID瓜.

Nerita, (Linnæus, I 75 8), Lamarck, 1799.

nigra, Gray. Dieffenb. N.Z. ii. $240 ; N$. melanotragus, E. A.

Snith; N. saturata, Hutton, P.L.S.N.S.W. 2, ii. 993 ;

Man. Conch. I, x. 26.

undata, Linnzeus. Man. Conch. I, X. 28.

Turbo, Linnæus, $175^{8 .}$

Family TURBONIDIDÆ.

helicinus, Born. I. smaragdus, Martyn. P.L.S.N.S.IV. I, ix. 355 ; Man. Conch. I, x. 217.

var. tricostata, Hutton. P.L.S.N.S.IV. I, ix. 355 .

granosus, Martyn. P.L.S.N.S.W. I. ix. 355 ; Man. Conch. I, $x .213$.

Leptothyra, (Cpr.) Dall, I 87 I.

fluctuata, Hutton. T.N.Z.I. xvi. 215 ; Man. Conch. I, x. 259.

Phasianella, Lamarck, I 804.

limbata, Hutton. Man. Conch. I, ix. 355.

huttoni, Pilsbry. Man. Conch. I, x. I 74 .

Astralium, Link, 1807 .

sulcatum, Martyn. P.L.S.N.S.IV. I, ix. 356 ; Man. Conch.

I, x. 242 .

var. davisii, Stowe. T.N.Z.I. iv. 218.

heliotropium, Martyn. P.L.S.N.S.IV. I, ix. $35^{6}$; Man. Conch. I, x. 192.

? shandi, Hutton. Man. N.Z. Moll. $9 \mathrm{r}$; Man. Conch. I, x. 2 I 2 .

Family TrochidE.

Trochus, Linnæus, 1758.

tiaratus, Quoy and Gaimard. P.Mal.S. ii. 260; Man. Conch. I, xi. 42 .

chathamensis, Hutton. P.Mal.S. ii. 260; Man. Conch. I, xi. 43 .

oppressus, Hutton. P.Mal.S. ii. 26r ; Man. Conch. I, xi. 232.

var. dunedinensis, Suter. P.Mal.S. ii. $26 r$.

viridis, Gmelin. P.Mal.S. ii. 26 r ; Man. Conch. I, xi. 43. ringens, Menke. P.Mal.S. ii. 262 ; Man. Conch. I, xi. 8I.

Monodonta, Lamarck, I8or.

æthiops, Gmelin. P.Mal.S. ii. 262 ; Man. Conch. I, xi. 98. nigerrima, Gmelin. P.Mal.S. ii. $264 ; M$. porcifera, Suter, l.c. non Adams, nec Watson. Man. Conch. 1, xi. 97.

morio, Troschel. P.Mal.S. ii. 263 ; M. porcifer, Watson, non Adams. Man. Conch. I, xi. 102, 105.

atrovirens, Philippi. P.Mal.S. ii. 263 ; Man. Conch. I,

xi. I 10. 
Monodonta coracina, Troschel. P.Mal.S. ii. $264 ; M$. niger rima, Hutton, non Gmelin; $M$. porcifera, A. Adams, non Watson. Man. Conch. I, xi. Iо3.

lugubris, Gmelin. P.Mal.S. ii. 265 ; Man. Conch. I, xi. 100. subrostrata, Gray. P.Mal.S. ii. 266 ; Man. Conch. I, xi. Io I. corrosa, A. Adams. P.Mal.S. ii. 266; Man. Conch. I, xi. I04; M. melanoloma, auct. non Menke.

var. undulosa, A. Adams. P.Mal.S. ii. 267 ; Man. Conch. I, xi. 105 .

var. plumbea, Hutton. P.Mal.S. ii. 267 ; Man. Conch. I, xi. I05.

excavata, Adams and Angas. P.Mal.S. ii. 265; Man. Conch. I, xi. Io9.

crinita, Philippi. P.Mal.S. ii. 267 ; Man. Conch. I, xi. I I I.

\section{Cantharidus, Montfort, I810.}

purpuratus, Martyn. P.Mal.S. ii. 270; Man. Conch. I,

xi. 125 .

var. texturata, Gould. P.Mal.S. ii. 270 ; Man. Conch. I, xi. I 25. iris, Gmelin. P.Mal.S. ii. 268 ; Man. Conch. I, xi. I 22.

sanguineus, Gray. P.Mal.S. ii. 27 I ; Man. Conch, r, xi. I 3 I. var. cælata, Hutton. P.Mal.S. ii. 272 ; Man. Conch. T, xi. $13 \mathrm{r}$.

var. elongata, Suter. P.Mal.S. ii. 272.

pruinius, Gould. P.Mal.S. ii. 268 ; Man. Conch. I, xi. I 22. var. perobtusa, Pilsbry. P.Mal.S. ii. 269; Man. Conch. I, xi. 123.

tenebrosus, A. Adams. P.Mal.S. ii. 269; Man. Conch. I, xi. I 23.

var. huttoni, E. A. Smith. P.Mal.S. ii. 269 ; Man. Conch. I, xi. 123 .

rufozonas, A. Adams. P.Mal.S. ii. 273; Man. Conch. x, xi. 150.

dilatatus, Sowerby. P.Mal.S. ii. 272 ; Man. Conch. I, xi. 148. pupillus, Hutton. P.Mal.S. ii. 270; Man. Conch. I, xi. I 30. fasciatus, Menke. P.Mal.S. ii. 274; Man. Conch. I, xi. 139 picturatus, H. and A. Adams. P.Mal.S. ii. 274; Man. Conch. I, xi. I 40.

conicus, Gray. P.Mal.S. ii. 275 ; Man. Conch. I, xi. 275.

Gibbula, Risso, I 826 .

antipoda, Hombron and Jacquinot. P.Mal.S. ii. 277 ; Man.

Conch. I, xi. 217 .

nitida, Adams and Angas. P.Mal.S. ii. 277 ; Man. Conch. I, xi. 217 .

fulminata, Hutton. P.Mal.S. ii. 276 ; Man. Conch. I, xi. 2 I 0. rosea, Hutton. P.Mal.S. ii. 276 ; Man. Conch. I. xi. 216. scamnata, Fischer. P.Mal.S. ii. 278 ; Man. Conch. I, xi. 220. 
Gibbula tasmanica, Petterd. P.Mal.S. ii. 276 ; Man. Conch. I, xi. 236.

suteri, E. A. Smith. P.Mal.S. ii. 278.

micans, Suter. P.Mal.S. ii. 279.

Fossarina, Adams and Angas, I 863.

rimata, Hutton. P.I.S.N.S.IV. 1, ix. 369 (Minos).

Monilea, Swainson, 1840.

egena, Gould. P.Mal.S. ii. 279 ; Man. Conch. I, xi. 253.

Calliostoma, Swainson, I 840.

tigris, Martyn. P.Mal.S. ii. 280; Man. Conch. I, xi. 333.

punctulatum, Martyn. P.Mal.S. ii. 280 ; Man. Conch. 1 , xi. 334 .

selectum, Chemnitz. P.Mal.S. ii. 28 I : Man. Conch. I, xi. 335. pellucidum, Valenciennes. P.Mal.S. ii. 28 I ; Man. Conch.

I, xi. 334 .

spectabile, A. Adams. P.Mal.S. ii. 280; Man. Conch. I, xi. $33^{2}$.

Euchelus, Philippi, I 847 .

bellus, Hutton. P.Mal.S. ii. 282 ; Man. Conch. I, xi. 435 .

var. iricolor, T. W. Kirk. P.Mal.S. ii. 282 ; Man. Conch. I, xi. 436 .

hamiltoni, T. W. Kirk. P.Mal.S. ii. 282 ; Man. Conch. x, xi. $43^{6 .}$

Ethalia, A. Adams, 1853 .

zelandica, Hombron and Jacquinot. P.Mal.S. ii 283 ; Man. Conch. I, xi. 459.

Family Crclostrematide.

Cirsonella, Angas, I 877 . neozealanica, Murdoch. P.Mal.S. iii. 320.

Family Pleurotomariide.

Scissurella, d Orbigny, I 823 .

mantelli, Woodward. P.Z.S. I 859, 202 ; Man. Conch. I, xii. 54 .

lytteltonensis, E. A. Smith. P.Mal.S. i. 57.

Haliotis, Linnæus, 1758 .

Family HaLIOTID

iris, Martyn. P.L.S.N.S.W. I, ix. 369 ; Man. Conch. I, xii. ı го. rugoso-plicata, Chemnitz. P.L.S.N.S.W. I, ix. 369 ; Man. Conch. I, xii. I Io.

virginea, Chemnitz. P.L.S.N.S.IV. I, ix. 370: H. gibha, Hutton, non Philippi, Man. Conch. I, xii. I 07.

var. huttoni, Filhol. Mission Ile Campbell, 527 ; Man. Conch. I, xii. 108. 
Family FisSURELLide.

Fissurella, Bruguière, I791.

squamosa, Hutton. Man. N.Z. Moll. I05.

Megatebennus, Pilsbry, I89o.

moniliferus, Hutton. Man. N.Z. Moll. Io6; Man. Conch.

I, xii. 227 .

Emarginula, Lamarck, I8o I.

striatula, Quoy and Gaimard. Cat. Tert. Moll. B.M. i. 288 ;

Man. Conch. I, xii. 259.

Subemarginula, Blainville, I 825 .

rugosa, Quoy and Gaimard, Voy. Astrol. iii. 33I ; Man.

Conch. 1 , xii. 278 .

parmophoidea, Quoy and Gaimard. Cat. Tert. Moll. B.M.

i. 290 ; Man. Conch. I, xii. 285.

intermedia, Reeve. P.L.S.N.S.W. I, ix. 37 r ; Man. Conch.

r, xii. 285 .

Scutum, Montfort, I8Io.

ambiguum, Chemnitz. Man. Conch. I, xii. 289.

Patella, Linnæus, I 758 .

Sub-order Docoglossa.

Family Patillide.

tramoserica, Martyn. P.L.S.N.S.IV. I, ix. 377 ; Man. Conch. I, xiii. I 42 . denticulata, Martyn. Man. Conch. I, xiii. I 38 . stellifera, Gmelin. P.L.S.N.S.W. I, ix. 378 ; Man. Conch. I, xiii. I 4 I.

radians, Gmelin. P.L.S.N.S.W. I, ix. 376 ; Man. Conch. I, xiii. I39; Newell, T.N.Z.I. xix. I 57 ; P. argyropsis, Lesson; $P$. radiatilis, Hombron and Jacquinot ; $P$. decora, Philippi; $P$. sagritata, Donovan.

var. pholidota, Lesson. Man. N.Z. Moll. ro8; Man. Conch. I, xiii. 140.

var. argentea, Quoy and Gaimard. Voy. Astrol. iii. $3+5$.

var. earlii, Reeve. P.I.S.N.S.W. I, ix. 376.

var. flava, Hutton. P.L.S.N.S.W. I, ix. 378 ; Man. Conch. $\mathrm{r}$, xiii. $\mathrm{I} 42$.

var. olivacea, Hutton. T.N.Z.I. xv. I33; Man. Conch. I, xiii. I 4 I.

ornata, Dillwyn. Man. Conch. I, xiii. I37.

var. inconspicua, Gray. Man. N.Z. Moll. Io7; Man. Conch. I, xiii. I 38 .

strigilis, Hombron and Jacquinot. P.L.S.N.S.IV. I, ix. 375 ; Man. Conch. I, xiii. I37.

var. redimiculum, Reeve. P.L.S.N.S.W. I, ix. 375 ; Man.

Conch. I, xiii. 136 . 
Patella floccata, Reeve. Conch. Icon. sp. 106.

illuminata, Gould. P.L.S.N.S.IV. I, ix. 376 ; Man. Conch. I, xiii. I 42 .

kermadecensis, Pilsbry. Nautilus, vii. 106 ; P. Acad. N.S. Philad. I 894, 208 : P. pilsbryi, Brazier.

Family ACMÆID心.

Acmæa, Eschscholtz, (1 828) i 830.

fragilis, Chemnitz, P.L.S.N.S.W. I, ix. 374 ; Man. Conch. I, xiii. 59 .

pileopsis, Quoy and Gaimard. P.L.S.N.S.W. I. ix. 373; Man. Conch. I, xiii. 57.

septiformis, Quoy and Gaimard, Voy. Astrol. iii. 362 ; Man. Conch. I, xiii. 55.; A. scabrilirata, Angas ; $A$. petterdi, T.Wroods.

lacunosa, Reeve. Conch. Icon. Patella, sp. 120;A. rubiginosa, Hutton.

cantharus, Reeve. Conch. Icon. Patella, sp. I3I ; Man. Conch. I, xiii. 55 .

octoradiata, Hutton. Cat. M.M.N. Zeal. $44 ; A$. perplexa Pils. viii. 50 .

corticata, Hutton. P.L.S.N.S.IV. I, ix. $372 ; A$. lacunosa, Pilsbry, non Reeve.

flammea, Hutton, non Quoy and Gaimard. T.N.Z.I. xv. i 28. cingulata, Hutton. P.L.S.N.S.IV. I, ix. 372 ; Man. Conch. I, xiii. 53 .

conoidea, Hutton, non Quoy and Gaimard. T.N.Z.I. xv. 127. chathamensis, Pilsbry. Man. Conch. I, xiii. 56.

helmsi, E. A. Smith. P.Mal.S. i. 58 .

? campbelli, Filhol. Mission Ile Campbell, 530.

CLASS, SCAPHOPODA.

Family Dentalid

Dentalium, Linnæus, $175^{8}$.

nanum, Hutton. Cat. Tert. M.N. Zeal. I ; Man. Conch, I, xvii. 9 .

opacum, Sowerby. Man. Conch. I, xvii. 70.

zelandicum, Sowerby. Thes. Conch. iii. I01 ; Man. Conch I, xvii. $70 ; D$. pacificum, Hutton.

huttoni, T. W. Kirk. T.N.Z.I. xii. 306; Man. Conch. I, xvii. 7 I.

ecostatum, T. W. Kirk. T.N.Z.I. xii. 306 ; Man. Conch. I, xvii. 102.

diarrhox, Watson. Challenger Reports, xv. iv. ; Man. Conch. I, xvii. Iog. 
Cadulus, Philippi, I 844 .

colubridens, Watson. Challenger Reports, xv. I 8 ; Man.

Conch. I, xvii. I 84 .

\section{CLASS, AMPHINEURA.}

Order PlacophoRa.

Chiton, Linnæus, I 758 .

Family CHITONIDE.

pellisserpentis, Quoy and Gaimard. P.Mal.S. ii. I95; Man.

Conch. I, xiv. I 73 .

canaliculatus, Quoy and Gaimard. P.Mal.S. ii. 196; Man.

Conch. I, xiv. I 77 .

quoyi, Deshayes. P.Mal.S. ii. I94; Man. Conch. I, xiv. 172. sinclairi, Gray. P.Mal.S. ii. I96; Man. Conch. I, xiv. I 74. æreus, Reeve. Conch. Icon. sp. 36 ; Man. Conch. 1 , xiv. 179. stangeri, Reeve. P.Mal.S. ii. I 96.

limans, Sykes. P.Mal.S. ii. I 97 ; Man. Conch. i. xiv. I 75.

Eudoxochiton, Shuttleworth, I 853 .

nobilis, Gray. P.Mal.S. ii. 197 ; Man. Conch. I, xiv. 193.

huttoni, Pilsbry. Man. Conch. I, xiv. 194.

Acanthopleura, Guilding, I 835 .

granuluta, Gmelin. Man. Conch. I, xiv. 227 ; A. corticata, Hutton, P.Mal.S. ii. 198.

Onithochiton, Gray, 1847 .

undulatus, Quoy and Gaimard. P.Mal.S. ii. I99; Man.

Conch. I, xiv. 245 .

semisculptus, Pilsbry. Man. Conch. I, xiv. 247.

Family AcanthochitıDÆ.

Spongiochiton, Carpenter, I873.

productus, (Cpr.) Pilsbry. Man. Conch. I, xiv. 26.

Acanthochites, Risso, 1826 .

porosus, Burrow. P.Mal.S. ii. I93; Man. Conch, I, xv. 36. zelandicus, Quoy and Gaimard. P.Mal.S. ii. I92; Man. Conch. I, xv. I6.

violaceus, Quoy and Gaimard. P.Mal.S. ii. 193; Man.

Conch. I, Xv. 39 .

rubiginosus, Hutton. Man. N.Z. Moll. II 4; Man. Conch. I. xv. I07; A. costatus, Suter, non Ad. and Ang., P.Mal.S. ii. 194 .

Plaxiphora, Gray, 1847 .

Family Mopalindæ.

biramosa, Quoy and Gaimard. P.Mal.S. ii. I88; Man Conch. I, xiv. 319. 
Plaxiphora superba, (Cpr.) Pilsbry. P.Mal.S. ii. I 88 ; Man.

Conch. I, xiv. 3 I9.

cælata, Reeve. P.Mal.S. ii. I89; Man. Conch. I, xiv. 328. suteri, Pilsbry. Nautilus, viii. 8 ; P.Mal.S. ii. I 90.

subatrata (Pilsbry), Suter. P.Mal.S. ii. I 9o.

obtecta, (Cpr.) Pilsbry. Man. Conch. I, xiv. $33^{\circ}$; P.Mal.S. ii. 192.

ovata, Hutton. P.Mal.S. ii. I92; Man. Conch. I, xiv. $33^{2}$.

Family IschNochITONIDÆ.

Callochiton, Gray, i 847 .

platessa, Gould. P.Mal.S. ii. I 84 ; Man. Conch. I, xiv. 49. illuminatus, Reeve. P.Mal.S. ii. I 85 ; Man. Conch. I, xiv. 5 I. empleurus, Hutton. Man. N.Z. Moll. I 3 ; P.Mal.S. ii. 185.

Ischnochiton, Gray, I 847 .

longicymba, Quoy and Gaimard. P.Mal.S. ii. I86; Man. Conch. r, xiv. 87 .

parkeri, Suter. P.Mal.S. ii. I 86.

Lepidopleurus, Risso, 1826 .

Family LePIDOPLEURIDÆ.

inguinatus, Reeve. P.Mal.S. ii. I 84 ; Man. Conch. I, xiv. $90 ;$ L. liratus, Adans and Angas.

Cuspidaria, Nardo, 1840.

\section{CLASS, PELECYPODA.}

Order Septibranchia.

Family CUSPIDARIIDE.

trailli, Hutton. Man. N.Z. Moll. I 37.

Order Eulamellibranchia.

Sub-order Sinupalliata.

Family PERIPLOMIDE.

Cochlodesma, Couthouy, 1839.

angasi, Crosse and Fischer. Journ. de Conch. xii. 349; xiii. 427 ; Anatina tasmanica, Hutton, non Reeve.

Thracia, Blainville, 1824 .

Family ThRACIIDE.

vitrea, Hutton. Plioc. M.N.Z. 75.

Family Chanostreide.

Chamostrea, F. de Roissy, 1825 .

albida, Lamarck. Man. N.Z. Moll. I38; Fischer, Man. de

Conch. pl. xxiii. fig. 14 ; Cheidotharus chamoides, Sowerby. 
Myodora, Gray, 1840 .

Family Мүоснамı®.

striata, Quoy and Gaimard, Voy. Astrol.iii. 537 ; P.L.S.N.S.IV. I, ix. 5 I 4 .

rotundata, Sowerby. P.Z.S. s 875 , I 29 ; P.L.S.N.S.W. I, ix. 5 I 5 .

brevis, Sowerby. P.L.S.N.S.W. I, ix. 515.

pandoriformis, Stutchbury. P.L.S.N.S.W. I, ix. 5 I 5 .

crassa, Stutchbury. P.L.S.N.S.W. I. ix. 5 I6.

novæ - zealandiæ, E. A. Smith. P.Z.S. r880, 584; P.L.S.N.S.IV. I, ix. 5 I6.

subrostrata, E. A. Smith. P.Z.S. 1880,584 ; P.L.S.N.S.W. I, Ix. 5 I 6 .

antipodum, E. A. Smith. P.Z.S. 1880,585 ; P.L.S.N.S.W. I, ix. 5 I 6 .

boltoni, E. A. Smith. P.Z.S. 585 ; P.L.S.N.S.IW. I, ix. 5 I 7 .

Nausitora, Wright, I 864 .

antarctica, Hutton. Man. N.Z. Moll. r33; P.L.S.N.S.W. 2, ix. pl. xxxii. figs. 6,7 .

Family PHOLADIDÆ.

Barnea, Leach, 1826 . similis, Gray. Man. N.Z. Moll. I32 ; P.L.S.N.S.IV. I, ix. 512.

Pholadidea, Goodall, I 819.

tridens, Gray. Man. N.Z. Moll. I33; P.L.S.N.S.W. I, ix. 5 I 2.

Family Saxicavide.

Panopea, M. de la Groye, I 807 . zelandica, Quoy and Gaimard, Voy. Astrol. iii. 547 ; P.L.S. N.S.W. I, ix. 5 I 3 .

Saxicava, F. de Belluvue, I8oz.

arctica, Linnæus. Man. N.Z. Moll. I34; S. australis, Lamarck, Challenger Reports, xiii. 78.

Family CORBUlidæ.

Corbula (Bruguière), Lamarck, I 797.

zelandica, Quoy and Gaimard, Voy. Astrol. iii. 5 I I ; Man.

N.Z. Moll. I 35 .

erythrodon, Lamarck. Man. N.Z. Moll. I35 ; P.L.S.N.S.IW. I, ix. 5 I 3 .

haastiana, Hutton. Man. N.Z. Moll. I35.

Family PSammobidid.

Psammobia, Lamarck, i 8 I8. stangeri, Gray. Man. N.Z. Moll. I 4 ı. 
Psammobia lineolata, Gray. Man. N.Z. Moll. 142. zealandica, Deshayes. Man. N.Z. Moll. 142.

affinis, Reeve. Man. N.Z. Moll. I42.

Solenotellina, Blainville, I824.

nitida, Gray. Man. N.Z. Moll. 142.

radiata, Deshayes. Man. N.Z. Moll. 145 (Capsella).

siliqua, Reeve. Man. N.Z. Moll. I42.

incerta, Reeve. Man. N.Z. Moll. I43; S. nitens, Tryon.

Cardium, Linnæus, $175^{8}$.

Family CaRdide.

pulchellum, Gray (non Reeve). Man. N.Z. Moll. I53;

C. striatulum, Sowerby, non Brocchi.

Family VeNerid «.

Meretrix, Lamarck, I 799 .

multistriata, Sowerby. Man. N.Z. Moll. 149; Challenger

Reports, xiii. pl. i. fig. 5 .

Dosinia, Scopoli, 1777 .

subrosea, Gray. Man. N.Z. Moll. I50; Ereb. and Terr. pl. iii. fig. 2.

australis, Gray. Man. N.Z. Moll. I50; Reeve, Conch. Icon. sp. 10.

lambata, Gould. Man. N.Z. Moll. I50; D. carpenteri, Roemer; Cyclina kroyeri, Philippi.

grayi, Zittel. Man. N.Z. Moll. I5I.

Chione, M. von Muehlfeldt, isiı.

oblonga, Hanley. Man. N.Z. Moll. I47; Ereb. and Terr. pl. ii. fig. I.

stutchburyi, Gray. Man. N.Z. Moll. ${ }_{4} 8$; Ereb. and Terr. pl. iii. fig. 4 .

costata, Quoy and Gaimard. Man. N.Z. Moll. I48; Voy. Astrol. pl. lxxxiv. figs. I, 2.

crassa, Quoy and Gaimard. Man. N.Z. Moll. I48; Voy.

Astrol. pl. lxxiviv. figs. 7,$8 ; C$. denticulata, C. mesodesma, Quoy and Gaimard.

crebra, Hutton. Man. N.Z. Moll. I 47.

Anaitis, Roemer, 1857 .

disjecta, Perry=lamellata, Lamarck. Man. N.Z. Moll. 447. yatei, Gray. Man. N.Z. Moll. I47; Ereb. and Terr. pl. iii. fig. I I.

Tapes, M. von Muehlfeldt, I 8 I I.

intermedia, Quoy and Gaimard. Man. N.Z. Moll. I5 I;

Voy. Astrol. pl. lxxxiv. figs. 9, го. 
Venerupis, Lamarck, i8 8 8.

reflexa, Gray. Man. N.Z. Moll. $5^{2}$; Ereb. and Terr. pl. ii. fig. $3 ; V$. paupercula, Deshayes.

siliqua, Deshayes. Man. N.Z. Moll. 152; P.Z.S. I 853 , pl. xviii. fig. I.

elegans, Deshayes. Man. N.Z. Moll. I52 ; Ereb. and Terr. pl. ii. fig. 6.

insignis, Deshayes. Man. N.Z. Moll. $152 ;$ P.Z.S. I853, pl. xviii. fig. 4 .

Family Mactride.

Mactra (Linnæus, I 758), Lamarck.

discors, Gray. Man. N Z. Moll. I38; Ereb. and Terr. pl. ii. fig. 4 ; $M$. murchisoni. Deshayes.

æquilatera, Deshayes. Man. N.Z. Moll. I39; Ereb. and Terr. pl. ii. fig. Io.

scalpellum, Deshayes. Man. N.Z. Moll. $3_{3} 8$; T.N.Z.I. xxxii. pl. xx. fig. Io.

lavata, Hutton. Plioc. Moll. N.Z. 76, pl. viii. fig. 81. ordinaria, E. A. Smith. P.Mal.S. iii. 23.

Standella, Gray, I 852 .

elongata, Quoy and Gaimard. Man. N.Z. Moll. I39; Voy. Astrol. pl. lxxxiii. figs. I, 2 ; S. notata, Hutton.

ovata, Gray. Man. N.Z. Moll. I39; Ereb. and Terr. pl ii. fig. 2 .

rudis, Hutton. Plioc. Moll. N.Z. 77 , pl. viii. fig. 83 .

Resania, Gray, I 852 . Vanganella, Gray (1851), i 8.53 .

lanceolata, Gray. Man. N.Z. Moll. I40; Ereb. and Terr.

pl. ii. fig. 5. ; $V$. taylori, Gray.

Zenatia, Gray, $185^{2}$.

acinaces, Quoy and Gaimard. Man. N.Z. Moll. 140; Voy.

Astrol. pl. lxxxiii. figs. 5,6 ; $Z$. deshayesi, Reeve.

Raëta, Gray, 1852 .

perspicua, Hutton. Man. N.Z. Moll. I4 I.

Cyamiomactra, Bernard, I 898 .

problematica, Bernard. Bull. Mus. Paris, iii. 3 I I.

Perrierina, Bernard, I 898.

taxodonta, Bernard. Bull. Mus. Paris, iii. 3 I 2.

Family Mesodesnatide.

Mesodesma, Deshayes, I8 31 .

novæ-zealandiæ, Chemnitz. Man. N.Z. Moll. I45; Voy. Astrol. pl. lxxxii. figs. 9-I I ; $M$. ovalis, Deshayes.

ventricosa, Gray. Man. N.Z. Moll. I45; Ereb. and Terr. pl. iii. fig. 6. 
Atactodea, Dall, i 895 .

subtriangulata, Gray. Man. N.Z. Moll. 146; Griffith's

Cuvier Anim. Kingd. xii. pl. xxii. fig. 4; Mesodesma spissa,

Reeve, Conch. Icon. sp. I8.

Family Tellinide.

Tellina (Linnæus, I 758), Lamarck.

alba, Quoy and Gaimard. Man. N.Z. Moll. 143 : Voy. Astrol. pl. lxxxi. figs. 1-3.

lactea, Quoy and Gaimard. Voy. Astrol. iii. 501 ; pl. Ixxxi. figs. 14-16;T. deltoidalis, Hutton, non Lamarck.

ticaonica, Deshayes. Man. N.Z. Moll. 144 ; Reeve, Conch. Icon. sp. 304.

disculus, Deshayes. Man. N.Z. Moll. 143 ; Reeve, Conch. Icon. sp. 306.

strangei, Deshayes. Man. N.Z. Moll. 144; T. subovata, Sowerby; $T$. lintea, $T$. retiaria, Hutton.

glabrella, Deshayes. Man. N.Z. Moll. I44; Ereb. and Terr. pl. ii. fig. 7 .

charlottæ, E. A. Smith. Challenger Reports, xiii. Iо०; pl. iv. fig. 1 .

huttoni, E. A.Smith. Challenger Reports, xiii. I о ; pl.iv. fig. 2.

Macoma, Leach, I819.

suteri, E. A. Smith. P.Mal.S. iii. 23.

Sub-order INTEgRipaLl1ata.

Pisidium, C. Pfeiffer, 1821 .

Family SPHÆRIIDж.

novæ-zealandiæ, Prime. P.L.S.N.S.W. 2, vii. $66_{4} ;$ P. lenticulata, Dunker.

Sphærium, Scopoli, 1777 .

novæ-zelandiæ, Deshayes. P.L.S.N.S.W. 2, vii. 664.

Diplodon, Spix, I827.

Family UNiONidE.

menziesi, Gray. P.L.S.N.S.IV. 2, vii. 662 ; Simpson, Pro.

U.S. Nat. Mus. xxii. 889 ; D. aucklandicus, Gray.

var. waikarensis, Colenso. P.L.S.N.S.W. 2, vii. 663.

var. hochstetteri, Dunker. P.L.S.N.S.W. 2, vii. 663 .

var. rugata, Hutton. P.L.S.N.S.W. 2, vii. $66_{3}$.

var. depauperata, Hutton, P.L.S.N.S.W. 2, vii. 663.

lutulentus, Gould. P.L.S.N.S.W. 2, vii. 663 .

zelebori, Dunker, P.L.S.N.S.IV. 2, vii. 662.

Kellya, Turton, I 822.

Family LePTONIDE.

cycladiformis, Deshayes. Man. N.Z. Moll. ${ }_{57} 7$.

citrina, Hutton. T.N.Z.I. xvi. 215. 
Neolepton, Monterosato, i 875.

antipodum, Filhol. Mission Ile Campbell, 543.

sanguineum, Hutton. T.N.Z.I. xri. 215.

Lasea, Leach, I 827.

miliaris, Philippi. Wiegman's Archiv. f. Naturgeschicte, I $845,5 \mathrm{I}$.

Mylitta, d'Orbigny and Récluz, i $85^{\circ}$.

stowei, Hutton. Man. N.Z. Moll. I57; A.M.N.H. 6, viii. pl. xiii.

Pachykellya, Bernard, I 898.

edwardsi, Bernard. Bull. Mus. Paris, iii. 310.

Family KeLLYeLLIDÆ.

Cyamium, Philippi, I 845 .

oblongum, E. A. Smith. P.Mal.S. iii. 24.

Family DiPLODONTIDÆ.

Diplodonta, Bronn, I83 I.

zelandica, Gray. Man. N.Z. Moll. $5_{56}^{6}$; Ereb. and Terr. pl. iii. fig. 8 .

globularis, Lamarck. Man. N.Z. Moll. ${ }_{56} 6$.

striata, Hutton. Man. N.Z. Moll. I 56.

Divaricella, v. Martens, I 880.

Family LuCiNid Æ.

cumingi, Adams and Angas. P.Z.S. 1863, 426; pl. xxxvii. fig. 20.

var. huttoni, Vanatta. Proc. Acad. Nat. Sci. Philadelphia, I 90 I, I 84 ; pl. v. figs. I 4, I 5 .

Family Crassatellitid Æ.

Crassatellites, Krueger, I 82.3 . obesa, Adams. P.Z.S. 1852,90 ; pl. xvi. fig. 2. bellula, Adams. P.Z.S., I852,95; Gouldia isabella, Hutton.

Family Condylocardide.

Condylocardia, Bernard, I 897.

crassicosta, Bernard. Jour. de Conch. xliv. I 75 ; pl. vi. fig. I.

concentrica, Bernard. Journ. de Conch. xliv. 176 ; pl. vi. fig. 2 .

Family CARUITIDE.

Cardita, Lamarck, 1799 .

aviculina, Lamarck. C. excavata, Deshayes ; C. tasmanica,

J. Woods: Man. N.Z. Moll. I6o. 
Venericardia, Lamarck, I80I.

australis, Lamarck. Man. N.Z. Moll. I 58 ; Voy. Astrol. pl. lxxviii. figs. I I-I 4.

difficilis, Deshayes. Man. N.Z. Moll. I59; P.Z.S. I852, pl. xvii. figs. I6-I 7 .

compressa, Reeve. P.L.S.N.S.IV. I, ix. 257.

\section{Order Pseudo-Lanellibranchia.}

Family SPONDYLID心.

Spondylus, Linnæus, $75^{8}$.

ostreoides, E. A. Smith, Challenger Reports, xiii. 326.

Family LIM1Dж.

Lima (Bruguière), Cuvier, I 798.

lima, Linnæus = zealandica, Sowerby. P.L.S.N.S.W. I, ix. $53 \mathrm{I}$.

angulata, Sowerby. Man. N.Z. Moll. I 72.

bullata, Born. P.L.S.N.S.IV. I, ix. $53 \mathrm{I}$.

Pecten, Mueller, I 776.

Family Pectinide.

medius, Lamarck = laticostatus, Gray. Man. N.Z. Moll. I 7 I. asperrimus, Lamarck. P.L.S.N.S.IV. I, ix. 53 I.

zelandiæ, Gray. P.L.S.N.S.W. I, ix. 53I.

var. gemmulata, Reeve. Conch. Icon. sp. I I I.

radiatus, Hutton. Man. N.Z. Moll. i 7 I.

convexus, Quoy and Gaimard. P.L.S.N.S.IV. I, ix. 532.

kermadecensis, E. A. Smith. Challenger Reports, xiii. 302 ;

pl. xxi. fig. 7 .

Ostrea, Linnæus, I $75^{8 .}$

Family OSTREIDE.

angasi, Sowerby. Reeve, Conch. Icon. sp. 28 ; P.L.S.N.S.IV.

I, ix. 533 ; O. edulis, auct., non Linnæus.

purpurea, Hanley. P.Z.S. I $867,934$.

glomerata, Gould. P.L.S.N.S.W. I, ix. 533 ; O. cucullata,

Angas ; O. mordax, T. IWnods.

reniformis, Sowerby. Reeve, Conch. Icon. sp. 57.

\section{Family PTERIIDÆ.}

Philobrya, P. Carpenter, I872.

meleagrina, Bernard. Journ. de Conch. xlv. i 2 ; pl. i. fig. 3 . costata, Bernard. Journ. de Conch. xlv. 15 ; pl. i. fig. 5.

filholi, Bernard. Journ. de Conch. xlv. 16 ; pl. i. fig. 6.

Hochstetteria, M. Chalmas and Vélain, I 876.

trapezina, Bernard. Journ. de Conch. xlv. I8; pl. 1, fig. 7 . 


\section{Family Pinnide.}

Pinna (Linnæus), Lamarck, I 799.

zelandica, Gray. Man. N.Z. Moll. I69; Ereb. and Terr. pl. iii. fig. 7 .

\section{Order Filibranchia.}

Family Mrtilide.

Mytilus (Linnæus, I 758), Bolten, I 798.

edulis, Linnæus. Man. N.Z. Moll. I67 ; M. latus, Lamarck, non Chemnitz; M. chorus, Hutton, non Molina.

latus, Chemnitz. P.L.S.N.S.W. I, 529.

magellanicus, Chemnitz. P.L.S.N.S.W. ix. 529.

Volsella, Scopoli, I777. Modiolus, Risso, I 799.

australis, Gray. P.L.S.N.S.W. I, ix. 530.

ater, Frauenfeld. P.L.S.N.S.W. 1, ix. 530.

fluviatilis, Hutton. Man. N.Z. Moll, 168.

Modiolaria, Beck, 1840.

barbata, Reeve. Conch. Icon. Lithodomus, sp. 27 ; P.Z.S. I867, 9 I I ; Lith. lanigerus, Reeve.

impacta, Herrmannsen. Man. N.Z. Moll. 168.

Adula, H. and A. Adams, 1857 . Myrina, H. and A. Adams, 1857 .

minuta, E. A. Smith. P.Mal.S. iii. 24.

Lithophago, Bolten, I 798. Lithodomus, Cuvier, I 8 I 7 .

truncata, Gray. Man. N.Z. Moll. I68; Ereb. and Terr. pl. ii. fig. 12.

\section{Family ModIOLARCIDÆ.}

Modiolarca, Gray, 1847 .

pusilla, Gould. U.S. Expl. Exp. xii. 455.

trapezina, Lamarck. Anim. s. Vert. 2nd ed. vii. 24.

bicolor, E. A. Smith; P. Mal. S. iii. 25.

Barbatia, Gray, 1840 .

\section{Family ARCIDE.}

decussata, Sowerby. Man. N.Z. Moll. I62.

reticulata, Chemnitz. Lamarck, Anim. s. Vert. and ed. vi.

475 ; A. domingensis, Lamarck, etc.

Glycymeris, Da Costa, I 778 . Pectunculus, Lamarck, 1799. laticostata, Quoy and Gaimard. Man. N.Z. Moll. I63. striatularis, Lamarck. Man. N.Z. Moll. 163.

Family LimopsidÆ.

Limopsis, Sassi, I827.

lata, E. A. Smith. Challenger Report, xiii. 257. 
Family ANOMHID:

Anomia, (Linnreus, I 758) O. F. Mueller, 1775.

cytæum, Gray Man. N.Z. Moll. I 74.

alectus, Gray. Man. N.Z. Moll. I 73 .

stowei, Hutton. Man. N.Z. Moll. I 73.

walteri, Hector. T.N.Z.I. xwiii. 292.

Placunanomia, Broderip, I 832 .

zelandica, Gray. Man. N.Z. Moll. 174 ; Ereb. and Terr. pl. iii. fig. Io.

ione, Gray. Man. N.Z. Moll. I 74.

Order Protobranch1ata.

Nucula, Lamarck, I 799 .

Family Nuculide.

nitidula, A. Adams. Man. N.Z. Moll. r64.

strangei, A. Adams. Man. N.Z. Moll. I64; Ereb. and Terr. pl. ii. fig. I 4 .

lacunosa, Hutton. P.L.S.N.S.IV. I, ix. 528 .

castanea, A. Adams. Man. N.Z. Moll. I64.

Leda, Schumacher, I 8 I 7 .

Family LEDIDE.

concinna, A. Adams. Man. N.Z. Moll. I65.

Malletia, C. des Moulins, I 832 . Solenella, Sowerby, I832. australis, Quoy and Gaimard. Man. N.Z. Moll. I66.

Family SOlenomyacide.

Solenomya, Lamarck, i8 I8.

parkinsoni, E. A. Smith. Man. N.Z. Moll. 57 ; Ereb. and Terr. pl. iii. fig. I. 


\title{
PHYLUM, ARTHROPODA.
}

Leioproctus, Smith,

\author{
CLASS, INSEC'TA. \\ Order HyMenoptera. \\ Sub-order Petiolata. \\ Series Tubulifera. \\ Family ANTHOPHILA.
}

imitatus, Smith. Cat. Hymen. B.M. i. 9.

Lamprocolletes, Smith, 1853 .

fulvescens, Smith. T.E.S. I 876,486 .

obscurus, Smith. Cat. Hymen. B.M. I I.

Dasycolletes, Smith, 1853 .

hirticeps, Smith. T.E.S. 1878,7 .

vestitus, Smith. T.E.S. I 876,485 .

purpureus, Smith. Cat. Hymen. B.M. 15.

metallicus, Smith. Cat. Hymen. I5; A. trichopus, White,

Voy. Ereb. and Terr. Ins. pl. vii. fig. I 2.

Halictus, Latreille, I804.

sordidus, Smith. Cat. Hymen. B.MI. 56.

familiaris, Smith. T.E.S. 1876,486 .

huttoni, Cameron. T.N.Z.I. xxxii. I 7 .

Prosopis, Fabricius, $x 804$.

agilis, Smith. T.E.S. 1876,484 .

regelatus, Smith. T.E.S. I 876,485 .

capitosus, Smith. T.E.S. 1876,485 .

lævigatus, Smith. Cat. Hymen. B.M. ii. 420.

sulcifrons, Cameron. Man. Mem. I895, 51.

innocens, Cameron. Man. Mem. I895, 52.

Series Aculeata.

Family FORMICID有.

Sub-family CaMPONOSTIN瓜.

Lasius, Fabricius, $\mathrm{x} \mathrm{SO}_{4}$.

advena, Smith. T.E.S. I 862,53 ; W. IV. Smith, T.N.Z.I.

xxviii. 477 .

zealandica, Smith. T.E.S. 1878,6 . 
Sub-family PONERINÆ.

Ponera, Latreille, I 804.

castanea, Mayr. Reise Norara, Formicidæ, 69.

Ectatomma, Smith, I 858 .

brounii, Forel. Nitt. Schw. Ent. Ges. I 892, 330.

Discothyrea, Rogers, I 863 . antarctica, Emery. T.N.Z.I. xxvii. 635 .

Amblypone, Erichson, 1842.

cephalotes, Smith. T.E.S. 1876, 490 .

saundersii, Forel. Mitt. Sch. Ent. Ges. 1892, 336.

\section{Sub-family MYRMICINÆ.}

Orectognathus, Smith, I 854 .

antennatus, Smith. T.E.S. 2, ii. 228.

perplexus, Smith. T.E.S. I 876,49 I.

Strumigenys, Smith, I86I.

antarctica, Forel. Mitt. Schw. Ent. Ges. 1892, 338.

Huberia, Forel, ז 890.

striata, Smith. T.E.S. $1876,48 \mathrm{I}$; T.N.Z.I. xxiv. 304 ;

IV. W. Smith, T.N.Z.I. xxviii. 470 .

Monomorium, Mayr, I 855 .

fulvum, Mayr. Reise Novara, Formicidæ, 93; W. W. Smith, T.N.Z.I. xxviii. 473.

nitidum, Smith. T.E.S. 1876,480 ; W. W. Smith, T.N.Z.I. xxviii. 473 .

suteri, Forel. Mitt. Schw. Ent. Ges. I892, 340; IV. IV.

Smith, T.N.Z.I. xxviii. 475 .

smithii, Forel. Mitt. Schw. Ent. Ges. 1892, 342.

Aphaenogaster, Mayr, I852.

antarcticus, Smith. Cat. Hymen. B.M. vi. 167.

\section{Family Pompilide.}

Pseudagenia, Kohl, I 884 . huttoni, Cameron. Man. Mem. I898, 49.

Salius, Fabricius, r 804 .

monachus, Smith. Cat. Hymen. B.M. iii. I64. carbonarius, Smith. Cat. Hymen. B.M. iii. I62. nitidiventris, Smith. T.E.S. 1878,6 . marginatus, Smith. T.E.S. 1876,483 . conformis, Smith. T.E.S. I 876,482 . triangularis, Cameron. Man. Mem. I $898,45$. diligens, Smith. T.E.S. I 876,483 . wakefieldi, Kirby. T.E.S. I88 r, 39. 
Salius fugax, Fabricius. Syst. Ent. $350 ; P$. maculipennus, Smith, T.E.S. 1876,482 .

huttoni, Kirby. T.E.S. 1883 , 199.

xenos, Kirby. T.E.S. $1883,200$.

brouni, Gribodo. Bull. Ent. Ital. xvi. 280.

Family SPHEGIDæ.

Rhopalum, Stephens, I 829.

carbonarium, Smith. Cat. Hymen. B.M. iv. 424.

perforator, Smith. T.E.S. 1876,483 .

albipes, Smith. T.E.S. I 878,7 .

cora, Cameron. Man. Mem. I 888, I8 I.

Gorytes, Latreille, I 804 .

carbonarius, Smith. Cat. Hymen. B.M. iv. 366.

trichiosoma, Cameron. Man. Mem. i888, I80.

Tachytes, Panzer, I806.

nigerrimus, Smith. Cat. Hymen. B.M. iv. 302 ; White, Voy.

Ereb. and Terr. Insects, pl. xii. fig. I 4.

sericops, Smith. Cat. Hymen. B.M. iv. 302.

depressus, Saussure. Reise Novara, Hym. 69.

helmsi, Cameron. Man. Mem. I888, 182.

Pison, Spinola, 1808 .

pruinosus, Cameron. Man. Mem. I898, 44.

morosus, Smith. Cat. Hymen. B.M. iv. 3I5.

tuberculatus, Smith. T.E.S. I 869, 296.

dubius, Kirby. T.E.S. I 883,201 (Taranga).

Sub-order Parasitica.

Family Proctotrupide.

Sub-family ProctotruPINÆ.

Proctotrupes, Latreille, 1796.

maculipennis, Cameron. Man. Mem. 1888, I 75 .

intrudens, Smith. T.E.S. 1878,5 .

Sub-family Betylinæ.

Betyla, Cameron, I889.

fulva, Cameron. Man. Mem. I 889 , I3; Hudson, T.N.Z.I. xxv. I69; Tanyzonus bolitophile, Marshall, Ent. Mon. Mag. I 892.

Sub-family DiaPRINE.

Diapria, Latreille, I804. coccophaga, Maskell. T.N.Z.I. xi. 228.

Spilomicrus, Westwood, 1832.

quadriceps, Smith. T.E.S. 1878,6 . 
Prosacantha, Nees, I 834 .

Sub-family MYMaRINe.

crimitaeri, Quail. 'T.N.Z.I. xxxiii. I53 (Mymar).

Sierola, Cameron, I88I.

Sub-family Bethylinæ.

antipoda, Asmead. P.L.S.N.S.IV. 1900, 326 ; Fauna Hawaiiensis, Hymen. 286.

Family Chalcidide.

Sub-family TORYMiNæ.

Torymus, Dalman, i 820 .

antipoda, Kirby. T.E.S. $188_{3}, 202$.

Eurytoma, Rossi, i 807 .

Sub-family EuRYToninæ.

oleariæ, Maskell. T.N.Z.I. xxi. 255.

Aphobetus, Howard, 1896 .

maskelli, Howard. Canadian Ent. xxviii. I66.

Sub-family APHeLininæ.

Pteroptrix, Westwood, I833.

maskelli, Ashmead. P.L.S.N.S.W. I 900, 346.

Sub-family EuPELMinæ.

Eupelmus, Dalman, I820.

messene, Walker. Mon. Chal. ii. 95; Kirby, T.E.S. I88 I, 48.

Pteromalus, Swederus, I 795 .

lelex, Walker. Mon. Chal. ii. 95 ; Kirby, T.E.S. I88 1, 48.

iambe, Walker. Mon. Chal. ii. 95 ; Kirby, T.E.S. I88 I, 49.

Gasteruption, Latreille, I 796.

Family Evanidæ.

pedunculatum, Schletterer. Ann. K.K. Nat. Wien, I890, $467 ;$ F. unguiculatus, Smith. T.E.S. I $869,480$.

Family BRACONIDE.

Sub-family RHOGADINE.

Doryctomorpha, Ashmead, i 900.

antipoda, Ashmead. Ent. News, I900, 630.

Rhogas, Nees, I8I8.

penetrator, Smith. T.E.S. 1878,5 .

Sub-family METEORINE.

Meteorus, Haliday, I 835 .

nova-zealandicus, Cameron. Man. Mem. I898, 38 . 
Fhogra, Cameron, I89r.

rubromaculata, Cameron. T.N.Z.I. xxxiii. I05.

Sub-family CheLoninæ.

Ascogaster, Wesmael, 1835 .

crenulatus, Cameron. Man. Mem. I 898, 37.

Sub-family Heliconine.

Schauinslandica, Ashmead, 1900.

femorata, Ashmead, Ent. News, I900, 627.

alfkenii, Ashmead. Ent. News, r900, 628.

pallidipes, Ashmead. Ent. News, I900, 628 .

Family Opınґ.

Diachasma, Forster, I 862.

carpocapsæ, Ashmead. P.L.S.N.S.W. $1900,357$.

Alysia, Latreille, 1804 .

Sub-family Alysinææ.

stramineipes, Cameron. Man. Mem. I898, 37.

Asobara, Forster, I 862.

antipoda, Ashmead. Ent. News, 1900, 625.

Family ICHNEUMIONIDÆ.

Sub-family ICHNEUMONINæ.

Ichneumon, Linnæus, 1735.

pyrastis, Cameron. T.N.Z.I. xxxiii. I I 5 .

frederici, Cameron. T.N.Z.I. xxxiii. I I6.

richardi, Cameron. T.N.Z.I. xxxiii. I I 7 .

wellingtoni, Cameron. T.N.Z.I. xxxiii. I I 7 .

actista, Cameron. Man. Mem. I898, 8.

helmsi, Cameron. Man. Mem. I898, 9.

atarxidia, Cameron. Man. Mem. I898, I3.

ixia, Cameron. Man. Mem. I898, I 3 .

colensii, Cameron. Man. Mem. $1898, \mathbf{r} 5$.

ursula, Cameron. Man. Mem. I 898 , I6.

brounii, Cameron. Man. Mem. I 898, i 7 .

leodacus, Cameron. Man. Mem. i898, i 9.

falsus, Cameron. Man. Mem. I898, i 8.

machimia, Cameron. Man. Mem. IS98, 20.

utetes, Cameron. Man. Mem. I 898, 2 I.

thyelma, Cameron. Man. Mem. I898, 22.

nova zealandicus, Cameron. Man. Mem. 23.

The following species require generic revision.

decoratorius, Fabricus. Syst. Ent. 333.

insidiator, Smith. T.E.S. I $876,476$.

deceptus, Smith. T.E.S. 1876,477 . 
Ichneumon exhilaratus, Smith. T.E.S. 477.

consanguineus, Smith. T.E.S. 476 .

placidus, Smith. T.E.S. 476 .

conspiratus, Smith. T.E.S. 476 .

perfidiosus, Smith. T.E.S. 475 .

huttoni, Kirby. T.E.S. I $881,44$.

hersilia, Cameron. Man. Mem. I898, 7 .

Probolus, Wesmael, i 844 .

lotatorius, Fabricius. Syst. Ent. 330 ; Priocnemis pascai,

Kirby; T.E.S. I 883, 200; Eristicus cinctus, Ashmead;

Pro. U.S. Nat. Mus. xii. $3^{89}$.

invectus, Smith. T.E.S. I 876,475 ; Eristicus basilaris, Ashmead, Pro. U.S. Nat. Mus. xii. 389 .

sollicitorius, Fabricius. Syst. Ent. $33^{2}$; E. apicalis,

Ashmead, Pro. U.S. Nat. Mus. xii. 388.

Amblytetes, Wesmael, I 844.

zealandicus, Cameron. T.N.Z.I. xxxiii. ro8.

hudsoni, Cameron, T.N.Z.I. xxxiii. rog.

Colobacis, Cameron, I 900.

forticornis, Cameron. T.N.Z.I. xxxiii. I 10.

Degithina, Cameron, I 900.

buchanani, Cameron. T.N.Z.I. xxxiii. 112.

caroli, Cameron. T.N.Z.I. xxxiii. II3.

davidi, Cameron. T.N.Z.I. xxxiii. i I 4.

hectori, Cameron. T.N.Z.I. xxxiii. I I4.

Zestocormus, Cameron, I 900.

melanoplus, Cameron. T.N.Z.I. xxxiii. I 19.

Dicœlotus, Westmael, i 844 .

striatifrons, Cameron. Man. Mem. 1898, 24.

Cryptus, Fabricius, I804.

Sub-family CRYPTinÆ.

penetrator, Smith. T.E.S. I $878,2$.

Mesostenus, Gravenhorst, I829.

albopictus, Smith. T.E.S. I 876,477 .

Hemiteles, Gravenhorst, I82g.

destructivorus, Cameron. Man. Mem. I 898,26

Bathymetis, Forster, I 862 .

antipoda, Ashmead. Ent. News, 1900, 624.

Sub-family OPHIONıN $\leftleftarrows$.

Ophion, Gravenhorst, I 829.

inutilis, Smith. T.E.S. 1876,478 .

punctatus, Cameron. Man. Mem. I898, 39. 
Ophion ferrugineus, Smith. T.E.S. I878, 2.

peregrinus, Smith. T.E.S. 1876,478 .

skelloni, Kirby. T.E.S. I 88 I, 46.

insularis, Kirby. T.E.S. I88 I, 46.

Paniscus, Gravenhorst, I 829.

productus, Brullé. Hist. Nat. des. Hym. iv. ; P.ephippia us, Smith, T.E.S. 1876,478 .

foveatus, Cameron. Man. Mem. 1898,36 .

Limnerium, Holmgren, I860.

zealandicum, Cameron. Man. Mem. I 898, 36.

hudsoni, Cameron. T.N.Z.I. xxxiii. 105.

Sub-family PimplidinÆ.

Rhyssus, Gravenhorst, 1829 .

fractinervis, Vollenhoven. Tijd Ent. 2, viii. $67 ; R$. antipodum, Smith, T.E.S. $1876,479$.

clavula, Colenso. T.N.Z.I. xvii. 158.

Allotheronia, Ashmead, I 900.

I2-guttata, Ashmead. P.L.S.N.S.W. I 900, 35 I.

Lissonota, Gravenhorst, I 829.

flavopicta, Smith. T.E.S. I 878,4 .

albopicta, Smith. T.E.S. 1878,4 .

multicolor, Colenso. T.N.Z.I. xvii. I 59.

tinctipennis, Cameron. Man. Mem. 1898, 28.

rubriplagiata, Cameron. T.N.Z.I. xxxiii. 106.

Euctenopus, Ashmead, I 900.

nova-zealandica, Ashmead. P.L.S.N.S.W. I $900,35 \mathrm{I}$.

Sub-family TryphoninÆ.

Tryphon, Fallen, I 8 I 3 . obstructor, Smith. T.E.S. I 878,4 .

Mesoleptus, Gravenhorst, I 829 .

mulleri, Butler. Voy. Erebus and Terror, Insects, 27.

sybarita, Cameron. Man. Mem. I 898, 32.

comparatus, Cameron. Man. Mem. I 898, 33.

Chorinæus, Holmgren, I 855 .

forticeps, Cameron. Man. Mem. I898, 29.

nigripes, Cameron. Man. Mem. I898, 33 .

Scolobates, Gravenhorst, I 829.

intrudens, Smith. T.E.S. 1878,3 ; genus doubtful.

Anacharis, Dalman, 1823 .

Family CynipIDÆ.

zealandica, Ashmead.

P.L.S.N.S.W. I 900, 329. 
Sub-order Sessiliventres.

Xiphydria, Latreille, I 802.

Family SIRICIDE.

decepta, Smith. T.E.S. I876, 474; X. Alavopicta, Smith, T.E.S. I878, I ; Brachyixiphus deceptus, Kirby, T.E.S. I88 r, 49.

Order LePIDOPTERA. Section Papilionina. Family NyMPHALIDÆ. Anosia, Hübner, 1816 .

Sub-family DaNainæ.

erripus, Cramer. Hudson, N.Z. Lep. ıо2 ; Fereday, T.N.Z.I. xxx. 327 (Danais archippus).

Sub-family SATYRINÆ.

Percnodaimon, Butler, i 876. pluto, Fereday. T.N.Z.I. xxx. 327 ; Hudson, I I4 (Erebia).

Erebiola, Fereday, I879.

butleri, Fereday. L.c. 327 ; Hudson, I 15.

Argyrophenga, Doubleday, 1845 .

antipodum, Doubleday. Fereday, 1.c. 327 ; Hudson, I I o.

Dodonidia, Butler, r 884 .

helmsi, Fereday. L.c. 327 ; Hudson, I 2.

Vanessa, Fabricius, 1805 .

Sub-family NyMPHALinæ.

gonerilla, Fab. Hudson, I05; Fereday, 1.c. 327 (Pyrameis);

T.N.Z.I. ix. 355 .

ida, Alfken. Zool. Anz. xxii. 5 (1899).

itea, Fab. Hudson, 107; Fereday, l.c. 328.

cardui, Linnæus. Hudson, 108 ; P. kershawi, Fereday.

Junonia, Hübner, I 8 I 6 .

vellida, Fabricius. Hudson, ro9; T.E.S. I 888 , I 46, pl. 6, fig. I I.

Family LyCæNIDÆ.

Chrysophanus, Hübner, I 816.

salustius, Fab. Hudson, 1 I6 ; Fereday, 1.c. 328 ; C. mani, Fereday.

feredayi, Bates. Fereday, 1.c. 329 ; Hutton, T.N.Z.I. xxxiii.

97 ; C. enysi, Hudson, l.c. I I 7.

rauparaha, Fereday. L.c. 328 ; Hutton, T.N.Z.I. xxxiii. 97.

boldenarum, White. Fereday, l.c. 329 ; Hudson, i 8.

Lycæna, Fabricius, I 808 .

oxleyi, Felder. Fereday, 1.c. 329 ; Hudson, I I9.

phœbe, Murray. Fereday, 1.c. 329 ; Hudson, Irg. 


\section{Section CARADrinina.}

Family ARCTIADE.

Metacrias, Meyrick, i 886.

strategica, Hudson, 4. Fereday, T.N.Z.I. xxx. 33I ; Howes,

T.N.Z.I. xxxiii. I 88; Quail, T.N.Z.I. xxxiv. 227.

erichrysa, Meyrick. Hudson, 4 ; Fereday, l.c. 331 .

huttoni, Butler. Hudson, 5 ; Fereday, l.c. 33 I ; Hampton,

Cat. Lep. Phal. in Brit. Mus. iii. 468.

Family HyPSIDE.

Nyctemera, Hübner, I 8 I 6 .

annulata, Boisduval. Hudson, 2 ; Fereday, l.c. 33 I ; T.N.Z.I.

ix. 355 .

Fanily Caradrinide.

Sub-family Polianæ.

Miselia, Ochsenheimer, 1816. pessota, Meyrick. Hudson, 6; Fereday, 1.c. 335 .

Orthosia, Ochsenheimer, 1816.

margarita, Hawthorne. T.N.Z.I. xxix. 283 ; Hudson, 6. comma, Walker. Hudson, 7 ; Fereday, 1.c. 335 ; T.N.Z.I. ix. 356 .

immunis, Walker. Hudson, 7 ; Fereday, l.c. 335 .

fortis, Butler. (Toxocampa), Meyrick, T.E.S. I90I, 565 .

Xanthia, Ochsenheimer, I 8 r6.

purpurea, Butler. Hudson, 8; Fereday, 1.c. 335 .

Sub-family MeLanchrine.

Physetica, Meyrick, I 887 .

cærulea, Guenée. Hudson, 8 ; Fereday, 1.c. 332.

Leucania, Hübner, I 816.

griseipennis, Felder. Hudson, 9 ; Fereday, 1.c. 332. moderata, Walker. Hudson, 9 ; Fereday, l.c. 332. temperata, Walker. Hudson, 9 ; Fereday, l.c. 332. nullifera, Walker. Hudson, 9 ; Fereday, l.c. 332. purdii, Fereday. Hudson, Io; Fereday, l.c. 332. atristriga, Walker. Hudson, Io : Fereday, l.c. 332. propria, Walker. Hudson, I1 ; Fereday, I.c. $33^{2}$. acontistis, Meyrick. Hudson, I I ; Fereday, l.c. 332. phaula, Meyrick. Hudson, I I ; Fereday, l.c. 332. alopa, Meyrick. Hudson, I 2 ; Fereday, l.c. 332. micrastra, Meyrick. Hudson, I2. unica, Walker. Hudson, I 2 ; Fereday, l.c. 332. arotis, Meyrick. Hudson, I2 ; Fereday, 1.c. 333. aulacias, Meyrick. Hudson, I 2, note; Fereday, l.c. 333. sulcana, Fereday. Hudson, I3; Fereday, 1.c. 333. semivittata, Walker. Hudson, I3; Fereday, l.c. 333. 
Leucania blenheimensis, Fereday. Hudson, 13; Fereday, T.N.Z.I. xxx. 333 .

unipuncta, Hawthorne. Hudson, 13 ; Fereday, l.c. 333 (extranea).

toroneura, Meyrick. T.E.S. $1901,565$.

Ichneutica, Meyrick, I 887.

dione, Hudson, i 4.

ceraunias, Meyrick. Hudson, 14 ; Fereday, 1.c. 333.

Melanchra, Hübner, i 8 I 6 .

disjungens, Walker. Hudson, I 5 ; Fereday, 1.c. 333 ( $M a-$ mestra).

paracausta, Meyrick. Hudson, I5 ; Fereday, l.c. 333 (Mamestra).

insignis, Walker. Hudson, 16 ; Fereday, l.c. 333 (Mamestra). maya, Hudson, I 7 .

plena, Walker. Hudson, 17 ; Fereday, l.c. 333 (.hamestra). lithias, Meyrick. Hudson, I 7 ; Fereday, l.c. 333 (Mamestra). mutans, Walker. Hudson, I8; Fereday, l.c. 333 ( $\mathrm{Ma}$ mestra); T.N.Z.I. xii. 273; Quail, T.N.Z.I. xxxiv 232. agorastis, Meyrick. Hudson, I 8 ; Fereday, l.c. 334 (Mamestra).

pictula, White. Hudson, 19 ; Fereday, l.c. 334 (Mamestra). rhodopleura, Meyrick. Hudson, I9; Fereday, l.c. 334 (Manestra).

merope, Hudson, 19.

pelistis, Meyrick. Hudson, 19 ; Fereday, l.c. 334 (Mamestra). proteastis, Meyrick. Hudson, 20; Fereday, l.c. 334 (Mamestra).

vitiosa, Butler. Hudson, 20; Fereday, l.c. 334 (Mamestra). diatmeta, Meyrick. Hudson, 2 I.

tartarea, Butler. Hudson, 2 I ; Fereday, l.c. 334 (Mamestra). homoscia, Meyrick. Hudson, 2 I ; Fereday, l.c. 334 (Mamestra).

omicron, Hudson, 22.

composita, Guenée. Hudson, 22 ; Fereday, l.c. 334 ( $M a$ mestra); T.N.Z.I. ix. 357 .

steropastis, Meyrick. Hudson, 23; Fereday, l.c. 334 ( $M a$ mestra).

infensa, Walker. Hudson, 23 ; Fereday, l.c. 334 (Mamestra). omoplaca, Meyrick. Hudson, 23 ; Fereday, l.c. 334 ( $\mathrm{Ma}$ mestra).

alcyone, Hudson, 24.

dotata, Walker. Hudson, 24 ; Fereday, l.c. 334 (Mamestra). asterope, Hudson, 24.

stipata, Walker. Hudson, 25 ; Fereday, l.c. 334 (Mamestra). octans, Hudson, 25. 
Melanchra rubescens, Butler..$_{\underline{\Sigma}}^{\leftarrow}$ Hudson, 25 ; Fereday, T.N.Z.I. xxx. 334 (Mamestra).

lignana, Walker. Hudson, 26 ; Fereday, l.c. 335 (Mamestra); T.N.Z.I. ix. 357 ( $H$. lignifurea).

cœleno, Hudson, 26.

ustistriga, Walker. Hudson, 26 ; Fereday, l.c. 335 (Mamestra); E. insignis, T.N.Z.I. ix. $35^{6}$.

prionistis, Meyrick. Hudson, 27 ; Fereday, l.c. 335 (Mamestra).

phricias, Meyrick. Hudson, 27 ; Fereday, l.c. 335 (Mamestra).

cucullina, Guenée. Hudson, 27 ; Fereday, l.c. 335 ( $M a-$ mestra).

bromias, Meyrick. T.E.S. I900, 273 .

Erana, Walker, I 857 .

graminosa, Walker. Hudson, 28 ; Fereday, l.c. 335.

Bityla, Walker, I 865 .

Sub-family CARADRININe.

defigurata, Walker. Hudson, 29 ; Fereday, l.c. 335.

sericea, Butler. Hudson, 29 ; Fereday, l.c. 336.

Agrotis, Ochsenheimer, I 8 I 6.

ypsilon, Bott. Hudson, 30 ; Fereday, l.c. 336.

admirationis, Guenée. Hudson, 31 ; Fereday, l.c. 336.

innominata, Hudson, $3 \mathrm{I}$.

sericea, Butler, $3 \mathrm{I}$; Fereday, l.c. 336.

ceropachoides, Guenée. Hudson, 32 ; Fereday, l.c. 336.

Heliothis, Ochsenheimer, 1816 .

armigera, Hübner. Hudson, 32 ; Fereday, l.c. 336.

Cosmodes, Guenée, 1852 .

elegans, Donovan: Hudson, 33 ; Fereday, l.c. 336.

Family Plusiade.

Sub-family HYPNine.

Hypenodes, Guenée, I 882 .

exsularis, Meyrick. Hudson, 34 ; Fereday, l.c. 337.

anticlina, Meyrick. T.E.S. I90I, 566; R. octias, Hudson, not of Meyrick.

Sub-family Plusianæ.

Plusia, Ochsenheimer, I 8 i 6.

chalcites, Esper. Hudson, 35 ; Fereday, l.c. 336 (eriosoma).

Dasypodia, Guenée, 1852.

selenophora, Guenée. Hudson, 35 ; Fereday, 1.c. 336 ; Colenso, T.N.Z.I. xi. 300.

Rhapsa, Walker, I 865.

scotosialis, Walker. Hudson, 36 ; Fereday, l.c. 337 . 
Series Notodontina.

Tatosoma, Butler, 1874 .

Family HYDRIOMENIDE.

lestevata, Walker. Hudson, 39; Fereday, T.N.Z.I. xxx. 339. agrionata, Walker. Hudson, 40 ; Fereday, l.c. 340.

timora, Meyrick. Hudson, 40 ; Fereday, l.c. 340.

Paradetis, Meyrick, r 886.

porphyrias, Meyrick. Hudson, $4 \mathrm{r}$; Fereday, l.c. 338.

Microdes, Guenée, 1857.

epicryptis, Meyrick. T.E.S. 1897,384 .

Chloroclystis, Hübner, I8I 6 .

plinthina, Meyrick. Hudson, 4I ; Fereday, l.c. 339 (Pasiphila).

bilineolata, Walker. Hudson, 4 I ; Fereday, l.c. 339 (Pasiphila).

antarctica, Hudson, 42 .

aristias, Meyrick. Hudson, 42.

nereis, Meyrick. Hudson, 43; Fereday, l.c. 339 (Pasiphila). dryas, Meyrick. Hudson, 43; Fereday, l.c. 339 (Pasiphila). sphragitis, Meyrick. Hudson, 43 ; Fereday, l.c. 339 (Pasiphila).

lichenodes, Purdie. Hudson, 44; Fereday, l.c. 339 (Pasiplita).

indicataria, Walker. Hudson, 44; Fereday, l.c. 339 (Pasiphila).

inductata, Walker. Hudson, 44; Fereday, l.c. 339 (Pasiphila). maculata, Hudson, 44.

rectilineata, Hudson, 45 .

Phrissogonus, Butler, I 882.

denotatus, Walker. Hudson, 45 ; Fereday, l.c. 339.

Elvia, Walker, r862.

glaucata, Walker. Hudson, 46 ; Fereday, l.c. 339 .

Hydriomena, Hübner, I8I6.

globiata, Felder. Hudson, 47 ; Fereday, l.c. 340 (Scotosia). prionota, Meyrick. Hudson, 47.

deltoidata, Walker. Hudson, 47 ; Fereday, 1.c. $340(\mathrm{Ce}-$ phalissa).

hemizona, Meyrick. Hudson, 48 .

subochraria, Doubleday. Hudson, 48 ; Fereday, l.c. $34 \mathbf{I}$ (Anachloris).

triphragma, Meyrick. Hudson, 49; Fereday, l.c. 34I (Cidaria).

rixata, Felder. Hudison, 49 ; Fereday, l.c. 34 I (Cidaria). purpurifera, Fereday. Hudson, 49; Fereday, l.c. 34I (Cidaria). 
Hydriomena similata, Walker. Hudson, 50; Fereday, T.N.Z.I. xxx. 34 I (Cidaria).

callichlora, Butler. Hudson, 50; Fereday, l.c. 34 I (Cidaria). arida, Butler. Hudson, 50 ; Fereday, l.c. 34 I (Cidaria).

siria, Meyrick. Hudson, 5 I ; Fereday, l.c. 344 (Cephalissa).

Euchœca, Hübner, I8I6.

rubropunctaria, Doubleday. Hudson, 5 I ; Fereday, l.c. 338 . (Epicyme).

Asthena, Hübner, I8I6.

pulchraria, Doubleday. Hudson, 52 ; Fereday, l.c. 340.

schistaria, Walker. Hudson, 52 ; Fereday, l.c. 34c.

Venusia, Curtis, I839.

verriculata, Felder. Hudson, 53 ; Fereday, l.c. $33^{8}$ ( $\mathrm{Pa}$ neyma).

xanthaspis, Meyrick. Hudson, 54; Fereday, 1.c. $33^{8}$ (Aulopola).

undosata, filder. Hudson, 54; Fereday, 1.c. 338 (Epiphryne)." " hitu

Asaphodes, Meyrick, 1886.

abrogata, Walker. Hudson, 55 ; Fereday, l.c. $33^{8}$.

siris, Hawthorn. Hudson, 55.

megaspilata, Walker. Hudson, 55 ; Fereday, l.c. $33^{8}$ (Probolcea); Quail, T.N.Z.I. xxxiv. 235 .

parora, Meyrick. Hudson, 56 ; Fereday, 1.c. $33^{8}$ (Probolcea). rufescens, Butler. Hudson, 56 ; Fereday, 1.c. $33^{8}$ (Homodotis).

Xanthorhoë, Hubner, I 8 I 6 .

limonodes, Meyrick. Hudson, 57; Fereday, l.c. $34 \mathrm{I}$ (Epyaxa).

subductata, Walker. Hudson, 57; Fereday, l.c. 34I (Epyaxa).

rosearia, Doubleday. Hudson, 57 ; Fereday, 1.c. 340 (Epyaxa).

orophyla, Meyrick. Hudson, 58; Fereday, l.c. 34 I (Epyaxa). semifissata, Walker. Hudson, 59; Fereday, l.c. 34 I (Epyaxa).

lophogramma, Meyrick. Hudson, 59.

chlamydota, Meyrick. Hudson, 59; Fereday, 1.c. 341 (Epyaxa).

stinaria, Guenée. Hudson, 60; Fereday, l.c. 342 (Larentia). mnesichola, Meyrick. Hudson, 60; Fereday, l.c. 343 (Larentia).

præfectata, Walker. Hudson, 6o; Fereday, l.c. 342 (Larentia).

nephelias, Meyrick. Hudson, 6r ; Fereday, 1.c. 342 ( $L a-$ rentia). 
Xanthorhoë cataphracta, Meyrick. Hudson, 61 ; Fereday, T.N.Z.I. xxx. 342 (Larentia).

clarata, Walker. Hudson, 6r; Fereday, l.c. 342 (Larentia). cosmodosa, Meyrick. Hudson, 62 ; Fereday, l.c. 343 ( $L a-$ rentia).

bryopis, Meyrick. Hudson, 62 ; Fereday, 1.c. 343 (Larentia). beata, Butler. Hudson, 63 ; Fereday, l.c. 342 (Larentia). adonis, Hudson, 63 .

chlorias, Meyrick. Hudson, 63; Fereday, l.c. 342 (Larentia). ægrota, Butler. Hudson, 64 ; Fereday, l.c. 342 (Larentia). lucidata, Walker. Hudson, 64 ; Fereday, l.c. 342 (Larentia). helias, Meyrick. Hudson, 64; Fereday, l.c. 342 (Larentia). prasinias, Meyrick. Hudson, 65 ; Fereday, l.c. 342 ( $L a-$ rentia).

chionogramma, Meyrick. Hudson, 65 ; Fereday, l.c. 342 (Larentia).

camelias, Meyrick. Hudson, 65 ; Fereday, l.c. 343 (Larentia). falcata, Butler. Hudson, 66; Fereday, l.c. 343 (Larentia). obarata, Felder. Hudson, 66; Fereday, l.c. 342 (Larentia). chorica, Meyrick. Hudson, 66; Fereday, l.c. 343 (Larentia). subobscurata, Walker. Hudson, 66; Fereday, l.c. 342 (Larentia).

cinerearia, Doubleday. Hudson, 67; Fereday, l.c. 343 (Larentia).

anthracias, Meyrick. Hudson, 67 ; Fereday, l.c. 343 ( $L a-$ rentia).

bulbulata, Guenée. Hudson, 68; Fereday, 1.c. 343 (Larentic.)

homalocyma, Meyrick. T.E.S. 1902, 274.

Lythria, Hübner, I8I6.

chrysopeda, Meyrick. Hudson, 68; Fereday, l.c. 339 (Arcteuthes).

euclidiata, Guenée. Hudson, 58 ; Fereday, 1.c. $33^{8}$ (Arcteuthes).

Dasyuris, Guenée, i 868.

enysii, Butler. Hudson, 69; Fereday, l.c. 344 (Strathmonyma).

anceps, Butler. Hudson, 69; Fereday, l.c. 344 (Strathmonyma).

partheniata, Guenée. Hudson, 70 ; Fereday, 1.c. 344.

hectori, Butler. Hudson, 70; Fereday, l.c. 344 (Strathmonyma).

Notoreas, Meyrick, 1886.

insignis, Butler. Hudson, $7 \mathrm{I}$; Fereday, l.c. 343.

orphnæa, Meyrick. Hudson, 7 I ; Fereday, l.c. 343 .

isoleuca, Meyrick. Hudson, 72. 
Notoreas mechanitis, Meyrick. Hudson, 72; Fereday, T.N.Z.I. xxx. 343 . paradelpha, Meyrick. Hudson, 72 ; Fereday, 1.c. 343. perornata, Walker. Hudson, 72 ; Fereday, l.c. 344. strategica, Meyrick. Hudson, 73 ; Fereday. 1.c. 343. callicrena, Meyrick. Hudson, 73 ; Fereday, l.c. 344. niphocrena, Meyrick. Hudson, 74 ; Fereday, 1.c. 344. simplex, Hudson, 74. ferox, Butler. Hudson, 74 ; Fereday, 1.c. 344 . zopyra, Meyrick. Hudson, 74 ; Fereday, l.c. 344. vulcanica, Meyrick. Hudson, 75; Fereday, l.c. 344 . brephosata, Walker. Hudson, 75 ; Fereday, l.c. 344 . omichlias, Meyrick. Hudson, 76 ; Fereday, l.c. 344.

Samana, Walker, i $86_{3}$.

falcatella, Walker. Hudson, 76 ; Fereday, l.c. 344 . acutata, Butler. Hudson, 76 ; Fereday, l.c. 344 .

Family Sterrh1DÆ.

Leptomeris, Hübner, r 6 .

rubraria, Doubleday. Hudson, 77 ; Fereday, 1.c. 337 (Acidalia).

Family Monocteniade.

Dichromodes, Guenée, I 857 .

nigra, Butler. Hudson, 78 ; Fereday, l.c. 345 .

gypsotis, Meyrick. Hudson, 78 ; Fereday, l.c. 345 .

petrina, Meyrick. Hudson, 78 ; Fereday, l.c. 345 .

Theoxena, Meyrick, I884.

scissaria, Guenée. Hudson, 79 ; Fereday, 1.c. 337.

Epirranthis, Hübner, 1816.

Family ORTHOSTIXID瓜.

alectoraria, Walker. Hudson, 8o; Fereday, 1.c. 345 (Lyrcea);

Marriner, T.N.Z.I. xxxiii. I 47.

hemipteraria, Guenée. Hudson, 80; Fereday, 1.c. 337

(Xyridacma).

Family Selidosenide.

Selidosema, Hübner, I 8 I6.

fenerata, Felder. Hudson. 82 ; Fereday, 1.c. 345.

rudisata, Walker. Hudson, 82 ; Fereday, l.c. 346.

suavis, Butler. Hudson, 83 ; Fereday, l.c. 345 .

humillima, Hudson, 83 .

cremnopa, Meyrick. T.E.S. 1897,387 .

productata, Walker. Hudson, 84 ; Fereday, 1.c. 345 .

aristarcha, Meyrick. Hudson, 85 ; Fereday, l.c. 346 .

melinata, Felder. Hudson, 85 ; Fereday, l.c. 346.

dejectaria, Walker. Hudson, 85 ; Fereday, l.c. 346 .

panagratta, Walker. Hudson, 87 ; Fereday, 1.c. 346 .

ombrodes, Meyrick. T.E.S. 1902, 275. 
Hybernia, Latreille, 1825 .

indocilis, Walker. Hudson, 88 ; Fereday, T.N.Z.I. xxx. 345 .

Chalastra, Walker, i 862.

pelurgata, Walker. Hudson, 88 ; Fereday, l.c. 347.

Sestra, Walker, I862.

humeraria, Walker. Hudson, 89 ; Fereday, l.c. 347.

flexata, Walker. Hudson, 90.

Gonophylla, Meyrick, i 886 .

nelsonaria, Felder. Hudson, 90; Fereday, l.c. 347.

gallaria, Walker. Hudson, 92 ; Feredáa l.c. 347.

ophiopa, Meyrick. Hudson, 93.

fortinata, Guenée. Hudson, 93 ; Fereday, l.c. 347.

Drepanodes, Guenée, I 856 .

muriferata, Walker. Hudson, 9I ; Fereday, l.c. 347.

Ipana, Walker, 1858 .

leptomera, Walker. Hudson, 94 ; Fereday, l.c. $34 \%$

Declana, Walker, i 858 .

atronivea, Walker. Hudson, 95 ; Fereday, 1.c. 346 (Detunda).

egregia, Walker. Hudson, 96 ; Fereday, l.c. 346 (Detunda). floccosa, Walker. Hudson, 96 ; Fereday, l.c. 346 ; T.N.Z.I. ix. 356 , and xii. 273 .

junctilinea, Felder. Hudson, 98 ; Fereday, l.c. 347.

hermione, Hudson, 98.

griseata, Hudson, 98 .

niveata, Butler. Hudson, 98 ; Fereday, 347 (Epicasis).

Sphinx, Linnæus, 1767 .

Family SPHINGIDÆ.

convolvuli, Linnæus. Hudson, 99; Fereday, l.c. 33 I.

\section{Section Pyralidina.}

Family PhycitidÆ.

Crocydopora, Meyrick, i 888 .

cinigerella, Walker. Fereday, T.N.Z.I. xxx. 357 .

œnospora, Meyrick. T.E.S. I $897,388$.

Homœosoma, Curtis, 1833 .

anaspila, Meyrick. T.E.S. I 901, 566.

Family CRANBIDE.

Tauroscopa, Meyrick, I888.

gorgopis, Meyrick. Fereday, l.c. 355 .

Hednota, Meyrick, I 886 .

leucophthalma, Meyrick. Fereday, T.N.Z.I. xxx. 354 . 
Diptychophora, Zeller, r 866.

pyrsophanes, Meyrick. Fereday, T.N.Z.I. xxx. 354.

chrysochyta, Meyrick. Fereday, 1.c. 354.

interrupta, Felder. Fereday, l.c. 354 .

lepidella, Walker. Fereday, l.c. 354 .

leucoxantha, Meyrick. Fereday, l.c. $35 \%$.

metallifera, Butler. Fereday, l.c. 354 .

auriscriptella, Walker. Fereday, l.c. 354 .

helioctypa, Meyrick. Fereday, l.c. 355.

elaina, Meyrick. Fereday, l.c. 355 .

selenæa, Meyrick. Fereday, l.c. 355 .

holanthes, Meyrick. Fereday, l.c. 355 .

bipunctella, Walker. Fereday, 1.c. 355 .

epiphæa, Meyrick. Fereday, 1.c. 355 .

harmonica, Meyrick. Fereday, l.c. 355.

Cryptomima, Meyrick, I 883.

acerella, Walker. Fereday, l.c. 354 .

Scenoploca, Meyrick, 1883 .

petraula, Meyrick. Fereday, 1.c. 354 .

Thinasotia, Heinemann.

claviferella, Walker. Fereday, 1.c. 355.

Crambus, Fabricius, 1798.

æthonellus, Meyrick. Fereday, 1.c. 355.

corruptus, Butler. Fereday, l.c. 355 .

ramosellus, Walker. Fereday, l.c. 356.

angustipennis, Zeller. Fereday, l.c. 356 .

dicrenellus, IValker. Fereday, l.c. $35^{6}$.

haplotomus, Meyrick. Fereday, 1.c. 356 .

callirrhoiis, Meyrick. Fereday, 1.c. 356 .

simplex, Butler. Fereday, 1.c. 356 .

seriellus, Meyrick. Fereday, 1.c. 356 .

apicellus, Zeller. Fereday, l.c. 356 .

vitellus, Doubleday. Fereday, l.c. 356 .

flexuosellus, Doubleday. Fereday, l.c. 356 .

tuhualis, Felder. Fereday, 1.c. 356 .

cyclopicus, Meyrick. Fereday, l.c. 356.

harpophorus, Meyrick. Fereday, 1.c. 356.

xanthogrammus, Meyrick. Fereday, l.c. 356.

ephorus, Meyrick. Fereday, l.c. 356 .

crenæus, Meyrick. Fereday. 1.c. $35^{6}$.

enchophorus, Meyrick. Fereday, l.c. 357.

diplorrhous, Meyrick. Fereday, l.c. 357.

pedias, Meyrick. Fereday, 1.c. 357 .

paraxenus, Meyrick. Fereday, l.c. 357.

sophronellus, Meyrick. Fereday, l.c. 357.

oncobolus, Meyrick. Fereday, l.c. 357 . 
Crambus heliotes, Meyrick. Fereday, T.N.Z.I. xxx. 357. isochytus, Meyrick. Fereday, l.c. 357. heteranthes, Meyrick. T.E.S. I $901,568$. antimorus, Meyrick. T.E.S. I901, 567. horistes, Meyrick. T.E.S. I902, 276.

Orocrambus, Meyrick, I 885 .

melampetrus, Meyrick. Fereday, l.c. 355 . catacaustus, Meyrick. Fereday, l.c. 355. tritonellus, Meyrick. Fereday, l.c. 355 . mylites, Meyrick. Fereday, l.c. 355 . thymniastes, Meyrick. T.E.S. I $901,567$.

Hymenia, Hubner, I 8 I 6.

Family Pyraustide. recurvalis, Fabricius. Fereday, l.c. 349.

Heliothela, Guenée, I 854 . atra, Butler. Fereday, l.c. 349.

Scoparia, Hawthorn, i 8 I I. oreas, Meyrick. Fereday, l.c. 349. philerga, Meyrick. Fereday, l.c. 350. chlamydota, Meyrick. Fereday, l.c. $35^{\circ}$. minusculalis, Walker. Fereday, l.c. $35^{\circ}$. hemiplaca, Meyrick. Fereday, l.c. $35^{\circ}$. linealis, Walker. Fereday, l.c. 350 . (sp. dub.) minualis, Walker. Fereday, l.c. $35^{\circ}$. chimeria, Meyrick. Fereday, l.c. $35^{\circ}$. dinodes, Meyrick. Fereday, 1.c. 350. acharis, Meyrick. Fereday, 1.c. $35^{\circ}$. cymatias, Meyrick. Fereday, l.c. $35^{\circ}$. microphthalma, Meyrick. Fereday, l.c. 350. hemicycla, Meyrick. Fereday, l.c. $35^{\circ}$. ergatis, Meyrick. Fereday, 1.c. $35^{\circ}$. encapna, Meyrick. Fereday, l.c. 350. critica, Meyrick. Fereday, l.c. 350. characta, Meyrick. Fereday, l.c. $35^{\circ}$. ustimacula, Felder. Fereday, l.c. $35^{\circ}$. pongalis, Felder. Fereday, l.c. 350 . melanægis, Meyrick. Fereday, l.c. 350 . trapezophora, Meyrick. Fereday, l.c. $35^{\circ}$. philetachra, Meyrick. Fereday, l.c. $35^{\text {I. }}$ colpota, Meyrick. Fereday, l.c. $35 \mathrm{I}$. periphanes, Meyrick. Fereday, l.c. 35 I. diphtheralis, Walker. Fereday, l.c. $35 \mathrm{I}$. submarginalis, Walker. Fereday, l.c. $35 \mathrm{I}$. cataxesta, Meyrick. Fereday, 1.c. 35I. tetracycla, Meyrick. Fereday, l.c. 35 I. 
Scoparia indistinctalis, Walker. Fereday, T.N.Z.I. xxx. $35 \mathrm{I}$ -

chalicodes, Meyrick. Fereday, 1.c. 35 r.

leptalea, Meyrick. Fereday, l.c. 35I.

psammitis, Meyrick. Fereday, 1.c. 35 I.

epicomia, Meyrick. Fereday, 1.c. 35 I.

feredayi, Knaggs. Fereday, l.c. 35I.

acompa, Meyrick. Fereday, 1.c. 35 I.

manganentis, Meyrick. Fereday, 1.c. $35 \mathrm{I}$.

crypsinoa, Meyrick. Fereday, l.c. $35 \mathrm{r}$.

axena, Meyrick. Fereday, l.c. $35 \mathrm{I}$.

steropæa, Meyrick. Fereday, l.c. $35^{2}$.

exilis, Knaggs. Fereday, 1.c. $35^{2}$.

elaphra, Meyrick. Fereday, $35^{2}$.

paltomacha, Meyrick. Fereday, 1.c. $35^{2}$.

deltophora, Meyrick. Fereday, 1.c. $35^{2}$.

sabulosella, Walker. Fereday, l.c. 352 .

panopla, Meyrick. Fereday, l.c. $35^{2}$.

trivirgata, Felder. Fereday, 1.c. 352 .

petrina, Meyrick. Fereday, l.c. 352 .

cyamenta, Meyrick. Fereday, l.c. $35^{2}$.

astragalota, Meyrick. Fereday, 1.c. $35^{2}$.

rotuella, Felder. Fereday, l.c. 352 .

harpalea, Meyrick. Fereday, 1.c. 352.

ejuncida, Knapps. Fereday, 1.c. $35^{2}$.

niphospora, Meyrick. Fereday, l.c. $35^{2}$.

apheles, Meyrick. Fereday, l.c. $35^{2}$.

aspidota, Meyrick. Fereday, l.c. 352 .

nomentis, Meyrick. Fereday, l.c. 353 .

epicremna, Meyrick. Fereday, l.c. 353 .

legnota, Meyrick. Fereday, l.c. 353.

octophora, Meyrick. Fereday, l.c. 353 .

asterisca, Meyrick. Fereday, l.c. 353.

leucogramma, Meyrick. Fereday, l.c. 353.

torodes, Meyrick. T.E.S. I901, 568.

parachalca, Meyrick. T.E.S. I90I, 569.

organæa, Meyrick. T.E.S. I90 r, 569 .

alopecias, Meyrick. T.E.S. I90 I, 570.

leptophæa, Meyrick. T.E.S. I902, 277.

Clepsicosma, Meyrick, i 888.

iridia, Meyrick. Fereday, 1.c. 354 .

Paraponyx, Hubner, i8 86 .

nitens, Butler. Fereday, 1.c. 354 .

Musotima, Meyrick, I 884.

aduncalis, Felder. Fereday, 1.c. 348 .

nitidalis, Walker. Fereday, l.c. 348 .

Diasemia, Guenée, 1854 .

grammalis, Doubleday. Fereday, 1.c. 348. 
Sceliodes, Guenée, I 854 .

cordalis, Doubleday. Fereday, T.N.Z.I. xxx. 348.

Mecyna, Guenée, 1854 .

deprivalis, Walker. Fereday, l.c. 349 .

marmarina, Meyrick. Fereday, l.c. 349 ; Meyrick, T.E.S. $1902,276$.

flavidalis, Doubleday. Fereday, l.c. 349 .

notata, Butler. Fereday, l.c. 349 .

daiclesalis, Walker. Fereday, l.c. 349 .

Protenia, Meyrick, I 884.

philocapna, Meyrick. Fereday, 1.c. 349.

Nesarcha, Meyrick, is 84 .

hybreadalis, Walker. Fereday, l.c. 349.

Deana, Butler.

Family Pyralidide.

paronalis, Walker. Fereday, l.c. 348.

Diplopseustis, Meyrick, I 884 .

minima, Butler. Fereday, l.c. 348 .

Platyptilia, Hubner, 18I6.

Family PTEROPHORIDE.

haasti, Felder. Fereday, 1.c. 353 .

falcatalis, Walker. Fereday, l.c. 353 .

heliastis, Meyrick. Fereday, l.c. 353 .

ælodes, Meyrick. T.E.S. I902, 278.

Stenoptilia, Hubner, I8I 6.

orites, Meyrick. Fereday, l.c. 353 .

charadrias, Meyrick. Fereday, l.c. 353.

lithoxesta, Meyrick. Fereday, 1.c. 353 .

Pterophorus, Geoffroy, i 76

furcatilis, Walker. Fereday, l.c. 353 .

lycosemus, Meyrick. Fereday, l.c. 353 .

monospilalis, Walker. Fereday, l.c. 353.

innotatalis, Walker. Fereday, l.c. 353 .

celidotus, Meyrick. Fereday, l.c. 353 .

Family SiculOdIDÆ.

Siculodes, Schaeffer, I 855 .

subfasciata, Walker. Fereday, l.c. 348.

Section Psychina.

Family Psychide.

Eceticus, Guilding, I 827 .

omnivorus, Fereday. Hudson, N.Z. Lep. I23; Fereday, l.c. 330 .

Orophora, Fereday, 1878 .

unicolor, Butler. Hudson, l.c. I 26 ; Fereday, l.c. 330. 
Section TorTRICINA. Family EPIBLEMIDA.

Epiblema, Hubner, I8 6.

aphrias, Meyrick. T.E.S. 190 r, 578 .

Epalxiphora, Meyrick, I 88I.

axenana, Meyrick. Fereday, T.N.Z.I. xxx. 360.

Bactra, Stephens, I 829.

lanceolana, Hubner. Fereday, l.c. 360.

Protithona, Meyrick, 1883 .

fugitivana, Meyrick. Fereday, l.c. 36 I.

Noteraula, Meyrick, I892.

straminea, Meyrick. Fereday, l.c. $36 \mathrm{I}$.

Strepticrates, Meyrick, I88 I.

ejectana, Walker. Fereday, l.c. 36 I.

zopherana, Meyrick. Fereday, 1.c. $36 \mathrm{I}$.

charopa, Meyrick. Fereday, l.c. 361.

parthenia, Meyrick. Fereday, l.c. $3^{6} \mathrm{I}$.

emplasta, Meyrick. T.E.S. I $901,571$.

Hendecasticha, Meyrick, I88 I.

æthaliana, Meyrick. Fereday, l.c. 36 r.

Exoria, Meyrick, I883.

mochlophorana, Meyrick. Fereday, l.c. $36 \mathbf{I}$.

Capua, Stephens, I834.

Family TORTRICIDE.

semiferana, Walker. Fereday, l.c. 357 .

Pyrgotis, Meyrick, I883.

plagiastana, Walker. Fereday, l.c. $35^{8}$.

zygiana, Meyrick. Fereday, l.c. $35^{8}$.

endorana, Meyrick. Fereday, l.c. 358 .

plinthoglypta, Meyrick. Fereday, l.c. 358.

pyramidias, Meyrick. T.E.S. I90 I, 57 I.

Adoxophyes, Meyrick, I883.

lotiana, Meyrick. Fereday, l.c. 358 .

conditana, Walker. Fereday, l.c. $35^{8}$.

camelina, Meyrick. Fereday, l.c. 358 .

trichroa, Meyrick. T.E.S. I901, 578.

Proselena, Meyrick, I 883.

aspistana, Meyrick. Fereday, l.c. 358 .

hermionana, Meyrick. Fereday, l.c. 358 .

siriana, Meyrick. Fereday, l.c. 358.

elephantina, Meyrick. Fereday, l.c. 358.

eremana, Meyrick. Fereday, l.c. 358 . 
Proselena loxias, Meyrick. Fereday, T.N.Z.I. xxx. $35^{8}$. eribola, Meyrick. Fereday, l.c. $35^{8}$.

zatrophana, Meyrick. Fereday, l.c. $35 \mathrm{~S}$. emphanes, Meyrick. T.E.S. I90I, 57 I.

Harmologa, Meyrick, $188_{3}$.

sityrana, Meyrick. Fereday, l.c. 358 . oblongana, Walker. Fereday, l.c. 359 . ænea, Butler. Fereday, l.c. 359. amplexana, Zeller. Fereday, l.c. 359. latomana, Meyrick. Fereday, l.c. 359. sirea, Meyrick. Fereday, 1.c. 359. petrias, Meyrick. T.E.S. 1901, 572. achrosta, Meyrick. T.E.S. I90I, 572 .

Ctenopseustris, Meyrick, I 885. obliquana, Walker. Fereday, l.c. 359.

Cacœcia, Hubner, i 8 I 6.

excessana, Walker. Fereday, l.c. 359 . enoplana, Meyrick. Fereday, l.c. 359 . alopecana, Meyrick. Fereday, l.c. 359. astrologana, Meyrick. Fereday, l.c. 359. torogramma, Meyrick. T.E.S. I 897, 388.

fervida, Meyrick. T.E.S. I $901,572$.

orthropis, Meyrick. T.E.S. I901, 573.

Tortrix, Treitschke, I 829 .

characterana, Meyrick. Fereday, l.c. 359.

demiana, Meyrick. Fereday, l.c. 359. pictoriana, Felder. Fereday, l.c. 360. philopoana, Meyrick. Fereday, l.c. 360. leucaniana, Walker. Fereday, l.c. 360. ærodana, Meyrick. Fereday, l.c. 360.

Dipterina, Meyrick, 1883 .

jactatana, Walker. Fereday, l.c. 360 . incessana, Walker. Fereday, l.c. 360 . imbriferana, Meyrick. Fereday, l.c. 360.

Eurythecta, Meyrick, I 883 . robusta, Butler. Fereday, l.c. 360 .

Prothelymna, Meyrick, I 883 . nephelotana, Meyrick. Fereday, l.c. 360.

Family Phalonide.

Heterocrossa, Meyrick, I $88_{3}$. adreptella, Walker. Fereday, l.c. 36r. gonosemana, Meyrick. Fereday, 1.c. 36 I. cryodana, Meyrick. Fereday, l.c. 36 r. 
Heterocrossa epomiana, Meyrick. Fereday, T.N.Z.I. xxx. 361 .

exochana, Meyrick. Fereday, l.c. 36r. eriphylla, Meyrick. Fereday, l.c. 362 . charaxias, Meyrick. Fereday, l.c. 362.

Isonomeutis, Meyrick, I 888 .

amauropa, Meyrick. Fereday, l.c. 362 .

Section Tineina.

Family Gelechiade.

Megacraspedus, Zeller, I 839 .

calamogonus, Meyrick. Fereday, 1.c. 362 .

Isochasta, Meyrick, 1886.

paradesma, Meyrick. Fereday, 1.c. 362 .

Thiotrichia, Meyrick, 1886.

tetrophala, Meyrick. Fereday, 1.c. 302.

thorybodes, Meyrick. Fereday, l.c. 362 .

Scieropepla, Meyrick, I 886.

typhicola, Meyrick. Fereday 1.c. 362 .

Gelechia, Hubner, I816.

solanella, Boisduval. Fereday, 1.c. 362 .

thyraula, Meyrick. Fereday, l.c. 362 .

brontophora, Meyrick. Fereday, l.c. 362.

schematica, Meyrick Fereday, 1.c. 362.

paraplura, Meyrick. Fereday, l.c. 362 .

pharetria, Meyrick. Fereday, l.c. 362 .

monophragma, Meyrick. Fereday, l.c. 362 .

lithodes, Meyrick. Fereday, 1.c. 363 .

achyrota, Meyrick. Fereday, l.c. 363 .

hippeis, Meyrick. T.E.S. I901, 573 .

Anisoplaca, Meyrick, I 886.

ptyoptera, Meyrick. Fereday, l.c. 363 .

Epiphthora, Meyrick, I 888.

melanombra, Meyrick. Fereday, 1.c. 363 .

Phæosaces, Meyrick, I 886.

Family CECOPHORIDE.

compsotypa, Meyrick. Fereday, 1 c. 363 .

apocrypta, Meyrick. Fereday, l.c. 363 .

liochroa, Meyrick. Fereday, l.c 363 .

Nymphostola, Meyrick, s 884 .

galactina, Felder. Fereday, 1.c. 363 ; Hutton, T.N.Z.I. xii. 274 .

Proteodes, Meyrick, I882.

carnifex, Butler. Fereday, l.c. $3^{6} 3$. 
Eulechria, Meyrick, r882.

zophoëssa, Meyrick. Fereday, T.N.Z.I. xxx. 363 .

photinella, Meyrick. Fereday, 1.c. 363 .

Atomotricha, Meyrick, 1884 .

ommatias, Meyrick. Fereday, l.c. $3^{6} 3$.

Brachysara, Meyrick, I 884 .

sordida, Butler. Fereday, l.c. $3^{6} 3$.

Phlœopola, Meyrick, I $88_{3}$.

dinocosma, Meyrick. Fereday, l.c. $3^{6} 4$.

Trachypepla, Meyrick, i 884

leucoplanetis, Meyrick. Fereday, 1.c. 364.

euryleucota, Meyrick. Fereday, 1.c. 364 . conspicuella, Walker. Fereday, l.c. 364 . spartodeta, Meyrick. Fereday, l.c. 364 . nyctopis, Meyrick. Fereday, 1.c. 364 . galaxias, Meyrick. Fereday, 1.c. 364 . hieropis, Meyrick. Fereday, 1.c. 364 . protochlora, Meyrick. Fereday, l.c. 364 . aspidephora, Meyrick. Fereday, l.c. 364 . anastrella, Meyrick. Fereday, l.c. 364 . lichenodes, Meyrick. Fereday, l.c. 364 .

Aschleta, Meyrick, I 884 . psychra, Meyrick. Fereday, 1.c. 364 .

Peltophora, Meyrick, is8s. amenena, Meyrick. Fereday, 1.c. 364 .

Semicosma, Meyrick, I 884 . mystis, Meyrick. Fereday, 1.c. 364 . peroneanella, Walker. Fereday, l.c. 364 . picarella, Walker. Fereday, l.c. 365 . epiphanes, Meyrick. Fereday, l.c. 365 . prasophyta, Meyrick. Fereday, l.c. 365 . austera, Meyrick. Fereday, l.c. 365 . apodoxa, Meyrick. Fereday, l.c. 365 . platyptera, Meyrick. Fereday, l.c. 365. caustopa, Meyrick. Fereday, l.c. 365 . paraneura, Meyrick. Fereday, l.c. 365 . balanophora, Meyrick. T.E.S. I 897, 389 .

Lathicrossa, Meyrick, r 884. leucocentra, Meyrick. Fereday, 1.c. 365 .

Thamnosara, Meyrick, I884. chirista, Meyrick. Fereday, l.c. 365 .

Gymnobathra, Meyrick, 1884 . coarctatella, Walker. Fereday, 1.c. 365 . 
Gymnobathra sarcoxantha, Meyrick. Fereday, T.N.Z.I. xxx. 365 .

parca, Butler. Fereday, l.c. 365 .

tholodella, Meyrick. Fereday, l.c. 365 .

calliploca, Meyrick. Fereday, l.c. 365 .

flavidella, Walker. Fereday, l.c. 365 .

hamatella, Walker. Fereday, l.c. 365 .

hyetodes, Meyrick. Fereday, l.c. 366 .

philadelpha, Meyrick. Fereday, l.c. 366.

habropis, Meyrick. Fereday, 1.c. 366.

omphalota, Meyrick. Fereday, l.c. 366.

thetodes, Meyrick. T.E.S. I 901, 574 .

Ecophora, Latreille, I 796.

scholæa, Meyrick. Fereday, l.c. 366.

letharga, Meyrick. Fereday, l.c. 366.

chloritis, Meyrick. Fereday, l.c. 366.

epimylia, Meyrick. Fereday, l.c. 366.

contextella, Walker. Fereday, l.c. 366.

hemimochla, Meyrick. Fereday, l.c. 366.

griseata, Butler. Fereday, l.c. 366 .

phlegophylla, Meyrick. Fereday, l.c. 366.

oporæa, Meyrick. Fereday, l.c. 366.

horæa, Meyrick. Fereday, l.c. 366 .

armigerella, Walker. Fereday, l.c. 366.

apanthes, Meyrick. Fereday, l.c. 366.

anæma, Meyrick. Fereday, l.c. 366.

macarella, Meyrick. Fereday, l.c. 366.

homodoxa, Meyrick. Fereday, l.c. 366.

siderodeta, Meyrick. Fereday, l.c. 366.

hoplodesma, Meyrick. Fereday, l.c. 367 ; T.E.S. 1901,574 -

chrysogramma, Meyrick. Fereday, l.c. 367 .

politis, Meyrick. Fereday, l.c. 367 .

nycteris, Meyrick. Fereday, l.c. 367 .

actinias, Meyrick. T.E.S. I901, 574 .

Cremnogenes, Meyrick, i 884.

oxyina, Meyrick. Fereday, l.c. 367 .

aphrontis, Meyrick. Fereday, l.c. 367 .

siderodeta, Meyrick. Fereday, l.c. 367 .

Vanicela, Walker, I864.

Family Elachistide.

disjunctella, Walker. Fereday, l.c. 372 .

xenadelpha, Meyrick. Fereday, l.c. 372.

Stathmopoda, Stainton, I 854 .

holochra, Meyrick. Fereday, l.c. 372.

phlegra, Meyrick. Fereday, l.c. 372.

campylocha, Meyrick. Fereday, l.c. 372. 
Stathmopoda skelloni, Butler. Fereday, T.N.Z.I. xxx. 372. epichlora, Meyrick. Fereday, l.c. 372. caminora, Meyrick. Fereday, l.c. 372 . aposema, Meyrick. T.E.S. I90I, 575 . mysteriastis, Meyrick. T.E.S. I90I, 575 .

Calicotis, Meyrick, I889. crucifera, Meyrick. Fereday, l.c. 372.

Thylacosceles, Meyrick, I889.

acridomima, Meyrick. Fereday, l.c. 372.

Zapyrastra, Meyrick, r889. calliphana, Meyrick. Fereday, l.c. 372 .

Limnœcia, Scudder, I 872. phragmitella, Stainton. Fereday, l.c. 372.

Syntomactis, Meyrick, 1889 . deamatella, Walker. Fereday, l.c. 372 .

Proterocosma, Meyrick, I889. apparitella, Walker. Fereday, l.c. 373 . aëllotricha, Meyrick. Fereday, l.c. 373 . anarithma, Meyrick. Fereday, 1.c. 373 .

Elachista, Treitschke, I 833 .

melanura, Meyrick. Fereday, l.c. 373 . gerasmia, Meyrick. Fereday, l.c. 373 . thallophora, Meyrick. Fereday, l.c. 373. helonoma, Meyrick. Fereday, l.c. 373 . exaula, Meyrick. Fereday, l.c. 373 . ombrodoca, Meyrick. Fereday, l.c. 373. archæonoma, Meyrick. Fereday, l.c. 373.

Batrachedra, Stainton, I 854 . eucola, Meyrick. Fereday, 1.c. 373 . arenosella, Walker. Fereday, l.c. 373 . psithyura, Meyrick. Fereday, l.c. 373 . tristicta, Meyrick. T.E.S. г 90 I, 579. agaura, Meyrick. T.E.S. I901, 579.

Endrosis, Hubner, I $\mathrm{S}$ I6. lacteella, Schiff. Fereday, l.c. 37 I.

Butalis, Treitschke, 1833 . epistrota, Meyrick. Fereday, l.c. 37 I.

Lysiphragma, Meyrick, I888. mixochlora, Meyrick. Fereday, l.c. 37 I. epixyla, Meyrick. Fereday, l.c. 37 I. howesii, Quail. T.N.Z.I. xxxiii. I 54 . 
Family Plutellide.

Sub-family GLYPHIPTERYGINÆ.

Heliostribes, Zeller, I874.

illita, Fëlder. Fereday, T.N.Z.I. xxx. 367 .

atychioides, Butler. Fereday, l.c. 367 .

electrica, Meyrick. Fereday, l.c. 367 .

Simæthis, Leach, I 8 I 9.

combinatana, Walker. Meyrick, P.L.S.N.S.IV. iv. 2 I 3 .

microlitha, Meyrick. Fereday, l.c. 367 .

marmarea, Meyrick. Fereday, l.c. 367 .

symbolæa, Meyrick. Fereday, l.c. 367 .

Glyphipteryx, Zeller, I 839 .

triselena, Meyrick. P.L.S.N.S.W. iv. 234.

euastera, Meyrick. P.L.S.N.S.W. 236.

asteronocta, Meyrick. P.L.S.N.S.W. iv. 249.

iocheæra, Meyrick. P.L.S.N.S.W. iv. 243.

acrothecta, Meyrick. P.L.S.N.S.IV. iv. 244.

astrapæa, Meyrick. P.I.S.N.S.IV. iv. 245.

transversella, Walker. Meyrick, P.L.S.N.S.W. iv, 246.

zelota, Meyrick. Fereday, T.N.Z.I. xxx. 367 .

acronoma, Meyrick. Fereday, T.N.Z.I. xxx. 367 .

leptosema, Meyrick. Fereday, T.N.Z.I. xxx. 367.

nephroptera, Meyrick. Fereday, T.N.Z.I. xxx. 367 .

Phryganostola, Meyrick, I88o.

oxymachæra, Meyrick. P.I.S.N.S.IV. iv. 25 I.

achlyoessa, Meyrick. P.L.S.N.S.W. iv. $25^{2}$.

ataracta, Meyrick. Fereday, l.c. 368 .

Circica, Meyrick, I 888.

cionophora, Meyrick. Fereday, l.c. 368.

xestobela, Meyrick. Fereday, l.c. 368 .

Pantosperma, Meyrick, r 888.

holochalea, Meyrick. Fereday, l.c. 368.

Sub-family Plutellin:.

Protosynæma, Meyrick, I 886 . eratospis, Meyrick. Fereday, l.c. 368 . steropucha, Meyrick. Fereday, l.c. 368.

Orthenches, Meyrick, r 886.

chlorocoma, Meyrick. Fereday, l.c. 368 .

prasinodes, Meyrick. Fereday, l.c. 368.

porphyritis, Meyrick. Fereday, l.c. 368.

Yponomeuta, Latreille, I 796.

cuprea, Meyrick. T.E.S. I90I, 575. 
Plutella, Schranck, ISo2.

sera, Meyrick. Fereday, T.N.Z.I. xxx. 368. psammochroa, Meyrick. Fereday, l.c. 368. antiphona, Meyrick. T.E.S. I901, 576 .

Dolichermis, Meyrick, I 89 I. chloroleuca, Meyrick. Fereday, l.c. 369.

Compristis, Meyrick, I 888 .

bifaciella, Walker. Fereday, l.c. 368 .

Hypeuryntis, Meyrick, I 897.

coricopa, Meyrick. T.E.S. 1897,389 .

Eutora, Meyrick, x 889 .

caryochroa, Meyrick. Fereday, l.c. 369. symmophora, Meyrick. Fereday, l.c. $3^{69}$.

Family Tineide.

Sub-family NepTiculin

Nepticula, Heyden, I 842.

tricentra, Meyrick. Fereday, l.c. 374 .

ogygia, Meyrick. Fereday, l.c. 37.4 .

propalæa, Meyrick. Fereday, l.c. 374 .

Sub-family GRACILIARIINE.

Gracillaria, Hawthorn, I 828 .

chrysitis, Felder. Fereday, l.c. 373 .

chalcodelta, Meyrick. Fereday, l.c. 373.

linearis, Butler. Fereday, l.c. 373 .

leucocyma, Meyrick. Fereday, l.c. 374.

aëllomacha, Meyrick. Fereday, l.c. 374.

æthalota, Meyrick. Fereday, l.c. 374 .

Coriscium, Zeller, 1839 .

miniellum, Felder. Fereday, l.c. 374 .

Conopomorpha, Meyrick, I 886.

cyanospila, Meyrick. Fereday, l.c. 374 .

Sub-family ArgyresthinNÆ.

Hofmannia, Heinemann and Wocke, 1876.

sphenota, Meyrick. Fereday, l.c. $37 \mathrm{I}$.

Circostola, Meyrick, r889.

copidota, Meyrick. Fereday, l.c. 37 I.

Cateristis, Meyrick, I889.

eustyla, Meyrick. Fereday, l.c. 37 I.

Bedellia, Stainton, I 849 .

psamminella, Meyrick. Fereday, l.c. 372. 
Sub-family ANAPHORINÆ.

Titanomis, Meyrick, 1888 .

sisyrota, Meyrick. Fereday, T.N.Z.I. xxx. $37 \mathrm{I}$.

Scoriodyta, Meyrick, r 888 .

conisalia, Meyrick. Fereday, 1.c. 370.

Mallobathra, Meyrick, 1888 .

cratæa, Meyrick. Fereday, l.c. 37 I.

metrosema, Meyrick. Fereday, l.c. 37 I.

microphanes, Meyrick. Fereday, l.c. 37 I.

homalopa, Meyrick. Fereday, l.c. 37 I.

Sub-family TrNeıNæ.

Amphixystis, Meyrick, I 90 r.

hapsimacha, Meyrick, 'T.E.S. I90I, 577.

Dryadaula, Meyrick, I 893.

pactolia, Meyrick. T.E.S. I901, 577 .

Ereunetis, Meyrick, I 888 .

technica, Meyrick. Fereday, l.c. 369 .

exospila, Meyrick. T.E.S. I 90 I, 577 .

Eschatotypa, Meyrick, r88o.

melichrysa, Meyrick, P.L.S.N.S.W. iv. 257.

Erechthias, Meyrick, I88o.

chasmatias, Meyrick. P.L.S.N.S.IV. iv. 264.

stilbella, Walker. Meyrick, P.L.S.N.S.W. iv. 265 .

charadrota, Meyrick. P.L.S.N.S.IV. iv. 268.

chionodira, Meyrick. P.L.S.N.S.W. iv. 268.

sub-pavonella, Walker. Meyrick, P.L.S.N.S.W. iv. 269 .

melanotricha, Meyrick. Fereday, l.c. 369 .

erebistis, Meyrick. Fereday, l.c. 269.

Decadarchis, Meyrick, i 89 I.

monastra, Meyrick. Fereday, l.c. 369 .

Endophthora, Meyrick, i 888 .

omogramma, Meyrick. Fereday, 1.c. 369.

pharotoma, Meyrick. Fereday, l.c. 369.

mesotypa. Meyrick. Fereday, l.c. 369 .

agriopa, Meyrick. Fereday, l.c. 369 .

Habrophila, Meyrick, I 889.

compseuta, Meyrick. Fereday, 1.c. 370.

Sagephora, Meyrick, I 888.

phortegella, Meyrick. Fereday, l.c. 370.

steropastis, Meyrick. Fereday, l.c. 370. 
Blabophanes, Zeller. Lep. Holland. ethelella, Newman. Fereday, T.N.Z.I. xxx. 370. ornithias, Meyrick. Fereday, l.c. 370.

Tinea, Fabricius, 1775 .

grammocosma, Meyrick. Fereday, l.c. 370.

belonota, Meyrick. Fereday, l.c. 370. certella, Walker. Fereday, l.c. 370. mochlota, Meyrick. Fereday, l.c. 370 . terranea, Butler. Fereday, l.c. 370 .

\section{Series Micropterygina.}

Family HePialide.

Hepialus, Fabricius, 1776 . virescens, Doubleday. Hudson, N.Z. Lep. I 29; Fereday, l.c. $33^{\circ}$; Gossett, T.N.Z.I. xi. 347 .

Porina, Walker, $185^{6}$.

dinodes, Meyrick. Hudson, l.c. I32 ; Fereday, l.c. 329 . mairi, Buller. Hudson, l.c. 132 ; Fereday, 1.c. 329. enysii, Butler. Hudson, l.c. I 33 ; Fereday, l.c. 329. characterifera, Walker. Hudson, l.c. I33; Fereday, l.c. 329. cervinata, Walker. Hudson. 1.c. 133 ; Fereday, l.c. 329 . despecta, Walker. Hudson, l.c. I34 ; Fereday, l.c. 33 o. umbraculata, Guenée. Hudson, l.c. I34; Fereday, l.c. $330 ;$ P. variolaris, Hutton, T.N.Z.I. ix. 356. signata, Walker. Hudson, l.c. I 34 ; Fereday, l.c. 330. variolaris, Guenée. Fereday, l.c. 330.

Family Micropterygidæ.

Mnesarchæa, Meyrick, 1886.

paracosma, Meyrick. Fereday, l.c. 368.

loxoscia, Meyrick. Fereday, l.c. 368.

hamadelpha, Meyrick. Fereday, 1.c. 369 .

Palæomicra, Meyrick, I886.

chalcophanes, Meyrick. Fereday, l.c. 369.

chrysargyra, Meyrick. Fereday, l.c. 369.

zonodoxa, Meyrick. Fereday, l.c. 369 .

doroxena, Meyrick. Fereday, l.c. 369 .

\section{APpendix.}

The following Butterfly has been found several times in New Zealand, but does not appear to be naturalised :-

Diadema nerina, Fabricius. Fereday, l.c. 328 ; Anosia bolina, Hudson, N.Z. Lep. I04. 
Order DIPTERA.

Series Cyclorrhapha Schizophora.

Family TACHINIDÆ.

Macquartia, R. Desvoidy, I 830 .

subtilis, Hutton. T.N.Z.I. xxxiii. 46.

vexata, Hutton. T.N.Z.I. xxxiii. 46.

Tryphera, Meigen, $\mathrm{r}_{3} 8$.

sosilus, Walker. Hutton, T.N.Z.I. xxxiii. 47.

Calcager, Hutton, $190 \mathrm{x}$.

apertum, Hutton. T.N.Z.I. xxxiii. 48 .

turbidum, Hutton. T.N.Z.I. xxxiii. 49.

temerarium, Hutton. T.N.Z.I. xxxiii. 50.

humeratum, Hutton. T.N.Z.I. xxxiii. 50.

Nemoræa, R. Desvoidy, 1830.

mestor, Walker. Hutton, T.N.Z.I. xxxiii. $5 \mathrm{I}$.

Hystricia, Macquart, $\mathrm{r} 843$.

lupina, Swederus. Hutton, T.N.Z.I. xxxiii. 51.

pachyprocta, Nowicki. Hutton, T.N.Z.I. xxxiii. 52.

Occisor, Hutton, r 90 I.

inscitus, Hutton. T.N.Z.I. xxxiii. 52. versutus, Hutton. T.N.Z.I. xxxiii. 53.

Tachina, Meigen, $\mathrm{x} 803$.

clarkii, Hutton. T.N.Z.I. xxxiii. 53 .

Procissio, Hutton, r $90 \mathrm{r}$.

cana, Hutton. T.N.Z.I. xxxiii. 54 .

modica, Hutton. T.N.Z.I. xxxiii. 55 .

valida, Hutton. T.N.Z.I. xxxiii. 55 .

montana, Hutton. T.N.Z.I. xxxiii. 55 .

Peremptor, Hutton, rgor.

pavida, Hutton. T.N.Z.I. xxxiii. 56.

egmonti, Hutton. T.N.Z.I. xxxiii. 56 .

Cerosomyia, Hutton, x90 I.

usitata, Hutton. T.N.Z.I. xxxiii. 57 .

Phorocera, R. Desvoidy, 1830.

feredayi, Hutton. T.N.Z.I. xxxiii. $5^{8}$.

clathrata, Nowicki. Hutton, T.N.Z.I. xxxiii. 5 S.

nyctemeriana, Hudson. Hutton, T.N.Z.I. xxxiii. 58.

marginata, Hutton. T.N.Z.I. xxxiii. 59.

nefaria, Hutton. T.N.Z.I. xxxiii. 59.

efferata, Hutton. T.N.Z.I. xxxiii. 59.

orasus, Walker. Hutton, T.N.Z.I. xxxiii. 6o.

atrox, Hutton. T.N.Z.I. xxxiii. 60.

funesta, Hutton. T.N.Z.I. xxxiii. 60.

perniciosa, Hutton. T.N.Z.I. xxxiii. 60. 
Gymnophania, Brauer and Bergenstamm, I889.

pernix, Hutton. T.N.Z.I. xxxiii. 61.

Phania, Meigen, I 824 .

verecunda, Hutton. T.N.Z.I. xxxiii. $6 \mathrm{r}$.

Family Muscidæ.

Calliphora, R. Desvoidy, I 830 .

quadrimaculata, Swederus. Hutton, T.N.Z.I. xxxiii. 64 ;

Travers, T.N.Z.I. iii. I I 6.

hortona, Walker. Hutton, T.N.Z.I. xxxiii. 65.

icela, Walker. Hutton, T.N.Z.I. xxxiii. 65.

antennatis, Hutton. T.N.Z.I. xxxiii. 65 .

læmica, White. Hutton, T.N.Z.I. xxxiii. 66.

eudypti, Hutton. T.N.Z.I. xxxiv. I 70.

antipodea, Hutton. T.N.Z.I. xxxiv. I 70.

Sepimentum, Hutton, ז 90 I.

fumosum, Hutton. T.N.Z.I. xxxiii. 67 .

demissum, Hutton. T.N.Z.I. xxxiii. 67 .

Family Anthomyıdæ.

Trichophthicus, Rondani, i 870 .

melas, Schiner. Hutton, T.N.Z.I. xxxiii. 70.

dolosus, Hutton. T.N.Z.I. xxxiii. 70.

carbonarius, Hutton. T.N.Z.I. xxxiii. 71 .

maculipennis, Hutton. T.N.Z.I. xxxiii. 7 I.

limpidus, Hutton. T.N.Z.I. xxxiii. 7 I.

ordinatus, Hutton. T.N.Z.I. xxxiii. 72.

villosus, Hutton. T.N.Z.I. xxxiv. 17 ז.

Limnophora, R. Desvoidy, I8zo.

rapax, Hutton. T.N.Z.I. xxxiii. 72.

aucklandica, Hutton. T.N.Z.I. xxxiv. I 72.

Homalomyia, Bouché, I 834.

fulvescens, Hutton. T.N.Z.I. xxxiii. 73 .

fuliginosa, Hutton. T.N.Z.I. xxxiii. 74 .

badia, Hutton. T.N.Z.I. xxxiii. 74 .

rava, Hutton. T.N.Z.I. xxxiii. 74 .

fraxinea, Hutton. T.N.Z.I. xxxiii. 75 .

Exsul, Hutton, I 901 .

singularis, Hutton. T.N.Z.I. xxxiii. 75 .

Phorbia, R. Desvoidy, I 830 .

novæ-zealandiæ, Hutton. T.N.Z.I. xxxiii. 76 .

Cœnosia, Meigen, 1826 .

spinipes, Walker. Hutton, T.N.Z.I. xxxiii. 76.

smithii, Hutton. T.N.Z.I. xxxiii. 77 .

rubriceps, Hutton. T.N.Z.I. xxxiii. 77 . 
Cœnosia purgatoria, Hutton. T.N.Z.I. xxxiii. 77 . algivora, Hutton. T.N.Z.I. xxxiii. 78 .

Family CORDyluride.

Cordylura, Fallen, I 819.

debilis, Hutton. T.N.Z.I. xxxiii. 79.

Family Phycodromide.

Cœlopa, Meigen, I 830 . littoralis, Hutton. T.N.Z.I. xxxiii. So. monstruosa, Hutton. T.N.Z.I. xxxiii. So. curvipes, Hutton. T.N.Z.I. xxxiv. 172. rufa, Hutton. T.N.Z.I. xxxiv. 173.

Helomyza, Fallen, I 839 .

Family HeLonyzide. scutellata, Hutton. T.N.Z.I. xxxiii. 81 . hudsoni, Hutton. T.N.Z.I. xxxiii. 8 I.

Heteromyza, Fallen, $\mathrm{I} 820$.

laquei, Hutton. T.N.Z.I. xxxiv. I73.

Leria, R. Desvoidy, i 830 .

placata, Hutton. T.N.Z.I. xxxiii. 82 .

fulva, Hutton. T.N.Z.I. xxxiii. 82 .

Family Scionyzide.

Sciomyza, Fallen, i 839 . nigricornis, Macquart. Hutton, T.N.Z.I. xxxiii. 83 .

Tetanocera, Dumeril, I $8 \mathrm{cg}$.

rara, Hutton. T.N.Z.I. xxxiii. 84 .

Limnia, R. Desvoidy, I 830 .

sigma, Walker. Hutton. T.N.Z.I. xxxiii. 84.

tranquilla, Hutton. T.N.Z.I. xxxiii. 85 .

transmarina, Schiner. Hutton, T.N.Z.I. xxxiii. 85 .

obscura, Hutton. T.N.Z.I. xxxiii. 85 .

Trigonometopus, Macquart, 1835 .

bipunctatus, Hutton. T.N.Z.I. xxxiii. 86.

Sapromyza, Fallen, i 820 .

Family SaPromyzide.

dichromata, Walker. Hutton, T.N.Z.I. xxxiii. 87 . sciomyzina, Schiner. Hutton, T.N.Z.I. xxxiii. 87 . decora, Schiner. Hutton, T.N.Z.I. xxxiii. 87.

Lauxania, Latreille, I 805 .

bilineata, Hutton. T.N.Z.I. xxxiii. 88 .

carbonaria, Hutton. T.N.Z.I. xxxiv. I73. 
Lonchæa, Fallen, I $\delta_{2}$.

aucklandica, Hutton. T.N.Z.I. xxxiv. 173.

Opomyza, Fallen, i $\delta z 0$.

Family OpOnyzide.

apicalis, Walker. Hutton, T.N.Z.I. xxxiii. 88.

Prophila, Fallen, I 820 .

Family PROPHILIDÆ.

smithii, Hutton. T.N.Z.I. xxxiii. 89 .

Family EPHYdrinide.

Ephydra, Fallen, i $8 \mathbf{I} 3$.

aquaria, Hutton. T.N.Z.I. xxxiii. 90.

Domina, Hutton, I90I.

metallica, Hutton. T.N.Z.I. xxxiii. 90.

Family DrosophILIDE.

Drosophila, Fallen, $\mathrm{x} 820$.

brouni, Hutton. T.N.Z.I. xxxiii. 9I.

marmoria, Huttoni. T.N.Z.I. xxxiii. 9x.

clarkii, Hutton. T.N.Z.I. xxxiii. 9I.

enderbyi, Hutton. T.N.Z.I. xxxiv. 174 .

Asteia, Meigen, I8I8.

levis, Hutton. T.N.Z.I. xxxiv. 175 .

Family OsCinide.

Oscinis, Latreille, $\mathrm{I} 804$.

badia, Hutton. T.N.Z.I. xxxiii. 92.

Family Agromyzidæ.

Agromyza, Fallen, I 823 . australensis, Mik. Hutton, T.N.Z.I. xxxiii. 93. fulvifrons, Hutton. T.N.Z.I. xxxiii. 93.

Milichia, Meigen, I 830 . picata, Hutton. T.N.Z.I. xxxiv. 195 . littorea, Hutton. T.N.Z.I. xxxiv. I 74 .

Ochthiphila, Fallen, I 823 . australis, Hutton. T.N.Z.I. xxxiv. 174.

Family BORBORIDE.

Borborus, Meigen, $\mathrm{I} 8 \mathrm{O}_{3}$.

empiricus, Hutton. T.N.Z.I. xxxiii. 94.

Apterina, Macquart, 1835 .

trilineata, Hutton. T.N.Z.I. xxxiii. 93. 


\section{Series Cyclorrhapha Aschiza.}

Family SIRPHIDæ.

Helophilus, Meigen, 1822 .

cingulatus, Fabricius. Hutton, T.N.Z.I. xxxiii. 37. trilineatus, Fabricius. Hutton, T.N.Z.I. xxxiii. 38 . vicinus, Hutton. T.N.Z.I. xxxiii. 38 .

antipodus, Schiner. Hutton, T.N.Z.I. xxxiii. 38. ineptus, Walker. Hutton, T.N.Z.I. xxxiii. 39. chathamensis, Hutton. T.N.Z.I. xxxiii. 39. campbellicus, Hutton. T.N.Z.I. xxxiv. I 70. latifrons, Schiner. Hurton, T.N.Z.I. xxxiii. 39.

Milesia, Latreille, ISO2. bilineata, Walker. Hutton, T.N.Z.I. xxxiii. 40.

Syrphus, Fabricius, I 775 .

novæ-zealandiæ, Macquart. Hutton, T.N.Z.I. xxxiii. 40. ortas, Walker. Hutton, T.N.Z.I. xxxiii. $4 \mathrm{I}$. ropalus, Walker. Hutton, T.N.ZI. xxxiii. 4I. obesus, Hutton. T.N.Z.I. xxxiii. 4 I.

Melanostoma, Schiner, I 860. fasciatum, Macquart. Hutton, T.N.Z.I. xxxiii. 42. apertum, Hutton. T.N.Z.I. xxxiii. 42. decessum, Hutton. T.N.Z.I. xxxiii. 43.

Series OrthorRapha Brachycera. Family Dolichopodide.

Psilopus, Meigen, 1824 . mobilis, Hutton. T.N.Z.I. xxxiii. 32. fuscatus, Hutton. T.N.Z.I. xxxiii. $3^{2}$. restrictus, Hutton. T.N.Z.I. xxiii. 33 . malitiosus, Hutton. T.N.Z.I. xxxiii. 33 . gemmatus, Walker. Hutton, T.N.Z.I. xxxiii. 33 .

Liancalus, Loëw, I $S_{57}$. vagus, Hutton. T.N.Z.I. xxxiii. 34 .

Ostenia, Hutton, I90I.

robusta, Hutton. T.N.Z.I. xxxiii. 34 .

Family EMPIDE.

Empis, Linnæus, I 767 . hudsoni, Hutton. T.N.Z.I. xxxiii. 30. smithii, Hutton. T.N.Z.I. xxxiii. 30 . brouni, Hutton. T.N.Z.I. xxxiii. 30.

Hilara, Meigen, ISz2. fulvipes, Hutton. T.N.Z.I. xxxiii. $3 \mathbf{I}$.

Clinocera, Meigen, ISz2. fumosa, Hutton. T.N.Z.I. xxxiii. 3 I. 
Apsoma, Westwood, 1876 .

Family Cyrtidæ.

muscaria, Westwood. Hutton, T.N.Z.I. xxxiii. 28.

Helle, Osten-Sacken. I 896.

longirostris, Hudson. Hutton, T.N.Z.I. xxxiii. 28.

Henops, Illiger, 1806 .

brunneus, Hutton. T.N.Z.I. xxxiii. 29.

nitens, Hutton. T.N.Z.I. xxxiii. 29.

Family Therevide.

Anarbarhynchus, Macquart, I 848 .

bilineatus, Fabricius. Hutton, T.N.Z.I. xxxiii. 24.

maori, Hutton. T.N.Z.I. xxxiii. 25.

innotatus, Walker. Hutton, T.N.Z.I. xxxiii. 25.

luridus, Schiner. Hutton, T.N.Z.I. xxxiii. 25.

exiguus, Hutton. T.N.Z.I. xxxiii. 26.

nebulosus, Hutton. T.N.Z.I. xxxiii. 26.

castaneus, Hutton. T.N.Z.I. xxxiii. 26.

micans, Hutton. T.N.Z.I. xxxiii. 27.

cupreus, Hutton. T.N.Z.I. xxxiii. 27.

Fraudator, Hutton, I $90 \mathrm{I}$,

Family Bombylide.

perspicuus, Hutton. T.N.Z.I. xxxiii. 23.

Saropogon, Loëw, I 847 .

Family Asilide.

viduus, Walker. Hutton, T.N.Z.I. xxxiii. 19.

clarkii, Hutton. T.N.Z.I. xxxiii. I9.

discus, Walker. Hutton, T.N.Z.I. xxxiii. I9.

proximus, Hutton. T.N.Z.I. xxxiii. I 9.

fugiens, Hutton. T.N.Z.I. xxxiii. 20.

antipodus, Schiner. Hutton, T.N.Z.I. xxxiii. 20.

hudsoni, Hutton. T.N.Z.I. xxxiii. 20.

chathamensis, Hutton. T.N.Z.I. xxxiii. 20. extenuatus, Hutton. T.N.Z.I. xxxiii. $2 \mathrm{I}$.

fascipes, Hutton. T.N.Z.I. xxxiv. 195.

Asilus, Linnæus, 1748 .

smithii, Hutton. T.N.Z.I. xxxiii. 2 I.

Itamus, Loëw, I 849 .

varius, Walker. Hutton, T.N.Z.I. xxxiii. 22.

bulbus, Walker. Hutton, T.N.Z.I. xxxiii. 22.

Senoprosopis, Macquart, I 839 .

lascus, Walker. Hutton, T.N.Z.I. xxxiii. 22. meridionalis, Hutton. T.N.Z.I. xxxiii. 23. 
Family TaBanide.

Tabanus, Linnæus, 1735 .

impar, Walker. Hutton, T.N.Z.I. xxxiii. I I.

oplus, White. Hutton, T.N.Z.I. xxxiii. I 2.

sordidus, Walker. Hutton, T.N.Z.I. xxxiii. I 2.

bratranchii, Nowicki. Hutton, T.N.Z.I. xxxiii. I 2.

gravis, Hutton. T.N.Z.I. xxxiii. I 3 .

sarpa, White. Hutton, T.N.Z.I. xxxiii. I3.

viridis, Hudson. Hutton, T.N.Z.I. xxxiii. I 3.

transversus, Walker. Hutton, T.N.Z.I. xxxiii. I4.

truncatus, Walker. Ricardo, A.M.N.H. 7, viii. 297.

Pangonia, Latreille, I806.

adrel, Walker. Hutton, T.N.Z.I. xxxiii. I5.

lerda, Walker. Hutton, T.N.Z.I. xxxiii. I6.

hirticeps, Nowicki. Hutton, T.N.Z.I. xxxiii. I6.

ricardoi, Hutton. T.N.Z.I. xxxiii. I6.

montana, Hutton. T.N.Z.I. xxxiii. I 7 .

Apatolestes, Williston, 1885 .

lutulentus, Hutton. T.N.Z.I. xxxiii. I8.

Family StRatiomyide.

Exaireta, Schiner, 1867 .

spiniger, Weidemann. Hutton, T.N.Z.I. xxxiii. 4. apicalis, White. Hutton, T.N.Z.I. xxxiii. 4. straznitzkii, Nowicki. Hutton, T.N.Z.I. xxxiii. 4. opposita, Walker. Hutton, T.N.Z.I. xxxiii. 5. alpina, Hutton. T.N.Z.I. xxxiii. 5.

Beris, Latreille, $\mathrm{I} 802$.

violacea, Hutton. T.N.Z.I. xxxiii. 6. micans, Hutton. T.N.Z.I. xxxiii. 6.

substituta, Walker. Hutton, T.N.Z.I. xxxiii. 6. cuprea, Hutton. T.N.Z.I. xxxiii. 6.

Odontomyia, Latreille, I809.

atrovirens, Bigot. Hutton, T.N.Z.I. xxxiii. 7. chloris, Walker. Hutton, T.N.Z.I. xxxiii. 8. collina, Hutton. T.N.Z.I. xxxiii. 8 . chathamensis, Hutton. T.N.Z.I. xxxiii. 8. fulviceps, Walker. Hutton, T.N.Z.I. xxxiii. 9. australiensis, Schiner. Hutton, T.N.Z.I. xxxiii. 9. angusta, Walker. Hutton, T.N.Z.I. xxxiii. 9.

Clitellaria, Meigen, I822. aberrans, Schiner. Hutton, T.N.Z.I. xxxiii. 10.

Cyclogaster, Macquart, 1834 . peregrinus, Hutton. T.N.Z.I. xxxiii. Iо. 
Series Orthorrhapha Nemocera.

Family SINULIDE.

Simulium, Latreille, I 802.

australiense, Schiner. Marshall, T.N.Z.I. xxviii. 3 I I ; Hudson, Man. N.Z. Ent. 53.

vexans, Mik. Hutton, T.N.Z.I. xxxiv. 170.

Dilophus, Meigen, 1803 .

Family Bibionid

nigrostigma, Walker. Hutton, T.N.Z.I. xxxiv. I93; Hudson, Man. N.Z. Ent. 52.

zealandicus, Walker. Hutton, T.N.Z.I. xxxiv. I93. insolitus, Hutton. T.N.Z.I. xxxiv. 193. segnis, Hutton. T.N.Z.I. xxxiv. I 94.

Scatopse, Geoffroy, I 764 .

carbonaria, Hutton. T.N.Z.I. xxxiv. I94.

Family Mycetophilide.

Arctoneura, Hutton, I903. New name for Cyrtoneura, which is preoccupied.

hudsoni, Marshall. T.N.Z.I. xxviii. 263 .

Nervijuncta, Marshall, I 896 .

nigrescens, Marshall. T.N.Z.I. xxviii. 266.

Casa, Hutton, 1903. New name for Huttonia, which is preoccupied.

tridens, Hutton. Marshall, T.N.Z.I. xxviii. 268.

Macrocera, Meigen, 1803.

montana, Marshall. T.N.Z.I. xxviii. 270.

howletti, Marshall. 'T.N.Z.I. xxviii. 270.

antennatis, Marshall. T.N.Z.I. xxriii. 27 I.

scoparia, Marshall. T.N.Z.I. xxviii. 272.

Bolitophila, Meigen, I 8 I 8.

luminosa, Skuse. Marshall, T.N.Z.I. xxviii. 273 ; Hudson,

Man. N.Z. Ent. 49; T.N.Z.I. xxiii. 43.

Ceroplatus, Meigen, 1818 .

dendyi, Marshall. T.N.Z.I. xxviii. 275.

hudsoni, Marshall. T.N.Z.I. xxviii. 276.

leucoceras, Marshall. T.N.Z.I. xxviii. 276 .

Platyura, Meigen, 1803 .

magna, Marshall. T.N.Z.I. xxviii. 278.

agricolæe, Marshall. T.N.Z.I. xxviii. 279.

flava, Marshall. T.N.Z.I. xxviii. $28 \mathrm{I}$.

Sciophila, Meigen, I8I8.

fagi, Marshall. T.N.Z.I. xxviii. 282.

hirta, Marshall. T.N.Z.I. xxviii. 283 . 
Parvicellula, Marshall, 1896 .

triangula, Marshall. T.N.Z.I. xxviii. 285.

Tetragoneura, Winnertz, I 846 .

nigra, Marshall. T.N.Z.I. xxviii. 286.

Aneura, Marshall, I896.

boletinoides, Marshall. T.N.Z.I. xxviii. 288.

Cycloneura, Marshall, 1896.

flava, Marshall. T.N.Z.I. xxviii. 289.

Paradoxa, Marshall, i 896 .

fusca, Marshall. T.N.Z.I. xxviii. 290.

Euryceras, Marshall, i 896.

anaclanoides, Marshall. T.N.Z.I. xxviii. 292.

Anomalomyia, Hutton, I903. New name for Anomala, which is preoccupied.

guttata, Hutton. Marshall, T.N.Z.I. xxviii. 294.

ninor, Marshall. T.N.Z.I. xxviii. 295.

Aphelomera, Skuse, 1888 .

skusei, Marshall. T.N.Z.I. xxviii. 296.

Zygomyia, Winnertz, I 863 .

flavicoxa, Marshall. T.N.Z.I. xxviii. 297.

fusca, Marshall. T.N.Z.I. xxviii. 298.

Brachidicrania, Skuse, I 888.

hiemalis, Marshall. T.N.Z.I. xxviii. 300.

Nycetophila, Meigen, I 803 .

antarctica, Hudson. Man. N.Z. Ent. 46.

sylvatica, Marshall. T,N.Z.I. xxviii. $30 \mathrm{I}$.

howletti, Marshall. T.N.Z.I. xxviii. 302.

fagi, Marshall. T.N.Z.I. xxviii. $3 \circ 3$.

variabilis, Marshall. T.N.Z.I. xxviii. 304. robusta, Marshall. T.N.Z.I. xxviii. 305 .

maculata, Marshall. T.N.Z.I. xxviii. 306.

Brevicornu, Marshall, I896.

flava, Marshall. T.N.Z.I. xxviii. 307.

fragilis, Marshall. T.N.Z.I. xxviii. 308 .

Family SCIARIDE.

Sciara, Meigen, I803. rufescens, Hutton. Cat. Dipt. N.Z. I3 (I88I).

marcilla, Hutton. T.N.Z.I. xxxiv. I92.

Trichosia, Winnertz, 1867 .

remota, Hutton. T.N.Z.I. xxxiv. I 92. 
Miastor, Meinert, 1864 .

Family Cecidomyide.

agricolæ, Marshall. T.N.Z.I. xxviii. 232.

difficilis, Marshall. T.N.Z.I. xxviii. 233.

Cecidomyia, Meigen, 1803 .

oleariæ, Maskell. T.N.Z.I. xxi. 257.

Diplosis, Loëw, 1850.

dubia, Marshall. T.N.Z.I. xxviii. 235.

difficilis, Marshall. T.N.Z.I. xxviii. 235 .

melana, Marshall. T.N.Z.I. xxviii. 236.

minuta, Marshall. T.N.Z.I. xxviii. 236.

fragilis, Marshall. T.N.Z.I. xxviii. 237.

hirta, Marshall. T.N.Z.I. xxviii. 237.

scoparia, Marshall. T.N.Z.I. xxviii. 238.

wanganuiensis, Marshall. T.N.Z.I. xxviii. 238 .

flava, Marshall. T.N.Z.I. xxviii. 239.

Epidosis, Loëw, I850.

magna, Marshall. T.N.Z.I. xxviii. 239.

agricolæ, Marshall. T.N.Z.I. xxviii. 240.

ordinaria, Marshall. T.N.Z.I. xxviii. 241.

aurea, Marshall. T.N.Z.I. xxviii. $24 \mathrm{I}$.

Campylomyza, Meigen, I $8 \circ 3$.

tenuis, Marshall. T.N.Z.I. xxviii. 242.

lincolnensis, Marshall. T.N.Z.I. xxviii. 243.

minuta, Marshall. T.N.Z.I. xxviii. 243.

nitida, Marshall. T.N.Z.I. xxviii. 244.

hirta, Marshall. T.N.Z.I. xxviii. 244.

squamata, Marshall. T.N.Z.I. xxviii. 245.

magna, Marshall. T.N.Z.I. xxviii. 245.

robusta, Marshall. T.N.Z.I. xxviii. 246.

ordinaria, Marshall. T.N.Z.I. xxviii. 247.

Lestremia, Macquart, 1827 .

novæ-zealandiæ, Marshall. T.N.Z.I. xxviii. 247.

Rhyphus, Latreille, I 804.

Family RHyPнide.

neozealandicus, Schiner. Hutton, Cat. Dipt. N.Z. го (г 88 г).

notatus, Hutton. T.N.Z.I. xxxiv. I 9 I.

Family TIPULıD有.

Dolichopeza, Curtis, i 825 . atropos, Hutton. T.N.Z.I. xxxii. 24.

Pachyrhina, Macquart, I 834 .

hudsoni, Hutton. T.N.Z.I. xxxii. 24. 
Tipula, Linnæus, I 740.

novaræ, Schiner. Hutton, T.N.Z.I. xxxii. 26.

fulva, Hutton. T.N.Z.I. xxxii. 26.

viridis, Walker. Hutton, T.N.Z.I. xxxii. 27.

obscuripennis, White. Hutton. T.N.Z.I. xxxii. 27.

dux, White. Hutton, T.N.Z.I. xxxii. 28.

orion, Hudson. Hutton, T.N.Z.I. xxxii. 28.

clara, White. Hutton, T.N.Z.I. xxxii. 29.

tenera, Hutton. T.N.Z.I. xxxii. 29.

Macromastix, Osten-Sacken, I 886.

vulpina, Hutton. T.N.Z.I. xxxii. 30.

fucata, Hutton. T.N.Z.I. xxxii. 3 r.

pallida, Hutton. T.N.Z.I. xxxii. 3 I.

montana, Hutton. T.N.Z.I. xxxii. 3 I.

lunata, Hutton. T.N.Z.I. xxxii. 32 .

binotata, Hutton. T.N.Z.I. xxxii. 32 .

Dicranomyia, Stephens, I8z9.

monilicornis, Osten-Sacken. Hutton, T.N.Z.I. xxxii. 34 . insularis, Mik. Hutton, T.N.Z.I. xxxii. 50.

kronii, Mik. Hutton, T.N.Z.I. xxxii. 50.

vicarians, Walker. Hutton, T.N.Z.I. xxxii. 33.

fasciata, Hutton. T.N.Z.I. xxxii. 34 .

nigrescens, Hutton. T.N.Z.I. xxxii. 34 .

Geranomyia, Haliday, I 833 .

annulipes, Hutton. T.N.Z.I. xxxii. 35 .

Limnobia, Meigen, I818.

fumipennis, White. Hutton, T.N.Z.I. xxxii. 35 .

conveniens, Walker. Hutton, T.N.Z.I. xxxii. $3^{6}$.

Trochobola, Osten-Sacken, I 868.

variegata, Hutton. T.N.Z.I. xxxii. 36 .

picta, Hutton. T.N.Z.I. xxxii. 37.

dohrni, Osten-Sacken. Hutton, T.N.Z.I. xxxiv. r89.

venusta, Osten-Sacken. Hutton, T.N.Z.I. xxxiv. I90.

Rhamphidia, Meigen, i 830 .

levis, Hutton. T.N.Z.I. xxxii. 38 .

Rhypholopus, Kolenati, I86o.

insulsus, Hutton. T.N.Z.I. xxxiv, I88.

fatuus, Hutton. T.N.Z.I. xxxiv. I 88.

Opifex, Hutton, I 902.

fuscus, Hutton. T.N.Z.I. xxxiv. I 88.

Gnophomyia, Osten-Sacken, I 859.

rufa, Hudson. Hutton, T.N.Z.I. xxxii. 39.

Trichocera, Meigen, 1803 .

antipodum, Mik. Hutton, T.N.Z.I. xxxii. 50. 
Limnophila, Macquart, I 834 .

sinistra, Hutton. T.N.Z.I. xxxii. 40.

argus, Hutton. T.N.Z.I. xxxii. 4I.

crassipes, Hutton. T.N.Z.I. xxxii. 4I.

denticulata, Hutton. T.N.Z.I. xxxii. 42.

marshalli, Hutton. T.N.Z.I. xxxii. 42.

umbrosa, Hutton. T.N.Z.I. xxxii. 43 .

geographica, Hutton. T.N.Z.I. xxxii. 43 .

bryobia, Mik. Hutton, T.N.Z.I. xxxii. 50.

skusei, Hutton. T.N.Z.I. xxxiv. 190.

Tinemyia, Hutton, 1900.

margaritifera, Hutton. T.N.Z.I. xxxii. 44.

Gynoplistia, Westwood, I835.

subfasciata, IValker. Hutton, T.N.Z.I. xxxii. 45.

wakefieldi, Westwood. Hutton, T.N.Z.I. xxxii. 45.

cuprea, Hudson. Hutton, T.N.Z.I. xxxii. 46.

formosa, Hutton. T.N.Z.I. xxxii. 46.

fulgens, Hutton. T.N.Z.I. xxii. 47 .

Cerozodia, Westwood, i 835 .

plumosa, Osten-Sacken. Hutton, T.N.Z.I. xxxii. 47.

Tanyderus, Philippi, r 865.

annuliferus, Hutton. T.N.Z.I. xxxii. 48.

forcipatus, Osten-Sacken. Hutton, T.N.Z.I. xxxii. 49.

Family Chironomide.

Ceratopogon, Meigen, $\mathrm{ISO}_{3}$.

antipodum, Hudson. Man. N.Z. Ent. 45.

Chironomus, Meigen, iso I.

zealandicus, Hudson. Hutton, T.N.Z.I. xxxiv. I 8 I.

lentus, Hutton. T.N Z.I. xxxiv. I 82 .

opimus, Hutton. 'T.N.Z.I. xxxiv. I 82 .

pavidus, Hutton. T.N.Z.I. xxxiv. I $8_{3}$.

ignavus, Hutton. T.N.Z.I. xxxiv. 183 .

Orthocladius, Van der Wulp, i 874 .

publicus, Hutton. T.N.Z.I. xxxiv. 184.

cingulatus, Hutton. T.N.Z.I. xxxiv. 184.

Camptocladius, Van der Wulp, 1874.

vernus, Hutton. T.N.Z.I. xxxiv. 185 .

Tanytarsus, Van der IVulp, 1874.

vespertinus, Hutton. T.N.Z.I. xxxiv. 185 .

Tanypus, Meigen, $\mathrm{r} 8 \mathrm{O}_{3}$.

languidus, Hutton. T.N.Z.I. xxxiv. I 86.

debilis, Hutton. T.N.Z.I. xxxiv. 186.

malus, Hutton. T.N.Z.I. xxxiv. 187. 
Culex, Linnæus, 1835 .

\section{Family CUlicidæ.}

albirostris, Macquart. Theobald, Mon. Culicidæ B.M. i. 382.

pervigilans, Bergroth. Theobald, Mon. Culicidæ B.M. ii. 88.

Tæniorhynchus, Arribalzaga, r899.

acer, Walker. Theobald, Mon. Culicidæ B.M. ii. 2 I I.

iracundus, Walker. Theobald, Mon. Culicidæ B.M. ii. 47.

Uranotænia, Arribalzaga, 1899.

argyropus, Walker. Theobald, Mon. Culicidæ B.M. ii. 264.

Corethra, Meigen, iso8.

antarctica, Hudson. Man. N.Z. Ent. 43; Theobald, Mon.

Culicidæ B.M. ii. 301 .

\section{Family PsychodidÆ.}

Psychoda, Latreille, 1796.

conspicillata, Hutton. Cat. Dipt. N.Z. I 3 (1881).

Pericoma, Walker, 1856.

funebris, Hutton. T.N.Z.I. xxxiv. I 80.

variegata, Hutton. T.N.Z.I. xxxiv. I 80.

Series Pupipara.

Family Hippoboscide.

Ornithomyia, Latreille, r 802.

opposita, Walker. Hutton, Cat. Dipt. N.Z. 70.

Series Aphaniptera.

Family Pulicide.

Pulex, Linnæus, I 735 .

kerguelensis, Rothschild. Nov. Zool. ii. 66 (r895).

Order Coleoptera.

Series LAMELlicornia.

Family LUCANIDE.

Lissotes, Westwood, 1855 .

reticulatus, Westwood. Man. N.Z. Coleop. 255 , No. $45^{\circ}$.

rufipes, Sharp. Man. N.Z. Coleop. I I09, No. I967.

elegans, Broun. Man. N.Z. Coleop. rr ro, No. r968. squamidorsis, White. Man. N.Z. Coleop. 25 r, No. 442. punctulatus, White. Man. N.Z. Coleop. 25 I, No. 443.

planus, Broun. Man. N.Z. Coleop. 252, No. 444.

stewarti, Broun. Man. N.Z. Coleop. 673, No. I 74. capito, Deyrolle. T.E.S. I 873,339 .

abditus, Broun. Man. N.Z. Coleop. 673 , No. I 175 . demaresti, Deyrolle. Ann. Soc. Ent. France, r 88 I, 239. helmsi, Sharp. Man. N.Z. Coleop. 770, No. 1365 . 
Lissotes ithagınis, Broun. Man. N.Z. Coleop. I ro8, No. I 966.

æmulus, Broun. Man. N.Z. Coleop. i 109, No. 2322. acmenus, Lewis. T.N.Z.I. vol. xxxiv. 203 . menalcas, Westwood. N.Z. Jour. Sci. ii. 221.

Ceratognathus, Westwood, ${ }_{1} \delta_{3} 8$. helotoides, Thomson. Man. N.Z. Coleop. 254, No. 449. alboguttatus, Bates. Man. N.Z. Coleop. 253 , No. 447. dispar, Sharp. Man. N.Z. Coleop. i r I I, No. I 969.

Mitophyllus, Parry, I 845 .

irroratus, Parry. Man. N.Z. Coleop. 253 , No. 445. parryanus, Hope. Man. N.Z. Coleop. 253 , No. 446. marmoratus, Watson. Ent. Mo. Mag. xi. 8. foveolatus, Broun. Man. N.Z. Coleop. 253 , No. 448. macrocerus, Broun. Man. N.Z. Coleop. 837, No. 1488. gibbosus, Broun. Man. N.Z. Coleop. 928, No. I666. tuberculatus, Broun. Man. N.Z. Coleop. i i i I, No. I970. cylindricus, Broun. A.M.N.H. 6, xv. 199. angusticeps, Broun. A.M.N.H. 6, xv. I99. fusculus, Broun. Man. N.Z. Coleop. 837 , No. 1489 .

Dendroblax, White, ${ }_{1} 8_{3} 6$.

earlianus, White. Man. N.Z. Coleop. $25 \mathrm{I}$, No. $44 \mathrm{I}$.

Family COPRIDE.

Saphobius, Sharp, I 873 .

edwardsi, Sharp. Man. N.Z. Coleop. 256 , No. 45 I. wakefieldi, Sharp. Man. N.Z. Coleop. 256 , No. $45^{2}$. nitidulus, Broun. Man. N.Z. Coleop. 256 , No. 453 . squamulosus, Broun. Man. N.Z. Coleop, 954, No. 1720. inflatipes, Broun. Man. N.Z. Coleop. i I I 2, No. 197 I. fuscus, Broun. Man. N.Z. Coleop. I I I3, No.. I 972. setosus, Sharp. Man. N.Z. Coleop. I I I3, No. 1973. fulvipes, Broun. Man. N.Z. Coleop. i i i 4 , No. 1974. curvipes, Broun. Man. N.Z. Coleop. I 349 , No. 2365. tibialis, Broun. A.M.N.H. 6, xv. 198 .

Aphodius, Illiger, I 798 .

Family ApHOdIDÆ.

exsculptus, White. Man. N.Z. Coleop. 258 , No. 454. candens, Broun. Man. N.Z. Coleop. 258 , No. 455. suspectus, Sharp. Man. N.Z. Coleop. 259 , No. $45^{6}$. pascoei, Sharp. Man. N.Z. Coleop. 259, No. 457. communis, Broun. Man. N.Z. Coleop. 260 , No. $45^{8}$. brouni, Sharp. Man. N.Z. Coleop. 260, No. 459. fortipes, Broun. Man. N.Z. Coleop. 954, No. I 72 I. distans, Sharp. 
Phycochus,, Broun, I 886.

graniceps, Broun. Man. N.Z. Coleop. 77 I, No. I 366.

lobatus, Broun. Man. N.Z. Coleop. x I I 4, No. 1975.

Family Melolonthide.

Stethaspis, Hope, I 837 .

suturalis, Hope. Man. N.Z. Coleop. 26 I, No. 460.

prasinus, Broun. Man. N.Z. Coleop. II I 5, No. 1976.

Costleya, Broun, i 886.

discoidea, Broun. Man. N.Z. Coleop. I I I6, No. 1977.

Pœcilodiscus, Broun, I 895 .

pulcher, Broun. A.M.N.H. 6, xv. 202.

Scythrodes, Broun, I 886.

squalidus, Broun. Man. N.Z. Coleop. 955, No. I 722.

Xylostygnus, Broun, I 886.

piceus, Broun. Man. N.Z. Coleop. 956, No. 1723.

Psilodontria, Broun, r895.

viridescens, Broun. A.M.N.H. 6, xv. 201.

Pyronota, Hope, I837.

festiva, Fabricius. Man. N.Z. Coleop. 262, No. 46 I. læta, Fabricius.

edwardsi, Sharp. Man. N.Z. Coleop. 263 , No. 462. sobrina, Sharp. Man. N.Z. Coleop. 263 , No. 463. munda, Sharp. Man. N.Z. Coleop. 264 , No. 464. electa, Broun. Man. N.Z. Coleop. i i 6 , No. 1978. regalis, Broun. Man. N.Z. Coleop. I i i6, No. I979. dives, Broun. Man. N.Z. Coleop. I I I 7, No. 1980. aurata, Broun. Man. N.Z. Coleop. I I 7 , No. I98I. purpurata, Broun. Man. N.Z. Coleop. i i 7 , No. 1982. cærulea, Broun. Man. N.Z. Coleop. i i 7 , No. I 983. lugubris, Sharp. Man. N.Z. Coleop. iा 8 , No. I 984. pallida, Broun. Man. N.Z. Coleop. i i 18 , No. I985.

Heteronyx, Guerin-Meneville, i 830 . pumilus, Sharp. Man. N.Z. Coleop. 264 , No. 465.

Eusoma, White, I 846 . rossii, White. Man. N.Z. Coleop. 268, No. 472. costella, Broun. Man. N.Z. Coleop. 269, No. 473.

Sericospilus, Sharp, 1882. advena, Sharp. Man. N.Z. Coleop. i i i 9, No. I987.

Odontria, White, I 846 . striata, White. Man. N.Z. Coleop. 265, No. 466. suavis, Broun. Man. N.Z. Cnleop. 266, No. 467. 
Odontria punctulata, Broun. Man. N.Z. Coleop. 266, No. 468.

cinnamonea, White. Man. N.Z. Coleop. 267 , No. 469. xanthosticta, White. Man. N.Z. Coleop. 267 , No. 470. sandageri, Broun. Man. N.Z. Coleop. 929, No. 1667. albonotata, Broun. Man. N.Z. Coleop. ri 18 , No. 1986. smithii, Broun. Man. N.Z. Coleop. I450, No. 2514. piciceps, Broun. Man. N.Z. Coleop. I450, No. 25 r 5. occiputalis, Broun. Man. N.Z. Coleop. I45I, No. 25 I6. marmorata, Broun. Man. N.Z. Coleop. I 45 I, No. 25 I 7. fusca, Broun. Man. N.Z. Coleop. 1452, No. 2518. glabrata, Broun. Man. N.Z. Coleop. I 452 , No. 25 I 9. obscura, Broun. A.M.N.H. 6, xv. 203. zealandica, White. Man. N.Z. Coleop. 270 , No. 474. brunnea, Broun. Man. N.Z. Coleop. 270, No. 475. sylvatica, Broun. Man. N.Z. Coleop. 268, No. 47 I.

Family Dynastide.

Pericoptus, Burmeister, I 8.47 . truncatus, Fabricius. Man. N.Z. Coleop. 27 I, No. 476. punctatus, White. Man. N.Z. Coleop. 272 , No. 477. stupidus, Sharp. Man. N.Z. Coleop. 272, No. 478. nitidulus, Broun. Man. N.Z. Coleop. 273, No. 479.

Series Adephaga.

Family CICINDELID球.

Cicindela, Linnæus, I 735 .

tuberculata, Fabricius. Man. N.Z. Coleop. 2, No. I. huttoni, Broun. Man. N.Z. Coleop. 2, No. 2. laticincta, White. Man. N.Z. Coleop. 3, No. 3. wakefieldi, Bates. Man. N.Z. Coleop. 3 , No. 4. douei, Chenu. Man. N.Z. Coleop. 3, No. 5. parryi, White. Man. N.Z. Coleop. 4, No. 6. dunedinensis, Castelnau. Man. N.Z. Coleop. 4, No. 7. feredayi, Bates. Man. N.Z. Coleop. 4, No. 8. austromontana, Bates. Man. N.Z. Coleop. 5, No, ro. perhispida, Broun. Man. N.Z. Coleop. 4, No. 9. campbelli, Broun. Man. N.Z. Coleop. 8I 7. No. I45I. helmsi, Sharp. Man. N.Z. Coleop. 975, No. I 757. incognita, Horn. Deutsche Ent. Zeit. 1892,82 . novaseelandica, Horn. Deutsche Ent. Zeit. I 892, 83 . circumpictoides, Horn. Deutsche Ent. Zeit. I 900, 207.

Family Carabide. Sub-family MigadopinÆ.

Amarotypus, Bates, 1872. edwardsi, Bates. Man. N.Z. Coleop. 6, No. I I. 
Clivina, Latreille, r 8 ro.

Sub-family SCARILINÆ.

rugithorax, Putzeys. Man. N.Z. Coleop. 7, No. I 2.

\section{Sub-family Cnemacanthinæ.}

Mecodema, Blanchard, i 853 .

sculpturatum, Blanchard. Man. N.Z. Coleop. 8, No. I3.

howittii, Castelnau. Man. N.Z. Coleop. 8, No. I4. rectolineatum, Castelnau. Man. N.Z. Coleop. 8, No. I 5. impressum, Castelnau. Man. N.Z. Coleop. 9, No. I6. lucidum, Castelnau. Man. N.Z. Coleop. 9, No. I 7. crenicolle, Castelnau. Man. N.Z. Coleop. 9, No. is. simplex, Castelnau. Man. N.Z. Coleop. 9, No. r 9. alternans, Castelnau. Man. N.Z. Coleop. Io, No. 20. crenaticolle, Redtenbacher. Man. N.Z. Coleop. Io, No. 2 I. spinifer, Broun. Man. N.Z. Coleop. Iо, No. 22. fulgidum, Broun. Man. N.Z. Coleop. 653 , No. I 142. constrictum, Broun. Man. N.Z. Coleop. 653 , No. 1 I 43. rugicolle, Broun. Man. N.Z. Coleop. 745, No. I 322. venator, Broun. Man. N.Z. Coleop. 81 7 , No. 1452. litoreum, Broun. Man. N.Z. Coleop. 875, No. I 557 a. ducale, Sharp. Man. N.Z. Coleop. 976, No. I $75^{8}$. rugiceps, Sharp. Man. N.Z. Coleop. 976, No. I759; N.Z.J.S. i. 2 I 5.

metallicum, Sharp. Man. N.Z. Coleop. 977, No. I 760. suteri, Broun. Man. N.Z. Coleop. 977, No. I 76 I.

scitulum, Broun. A.M.N.H. 6, xiv. 302 .

lineatum, Broun. A.M.N.H. 6, xiv. 303 . punctifer, Broun. Man. N.Z. Coleop. 745, No. I323. æneoniger, Broun. Man. N.Z. Coleop. Si 8 , No. I453. bullatum, Lewis. T.N.Z.I. xxxiv. 202. infimate, Lewis. T.N.Z.I. xxxiv. 202.

Metaglymma, Bates, I 867.

tibiale, Castlenau. Man. N.Z. Coleop. I I, No. 23. monilifer, Bates. Man. N.Z. Coleop. I I, No. 24. punctatum, Putzeys. Man. N.Z. Coleop. I I, No. 25. morio, Castelnau. Man. N.Z. Coleop. i I, No. 26. elongatum, Castelnau. Man. N.Z. Coleop. I 2, No. 27. aberrans, Putzeys. Man. N.Z. Coleop. i 2, No. 28. dyschirioides, Castelnau. Man. N.Z. Coleop. I 2, No. 30. oblongum, Broun. Man. N.Z. Coleop. 746, No: 1324. asperum, Broun. Man. N.Z. Coleop. 978 , No. 1762 . junctum, Broun. Man. N.Z. Coleop. 979, No. I 763. sulcatum, Sharp. Man. N.Z. Coleop. 979, I 764. tersatum, Broun. Man. N.Z. Coleop. 980, No. 1765 . rugipenne, Broun. Man. N.Z. Coleop. i321, No. 2323. thoracicum, Broun. Man. N.Z. Coleop. I322, No. 2324. 
Metaglymma modicum, Broun. A.M.N.H. 6, xiv. 305. oregoide, Broun. A.M.N.H. 6, xiv. 305.

rufipes, Broun. Man. N.Z. Coleop. 876 , No. 1558 .

Oregus, Putzeys, i 868 .

æreus, White. Man. N.Z. Coleop. I3, No. 3 I.

inæqualis, Castelnau. Man. N.Z. Coleop. I3, No. 32.

Brullea, Castelnau, i 867 .

antarctica, Castelnau. Man. N.Z. Coleop. 14, No. 33.

Diglymma, Sharp, i 886.

ovipenne, Sharp. Man. N.Z. Coleop. 98I, No. I 766.

dubium, Sharp. Man. N.Z. Coleop. 981 , No. I 767.

punctipenne, Broun. Man. N.Z. Coleop. 982 , No. 1768.

nigripes, Broun. Man. N.Z. Coleop. 982, No. 1769.

obtusum, Broun. Man. N.Z. Coleop. 819, No. I 454.

clivinoides, Castelnau. Man. N.Z. Coleop. r2, No. 29.

Acallistus, Sharp, I 886 .

simplex, Sharp. Man. N.Z. Coleop. 983 , No. I 770.

Rembus, Latreille, i 822 .

Sub-family LicıNıNe.

zeelandicus, Redtenbacher. Man. N.Z. Coleop. I5, No. 34.

Physolæsthus, Chaudoir, 1850.

insularis, Bates. Man. N.Z. Coleop. 15, No. 35 .

limbatus, Bates. Man. N.Z. Coleop. I 7, No. 39.

Dichrochile, Guérin, 1846 .

Sub-family ANCHOMENinÆ.

subopaca, Bates. Man. N.Z. Coleop. 16, No. 36 .

aterrima, Bates. Man. N.Z. Coleop. I6, No. 37.

ovicollis, Motschulsky. Man. N.Z. Coleop. I6, No. 38.

maura, Broun. Man. N.Z. Coleop. i8, No. 40.

nitida, Broun. Man. N.Z. Coleop. 746, No. I 325.

cinctiger, Broun. Man. N.Z. Coleop. 747 , No. 1326.

cephalotes, Broun. A.M.N.H. 6, xiv. 306.

fabrei, Guérin. Ann. Fr. 1846, Bull. I03.

anthracina, Broun. A.M.N.H. 6, xii. I6I.

Calathus, Bonelli, i 809 .

rubromarginatus, Blanchard. Voy. Pole Sud, Zool. iv. 624 .

Anchomenus, Bonelli, i 809 .

novæ-zealandiæ, Fairmaire. Ann. Soc. Ent. I 843 .

elevatus, White. Man. N.Z. Coleop. 2 I, No. 45 ; N.Z.J.S.

ii. 297 .

montivagus, Broun. Man. N.Z. Coleop. 22, No. 46.

feredayi, Bates. Man. N.Z. Coleop. 23 , No. 48.

lawsoni, Bates. Man. N.Z. Coleop. 23 , No. 49. 
Anchomenus submetallicus, White. Man. N.Z. Coleop. 24, No. 50.

deplanatus, White. Man. N.Z. Coleop. 25, No. 53. colensonis, White. Man. N.Z. Coleop. 25, No. 55. otagoensis, Bates. Man. N.Z. Coleop. 25 , No. 54 ; N.Z.J.S. ii. 298.

edwardsi, Bates. Man. N.Z. Coleop. 26, No. 56. sulcitarsis, Broun. Man. N.Z. Coleop. 27 , No. 58 . helmsi, Sharp. Man. N.Z. Coleop. 749, No. I 328. haasti, Broun. Man. N.Z. Coleop. 747, No. 1327. sandageri, Broun. Man. N.Z. Coleop. 748 , No. $46 a$. oreobius, Broun. Man. N.Z. Coleop. 876, No. I 559. atratus, Blanchard. Voy. Pole Sud, iv. 2r. raptor, Redtenbacher. Reise Novara, Coleop. I 8. marginellus, Erichson. Archives, I 842, I 30.

Ctenognathus, Fairmaire, $\mathrm{I} 843$.

latipennis, Sharp. Man. N.Z. Coleop. 984 , No. 45. parabilis, Broun. Man. N.Z. Coleop. 20 , No. 43. politulus, Broun. Man. N.Z. Coleop. 20, No. 46. batesi, Broun. Man. N.Z. Coleop. 20, No. 44. deformipes, Broun. Man. N.Z. Coleop. 20, No. 42. cheesemani, Broun. Man. N.Z. Coleop. 20 , No. 57. suborbithorax, Broun. Man. N.Z. Coleop. 20, No. 5 I. perrugithorax, Broun. Man. N.Z. Coleop. 20, No. 52. lucifugus, Broun. Man. N.Z. Coleop. 20, No. 1455. adamsi, Broun. Man. N.Z. Coleop. 20, No. 1686. pictonensis, Sharp. Man. N.Z. Coleop. 985, No. 1772. bidens, Chaudoir. Ann. Soc. Ent. Fr. 1875, 303. munroi, Broun. Man. N.Z. Coleop. 984, No. I 77 I. actochares, Broun. A.M.N.H. 6, xiv. 307. novæ-zealandiæ, Fairmaire. Ann. Soc. Ent. Fr. I843, I 2. neozealandicus, Chaudoir. Ann. Soc. Ent. Fr. I875, 294. crenatus, Chaudoir. Ann. Soc. Ent. Fr. 1 875, 304. cardiophorus, Chaudoir. Ann. Soc. Ent. Fr. I 875, 305. macropterus, Chaudoir. Ann. Soc. Ent. Fr. I $875,370$.

Heterodactylus, Guérin, I84I. nebrioides, Guérin. Rev. Zool. i 84 I. castaneus, Blanchard. Voy. Pol. Sud, Zool. iv. 23.

Pristancylus, Blanchard, I 853 . brevis, Blanchard. Voy. Pole Sud, Zool. iv. 23.

Drimostoma, Dejean, I8 3 I. antarctica, Castelnau. Man. N.Z. Coleop. 30, No. 63.

Sub-family FERONINe.

Prosopogmus, Chaudoir, $\mathbf{x} 865$. impressifrons, Chaudoir. Man. N.Z. Coleop. 30, No. 64 . 
Trichosternus, Chaudoir, 1865 .

antarcticus, Chaudoir. Man. N.Z. Coleop. 31, No. 65 . guerinii, Chaudoir. Man. N.Z. Coleop. 32, No. 66. capito, White. Man. N.Z. Coleop. 32, No. 67. sylvius, Bates. Man. N.Z. Coleop. 35, No. 72. aucklandicus, Broun. Man. N.Z. 33, No. 68. difformipes, Bates. Man. N.Z. Coleop. 33, No. 69. lobipes, Bates. Man. N.Z. Coleop. 34, No. 70. temukensis, Bates. Man. N.Z. Coleop. 34, No. 7 I. rectangulus, Chaudoir. Man. N.Z. Coleop. 35, No. 73. planiusculus, White. Man. N.Z. Coleop. 36, No. 75. humeralis, Broun. Man. N.Z. Coleop. 750, No. I 332. enysi, Broun. Man. N.Z. Coleop. 752, No. I334. cephalotes, Broun. Man. N.Z. Coleop. 825, No. 466. alternus, Broun. Man. N.Z. Coleop. 877 , No. I 560. urquharti, Broun. Man. N.Z. Coleop. 877 , No. r 56 r. virens, Broun. Man. N.Z. Coleop. 937, No. I687. compressus, Sharp. Man. N.Z. Coleop. 987 , No. 1774. polychætus, Broun. Man. N.Z. Coleop. 987 , No. I 775 . smithii, Broun. Man. N.Z. Coleop. 1322, No. 2325. hampdenensis, Broun. Man. N.Z. Coleop. I 323, No. 2326. crassalis, Broun. Man. N.Z. Coleop. I395, No. 2434 .

Zeopœcilus, Sharp, 1886.

calcaratus, Sharp. Man. N Z. Coleop. 989, No. 1778. achilles, Sharp. Man. N.Z. Coleop. 990, No. I 779. putus, Broun. Man N.Z. Coleop. 750, No. I $33^{\circ}$. opulentus, Broun. Man. N.Z. Coleop. 820, No. I 457.

Pterostichus, Bonelli, r 809 .

Species like Trichosternus, but without prosternal setce:fultoni, Broun. Man. N.Z. Coleop. 751, No. I333. waihorensis, Broun. Man. N.Z. Coleop. 821 , No. I 458. monticola, Broun. Man. N.Z. Coleop. S21, No. I 459. æruginosus, Broun. Man. N.Z. Coleop. 822 , No. 1460. asperatus, Broun. Man. N.Z. Coleop. 822, No. I46r. chloris, Broun. Man. N.Z. Coleop. 823, No. 1462. grassator, Broun. Man. N.Z. Coleop. 823 , No. 1463 .

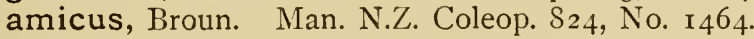
fusulus, Broun. Man. N.Z. Coleop. 824 , No. I 465. curvipes, Broun. Man. N.Z. Coleop. 878 , No. I 562. optabilis, Broun. Man. N.Z. Coleop. 913, No. I634. meritus, Broun. Man. N.Z. Coleop. 9I 4, No. I635. angulatus, Broun. Man. N.Z. Coleop. 914, No. I636. meliusculus, Broun. Man. N.Z. Coleop. 915, No. I637. agriolis, Broun. Man. N.Z. Coleop. 915, No. 1638. erraticus, Broun. Man. N.Z. Coleop. 9I 5, No. I639. deceptus, Broun. Man. N.Z. Coleop. 9I6, No. IG 4 C. 
Pterostichus curtulus, Broun. Man. N.Z. Coleop. 9i6, No. I64I.

suspicax, Broun. Man. N.Z. Coleop. 917, No. I642. amplicollis, Broun. Man. N.Z. Coleop. 918, No. I643. sandageri, Broun. Man. N.Z. Coleop. 988, No. 1776. haplopus, Broun. Man. N.Z. Coleop. I 323 , No. 2327. vagans, Broun. Man. N.Z. Coleop. 825 , No. 1467. arduus, Broun. Man. N.Z. Coleop. I 395, No. 2435. dentiferus, Broun. Man. N.Z. Coleop. 36, No. 76.

Holcaspis, Chaudoir, I 843 .

Thorax with trey sete on each side :-

prolixus, Broun. Man. N.Z. Coleop. 35, No. 74. integratus, Bates. Man. N.Z. Coleop. 40, No. 83 . cavelli, Broun. Man. N.Z. Coleop. 991, No. I780. procerulus, Broun. Man. N.Z. Coleop. 827, No. I47 I. ovatellus, Chaudoir. Man. N.Z. Coleop. 39, No. 82. placidulus, Broun. Man. N.Z. Coleop. 658, No. I 50. impiger, Broun. Man. N.Z. Coleop. 879, No. I563. mordax, Broun. Man. N.Z. Coleop. 938, No. 1688. longiformis, Sharp. Man. N.Z. Coleop. 99 I, No. I 78 I. sternalis, Broun. Man. N.Z. Coleop. 658, No. I I 49. oscillator, Sharp. Man. N.Z. Coleop. 995, No. I 786. perfidiosus, Broun. Man. N.Z. Coleop. 995, No. 7787. hunuensis, Broun. Man. N.Z. Coleop. 996, No. I 788. maorinus, Bates. Man. N.Z. Coleop. 4I, No. 88. disparalis, Broun. Man. N.Z. Coleop. I 324, No. 2328. edax, Broun. Man. N.Z. Coleop. I 326 , No. 2332. arđuus, Broun. Man. N.Z. Coleop. I 395, No. 2435. scitipennis, Broun. Man. N.Z. Coleop. I 396, No. 2436. sharpianus, Broun. A.M.H.N. 6, xii. I63.

Thorax with three sete on each side:elongellus, White.

Thorax with four setce on each side :-

hispidulus, Broun. Man. N.Z. Coleop. 40, No. 84 . cribralis, Broun. Man. N.Z. Coleop. 752, No. I 335. myrmidon, Sharp. Man. N.Z. Coleop. 993, No. 1783. hybridus, Broun. Man. N.Z. Coleop. 826, No. I469. præcox, Broun. Man. N.Z. Coleop. 827, No. 1470. brounianus, Sharp. Man. N.Z. Coleop. 992, No. 1782. constrictellus, Sharp. Man. N.Z. Coleop. 994, No. 1784. detractus, Broun. Man. N.Z. Coleop. 996, No. I 789. subæneus, Guérin. Man. N.Z. Coleop. 38, No. 79. $œ$ dicnemus, Bates. Man. N.Z. Coleop. 39, No. 8 . pastoricius, Broun. Man. N.Z. Coleop. 994, No. I 785. inconstans, Broun. Man. N.Z. Coleop. 999, No. I 793. 
Holcaspis egmontensis, Broun. Man. N.Z. Coleop 997, No. 1790.

irregularis, Broun. Man. N.Z. Coleop. 998, No. I 79 I. suteri, Broun. Man. N.Z. Coleop. I324, No. 2329. sinuellus, Broun. Man. N.Z. Coleop. I 325 , No. 2330. delator, Broun. Man. N.Z. Coleop. I 397, No. 2437. rugifrons, Sharp. Man. N.Z. Coleop. I397, No. 2438. ithaginis, Broun. A.M.N.H. 6, xii. 162. obsoletus, Broun. A.M.N.H. 6, xii. I63. lewisi, Broun. A.M.N.H. 6, xiv. 3ro.

Thorax with five or more setce on each side:catenulatus, Broun. Man. N.Z. Coleop. 753, No. 1336. mucronatus, Broun. Man. N.Z. Coleop. 826, No. I468. insidiosus, Broun. Man. N.Z. Coleop. 999, No. I 792. sculptipes, Broun. Man. N.Z. Coleop. i325, No. 233 I.

Species of doubtful position:-

thoracicus, Broun. Man. N.Z. Coleop. 657 , No. I I 48. angustulus, Chaudoir. Man. N.Z. Coleop. 38 , No. 77. sylvaticus, Chaudoir. Man. N.Z. Coleop. 38 , No. 78 . vigil, White. Man. N.Z. Coleop. 4r, No. 85 . politissima, White. Man. N.Z. Coleop. 4r, No. 86. pellax, White. Man. N.Z. Coleop. 657, No. I 447. reflexum, Chaudoir. Bull. Mosc. xxxviii. 74 . convexidorsis, Tschitschérine. Hor. Ent. Ross, I891, I66.

Alogus, Motschulsky, I $86_{5}$.

monachicus, Motschulsky. Man. N.Z. Coleop. 44, No. 92.

Cerabilia, Castlenau, i 868.

maori, Castlenau. Man. N.Z. Coleop. 44, No. 93. punctigera, Broun. Man. N.Z. Coleop. 753 , No. 1337. ruficornis, Broun. Man. N.Z. Coleop. 754 , No. $133^{8}$.

Rhytisternus, Chaudoir, I 865 . rugifrons, Broun. Man. N.Z. Coleop. 4I, No. 87. erythrognathus, Broun. Man. N.Z. Coleop. 986, No. 1773.

Steropus, Megerle, r82 I.

helmsi, Sharp. Man. N.Z. Coleop. 989, No. 1777.

Argutor, Megerle, I 837 .

erythropus, Blanchard. Man. N.Z. Coleop. 42, No. 89. piceus, Blanchard. Man. N.Z. Coleop. 43, No. 90. pantomelas, Blanchard. Voy. Pole Sud, Zool. iv. 27. calathoides, Broun. Man. N.Z. Coleop. 879, No. ${ }_{5} 54$.

Triplosarus, Bates, 1874 .

Sub-family Anisodactylinæ.

fulvescens, Bates. Man. N.Z. Coleop. 45, No. 94. novæ-zealandiæ, Castelnau. Man. N.Z. Coleop. 659,

No. I I 5 I. 
Lecanomerus, Chaudoir, 1850.

latimanus, Bates. Man. N.Z. Coleop. 45, No. 95. insignitus, Broun. Man. N.Z. Coleop. 47. No. 96. obesulus, Bates. Man. N.Z. Coleop. 47, No. 97. fuliginosus, Broun. Man. N.Z. Coleop. 48 , No. 98. fallax, Broun. Man. N.Z. Coleop. 48, No. 99. stenopus, Broun. Man. N.Z. Coleop. 88o, No. r 565. marginatus, Sharp. Man. N.Z. Coleop. Iоo0, No. I794. pallipes, Broun. A.M.N.H. 6, xiv. 379 .

Zabronothus, Broun, 1893 .

striatulus, Broun. Man. N.Z. Coleop. I 327 , No. 2333. rufipes, Broun. Man. N.Z. Coleop. I328, No. 2334.

Hypharpax, MacLeay, i 825 . antarcticus, Castelnau. Man. N.Z. Coleop. 49, No. 100. abstrusus, Bates. Man. N.Z. Coleop. 49, No. IoI.

Syllectus, Bates, 1878 . anomalus, Bates. Man. N.Z. Coleop. 52, No. 102.

Sub-family HarPalinæ.

Harpalus, Latreille, I 804.

novæ-zealandiæ, Castlenau. Trans. R. Soc. Vict. I867, Io8. antarcticus, Castelnau. Trans. R. Soc. Vict. $1867,107$.

Euthenarus, Bates, I874. brevicollis, Bates. Man. N.Z. Coleop. 53, No. 103. puncticollis, Bates. Man. N.Z. Coleop. 53, No. I04. cilicollis, Broun. T.N.Z.I. xxxiv. $\mathrm{I} 76$. huttoni, Broun. T.N.Z.I. xxxiv. 177 .

Zolus, Sharp, r 886 .

Sub-family ZoLiNe. helmsi, Sharp. Man. N.Z. Coleop. I00I, No. I 795. atratus, Broun. Man. N.Z. Coleop. 1002, No. 1796. femoralis, Broun. A.M.N.H. 6, xiv. 3r1. carinatus, Broun. Man. N.Z. Coleop. 754. No. I 339.

Megadromus, Motschulsky, I $\$ 65$. viridilimbatus, Motschulsky. Bull. Mosc. iv. 25 I.

Sub-family Pogonine.

Oöpterus, Guérin, I 84I.

laevicollis, Bates. Man. N.Z. Coleop. 54, No. ro5. patulus, Broun. Man. N.Z. Coleop. 655, No. I I 45. fulvipes, Broun. Man. N.Z. Coleop. 936, No. 1684. sobrinus, Broun. Man. N.Z. Coleop. 936, No. 1685. collaris, Broun. Man. N.Z. Coleop. 1002, No. I 797. pallidipes, Broun. Man. N.Z. Coleop. roo3, No. I 798. 
Oöpterus puncticeps, Broun. Man. N.Z. Coleop. г 398, No. 2440.

clivinoides, Guérin. Voy. Pole Sud, Zool. iv. 43.

plicaticollis, Blanchard. Voy. Pole Sud, Zool. iv. 44.

Cyclothorax, MacLeay, i $8_{73}$.

rotundicollis, White. Man. N.Z. Coleop. 54, No. I05. insularis, Motschulsky. Man. N.Z. Coleop. 29, No. 62.

Tarastethus, Sharp, $188_{3}$.

puncticollis, Sharp. Man. N.Z. Coleop. I004, No. I 799. læviventris, Sharp. Man. N.Z. Coleop. 1004, No. ISoo. alpinalis, Broun. Man. N.Z. Coleop. roo5, No. ISor. debilis, Sharp. Man. N.Z. Coleop. 1005, No. isoz. cinctus, Broun. Man. N.Z. Coleop. 1005, No. I 803. sulcicollis, Bates. Man. N.Z. Coleop. 28 , No. 59. seriatoporus, Bates. Man. N.Z. Coleop. 28 , No. 60. placens, Broun. Man. N.Z. Coleop. 2S, No. 6I.

Sympiestus, Sharp, r 866 .

syntheticus, Sharp. Man. N.Z. Coleop. roo6, No. 1804. fallax, Broun. Man. N.Z. Coleop. roo7, No. r 805. oculator, Broun. Man. N.Z. Coleop. I398, No. 2439. modestus, Broun. A.M.N.H. 6, xiv. 3II.

Sub-family BEMBIDHNÆ.

Bembidium, Latreille, 1804 . charile, Bates. Man. N.Z. Coleop. 55, No. 107. maorinum, Bates. Man. N.Z. Coleop. 55, No. ro8. tairuense, Bates. Man. N.Z. Coleop. 55, No. rog. rotundicolle, Bates. Man. N.Z. Coleop. 56, No. i Io. parviceps, Bates. Man. N.Z. Coleop. 56 , No. i ir. anchonoderum, Bates. Man. N.Z. Coleop. 57, No. I I 2. eustictum, Bates. Man. N.Z. Coleop. 57, No. I I 3 . callipeplum, Bates. Man. N.Z. Coleop. 57, No. I I 4 . orbiferum, Bates. Man. N.Z. Coleop. 58 , No. i I 5. chalceipes, Bates. Man. N.Z. Coleop. 58 , No. i I6. hokitikense, Bates. Man. N.Z. Coleop. 59, No. I 7 . musæ, Broun. Man. N.Z. Coleop. 755, No. 1340. latiusculum, Broun. Man. N.Z. Coleop. 755, No. I 34I. nesophilum, Broun. Man. N.Z. Coleop. 828, No. I 472. tantillum, Broun. Man. N.Z. Coleop. 828 , No. I 473. tekapoense, Broun. Man. N.Z. Coleop. 88o, No. ${ }_{5} 66$. attenuatum, Broun. Man. N.Z. Coleop. 881, No. I 567. clevedonense, Broun. Man. N.Z. Coleop. 1007 , No. r8o6. diaphanum, Broun. Man. N.Z. Coleop. 1007, No. I 807. dehiscens, Broun. Man. N.Z. Coleop. roo9, No. 1808. antipodum, Broun. Man. N.Z. Coleop. I 009 , No. I 809. tersatum, Broun. Man. N.Z. Coleop. roro, No. I8ro. 
Cillenum, Samouelle, I8I 9.

albescens, Bates. Man. N.Z. Coleop. 59, No. i I 8.

chalmeri, Broun. Man. N.Z. Coleop. 88 I, No. I 568.

subcæcum, Sharp. Man. N.Z. Coleop. ıо Iо, No. I 8 I I.

Tachys, Shaum, i 860.

antarcticus, Bates. Man. N.Z. Coleop. 60, No. i i 9.

latipennis, Sharp. Man. N.Z. Coleop. Io I I, No. I8 2.

oreobius, Broun. Man. N.Z. Coleop. I399, No. 2441.

cavelli, Broun. Man. N.Z. Coleop. I 400, No. 2442.

Anillus, Duval, $185 \mathrm{I}$.

pallidus, Broun. Man. N.Z. Coleop. 918, No. I644.

phyllobius, Broun. A.M.N.H. 6. xii. I64.

Actenonyx, White, $\mathrm{I} 846$.

Sub-family LEBIINÆ.

bembidioides, White. Voy. E. \& T. 6I, No. I 20.

Lebia, Latreille, I 804.

bembidioides, Fairmaire. Rev. Zool. I849, 32.

posticalis, Guérin. Voy. Coquille, Col. 58.

Wakefieldia, Broun, r880.

vittata, Broun. Man. N.Z. Coleop. 62, No. I2I.

Agonochila, Chaudoir, I 848 .

binotata, White. Man. N.Z. Coleop. 63, No. I 22.

Demetrida, White, I 846 .

lineella, White. Man. N.Z. Coleop. 64, No. I23.

nasuta, White. Man. N.Z. Coleop. 64, No. I24. picea, Chaudoir. Man. N.Z. Coleop. 65 , No. I 25. ater, Broun. Man. N.Z. Coleop. 66, No. I 26.

moesta, Sharp. Man. N.Z. Coleop. 66, No. I27.

Pedalopia, Castelnau, 1867.

novæ-zealandiæ, Castelnau. Man. N.Z. Coleop. 67 , No. I 28.

Scopodes, Erichson, I 842 .

Sub-family Pericaline.

fossulatus, Blanchard. Man. N.Z. Coleop. 68, No. I 29.

elaphroides, White. Man. N.Z. Coleop. 68, No. I 30.

edwardsi, Bates. Man. N.Z. Coleop. 68, No. I3 I.

versicolor, Bates. Man. N.Z. Coleop. 68, No. I32.

prasinus, Bates. Man. N.Z. Coleop. 69, No. I 33 .

multipunctatus, Bates. Man. N.Z. Coleop. 69, No. I34.

lævigatus, Bates. Man. N.Z. Coleop. 70, No. I 35 .

venustus, Broun. Man. N.Z. Coleop. 756, No. I342.

planus, Broun. Man. N.Z. Coleop. 756, No. I 343 .

tardus, Broun. Man. N.Z. Coleop. 757, No. I 344. 
Scopodes pustulatus, Broun. Man. N.Z. Coleop. 757, No. I 345 .

lævistriatus, Broun. Man. N.Z. Coleop. 829 , No. 1474. bryophilus, Broun. Man. N.Z. Coleop. 882, No. I 569. cognatus, Broun. Man. N.Z. Coleop. 882, No. 1570. antennalis, Broun. Man. N.Z. Coleop. 882 , No. I 571 . nigrinus, Sharp. Man. N.Z. Coleop. Ior I, No. r813. basalis, Broun. Man. N.Z. Coleop. Ior 2, No. I8I 4. speciosus, Broun. Man. N.Z. Coleop. IO 2, No. I8I5.

Family DyTiscide.

Homœodytes, Sharp, I 882 . hookeri, White. Man. N.Z. Coleop. 75, No. I4I. scutellaris, Sharp. Man. N.Z. Coleop. I 33I, No. 2338.

Rhantus, Sharp, I 882.

pulverosus, Stephens $=C$. rufimanus, White. Man. N.Z. Coleop. 74, No. I 70 ; Man. N.Z. Coleop. I 333, No. 2340. plantaris, Sharp. Man. N.Z. Coleop. I333, No. 2339.

Lancetes, Sharp, I 882 .

lanceolatus, Clark. Man. N.Z. Coleop. I335, No. 234 r.

Family Hydroporidæ.

Antiporus, Sharp, r $\$ S 2$.

uncifer, Sharp. Man. N.Z. Coleop. 1330, No. 2337. wakefieldi, Sharp. Man. N.Z. Coleop. 71, No. I36. duplex, Sharp. Man. N.Z. Coleop. 72, No. I 37. strigosulus, Broun. Man. N.Z. Coleop. 72 , No. ${ }_{1} 8$. nitidicornis, Broun. Man. N.Z. Coleop. 73, No. r 39.

Bidessus, Sharp, r 882.

impressus, Sharp. Man. N.Z. Coleop. I329, No. 2334. plicatus, Sharp. Man. N.Z. Coleop. I 329 , No. 2335. huttoni, Sharp. Man. N.Z. Coleop. 1329 , No. 2336.

Huxelhydrus, Sharp, i 882.

syntheticus, Sharp. Trans. Roy. Dub. Soc. 1882. virgatus, Broun. Man. N.Z. Coleop. Ior 4, No. i 8 I6.

Copelatus, Erichson. Sharp, I882.

sharpi, Broun. Man. N.Z. Coleop. ror 4 and 939, No. 1689.

Series Polymorpha.

Family GYRINIDE.

Gyrinus, Linnæus, 1733 . huttoni, Pascoe. Man. N.Z. Coleop. 76, No. $14^{2}$.

Family Hydroph1Lide.

Philhydrus, Solier, I 834 . tritus, Broun. Man. N.Z. Coleop. 78 , No. I 45. 
Philhydrus variolosus, Broun. Man. N.Z.Coleop. 79, No. I 46. abditus, Sharp. Man. N.Z. Coleop. Iо I8, No. I 824.

Berosus, Leach, i 8 r 7 .

mergus, Broun. Man. N.Z. Coleop. 883, No. I 572.

Phelerosus, Sharp, I884. pallidipennis, Sharp. Man. N.Z. Coleop. 1023 , No. 1834.

Saphydrus, Sharp, i 884 .

oblongus, Broun. Man. N.Z. Coleop. 8r, No. I50. suffusus, Sharp. Man. N.Z. Coleop. Ior 5, No. I I $_{7} 7$. obesus, Sharp. Man. N.Z. Coleop. го I6, No. I 8 I 8 . antennatus, Sharp. Man. N.Z. Coleop. Iог6, No. i 8 I 9. longulus, Sharp. Man. N.Z. Coleop. Ior6, No. I820. monticola, Broun. Man. N.Z. Coleop. I40I, No. 2443.

Rygmodus, White, i 846 .

modestus, White. Man. N.Z. Coleop. So, No. I 47. pedinoides, White. Man. N.Z. Coleop. So, No. I 48. incertus, Broun. Man. N.Z. Coleop. 8r, No. 149. opimus, Broun. Man. N.Z. Coleop. 82, No. 15 I. cyaneus, Broun. Man. N.Z. Coleop. 659, No. I I 52. puncticeps, Broun. Man. N.Z. Coleop. 757 , No. I346. femoratus, Sharp. Man. N.Z. Coleop. Io I6, No. I 82 I. tibialis, Broun. Man. N.Z. Coleop. I017. No. I822. ovalis, Sharp. Man. N.Z. Coleop. Iо I 7 , No. I 823. limbatus, Broun. Man. N.Z. Coleop. I335, No. 2342. alienus, Broun. Man. N.Z. Coleop. I 401 , No. 2444.

Tormus, Sharp, I 884 .

helmsi, Sharp. Man. N.Z. Coleop. I I I9, No. I $\delta 25$. nitidus, Broun. Man. N.Z. Coleop. I402, No. 2445.

Tormissus, Broun, I 893 . marginatus, Broun. Man. N.Z. Coleop. го2 I, No. 1829. magnulus, Broun. Man. N.Z. Coleop. I02 I, No. I830.

Hydrostygnus, Sharp, i 884 . linsi, Sharp. Man. N.Z. Coleop. I020, No. I 826. pontalis, Broun. Man. N.Z. Coleop. 84 , No. I 55 . minor, Broun. Man. N.Z. Coleop. I020, No. 1827. bifoveatus, Broun. Man. N.Z. Coleop. I02 I, No. I 828 .

Exydrus, Broun, I 886. flavicornis, Broun. Man. N.Z. Coleop. 84 , No. I 53. gibbosus, Broun. Man. N.Z. Coleop. 84, No. I 54 .

Stygnohydrus, Broun, I 893. nitidus, Broun. Man. N.Z. Coleop. I336, No. 2343.

Cyloma, Sharp, 1872 . lawsonus, Sharp. Man. N.Z. Coleop. 85 , No. ${ }_{5} 6$. 
Cyloma thomsonus, Sharp. Man. N.Z. Coleop. roz2, No. I $\delta_{3} \mathrm{I}$.

guttulatus, Sharp. Man. N.Z. Coleop. 1022, No. 1832. stewarti, Broun. A.M.N.H. 6, xiv. 379.

Cyclonotum, Dejean, i 833 .

marginale, Sharp. Man. N.Z. Coleop. 83 , No. ${ }_{5} 2$.

Psephoboragus, Broun, I 893 .

signatus, Broun. Man. N.Z. Coleop. $1_{403}$, No. 2446.

lineatus, Broun. Man. N.Z. Coleop. 1404, No. 2447.

Adolopus, Sharp, I884.

helmsi, Sharp. Man. N.Z. Coleop. 1023, No. I833.

badius, Broun. Man. N.Z. Coleop. 86, No. 57.

altulus, Broun. Man. N.Z. Coleop. 86, No. 158.

vicinus, Broun. Man. N.Z. Coleop. 939, No. 1690.

rugipennis, Broun. Man. N.Z. Coleop. 939, No. I691.

montanus, Broun. Man. N.Z. Coleop. I336, No. 2344.

convexus, Broun. Man. N.Z. Coleop. I 404 , No. 2448.

Cercyodes, Broun, 1893 .

lævigatus, Broun. Man. N.Z. Coleop. 94I, No. 1692.

Potaminus, Sturm, i 837 .

Family PARNIDE.

angusticollis, Pascoe. Man. N.Z. Coleop. 248 , No. 439.

Alloparnus, Broun, I893. Substituted for Parnida.

agrestis, Broun. Man. N.Z. Coleop. I 107 and 249, No. 440.

Protoparnus, Sharp, is $\$ 6$.

longulus, Sharp. Man. N.Z. Coleop. I107, No. 1965.

Family ELmide.

Hydora, Broun, I 886. Substituted for Pachycephala. picea, Broun. Man. N.Z. Coleop. 672, No. II 73. nitida, Broun. Man. N.Z. Coleop. 927 , No. I664. obsoleta, Broun. Man. N.Z. Coleop. 928, No. I665.

Family BuPRESTIDE.

Nascio, Castelnau and Gory, 1835 .

eremita, White. Man. N.Z. Coleop. 276, No. 481.

enysi, Sharp. Man. N.Z. Coleop. 276 , No. 482.

Neocharis, Sharp, i 877.

Family Eucuenide.

varia, Sharp. Man. N.Z. Coleop. 277 , No. 483 .

pubescens, Sharp. Man. N.Z. Coleop. 278 , No. 484.

simplex, Sharp. Man. N.Z. Coleop. 278 , No. 485 .

concolor, Sharp. Man. N.Z. Coleop. 278 , No. 486.

osculans, Broun. Man. N.Z. Coleop. 674 , No. i 76 . 
Talerax, Sharp, 1877 .

distans, Sharp. Man. N.Z. Coleop. 279, No. 487. capax, Broun. Man. N.Z. Coleop. 674, No. I I 77 . niger, Broun. Man. N.Z. Coleop. 675 , No. I I 78 . rusticus, Broun. Man. N.Z. Coleop. 675, No. I I 79. foveatus, Broun. Man. N.Z. Coleop. 676, No. ir 80. tenuis, Broun. Man. N.Z. Coleop. 772, No. I 368. micans, Broun. Man. N.Z. Coleop. I 350, No. 2366.

Drasterius, Eschscoltz, I829. nigellus, White. Man. N.Z. Coleop. 279, No. 488.

Melanus, Broun, i 88I. sculptus, Broun. Man. N.Z. Coleop. 677 , No. I I 8 I.

Agalba, Broun, 1893 . ruficornis, Broun. Man. N.Z. Coleop. i I 20, No. IgSS. nigrescens, Broun. Man. N.Z. Coleop. I I 2 , No. I 989. cylindrata, Broun. Man. N.Z. Coleop. 77 I, No. 1367 .

Family Elateride.

Protelater, Sharp, I 877 . elongatus, Sharp. Man. N.Z. Coleop. 305 , No. 545 . huttoni, Sharp. Man. N.Z. Coleop. 305, No. 546. guttatus, Sharp. Man. N.Z. Coleop. $3 \circ 5$, No. 547. picticornis, Sharp. Man. N.Z. Coleop. 306, No. 548. opacus, Sharp. Man. N.Z. Coleop. 306, No. 549. nigricans, Sharp. Man. N.Z. Coleop. 772, No. 1369. vitticollis, Broun. Man. N.Z. Coleop. 956, No. I 724 . atriceps, Broun. Man. N.Z. Coleop. 957, No. 1725. urquharti, Broun. Man. N.Z. Coleop. I I 2 I, No. I 990. costiceps, Broun. Man. N.Z. Coleop. I I 2 I, No. I 99 I. pubescens, Broun. Man. N.Z. Coleop. II 22, No. I992.

Geranus, Sharp, I 877 . collaris, Pascoe. Man. N.Z. Coleop. 302, No. 540. lineicollis, White. Man. N.Z. Coleop. 304, No. 544. fulvus, Sharp. Man. N.Z. Coleop. 303, No. 542. similis, Sharp. Man. N.Z. Coleop. 303 , No. 543.

Parinus, Sharp, 1877 . villosus, Sharp. Man. N.Z. Coleop. 301 , No. $53^{8}$.

Corymbites, Latreille, I 834 . antipodus, Candèze. Man. N.Z. Coleop. 299, No. 532. dubius, Sharp. Man. N.Z. Coleop. 299, No. 533. strangulatus, White. Man. N.Z. Coleop. 300, No. 534. myops, White. Man. N.Z. Coleop. 300, No. 535. olivascens, White. Man. N.Z. Coleop. $30^{\circ}$, No. 536. agriotoides, Sharp. Man. N.Z. Coleop. 30I, No. 537. irregularis, Sharp. Man. N.Z. Coleop. I I 2 , No. 2006. 
Corymbites mundus, Sharp. Man. N.Z. Coleop. I I29, No. 2007.

canaliculatus, Broun. Man. N.Z. Coleop. i i 29, No. 2008. munroi, Broun. Man. N.Z. Coleop. I I 30, No. 2009.

Asymphus, Sharp, I 886 .

insidiosus, Sharp. Man. N.Z. Coleop. I I32, No. 20 I.

Exœolus, Broun, I 893 .

rufescens, Broun. Man. N.Z. Coleop. I I 34, No. 2013. punctatus, Broun. Man. N.Z. Coleop. II 34, No. 20 I 4. obsoletus, Broun. Man. N.Z. Coleop. Ir 35, No. 20 I 5.

Ochosternus, Candèze, $\mathrm{I} \mathrm{S} 63$.

zealandicus, White. Man. N.Z. Coleop. 298 , No. 53 I. parryi, Candèze. Mon. iv. 447.

Chrosis, Candèze, I 863.

polita, Sharp. Man. N.Z. Coleop. 296, No. $5^{27}$.

reversa, Sharp. Man. N.Z. Coleop. 297, No. 528.

barbata, Candèze. Man. N.Z. Coleop. 297 , No. 529.

elongata, Sharp. Man. N.Z. Coleop. 298, No. 530.

livens, Broun. Man. N.Z. Coleop. 68I, No. i I88. valida, Broun. Man. N.Z. Coleop. 68ı, No. i is9. setigera, Broun. Man. N.Z. Coleop. 682, N'o. i i 90. brevicollis, Broun. Man. N.Z. Coleop. 682, No. I I9I. castanea, Broun. Man. N.Z. Coleop. 683, No. Ir92. certa, Broun. Man. N.Z. Coleop. $68_{3}$, No. i 193.

fulvipes, Broun. Man. N.Z. Coleop. $68_{3}$, No. i I 94. impressa, Broun. Man. N.Z. Coleop. I I33, No. 2012. æneola, Candèze. Mem. Belg. xvii. I $865,54$. eximia, Broun. A.M.N.H. 6, xii. 288.

Cryptohypnus, Eschscholtz, 1833 . powelli, Sharp. Man. N.Z. Coleop. 295, No. 522. humilis, Sharp. Man. N.Z. Coleop. 295, No. 523. frontalis, Sharp. Man. N.Z. Coleop. 295, No. 524. longicornis, Sharp. Man. N.Z. Coleop. 296, No. 525. thoracicus, Sharp. Man. N.Z. Coleop. 296, No. 526. meinertzhageni, Broun. Man. N.Z. Coleop. 680, No. i i 87. montanus, Broun. Man. N.Z. Coleop. 773, No. I37I. sandageri, Broun. Man. N.Z. Coleop. 774 , No. I 372. pallipes, Sharp. Man. N.Z. Coleop. I I 22, No. I I 94. picticornis, Broun. Man. N.Z. Coleop. I I 22, No. I 195. monticola, Broun. Man. N.Z. Coleop. i I 24, No. i 96. sternalis, Broun. Man. N.Z. Coleop. I I 24 , No. I 197. basalis, Broun. Man. N.Z. Coleop. I I 25, No. i i 98. fuscipennis, Broun. Man. N.Z. Coleop. I I 25, No. I 99. attenuatus, Broun. Man. N.Z. Coleop. I453, No. 252 I. deterius, Broun. Man. N.Z. Coleop. I454, No. 2522. 
Oxylasma, Broun, ISSI.

pannosum, Broun. Man. N.Z. Coleop. 679, No. iI $S_{5}$. tectum, Broun. Man. N.Z. Coleop. 680, No. I I 86. basale, Broun. Man. N.Z. Coleop. 957, No. I 726. carinale, Broun. Man. N.Z. Coleop. I r 30, No. 2010. vittiger, Broun. Man. N.Z. Coleop. I452, No. 2520.

Silene, Broun, I893.

brunnea, Broun. Man. N.Z. Coleop. i I 36, No. 2017.

Monocrepidius, Eschscholtz, I 829.

exsul, Sharp. Man. N.Z. Coleop. 294, No. 520.

subrufus, Broun. Man. N.Z. Coleop. 294, No. 52 I.

maritimus, Broun. Man. N.Z. Coleop. I I 35, No. 2016.

Mecastrus, Sharp, I877.

convexus, Sharp. Man. N.Z. Coleop. 293, No. 5 I 7.

vicinus, Sharp. Man. N.Z. Coleop. 293 , No. 5 I 8.

discedens, Sharp. Man. N.Z. Coleop. 293, No. 5 I9.

lateristrigatus, White. Man. N.Z. Coleop. 306, No. 550. intermedius, Broun. Man. N.Z. Coleop. i I 28 , No. 2005.

Zeaglophus, Broun, I 895 .

pilicornis, Broun. A.M.N.H. 6, xv. 235 .

Lomemus, Sharp, i 877 .

pilicornis, Sharp. Man. N.Z. Coleop. 290, No. 509.

pictus, Sharp. Man. N.Z. Coleop. 290 , No. 510.

suffusus, Sharp. Man. N.Z. Coleop. 291, No. 5 I I.

flavipes, Sharp. Man. N.Z. Coleop. 29I, No. 5 I 2.

similis, Sharp. Man. N.Z. Coleop. 29I, No. $5^{\mathrm{I}} 3$.

obscuripes, Sharp. Man. N.Z. Coleop. 292, No. 5 I 4.

elegans, Sharp. Man. N.Z. Coleop. 292, No. 5 I 5.

collaris, Sharp. Man. N.Z. Coleop. 292, No. 5 I 6.

rectus, Broun. Man. N.Z. Coleop. 774, No. I 373. vittatus, Broun. Man. N.Z. Coleop. 775 , No. I 374. fulvipennis, Broun. Man. N.Z. Coleop. i I 26, No. 2000. fuscicornis, Broun. Man. N.Z. Coleop. I I 26, No. 200 I. maurus, Broun. Man. N.Z. Coleop. I I 27, No. 2002. sculpturatus, Broun. Man. N.Z. Coleop. I I 27, No. 2003. frontalis, Broun. Man. N.Z. Coleop. I 28 , No. 2004. fuscipes, Broun. Man. N.Z. Coleop. 1454, No. 2523. puncticollis, Broun. A.M.N.H. 6, xv. 234 .

Psorochroa, Broun, I886.

granulata, Broun. Man. N.Z. Coleop. 773, No. I 370.

Aglophus, Sharp, 1877 .

modestus, Sharp. Man. N.Z. Coleop. 289 , No. 508.

Panspœus, Sharp, 1877 .

guttatus, Sharp. Man. N.Z. Coleop. 288, No. 506.

tenebrosus, Broun. Man. N.Z. Coleop. 288, No. 507. 
Betarmon, Kiesenwetter, I $85^{8 .}$

gracilipes, Sharp. Man. N.Z. Coleop. 287 , No. 502. frontalis, Sharp. Man. N.Z. Coleop. 287 , No. $5 \circ 3$. lætus, Sharp. Man. N.Z. Coleop. 287 , No. 504. obscurus, Sharp. Man. N.Z. Coleop. 287 , No. 505. flavipilus, Broun. Man. N.Z. Coleop. I 350, No. 2367.

Amphiplatys, Sharp, i 877 .

lawsoni, Sharp. Man. N.Z. Coleop. 286 , No. 50 I.

Metablax, Sharp, I877.

brouni, Sharp. Man. N.Z. Coleop. 284 , No. 497. acutipennis, White. Man. N.Z. Coleop. 284 , No. 498. approximans, White. Man. N.Z. Coleop. $28_{5}$, No. 499. cinctiger, White. Man. N.Z. Coleop. 285 , No. 500.

Thoramus, Sharp, 1877 .

wakefieldi, Sharp. Man. N.Z. Coleop. 280, No. 489. rugipennis, Broun. Man. N.Z. Coleop. $28 \mathrm{I}$, No. $49 \mathrm{I}$. feredayi, Sharp. Man. N.Z. Coleop. 282, No. 493. lævithorax, White. Man. N.Z. Coleop. 282 , No. 494. perblandus, Broun. Man. N.Z. Coleop. 282 , No. 495. foveolatus, Broun. Man. N.Z. Coleop. 283 , No. 496. cervinus, Broun. Man. N.Z. Coleop. 677, No. ir82. angustus, Broun. Man. N.Z. Coleop. 678 , No. i 83 . parvulus, Broun. Man. N.Z. Coleop. 678 , No. I I 84. huttoni, Sharp. Man. N.Z. Coleop. I I 22, No. 1993.

\section{Genera of doubtful position:-}

Brounia, Sharp, 1878 .

thoracica, Sharp. Man. N.Z. Coleop. 274, No. 480.

Byrrhocryptus, Broun, I 893.

urquharti, Broun. Man. N.Z. Coleop. I 137, No. 20 I 8.

Amplectopus, Sharp, 1886.

ovalis, Sharp. Man. N.Z. Coleop. I 38 , No. 2019. latulus, Broun. Man. N.Z. Coleop. I 35 I, No. 2368. fuscus, Broun. Man. N.Z. Coleop. I351, No. 2369. pallicornis, Broun. Man. N.Z. Coleop. I 456, No. 2526.

Byrrhodes, Sharp, 1878 .

Family Dascillide. gravidus, Sharp. Man. N.Z. Coleop. 307 , No. 55 I.

Cyprobius, Sharp, 1878 .

nitidus, Sharp. Man. N.Z. Coleop. 308, No. $55^{2}$. undulatus, Broun. Man. N.Z. Coleop. 775 , No. 1375 . terrenus, Broun. A.M.N.H. 6, xv. 236.

Cyphanus, Sharp, 1878 . laticeps, Sharp. Man. N.Z. Coleop. 3०9, No. 553. 
Cyphanus punctatus, Sharp. Man. N.Z. Coleop. 309, No. 554. capax, Broun. Man. N.Z. Coleop. 309, No. 555. mollis, Sharp. Man. N.Z. Coleop. 3 ro, No. 556. debilis, Sharp. Man. N.Z. Coleop. 310, No. 557. granulatus, Broun. Man. N.Z. Coleop. 3 I I, No. $55^{8}$. medius, Broun. Man. N.Z. Coleop. 3 I I, No. 559. mandibularis, Broun. Man. N.Z. Coleop. 776, No. 1376. dubius, Broun. Man. N.Z. Coleop. I I39, No. 2020. scaber, Broun. Man. N.Z. Coleop. Ir 39, No. 202 I.

Atopida, White, 1846 .

lawsoni, Sharp. Man. N.Z. Coleop. 3I 2, No. 560. suturalis, White. Man. N.Z. Coleop. 338, No. 6 5 . brouni, Sharp. Man. N.Z. Coleop. 31 2, No. 56r. proba, Sharp. Man. N.Z. Coleop. 31 3, No. $5^{6} 2$. castanea, White. Man. N.Z. Coleop. 3I 3, No. 563. hirta, Broun. Man. N.Z. Coleop. 31 3, No. 564. testacea, Broun. Man. N.Z. Coleop. 314, No. 565 . dorsalis, Broun. Man. N.Z. Coleop. I I 40, No. 2023. sinuata, Broun. Man. N.Z. Coleop. I 455, No. 2524.

Veronatus, Sharp, I 878 .

longicornis, Sharp. Man. N.Z. Coleop. 314, No. 566. longipalpis, Sharp. Man. N.Z. Coleop. 315, No. $5^{67}$. sharpi, Broun. Man. N.Z. Coleop. 31 5, No. 568. frontalis, Broun. Man. N.Z. Coleop. 316, No. 569. scabiosus, Broun. Man. N.Z. Coleop. 316, No. 570. capito, Broun. Man. N.Z. Coleop. 316, No. 571. antennalis, Broun. Man. N.Z. Coleop. 776 , No. 1377. nubilus, Broun. Man. N.Z. Coleop. 1455, No. 2525. amplus, Broun. A.M.N.H. 6, xv. 237 .

Mesocyphon, Sharp, 1878 .

marmoratus, Sharp. Man. N.Z. Coleop. 317 , No. 572. setiger, Sharp. Man. N.Z. Coleop. 317, No. 573. wakefieldi, Sharp. Man. N.Z. Coleop. 318 , No. 574. divergens, Sharp. Man. N.Z. Coleop. 318, No. 575. monticola, Broun. Man. N.Z. Coleop. 958, No. 1727. granulatus, Broun. Man. N.Z. Coleop. 958 , No. I 728. pallidus, Broun. Man. N.Z. Coleop. I I 4I, No. 2024. laticeps, Broun. Man. N.Z. Coleop. I I42, No. 2025.

Cyphon, Paykull, I 798.

huttoni, Sharp. Man. N.Z. Coleop. 320, No. 576. parviceps, Sharp. Man. N.Z. Coleop. 320 , No. 577. pumilio, Sharp. Man. N.Z. Coleop. 320 , No. 578 . arduus, Sharp. Man. N.Z. Coleop. 320, No. 579. oscillans, Sharp. Man. N.Z. Coleop. 32 I, No. 580. æqualis, Sharp. Man. N.Z. Coleop. 32 I, No. 58 I. amplius, Broun. Man. N.Z. Coleop. 322 , No. 582. 
Cyphon viridipennis, Broun. Man. N.Z. Coleop. 322 , No. $5^{8} 3$ graniger, Sharp. Man. N.Z. Coleop. 322, No. 584. pictulus, Sharp. Man. N.Z. Coleop. $3^{22}$, No. $5^{8} 5$. zealandicus, Sharp. Man. N.Z. Coleop. 323, No. 586. suffusus, Sharp. Man. N.Z. Coleop. 323 , No. 587 . laticeps, Sharp. Man. N.Z. Coleop. 323, No. 588. genalis, Sharp. Man. N.Z. Coleop. 324 , No. $5^{89}$. remotus, Broun. Man. N.Z. Coleop. 776 , No. I 378. nigropictus, Broun. Man. N.Z. Coleop. 777 , No. I379. laticollis, Broun. Man. N.Z. Coleop. 777, No. 1380. rectangulus, Broun. Man. N.Z. Coleop. 777 , No. I38I. plagiatus, Broun. Man. N.Z. Coleop. 778 , No. ${ }_{3} 382$. propinquus, Broun. Man. N.Z. Coleop. 778 , No. ${ }^{2} 383$. acerbus, Broun. Man. N.Z. Coleop. 778 , No. ${ }_{3} 84$. crassus, Broun. Man. N.Z. Coleop. 779, No. 1385. suturalis, Broun. Man. N.Z. Coleop. 779, No. I 386. nitidus, Broun. Man. N.Z. Coleop. 779, No. I 387. lateralis, Broun. Man. N.Z. Coleop. 780 , No. I 388. molestus, Broun. Man. N.Z. Coleop. 780 , No. I 389. dilutus, Broun. Man. N.Z. Coleop. 780 , No. I 390. rectalis, Broun. Man. N.Z. Coleop. 837 , No. I 490. waikatoensis, Broun. Man. N.Z. Coleop. 838 , No. I49r. signatus, Broun. Man. N.Z. Coleop. 958, No. I 729. æthiops, Broun. Man. N.Z. Coleop. 959, No. I 730. trivialis, Broun. Man. N.Z. Coleop. 959, No. I 73 I. pauper, Broun. Man. N.Z. Coleop. I I 43, No. 2026. điscedens, Broun. Man. N.Z. Coleop. I I43, No. 2027. flavescens, Broun. Man. N.Z. Coleop. I I 43, No. 2028. nigritulus, Broun. Man. N.Z. Coleop. I I 44, No. 2029. fuscifrons, Broun. Man. N.Z. Coleop. I I 44, No. 2030. fulvicornis, Broun. Man. N.Z. Coleop. I I 44, No. 203 I. ornatus, Broun. Man. N.Z. Coleop. I I 45, No. 2032. variegatus, Sharp. Man. N.Z. Coleop. I I 45, No. 2033. mackerrowi, Broun. A.M.N.H. 6, xv. 237.

Cyphotelus, Sharp, 1878 .

angustifrons, Sharp. Man. N.Z. Coleop. 325 , No. 590.

Paupris, Sharp, 1877 .

Family Cleridæ. aptera, Sharp. Man. N.Z. Coleop. 331, No.60I.

Parmius, Sharp, 1877 . longipes, Sharp. Man. N.Z. Coleop. 331, No. 602. debilis, Sharp. Man. N.Z. Coleop. 332, No. 603. rugosus, Broun. Man. N.Z. Coleop. I I 48 , No. 2037.

Pelonium, Spinola, I 844 . pustuliferum, Westwood. P.Z.S. I $85^{2}$, p. 52, pl. 24 , fig. 8 . 
Balcus, Sharp, 1877 .

niger, Sharp. Man. N.Z. Coleop. 333, No. 604.

signatus, Broun. Man. N.Z. Coleop. 333, No. 605 .

Eumede, Pascoe, I876.

æraria, Pascoe. Man. N.Z. Coleop. 334, No. 606.

Phymalophæa, Pascoe, i 876.

electa, Pascoe. Man. N.Z. Coleop. 334, No. 607.

violacea, Fabricius. Man. N.Z. Coleop. 335, No. 608.

hilaris, Sharp. Man. N.Z. Coleop. 335, No. 609.

pantomelas, Boisduval. Man. N.Z. Coleop. 335, No. 6ro.

longula, Sharp. Man. N.Z. Coleop. 336, No. 6 I I.

opiloides, Pascoe. Man. N.Z. Coleop. 336, No. 6 I 2.

fulvipalpis, Broun. Man. N.Z. Coleop. 336, No. 6r 3 . picta, Broun. Man. N.Z. Coleop. $68_{5}$, No. irgS.

dorsalis, Broun. Man. N.Z. Coleop. 685, No. I I 99. viridans, Broun. Man. N.Z. Coleop. 686, No. I 200. testacea, Broun. Man. N.Z. Coleop. 686, No. I 201. atrata, Broun. Man. N.Z. Coleop. 686, No. 1202. ignea, Broun. Man. N.Z. Coleop. 784 , No. I 397. opacula, Broun. Man. N.Z. Coleop. I 352, No. 2372.

Mathesis, Waterhouse, I 877 . guttigera, Waterhouse. T.E.S. 1877, p. 8 .

Dasytes, Paykull, I 798. subcyaneus, Broun. Man. N.Z. Coleop. 328, No. 596. wakefieldi, Sharp. Man. N.Z. Coleop. 329 , No. 597. cinerohirtus, Broun. Man. N.Z. Coleop. 329, No. 598. obscuricollis, Broun. Man. N.Z. Coleop. 330, No. 599. laticeps, Broun. Man. N.Z. Coleop. 330, No. 600. stewarti, Broun. Man. N.Z. Coleop. 684, No. I 197. constrictus, Broun. Man. N.Z. Coleop. $7 \delta_{1}$, No. 1392. occiputalis, Broun. Man. N.Z. Coleop. 782, No. I393. cheesemani, Broun. Man. N.Z. Coleop. 902, No. i6 i r. opaculus, Broun. Man. N.Z. Coleop. 202, No. i6 I 2. helmsi, Sharp. Man. N.Z. Coleop. I I 47, No. 2035. oreocharis, Broun. Man. N.Z. Coleop. I I $4 S$, No. 2036. littoralis, Broun. Man. N.Z. Coleop. I 35 I, No. 2370. æthiops, Broun. Man. N.Z. Coleop. I 352, No. 237 I. nigripes, Broun. A.M.N.H. 6, xv. 238 .

Arthracanthus, Broun, I 886 . planifrons, Broun. Man. N.Z. Coleop. 78 I, No. I39I.

Halyles, Broun, i 886 . nigrescens, Broun. Man. N.Z. Coleop. 783 , No. I394. brevicornis, Broun. Man. N.Z. Coleop. $78_{3}$, No. ${ }_{3} 395$. semidilutus, Broun. Man. N.Z. Coleop. $7 s_{3}$, No. 1396. 
Asilis, Broun, I 893 .

\section{Family TELEPHORIDÆ.}

fulvithorax, Broun. Man. N.Z. Coleop. 326, No. 59 I. nigricans, Broun. Man. N.Z. Coleop. 327 , No. 592. subnudus, Broun. Man. N.Z. Coleop. 327 , No. 593. tenuiculus, Broun. Man. N.Z. Coleop. 327 , No. 594. striatus, Broun. Man. N.Z. Coleop. 328, No. 595. tumidus, Broun. Man. N.Z. Coleop. 684, No. I 195. piliventer, Broun. Man. N.Z. Coleop. 684, No. I 196. lævigatus, Broun. Man. N.Z. Coleop. 90I, No. I6 Iо. coloratus, Broun. Man. N.Z. Coleop. I I 47, No. 2034.

Ptinus, Linnæus, 1767 .

Family Ptinide.

speciosus, Broun. Man. N.Z. Coleop. 338, No. 6 I 4. murinus, White. Man. N.Z. Coleop. 338, No. 6 I 6. pilosus, White. Man. N.Z. Coleop. 338, No. 6 I 7 . littoralis, Broun. Man. N.Z. Coleop. I353, No. 2373.

Sub-family ANoBıinæ.

Anobium, Fabricius, I 775 .

amplicolle, Broun. Man. N.Z. Coleop. 339, No. 6 I8. ruficorne, Broun. Man. N.Z. Coleop. 340, No. 6I 9. tricostellum, White. Man. N.Z. Coleop. 34I, No. 623. undulatum, Broun. Man. N.Z. Coleop. 687 , No. I204.

Xenogonus, Broun, I 882 .

notatus, Broun. Man. N.Z. Coleop. 340, No. 620. granulatus, Broun. Man. N.Z. Coleop. 34I, No. 62 I. sericeus, Broun. Man. N.Z. Coleop. 34I, No. 622. pullus, Broun. Man. N.Z. Coleop. 688, No. I 205. furcus, Broun. Man. N.Z. Coleop. 689, No. г 206. versutus, Broun. Man. N.Z. Coleop. 689, No. I 207. plagiatus, Broun. Man. N.Z. Coleop. 689, No. I 208. ambiguus, Broun. Man. N.Z. Coleop. 689, No. 1209.

Ochina, Sturm, i 837 . vulgata, Broun. Man. N.Z. Coleop. 342, No. 624 .

Sphindoteles, Broun, I88 I.

atriventris, Broun. Man. N.Z. Coleop. 687 , No. 1203 .

Methemus, Broun, I903.

griseipilus, Broun (Capnodes). Man. N.Z. Coleop. 69o, No. I 2 I 0.

Dorcatoma, Herbst, I 790.

oblonga, Broun. Man. N.Z. Coleop. 343, No. 625. illustris, Broun. Man. N.Z. Coleop. 343 , No. 626 . lauta, Broun. Man. N.Z. Coleop. 690, No. I 2 I I. 
Euderia, Broun, r880.

squamosa, Broun. Man. N.Z. Coleop. 344, No. 627.

Family Bostrichide.

Apate, Fabricius, 1775 .

minuta, Fabricius. Man. N.Z. Coleop. 346, No. 628.

Cis, Latreille, 1796.

Family Crordæ.

undulatus, Broun. Man. N.Z. Coleop. 347 , No. 630. asimilis, Broun. Man. N.Z. Coleop. 347, No. 631. rufulus, Broun. Man. N.Z. Coleop. 348, No. 632. lineicollis, Broun. Man. N.Z. Coleop. 348, No. 633. flavitarsis, Broun. Man. N.Z. Coleop. 348, No. 634. asperimus, Broun. Man. N.Z. Coleop. 349, No. 635 . cornuticeps, Broun. Man. N.Z. Coleop. 349, No. 636 . illustris, Broun. Man. N.Z. Coleop. 349, No. 637 . perpinguis, Broun. Man. N.Z. Coleop. 350, No. 638 . anthracinus, Broun. Man. N.Z. Coleop. $35 \circ$, No. 639 . picturatus, Broun. Man. N.Z. Coleop. 838, No. 1492. obesulus, Broun. Man. N.Z. Coleop. 839, No. 1493. recurvatus, Broun. Man. N.Z. Coleop. 784 , No. 1398. picicollis, Broun. Man. N.Z. Coleop. 785 , No. I 399. viridiflavus, Broun. Man. N.Z. Coleop. 785 , No. I 400. fultoni, Broun. Man. N.Z. Coleop. 904, No. x614. piciceps, Broun. Man. N.Z. Coleop. 904, No. r6r 5.

Potaminus, Sturm, 1837 .

Family Parnide.*

angusticollis, Pascoe. Man. N.Z. Coleop. 248, No. 439.

Alloparnus, Broun, I 893 .

agrestis, Broun. Man. N.Z. Coleop. 249 and 1107 , No. 440.

Protoparnus, Sharp, 1886.

longulus, Sharp. Man. N.Z. Coleop. I 107, No. 1965.

Family BYRRHIDE.

Nosodendron, Latreille, $x 807$.

ovatum, Broun. Man. N.Z. Coleop. 242, No. 429. zealandicum, Sharp. Man. N.Z. Coleop. I ro6, No. 1694.

Curimus, Erichson, I 846 .

Sub-family BYRRHINÆ.

zealandicus, Redtenbacher. Man. N.Z. Coleop. 243, No. 430 .

anomalus, Broun. Man. N.Z. Coleop. 243 , No. 431 .

striatus, Broun. Man. N.Z. Coleop. 244, No. 432. squamiferus, Broun. Man. N.Z. Coleop. 1448 , No. 25 I 2. 
Pedilophorus, Steffahny, i 843 .

coruscans, Pascoe. Man. N.Z. Coleop. 245, No. 433. setarius, Broun. Man. N.Z. Coleop. 245, No. 434. rotundus, Broun. Man. N.Z. Coleop. 67 I, No. I I 7 I. gemmeus, Broun. Man. N.Z. Coleop. 769 , No. ${ }_{3} 63$. villosus, Broun. Man. N.Z. Coleop. 900, No. I608. lætus, Broun. Man. N.Z. Coleop. I 349, No. 2364. lævipennis, Broun. A.M.N.H. 6, xii. I 90. tibialis, Broun. A.M.N.H. 6, xii. I9I. puncticeps, Broun. A.M.N.H. 6, xii. I9I. picipes, Broun. A.M.N.H. 6, xii. I9I. creperus, Broun. A.M.N.H. 6, xii. 192. probus, Broun. A.M.N.H. 6, xii. I92. granulatus, Broun. A.M.N.H. 6, xii. I93.

Liochoria, Pascoe, 1875.

huttoni, Pascoe. Man. N.Z. Coleop. 246, No. 436. sternalis, Broun. A.M.N.H. 6, xii. I94. orbicularis, Broun. Man. N.Z. Coleop. 245 , No. 435 . nigricans, Broun. Man. N.Z. Coleop. 671, No. i i 78 . insueta, Broun. Man. N.Z. Coleop. 769, No. 1364. mixta, Broun. Man. N.Z. Coleop. 901, No. 1609. nigralis, Broun. Man. N.Z. Coleop. I 348 , No. 2363.

Cytilissus, Broun, I893.

claviger, Broun. A.M.N.H. 6, xii. 195.

Sub-family Limnichinæ.

Limnichus, Latreille, I 829.

decorus, Broun. Man. N.Z. Coleop. 247, No. 437. punctatus, Broun. Man. N.Z. Coleop. 247 , No. 438 . picinus, Broun. Man. N.Z. Coleop. 67 I, No. I I 72. nigripes, Broun. Man. N.Z. Coleop. I 449, No. $25^{\mathrm{I}} 3$.

Hydora, Broun, 1886.

Sub-family Elminæ.*

picea, Broun (Pachycephala). Man. N.Z. Coleop. 672, No. I I 73. nitida, Broun (Hydora). Man. N.Z. Coleop. 927 , No. 1664 . obsoleta, Broun (Hydora). Man. N.Z. Coleop. 928, No. 1665 .

Family Dermestide.

Trogoderma, Latreille, I 829.

serrigerum, Sharp. Man. N.Z. Coleop. 240, No. 426. signatum, Sharp. Man. N.Z. Coleop. 240, No. 427. mæstum, Broun. Man. N.Z. Coleop. 24I, No. 428. granulatum, Broun. Man. N.Z. Coleop. 900, No. I607. punctatum, Broun. Man. N.Z. Coleop. 953, No. i 7 I 8. suffusum, Broun. Man. N.Z. Coleop. 953, No. I 719. 
Trogoderma 4-fasciatum, Broun. Man. N.Z. Coleop. I105, No. 1962 .

antennale, Broun. Man. N.Z. Coleop. i 106, No. I963.

Family LATHRIDIIDE.

Holoparamecus, Curtis, 1833 .

lucidus, Broun. Man. N.Z. Coleop. 232, No. 408.

castaneus, Broun. Man. N.Z. Coleop. I448, No. $25^{1} 5$.

Lathridius, Herbst, I 793 .

antipodeus, White. Man. N.Z. Coleop. 233, No. 409. sculpturatus, Broun. Man. N.Z. Coleop. 233, No. $4 \mathrm{I} 0$. marginalis, Broun. Man. N.Z. Coleop. 233, No. 4I I. costulatus, Broun. Man. N.Z. Coleop. 234, No. 4I 2. floridus, Broun. Man. N.Z. Coleop. 234, No. 4I3. bifoveatus, Broun. Man. N.Z. Coleop. 834 , No. 1483 . castaneus, Broun. Man. N.Z. Coleop. 834 , No. 1484 . priopterus, Broun. Man. N.Z. Coleop. 898, No. I602. puncticeps, Broun. Man. N.Z. Coleop. 898, No. I603. caviceps, Broun. Man. N.Z. Coleop. 1447, No. 2510.

Corticaria, Marsham, I8oz.

angusticollis, Broun. Man. N.Z. Coleop. 235, No. 4I 4pubera, Broun. Man. N.Z. Coleop. 235, No. 415. fasciata, Broun. Man. N.Z. Coleop. 235, No. 4 I 6. hirtalis, Broun. Man. N.Z. Coleop. 236, No. 4I 7 . finitima, Broun. Man. N.Z. Coleop. 236, No. 4 I 8. variegata, Broun. Man. N.Z. Coleop. 236, No. 419. discoidea, Broun. Man. N.Z. Coleop. 236 No. 420. obesa, Broun. Man. N.Z. Coleop. 237 , No. $42 \mathrm{I}$. pudibunda, Broun. Man. N.Z. Coleop. 237 , No. 422. alacer, Broun. Man. N.Z. Coleop. 237, No. 423. tarsalis, Broun. Man. N.Z. Coleop. 768, No. I 362. pacata, Broun. Man. N.Z. Coleop. 899, No. r604. erythrocephala, Broun. Man. N.Z. Coleop. 899 Nu. 1605. platyptera, Broun. Man. N.Z. Coleop. 899, No. i 606. semirufa, Broun. Man. N.Z. Coleop. 952, No. i 7 i6. gilvipes, Broun. Man. N.Z. Coleop. 953, No I 7 I 7 . amplipennis, Broun. Man. N.Z. Coleop. I 104, No. 1960. clarula, Broun. A.M.N.H. 6, xv. 197.

Rethusus, Broun, I886. pictulus, Broun. Man. N.Z. Coleop. 835, No. 1485 . lachrymosus, Broun. Man. N.Z. Coleop. 836, No. 1486 .

Diarthrocera, Broun, r 893.

formicæphila, Broun. Man. N.Z. Colesp. I 348, No. 2362.

Family CocCineLLide.

Coccinella, Linnæus. I 735 .

tasmanii, White. Man. N.Z. Coleop. 645, No. I I 26. 
Coccinella coriacea, Broun. Man. N.Z. Coleop. i 319 , No. 2321. novæ-zealandiæ, Colenso. T.N.Z.I. xx. 40.

Leis, Mulsant, I8 5 I. antipodum, Mulsant. Man. N.Z. Coleop. 645, No. II28.

Cranophorus, Mulsant, I $85 \mathrm{I}$. venustus, Pascoe. Man. N.Z. Coleop. 650, No. I I4I.

Scymnus, Kugelann, I 794 .

eximius, Broun. Man. N.Z. Coleop. 646, No. iा29. flavihirtus, Broun. Man. N.Z. Coleop. 646 , No. I I 30. acceptus, Broun. Man. N.Z. Coleop. 647 , No. I I 3 I. consors, Broun. Man. N.Z. Coleop. 647, No. I I32. tristis, Broun. Man. N.Z. Coleop. 647, No. I I 33 . pallidiceps, Broun. Man. N.Z. Coleop. 648, No. II 34 . fagus, Broun. Man. N.Z. Coleop. 648, No. I 35 . terrenus, Broun. Man. N.Z. Coleop. 648, No. I I 36. rarus, Broun. Man. N.Z. Coleop. 649, No. I I 37. suffusus, Broun. Man. N.Z. Coleop. 649 , No. i I 38 . minutulus, Broun. Man. N.Z. Coleop. 649, No. I I 39. picinus, Broun. Man. N.Z. Coleop. 649, No. I I 40. sedatus, Broun. Man. N.Z. Coleop. 8I 3, No. 1446. villosus, Broun. Man. N.Z. Coleop. 8I 4, No. I447. circularis, Sharp. Insect Life, I889, p. 365 .

Holopsis, Broun, I886.

nigellus, Broun. Man. N.Z. Coleop. 8I 4, No. I 448. pallidus, Broun. Man. N.Z. Coleop. 8 I 5, No. I 449. lawsoni, Broun. Man. N.Z. Coleop. 8I 5 , No. I $55^{\circ}$. pictulus, Broun. Man. N.Z. Coleop. I $5 \circ 3$, No. 2588. rotundatus, Broun. Man. N.Z. Coleop. $15 \circ 3$, No. 2589.

Veronicobius, Broun, I 893.

hirtus, Broun. Man. N.Z. Coleop. I393, No. 2430.

Typhæa, Stephens, I830.

Family Mycetophagide.

curvipes, Broun. Man. N.Z. Coleop. 238 , No. 424. hirta, Broun. Man. N.Z. Coleop. 239, No. 425.

Triphyllus, Latreille, I 829 . integritus, Broun Man. N.Z. Coleop. I I05, No. I96 I. fuliginosus, Broun. Man. N.Z. Coleop. 228 , No. 40 I. substriatus, Broun. Man. N.Z. Coleop. 229 No. 402. aciculatus, Broun. Man. N.Z. Coleop. 229, No. 403. serratus, Broun. Man. N.Z. Coleop. 229. No. 404. punctulatus, Broun. Man. N.Z. Coleop. 230, No. 405. adspersus, Broun. Man. N.Z. Coleop. 230, No. 406. hispidellus, Broun. Man. N.Z. Coleop. 231 No. 407. 
Family Erotylide.

Cryptodacne, Sharp, 1878 .

synthetica, Sharp. Man. N.Z. Coleop. 64I, No. I I 22.

lenis, Broun. Man. N.Z. Coleop. 64I, No. I I 23.

vagepunctata, Broun. Man. N.Z. Coleop. 8I 3, r 445 .

vittata, Broun. Man. N.Z. Coleop. 873, No. I 554.

pubescens, Broun. Man. N.Z. Coleop. I319, No. 2320.

Triplax, Paykull, I 798.

brouni, Pascoe. Man. N.Z. Coleop. 642, No. I I 24.

Thallis, Erichson, 1842 .

polita, White. Man. N.Z. Coleop. 643, No. I 125 .

Family Cryptophagide.

Telmatophilus, Heer, I 84 I. depressus, Sharp. Man. N.Z. Coleop. 224, No. 39 r. nitens, Sharp. Man. N.Z. Coleop. 224, No. 392. olivascens, Broun. Man. N.Z. Coleop. I 104, No. 1959.

Cryptophagus, Herbst, I 792.

australis, Redtenbacher. Man. N.Z. Coleop. 225, No. 393. rubellus, Broun. Man. N.Z. Coleop. 225, No. 394. rutilus, Broun. Man. N.Z. Coleop. 226, No. 395 . vestitus, Broun. Man. N.Z. Coleop. 226, No. 396. sylvanus, Broun. Man. N.Z. Coleop. 227, No. 397. angulifer, Broun. Man. N.Z. Coleop. 227 , No. 398. hispidulus, Broun. Man. N.Z. Coleop. 227 , No. 400. discoideus, Broun. Man. N.Z. Coleop. I 345, No. 2360. distinctus, Broun. Man. N.Z. Coleop. 1346, No. 236 I. tumidus, Broun. Man. N.Z. Coleop. 1445, No. 2507. obscurus, Broun. Man. N.Z. Coleop. I 446, No. 2508. anthracinus, Broun. Man. N.Z. Coleop. I446, No. 2509. rufescens, Blanchard. Voy. Pole Sud, iv. 53, pl. 4.

Salltius, Broun, 1893 . ruficeps, Broun. Man. N.Z. Coleop. 228 and I 347 , No. 399.

Paramecosoma, Curtis, I 833 . maculosum, Broun. Man. N.Z. Coleop. 670, No. i 69.

Family Cucujide.

Chætosoma, Westwood, i85 I. scaritides, Westwood. Man. N.Z. Coleop. 767 , No. 1360.

Diagrypnodes, Waterhouse, i 876 .

wakefieldi, Waterhouse. Man. N.Z. Coleop. 2 I 7 , No. 382.

Dryocora, Pascoe, i 868.

howittii, Pascoe. Man. N.Z. Coleop. 2 r8, No. 383 . 
Dendrophagus, Schönherr, I809.

umbrinus, Smith. Cat. Coleop. Brit. Mus. I 2.

capito, Pascoe. Man. N.Z. Coleop. 2 I9, No. $3^{84}$.

Parabrontes, Redtenbacher, i 867.

silvanoides, Redtenbacher. Man. N.Z. Coleop. 220, No. 386.

setiger, Broun. Man. N.Z. Coleop. 768, No. г $36 r$.

Cryptamorpha, Wollaston, I 854 .

brevicornis, White. Man. N.Z. Coleop. 221 , No. 387. curvipes, Broun. Man. N.Z. Coleop. 22 I, No. 388. suturalis, White. Man. N.Z. Coleop. 222 , No. 389 . lateritia, Broun. Man. N.Z. Coleop. 222 , No. 390.

Picrotus, Sharp, I 886.

thoracicus, Sharp. Man. N.Z. Coleop. Io98, No. 1952. sanguineus, Broun. Man. N.Z. Coleop. ro98, No. I 953.

Brontopriscus, Sharp, i 886.

sinuatus, Sharp. Man. N.Z. Coleop. Iо99, No. I 954. pleuralis, Sharp. Man. N.Z. Coleop. 2 I 9 , No. 385.

Cathartocryptus, Sharp, i 886.

obscurus, Sharp. Man. N.Z. Coleop. i i oo, No. 1955.

Saphophagus, Sharp, I 886.

minutus, Sharp. Man. N.Z. Coleop. г го I, No. 1956.

ferrugineus, Broun. Man. N.Z. Coleop. Iro2, No. 1957.

Tularthrum, Broun, I893.

lineatum, Broun. Man. N.Z. Coleop. ıго3, No. 1958.

Thortus, Broun, I 893 .

ovalis, Broun. Man. N.Z. Coleop. I345, No. 2359.

Family RHYSODIDE.

Rhysodes, Dalman, I823.

aterrimus, Broun. Man. N.Z. Coleop. 2 I 4 , No. 377. eminens, Broun. Man. N.Z. Coleop. 2 I 5 , No. 379. orbitosus, Broun. Man. N.Z. Coleop. 2 15, No. 380. proprius, Broun. Man. N.Z. Coleop. 2 I6, No. 38 I. pensus, Broun. Man. N.Z. Coleop. 2 I 5, No. 378.

Family COLYDIIDÆ.

Sub-family Trichopterygine.

Trichopteryx, Kirby, I 826 . inconspicua, Matthews. Man. N.Z. Coleop. I 436, No. 2492.

Ptenidium, Erichson, I 845 . lawsoni, Matthews. Man. N.Z. Coleop. I 437, No. 2493. posticale, Broun. Man. N.Z. Coleop. I 437, No. 2494. proprium, Broun. Man. N.Z. Coleop. I438, No. 2495. cavelli, Broun. Man. N.Z. Coleop. 1438, No. 2496. 
Actidium, Matthews, I 868.

lineare, Matthews. Man. N.Z. Coleop. I 439, No. 2497.

Actinopteryx, Matthews. I 872 .

australis, Matthews. Man. N.Z. Colesp. 1440, No. $249^{8}$.

Ptinella, Motschoulski, 1844 .

fauvelli, Matthews. Mon. Trichop. Suppl. 19.

Sub-family Colydine.

Tarphiomimus, Wollaston, I 873 .

indentatus, Wollaston. Man. N.Z. Coleop. 182 , No. 324. wollastoni, Sharp. Man. N.Z. Coleop. I 080, No. I 924.

Dryptops, Broun, I 886.

dorsalis, Broun. Man. N.Z. Coleop. 763, No. I 354. undosus, Broun. Man. N.Z. Coleop. 764, No. I 355 . acuminatus, Broun. Man. N.Z. Coleop. $x_{3}$, No. 325 .

Ulonotus, Erichson, I 845 .

antarcticus, White. Man. N.Z. Coleop. I87, No. 33 I.

discedens, Sharp. Man. N.Z. Coleop. I 87 , No. 332.

brouni, Sharp. Man. N.Z. Coleop. I 88, No. 333.

viridipictus, Wollaston. Man. N.Z. Coleop. I 88, No. 334.

asper, Sharp. Man. N.Z. Coleop. 189 , No. 335.

integer, Sharp. Man. N.Z. Coleop. I89, No. 336.

lawsoni, Wollaston. Man. N.Z. Coleop. I 89, No. 337.

aberrans, Broun. Man. N.Z. Coleop. I89, No. 338.

atratus, Broun. Man. N.Z. Coleop. I 90, No. 339.

punctatus, Broun. Man. N.Z. Coleop. 894, No. 1595.

isostictus, Broun. Man. N.Z. Coleop. 926, No. I66I.

carus, Broun. Man. N.Z. Coleop. 947, No. 1706.

fuscatus, Broun. Man. N.Z. Coleop. 948, No. I 707

rufescens, Broun. Man. N.Z. Coleop. 948, No. I 708.

cinereus, Broun. Man. N.Z. Coleop. 948, No. 1709.

integratus, Broun. Man. N.Z. Coleop. 949, No. I 7 I 0.

fulvus, Broun. Man. N.Z. Coleop. I 080 , No. 1925.

dissimilis, Sharp. Man. N.Z. Coleop. ro8I, No. I 926.

Notoulus, Broun, I 886 (substituted for Ablabus).

ornatus, Broun. Man. N.Z. Coleop. I 84, No. 326.

pallidipictus, Broun. Man. N.Z. Coleop. I 85, No. 327.

scabrus, Broun. Man. N.Z. Coleop. 185, No. 328.

fervidulus, Broun. Man. N.Z. Coleop. I86, No. 329.

punctipennis, Broun. Man. N.Z. Coleop. I86, No. 330.

brevis, Broun. Man. N.Z. Coleop. 763. No. 1353 .

nodosus, Broun. Man. N.Z. Coleop. 894, No. I 594.

sparsus, Broun. Man. N.Z. Coleop. 947, No. 1704.

libentus, Broun. Man. N.Z. Coleop. 947 , No. I 705.

facetus, Broun. Man. N.Z. Coleop. 1341, No. 2353. 
Bitoma, Herbst, 1793.

insularis, White. Man. N.Z. Coleop. 192, No. 343. vicina, Sharp. Man. N.Z. Coleop. 193, No. 344. distans, Sharp. Man. N.Z. Coleop. I 93, No. 345. rugosa, Sharp. Man. N.Z. Coleop. 193, No. 346. nana, Sharp. Man. N.Z. Coleop. 194, No. 347. distincta, Broun. Man. N.Z. Coleop. I 94, No. 348. discoidea, Broun. Man. N.Z. Coleop. I95, No. 349. lobata, Broun. Man. N.Z. Coleop. 833, No. ${ }_{4} 82$. scita, Broun. Man. N.Z. Coleop. 895, No. 1596. guttata, Broun. Man. N.Z. Coleop. 895, No. I 597. sellata, Sharp. Man. N.Z. Coleop. Io8 I, No. I 927. auriculata, Sharp. Man. N.Z. Coleop. I082, No. 1928. serraticula, Sharp. Man. N.Z. Coleop. Io83, No. I 929. mundula, Sharp. Man. N.Z. Coleop. Io83, No. I 930.

Coxelus, Latreille, I 829 .*

dubius, Sharp. Man. N.Z. Coleop. 196, No. $35^{\circ}$. similis, Sharp. Man. N.Z. Coleop. I96, No. 35 I. robustus, Broun. Man. N.Z. Coleop. 197, No. $35^{2}$. clarus, Broun. Man. N.Z. Coleop. 766, No. I 357. grossanus, Broun. Man. N.Z. Coleop. 927 , No. I662. chalmeri, Broun. Man. N.Z. Coleop. 949, No. I 7 I I. rufus, Broun. Man. N.Z. Coleop. I084, No. 1931. posticalis, Broun. Man. N.Z. Coleop. Io84, No. 1932. ovicollis, Broun. Man. N.Z. Coleop. 1084, No. I933. longulus, Broun. Man. N.Z. Coleop. I085, No. 1934. oculator, Broun. Man. N.Z. Coleop. I 342, No. 2354. picicornis, Broun. Man. N.Z. Coleop. I 342, No. 2355. graniceps, Broun. Man. N.Z. Coleop. I 343, No. 2356. regularis, Broun. Man. N.Z. Coleop. I 440, No. 2499. thoracicus, Broun. A.M.N.H. 6, xv. I94.

Heterargus, Sharp, I 886. rudis, Sharp. Man. N.Z. Coleop. Io86, No. I 935. serricollis, Broun. NIan. N.Z. Coleop. I44 I, No. 2500.

Gathocles, Broun, 1893 . nodosus, Broun. Man. N.Z. Coleop. ıо86, No. 1936.

Vitiacus, Broun, I893.

costatus, Broun. Man. N.Z. Coleop. ro87, No. I937. costicollis, Broun. Man. N.Z. Coleop. I 442, No. 25 I. incertus, Broun. A.M.N.H. 6, xv. 195.

Enarsus, Pascoe, i 866.

bakewellii, Pascoe. Man. N.Z. Coleop. I 99, No. 355. wakefieldi, Sharp. Man. N.Z. Coleop. 199, No. 356. 
Enarsus rudis, Sharp. Man. N.Z. Coleop. 200, No. 357. contractifrons, Broun. Man. N.Z. Coleop. 200, No. 358. probus, Broun. Man. N.Z. Coleop. 1088, No. 1938. cucullatus, Sharp. Man.»N.Z. Coleop. ${ }^{2}$ Io89, No. 1939.

Rytidinotus, Broun, I880.

squamulosus, Broun. Man. N.Z. Coleop. 204 , No. 364 .

Syncalus, Sharp, i 876 .

optatus, Sharp. Man. N.Z. Coleop. 201, No. 359. politus, Broun. Man. N.Z. Coleop. 201 , No. 360. hystrix, Sharp. Man. N.Z. Coleop. 202, No. 361. piciceps, Broun. Man. N.Z. Coleop. I092, No. 1942. monroi, Broun. Man. N.Z. Coleop. I 442, No. 2502.

Acosmetus, Broun, r880. oblongus, Broun. Man. N.Z. Coleop. 197, No. 353. granulatus, Broun. Man. N.Z. Coleop. I 97 , No. 354.

Protarphius, Broun, 1893 .

ruficornis, Broun. A.M.N.H. 6, xii. I 84. indentatus, Broun. A.M.N.H. 6, xii. I 85 . crassus, Broun. Man. N.Z. Coleop. 669, No. i I67.

Recyntus, Broun, r 886.

exiguus, Broun. Man. N.Z. Coleop. 765 , No. 1356. tuberculatus, Broun. Man. N.Z. Coleop. i 9 I, No. 340. insignis, Broun. Man. N.Z. Coleop. r 9 r, No. 34 r. salebrosus, Broun. Man. N.Z. Coleop. I92, No. $34^{2}$.

Epistranus, Sharp, 1876 .

lawsoni, Sharp. Man. N.Z. Coleop. 203, No. 362. humeralis, Broun. Man. N.Z. Coleop. 203 , No. 363 . parvus, Broun. Man. N.Z. Coleop. 950, No. I 7 I 2. optabilis, Broun. Man. N.Z. Coleop. r 343, No. 2357. hirtalis, Broun. A.M.N.H. 6, xii. I89.

Norix, Broun, I893.

crassus, Broun. Man. N.Z. Coleop. ı09r, No. I 94 I.

Glenentela, Broun, I 893 .

serrata, Broun. Man. N.Z. Coleop. ıо9o, No. r 940.

Ithris, Pascoe, r 864.

gracilis, Sharp. Man. N.Z. Coleop. 205, No. 365 .

Rhizonium, Sharp, i 877 .

antiquum, Sharp. Man. N.Z. Coleop. 206, No. 366.

Chorasus, Sharp, I882.

subcæcus, Sharp. Man. N.Z. Coleop. 1093, No. I 943.

Lithostygnus, Broun, I 886.

costatus, Broun. Man. N.Z. Colenp. 9.50, No. I 713 . 
Ciconissus, Broun, 1893 .

granifer, Broun. A.M.N.H. 6, xii. 186.

Sub-family BOTHRIDERINE.

Bothrideres, Erichson, I 845 .

mœstus, Sharp. Man. N.Z. Coleop. 207, No. 367.

cognatus, Sharp. Man. N.Z. Coleop. 1096, No. I 95 r.

obsoletus, Broun. A.M.N.H. 6, xv. r96.

Sub-family Pycnomerinæ.

Pycnomerus, Erichson, 1842.

sophoræ, Sharp. Man. N.Z. Coleop. 208, No. 368.

simulans, Sharp. Man. N.Z. Coleop. 209, No. 369.

simplex, Broun. Man. N.Z. Coleop. 209, No. 370.

minor, Sharp. Man. N.Z. Coleop. 209, No. 37 I.

ellipticus, Broun. Man. N.Z. Coleop. 2 10, No. 372.

rufescens, Broun. Man. N.Z. Coleop. 766, No. r $35^{8}$.

basalis, Broun. Man. N.Z. Coleop. 766, No. I359.

carinellus, Broun. Man. N.Z. Coleop. 896, No. 1598.

cæcus, Broun. Man. N.Z. Coleop. 896, No. 1599.

hirtus, Broun. Man. N.Z. Coleop. 897, No. r600. lateralis, Broun. Man. N.Z. Coleop. 897, No. r60 r. arboreus, Broun. Man. N.Z. Coleop. 927 , No. 1663. cognatus, Broun. Man. N.Z. Coleop. 951, No. I 7 I 4 . marginalis, Broun. Man. N.Z. Coleop. 1093, No. 1944. sinuatus, Broun. Man. N.Z. Coleop. ro94, No. r 945. impressus, Broun. Man. N.Z. Coleop. ro94, No. 1946. longulus, Sharp. Man. N.Z. Coleop. ro95, No. 1947. helmsi, Sharp. Man. N.Z. Coleop. 1095, No. r 948. sulcatissimus, Sharp. Man. N.Z. Coleop. ro95, No. I949. latitans, Sharp. Man. N.Z. Coleop. rog6, No. $195^{\circ}$. angulatus, Broun. Man. N.Z. Coleop. 1443, No. $25^{\circ} 3$. frontalis, Broun. Man. N.Z. Coleop. I 443, No. 2504. elongellus, Broun. Man. N.Z. Coleop. r 444, No. 2505. longipes, Broun. Man. N.Z. Coleop. 1444, No. 2506. depressiusculus, White. Voy. Ereb. and Terr. Ins. p. I8.

Pycnomerodes, Broun, r 886.

peregrinus, Broun. Man. N.Z. Coleop. 952, No. 1715 .

Sub-family Cerylonine.

Philothermus, Aubé, I 843 .

nitidus, Sharp. Man. N.Z. Coleop. 2 I I, No. 373. sanguineus, Broun. Man. N.Z. Coleop. 2 I r, No. 374. notabilis, Broun. Man. N.Z. Coleop. 2 I I, No. 375 .

Trogosita, Olivier, r 790.

Family Trogositide.

affinis, White. Man. N.Z. Coleop. 177 , No. 3 r4. 
Leperina, Erichson, I 844 .

nigrosparsa, White. Man. N.Z. Coleop. I78, No. 315.

brounii, Pascoe. Man. N.Z. Coleop. i 78 , No. 3 I 6.

sobrina, White. Man. N.Z. Coleop. I 78 , No. 31 7.

wakefieldi, Sharp. Man. N.Z. Coleop. r 79, No. 3 I 8.

ambigua, Broun. Man. N.Z. Coleop. I79, No. 3 I 9.

farinosa, Broun. Man. N.Z. Coleop. I 79, No. 320.

Promanus, Sharp, 1877 .

depressus, Sharp. Man. N.Z. Coleop. I8o, No. 32 I.

auripilus, Broun. Man. N.Z. Coleop. 1079, No. 1922.

Grynoma, Sharp, 1877 .

fusca, Sharp. Man. N.Z. Coleop. I81, No. 322.

diluta, Sharp. Man. N.Z. Coleop. I8I, No. 323.

viridescens, Broun. Man. N.Z. Coleop. 893, No. 1593.

regularis, Sharp. Man. N.Z. Coleop. 1079, No. 1923.

varians, Broun. Man. N.Z. Coleop. I340, No. 235 I.

rugosa, Broun. Man. N.Z. Coleop. I341, No. 2352.

Phycosecis, Pascoe, 1875 .

discoidea, Pascoe. Man. N.Z. Coleop. 359 , No. $65^{\circ}$.

atomaria, Pascoe. Man. N.Z. Coleop. 359, No. 65 I.

Family NitidULıDÆ.

Brachypeplus, Erichson, I 842 .

brevicornis, Sharp. Man. N.Z. Coleop. I68, No. 30 I.

Epuræa, Erichson, I 843 .

antarctica, White. Man. N.Z. Coleop. I69, No. 302. zealandica, Sharp. Man. N.Z. Coleop. I69, No. 303. signata, Broun. Man. N.Z. Coleop. 169, No. 304.

Homepuræa, Broun, 1893 .

amcena, Broun. Man. N.Z. Coleop. I 7 I and ro73, No. 307.

Nitidula, Fabricius, 1775 .

lateralis, White. Man. N.Z. Coleop. I 70 , No. 305.

abbreviata, Fabricius. Man. N.Z. Coleop. I 7 I, No. 306.

Soronia, Erichson, I 843 .

hystrix, Sharp. Man. N.Z. Coleop. I 72, No. 308.

optata, Sharp. Man. N.Z. Coleop. 172, No. 309.

morosa, Broun. Man. N.Z. Coleop. ro74, No. I9I 7.

micans, Broun. Man. N.Z. Coleop. 1074, No. I918.

Omosita, Erichson, I 843 .

spinipes, Broun. Man. N.Z. Coleop. I 73, No. 310.

scutellaris, Broun. Man. N.Z. Coleop. I 73, No. 3 I I.

Xenoscelis, Wollaston, 1864 .

prolixus, Sharp. Man. N.Z. Coleop. 1 74, No. 3 I 2. 
Priates, Broun, I88I.

optandus, Broun. Man. N.Z. Coleop. 669, No. i I 36.

Ips, Fabricius, 1776 .

minimus, Sharp. Man. N.Z. Coleop. 1078, No. I92т.

Cyclaxyra, Broun, I 893 (substituted for Cyclimorpha). politula, Broun. Man. N.Z. Coleop. 668, No. I 65.

Platipidia, Broun, 1893 . asperella, Broun. Man. N.Z. Coleop. ıо75, No. I 9 I 9.

Priasilpha, Broun, 1893 . obscura, Broun. Man. N.Z. Coleop. I078, No. 1920.

Lenax, Sharp, 1877 .

Sub-family RHIzOPHAGIN龙.

mirandus, Sharp. Man. N.Z. Coleop. I75, No. 3 I 3 .

Clambus, Fisher, $\mathrm{I} \$_{2} 0$.

Sub-family Anisotomine.

domesticus, Broun. Man. N.Z. Coleop. 762, No. I350. vestitus, Broun. Man. N.Z. Coleop. 762, No. I351. suffusus, Broun. Man. N.Z. Coleop. 762 , No. $135^{2}$.

Family HisTERIDÆ.

Platysoma, Leach, i 8 i 7.

bakewelli, Marseul. Man. N.Z. Coleop. ı6 I, No. 291.

cinnamoneus, White. Man. N.Z. Coleop. I6 I, No. 292.

Sternaulax, Marseul, I 862.

zealandicus, Marseul. Man. N.Z. Coleop. I62, No. 293.

Epierus, Erichson, I 834 .

sylvanus, Broun. Man. N.Z. Coleop. 163, No. 294.

purus, Broun. Man. N.Z. Coleop. 163, No. 295.

abrogatus, Broun. Man. N.Z. Coleop. 892 , No. 1588.

planiceps, Broun. Man. N.Z. Coleop. 892 , No. 1589.

crenulatus, Broun. Man. N.Z. Coleop. 892, No. I 590.

simplex, Broun. Man. N.Z. Coleop. 893, No. I59I. rusticus, Broun. Man. N.Z. Coleop. 893, No. I 592.

Saprinus, Erichson, I 834 .

pseudocyaneus, White. Man. N.Z. Coleop. I64, No. 296. punctulipennis, Broun. Man. N.Z. Coleop. I65, No. 297. latipes, Broun. Man. N.Z. Coleop. 666, No. I 163.

Pachylopus, Erichson, I 834 .

pedator, Sharp. Man. N.Z. Coleop. I65, No. 298. lepidulus, Broun. Man. N.Z. Coleop. 665, No. i 162.

Abræus, Leach, r8r 7 .

brouni, Lewis. Man. N.Z. Coleop. I66, No. 299. 
Abræus vividulus, Broun. Man. N.Z. Coleop. I66, No. 300. brunneus, Broun. Man. N.Z. Coleop. 666, No. 1164.

Family SCAPIDIIDE.

Scaphisoma, Leach, I8I 2. concinnum, Broun. Man. N.Z. Coleop. 158, No. 287. scutellare, Redtenbacher. Man. N.Z. Coleop. I 59, No. 288. tenellum, Pascoe. Man. N.Z. Coleop. I59, No. 289. apicellum, Broun. Man. N.Z. Coleop. i60, No. 290. actuosum, Broun. Man. N.Z. Coleop. 664, No. I I 59.

Bæocera, Erichson, I 845 . rufa, Broun. Man. N.Z. Coleop. 665, No. i I6r. rufipes, Broun. Man. N.Z. Coleop. 833 , No. I48I. fulvicollis, Broun. Man. N.Z. Coleop. 891, No. I 586 . armata, Broun. Man. N.Z. Coleop. 89i, No. I 587.

Brachynopus, Broun, I88I.

latus, Broun. Man. N.Z. Coleop. 664, No. r r6o.

Family CORYLOPHIDE.

Anisomeristes, Matthews, I 886. sharpi, Matthews. Ent. Mon. Mag. I886, xxii. 425. ater, Matthews. Ent. Mon. Mag. I886, xxii. 425.

Sacina, Broun, r 893 .

oblonga, Broun. Man. N.Z. Coleop. Io7 r, No. I9r 3.

Sericoderus, Stephens, I 829.

fulvicollis, Broun. Man. N.Z. Coleop. Io72, No. I9I4. scutellaris, Broun. Man. N.Z. Coleop. 1073, No. I915. basalis, Broun. Man. N.Z. Coleop. Iо73, No. rgr6.

Family SiLPHide.

Necrophilus, Latreille, I 829. prolongatus, Sharp. Man. N.Z. Coleop. 760, No. I349.

Camiarus, Sharp, I876.

thoracicus, Sharp. Man. N.Z. Coleop. I48, No. 270. indiscretus, Broun. Man. N.Z. Coleop. I49, No. 27 I. convexus, Sharp. Man. N.Z. Coleop. I 50, No. 272.

Inocatops, Broun, 1893 . compactus, Broun. Man. N.Z. Coleop. I066, No. 1907. flectipes, Broun. Man. N.Z. Coleop. 1067, No. 1908. nigrescens, Broun. Man. N.Z. Coleop. I339, No. 2349. concinnus, Broun. Man. N.Z. Coleop. I 50 , No. 273.

Microsilpha, Broun, x 886. littorea, Broun. Man. N.Z. Coleop. 895 , No. I 584.

Asphærites, Broun, 1893 . nitidus, Broun. Man. N.Z. Coleop. ı 668, No. I 909. 
Catopsolius, Sharp, I886.

lævicollis, Sharp. Man. N.Z. Coleop. ro69, No. I9ro.

Isocolon, Broun, 1893 .

hilaris, Broun. Man. N.Z. Coleop. ıо70, No. I 912.

Choleva, Latreille, I 796.

antennalis, Broun. Man. N.Z. Coleop. I 52, No. 274. alacris, Broun. Man. N.Z. Coleop. I 52, No. 275.

granifer, Broun. Nan. N.Z. Coleop. 890, No. I 585 . fulvitarsis, Broun. Man. N.Z. Coleop. 946, No. I 702. lugubris, Sharp. Man. N.Z. Coleop. I o69, No. I 9 I I. relata, Broun. Man. N.Z. Coleop. I339, No. 2350. phyllobia, Broun. Man. N.Z. Coleop. I 433, No. 2488. monticola, Broun. Man. N.Z. Coleop. I 434, No. 2489 . hunuensis, Broun. Man. N.Z. Coleop. I 434, No. 2490. marginalis, Broun. A.M.N.H. 6, xv. 87 . suturalis, Broun. A.M.N.H. 6, xv. 88.

Allocatops, Broun, I 893 .

ovalis, Broun. Man. N.Z. Coleop. I 435, No. 249 I.

Mesocolon, Broun, I880.

clathrata, Broun. Man. N.Z. Coleop. I 53, No. 276.

liturata, Broun. Man. N.Z. Coleop. I 54, No. 277. puncticeps, Broun. Man. N.Z. Coleop. I 54, No. 278. undulata, Broun. Man. N.Z. Coleop. I 54, No. 279. bicolor, Broun. Man. N.Z. Coleop. 155, No. 280.

nebulosa, Broun. Man. N.Z. Coleop. I55, No. 28 r. maculifer, Broun. Man. N.Z. Coleop. I 56, No. 282. hirtalis, Broun. Man. N.Z. Coleop. I 56, No. 283. punctulata, Broun. Man. N.Z. Coleop. I 57, No. 284. torva, Broun. Man. N.Z. Coleop. 157, No. 285. domestica, Broun. Man. N.Z. Coleop. I 58, No. 286. varia, Broun. Man. N.Z. Coleop. 946, No. I 703.

Bæosilpha, Broun, r 895 . rufescens, Broun. A.M.N.H. 6, xv. 85 .

Mesagyrtes, Broun, I 895 . scabripes, Broun. A.M.N.H. 6, xv. 86.

Silphotelus, Broun, I 895 . nitidus, Broun. A.M.N.H. 6, xv. 84 .

Family ScyDmænide.

Scydmænus, Latreille, iso2.

edwardsi, Sharp. Man. N.Z. Coleop. I44, No. 264. ambiguus, Broun. Man. N.Z. Coleop. I 45, No. 265. puncticollis, Broun. Man. N.Z. Coleop. I 46, No. 266. princeps, Broun. Man. N.Z. Coleop. 924, No. 1656. galerus, Broun. Man. N.Z. Coleop. 924, No. 1657. 
Scydmænus elongellus, Broun. Man. N.Z. Coleop. I338, No. 2347 .

lætans, Broun. Man. N.Z. Coleop. 663 , No. I I 58 . cilipes, Broun. A.M.N.H. 6, xii. I 78 .

cedius, Broun. A.M.N.H. 6, xii. I79.

allocerus, Broun. A.M.N.H. 6, xii. I 79 .

brachycerus, Broun. A.M.N.H. 6, xii. 180.

xanthopus, Broun. A.M.N.H. 6, xii. I8I.

heterarthus, Broun. A.M.N.H. 6, xii. I 8 I.

relatus, Broun. A.M.N.H. 6, xii. I 82 .

insignis, Broun. A.M.N.H. 6, xii. I 82 .

Phaganophana, King, I 864 .

setosa, Sharp. Man. N.Z. Coleop. I46, No. 267.

picicollis, Broun. Man. N.Z. Coleop. I47, No. 268.

calva, Broun. Man. N.Z. Coleop. I47, No. 269.

oreas, Broun. Man. N.Z. Coleop. 925, No. 1658.

lanosa, Broun. Man. N.Z. Coleop. 925, No. I659.

angustata, Broun. Man. N.Z. Coleop. 926, No. I66o. planiceps, Broun. Man. N.Z. Coleop. I063, No. I900. munroi, Broun. Man. N.Z. Coleop. Io63, No. I90 I. antennalis, Broun. Man. N.Z. Coleop. 1064, No. 1902. stenocera, Broun. Man. N.Z. Coleop. 1064, No. 1903. latiuscula, Broun. Man. N.Z. Coleop. Io64, No. 1904. sanguinea, Broun. Man. N.Z. Coleop. I065, No. I905. erythronota, Broun. Man. N.Z. Coleop. 1065, No. 1906. ovipennis, Broun. Man. N.Z. Coleop. I 339, No. 2348. russata, Broun. Man. N.Z. Coleop. I432, No. 2485. monilifer, Broun. Man. N.Z. Coleop. I 432, No. 2486. angulata, Broun. Man. N.Z. Coleop. I 433, No. 2487.

Sciacharis, Broun, I893.

fulva, Broun. Man. N.Z. Coleop. 1062, No. I 899.

Tyrus, Aubé, i 833 .

Family Pselaphide.*

crassipes, Broun. Man. N.Z. Coleop. I337, No. 2345.

Euglyptus, Broun, I 893 . elegans, Broun. Man. N.Z. Coleop. I4I2, No. 2460. costifer, Broun. A.H.N.H. 6, xii. I66.

Microtyrus, Broun, I 893 . punctatus, Broun. Man. N.Z. Coleop. I4I3, No. 246 I.

Tyrogetus, Broun, 1893 . optandus, Broun. A.H.N.H. 6, xii. г66. 
Hamotulus, Schaufuss, r 887 .

mutandus, Sharp. Man. N.Z. Coleop. I 2 I, No. 225. armatus, Broun. Man. N.Z. Coleop. I 043, No. 1866. spinipes, Broun. Man. N.Z. Coleop. I 4 I I, No. 2459. sternalis, Broun. A.M.N.H. 6, xii. 168 . curvipes, Broun. A.M.N.H. 6, xii. 170.

Pselaphus, Herbst, 1792.

pauper, Sharp. Man. N.Z. Coleop. 122, No. 226. pilistriatus, Broun. Man. N.Z. Coleop. I 23, No. 227. dulcis, Broun. Man. N.Z. Coleop. 660, No. I I 53. cæcus, Broun. Man. N.Z. Coleop. 943, No. I 696. delicatus, Broun. Man. N:Z. Coleop. 943, No. 1697. fuscopilus, Broun. Man. N.Z. Coleop. 944, No. 1698. citimus, Broun. Man. N.Z. Coleop. 1043, No. I 867. meliusculus, Broun. Man. N.Z. Coleop. 1044, No. 1868. cavelli, Broun. Man. N.Z. Coleop. 1414, No. 2462. sulcicollis, Broun. Man. N.Z. Coleop. 14I5, No. 2463.

Gastrobothrus, Broun, $\mathbf{x} 886=$ Physa, Raffray, 1890 (not of Draparnaud, I 80 I).

inflata, Sharp. Man. N.Z. Coleop. I 24, No. 228.

sharpi, Broun. Man. N.Z. Coleop. х 24, No. 229. abdominalis, Broun. Man. N.Z. Coleop. 125 and 758, No. 230.

Eupines, King, I 864 . rudicornis, Broun. Man. N.Z. Coleop. 759, No. I 347. micans, Sharp. Man. N.Z. Coleop. I 26, No. 23 I. clavata, Broun. Man. N.Z. Coleop. I26, No. 232. picipes, Broun. Man. N.Z. Coleop. 127 , No. 233. dispar, Sharp. Man. N.Z. Coleop. I 27 , No. 234. impressifrons, Broun. Man. N.Z. Coleop. I 28, No. 235. munda, Broun. Man. N.Z. Coleop. 129 , No. 236. crassicornis, Broun. Man. N.Z. Coleop. I29, No. 237. deformis, Sharp. Man. N.Z. Coleop. i 30 , No. 238. impar, Sharp. Man. N.Z. Coleop. I 30, No. 239. grata, Sharp. Man. N.Z. Coleop. I 3 I, No. 240. altula, Broun. Man. N.Z. Coleop. I 3 I, No. 24 I. nasuta, Broun. Man. N.Z. Coleop. I 32, No. 242. sanguinea, Broun. Man. N.Z. Coleop. I 32, No. 242. pagana, Broun. Man. N.Z. Coleop. 660, No. I I 54. ignota, Broun. Man. N.Z. Coleop. 66 I, No. I I 55. glabrata, Broun. Man. N.Z. Coleop. 830, No. I 476. nemoralis, Broun. Man. N.Z. Coleop. 83 I, No. 1477. punctata, Broun. Man. N.Z. Coleop. 83I, No. 1478. calcarata, Broun. Man. N.Z. Coleop. 83 I, No. I479. sylvicola, Broun. Man. N.Z. Coleop. 919, No. I645. fraudulenta, Broun. Man. N.Z. Coleop. 944, No. 1699. 
Eupines euplectoides, Broun. Man. N.Z. Coleop. I 045 , No. I 869 .

decens, Broun. Man. N.Z. Coleop. 1046, No. г870.

munroi, Broun. T.N.Z.I. xxii. 231 ; Man. N.Z. Coleop. I 046 , No. 187 I.

forficulida, Broun. T.N.Z.I. xxii. 232 ; Man, N.Z. Coleop. I 047, No. I 872.

foveatissima, Broun. T.N.Z.I. xxii. 233; Man. N.Z. Coleop. I 047 , No. 1873 .

platynota, Broun. Man. N.Z. Coleop. I33S, No. 2346.

conspicua, Broun. Man. N.Z. Coleop. I 415 , No. 2464. costata, Broun. Man. N.Z. Coleop. I4I6, No. 2465. platyarthra, Broun. Man. N.Z. Coleop. I4 I 7, No. 2466. mundula, Broun. Man. N.Z. Coleop. I4I 7 , No. 2467. fulvitarsis, Broun. Man. N.Z. Coleop. I 4 I 8 , No. 2468.

Parmipalpus, Broun, $\mathbf{1} 88 \mathbf{1}$.

montivagus, Broun. Man. N.Z. Coleop. 662, No. I 156.

Startes, Broun, 1886.

sculpturata, Broun. Man. N.Z. Coleop. 830, No. 1475 . foveata, Broun. A.M.N.H. 6, xii. I 70 .

Dalma, Sharp, I 874 . pubescens, Sharp. Man. N.Z. Coleop. I 33, No. 244.

Dalmisus, Sharp, i 886. batrisodes, Sharp. Man. N.Z. Coleop. I049, No. 1874 .

Sagola, Sharp, I 874 .

major, Sharp. Man. N.Z. Coleop. 135, No. 246. prisca, Sharp. Man. N.Z. Coleop. I 36, No. 247. misella, Sharp. Man. N.Z. Coleop. I 36, No. 248. parva, Sharp. Man. N.Z. Coleop. I 36, No. 249. pulcher, Broun. Man. N.Z. Coleop. I37, No. 250. notabilis, Broun. Man. N.Z. Coleop. I 37 , No. 25 I. deformipes, Broun. Man. N.Z. Coleop. I.38, No. 252. denticollis, Broun. Man. N.Z. Coleop. I 38 , No. 253. genalis, Broun. Man. N.Z. Coleop. 663 , No. I 57 . terricola, Broun. Man. N.Z. Coleop. 832, No. I480. castanea, Broun. Man. N.Z. Coleop. 884, No. 1573. excavata, Broun. Man. N.Z. Coleop. 884 , No. 1574 . sulcator, Broun. Man. N.Z. Coleop. 885, No. 1575. osculans, Broun. Man. N.Z. Coleop. 885 , No. 1576. fovealis, Broun. Man. N.Z. Coleop. 886, No. I577. elevata, Broun. Man. N.Z. Coleop. 886, No. 1578. brevitarsis, Broun. Man. N.Z. Coleop. 887 , No. 1579. bipunctata, Broun. Man. N.Z. Coleop. 887 , No. I 580. duplicata, Broun. Man. N.Z. Coleop. 888, No. I 58 I. tenuis, Broun. Man. N.Z. Coleop. 888 , No. ${ }_{5} 82$. 
Sagola convexa, Broun. Man. N.Z. Coleop. 889 , No. ${ }_{5}{ }^{8} 3$.

insignis, Broun. Man. N.Z. Coleop. 1049, No. 1875.

hirtalis, Broun. Man. N.Z. Coleop. 1050, No. 1876. sobrina, Broun. Man. N.Z. Coleop. 1050, No. 1877. rectipes, Broun. Man. N.Z. Coleop. I051, No. 1878 . insolens, Broun. Man. N.Z. Coleop. 105I, No. 1879. punctata, Broun. Man. N.Z. Coleop. ro52, No. 1880. fulva, Broun. Man. N.Z. Coleop. 1052, No. r 881 . ruficeps, Broun. Man. N.Z. Coleop. I053, No. I 882. parallela, Broun. Man. N.Z. Coleop. I053, No. I883. anisarthra, Broun. Man. N.Z. Coleop. 1053, No. I884. macronyx, Broun. Man. N.Z. Coleop. 1418, No. 2466. mimica, Broun. Man. N.Z. Coleop. I419, No. 2467. robusta, Broun. Man. N.Z. Coleop. I420, No. 2468. laminata, Broun. Man. N.Z. Coleop. I 42 I, No. 2469. immota, Broun. Man. N.Z. Coleop. r422, No. 2470. flavipes, Broun. Man. N.Z. Coleop. I 422, No. 247 I. elongata, Broun. Man. N.Z. Coleop. 1423, No. 2472. gracilis, Broun. Man. N.Z. Coleop. I424, No. 2473. lineata, Broun. A.M.N.H. 6, xii. 175 . pertinax, Broun. A.M.N.H. 6, xii. I 76 . citima, Broun. A.M.N.H. 6, xii. 177. rugifrons, Broun. A.M.N.H. 6, xv. 73 . spinifer, Broun. A.M.N.H. 6, xv. 75 . eminens, Broun. A.M.N.H. 6, xv. 75 . punctulata, Raffray. Rev. Ent. Fr. I 893, 22. frontalis, Raffray. Rev. Ent. Fr. I 893, 23. sharpi, Raffray. Rev. Ent. Fr. 1893, 26. brevicornis, Raffray. Rev. Ent. Fr. I893, 27.

Exeirarthra, Broun, 1893. enigma, Broun. Man. N.Z. Coleop. 1054, No. 1885. pallida, Broun. Man. N.Z. Coleop. I424, No. 247 .

Vidanus, Raffray, is 98 . convexus, Sharp. Man. N.Z. Coleop. I 39, No. 254.

Sagolonus, Raffray, r 898 . patronus, Broun. Man. N.Z. Coleop. ıо6о, No. 1896.

Zelandius, Raffray, r 898 . asper, Broun. Man. N.Z. Coleop. 140 , No. $25^{6}$.

Whitea, Hutton, $1894=$ Brounia, Raffray, 1898 (not of Sharp, I 878 ).

lævifrons, Broun. Man. N.Z. Coleop. I425, No. 2475.

Plectomorphus, Raffray, i 898. spinifer, Broun. Man. N.Z. Coleop. I426, No. 2476.

Euplectus (?), Leach, I8I 7 . trisulcicollis, Broun. Man. N.Z. Coleop. I 40, No. 255. 
Euplectus longulus, Broun. Man. N.Z. Coleop. I4I, No. 257.

opacus, Sharp. Man. N.Z. Coleop. 141, No. $25^{8}$. sculpturatus, Broun. Man. N.Z. Coleop. 142, No. 259. frontalis, Broun. Man. N.Z. Coleop. 142, No. 260. brevitarsis, Broun. Man. N.Z. Coleop. 143, No. 26 r. ovicollis, Broun. Man. N.Z. Coleop. I 43, No. 262. foveolatus, Broun. Man. N.Z. Coleop. I 43, No. 263. tuberigerus, Broun. Man. N.Z. Coleop. 760 , No. 1348. crassipes, Broun. Man. N.Z. Coleop. 919, No. 1646. patruelis, Broun. Man. N.Z. Coleop. 919, No. I647. monticola, Broun. Man. N.Z. Coleop. 920 , No. 1648. ovithorax, Broun. Man. N.Z. Coleop. 920, No. 1649. obnisus, Broun. Man. N.Z. Coleop. 921 , No. 1650. vacuus, Broun. Man. N.Z. Coleop. 921 , No. 1651. incomptus, Broun. Man. N.Z. Coleop. 921 , No. 1652. mirificus, Broun. Man. N.Z. Coleop. 922, No. 1653. u-impressus, Broun. Man. N.Z. Coleop. 923 , No. 1654 . cereus, Broun. Man. N.Z. Coleop. 923, No. 1655. eminens, Broun. Man. N.Z. Coleop. 945, No. I 700. auripilus, Broun. Man. N.Z. Coleop. 945 , No. I 701 . mœrens, Broun. Man. N.Z. Coleop. 1055, I 886. lepiphorus, Broun. Man. N.Z. Coleop. I056, No. I887. validus, Broun. Man. N.Z. Coleop. 1056, No. г 888. scruposus, Broun. Man. N.Z. Coleop. 1057, No. I 889. allocephalus, Broun. Man. N.Z. Coleop. 1057, No. I890. acuminatus, Broun. Man. N.Z. Coleop. 1058, No. I89r. clevedonensis, Broun. Man. N.Z. Coleop. ro58, No. I892. sandageri, Broun. Man. N.Z. Coleop. 1059, No. I893. personatus, Broun. Man. N.Z. Coleop. 1059, No. I894. unicus, Broun. Man. N.Z. Coleop. 1060, No. 1895. verticalis, Broun. Man. N.Z. Coleop. I06 I, No. i 897. antiquus, Broun. Man. N.Z. Coleop. 106r, No. I898. munroi, Broun. Man. N.Z. Coleop. 1426, No. 2477. obscurus, Broun. Man. N.Z. Coleop. I427, No. 2478. coxalis, Broun. Man. N.Z. Coleop. 1428, No. 2479. inscitus, Broun. Man. N.Z. Coleop. 1428 , No. 2480. iracundus, Broun. Man. N.Z. Coleop. 1429, No. 248r. caudatus, Broun. Man. N.Z. Coleop. I 429 , No. 2482. claviger, Broun. Man. N.Z. Coleop. 1480, No. 2483. tumipes, Broun. A.M.N.H. $6, x v .76$. parvulus, Broun. A.M.N.H. 6, xv. 77 . modestus, Broun. A.M.N.H. 6, xv. 78 . arohaensis, Broun. A.M.N.H. 6, xv. 79. foveiceps, Broun. A.M.N.H, 6, xv. 80 . semiopacus, Broun. A.M.N.H. 6, xv. 8I. pusillus, Broun. A.M.N.H. 6, xv. 82 . 
Family STAPHyLINIDE.

Sub-family Aleochariñ.

Falagria, Mannerheim, 1830 .

micans, Broun. Man. N.Z. Coleop. 96, No. i 76.

subopaca, Broun. Man. N.Z. Coleop. 1405, No. 2449.

Ocalea, Erichson, I 837 .

socialis, Broun. Man. N.Z. Coleop. 90, No. 167.

crepera, Broun. A.M.N.H. 6, xiv. $3^{80}$.

Aleochara. Gravenhorst, I 802.

puber, Broun. Man. N.Z. Coleop. 90, No. 166.

semifusca, Broun. A.M.N.H. 6, xiv. 386

subænea, Fauvel. Man. N.Z. Coleop. 91, No. I67.

Calodera, Mannerheim, 1830.

flavescens, Broun. Man. N.Z. Coleop. 93, No. 17 I.

optabilis, Broun. Man. N.Z. Coleop. 94, No. 172.

æraria, Broun. Man. N.Z. Coleop. 94, No. I 73.

thoracica, Broun. Man. N.Z. Coleop. 95, No. 174.

fulgens, Broun. Man. N.Z. Coleop. 95 , No. 175.

algophila, Broun. Man. N.Z. Coleop. 941, No. 1693.

sericophora, Broun. A.M.N.H. 6, xiv. 38 I.

granifer, Broun. A.M.N.H. 6, xiv. 381 .

diversa, Broun. A.M.N.H. 6, xiv. 382 .

vestita, Broun. A.M.N.H. 6 , xiv. $3^{8} 3$.

tumidella, Broun. A.M.N.H. 6, xiv. 383 .

bituberculata, Broun. A.M.N.H. 6, xiv. 384 .

fungicola, Broun. A.M.N.H. 6 , xiv. 384 .

rufipennis, Broun. Man. N.Z. Coleop. 92, No. 170.

Tachyusa, Erichson, 1837 .

fuscicornis, Broun. Man. N.Z. Coleop. 92, No. 169.

Homalota, Mannerheim, I 825.

cornigera, Broun. Man. N.Z. Coleop. 91, No. 165.

atriceps, Broun. Man. N.Z. Coleop. 9I, No. I64.

Polylobus, Solsky, I 85 I.

sternalis, Broun. Man. N.Z. Coleop. 88 , No. 160.

Aphytopus, Sharp, 1886.

gracilis, Sharp. Man. N.Z. Coleop. 1025 , No. 1835 .

Gyrophæna, Mannerheim, I830.

punctata, Broun. Man. N.Z. Coleop. 87 , No. 159.

nugax, Broun. Man. N.Z. Coleop. 88, No. I6I.

densicornis, Broun. Man. N.Z. Coleop. 89, No. 162. versicolor, Broun. Man. N.Z. Coleop. 89, No. 163. atriceps, Broun. Man. N.Z. Coleop. 89 , No. 164.

Encephalus, Stephens, 1832.

latulus, Broun. A.M.N.H. 6, xiv. 386 . 


\section{Sub-family Digramminæ.}

Digrammus, Fauvel, I 900 .

miricollis, Fauvel. Rev. Ent. Fr. xix. I 24.

\section{Sub-family TACHYPORINÆ.}

Conurus, Stephens, 1832 .

largulus, Broun. Man. N.Z. Coleop. 97, No. 177. austerus, Broun. Man. N.Z. Coleop. 97, No. 178. subruber, Broun. Man. N.Z. Coleop. 98, No. I79. badius, Broun. Man. N.Z. Coleop. 98, No. I 80. flavithorax, Broun. Man. N.Z. Coleop. 98, No. 181 . acerbus, Broun. Man. N.Z. Coleop. 99, No. I 82. atricapillus, Broun. Man. N.Z. Coleop. 99, No. 183. auricomus, Broun. Man. N.Z. Coleop. ıоo, No. I 84 . nubilus, Broun. Man. N.Z. Coleop. I oo, I 85. maculosus, Broun. Man. N.Z. Coleop. ıоo, No. I 86. maorinus, Broun. Man. N.Z. Coleop. 1025 , No. i 836 . brevicornis, Broun. Man. N.Z. Coleop. I 405 , No. 2450. niticollis, Broun. Man. N.Z. Coleop. I 406, No. $245 \mathrm{I}$.

Sub-family StaPhyLininÆ.

Quedius, Stephens, $183^{2}$.

ruficornis, Broun. Man. N.Z. Coleop. II I, No. 205. æneoceps, Broun. Man. N.Z. Coleop. i I I, No. 206. enodis, Broun. Man. N.Z. Coleop. i I I, No. 207. veteratorius, Broun. Man. N.Z. Coleop. I I 2, No. 208. vividus, Broun. Man. N.Z. Coleop. i I 2, No. 209. arctifrons, Broun. Man. N.Z. Coleop. I I 2, No. 2 го. fuscatus, Broun. Man. N.Z. Coleop. 1027, No. 1839 . antipodus, Sharp. Man. N.Z. Coleop. Io28, No. I840. edwardsi, Sharp. Man. N.Z. Coleop. 1029, No. I84I. insolitus, Sharp. Man. N.Z. Coleop. 1029, No. I842. latifrons, Sharp. Man. N.Z. Coleop. 1030, No. I 843. discrepans, Broun. Man. N.Z. Coleop. Io30, No. I 844. latimanus, Broun. Man. N.Z. Coleop. I031, No. I 845 . collinus, Broun. Man. N.Z. Coleop. Iо3 I, No. I846. cavelli, Broun. Man. N.Z. Coleop. 1032, No. 1847. agathis, Broun. Man. N.Z. Coleop. 1407, No. 2453. sciticollis, Broun. A.M.N.H. 6, xiv. 420. conspicuellus, Broun. A.M.N.H. 6, xiv. 42 I. scutellaris, Broun. A.M.N.H. 6, xiv. 422 . puncticollis, Broun. A.M.N.H. 6, xiv. 422 . ambiguus, Broun. A.M.N.H. 6, xiv. 423 . taieriensis, Broun. A.M.N.H. 6, xiv. 424 .

Cafioquedus, Sharp, i 866. gularis, Sharp. Man. N.Z. Coleop. ı033, No. 1848 . 
Staphylinus, Linnæus, $175^{8}$.

oculatus, Fabricius. Man N.Z. Coleop. 107, No. 199. huttoni, Broun. Man. N.Z Coleop. I08, No. 200.

Xantholinus, Serville, I 825 .

sharpi, Broun. Man. N.Z. Coleop. I02, No. I 88. cultus, Broun. Man. N.7. Coleop. I03, No. I 89. arecæ, Broun. Man. N.7. Coleop. I03, No igo. mediocris, Broun. Man. N 7. Coleop. I04, No. I9I. labralis, Broun. Man N.Z. Coleop. I04. No. I92. socius, Fauvel. Man. N.Z. Coleop. 104, Nu. I93. puncticeps, Broun. Man. N.Z. Coleop. 104, No. 195.

Pachycorynus, Motschulsky, 1858 . dimorphus, Fauvel. Rev. Ent. Soc. Fr. I $\$ 92,92$.

Metoponcus, Kraatz, I857. brouni, Sharp. Man. N.Z. Coleop. Io6, No. 196. fulvipes, Broun. Man. N.Z. Coleop. 106, No. 197. rufulus, Broun. Man. N.Z. Coleop. ı06, No. 198.

Cafius, Leach, i 830 .

litoreus, Broun. Man. N.Z. Coleop. ı08, No. 201. maritimus, Broun. Man. N.Z. Coleop. r 09, No. 202. quadri-impressus (fem.), White. Man. N.Z. Coleop. ro8, No. $20 \mathrm{I}=$ caviceps (male), Broun. Man. N.Z. Coleop. 942 , No. I 694. algophilus, Broun. A.M.N.H. 6, xiv. 419.

Othius, Stephens, I 832 . adustus, Broun. Man. N.7. Coleop. го I, No. I 87. angustus, Broun. Man. N.Z. Coleop. 1026, No. 1837. puncticeps, Broun. A.M.N.H. 6, xiv. 419.

Sub-family PÆDERıNÆ.

Lithocharis, Lacordaire, I 835 .

zealandicus, Redtenbacher. Man. N.Z. Coleop. I I 4, No. 2 I 2.

mandibularis, Broun. Man. N.Z. Coleop. I I 4, No. 2 I 3. ventralis, Broun. Man. N.Z. Coleop. II 5 , No. 214. cæcus, Broun. A.M.N.H. 6, xiv. 424 .

Phanophilus, Sharp, I 886.

comptus, Broun. Man. N.Z. Coleop. 1026 and I I4, No. 2 I I.

Hyperomma, Fauvel, I 878 .

dispersum, Fauvel. Man. N.Z. Coleop. I 408 , No. 2454. mandibulare, Broun. Man. N.Z. Coleop. I409, No. 2455. duplicatum, Broun. Man. N.Z. Coleop. I 409, No. 2456. sanguineum, Broun. A.M.N.H. 6, xiv. 425 . 


\section{Sub-family OXvTELINÆ.}

Coprostygnus, Sharp, I 886 sculptipennis, Sharp. Man. N.Z. Coleop. 1027 , No. 1838 . optandus, Broun. Man. N.Z. Coleop. I409, No. 2457.

Holotrochus, Erichson, 1840.

ferrugineus, Broun. Man. N.Z. Coleop. I 034 , No. I 849. brevipennis, Broun. Man. N.Z. Coleop. 1034, No. $185^{\circ}$.

Sub-family OMaLinæ.

Omalium, Gravenhorst, I 802 .

hebes, Broun. Man. N.Z. Coleop. i I6, No. 2 I 5.

sulcithorax, Broun. Man N.Z. Coleop. I 6 , No. 216.

agrestis, Broun. Man. N.Z. Coleop. I I 7, No. 217.

spadix, Broun. Man. N.Z. Coleop. I I 7, No. 218.

pullum, Broun. Man. N.Z. Coleop. I 20, No. 223.

tibiale, Broun. Man. N.Z. Coleop. I 20, No. 224.

littoreum, Broun. Man. N.Z. Coleop. 942, No. т695.

sagoloide, Sharp. Man. N.Z. Coleop. 1035, No. 185 I.

actobium, Broun. Man. N.Z. Coleop. 1035, No. 1852.

cariniferum, Broun. Man. N.Z. Coleop. 1036, No. 1853.

antipodum, Broun. Man. N.Z. Coleop. 1036, No. 1854.

chalmeri, Broun. Man. N.Z. Coleop 1037, No. 1855.

micıarthrum, Broun. Man. N.Z. Coleop. I037, No. 1856.

cognatum, Broun. Man. N.Z. Coleop. 1038, No. 1857.

monilifer, Broun. Man. N.Z. Coleop. 1038, No. 1858 .

debile, Broun. Man. N.Z. Coleop. 1039, No. 1859.

prolixum, Broun. Man. N.Z. Coleop. 1039, No. I860.

stenosoma, Broun. Man. N.Z. Coleop. 1040, No. 1861.

tenellum, Broun. Man. N.Z. Coleop. 1410 , No. 2458.

fusciventre, Broun. A.M.N.H. 6, xiv. 426 .

australe, Broun. A.M.N.H. 6, xiv. 426 .

philpoti, Broun. A.M.N.H. 6, xiv. 427 .

perplexum, Broun. A M.N.H. 6, xiv. 428 .

Ischnoderus, Fauvel, 1867.

tectus, Broun. Man. N.Z. Coleop. I17, No. 219. genalis, Broun. Man. N.Z. Coleop. i 18, No. 220. pictulus, Broun. Man. N.Z. Coleop. 1040, No. 1862. morosus, Broun. Man. N.Z. Coleop. I041, No. I863. fultoni, Broun. Man. N.Z. Coleop. I04I, No. 1864. politulus, Broun. Man. N.Z. Coleop. I19, No. 221.

Omalissus, Broun, 1893.

castaneus, Broun. Man. N.Z. Coleop 1042, No. 1865 .

Trogophlæus, Mannerheim, I830. zealandicus, Sharp. Ent. Mag. xxxvi. 233.

Blediotrogus, Sharp, 1900. guttiger, Sharp. Ent. Mag. xxxvi. 234. cribricollis, Fauvel. Rev. Ent. Fr. xix I 84. 
Series Heteromera.

Family TENEBRIONIDE.

Sub-family OPATRINe.

Opatrum, Fabricius, I 775 .

lævigatum, Fabricius. Ent. Syst. i. 89, 5.

Pseudopatrum, Sharp, I 886.

tuberculicostatum, White. Man. N.Z. Coleop. 353, No. 642 .

sordidum, Sharp. Man. N.Z. Coleop. I I52, No. 204 I.

Mesopatrum, Broun, 1893 .

granulosum, Broun. Man. N.Z. Coleop. I 355, No. 2376.

Periatrum, Sharp, I 886.

helmsi, Sharp. Man. N.Z. Colleop. 1153, No. 2042.

tumipes, Broun. Man. N.Z. Coleop. 1456, No. $25^{27}$.

Syrphetodes, Pascoe, 1875 .

marginatus, Pascoe. Man. N.Z. Coleop. 352 , No. 640.

crenatus, Broun. Man. N.Z. Coleop. 352 , No. 64 I.

decoratus, Broun. Man. N.Z. Coleop. 353 , No. 643 . bullatus, Sharp. Man. N.Z. Coleop. I I 50, No. 2039. sylvius, Broun. Man. N.Z. Coleop. I I 50, No. 2040. cordipennis, Broun. Man. N.Z. Coleop. I457, No. 2528. dorsalis, Broun. Man. N.Z. Coleop. I 458, No. 2529. punctatus, Broun. Man. N.Z. Coleop. 1458, No. $253^{\circ}$.

Paraphylax. Broun, i 880.

squamiger, Broun. Man. N.Z. Coleop. 355, No. 644 . varius, Broun. Man. N.Z. Coleop. 355 , No. 645 . binodosus, Broun. A.M.N.H. 6, xv. 240.

Exohadrus, Broun, I 893 .

volutithorax, Broun. Man. N.Z. Coleop. 356 and I I53, No. 646.

Sub-family TrachyscelinÆ.

Chærodes, White, I 846 .

trachyscelides, White. Man. N.Z. Coleop. 357, No. 647. lætus, Broun. Man. N.Z. Coleop. 358, No. 648. concolor, Sharp. Man. N.Z. Coleop. $35^{8}$, No. 649. fuscatus, Broun. A.M.N.H. 6, xv. 24 I.

Actizeta, Pascoe, 1875 . ammobioides, Pascoe. Man. N.Z. Coleop. 359, No. 652. albata, Pascoe. Man. N.Z. Coleop. 360, No. 653.

Sub-family DiaPERINe.

Menimus, Sharp, 1876 . batesi, Sharp. Man. N.Z. Coleop. 36r, No. 654 . 
Menimus puncticeps, Broun. Man. N.Z.Coleop. 36 I, No. 655 . oblongus, Broun. Man. N.Z. Coleop. 362 , No. 656 . cæcus, Sharp. Man. N.Z. Coleop. 362 , No. 657 . crinalis, Broun. Man. N.Z. Coleop. 363 , No. $65^{8}$. crassus, Sharp. Man. N.Z. Coleop. 363 , No. 659. dubius, Broun. Man. N.Z. Coleop. 363 , No. 660. obscurus, Broun. Man. N.Z. Coleop. 364 , No 66r. thoracicus, Broun. Man. N.Z. Coleop. 364. No. 662. curtulus, Broun. Man. N.Z. Coleop. 785 , No. 1401. piceus, Broun. Man. N.Z. Coleop. 786, No. I 402. striatulus, Broun. Man. N.Z. Coleop. 842 , No. 1497. sinuatus, Broun. Man. N.Z. Coleop. 842 , No. 1498. vicinus, Broun. A.M.N.H. 6, xii. 288. lævicollis, Broun. A.M.N.H. 6, xv. 242.

Bolitophagus, Illiger, 1798 . angulifer, Blanchard. Voy. Pole Sud. iv. p. I67.

Uloma, Castelnau, I 840 . tenebrionoides, White. Man. N.Z. Coleop. 366 , No. 663 lævicostata, Blanchard. Voy. Pole Sud, iv. I65.

Ulomotypus, Broun, I 886.

lævigatus, Broun. Man. N.Z. Coleop. 841, No. 1496.

Apthora, Bates, I 872 :

ruficeps, Bates. Man. N.Z. Coleop. 367, No. 664. glabritarsis, Sharp. Man. N.Z. Coleop. I I 53, No. 2043.

Cilibe, Brême, I 842.

opacula, Bates. Man. N.Z. Coleop. 368 , No. 665 . nitidula, Bates. Man. N.Z. Coleop. 369, No. 666. otagensis, Bates. Man. N.Z. Coleop. 370, No. 667. elongata, Brême. Man. N.Z. Coleop. 371, No. 668. pascoei, Bates. Man. N.Z. Coleop. 372, No. 669. humeralis, Bates. Man. N.Z. Coleop. 373, No. 670. thoracica, Bates. Man. N.Z. Coleop. 373, No. 671. brevipennis, Bates. Man. N.Z. Coleop. 374 , No. 672 . granulosa, Brême. Man. N.Z. Coleop. 375, No. 673. rugosa, Bates. Man. N.Z. Coleop. 375, No. 674. tibialis, Bates. Man. N.Z. Coleop. 376, No. 675 . impressifrons, Bates. Man. N.Z. Coleop. 376, No. 676 . buchanani, Broun. Man. N.Z. Coleop. 377 , No. 677 . huttoni, Sharp. Man. N.Z. Coleop. 378, No. 678 . marginalis, Broun. Man. N.Z. Coleop. 1155 , No. 2047. phosphugoides, White. Voy. Ereb. and Terr., Insects, 1 I.

Zolodinus, Blanchard, 1853 .

zealandicus, Blanchard. Man. N.Z. Coleop. 379, No. 679 .

Lorelus, Sharp, 1876 .

priscus, Sharp. Man. N.Z. Coleop. 381 , No. 680. 
Lorelus pubescens, Broun. Man. N.Z. Coleop. 38 I, No. 68 I. crassicornis, Broun. Man. N.Z. Coleop. 382, No. 682. quadricollis, Broun. Man. N.Z. Coleop. 786, No. 1403.

Demtrius, Broun, I 895 . carinulatus, Broun. A.M.N.H. 6, xv. 243.

Arthopus, Sharp, 1876 . brouni, Sharp. Man. N.Z. Coleop. 383 , No. 683.

Edalus, Broun, I 893 . alienus, Broun. Man. N.7. Coleop. 39r, No. 695. opacus, Broun. Man. N.Z. Coleop. I I60, No. 2057. pleuralis, Broun. A.M.N.H. 6, xii. 289.

\section{Sub-family CNODALONINA.}

Artystona, Bates, I 873 .

erichsoni, White. Man. N.Z. Coleop. 384 , No. 684. wakefieldi, Bates. Man. N.Z. Coleop. 385 , No. 685 . rugiceps, Bates. Man. N.Z. Coleop. 385 , No. 686. obscura, Sharp. Man. N.Z. Coleop. I I 54, No. 2044. collaris, Sharp. Man. N.Z. Coleop. I I 54 , No. 2045. obsoleta, Sharp. Man. N.Z. Coleop. I I54, No. 2046. interrupta, Redtenbacher. Reise Novara, Coleop. 128.

Adelium, Kirby, I8 8 .

Sub-family HELOPIN ※.

harpaloides, White. Voy. Ereb. and Terror, Insects, I I. bullatum, Pascoe. Man. N.Z. Coleop. 386, No. 687 . intricatum, Bates. Man. N.Z. Coleop. 387 , No. 688. aucklandicum, Broun. Man. N.Z. Coleop. 387 , No. 689. amaroides, White. Man. N.Z. Coleop. 388, No. 690. æratum, Broun. Man. N.Z. Coleop. 388, No. 69 I. lentum, Broun. Man. N.Z. Coleop. 389, No. 692. zealandicum, Bates. Man. N.Z. Coleop. 390, No. 693. thoracicum, Bates. Man. N.Z. Coleop. 390, No. 694. cheesemani, Broun. Man. N.Z. Coleop. 787, I 404. chalmeri, Broun. Man. N.Z. Coleop. 787 , No. 1405. indagator, Broun. Man. N.Z. Coleop. 839, No. 1494. rufilabrum, Broun. Man. N.Z. Coleop. 840 , No. 1495. hanseni, Broun. Man. N.Z. Coleop. 929, No 1668. nigritulum, Broun. Man. N.Z. Coleop. 930, No. I669. multistriatum, Sharp. Man. N.Z. Coleop. I I 55, No. 2048, simplex, Sharp. Man. N.Z. Coleop. i I 56, No. 2049. sericatum, Sharp. Man. N.Z. Coleop. I I 56, No. $205^{\circ}$. intermedium, Sharp. Man. N.Z. Coleop. I I 56, No. $205 \mathrm{I}$. dunedinis, Sharp. Man. N.Z. Coleop. I I 57, No. 2052. urquharti, Broun. Man. N.Z. Coleop. I I 57, No. 2053. miniatum, Broun. Man. N.Z. Coleop. i I 57 , No. 2054. gratiosum, Broun. Man. N.Z. Coleop. I I 58, No. 2055. 
Adelium turgidulum, Broun. Man. N.Z. Coleop. I 58 , No. 2056.

tuberculatum, Guerin. Voy. Pole Sud, iv. I75.

simulans, Redtenbacher. Reise Novara, 32.

Cerodolus, Sharp, i 886.

chrysomeloides, Sharp. Man. N.Z. Coleop. I I6 I, No. 2058. genialis, Broun. Man. N.Z. Coleop. I 162 , No. 2059.

æneus, Broun. Man. N.Z. Coleop. I I62, No. 2060.

Leiopeplus, Broun, I 893 .

expolitus, Broun. Man. N.Z. Coleop. 392 and 1 160, No. 696.

Sub-family AMARYGMINæ.

Amarygmus, Dalman, 1823 .

zealandicus, Bates. Man. N.Z. Coleop. 394, No. 697.

Family Cistelide.

Amarosoma, Redtenbacher, 1868.

metallicus, White. Man. N.Z. Coleop. 395, No. 698.

sophoræ, Broun. Man. N.Z. Coleop. 396, No. 699.

Xylochus, Broun, I88o.

substriatus, Broun. Man. N.Z. Coleop. 397, No. 700.

tibialis, Broun. Man. N.Z. Coleop. 397, No. 7 ог.

dentipes, Broun. Man. N.Z. Coleop. 788, No. 1406.

spiniter, Broun. Man. N.Z. Coleop. I I68, No. 2075.

Omedes, Broun, i 893.

nitidus, Broun. Man. N.Z. Coleop. I I69, No. 2076.

fuscatus, Broun. Man. N.Z. Coleop. i I 70, No. 2077.

apterus, Broun. A.M.N.H. 6, xv. 244.

Family LAGRIIDÆ.

Lagrioda, Fairmaire et Germain, I 860.

brounii, Pascoe. Man N.Z. Coleop. 408, No. 721.

Family MELANDRYide.

Ctenoplectron, Redtenbacher, r 868.

fasciatum, Redtenbacher. Man. N.Z. Coleop. 40 I, No. 706.

fuliginosum, Broun. Man. N.Z. Coleop. 402, No. 708.

maculatum, Broun. Man. N.Z. Coleop. 69I, No. I 213.

costatum, Broun. Man. N.Z. Coleop. 69I, No. I 2 I4.

vittatum, Broun. Man. N.Z. Coleop. 844 , No. 1500.

coloratum, Broun. Man. N.Z. Coleop. 959, No. I 732.

dignum, Broun. Man. N.Z. Coleop. 960, No. 1733.

Allopterus, Broun, I 886 .

reticulatus, Broun. Man. N.Z. Coleop. 790, No. I4Io.

ornatus. Broun. Man. N.Z. Coleop. 401, No. 707.

instabilis, Broun. Man. N.Z. Coleop. 844 , No. I50I.

cavelli, Broun. Man. N.Z. Coleop. I356, No. 2377 
Hylobia, Broun, I880.

velox, Broun. Man. N.Z. Coleop. 404, No. 7 ro. undulata, Broun. Man. N.Z. Coleop. 404, No. 7 I I. nubeculosa, Broun. Man. N.Z. Coleop. 404, No. 7 I 2. pulla, Broun. Man. N.Z. Coleop. 405, No. 7 I 3. nigricornis, Broun. Man. N.Z. Coleop. 405, No. 7 I 4. calida, Broun. Man. N.Z. Coleop. 405, No. 715. bifasciata, Broun. Man. N.Z. Coleop. 406, No. 7 I 6. minor, Broun. Man. N.Z. Coleop. 406, No. 7 I 7 . cylindrata, Broun. Man. N.Z. Coleop. 406, No. 7 I 8. usitata, Broun. Man. N.Z. Coleop. 407, No. 719. nigella, Broun. Man. N.Z. Coleop. 407, No. 720.

Chalcodrya, Redtenbacher, i 868.

variegata, Redtenbacher. Man. N.Z. Coleop. 403, No. 709. mollis, Broun. Man. N.Z. Coleop. 960, No. I 734.

Onysius, Broun, I 886.

anomalus, Broun. Man. N.Z. Coleop. 843 , No. I 499. pulcher, Broun. Man. N.Z. Coleop. I357, No. 2378.

Salpingus, Gyllenhal, i 8 I 0 .

Family Pythide.

perpunctatus, Broun. Man. N.Z. Coleop. 398, No. 702. bilunatus, Pascoe. Man. N.Z. Coleop. 398, No. 703. angusticollis, Broun. Man. N.Z. Coleop. 399 , No. $7 \circ 4$. unguiculus, Broun. Man. N.Z. Coleop. 399, No. 704a. lautus, Broun. Man. N.Z. Coleop. 400, No. 705. hirtus, Broun. Man. N.Z. Coleop. 789 , No. 1407. simplex, Broun. Man. N.Z. Coleop. 789 , No. 1408. quisquilius, Broun. Man. N.Z. Coleop. 789 , No. 1409. fossulatus, Broun. Man. N.Z. Coleop. I 459, No. 253I. ornatus, Broun. A.M.N.H. 6, xv. 245.

Family ANThicidæ. Sub-family ANTHICINÆ.

Cotes, Sharp, i 877 .

vestita, Sharp. Man. N.Z. Coleop. 4I I, No. 724. proba, Broun. Man. N.Z. Coleop. 69I, No. I 215. crispi, Broun. Man. N.Z. Coleop. 4I 2, No. 727. optima, Broun. Man. N.Z. Coleop. I I65, No. 2067. punctata, Broun. Man. N.Z. Coleop. 1 I65, No. 2068. dorsalis, Broun. Man. N.Z. Coleop. i i 66, No. 2069. distincta, Broun. Man. N.Z. Coleop. i i 66, No. 2070. proxima, Broun. Man. N.Z. Coleop. i i67, No. 207 I. rufa, Broun. Man. N.Z. Coleop. i 67 , No. 2072.

Anthicus, Paykull, r 798. obscuricornis, Broun. Man. N.Z. Coleop. 4 I I, No. 725. pellucidipes, Broun. Man. N.Z. Coleop. 4I 2, No. 726. 
Anthicus minor, Broun. Man. N.Z. Coleop. 930, No. 1670. anthracinus, Broun. Man. N.Z. Coleop. I I68, No. 2073. fallax, Broun. Man. N.Z. Coleop. i 68 , No. 2074.

\section{Sub-family PediLinæ.}

Macratria, Newman, I838.

exilis, Pascoe. Man. N.Z. Coleop. 409, No. 722.

verticalis, Sharp. Man. N.Z. Coleop. 409, No. 723.

Scraptogetus, Broun, I 893 .

anthracinus, Broun. Man. N.Z. Coleop. I $35^{8}$, No. 2379.

\section{Family CEDemer1de.}

Selenopalpus, White, $\mathbf{I} 846$.

cyaneus, Fabricius. Man. N.Z. Coleop. 420, No. 736. subviridis, White. Voy. Freb. and Terr., Insects, r 3.; aciphyllæ, Broun. Man. N.Z. Coleop. 845, No. I 502.

Sessinia, Pascoe, I863.

strigipennis, White. Man. N Z Coleop. 420, No. 737. latiuscula, Broun. Man. N.Z. Coleop. 42 I, No. $73^{8}$. lineata, Fabricius. Man. N.Z. Coleop. 421, No. 739. pauperata, Pascoe. Man. N.Z. Coleop. 422, No. 740. nemoralis, Broun. Man. N.Z. Coleop. 845 , No. 1503 . longicornis, Broun. Man. N.Z. Coleop. 1459, No. 2532. thoracica, Broun. A.M.N.H. 6, xii. 290. reversa, Broun. A.M.N.H. 6, xii. 291.

Thelyphassa, Pascoe, i 876 . diaphana, Pascoe. Man. N.Z. Coleop. 422, No. 74I. obscura, Broun. Man. N.Z. Coleop. 422, No. 742. conspicua, Broun. Man. N.Z. Coleop. 790, No. I4I I. limbata, Broun. Man. N.Z. Coleop. 961, No. I 735.

Dammarobius, Broun, 1886.

mollis, Broun. Man. N.Z. Coleop. 846 , No. I 504.

Baculipalpus, Broun, r8so.

rarus, Broun. Man. N.Z. Coleop. 423, No. 743.

Exocalopus, Broun, 1893 . pectinatus, Broun. Man. N.Z. Coleop. I I 70, No. 2078.

Phytilea, Broun, I 893 . propera, Broun. Man. N.Z. Coleop. II 72 , No. 2079.

Techmessa, Bates, 1874 .

concolor, Bates. Man. N.Z. Coleop. 424, No. 744. telephoroides, Bates. Man. N.Z. Coleop. 424, No. 745. attenuata, Broun. Man. N.Z. Coleop. i I 72 , No. 2080. varians, Broun. Man. N.Z. Coleop. I I 73, No. 208r. 
Techmessodes, Broun, 1893.

picticornis, Broun. Man. N.Z. Coleop. 424, No. 746.

versicolor, Broun. Man. N.Z. Coleop. I I 73, No. 2082.

Family MordeLlide.

Sub-family MORDELLINÆ.

Mordella, Linnæus, I $75^{8}$.

antarctica, White. Voy. Ereb. and Terror, Insects, $\mathbf{I} 2$.

funerea, Pascoe. Man. N.Z. Coleop. 414, No. 728.

tibialis, Broun. Man. N.Z. Coleop. 4I 4, No. 729.

detracta, Pascoe. Man. N.Z. Coleop. 4I 4, No. 730.

tairuensis, Broun. Man. N.Z. Coleop. 414, No. 73 I.

Mordellistena, Costa, I 855 .

jucunda, Broun. Man. N.Z. Coleop. 415, No. 732.

neglecta, Broun. Man. N.Z. Coleop. 4I5, No. 733.

Zeamordella, Broun, r88o.

monacha, Broun. Man. N.Z. Coleop. 847 , No. 1505 .

Apeosina, Broun, I88I.

stewarti, Broun. Man. N.Z. Coleop. 693, No. I 2 I6.

tener, Broun. Man. N.Z. Coleop. 693, No. I 217.

Cryptomera, Broun, i 893 .

nigra, Broun. Man. N.Z. Coleop. I359, No. 2380.

Sub-family RHIPIPHORINÆ.

Rhipistena, Sharp, 1878 .

lugubris, Sharp. Man. N.Z. Coleop. 4 I 7 , No. 734.

Sharpia, Broun, i88o (not of Tournier, I 873).

hirtella, Broun. Man. N.Z. Coleop. 4 I 8, No. 735.

Series Phytophaga.

Family CERAMBYCIDÆ.

Sub-family PrIONINE.

Prionoplus, White, 1846.

reticularis, White. Man. N.Z. Coleop. 566, No. 99I.

T.N.Z.I. xii. 284 .

Ochrocydus, Pascoe, 1876 .

huttoni, Pascoe. Man. N.Z. Coleop. 567, No. 992.

Sub-family Cerambycinæ.

Liogramma, Bates, I 874 .

zealandica, Blanchard. Man. N.Z. Coleop. 568, No. 993.

Didymocantha, Newman, i 840 .

sublineata, White. Man. N.Z. Coleop. 568, No. 994. 
Didymocantha diversicornis, White. Man. N.Z. Coleop. 569 , No. 995 .

picta, Bates. Man. N.Z. Coleop. 569, No. 996.

ægrota, Bates. Man. N.Z. Coleop. 569, No. 997.

brevicornis, Broun. Man. N.Z. Coleop. 570, No. 998.

clavipes, Broun. Man. N.Z. Coleop. 808, No. 1435. robusta, Sharp. Man. N.Z. Coleop. I 271, No. 2238. quadriguttata, Broun. Man. N.Z. Coleop. I 272, No. 2239. jucunda, Broun. Man. N.Z. Coleop. I 272 , No. 2240. binotata, Broun. Man. N.Z. Coleop. I 273, No. 224 I. vittata, Broun. Man. N.Z. Coleop. I 273, No. 2242. pallida, Broun. A.M.N.H. 6, xii. 388 .

Emona, Newman, i842.

hirta, Fabricius. Man. N.Z. Coleop. 570 and I 275 , No. 999. villosa, Fabricius. Man. N.Z. Coleop. 1 275, No. 2244. simplicollis, Broun. Man. N.Z. Coleop. $57 \mathrm{I}$ and 1276 , No. 1000 .

humilis, Newman. Man. N.Z. Coleop. I 274, No. 2243. inæqualis, Sharp. Man. N.Z. Coleop, I 275, No. 2245. mutica, Sharp. Man. N.Z. Coleop. I 276 , No. 2246. plicicollis, Sharp. Man. N.Z. Coleop. I 276, No. 2247. debilis, Sharp. Man. N.Z. Coleop. I 277 , No. 2248.

Leptachrous, Bates, 1874 .

strigipennis, Westwood. Man. N.Z. Coleop. 752, No. Ioor.

Votum, Broun, I88o.

mundum, Broun. Man. N.Z. Coleop. 753, No. 1002.

Pseudocalliprason, Broun, r880. marginatum, White. Man. N.Z. Coleop. 574, No. 1003.

Ambeodontus, tristis, Fabricius. Man. N.Z. Coleop. 574, No. roo4.

Agapanthida, White, 1846 . pulchella, White. Man. N.Z. Coleop. 575, No. 1005. scutellaris, Pascoe. Man. N.Z. Coleop. 575, No. I 006.

Ophryops, White, 1846 .

pallidus, White. Man. N.Z. Coleop. 576, No. 1007. dispar, Sharp. Man. N.Z. Coleop. I 277 , No. 2249. nigropictus, Broun. Man. N.Z. Coleop. 1 278, No. 2250. testaceus, Broun. Man. N.Z. Coleop. I 278 , No. 225 I. lentiginosus, Broun. Man. N.Z. Coleop. I 279, No. 2252.

Pseudosemnus, Broun, I 893. amabilis, Broun. Man. N.Z. Coleop. I280, No. 253.

Hesperophanes, Mulsant, I 839. unicolor, Fabricius. Mantissa Insectorum, 147. 
Astetholea, Bates, I 874 .

pauper, Bates. Man. N.Z. Coleop. 577, No. 1008.

lepturoides, Bates. Man. N.Z. Coleop. 577, No. 1009.

aubreyi, Broun. Man. N.Z. Coleop. 577, No. 1010.

Astetholida, Broun, I880.

lucida, Broun. Man. N.Z. Coleop. 578, No. Iо I I.

Epheus, Broun, I 886.

costifer, Broun. Man. N.Z. Coleop. 87 I, No. I55I.

Blosyropus, Redtenbacher, I 868.

spinosus, Redtenbacher. Man. N.Z. Coleop. 579, No. Iог 2.

Ptychopterus, Broun, I 886.

rugosus, Broun. Man. N.Z. Coleop. 809, No. Iо 3.

Psilomorpha, Saunders, I 850.

tenuipes, Saunders. Man. N.Z. Coleop. 580, No. Ior 4.

Xuthodes, Pascoe, I 875 .

punctipennis, Pascoe. Man. N.Z. Coleop. 580, No. ror 5 a. apicalis, Sharp. Man. N.Z. Coleop. 580, No. Io 5. batesi, Sharp. Man. N.Z. Coleop. $5^{8}$ г, No. 10 I 6. divergens, Broun. Man. N.Z. Coleop. $5^{81}$, No. Iо 7. lepidus, Broun. Man. N.Z. Coleop. I 280, No. 2254.

Calliprason, White, i 846 .

sinclairi, White. Man. N.Z. Coleop. 582, No. Ior 8.

Stenopotes, Pascoe, i 875. pallidus, Pascoe. Man. N.Z. Coleop. 583 , No. roig.

Cacodrotus, Broun, I 893 .

bifasciatus, Broun. Man. N.Z. Coleop. I 28 I, No. 2255.

Drotus, Sharp, 1877 .

elegans, Sharp. Man. N.Z. Coleop. $5^{8} 3$, No. 1020.

Zorion, Pascoe, i 867.

minutum, Fabricius. Man. N.Z. Coleop. 584 , No. Iо21. guttigerum, Westwood. Man. N.Z. Coleop. 585, No. 1022. castum, Broun. Man. N.Z. Coleop. I 281, Na. 2256.

Gnornodes, Broun, i 893 .

piceus, Broun. Man. N.Z. Coleop. I282, No. 2257.

Gastrosarus, Bates, 1874 .

nigricollis, Bates. Man. N.Z. Coleop. 586, No. Iо23. urbanus, Broun. Man. N.Z. Coleop. I 283 , No. $225^{8}$. lautus, Broun. Man. N.Z. Coleop. I283, No. 2259. picticornis, Broun. Man. N.Z. Coleop. I 284, No. 2260.

Eburida, Broun, I880.

sericea, Smith. Man. N.Z. Coleop. 587 , No. 1024. 
Acrocyrta, Pascoe, 1856. spinicornis, Newman. Man. N.Z. Coleop. 587 , No. 1025.

Clytus, Laichorting, I 784 .

rugulosus, Broun. Man. N.Z. Coleop. 588, No. 1026.

Coptomma, Newman, I 840 . variegata, Fabricius. Man. N.Z. Coleop. 589, No. 1027.

Navomorpha, Blanchard, i 853 .

lineatus, Fabricius. Man. N.Z. Coleop. 590, No. Iо28. sulcatus, Fabricius. Man. N.Z. Coleop. 590, No. I029. neglectus, Broun. Man. N.Z. Coleop. 59I, No. 1030. sticticus, Broun. Man. N.Z. Coleop. 1284, No. 226 I.

Ceresium, Newman, 1842 . zealandicum, Blanchard. Voy. Pole Sud, iv. 272.

Aphanasium, Thomson, 1860 .

australe, Boisduval. Voy. Astrolabe, ii. 480.

Phlyctænodes, Newman, i 840 . retifer, Lacordaire. Gen. Col. I869, 374.

Sub-family Lamine.

Hexatricha, Lacordaire, I869. pulverulenta, Westwood. Man. N.Z. Coleop. 592, No. I03I. heteromorpha, Boisduval. Voy. Astrolabe, ii. 2 I.

Xyloteles, Newman, I 840 .

lynceus, Fabricius. Man. N.Z. Coleop. 593, No. 1032. griseus, Westwood. Man. N.Z. Coleop. 593, No. I033. humeratus, Bates. Man. N.Z. Coleop. 593, No. I034. subpinguis, White. Man. N.Z. Coleop. 594, No. 1035. nudus, Bates. Man. N.Z. Coleop. 594, No. 1036. rugicollis, Bates. Man. N.Z. Coleop. 594, No. I037. inornatus, Broun. Man. N.Z. Coleop. 595, No. I038. lætus, White. Man. N.Z. Coleop. 595, No. ro39. gratus, Broun. Man. N.Z. Coleop. 595, No. I040. nanus, Bates. Man. N.Z. Coleop. 596, No. г о4 I. ægrotus, Bates. Man. N.Z. Coleop. 597, No. 1042. pulchellus, Bates. Man. N.Z. Coleop. 597, No. I043. scissicauda, Bates. Man. N.Z. Coleop. 597, No. I044. bullatus, Sharp. Man. N.Z. Coleop. 598, No. 1045. pictulus, Bates. Man. N.Z. Coleop. 598, No. 1046. sandageri, Broun. Man. N.Z. Coleop. 91 2, No. I6 62. maculosus, Broun. Man. N.Z. Coleop. 913, No. 1633. angustulus, Broun. Man. N.Z. Coleop. 973, No. 1756. huttoni, Sharp. Man. N.Z. Coleop. 1287, No. 2264. gaudens, Broun. Man. N.Z. Coleop. I 288, No. 2265. germanus, Sharp. Man. N.Z. Coleop. 1289 , No. 2266. 
Xyloteles fasciatus, Sharp. Man. N.Z. Coleop. г 289 , No. 2267. phormiobius, Broun. Man. N.Z. Coleop. I 388, No. 2422. prolongatus, Broun. Man. N.Z. Coleop. I389, No. 2423. costatus, Pascoe. A.M.N.H. 4, xvi. 2 I 7.

parvulus, White. Voy. Ereb. and Terror, Insects, 22.

Microlamia, Bates, I 874 .

pygmæa, Bates. Man. N.Z. Coleop. 599, No. 1047. æmula, Broun. Man. N.Z. Coleop. 8 I o, No. 1437.

Mesolamia, Sharp, I882.

marmorata, Sharp. Man. N.Z. Coleop. I295, No. 2278. ærata, Broun. Man. N.Z. Coleop. I 296, No. 2279.

Somatidia, Thomson, I 864 . antarctica, White. Man. N.Z. Coleop. 600, No. 1048. assimilata, Broun. Man. N.Z. Coleop. 600, No. 1049. terrestris, Broun. Man. N.Z. Coleop. 60 I, No. I050. ptinoides, Bates. Man. N.Z. Coleop. 60 I, No. ro5 I. angusta, Broun. Man. N.Z. Coleop. 601, No. 1052. pictipes, Broun. Man. N.Z. Coleop. 602, No. I053. longipes, Sharp. Man. N.Z. Coleop. 602, No. Iо54. diversa, Broun. Man. N.Z. Coleop. 603, No. 1055. nitida, Broun. Man. N.Z. Coleop. 603, No. 1056. crassipes, Broun. Man. N.Z. Coleop. 8 I o, No. I 438. elongata, Broun. Man. N.Z. Coleop. 8 i I, No. I 439. grandis, Broun. Man. N.Z. Coleop. I 290, No. 2269. helmsi, Sharp. Man. N.Z. Coleop. I 290 , No. 2270. spinicollis, Broun. Man. N.Z. Coleop. I 29I, No. 227 I. costifer, Broun. Man. N.Z. Coleop. I 29I, No. 272. variegata, Broun. Man. N.Z. Coleop. 1292, No. 2273. simplex, Broun. Man. N.Z. Coleop. I 292, No. 2274. signata, Broun. Man. N.Z. Coleop. I 293, No. 2275. convexa, Broun. Man. N.Z. Coleop. I 498, No. 258 I. latula, Broun. Man. N.Z. Coleop. I 499, No. 2582. lævior, Broun. Man. N.Z. Coleop. I 499, No. $25^{8} 3$. tenebrica, Broun. Man. N.Z. Coleop. ${ }_{1500,}$ No. $25^{8} 4$. albicoma, Broun. Man. N.Z. Coleop. I 500, No. 2585. proxima, Broun. A.M.N.H. 6, xii. 390. picticornis, Broun. A.M.N.H. 6, xv. $4 \mathrm{I} 8$.

Stenellipsis, Bates, I874.

bimaculata, White. Man. N.Z. Coleop. 604, No. 1057. gracilis, White. Man. N.Z. Coleop. 604, No. 1058. latipennis, Bates. Man. N.Z. Coleop. 605, No. Iо59. pumila, Pascoe. Man. N.Z. Coleop. 605, No. Iо60. cuneata, Sharp. Man. N.Z. Coleop. I 289, No. 2268.

Psilocnæia, Bates, r874. linearis, Bates. Man. N.Z. Coleop. 606, No. I 06 I. brouni, Bates. Man. N.Z. Coleop. 606, No. 1062. 
Spilotrogia, Bates, 1874 .

maculata, Bates. Man. N.Z. Coleop. 6o7, No. 1063 . hilarula, Broun. Man. N.Z. Coleop. 607, No. 1062.

Eurychæna, Bates, I874.

fragilis, Bates. Man. N.Z. Coleop. 608, No. xo65.

feredayi, Bates. Man. N.Z. Coleop. 608, No. 1066.

acutula, Broun. Man. N.Z. Coleop. 608, No. 1067.

Tetrorea, White, 1846 .

cilipes, White. Man. N.Z. Coleop. 609, No. 1068.

discedens, Sharp. Man. N.Z. Coleop. I 294, No. 1440. longipennis, Sharp. Man. N.Z. Coleop. I 293, No. 2276. sellata, Sharp. Man. N.Z. Coleop. I 294, No. 2277.

Hybolasius, Bates, 1874 .

crista, Fabricius. Man. N.Z. Coleop. 61 о, No. 1069. lanipes, Sharp. Man. N.Z. Coleop. 6 го, No. 1070. pedator, Sharp. Man. N.Z. Coleop. 6ro, No. ro7r. wakefieldi, Bates. Man. N.Z. Coleop. 6r I, No. Iо72. concolor, Broun. Man. N.Z. Coleop. 6i I, No. 1073. viridescens, Bates. Man. N.Z. Coleop. 6 i I, No. Io74. promissus, Broun. Man. N.Z. Coleop. 61 2, No. I075. simplex, Bates. Man. N.Z. Coleop. 6 г 2, No. 1076. modestus, Broun. Man. N.Z. Coleop. 613, No. 1077. variegatus, Broun. Man. N.Z. Coleop. 6I3, No. 1078. cristatellus, Bates. Man. N.Z. Coleop. 6r 4, No. 1079. bellicosus, Broun. Man. N.Z. Coleop. 6r 4 , No. Io8o. parvus, Broun. Man. N.Z. Coleop. 6r4, No. I08I. vegetus, Broun. Man. N.Z. Coleop. 744, No 1320. fasciatus, Broun. Man. N.Z. Coleop. 744, No. I32 I. brevicollis, Broun. Man. N.Z. Coleop. 8 I I, No. I44I. picitarsis, Broun. Man. N.Z. Coleop. 8 I 2, No. 1442. pusillus, Broun. Man. N.Z. Coleop. 81 2, No. I443. apicalis, Broun. Man. N.Z. Coleop. 872, No. I $55^{2}$. piceus, Broun. Man. N.Z. Coleop. 872 , No. I 553 . deplanatus, Sharp. Man. N.Z. Coleop. I 297, No. 2280. castaneus, Broun. Man. N.Z. Coleop. I 297, No. 228I. finitimus, Broun. Man. N.Z. Coleop. I 298, No. 2282. albihirtus, Broun. Man. N.Z. Coleop. I 298, No. 2283. gnarus, Broun. Man. N.Z. Coleop. r 298, No. 2284 . femoralis, Broun. Man. N.Z. Coleop. I 299, No. 2285. thoracicus, Broun. Man. N.Z. Coleop. I 299, No. 2286. rufescens, Broun. Man. N.Z. Coleop. I 300, No. 2287. dubius, Broun. Man. N.Z. Coleop. I389, No. 2424. optatus, Broun. A.M.N.H. 6, xii. $3^{89}$. trigonellus, Hutton. T.N.Z.I. xxx. 158 .

Pœcilippe, Bates, 1874 . stictica, Bates. Man. N.Z. Coleop. 6I5, No. 1082. 
Pœcilippe flavipes, White. Man. N.7. Coleop. 615, No. 1083. medialis, Sharp. Man. N.Z. Coleop. 1 301, No. 2288. femoralis, Sharp. Man. N.Z. Coleop. I30 I, No. 2289. simplex, Bates. Man. N.Z. Coleop. 612, No. 1076.

Diastamerus, Redtenbacher, 1868.

tomentosus, Redtenbacher. Man. N.\%. Coleop. 616, No. 1084 .

Tympanopalpus, Redtenbacher, 1868. dorsalis, Redtenbacher. Man. N.Z. Coleop. 617, No. 1085.

Family Chryomelide. Sub-family EUMOLPIN E.

Eucolaspis, Sharp, I 886.

brunneus, Fabricius. Man. N.Z. Coleop. 622, No. 1090. pallidipennis, White. Man. N.Z. Coleop. 622, No. 1091. puncticollis, Broun. Man. N.Z. Coleop. 62.3, No. 1092. jucundus, Broun. Man. N.Z. Coleop 623, No. 1093. subæneus, Broun. Man. N.Z. Coleop. 624, No. 1094. sculptus, Broun. Man. N.Z. Coleop. 624, No. 1095. merus, Broun. Man. N.Z. Coleop. 624, No. 1096. brevicollis, Broun. Man. N.Z. Coleop. 625, No. 1097. atroceruleus, Broun. Man. N.Z. Coleop. 625, No 1098. ochraceus, Broun. Man. N.Z. Coleop I 303 , No. 2290. coloratus, Broun. Man. N.Z. Coleop. I 303, No. 2291. montanus, Broun. Man. N.Z. Coleop. I304, No. 2292. vittiger, Broun. A.M.N H. 6, xii. 391 . picticornis, Bromn. A M.N.H. 6, xii. 391.

Atrichatus, Sharp, I886.

ochraceus, Broun. Man. N.Z. Colcop. 625 and 1305, No. 1099 .

æneicollis, Broun. A.M.N.H. 6, xv. 419.

Pilacolaspis, Sharp, i 886.

wakefieldi, Sharp. Man. N.Z. Coleop. 1 306, No. 2293. huttoni, Broun. Man. N.Z. Coleop. 626, No. 1100.

Peniticus, Sharp, 1876 suffusus, Sharp. Man. N.Z. Coleop. 627, No. 1101. antiquus, Sharp. Man. N.Z. Coleop. 627, No. 1102. robustus, Broun. Man. N.Z. Coleop. 628, No 1103.

Sub-family Cryptocephaline.

Arnomus, Sharp, i 876 .

brouni, Sharp. Man. N.Z. Coleop. 6i 9, No. 1086. curtipes, Broun. Man. N.Z. Coleop. 1390 , No. 2425 . marginalis, Broun. Man. N.\%. Coleop. I 390, No. 2426. 
Alema, Sharp, 1876 .

paradoxa, Sharp. Man. N.Z. Coleop. 620, No. 1087. puncticollis, Broun. Man. N.Z. Coleop. 620, No. 1088. spatiosa, Broun. Man. N.Z. Coleop. 62 I, No. Io89.

Scaphodius, Chapuis, I 874

compactus, Sharp. Man. N.Z. Coleop. 1302, No. 1444.

Sub-family Chrysoneline.

Allocharis, Sharp, I882.

marginata, Sharp. Man. N.Z. Coleop. I 306, No. 2294.

morosa, Broun. Man. N.Z. Coleop. I 307 , No. 2295.

limbata, Broun. Man. N.Z. Coleop. I 307, No. 2296.

Aphilon, Sharp, 1876 .

enigma, Sharp. Man. N.Z. Coleop. 629, No. I Io4. pretiosa, Broun. Man. N.Z. Coleop. 630, No. I 105. punctata, Broun. Man. N.Z. Coleop. 630 , No. I 106. minuta, Broun. Man. N.Z. Coleop. 631, No. I 107. monstrosa, Broun. Man. N.Z. Coleop. 874 , No. 1556. sobrina, Broun. Man. N.Z. Coleop. 875 , No. 1557. præstans, Broun. Man. N.Z. Coleop. I309, No. 230 I. convexa, Broun. Man. N.Z. Coleop. I 3 Iо, No. 2302. latulum, Broun. Man. N.Z. Coleop. I 3 Iо, No. $23 \circ 3$. scutellaris, Broun. Man. N.Z. Coleop. I 50 I, No. 2586. laticollis, Broun. A.M.N.H. 6, xii. 392.

Caccomolpus, Sharp, I 886.

globosus, Sharp. Man. N.Z. Coleop. r 308 , No. 2297. plagiatus, Sharp. Man. N.Z. Coleop. I 308, No. 2298. pullatus, Broun. Man. N.Z. Coleop. I 309, No. 2299. maculatus, Broun. Man. N.Z. Coleop. I 309, No. 2300.

Sub-family GaLerUCinæ.

Luperus, Geoffroy, 1764 .

vulgaris, Broun. Man. N.Z. Coleop. 632, No. I 108. attenuatus, Broun. Man. N.Z. Coleop. 633, No. I Io9. thoracicus, Broun. Man. N.Z. Coleop. 63.3, No. I I 10. nigripes, Broun. Man. N.Z. Coleop. 634, No. I I I I. nigricans, Broun. Man. N.Z. Coleop. 634, No. I I 12. nitidicollis, Broun. Man. N.Z. Coleop. 634, No. I I I 3. æneus, Broun. Man. N.Z. Coleop. 635 , No. I I I 4. viridis, Broun. Man. N.Z. Coleop. 635, No. I I 15. oleareæ, Broun. Man. N.Z. Coleop. I312, No. 2304. rugicollis, Broun. Man. N.Z. Coleop. I3 13 , No. 2305. brevicollis, Broun. Man. N.Z. Coleop. I3 33 , No. 2306. sulcifer, Broun. Man. N.Z. Coleop. I3I3, No. 2307. rectipes, Broun. Man. N.Z. Coleop. 1314 , No. 2308. calcaratus, Broun. Man. N.Z. Coleop. I314, No. 2309. 
Luperus princeps, Broun. Man. N.Z. Coleop. I3 I4, No. 23 ro. monticola, Broun. Man. N.Z. Coleop. I $3^{1}$ 5, No. 23 I I. fuscatus, Broun. Man. N.Z. Coleop. I3 15 , No. 23 I 2. sordidus, Broun. Man. N.Z. Coleop. I316, No. 2313. truncatus, Broun. Man. N.Z. Coleop. I3I6, No. 2314. nigricornis, Sharp. Man. N.Z. Coleop. I3I6, No. 2315. ænescens, Sharp. Man. N.Z. Coleop. I3 17 , No. 2316. puncticollis, Sharp. Man. N.Z. Coleop. I3I 7, No. 2317. mollis, Broun. Man. N.Z. Coleop. 1502 , No. 2587.

Allastena, Broun, i 893 .

nitida, Broun. Man. N.Z. Coleop. г 3 I8, No. 23 I 8. quadrata, Broun. Man. N.Z. Coleop. I319, No. 23 I9.

Bryobates, Broun, 1886.

coniformis, Broun. Man. N.Z. Coleop. 874 , No. 1555 .

Trachytetra, Sharp, i 886.

Sub-family HaLticine.

rugulosa, Broun. Man. N.Z. Coleop. 636 and i 3 I I, No. I 1 I 6 .

Inopelonia, Broun, 1893 .

testacea, Broun. Man. N.Z. Coleop. 637, No. i i 8 .

fuliginosa, Broun. Man. N.Z. Coleop. 637, No. i i 9.

Pleuraltica, Sharp, I 886.

cyanea, Broun. Man. N.Z. Coleop. I 3 I 2 and 638, No. I 20.

Phyllotreta, Chevrolat, I 839 .

nitida, Broun. Man. N.Z. Coleop. 636, No. I I 7 .

graminicola, Broun. Man. N.Z. Coleop. I 39I, No. 2427.

littoralis, Broun. Man. N.Z. Coleop. I391, No. 2428.

vittigera, Broun. Man. N.Z. Coleop. I 392, No. 2429.

Series RhyncophoRa.

Family CURCULIONIDÆ.

Sub-family OTIORHYNCHINA.

Nicæana, Pascoe, i 877 .

modesta, Pascoe. Man. N.Z. Coleop. 428, No. 749. concinna, Broun. Man. N.Z. Coleop. 847 , No. 1506. cinerea, Broun. Man. N.Z. Coleop. 93 I, No. 1673. cervina, Broun. Man. N.Z. Coleop. 1360, No. 238 I. tarsalis, Broun. Man. N.Z. Coleop. I 460, No. 2533.

Stygeopetes, Broun, 1893 .

littoralis, Broun. Man. N.Z. Coleop. I I 76, No. 2085 .

Cecyropa, Pascoe, i 875.

tychioides, Pascoe. Man. N.Z. Coleop. 437 , No. 768. maritima, Broun. Man. N.Z. Coleop. 438 , No. 769 . 
Cecyropa brevipennis, Broun. Man. N.Z. Coleop. 438, No. 770. alba, Broun. Man. N.Z. Coleop. 698, No. I 228. varia, Broun. Man. N.Z. Coleop. 698, No. I 229. discors, Broun. Man. N.Z. Coleop. 699, No. I 230. setigera, Broun. Man. N.Z. Coleop. 905, No. I6 I 7 . macularia, Broun. Man. N.Z. Coleop. 961, No. 1736. albicans, Sharp. Man N.Z. Coleop. i i 75, No. 2083. fumosa, Broun. Man. N.Z. Coleop. I I 75, No. 2084 .

Nonnotus, Sharp, i 886 .

griseolus, Sharp. Man. N.Z. Coleop. I I 77, No. 2086. eclectus, Broun. Man. N.Z. Coleop. i I 77, No. 2087. pallescens, Broun. Man. N.Z. Coleop. i 78 , No. 2088. albicans, Broun. Man. N.Z. Coleop. 443, No. 78 I. albatus, Broun. Man. N.Z. Coleop. 694, No. I 2 I 9.

Tigones, Broun, i 886.

caudata, Broun. Man. N.Z. Coleop. 855, No. I 52 I. osculans, Broun. Man. N.Z. Coleop. 856, No. I522. certa, Broun. Man. N.Z. Coleop. 856, No. 1523. grisea, Broun. Man. N.7. Coleop. 856 , No. 1524. cervina, Broun. Man. N.Z. Coleop. 857 , No. 1525 . aulica, Broun. Man. N.Z. Coleop. i I 80, No. $209^{2}$. variegata, Broun. Man. N.Z. Coleop. I I 8 I, No. 2093. scutellaris, Broun. Man. N.Z. Coleop. I I \& I, No. 2094. cruda, Broun. Man. N.\%. Coleop. i i 82, No. 2095. antennalis, Broun. Man. N.Z. Coleop. i i 82, No. 2096. diversa, Broun. Man. N.Z. Coleop. I 183 , No. 2097. rufula, Broun. Man. N.Z. Coleop. i 183 , No. 2098. cavelli, Broun. Man. N.Z. Coleop. I $3^{61}$, No. $23^{82}$. obscura, Broun. Man. N.Z. Coleop. I 36 I, No. 2383 . cuspidata, Broun. Man. N.Z. Coleop. 694, No. I 220. plana, Broun. Man. N.Z. Coleop. 700, No. I 233.

Protophormus, Sharp, i $\$ 86$.

gracilis, Sharp. Man. N.Z. Coleop. I I 79, No. 2089. binodulus, Sharp. Man. N.Z. Coleop. I I 79, No. 2090. robustus, Sharp. Man. N.Z. Coleop. i I 80, No. 209I.

Epitimetes, Pascoe, 1877.

lutosus, Pascoe. Man. N.Z. Coleop. 435 , No. 763 . wakefieldi, Sharp. Man. N.Z. Coleop. I i 83, No. 2099.

Platyomida, White, i $\delta_{4} 6$.

binodes, White. Man. N.Z. Coleop. 442, No. 776. censoria, Pascoe. Man. N.Z. Coleop. 442, No. 777 . aculeata, Broun. Man. N.Z. Coleop. 442 , No. 778 . perniciosa, Broun. Man. N.Z. Coleop. 442, No. 779. apicalis, Broun. Man. N.Z. Coleop. 701, No. I 235. 
Platyomida amota, Broun. Man. N.Z. Coleop. 793, No. I 45. enysi, Broun. Man. N.Z. Coleop. 852 , No. I 516. æquua, Broun. Man. N.Z. Coleop. 932, No. 1677. hochstetteri, Redtenbacher. Man. N.Z. Coleop. 476, No. 842 . simulatrix, Sharp. Man. N.Z. Coleop. I I 85 , No. 2 I 00. depressa, Sharp. Man. N.Z. Coleop. I 185 , No. 2 เ 1.

Rhynchogonus, germanus, Broun. A.M.N.H. 6, xii. 293.

Lyperobates, Broun, I $\delta_{93}$. asper, Broun. Man. N.Z. Coleop. I 462, No. 2534.

Hygrochus, Broun, i 88 I. oscitans, Broun. Man. N.Z. Coleop. 703, No. 1237. verrucosus, Broun. Man. N.Z. Coleop. I 220 , No. 2 I 49. illepidus, Broun. Man. N.Z. Coleop. I220, No. 2150. oculatus, Broun. $\Lambda$ M.N.H. 6, xii. 292.

Homodus, Broun, is 88 I. fumeus, Broun. Man. N.Z. Coleop. 703, No. 1238.

Aporolobus, Sharp, i 886.

irritus, Pascoe. Man. N.Z. Coleop. 43I, No. 754. hariolus, Broun. Man. N.Z. Coleop. 848 , No. 1507. cecyropioides, Broun. Man. N.Z. Coleop. 905, No. I6 6. rugosus, Broun. Man. N.Z. Coleop. 930, No. I67 I. pallidus, Broun. Man. N.Z. Colcop. I 365, No. 2388.

Notiopatæ, Broun, I893.

setifer, Broun, Man. N.Z. Coleop. I 187, No. 2 I 02. sternalis, Broun. Man. N.Z. Coleop. 1462 , No. 2535. clarus, Broun. Man. N.Z. Coleop. 43I, No. 755 .

Pælocharis, Broun, I 893 . inflata, Broun. Man. N.Z. Coleop. 1188 , No. 2 103. vestita, Broun. Man. N.Z. Coleop. 1463 , No. 2536. corpulenta, Broun. Man. N.Z. Coleop. 431, No. $75^{6}$.

Protolobus, Sharp, i 886.

obscurus, Sharp. Man. N.Z. Coleop. I 188. No. 2 104. porculus, Pascoe. Man. N.Z. Coleop. 430, No. 753.

Catoptes, Schönherr, 1842.

obliquis, Schönherr. Man. N.Z. Coleop. 428, No. 750. parilis, Pascoe. Man. N.Z. Coleop. 443, No. 780. compressus, Broun. Man. N.Z. Coleop. 429, No. $75 \mathrm{I}$. obliquisignatus, Bohem. Man. N.Z. Coleop. (93, No. I 2 I 8. attenuatus, Broun. Man. N.Z. Coleop. 791, No. I4I 2. pilosellus, Broun. Man. N.Z. Coleop. 853 , No. 15 I 7. bicostatus, Broun. Man. N.Z. Coleop. 853, No. I 518. 
Catoptes tibialis, Broun. Man. N.Z. Coleop. 854 , No. 1519 . stolidus, Broun. Man. N.Z. Coleop. 854, No. 1520. caliginosus, Broun. Man. N.Z. Coleop. I189, No. 2 105. posticalis, Broun. Man. N.Z. Coleop. i I89, No. 2 ro6. humeralis, Broun. Man. N.Z. Coleop. I I90, No. 2107. chalmeri, Broun. Man. N.Z. Coleop. I r go, No. 2 ro8. asperellus, Broun. Man. N.Z. Coleop. II9I, No. 2 rog. cheesemani, Broun. Man. N.7. Coleop. II9I, No. 2 Iro. decorus, Broun. Man. N.Z. Coleop. I I92, No. 2 I Ir. brevicornis, Sharp. Man. N.Z. Coleop. I I93, No. 2 II 2. æmulator, Broun. Man. N.Z. Coleop. Ir93, No. 2 I I 3. tenebricus, Broun. Man. N.Z. Coleop. I I 94 , No. 2 I 4. latipennis, Broun. Man N.Z. Coleop. 1362 , No. 2384. furvus, Broun. Man. N.Z. Coleop. 1362 , No. 2385 . vastator, Broun. Man. N.Z. Coleop. 1463, No. 2537. scutellaris, Sharp. Man. N.Z. Coleop. I 503, No. 259 I. longulus, Sharp. Man. N.Z. Coleop. I 504, No. 2592.

Haplolobus, Broun, 1893 .

sævus, Broun. Man. N.Z. Coleop. I 199, No. 2 I 22. æthiops, Broun. Man. N.Z. Coleop. I I99, No. 2 I 23. gregalis, Broun. Man. N.Z. Coleop. I I99, No. 2 I 24.

Brachylous, White, I 846. punctatus. White. Man. N.Z. Coleop. 432, No. 757. breviusculus, Broun. Man. N.Z. Coleop. 440, No. 774 . elegans, Broun. Man. N.Z. Coleop. I I 94, No. 2 I 5. inæqualis, Sharp. Man. N.Z. Coleop. I 195 , No. 2 I 6. huttoni, Sharp. Man. N.Z. Coleop. I 195 , No. 2 I 7. bagooides, Sharp. Man. N.Z. Coleop. I 195, No. 2 I I 8. punctipennis, Sharp. Man. N.Z. Coleop. I196, No. 2 II9. longicollis, Sharp. Man. N.Z. Coleop. I 196, No. 2 I 20. viridescens, Broun. Man. N.Z. Coleop. I 363 , No. 2386. posticalis, Broun. Man. N.Z. Coleop. 1364 , No. 2387.

Inocatoptes, Broun, 1902. incertus, Broun. T.N.Z.I. xxxiii. I 78 .

Inophløus, Pascoe, 1875. traversii, Pascoe. Man. N.Z. Coleop. 439, No. $770 a$. inuus, Pascoe. Man. N.Z. Coleop. 439, No. 77 I. rhesus, Pascoe. Man. N.Z. Coleop. 439, No. 772. vitiosus, Pascoe. Man. N.Z. Coleop. 440, No. 773. rubidus, Broun. Man. N.Z. Coleop. 699, No. I 231. nigellus, Broun. Man. N.Z. Coleop. 700, No. I 232. prælatus, Broun. Man. N.Z. Coleop. 851 , No. 15 r4. sulcifer, Broun. Man. N.Z. Coleop. 905, No. 1618. costifer, Broun. Man. N.Z. Coleop. 932, No. I676. egregius, Broun. Man. N.Z. Coleop. 962, No. 1737. nodifer, Broun. Man. N.Z. Coleop. I 200, No. 2125. 
Inophlœus alacer, Broun. Man. N.Z. Coleop. I200, No. 2126. albonotatus, Broun. Man. N.Z. Coleop. I 201 , No. 2127. vestitus, Broun. Man. N.Z. Coleop. I 202, No. 2 I 28. suturalis, Broun. Man. N.Z. Coleop. 1464, No. $253^{8}$. villaris, Pascoe. A.M.N.H. 4, xvi. 220. medius, Broun. A.M.N.H. 6, xii. 294.

Phæophanus, Broun, i 886. Sub-family CyLindrorHinte. rugosus, Broun. Man. N.Z. Coleop. 793, No. I4I4. similis, Broun. Man. N.Z. Coleop. I 216 , No. 2145. lituratus, Broun. Man. N.Z. Coleop. I216, No. 2146.

Heteraomus, Broun, 1893 .

longipes, Broun. Man. N.Z. Coleop. 1218 , No. 2147.

Anagotus, Sharp, r882.

helmsi, Sharp. Man. N.Z. Coleop. I 2 I5, No. 2 I 44.

Phoxoteles, Broun, r 893 .

graniger, Broun. Man. N.Z. Coleop. 850 , No. ${ }^{5}{ }^{1} 3$.

Heterotyles, Broun, r 886.

argentatus, Broun. Man. N.Z. Coleop. 795, No. I4I 7.

Rhadinosomus, Schönherr, I 840 .

acuminatus, Schönherr. Man. N.Z. Coleop. 430, 752.

Genus of doubtful position:-

Geochus, Broun, I882.

inæqualis, Broun. Man. N.Z. Coleop. 446 , No. 785 . politus, Broun. Man. N.Z. Coleop. 704, No. I 239. rugulosus, Broun. Man. N.Z. Coleop. 931, No. I672. similis, Broun. Man. N.Z. Coleop. I221, No. 2 I 5 I. nodosus, Broun. Man. N.Z. Coleop. 1221 , No. 2152. plagiatus, Broun. Man. N.Z. Coleop. 1365 , No 2389. puncticollis, Broun. Man. N.Z. Coleop. I 367 , No. 2390. squamosus, Broun. Man. N.Z. Coleop. 1465, No. 2539. tibialis, Broun. Man. N.Z. Coleop. 1465 , No. 2540. marginatus, Broun. Man. N.Z. Coleop. I 466, No. 254 I. frontalis, Broun. Man. N.Z. Coleop. I466, No. 2542. nigripes, Broun. Man. N.Z. Coleop. 1467, No. 2543. setiger, Broun. A.M.N.H. 6, xii. 295.

Lyperobius, Pascoe, 1876 .

huttoni, Pascoe. Man. N.Z. Coleop. 445 , No. 783 . tuberculatus, Pascoe. Man. N.Z. Coleop. 445 , No. 784. carinatus, Broun. Man. N.Z. Coleop. 702 , No. 1236. cupiendus, Broun. Man. N.Z. Coleop. 962, No. $173^{8 .}$ læviusculus, Broun. T.N.Z.I. xxiii. I 79. 
Lyperopais, Broun, I 893.

mirus, Broun. Man. N.Z. Coleop. I 222 , No. 2 I 53.

Sub-family HyLOBHNÆ.

Eiratus, Pascoe, 1877.

parvulus, Pascoe. Man. N.Z. Coleop. 447 , No. 786.

tetricus, Broun. Man. N.Z. Coleop. 447, No. 787. costatus, Broun. Man. N.Z. Coleop. 794, No. I 4 I6. ornatus, Broun. Man. N.Z. Coleop. 906, No. r6 I9. suavis, Broun. Man. N.Z. Coleop. 934, No. I679. pyriformis, Broun. Man. N.Z. Coleop. I 223 , No. 2154 . rugosus, Broun. Man. N.Z. Coleop. I 223 , No. 2 I 55.

Dryopais, Broun, I 886.

variabilis, Broun. Man. N.Z. Coleop. 933, No. 1678 .

Pædaretus, Pascoe, I 876 .

hispidus, Pascoe. Man. N.Z. Coleop. 444, No. 782.

Sub-family RHYPAROSOMIN.

Phrynixus, Pascoe, I 875 .

terreus, Pascoe. Man. N.Z. Coleop. 433, No. $75^{8}$.

astutus, Pascoe. Man. N.Z. Coleop. 433, No. 759. celatus, Broun. Man. N.Z. Coleop. 433, No. 760. modicus, Broun. Man. N.Z. Coleop. 434, No. 76 I. facetus, Broun. Man. N.Z. Coleop. 695, No. I 22 I. intricatus, Broun. Man. N.Z. Coleop. 848 , No. 1508. tuberculatus, Broun. Man. N.Z. Coleop. 849 , No. I509. differens, Broun. Man. N.Z. Coleop. 849 , No. I 5 Io. rufipes, Broun. Man. N.Z. Coleop. 849 , No. I 5 I I. simplex, Broun. Man. N.Z. Coleop. I 202, No. 2 I 29. costirostris, Broun. Man. N.Z. Coleop. I 203 , No. 2 I 30. humeralis, Broun. Man. N.Z. Coleop. 1467, No. 2544. brevipennis, Broun. Man. N.Z. Coleop. I 468, No. 2545. cedius, Broun. Man. N.Z. Coleop. I 468, No. 2546.

Bradypatæ, Broun, I 893 .

capitalis, Broun. Man. N.Z. Coleop. 1206 and 850, No. I 5 I 2.

armiger, Broun. A.M.N.H. 6, xii. 296.

Erymneus, Pascoe, 1877 .

sharpi, Pascoe. Man. N.Z. Coleop. 436, No. 764. scabiosus, Broun. Man. N.Z. Coleop. 436, No. 765 . castaneus, Broun. Man. N.Z. Coleop. 436, No. 766. granulatus, Broun. Man. N.Z. Coleop. 437, No. 767. longulus, Broun. Man. N.Z. Coleop. 931, No. 1674. cœnosus, Broun. Man. N.Z. Coleop. 932, No. 1675. ferrugatus, Broun. Man. N.Z. Coleop. I 203, No. 2 I 3 I. irregularis, Broun. Man. N.Z. Coleop. 1367 , No. 2391. 
Erymneus crassipes, Broun. Man. N.Z. Coleop. I469, No. 2547 .

firmus, Broun. Man. N.Z. Coleop. I 470, No. 2548. probus, Broun. A.M.N.H. 6, xii. 299.

Lithocia, Broun, I893.

fimbriata, Broun. Man. N.Z. Coleop. 147 I, No. 2549.

Pachyprypnus, Broun, I886.

pyriformis, Broun. Man. N.Z. Coleop. 792, No. I4I3. longiusculus, Broun. Man. N.Z. Coleop. 434, No. 762.

Dolioceuthus, Broun, i 893 .

dumetosus, Broun. Man. N.Z. Coleop. I 208, No. 2 I 35. vestitus, Broun. Man. N.Z. Coleop. I 209, No. 2 I 36.

Astyplus, Broun, I893.

conicus, Broun. Man. N.Z. Coleop. 1210, No. 2 I 37.

Styphlotelus, Broun, I 893.

foveatus, Broun. Man. N.Z. Coleop. I 368, No. 2392.

fascicularis, Broun. Man. N.Z. Coleop. I 369 , No. 2393.

Cuneopterus, Sharp, i 886 .

conicus, Sharp. Man. N.Z. Coleop. I 204, No. 2 I 32.

tenuicornis, Broun. A.M.N.H. 6, xii. 297.

Chamæpsephis, Broun, I893.

aurisetifer, Broun. Man. N.Z. Coleop. 1207, No. 2 I34.

Aræoscapus, Broun, 1893 .

ovipennis, Broun. A.M.N.H. 6, xii. 300.

flavipes, Broun. Man. N.Z. Coleop. I 473, No. 2552.

thoracicus, Broun. Man. N.Z. Coleop. 1474, No. 2553.

obscurus, Broun. Man. N.Z. Coleop. I 475, No. 2554.

Bantiades, Broun, I893.

fuscatus, Broun. Man. N.Z. Coleop. I371, No. 2396.

valgus, Broun. Man. N.Z. Coleop. 1472 , No. $255 \mathrm{I}$.

Rachidiscus, Broun, i 893.

granicollis, Broun. Man. N.Z. Coleop. 1478, No. 2557.

Dermotrichus, Sharp, i 886.

mundulus, Sharp. Man. N.Z. Coleop. I 205, No. 2133.

Clypeorhynchus, Sharp, I $88_{3}$.

gracilipes, Sharp. Man. N.Z. Coleop. I 2 I I, No. 2 I 38. thoracicus, Broun. Man. N.Z. Coleop. Iz I , No. 2 I 39. brevicornis, Broun. Man. N.Z. Coleop. I2I2, No. 2 I 40. impressus, Broun. Man. N.Z. Coleop. I 2 I 2, No. 2 I 4 I. ovipennis, Broun. Man. N.Z. Coleop. I 2 I 3, No. 2142. cordipennis, Broun. Man. N.Z. Coleop. I 370 , No. 2394. cristatus, Broun. Man. N.Z. Coleop. 1370, No. 2395. 
Dacnophylla, Broun, I 893 .

setosa, Broun. Man. N.Z. Coleop. 1472 , No. $255^{\circ}$.

variegata, Broun. A.M.N.H. 6, xii. 301.

Nestrius, Broun, 1893 .

serripes, Broun. Man. N.Z. Coleop. 1480, No. 2559, and A.M.N.H. 6, xii. 302 .

Plotnus, Broun, I 893 .

ovithorax, Broun. Man. N.Z. Coleop. 148I, No. 2560.

Phemus, Broun, I893.

scabralis, Broun. Man. N.Z. Coleop. I 2 I 4, No. 2 I 43.

rufipes, Broun. Man. N.Z. Coleop. I482, No. 256r.

Rystheus, Broun, I893.

ocularius, Broun. Man. N.Z. Coleop. I 2 I 9, No. 2 I 48.

Sosgenes, Broun, I 893 .

carinatus, Broun. Man. N.Z. Coleop. 1477 , No. 2556.

Phyllodytes, Broun, I893.

foveatus, Broun. Man. N.Z. Coleop. 1479 , No. $255^{8}$.

Phronira, Broun, I 893 .

sulcirostre, Broun. Man. N.Z. Coleop. 696, No. 122.

asper, Broun. Man. N.Z. Coleop. 696, No. I223.

costosum, Broun. Man. N.Z. Coleop. 696, No. I 224.

osculans, Broun. Man. N.Z. Coleop. 697, No. I 225.

simplex, Broun. Man. N.Z. Coleop. 697, No. I 226.

striatum, Broun. Man. N.Z. Coleop. 697, No. 1227.

nodosum, Broun. Man. N.Z. Coleop. I476, No. 2555.

Philacta, Broun, I 880 .

Sub-family ERIRHININÆ.

testacea, Broun. Man. N.Z. Coleop. 449, No. 789.

Erirhinus, Schönherr, I 826 .

acalyptoides, Pascoe. Man. N.Z. Coleop. $45^{\circ}$, No. 790.

limbatus, Pascoe. Man. N.Z. Coleop. 450, No. 791. glottis, Pascoe. Man. N.Z. Coleop. 450, No. $79^{2}$.

discoideus, Broun. Man. N.Z. Coleop. 45 I, No. 794.

flavitarsis, Broun. Man. N.Z. Coleop. 45I, No. 795 .

fasciatus, Broun. Man. N.Z. Coleop. 452, No. 796.

viridipennis, Broun. Man. N.Z. Coleop. 452, No. 798.

sexmaculatus, Broun. Man. N.Z. Coleop. 706, No. I 243 .

dolosus, Broun. Man. N.Z. Coleop. 707, No. г 244.

fascialis, Broun. Man. N.Z. Coleop. 707, No. I 245.

crucigerus, Broun. Man. N.Z. Coleop. 708, No. I 246.

anchoralis, Broun. Man. N.Z. Coleop. 708, No. I 247.

gracilirostris, Broun. Man. N.Z. Coleop. 708, No. I 248.

stramineus, Broun. Man. N Z. Coleop. 709, No. I 249. 
Erirhinus nocens, Broun. Man. N.Z. Coleop. 709, No. I 250. acceptus, Broun. Man. N.Z. Coleop. 7 Io, No. 125 I. femoralis, Broun. Man. N.Z. Coleop. 7 ro, No. 1252. concolor, Broun. Man. N.Z. Coleop. 7 Io, No. 1253. eustictus, Broun Man. N.Z. Coleop. 857 , No. 1526 . veronicæ, Broun. Man. N.Z. Coleop. 858, No. 1527 . thomsoni, Broun. Man N.Z. Coleop. 858, No. 1528 . bicavus, Broun. Man. N.Z. Coleop. 859 , No. I 529 . melastomus, Broun. Man. N.Z. Coleop. 859, No. 1530. confusus, Broun. Man. N.Z. Coleop. 860, No. I 531. xenorhinus, Broun. Man. N.Z. Coleop. 860, No. 1532. durus, Broun. Man. N.Z. Coleop. 861, No. 1533. fuscoventris, Broun. Man. N.Z. Coleop. 86I, No. I 534. fulvus, Broun. Man. N.Z. Coleop. 862, No. I 535. cheesemani, Broun. Man. N.Z. Coleop. 963, No. 1740. difformipes, Broun. Man. N.Z. Coleop. 964, No. I 741 . nesobius, Broun. Man. N.Z. Coleop. 965 , No. 1742. anxius, Broun. Man. N.Z. Coleop. I372, No. 2397. fuscipes, Broun. Man. N.Z. Coleop. 1373, No. 2398.

Dorytomus, Stephens, 1831 .

trilobus, Pascoe. Man. N.Z. Coleop. 453, No. 799. rufirostris, Broun. Man. N.Z. Coleop. 453 , No. 801. lateralis, Broun. Man. N.Z. Coleop. 711 , No. 1254. sudus, Broun. Man. N.Z. Coleop. 7 I I, No. 1255. ochraceus, Broun. Man. N.Z. Coleop. 712 , No. 1256. æricomus, Broun. Man. N.Z. Coleop. 795, No. I4I8. aciphyllæ, Broun. Man. N.Z. Coleop. 965, No. I $7+3$.

Neomycta, Pascoe, 1877 . pulicaris, Pascoe. Man. N.Z. Coleop. 457, No. 807 . rubida, Broun. Man. N.Z. Coleop. 457 , No. 808.

Hypotagea, Pascoe, i 876.

rubida, Pascoe. Man. N.Z. Coleop. 456, No. 804. testaceipennis, Broun. Man. N.Z. Coleop. 456, No. 805 . variegata, Broun. Man. N.Z. Coleop. 456 , No. 806. creperus, Broun. Man. N.Z. Coleop. 706, No. I 241. simulans, Broun. Man. N.Z. Coleop. 706, No. 1242. castanea, Broun. Man. N.Z. Coleop. 714 , No. I 262. vestita, Broun. Man. N.Z. Coleop. 7 I 5, No. I 263. dissona, Broun. Man. N.Z. Coleop. 863 , No. 1538. tibialis, Broun. Man. N.Z. Coleop. 1376 , No. 2403.

Euprocas, Broun, 1886. scitulus, Broun. Man. N.Z. Coleop. 1375, No. 2402.

Alloprocas, Broun, i 886.

rufus, Broun. Man. N.Z. Coleop. I 374, 2399. 
Celetotelus, Broun, I886.

fulvus, Broun. Man. N.Z. Coleop. I375, No. 240 I.

Phorostichus, Broun, I 882.

linearis, Broun. Man. N.Z. Coleop. 705, No. I 240.

Simachus, Broun, г 886.

montanus, Broun. Man. N.Z. Coleop. 963, No. I 739.

Aganeuma, Broun, i 886 .

rufula, Broun. Man. N.Z. Coleop. 1484 , No. $25^{6} 3$.

Stilbopsis, Broun, i 886 .

politus, Broun. Man. N.Z. Coleop. 1483 , No. 2562.

Etheophanus, Broun, I 886.

pinguis, Broun. Man. N.Z. Coleop. I 233, No. 2 I 7 I.

Praolepra, Pascoe, i 88 o.

squamosa, Pascoe. Man. N.Z. Coleop. 454, No. Sor. infusca, Broun. Man. N.Z. Coleop. 452, No. 802.

albopicta, Broun. Man. N.Z. Coleop. 7 I 2, No. 1257. rufescens, Broun. Man. N.Z. Coleop. 713 , No. I 258 . varia, Broun. Man. N.Z. Coleop. 7 I 3, No. I 259. asperirostris, Broun. Man. N.Z. Coleop. 7 I 3 , No. 1260. pallida, Broun. Man. N.Z. Coleop. 7 I4, No. I26r.

Megacolabus, Broun, i 886.

sculpturatus, Broun. Man. N.Z. Coleop. I 378 , No. 2407.

Aneuma, Pascoe, i 876.

fulvipes, Pascoe. Man. N.Z. Coleop. 455, No. 803 . rubricalis, Broun. Man. N.Z. Coleop. 452, No. 797. stramineipes, Broun. Man. N.Z. Coleop. 862, No. 1536. ferruginea, Broun. Man. N.Z. Coleop. 862, No. I537. compta, Broun. Man. N.Z. Coleop. 934, No. 1680.

Tysius, Pascoe, I 875 .

amplipennis, Pascoe. Man. N.Z. Coleop. 458, No. 809. purus, Broun. Man. N.Z. Coleop. $\{224$, No. 2157.

Cyttalia, Pascoe, ${ } S_{73}$. dispar, Broun. Man. N.Z. Coleop. 907, No. I620.

Cænophanus, Broun, i 886. flavipilus, Broun. Man. N.Z. Coleop. 796, No. I 4 I 9.

Eugnomus, Schönherr, I 847 .

elegans, Pascoe. Man. N.Z. Coleop. 458, No. Sio. picipennis, Pascoe. Man. N.Z. Coleop. 459. No. 8I I. fervidus, Pascoe. Man. N.Z. Coleop. 459, No. Si 2. wakefieldi, Pascoe. Man. N.Z. Coleop. 459, No. 8 I 3. fucosus, Pascoe. Man. N.Z. Coleop. 459, No. Si 4. interstitialis, Broun. Man. N.Z. Coleop. 460, No. 8I 5 . 
Eugnomus discolor, Broun. Man. N.Z. Coleop. 460, No. 8I 6. cinnamoneus, White. Man. N.Z. Coleop. 46I, No. 8 I 7 . maculosus, Broun. Man. N.Z. Coleop. 7 I 5 , No. I 264. nubilans, Broun. Man. N.Z. Coleop. 7 I5, No. 1265. fasciatus, Broun. Man. N.Z. Coleop. 7 I6, No. 1266. luctuosus, Broun. Man. N.Z. Coleop. $86_{3}$, No. 1539. monachus, Broun. Man. N.Z. Coleop. 966, No. I 744. argutus, Sharp. Man. N.Z. Coleop. I 225, No. 2 I 58. nobilis, Broun Man. N.Z. Coleop. 2225 , No. 2159 . ænescens, Broun. Man. N.Z. Coleop. 1226, No. 2 I60. cyaneus, Broun. Man. N.Z. Coleop. I 227 , No. 2 г6 г. tarsalis, Broun. Man. N.Z. Coleop. I227, No. 2 162. lituratus, Broun. Man. N.Z. Coleop. I 227 , No. 2 I63. aspersus, Broun. Man. N.Z. Coleop. I 376, No. 2404. squamifer, Broun. Man. N.Z. Coleop. I 377 , No. 2405. maurus, Broun. Man. N.Z. Coleop. I 377, No. 2406.

Oreocharis, Broun, I 886.

nigriceps, Broun. Man. N.Z. Coleop. 864 , No. I 540. picigularis, Broun. Man. N.Z. Coleop. 865 , No. I 54 I. nigrescens, Broun. Man. N.Z. Coleop. 865, No. I 542. cyanea, Broun. Man. N.Z. Coleop. 7 I6, No. I 267. vittata, Broun. Man. N.Z. Coleop. I228, No. 2164. dealbata, Broun. Man. N.Z. Coleop. I228, No. 2 I65.

Hoplocneme, White, I 846 . hookeri, White. Man. N.Z. Coleop. 46I, No. 8 I 8. punctatissima, Pascoe. Man. N.Z. Coleop. 46r, No. 8r g. squamosa, Broun. Man. N.Z. Coleop. 462, No. 820. inæqualis, Broun. Man. N.Z. Coleop. I 229, No. 2 I 66. cyanea, Broun. Man. N.Z. Coleop. I230, No. 2 I67.

Stephanorhynchus, White, i 846 . curvipes, White. Man. N.Z. Coleop. 462, No. 821 . brevipennis, Pascoe. Man. N.Z. Coleop. 463 , No. 822 . lawsoni, Sharp. Man. N.Z. Coleop. 463 , No. 823 . crassus, Broun. Man. N.Z. Coleop. 464, No. 824. tuberosus, Broun. Man. N.Z. Coleop. 7 I 7 , No. I 268. fatuus, Broun. Man. N.Z. Coleop. 718, No. 1269. griseipictus, Broun. Man. N.Z. Coleop. 866, No. I $5+3$. nigrosparsus, Broun. Man. N.Z. Coleop. 1230, No. 2 I 68. insolitus, Broun. Man. N.Z. Coleop. I 231 , No. 2 I69. costifer, Broun. Man. N.Z. Coleop. I 23 I, No. 2 I 70. attelaboides, Fabricius. Ent. Syst. p. 156.

Pactola, Pascoe, 1876. variabilis, Pascoe. Man. N.Z. Coleop. 465 , No. 825 . demissa, Pascoe. Man. N.Z. Coleop. 465 , No. 826 . humeralis, Broun. A.M.N.H. 6, xv. 407 . 
Brexius, Pascoe, 1870 .

ascitus, Pascoe. Man. N.Z. Coleop. 465, No. 827 .

Sub-family APIONinæ.

Apion, Herbst, 1797 .

metrosideros, Broun. Man. N.Z. Coleop. 466, No. $82 S$.

Sub-family RHinomacerinæ.

Rhinorhynchus, Sharp, I882.

rufulus, Broun. Man. N.Z. Coleop. 467 , No. 829 .

Pachyura, Hope, I833.

Sub-family BELIN

metallica, Pascoe. Man. N.Z. Coleop. 469, No. 830 . rubicunda, Broun. Man. N.Z. Coleop. 469, No. 83 I. sumptuosa, Broun. Man. N.Z. Coleop. 470, No. 832 . albocoma, Broun. Man. N.Z. Coleop. I 233, No. 2 I 72. stictica, Broun. Man. N.Z. Coleop. I379, No. 2408.

Agathinus, Broun, I880.

tridens, Fabricius. Man. N.Z. Coleop. 471, No. 834 .

Sub-family SCOLOPTERINÆ.

Scolopterus, White, I 846 .

tetracanthus, White. Man. N.Z. Coleop. 472, No. 835-

æquus, Broun. Man. N.Z. Coleop. 473 , No. 836 .

penicillatus, White. Man. N.Z. Coleop. 473 , No. 837 . pectoralis, Broun. Man. N.Z. Coleop. 474 , No. 838 .

æneorufus, Broun. Man. N.Z. Coleop. 7 I8, No. 1270.

Icmalius, Broun, 1893 .

abnormis, Broun. Man. N.Z. Coleop. 908, No. 1623 .

helmsi, Sharp. Ent. Mon. Mag. xviii. 47.

Nyxetes, Pascoe, 1870.

bidens, Fabricius. Man. N.Z. Coleop. 474 , No. $\$_{39}$.

rufipes, Broun. Man. N.Z. Coleop. 718 , No. I 27 I.

Ancistropterus, White, 1846.

quadrispinosus, White. Man. N.Z. Coleop. 475 , No. 840.

brouni, Sharp. Man. N.Z. Coleop. 475, No. 84 I.

pilosus, Broun. Man. N.Z. Coleop. 476 , No. 843 .

Amylopterus, Broun, I 886.

prasinus, Broun. Man. N.Z. Coleop. 797, No. 1420.

Oropterus, White, 1846 .

coniger, White. Man. N.Z. Coleop. 477 , No. 844 .

Sub-family TychinNe.

Sibinia, Germar, 1824 .

tychioides, Pascoe. Man. N.Z. Coleop. 478 , No. 845 . 
Psepholax, White, 1846 .

Sub-family CRYPTORHYNCHIN:E.

sulcatus, White. Man. N.Z. Coleop. 479, No. 846.

granulatus, Broun. Man. N.Z. Coleop. 479, No. 847 .

cornutus, Broun. Man. N.Z. Coleop. 479 , No. 848 .

barbifrons, White. Man N.Z. Coleop. 480, No. 849.

punctulatus, Broun. Man. N.Z. Coleop. 480 , No. 850.

rostralis, Broun. Man. N.Z. Coleop. 480 , No. 85 I.

coronatus, White. Man. N.Z. Coleop. $48 \mathrm{r}$, No. 852 .

femoratus, Broun. Man. N.Z. Coleop. 481 , No. 853.

simplex, Pascoe. Man. N.Z. Coleop. 48 r, No. 854 . mystacinus, Broun. Man. N.Z. Coleop. 866, No. 1544. mediocris, Broun. Man. N.Z. Coleop. 909, No. 1624. brevicornis, Broun. Man. N.Z. Coleop. r382, No. 24 I3. crassicornis, Broun. A.M.N.H. 6, xv. 408.

Pseudoreda, Broun, r 893 .

tibialis, Broun. Man. N.Z. Coleop. $\mathrm{I}_{3} 83$ and 482 , No. 855 .

Oreda, White, $\mathrm{r} 846$.

notata, White. Man. N.Z. Coleop. 487 , No. 862.

brevis, Pascoe. Man. N.Z. Coleop. 487 , No. 863.

murina, Broun. Man. N.Z. Coleop. 487 , No. 864.

Mesoreda, Broun, I893.

setigera, Broun. Man. N.Z. Coleop. 1235 and 488 , No. 865 .

orthorhina, Broun. Man. N.Z. Coleop. 909, No. 1625.

Homoreda, Broun, I893.

punctata, Broun. Man. N.Z. Coleop. 1383 , No. 2414.

Aldonus, White, r 846 .

hylobioides, White. Man. N.Z. Coleop. 483 , No. $85^{6}$. celator, Pascoe. Man. N.Z. Coleop. 483 , No. 857 .

rostratus, Broun. Man. N.Z. Coleop. 483, No. 858 .

Nothaldonus, Broun, I893.

peacei, Broun. Man. N.Z. Coleop. 484 , No. $8_{59}$.

Incentia, Broun, r 88 o.

nubila, Broun. Man. N.Z. Coleop. 485 , No. 860.

straminea, Broun. Man. N.Z. Coleop. 486, No. 86 I.

Ectopsis, Broun, r88r.

ferrugalis, Broun. Man. N.Z. Coleop. 7 I9, No. 1272.

simplex, Broun. A.M.N.H. 6, xii. 383 .

Tychanus, Pascoe, 1876 .

gibbus, Pascoe. Man. N.Z. Coleop. 498, No. 885.

ferrugatus, Pascoe. Man. N.Z. Coleop. 498, No. 886.

densus, Broun. Man. N.Z. Coleop. 499, No. 887.

verrucosus, Pascoe. Man. N.Z. Coleop. 499, No. 888. 
Tychanus lachrymosus, Broun. Man. N.Z. Coleop. 729, No. I 294.

scabiosus, Broun. Man. N.Z. Coleop. 801, No. 1429. quadratus, Broun. Man. N.Z. Coleop. 867 , No. I545. ventralis, Broun. Man. N.Z. Coleop. 935, No. I682. bufo, Sharp. Man. N.Z. Coleop. I 239, No. 2 I 82. dux, Broun. Man. N.Z. Coleop. I 240, No. 2 I 83.

Sympedius, Pascoe, 1876 .

testudo, Pascoe. Man. N.Z. Coleop. 500, No. 889. vexatus, Pascoe. Man. N.Z. Coleop. 500, No. 890. curtus, Broun. Man. N.Z. Coleop. 728, No. I 293. lepıdus, Broun. Man. N.Z. Coleop. 935, No. I683.

Crisius, Pascoe, 1876.

binotatus, Pascoe. Man. N.Z. Coleop. 500, No. 89 I. variegatus, Broun. Man. N.Z. Coleop. 501, No. 892. scutellaris, Broun. Man. N.Z. Coleop. 501, No. 893. obesulus, Sharp. Man. N.Z. Coleop. I 24I, No. 2 I84. picicollis, Broun. Man. N.Z. Coleop. I24I, No. 2 I 85. signatus, Broun. Man. N.Z. Coleop. I 242, No. 2 I 86. ornatus, Broun. Man. N.Z. Coleop. I 242, No. 2 I 87. fasciculatus, Broun. Man. N.Z. Coleop. I 492, No. 2574.

Agacalles, Broun, I886. formosus, Broun. Man. N.Z. Coleop. 967 , No. I 746.

Getacalles, Broun, 1893 . rostralis, Broun. Man. N.Z. Coleop. I380, No. 2410. minor, Broun. Man. N.Z. Coleop. I $38 \mathrm{I}$, No. 24 II. humeratus, Broun. Man. N.Z. Coleop. I 38 I, No. 24 I 2. inæqualis, Broun. Man. N.Z. Coleop. I 493, No. 2575.

Tychanopais, Broun, r 893 . pictulus, Broun. Man. N.Z. Coleop. I380, No. 24 го.

Dendrostygnus, Broun, I 895 . calcaratus, Broun. A.M.N.H. 6, xv. 410.

Hadracalles, Broun, I 893 . fuliginosus, Broun. Man. N.Z. Coleop. 1384 , No. 2415.

Acalles, Schönherr, I 826 .

intutus, Pascoe. Man. N.Z. Coleop. 489 , No. 866. erroneus, Pascoe. Man. N.Z. Coleop. 489 , No. 867. hystriculus, Pascoe. Man. N.Z. Coleop. 489 , No. 868. impexus, Pascoe. Man. N.Z. Coleop. 490, No. 869. perpusillus, Pascoe. Man. N.Z. Coleop. 490, No. 870. trinotatus, Broun. Man. N.Z. Coleop. 490, No. 87 I. pascoei, Broun. Man. N.Z. Coleop. 491, No. 872. signatus, Broun. Man. N.Z. Coleop. 49I, No. 873. 
Acalles tortipes, Broun. Man. N.Z. Coleop. 492, No. 874 . crisioides, Broun. Man. N.Z. Coleop. 492, No. 875 . vividus, Broun. Man. N.Z. Coleop. 493, No. 876. certus, Broun. Man. N.Z. Coleop. 493, No. 877. scitus, Broun. Man. N.Z. Coleop. 494, No. 878 . ovatellus, Broun. Man. N.Z. Coleop. 720 , No. 1273. læviculus, Broun. Man. N.Z. Coleop. 721 , No. I 275. cristatus, Broun. Man. N.Z. Coleop. 72 I, No. 1276. rudis, Broun. Man. N.Z. Coleop. 721 , No. 1277. dorsalis, Broun. Man. N.Z. Coleop. 722 , No. I 278. volens, Broun. Man. N.Z. Coleop. 722 , No. I 279. horridus, Broun. Man. N.Z. Coleop. 723, No 1280. rubricus, Broun. Man. N.Z. Coleop. 723 , No. I 28 I. spurcus, Broun. Man. N.Z. Coleop. 724, No. 1282. mundus, Broun. Man. N.Z. Coleop. 724, No. I 283. canescens, Broun. Man. N.Z Coleop. 725, No. I 284 . arctus, Broun. Man. N.Z. Coleop. 725, No. 1285. vafrus, Broun. Man. N.Z. Coleop. 725 , No. I 286. griseus, Broun. Man. N.Z. Coleop. 797, No. I 42 I. diversus, Broun. Man. N.Z. Coleop. 798, No. I422. cingulatus, Broun. Man. N.Z. Coleop. 798 , No. I423. sentus, Broun. Man. N.Z. Coleop. 799, No. I 424. latirostris, Broun. Man. N.Z. Coleop. 799, No. 1425. decemcristatus, Broun. Man. N.Z. Coleop. 800, No. 1426. triangulatus, Broun. Man. N.Z. Coleop. 800 , No. 1427. huttoni, Brown. Man. N.Z. Coleop. 801, No. I 428. setifer, Broun. Man. N.Z. Coleop. 867, No. I 546. posticalis, Broun. Man. N.Z. Coleop. 868, No. 1547. terricola, Broun. Man. N.Z. Coleop. 934, No. I68I. floricola, Broun. Man. N.Z. Coleop. 966, No. 3745 . veratrus, Broun. Man. N.Z. Coleop. I235, Nu. 2 I 73. maritimus, Broun. Man. N.Z. Coleop. I 236 , No. 2 I 74. cryptobius, Broun. Man. N.Z. Coleop. I 236, No. 2175. ingens, Broun. Man. N.Z. Coleop. I236, No. 2 I 76. xanthostictus, Broun. Man. N.Z. Coleop. I 237, No. 2 I 77. adamsi, Broun. Man. N.Z. Coleop. I 237 , No. 2178. concinnus, Broun. Man. N.Z. Coleop. I238, No. 2 I 79. australis, Broun. Man. N.Z. Coleop. I 239, No. 2 I 80. mimus, Broun. Man. N.Z. Coleop. ${ }_{3} 385$, No. 2416. lepirhinus, Broun. Man. N.Z. Coleop. ${ }_{3} 8_{5}$, No. 2417. sympedioides, Broun. Man. N.Z. Coleop. I 386, No. 2418. dolosus, Broun. Man. N.Z. Coleop. 1484 , No. 2564. comptus, Broun. Man. N.Z. Coleop. I 486 , No. 2565. facilis, Broun. Man. N.Z. Coleop. I 486, No. 2566. quietus, Broun. Man. N.Z. Coleop. 1487 , No. 2567. aulacus, Broun. Man. N.Z. Coleop. I489, No. 2570. allostethus, Broun. Man. N.Z. Coleop. I490, No. 257 I. 
Acalles incultus, Broun. Man. N.Z. Coleop. I 490, No. 2572. integer, Broun. A.M.N.H. 6, xii. 374.

formosus, Broun. A.M.N.H. 6, xii. 375 . ruficollis, Broun. A.M.N.H. 6, xii. 376 . porcatus, Broun. A.M.N.H. 6, xii. 377 . puncticollis, Broun. A.M.N.H. 6, xii. 378 . farinosus, Broun. A.M.N.H. 6, xii. 378 . fougeri, Hutton. T.N.Z.I. xxx. 157.

Acallopais, Pascoe. I 877 . rudis, Pascoe. Man. N.Z. Coleop. 495, No. 879. sculpturatus, Broun. Man. N.Z. Coleop. 495 , No. 880.

Scelodolichus, Broun, 1880. celsus, Broun. Man. N.Z. Coleop. 496, No. 88I. lineithorax, Broun. Man. N.Z. Coleop. 496, No. S8z. setosus, Broun. Man. N.Z. Coleop. 726 , No. I 287. villosus, Broun. Man. N.Z. Coleop. 726 , No. I 288 . denotans, Broun. Man. N.Z. Coleop. 726, No. I 289. altulus, Broun. Man. N.Z. Coleop. 968, No. 1747. juncobius, Broun. Man. N.Z. Coleop. I239, No. 218 I. hilaris, Broun. Man. N.Z. Coleop. I491, No. 2573. politus, Broun. A.M.N.H. 6, xv. 4I 2. squamosus, Broun. A.M.N.H. 6, xv. 4I3.

Zeacalles, Broun, 1893 .

flavescens, Broun. A.M.N.H. 6, xii. 3 So. varius, Broun. A.M.N.H. 6, xii. 38 I. alpestris, Broun. Man. N.Z. Coleop. I 488, No. 2568. picatus, Broun. Man. N.Z. Coleop. I 488 , No. 2569.

Cyclacalles, Broun, i 880.

setiger, Broun. Man. N.Z. Coleop. 497, No. 883. vestitus, Broun. Man. N.Z. Coleop. 497 , No. 884 .

Schylus, Bruun, i 895 . nigricollis, Broun.

Metacalles, Broun, 1893 . aspersus, Broun. A.M.N.H. 6, xii. 382 . rugicollis, Broun. A.M.N.H. 6, xii. 383 . cordipennis, Broun. Man. N.Z. Coleop. 720 , No. 1274. latus, Broun. Man. N.Z. Coleop. 727 , No. I 290. crinitus, Broun. Man. N.Z. Coleop. 727, No. т29 I. exiguus, Broun. Man. N.Z. Coleop. 728, No. I 292.

Dermothrius, Broun, I882. sanguineus, Broun. Man. N.Z. Coleop. 730, No. I 295.

Euthryhinus, Schönherr, 1837 . squamiger, White. Man. N.Z. Coleop. 503, No. 896. 
Rhyncodes, White, 1846 .

ursus, White. Man. N.Z. Coleop. 502, No. 894. saundersi, White. Man. N.Z. Coleop. 503, No. 895 . squameus, Broun. Man. N.Z. Coleop. 730, No. I 296. atrus, Broun. Man. N.Z. Coleop. So2, No. I430. weberi, Colenso. T.N.Z.I. xiv. 28 I. rubipunctatus, Colenso. T.N.Z.I. xiv. 282.

Mitrastethus, Redtenbacher, 1868. bituberculatus, Fabricius. Man. N.Z. Coleop. 504, No. 897 .

Paranomocerus, Redtenbacher, I 868. spiculus, Redtenbacher. Man. N.Z. Coleop. 505, No. 898. maurus, Broun. Man. N.Z. Coleop. 505, No. 899.

Idotasia, Pascoe, 187 I.

egena, Pascoe. Man. N.Z. Coleop. 506, No. 900.

Mecistostylus, Lacordaire, I 866. douei, Lacordaire. Gen. Col. vii.

Sympiezoscelus, Waterhouse, 1853 .

spencei, Waterhouse. Trans. Ent. Soc. 2, ii. 204.

Sub-family Cossoninæ.

Pentarthrum, Wollaston, I 854 . apicale, Broun. Man. N.Z. Coleop. 509, No. 906. sculpturatum, Broun. Man. N.Z. Coleop. 73I, No. I 297. rufum, Broun. Man. N.Z. Coleop. 5 ro, No. 908. confinis, Broun. Man. N.Z. Coleop. 732, No. т 299. asperellum, Broun. Man. N.Z. Coleop. 733, No. I 30 I. punctatissimum, Broun. Man. N.Z. Coleop. I 246 , No. 2 I 94. porcatum, Sharp. Man. N.Z. Coleop. 1248, No. 2 I 98. antennale, Broun. A.M.N.H. 6, xv. 4I5. zealandicum, Wollaston. Man. N.Z. Coleop. 508, No. 903. subsericatum, Wollaston. Man. N.Z. Coleop. 509 , No. 905. cylindricum, Wollaston. Trans. Ent. Soc. 2, r. $39 \mathrm{~S}$. punctirostre, Broun. Man. N.Z. Coleop. 733, No. I 300. melanosternum, Broun. Man. N.Z. Coleop. 869, No. I 548. fultoni, Broun. Man. N.Z. Coleop. I 243, No. 2 I 89. amicum, Broun. Man. N.Z. Coleop. I 247, No. 2195. brunneum, Broun. Man. N.Z. Coleop. 5 I0, No. 907. brevirostre, Sharp. Man. N.Z. Coleop. 5 I 2, No. 9 I I. ruficorne, Broun. Man. N.Z. Coleop. 734, No. I304. rugirostre, Broun. Man. N.Z. Coleop. 734, No. I303. punctatum, Broun. Man. N.Z. Coleop. 5 I8, No. 925. piceum, Broun. Man. N.Z. Coleop. 515, No. 919. reductum, Broun. Man. N.Z. Coleop. 732, No. I298. assimilatum, Broun. Man. N.Z. Coleop. 5 I I, No. 909. 
Pentarthrum longirostre, Wollaston. Man. N.Z. Coleop. 508 , No. 904 .

morosum, Broun. Man. N.Z. Coleop. 969, No. I 749.

fulvum, Broun. Man. N.Z. Coleop. I 243, No. 2 I 88.

crenatum, Broun. Man. N.Z. Coleop. 804, No. I43I.

lateritium, Broun. Man. N.Z. Coleop. 5 I8, No. 924.

exile, Broun. Man. N.Z. Coleop. I 245, No. 2193.

nubilum, Broun. Man. N.Z. Coleop. I244, No. 219 I.

aurantiacum, Broun. Man. N.Z. Coleop. 1245, No. 2 I 92. macrocephalum, Broun. Man. N.Z. Coleop. 869, No. I 549. diversum, Broun. Man. N.Z. Coleop. I 244, No. 2 I 90. cephalotes, Sharp. Man. N.Z. Coleop. I 247 , No. 2197. angustum, Broun. Man. N.Z. Coleop. 910, No. 1626. proximum, Broun. Man. N.Z. Coleop. 91 , No. I627. spadiceum, Broun. Man. N.Z. Coleop. 9i I, No. I6z8. servuium, Broun. Man. N.Z. Coleop. 91 I, No. I629. comatum, Broun. Man. N.Z. Coleop. 968, No. 1748. philpotti, Broun. A.M.N.H. 6, xv. 4I5. helmsianum, Sharp. Man. N.Z. Coleop. I 247 , No. 2 I 96.

Sericotrogus, Wollaston, 1873 . subænescens, Wollaston. Man. N.Z. Coleop. 52 I, No. 929. ovicollis, Broun. Man. N.Z. Coleop. 522, No. $93 \mathrm{I}$. stramineus, Broun. Man. N.Z. Coleop. $5^{2} 3$, No. 932. simulans, Sharp. Man. N.Z. Coleop. 523, No. 933. vestitus, Broun. Man. N.Z. Coleop. 514, No. 9 r6. auricomus, Broun. Man. N.Z. Coleop. 733, No. 1302.

Eucossonus, Broun, i 886.

comptus, Broun. Man. N.Z. Coleop. 870, No. $155^{\circ}$. elegans, Broun. Man. N.Z. Coleop. I 386 , No. 24 I 9. gracilis, Broun. Man. N.Z. Coleop. I 387 , No. 2420. setiger, Sharp. Man. N.Z. Coleop. 523, No. 934. castus, Broun. Man. N.Z. Coleop. 736, No. 1307.

Agastegnus, Broun, I 886.

ruficollis, Broun. Man. N.Z. Coleop. So6, No. I $43^{2}$. coloratus, Broun. Man. N.Z. Coleop. 9i I, No. I630. femoralis, Broun. Man. N.Z. Coleop. 970, No. I 75 I. sericatus, Broun. Man. N.Z. Coleop. 970, No. I $75^{2}$. nitidirostris, Broun. Man. N.Z. Coleop. I 250 , No. 2202. distinctus, Broun. Man. N.Z. Coleop. I388. No. 242 I. longipes, Broun. Man. N.Z. Coleop. 522, No. 930.

Microtribus, Wollaston, I 873 . huttoni, Wollaston. Man. N.Z. Coleop. $5^{27}$, No. 937. pictonensis, Sharp. Man. N.Z. Coleop. I 250, No. 2203 .

Mesoxenophasis, Wollaston, I 874 .

brouni, Wollaston. Man. N.Z. Coleop. 525, No. 935. 
Entium, Sharp, I 878 .

aberrans, Sharp. Man. N.Z. Coleop. 520, No. 927.

Agrilochilus, Broun, I88o.

prolixus, Broun. Man. N.Z. Coleop. 521 , No. 928.

Novitas, Broun, I880.

rufus, Broun. Man. N.Z. Coleop. 528, No. $93^{8}$.

nigrans, Broun. A.M.N.H. 6 , xii. 385 ; Man. N.Z. Coleop. 528, No. 939.

dispar, Broun. Man. N.Z. Coleop. I 25I, No. 2204.

Rhinanisus, Broun, I 886 .

fulvicornis, Broun. Man. N.Z. Coleop. 512, No. 912.

parvicornis, Sharp. Man. N.Z. Coleop. 513, No. 913.

contiguus, Broun. Man. N.Z. Coleop. 517, No. 9?3.

confertum, Sharp. Man. N.Z. Coleop. I 248 , No. 2 I 99.

constrictus, Sharp. Man. N.Z. Coleop. I 249, No. 2200.

cheesemani, Broun. Man. N.Z. Coleop. I249, No. 2201.

sagax, Broun. Man. N.Z. Coleop. 969, No. 1750.

Macroscytalus, Broun, i 886.

laticollis, Broun. Man. N.Z. Coleop. 737, No. I 308.

russulus, Broun. Man. N.Z. Coleop. 737, No. I 309.

remotus, Sharp. Man. N.Z. Coleop. 5 I3, No. 9 I4.

æneopiceus, Broun. Man. N.Z. Coleop. 513, No. 9I5.

badius, Broun. Man. N.Z. Coleop. 516 , No. 920.

frontalis, Broun. A.M.N.H. 6, xii. 386.

Bæorhopalus, Broun, i 886.

glabrus, Broun. Man. N.Z. Coleop. 735, No. 1305.

Glyphoramphus, Broun, I 886.

rarus, Broun. Man. N.Z. Coleop. 97 I, No. 1.753.

Camptoscapus, Broun, I 893 .

sanguineus, Broun. A.M.N.H. 6, xii. 385 .

planiusculus, Broun. Man. N.Z. Coleop. 5 I I, No. 9 Iо.

conicollis, Broun. Man. N.Z. Coleop. 735, No. I 306.

Idus, Broun, I 893 .

cæcus, Broun. Man. N.Z. Coleop. I494, No. 2576.

Heteropsis, Wollaston, 1873 .

lawsoni, Wollaston. Man. N.Z. Coleop. 529, No. 940.

Proconus, Broun, 1886.

asperirostris, Broun. Man. N.Z. Coleop. 5 I 6, No. 92 I. crassipes, Broun. Man. N.Z. Coleop. 91 2, No. I63 I.

Diöedimorpha, Broun, I 886.

wollastonianum, Sharp. Man. N.Z. Coleop. 5I 4, No. 9I 7 .

debile, Sharp. Man. N.Z. Coleop. 514, No. 918.

Arecocryptus, Broun, 1903 (substituted for Canthorhynchus).

bellus, Broun. Man. N.Z. Coleop. 526, No. 936. 
Phlœophagosoma, Wollaston, I873. corvinum, Wollaston. Man. N.Z. Coleop. 530, No. 94 I. thoracicum, IVollaston. Man. N.Z. Coleop. 53I, No. 943. dilutum, Wollaston. Man. N.Z. Coleop. 53 I, No. 944. pedatum, Wollaston. Man. N.Z. Coleop. 532, No. 945. rugipenne, Broun. Man. N.Z. Coleop. 738 , No. I 3 Io. abdominale, Broun. Man. N.Z. Coleop. 738 , No. I 3 I I. serenum, Broun. Man. N.Z. Coleop. i25r, No. 2205.

Eutornus, Wollaston, 1873 . dubius, Wollaston. Man. N.Z. Coleop. 534, No. 948. vicinus, Broun. Man. N.Z. Coleop. 535 , No. 949. breviceps, Broun. Man. N.Z. Coleop. 535, No. 950. amplus, Broun. Man. N.Z. Coleop. 535, No. $95 \mathrm{I}$. littoralis, Broun. Man. N.Z. Coleop. 536, No. 952. cylindricus, Broun. Man. N.Z. Coleop. I 252, No. 2206 parvulus, Broun. Man. N.Z. Coleop. I 253, No. 2207

Arecophaga, Broun, I880. varia, Broun. Man. N.Z. Coleop. 534, No. 947.

Pogonohrinus, Broun, I 903 (substituted for Sasiorhinus, i 880). opacus, Broun. Man. N.Z. Coleop. 533, No. 946.

Euodontus, Broun, i 886 . punctithorax, Broun. Man. N.Z. Coleop. 808, No. 1434.

Exomesites, Broun, r 886. optimus, Broun. Man. N.Z. Coleop. 972, NTo. I 754.

Stilbocara, Broun, i 893 . nitida, Broun. A.M.N.H. 6 , xii. 387 . constricticollis, Broun. Man. N.Z. Coleop. 530, No. 942.

Allaorus, Broun, i 893.

urquharti, Broun. Man. N.Z. Coleop. I 495; No. 2577. pedatus, Broun. Man. N.Z. Coleop. I 496, No. 2578. sternalis, Broun. Man. N.Z. Coleop. I 496, No. 2579. ovatus, Broun. Man. N.Z. Coleop. I 497, No. 25 So. pyriformis, Broun. Man. N.Z. Coleop. I 223 , No. 2154. rugosus, Broun. Man. N.Z. Coleop. I 223 , No. 2 I 55. versutus, Broun. Man. N.Z. Coleop. 447 , No. 788.

Pselactus, Broun, I886. punctatus, Broun. Man. N.Z. Coleop. 972, No. 755

Xenocnema, Wollaston, I 873 . spinipes, Wollaston. Man. N.Z. Coleop. 537 , No. 953.

Inosomus, Broun, r 903 (substituted for Stenopus, 1880). rufopiceus, Broun. Man. N.Z. Coleop. 739, No. I $3{ }^{12 .}$ 
Family Scolytide.

Pachycotes, Sharp, 1877 .

Sub-family SCOLYTinæ.

ventralis, Sharp. Man. N.Z. Coleop. 538, No. 954.

Hylastes, Erichson, ${ } \delta_{3} 6$.

peregrinus, Chapuis. Man. N.Z. Coleop. 539, No. 955.

Acrantus, Broun, 1903 (substituted for Homarus, r88 r). mundulus, Broun. Man. N.Z. Coleop. 740, No. 13 I 3 . opacus, Broun. A.M.N.H. 6, xv. 4 I 7 .

Dendrotrupes, Broun, I $88 \mathrm{I}$. vestitus, Broun. Man. N.Z. Coleop. 74r, No. r 314. costiceps, Broun. Man. N.Z. Coleop. 74I, No. ${ }_{3} 15$.

Tomicus, Latreille, i 8 ro.

asper, Broun. Man. N.Z. Coleop. 742, No. r 316.

Mesoscolytus, Broun, 1903 (substituted for Apate). inurbanus, Broun. Man. N.Z. Coleop. 346, No. $6 z 9$.

Platypus, Herbst, I 793 .

Sub-family Platypide.

douei, Reich. Man. N.Z. Coleop. 540, No. 956.

apicalis, White. Man. N.Z. Coleop. 54 I, No. 957.

caviceps, Broun. Man. N.Z. Coleop. 54I, No. $95^{8}$.

castaneus, Broun. Man. N.Z. Coleop. 542, No. 959.

lobatus, Broun. Man. N.Z. Coleop. I 253, No. 2208.

gracilis, Broun. Man. N.Z. Coleop. I 254, No. 2209.

Family BRENTHIDe.

Lasiorhynchus, Lacordaire, I 866.

barbicornis, Fabricius. Man. N.Z. Coleop. 544, No. 961.

cylindricornis, Fabricius. Man. N.Z. Coleop. 543, No. 960.

Family ANThribide.

Anthribus, Geoffroy, I 762 .

spinifer, Sharp. Man. N.Z. Coleop. 545, No. 962.

sharpi, Broun. Man. N.Z. Coleop. 546, No. 963.

brouni, Sharp. Man. N.Z. Coleop. 547, No. 964.

sandageri, Broun Man. N.Z. Coleop. I261, No. 2224.

obsoletus, Broun. Man. N.Z. Coleop. 1262. Nu.

rudis, Sharp. Man. N.Z. Coleop. 547, No. 965.

bullatus, Sharp. Man. N.Z. Coleop. 548, No. y66.

vates, Sharp. Man. N.Z. Coleop. 548, No. 967.

inornatus, Sharp. Man. N.Z. Coleop. I 255, No 22 I 2.

concolor, Sharp. Man. N.Z. Coleop. I 256, No. 2213.

brunneus, Broun. Man. N.Z. Coleop. I 262, No. 2225.

flavipilus, Broun. A.M.N.H. 6, xv. 4I 7 . 
Anthribus tessellatus, Broun. Man. N.Z. Coleop. I263, No. 2226.

curvatus, Broun. Man. N.Z. Coleop. I 263, No. 2227. lanuginosus, Broun. Man. N.Z. Coleop. 549, No. 968. phymatodes, Redtenbacher. Man. N.Z. Coleop. 550, No. 969 .

hetæra, Sharp. Man. N.Z. Coleop. 550, No. 970. discedens, Sharp. Man. N.Z. Coleop. 55 I, No. 97 I. altus, Sharp. Man. N.Z. Coleop. 55 I, No. 972. ornatus, Sharp. Man. N.Z. Coleop. 552, No. 973. huttoni, Sharp. Man. N.Z. Coleop. 553, No. 974. decens, Broun. Man. N.Z. Coleop. 1264 , No. 2228. finitimus, Broun. Man. N.Z. Coleop. 1 264, No. 2229. anxius, Broun. Man. N.Z. Coleop. I 265 , No. 2230. lætabilis, Broun. Man. N.Z. Coleop. I265, No. 223 I. deterius, Broun. Man. N.Z. Coleop. i 266, No. 2232. impar, Broun. Man. N.Z. Coleop. 1266, No. 2233. picipictus, Broun. Man. N.Z. Coleop. 742, No. I3I 7. nigrescens, Broun. Man. N.Z. Coleop. 743 , No. I3 18. torulosus, Broun. Man. N.Z. Coleop. 743, No. I 3 I 9. crassus, Sharp. Man. N.Z. Coleop. 560, No. 983. nanus, Sharp. Man. N.Z. Coleop. 56 I, No. 984. atomus, Sharp. Man. N.Z. Coleop. $56 \mathrm{I}$, No. 985. purpureus, Broun. Man. N.Z. Coleop. 559, No. 982. minor, Broun. Man. N.Z. Coleop. I 260, No. 222 r. fungicola, Broun. Man. N.Z. Coleop. I 26r, No. 2222. thoracicus, Broun. Man. N.Z. Coleop. 126I, No. 2223.

Exilis, Pascoe, 1873 (=Lazesonia, Sharp).

lawsoni, Sharp. Man. N.Z. Coleop. 556, No. 978. variabilis, Sharp. Man. N.Z. Coleop. 557, No. 979.

Arecopais, Broun, 1893 .

spectabilis, Broun. Man. N.Z. Coleop. $55^{8}$, No. 980.

Proscoporhinus, Montrouzier, $\mathrm{x} 860$.

meinertzhageni, Broun. Man. N.Z. Coleop. 563, No. 989. signatus, Broun. Man. N.Z. Coleop. I 268, No. 2234. viridescens, Broun. Man. N.Z. Coleop. 1 269, No. 2235. albifrons, Sharp. Man. N.Z. Coleop. 1269, No. 2236.

Etnalis, Sharp, I873. spinicollis, Sharp. Man. N.Z. Coleop. 554 , No. 975.

Eugonissus, Broun, I 893 .

pictipes, Broun. Man. N.Z. Coleop. I257, No. 2215. conulus, Broun. Man. N.Z. Coleop. 555, No. 976. proximus, Broun. Man. N.Z. Coleop. 555 , No. 977.

Dysnocryptus, Broun, $\mathrm{x} 893$. plagiatus, Broun. Man. N.Z. Coleop. I 258, No. 22 г6. 
Dysnocryptus testaceus, Broun. Man. N.Z. Coleop. I259,

No. 2217.

pallidus, Broun. Man. N.Z. Coleop. 1259, No. 22 I 8.

maculifer, Broun. Man. N.Z. Coleop. I 260, No. 22 I 9.

nigricans, Broun. Man. N.Z. Coleop. I 260, No. 2220.

inflatus, Sharp. Man. N.Z. Coleop. 562, No. 986.

dignus, Broun. Man. N.Z. Coleop. 563 , No. 987.

rugosus, Sharp. Man. N.Z. Coleop. 563, No. 988.

Aræocerus, Pascoe, 1876 .

pardalis, Pascoe. Man. N.Z. Coleop. 559, No. 98 r.

Xenanthribus, Broun, I 893 .

hirsutus, Broun. Man. N.Z. Coleop. 1270, No. 2237.

Order HeMiptera.

Sub-order Heteroptera.

Family PENTATOMIDE.

Echalia, Stål, i862.

Sub-family AsOPıN

schellembergii, Guérin. Hutton, T.N.Z.I. xxx. 169.

Cermatulus, Dall, i85I.

nasalis, Westwood. Hutton, T.N.Z.I. xxx. I 70.

Sub-family SciocorinÆ.

Dictyotus, Dall, 1851 .

vilis, Walker. Hutton, T.N.Z.I. xxx. I 70.

Sub-family Pentatomine.

Nezara, Amyot and Serville, 1843 .

amoyti, White. Hutton, T.N.Z.I. xxx. I 70.

Sub-family Halydine.

Platycoris, Guérin, 1830 .

immarginatus, Dallas. Hutton, T.N.Z.I. xxx. I 7 I.

Rhopalimorpha,

Sub-family Acanthosomatinæ.

obscura, White. Hutton, T.N.Z.I. xxx. I7 I.

Anubis, Stål, 1864 .

vittatus, Fabricius. Hutton, T.N.Z.I. xxx. I 7 I.

Sub-family Cydnines.

Geotomus, Mulsant, I 866 .

leptospermi, White. Hutton, T.N.Z.I. xxx. I 72.

Pangœus, Stål, I 862 .

scotti, Signoret. Hutton, T.N.Z.I. xxx. I 72. 
Chœrocydnus, White, I 84 I.

nigrosignatus, Buchanan-White. Hutton, T.N.Z.I. xxx. 172.

Family LYGæIDÆ.

Sub-family BERYTINE.

Neides, Latreille, 1802 .

wakefieldi, Buchanan-White. Hutton, T.N.Z.I. xxx. 172.

Sub-family LYGæ1NÆ.

Arocatus, Spinola, 1337 . ruficollis, Walker. Hutton, T.N.Z.I. xxx. I 73.

Nysius, Dall, I 832 .

zealandicus, Dallas. Hutton, T.N.Z.I. xxx. I73.

anceps, Buchanan-White. Hutton, T.N.Z.I. xxx. 173.

huttoni, Buchanan-White. Hutton, T.N.Z.I. xxx. 173.

Paresuris, Reuter, I89o.

helmsi, Reuter, Hutton, T.N.Z.I. xxx. 173.

Sub-family Pachymerinæ.

Plociomerus, Luy, 1831 . inornatus, Walker. Hutton, T.N.Z.I. xxx. 174.

Metagerra, Buchanan-White, I 878 .

obscura, Buchanan-White. Hutton, T.N.Z.I. xxx. I74.

Targarema, Buchanan-White, $\mathbf{1} 878$.

electa, Buchanan-White. Hutton, T.N.Z.I. xxx. I 74 .

ståli, Buchanan-IVhite. Hutton, T.N.Z.I. xxx. I74.

Margareta, Buchanan-White, 1878 .

dominica, Buchanan-White. Hutton, T.N.Z.I. xxx. 174.

Scolopostethus, Fieber, I86r.

putoni, Buchanan-White. Hutton, T.N.Z.I. xxx. 175.

Family Phymatide.

Phymata, Latreille, I802.

feredayi, Scott. Hutton, T.N.Z.I. xxx. I 75.

conspicua, Scott. Hutton, T.N.Z.I. xxx. I75.

Family ARAdinidæ.

Aradus, Fabricius, 1803 .

australis, Erichson. Hutton, T.N.Z.I. xxx. 175.

Ctenoneurus, Bergroth, is88.

hochstetteri, Mayr. Hutton, T.N.Z.I. xxx. I 75 ; Distant, A.M.N.H. 7, xi. 361 .

Aneurus, Curtis, 1825 .

brouni, Buchanan-White. Hutton, T.N.Z.I. xxx. 175 . 
Family HYDrometrIDÆ.

Hydroessa, Burmeister, i 835 . macgregori, Kirkaldy. Rev. Ent. France, xviii. 9 I (I 899).

\section{Family CAPSIDE.}

Megalocerœa, Fieber, 1858 .

reuteriana, Buchanan-IVhite. Hutton, T.N.Z.I. xxx. 176.

Morna, Buchanan-White, I 878 .

capsoides, Buchanan-White. Hutton, T.N.Z.I. xxx. I 76. scotti, Buchanan-White. Hutton, T.N.Z.I. xxx. 177.

Reuda, Buchanan-White, i 878. mayri, Buchanan-IWhite. Hutton, T.N.Z.I. xxx. 177 .

Capsus, Fabricius, I 803 .

laticinctus, Walker. Hutton, T.N.Z.I. xxx. 177 . ustulatus, Walker. Hutton, T.N.Z.I. xxx. I 77 .

Leptomerocoris, Kirschb, 1855 .

maoricus, Walker. Hutton, T.N.Z.I. xxx. 177.

Family ANTHOCORIDE.

Cardiastethus, Buchanan-White, I 875 (?).

brounianus, Buchanan-White. Hutton, T.N.Z.I. xxx. 178. consors, Buchanan-White. Hutton, T.N.Z.I. xxx. I 78 . poweri, Buchanan-White. Hutton, T.N.Z.I. xxx. I 78 .

Pirates, Burmeister, 1835 .

Family REDUVIIDE.

ephippigera, White. Hutton, T.N.Z.I. xxx. I 78.

Family Emeside.

Emesodema, Spinola, 1837 .

huttoni, Scott. Hutton, T.N.Z.I. xxx. 179.

Nabis, Latreille, 1802.

Family NABIDE.

saundersi, Buchanan-White. Hutton, T.N.Z.I. xxx. 778 . maoricus, Walker. Hutton, T.N.Z.I. xxx. 178.

Family SALDIDÆ.

Salda, Fabricius, $18 \circ 3$.

australis, Buchanan-White. Hutton, T.N.Z.I. xxx. 179. bulteri, Buchanan-IWhite. Hutton, T.N.Z.I. xxx. 179. lælaps, Buchanan-IVhite. Hutton, T.N.Z.I. xxx. I 79 .

Family NotONECTIDE.

Anisops, Spinola, I 837 .

wakefieldi, Buchanan-White. Hutton, T.N.Z.I. xxx. I79. assimilis, Buchanan-White. Hutton, T.N.Z.I. xxx. r8o. 
Corixa, Geoffroy, 1762 .

Family CORIXID E.

arguta, Buchanan-White. Hutton, T.N.Z.I. xxx. 180.

Family Sigarilde.

Diaprepocoris, Kirkaldy, I 897 .

barycephala, Kirkaldy, A.M.N.H. 6, xx. 53.

Sub-order HOMOPTERA.

Series Trimera.

O Family Cicadide.

Melampsalta, Amyot, I 847 .

scutellaris, Walker. Hutton, T.N.Z.I. xxx. I 8 I.

0 cingulata, Fabricius. Hutton, T.N.Z.I. xxx. I 82.

obscura, Hudson. Hutton, T.N.Z.I. xxx. I 82.

0 muta, Fabricius. Hutton, T.N.Z.I. xxx. 182.

0 cutora, Walker. Hutton, T.N.Z.I. xxx. I 82.

0 cruentata, Fabricius. Hutton, T.N.Z.I. xxx. I 82.

o sericea, Walker. Hutton, T.N.Z.I. xxx. I 83 .

0 flavescens, Hudson. Hutton, T.N.Z.I. xxx. I83.

0 angusta, Walker. Hutton, T.N.Z.I. xxx. 183 .

cincta, Walker. Hutton, T.N.Z.I. xxx. $18_{3}$.

iolanthe, Hudson. Hutton, T.N.Z.I. xxx. 183 .

o nervosa, Walker. Hutton, T.N.Z.I. xxx. 183 .

$\sigma$ mangu, Buchanan-White. Hutton, T.N.Z.I. xxx. I84.

Ocassiope, Hudson. Hutton, T.N.Z.I. xxx. I 84.

Family Cercopide.

Sub-family APHROPHORINÆ.

Aphrophora, Germar, I82 I.

jactator, Buchanan-White. Hutton, T.N.Z.I. xxx. 184 .

Phlænus, Stål, i 864 .

fingens, Walker. Hutton, T.N.Z.I. xxx. 184 .

subvirescens, White. Hutton, T.N.Z.I. xxx. 184.

atrimaculatus, White. Hutton, T.N.Z.I. xxx. I 84 .

${ }^{\circ}$ Family JAsside.

Dorydium, Burmeister, I 835 .

westwoodi, Buchanan-White. Hutton, T.N.Z.I. xxx. 185 .

Athysanus, Burmeister, 1835 .

negatus, Buchanan-White. Hutton, T.N.Z.I. xxx. 185.

\footnotetext{
Family FUlgoride.

- Sub-family Cixiıne.
}

Cixius, Latreille, $x \mathrm{SO}_{4}$.

0 interior, Walker. Hutton, T.N.Z.I. xxx. I85.

o punctimargo, Walker. Hutton, T.N.Z.I. xxx. I 86. 
${ }^{\circ}$ Cixius rufifrons, Walker. Hutton, T.N.Z.I. xxx. I 86.

$\circ$ aspilus, Walker. Hutton, T.N.Z.I. xxx. 186.

- Oliarus, Stål, i 862.

oppositus, IValker. Hutton, T.N.Z.I. xxx. I $\$ 6$.

o marginalis, Walker. Hutton, T.N.Z.I. xxx. I 86.

Aka, Buchanan-White, I 879 .

o finitima, Walker. Hutton, T.N.Z.I. xxx. I 86.

o Semo, Buchanan-IVhite, I 879 .

clypeatus, Buchanan-IVhite. Hutton, T.N.Z.I. xxx. I 87 .

\section{o Sub-family AcHiLiNæ.}

Agandecca, Buchanan-White, I 879 .

$V \quad \bullet$ annectens, Buchanan-White. Hutton, T.N.Z.I. xxx. I 87 .

- Cona, Buchanan-IVhite, I 879.

- Sub-family Delphacinæ.

o cælata, Buchanan-IVhite. Hutton, T.N.Z.I. xxx. I 87 .

Series Dimera.

Family PSYLlide.

Sub-family APHALARINÆ.

Rhinocola, Förster, I 848 . eucalypti, Maskell. T.N.Z.I. xxii. I 60. fuchsiæ, Maskell. T.N.Z.I. xxii. I 62.

Trioza, Förster, I $\$ 48$.

Sub-family TrioziNæ.

pellucida, Maskell. T.N.Z.I. xxii. I64. panacis, Maskell. T.N.Z.I. xxii. 167.

Family Aleurodide:

Aleurodes, Amyot and Serville, I 843 . papillifer, Maskell. T.N.Z.I. xxii. I 73. asplenii, Maskell. T.N.Z.I. xxii. 173. melicyti, Maskell. T.N.Z.I. xxii. I 74 . fagi, Maskell. T.N.Z.I. xxii. I 75. simplex, Maskell. T.N.Z.I. xxii. I 75. cerata, Maskell. T.N.Z.I. xxviii. 425. fodiens, Maskell. T.N.Z.I. xxviii. 433 .

Series Monomera.

Family COCCIDÆ.

Sub-farnily DiaspidinÆ.

Aspidiotus, Bouché, I 834 . atherospermæ, Maskell. T.N.Z.I. xxvii. I. carpodeti, Maskell. T.N.Z.I. xxvii. 2. 
Aspidiotus corokiæ, Maskell. T.N.Z.I. xxvii. 3. dysoxyli, Maskell. T.N.Z.I. xxvii. 3 . sophoræ, Maskell. T.N.Z.I. xxvii. 4 .

Diaspis, Costa, $18{ }_{3} 6$.

santali, Maskell. T.N.Z.I. xxvii. 5.

Mytilaspis, Targioni-Tozzetti, 1868.

cordylinidis, Maskell. T.N.Z.I. xxvii. 6.

drimydis, Maskell. T.N.Z.I. xxvii. 6.

epiphytidis, Maskell. T.N.Z.I. xxvii. 6. intermedia, Maskell. T.N.Z.I. xxvii. 7 .

lactea, Maskell. T.N.Z.I. xxvii. 7 .

leptospermi, Maskell. T.N.Z.I. xxvii. 7 . metrosideri, Maskell. T.N.Z.I. xxvii. 7 . phymatodidis, Maskell. T.N.Z.I. xxvii. 7 . pyriformis, Maskell. T.N.Z.I. xxvii. 7 .

Poliaspis, Maskell, 1879 .

media, Maskell. T.N.Z.I. xxvii. 9.

Chionaspis, Signoret, 1869.

dubia, Maskell. T.N.Z.I. xxvii. 9.

dysoxyli, Maskell. T.N.Z.I. xxvii. 9.

minor, Maskell. T.N.Z.I. xxvii. Io.

Fiorinia, Signoret, I 869.

asteliæe, Maskell. T.N.Z.I. xxvii. ro.

grossulariæ, Maskell. T.N.Z.I. xxvii. I I.

minima, Maskell. T.N.Z.I. xxvii. I I.

stricta, Maskell. T.N.Z.I. xxvii. I r.

Sub-family LECANINÆ.

Lecanochiton, Maskell, I88I. metrosideri, Maskell. T.N.Z.I. xxvii. II. minor, Maskell. T.N.Z.I. xxvii. I I.

Ctenochiton, Maskell, 1878 .

dacrydii, Maskell. T.N.Z.I. xxvii. I 2. depressus, Maskell. T.N.Z.I. xxvii. I 2. elæocarpi, Maskell. T.N.Z.I. xxvii. I2. elongatus, Maskell. T.N.Z.I. xxvii. I 2. flavus, Maskell. T.N.Z.I. xxvii. I 3. fuscus, Maskell. T.N.Z.I. xxvii. I 3 . hymenantheræ, Maskell. T.N.Z.I. xxvii. I3. perforatus, Maskell. T.N.Z.I. xxvii. I 3. piperis, Maskell. T.N.Z.I. xxvii. I3. viridis, Maskell. T.N.Z.I. xxvii. I 3 .

Inglisia, Maskell, 1878 .

fagi, Maskell. T.N.Z.I. xxvii. I3. 
Inglisia inconspicua, Maskell. T.N.Z.I. xxvii. I4. leptospermi, Maskell. T.N.Z.I. xxvii. 14. ornata, Maskell. T.N.Z.I. xxvii. I 4. patella, Maskell. T.N.Z.I. xxvii. I4.

Lecanium, Burmeister, I 835 .

cassiniæ, Maskell. T.N.Z.I. xxvii. I 5.

Eriochiton, Maskell, i 878 .

hispidus, Maskell. T.N.Z.I. xxvii. 18. spinosus, Maskell. T.N.Z.I. xxvii. I8.

Sub-family Coccinæ.

Solenophora, Maskell, i 889.

corokiæ, Maskell. T.N.Z.I. xxvii. 19. fagi, Maskell. T.N.Z.I. xxvii. r 9.

Planchonia, Signoret, I 870 . epacridis, Maskell. T.N.Z.I. xxvii. 19.

Rhizococcus, Signoret, I 875 .

celmisiæ, Maskell. T.N.Z.I. xxvii. 20. fossor, Maskell. T.N.Z.I. xxvii. 20. intermedius, Maskell. T.N.Z.I. xxvii. 20. maculatus, Maskell. T.N.Z.I. xxvii. 20. pulchellus, Maskell. T.N.Z.I. xxvii. 2 I. totaræ, Maskell. T.N.Z.I. xxvii. 2 I.

Gossyparia, Signoret, I 875 .

cavellii, Maskell. T.N.Z.I. xxvii. 2 I.

Eriococcus, Targioni-Tozzetti.

danthoniæ, Maskell. 'T.N.Z.I. xxvii. 22. fagicorticus, Maskell. T.N.Z.I. xxvii. 22. hoheriæ, Maskell. T.N.Z.I. xxvii. 22. multispinus, Maskell. T.N.Z.I. xxvii. 22. pallidus, Maskell. T.N.Z.I. xxvii. 22. phyllocladi, Maskell. T.N.Z.I. xxvii. 23. raithbyi, Maskell. T.N.Z.I. xxvii. 23.

Ripersia, Signoret, I 874.

fagi, Maskell. T.N.Z.I. xxvii. 23.

formicicola, Maskell: T.N.Z.I. xxvii. 23 ; Smith, T.N.Z.I. xxviii. 474 . rumicus, Maskell. T.N.Z.I. xxvii. 24.

Dactylopius, Costa, 1836 .

alpinus, Maskell. T.N.Z.I. xxvii. 24.

arecæ, Maskell. T.N.Z.I. xxvii. $24 ;$ Smith, T.N.Z.I. xxviii. 470.

aurilanatus, Maskell. T.N.Z.I. xxvii. 24. calceolariæ, Maskell. T.N.Z.I. xxvii. 24. 
Dactylopius glaucus, Maskell. T.N.Z.I. xxvii. 25 . iceryoides, Maskell. T.N.Z.I. xxvii. 25. obtectus, Maskell. T.N.Z.I. xxvii. 26. poæ, Maskell. T.N.Z.I. xxvii. 26.

Pseudococcus, Westwood, 1848. asteliæ, Maskell. T.N.Z.I. xxvii. 26.

Sub-family MONOPHLEBINA.

Leachia, Signoret, 1875 . zealandica, Maskell. T.N.Z.I. xxvii. 29.

Cœlostoma, Maskell, i 879 . assimile, Maskell. T.N.Z.I. xxvii. 29. compressum, Maskell. T.N.Z.I. xxvii. 29. pilosum, Maskell. T.N.Z.I. xxvii. 29. wairoense, Maskell. T.N.Z.I. xxvii. 30. zealandicum, Maskell. T.N.Z.I. xxvii. 30.

Icerya, Signoret, 1874 . purchasi, Maskell. 'T.N.Z.I. xxvii. 30 .

Sub-order ANOPLuRA. Family Pediculide.

Echinophthirius, Gieb, $187 \mathrm{I}$. microchir, Trouessart. La Nature, 2, ii. 80.

Order NeUroptera.

Series TRICHOPTERA.

Family SERicostomatide.

Econesus, McLachlan, i 862. maori, McLachlan. Hutton, T.N.Z.I. xxxi. 235.

Pseudæconesus, McLachlan, 1894 . mimus, McLachlan. Hutton, T.N.Z.I. xxxi. 236. stramineus, McLachlan. Hutton, T.N.Z.I. xxxi. 236-

Olinga, McLachlan, I894.

feredayi, McLachlan. Hutton, T.N.Z.I. xxxi. 238.

Pycnocentria, McLachlan, i 866.

funerea, McLachlan. Hutton, T.N.Z.I. xxxi. 238. evecta, McLachlan. Hutton, T.N.Z.I. xxxi. 239. aureola, McLachlan. Hutton, T.N.Z.I. xxxi. 239.

Family LEPTOCERIDÆ.

Pseudonema, McLachlan, I862. obsoleta, McLachlan. Hutton, T.N.Z.I. xxxi. 240. amabilis, McLachlan. Hutton, T.N.Z.I. xxxi. 24 I. 
Notanatolica, McLachlan, I 866.

cognata, McLachlan. Hutton, T.N.Z.I. xxxi. 24 I.

cephalota, Walker. Hutton, T.N.Z.I. xxxi. 242.

Leptocerus, Leach, i 8 r 5 .

alienus, McLachlan (gen. dub.). Hutton, T.N.Z.I. xxxi. 242.

Setodes, Rambur, $\mathrm{r} 8_{42}$.

unicolor, McLachlan. Hutton, T.N.Z.I. xxxi. 243.

Philaniscus, Walker, $185^{2}$. plebejus, Walker. Hutton, T.N.Z.I. xxxi. 243.

Family HydropsYchIDE.

Hydropsyche, Pictet, I 834 .

fimbriata, McLachlan. Hutton, T.N.Z.I. xxxi. 244. colonica, McLachlan. Hutton, T.N.Z.I. xxxi. 245.

Polycentropus, Curtis, 1835 . puerilis, McLachlan. Hutton, T.N.Z.I. xxxi. 245.

Hydrobiosis, McLachlan, I870.

frater, McLachlan. Hutton, T.N.Z.I. xxxi. 246. umbripennis, McLachlan. Hutton, T.N.Z.I. xxxi. 246.

Psilochorema, McLachlan, r\$66. mimicum, McLachlan. Hutton, T.N.Z.I. xxxi. 247. confusum, McLachlan. Hutton, T.N.Z.I. xxxi. 248.

Family HydRoptiLIDE.

Oxyethra, Eaton, I 873 . albiceps, McLachlan. Hutton, T.N.Z.I. xxxi. 249.

Series Planipennia.

Family Sialide.

Chauliodes, Latreille, I 805. diversus, Walker. Hutton, T.N.Z.I. xxxi. 228.

Family Myrmeleontide.

Myrmeleon, Linneus, I 748 .

acutus, Walker. Hutton, T.N.Z.I. xxxi. 229.

Family Hemerobide

Stenosmylus, McLachlan, i 867 .

incisus, McLachlan. Hutton, T.N.Z.I. xxxi. 230. citrinus, McLachlan. Hutton, T.N.Z.I. xxxi. 230. latiusculus, McLachlan. Hutton, T.N.Z.I. xxxi. 23 I. stellæ, McLachlan. Ent. Mon. Mag. I 899, 259.

Drepanepteryx, Leach, I 835 . instabilis, McLachlan. Hutton, T.N.Z.I. xxxi. 232. humilis, McLachlan. Hutton, T.N.Z.I. xxxi. 232. 
Micromus, Rambur, 1866.

tasmaniæ, Walker. Hutton, T.N.Z.I. xxxi. 233.

Series Odonata.

Family LibelLulide.

Sympetrum, Newman, 1855 .

bipunctatum, var. novæ-zealandiæ, McLachlan.

Hutton, T.N.Z.I. xxxi. 22 I.

Family CORdulindæ.

Somatochlora, Selys, i 878.

smithii, White. Hutton, T.N.Z.I. xxxi. 222.

grayi, Selys. Hutton, 'T.N.Z.I. xxxi. 222.

braueri, Selys. Hutton, T.N.Z.I. xxxi. 223.

Family Æschnidæ.

Eschna, Fabricius, 1776 .

brevistyla, Rambur. Hutton, T.N.Z.I. xxxi. 224.

Uropetala, Selys, 1857 .

Family GOMPHINIDE.

carovei, White. Hutton, T.N.Z.I. xxxi. 224.

Lestes, Leach, I8I 7.

Family Agrionide.

colensonis, White. Hutton, T.N.Z.I. xxxi. 225.

Xanthagrion, Selys, 1876 .

zealandicum, McLachlan. Hutton, T.N.Z.I. xxxi. 226.

antipodum, Selys. Hutton, T.N.Z.I. xxxi. 227.

sobrinum, McLachlan. Hutton, T.N.Z.I. xxxi. 227.

Series Pseudo-Neuroptera.

Family Termitide.

Calotermes, Hagen, 1853 .

brouni, Froggart. Hutton, T.N.Z.I. xxxi. 209.

insularis, Walker. Hutton, T.N.Z.I. xxxi. 2 ro.

Stolotermes, Hagen, 1858 .

ruficeps, Brauer. Hutton, T.N.Z.I. xxxi. 2 ro.

Family Procide.

Myopsocus, Hagen, i 866 .

novæ-zealandiæ, Kolbe. Hutton, T.N.Z.I. xxxi. 2 I I.

Family PERLIDE.

Stenoperla, McLachlan, i 866.

prasina, Newman. Hutton, T.N.Z.I. xxxi. 2 I 2.

cyrene, Newman (gen. dub.). Hutton, T.N.Z.I. xxxi. 2 I 2. 
Leptoperla, Newman, I $\delta_{39}$.

opposita, Walker. Hutton, T.N.Z.I. xxxi. 2 I 3.

Family EPHEMERID代.

Ichthybotus, Eaton, 899 .

hudsoni, McLachlan. Hutton, T.N.Z.I. xxxi. 214 ; Eaton, Trans. Ent. Soc. I 899, 285.

Atalophlebia, Eaton, I $8 S \mathrm{I}$.

dentata, Eaton. Hutton, T.N.Z.I. xxxi. 215.

nodularis, Eaton. Hutton, T.N.Z.I. xxxi. 2 r6; Lillie, T.N.Z.I. xxxi. 168.

scita, IValker. Hutton, T.N.Z.I. xxxi. 2 I6; Lillie, T.N.Z.I.

xxxi. 167.

versicolor, Eaton. T.E.S. I 899, 286.

Deleatidium, Eaton, I 899.

lillii, Eaton. T.E.S. I $899,289$.

Coloburiscus, Eaton, i 887 .

humeralis, Walker. Hutton, T.N.Z.I. xxxi. 2 I 7.

Ameletus, Eaton, I 887 .

ornatus, Eaton. Hutton, T.N.Z.I. xxxi. 2 I 7.

perscitus, Eaton. T.E.S. 1 899, 29!.

Oniscigaster, McLachlan, I 873 .

wakefieldi, McLachlan. Hutton, T.N.Z.I. xxxi. 2 I 8.

intermedius, Eaton. T.E.S. I899, 292.

distans, Eaton. T.E.S. 1899, 293.

Order ORTHOPTERA.

Series SALTATORIA.

Family GRYLLIDÆ.

Lissotrachelus, Brunner, I 893.

maoricus, IValker. Hutton, T.N.Z.I. xxxii. 19.

Family Locustide.

Xiphidium, Serville, I 839.

semivittatum, IValker. Hutton, T.N.Z.I. xxx. I37.

Agræcia, Serville, I 839 .

solida, Walker. Hutton, T.N.Z.I. xxx. I 38.

Family Stenopelmatide.

Sub-family Anostostomine.

Deinacrida, White, I 842 .

heteracantha, White. Hutton, T.N.Z.I. xxix. 2 I 2.

rugosa, Buller. Hutton, T.N.Z.I. xxix. 213.

Hemideina, Walker, I 869.

parva, Buller. Hutton, T.N.Z.I. xxxii. 20. 
Hemideina armiger, Colenso. Hutton, T.N.Z.I. xxix. $2 \mathrm{I} 7$. thoracica, Walker. Hutton, T.N.Z.I. xxix. $2 \mathrm{I} 7 ; H$. producta, Walker.

megacephala, Buller. Hutton, T.N.Z.I. xxix. 2 I 5.

figurata, Walker. Hutton, T.N.Z.I. xxix. 2 I6.

femorata, Hutton. T.N.Z.I. xxix. 2 IS.

broughi, Buller. Hutton, T.N.Z.I. xxxii. 2 I.

maori, Pictet et Saussure. Hutton, T.N.Z.I. xxix. 219.

Onosandrus, Stål, I 876.

pallitarsis, Walker. Hutton, T.N.Z.I. xxix. 221.

focalis, Hutton. T.N.Z.I. xxix. 222.

maori, Pictet et Saussure. Hutton, T.N.Z.I. xxix. 223.

maculifrons, Walker. Hutton, T.N.Z.I. xxix. 223.

lanceolatus, Walker. Kirby MSS.

Talitropsis, Bolivar, I 882 .

Sub-family Dolichopodine.

sedilotti, Bolivar. Hutton, T.N.Z.I. xxix. 225.

irregularis, Hutton. T.N.Z.I. xxix. 227.

Grammaroparnops, Alfken, I90I.

crassicruris, Hutton. T.N.Z.I. xxix. 226.

Ischyroplectron, Hutton, 1897.

isolatum, Hutton. T.N.Z.I. xxix. 228.

Gymnoplectron, Hutton, I 897 .

longipes, Colenso. Hutton, T.N.Z.I. xxix. 229.

stephensiensis, Alfken. Erge. Reise Pacific, I 50.

Pachyrhamma, Brunner, I 888.

fascifer, Walker. H. speluncre, Colenso. Hutton, T.N.Z.I. xxix. 23 I.

altus, Walker. P. novce-zealandice, Brunner. Hutton, l.c. $23^{2}$.

Pleioplectron, Hutton, I 897.

simplex, Hutton. T.N.Z.I. xxix. 233.

pectinatum, Hutton. T.N.Z.I. xxix. 234.

hudsoni, Hutton. T.N.Z.I. xxix. 234.

edwardsi, Scudder. Hutton, T.N.Z.I. xxix. 240.

cavernæ, Hutton. T.N.Z.I. xxxii. 2 I.

Miotopus, Hutton, I 899 .

diversus, Hutton. T.N.Z.I. xxxi. 4I.

Neonetus, Brunner, I888.

variegatus, Brunner. Hutton, T.N.Z.I. xxix. 236.

pilosus, Hutton. T.N.Z.I. xxix. 236.

Isoplectron, Hutton, I 897 .

armatum, Hutton. T.N.Z.I. xxix. 237.

calcaratum, Hutton. T.N.Z.I. xxix. 238. 
Pharmacus, Pictet et Saussure, i 89 I.

montanus, Pictet et Saussure. Hutton, T.N.Z.I. xxix. 239.

Macropathus, Walker, I 870.

filifer, Hutton. T.N.Z.I. xxix. 239.

Family ACRIDID.E.

Sub-family CEDIPODINÆ.

Pachytylus, Fieber, ${ }^{8} 8_{53}$.

cinerascens, Fabricius. Hutton, T.N.Z.I. xxx. I49. migratoroides, Reich. Hutton, T.N.Z.I. xxx. I50.

Sub-family ACRidine.

Phaulacridium, Brunner, I89r. marginale, Walker. Hutton, T.N.Z.I. xxx. I4I. luteum, Hutton. T.N.Z.I. xxx. I42.

Paprides, Hutton, i 898 . nitidus, Hutton. T.N.Z.I. xxx. I46. australis, Hutton. T.N.Z.I. xxx. I47. furcifer, Hutton. T.N.Z.I. xxxi. 46. torquatus, Hutton. T.N.Z.I. xxxi. 47. armillatus, Hutton. T.N.Z.I. xxxi. 47 .

Sigaus, Hutton, 1898 .

- piliferus, Hutton. T.N.Z.I. xxx́. I40.

Trigoniza, Brunner, г 89 г.

campestris, Hutton. T.N.Z.I. xxx. 148.

directa, Hutton. T.N.Z.I. xxx. I 48 .

rugosa, Hutton. T.N.Z.I. xxx. I 48 .

Brachaspis, Hutton, I 899.

nivalis, Hutton (Pezotettix). T.N.Z.I. xxx. I 44 ; xxxi. 49.

collinus, Hutton. T.N.Z.I. xxx. I 44 ; xxxi. 49.

petricolus, Hutton. T.N.Z.I. xxx. 145 ; xxxi. 49.

terrestris, Hutton. T.N.Z.I. xxx. 145 .

Series Cursoria.

Family PHASMIDE.

Sub-family Clitun'Ninæ.

Pachymorpha, Gray, I 835 .

hystriculea, Westwood. Hutton, T.N.Z.I. xxxi. 52.

salebrosa, Hutton. T.N.Z.I. xxxi. 52.

annulata, Hutton. T.N.Z.I. xxxi. 53 .

acornuta, Hutton. T.N.Z.I. xxxi. 53 .

Clitarchus, Stăl, I875.

hookeri, White. Hutton, T.N.Z.I. xxxi. 54.

coloreus, Colenso. Hutton, T.N.Z.I. xxxi. 55 . 
Clitarchus reductus, Hutton. T.N.Z.I. xxxi. 55 . læviusculus, Stål. Hutton, T.N.Z.I. xxxi. 56 .

Acanthoderus, Gray, i $S_{35}$.

prasinus, Westwood. Hutton, T.N.Z.I. xxxi. 56.

suteri, Hutton. T.N.Z.I. xxxi. 56.

geisovii, Kaup. Hutton. T.N.Z.I. xxxi. 57.

fasciatus, Hutton. T.N.Z.I. xxxi. 58.

Argosarchus, Hutton, I 898 .

spiniger, White. Hutton, T.N.Z.I. xxxi. $59=$ B. horridus, White, which was printed last.

Family BLatTide.

Blatta, Linnæus, I 766.

Sub-family Blattine.

conjuncta, Walker. Hutton, Cat. Orth. N.Z. 7 I.

Sub-family PERIPLANetinæ.

Polyzosteria, Burmeister, i 829 .

novæ-zealandiæ, Brunner. Nov. Syst. Blatt. 218;

B. forticeps, Walker. Hutton. Cat. Orth. N.Z. 72 .

Platyzosteria, Brunner, I 865.

undulivitta, Walker. Hutton, Cat. Orth. N.Z. 73.

brunni, Alfken. Erge. Reise Pacific, I 90I, I 42.

Anisolabis, Fieber, 1853 .

Family ForFICULIDæ.

littorea, White. Hutton, Cat. Orth. N.Z. 93 ; Kirby, J.L.S. xxiii. 5 I 7 .

Order APTERA.

Series Collembola.

Family LIPURIDÆ.

Lipura, Burmeister, $\mathrm{I}_{3} 8$.

incerta, Moniez. Rer. Biol. Nord France, vi. 2 I I (1894).

Drepanura, Schott, i 893 .

brachycephala, Moniez. Rev. Biol. Nord France, vi. 208 (I 894).

Anoura, Gervais, 1842 .

spinosa, Lubbock. J.L.S. xxvii. 334 ; Dendy, T.N.Z.I. xxxiii. 97 .

CLASS, MYRIAPODA.

Order DipLOPODA.

Family SPHAROTHERIID瓜.

Cyliosoma, Pocock, I 895.

de Lacyi, White. A.M.N.H. 3, iii. 406 
Cyliosoma leiosoma, Hutton. T.N.Z.I. x. 29 I.

novæ-zealandiæ, Kirk. T.N.Z.I. xviii. I 39.

striolatum, Pocock. A.M.N.H. 6, xvi. 4I 4 (1895).

Julus, Linnæus, I 748 .

Family JULIDE.

berardi, Walckener. In Gervais' Aptēres, iv. 333.

Spirostreptus, Brandt, I 833 .

antipodarum, Newport. In Dieff. N.Z. ii. 270.

striatus, Hutton. T.N.Z.I. x. 290.

Family Polydesmide.

Polydesmus, Latreille, I 802.

haastii, Humbert and Saussure. Verh. z. b. Ges. Wien. xix. $68_{3}$; Hutton, T.N.Z.I. x. 292.

serratus, Hutton. T.N.Z.I. x. 290.

worthingtoni, Hutton. T.N.Z.I. x. 290.

Strongylosoma, Brandt, I 833 .

novaræ, Humbert and Saussure. Verh. z. b. Ges. Wien. xix. 689 (1869); Hutton, T.N.Z.I. x. 292.

macrocephala, Hutton. T.N.Z.I. x. 29I.

Icosidesmus, Humbert and Saussure, I869.

hochstetteri, Humbert and Saussure. Verh.z. b. Ges. Wien.

xix. 890 ; Hutton, T.N.Z.I. x. 292.

Craspedosoma, Leach, I8 8 .

trisetosa, Hutton. T.N.Z.I. x. 291.

Serangodes, Attems, I 899.

strongylosomoides, Attems. Denk. Ak. Wien. 1xvii. 273.

Order Chilopoda.

Family LiтновіIDÆ.

Lithobius, Leach, I8 2 .

argus, Newport. T.L.S. xix. 369 .

Henicops, Newport, I 845 .

maculatus, Newport. T.L.S. xix. 372 ; Pocock, A.M.N.H. 6 , xi. I 25.

impressus, Hutton. T.N.Z.I. x. 228 .

Lamyctes, Meinert, I868.

emarginatus, Newport. T.L.S. xix. 372 ; Pocock, A.M.N.H.

7 , viii. 449 .

Family ScOLOPENDRIDA.

Heterostoma, Newport, I 845 .

platycephala, Newport. T.L.S. xix. 4I5. 
Cosmocephalus, Newport, 1845 .

rubriceps, Newport. T.L.S. xix. $42 \mathrm{c}$.

pallipes, Newport. T.L.S. xix. 424.

purpureus, Pocock. A.M.N.H. 6, xi. $127 ;$ C. violacelts, Newport, not of Fabricius.

huttoni, Pocock. A.M.N.H. 6, xi. 128; C. violaceus, Hutton, not of Newport.

Cryptops, Leach, r8r2.

australis, Newport. T.L.S. xix. 408.

setosus, Pocock. A.M.N.H. 6, viii. I57 (I89r).

Geophilus, Leach, I 8 I 2.

Family GeOPHiLidæ.

huttoni, Pocock. A.M.N.H. 6, viii. 223.

morbosus, Hutton. T.N.Z.I. x. 289 (Himantarium).

provocator, Pocock. A.M.N.H. 6, viii. 225.

Necrophlœsophagus, Newport, I 845 .

antipodum, Pocock. A.M.N.H. 6, viii. 222 , and 7 , viii. 46 I.

Order Schizotarsia.

Family Cermatidde.

Cermatia, Rossi, 1807 .

smithii, Newport. T.L.S. xix. 359, and A.M.N.H. r, xiii. 96.

\section{CLASS, ONYCHOPHORA.}

Family PERIPATIDÆ.

Peripatoides, Pocock, I 895.

novæ-zealandiæ, Hutton. Sedgwick, Q.J.M.S. xxviii. 456 ;

Sheldon, Q.J.M.S. xxviii. 202.

suteri, Dendy. T.N.Z.I. xxvii. 190.

Oöperipatus, Dendy, r 900 .

viridimaculatus, Dendy. Zool. Anzeig, xxiii. 509; Q.J.M.S.

45 n.s., 399.

\section{CLASS, ARACHNIDA.}

Order ARANeida.

Family 'Theraphoside.

Migas, Koch, I873.

paradoxus, Koch. Arach. Aust. 467.

distinctus, Cambridge. P.Z.S. 1879,683 ; Goyen, T.N.Z.I. xix. 2 ro.

sandageri, Goyen. T.N.Z.I. xxiii. I 23. 
Arbanitis, Koch, $\times 875$.

gilliesii, Cambridge. T.N.Z.I. x. 284 (Nemesia); Gillies, T.N.Z.I. x. $30 \mathrm{I}$.

huttoni, Cambridge. P.Z.S. I 879,682 ; Goyen, T.N.Z.I. xxiv. 255 .

kirki, Urquhart. T.N.Z.I. xxvi. 204.

Maoriana, Hogg, I gor.

dendyi, Hogg. P.Z.S. 1901, 237.

Hexathele, Ausserer, i 87 I.

hochstetteri, Ausserer. Koch's Arach. Aust. 459; Hogg,

P.Z.S. I $901,276$.

petriei, Goyen. T.N.Z.I. xix. 207.

Porrhothele, Simon, I892.

antipodiana, Walckenaer. Simon, Zool. Jahrb. Syst. xii. 420 (Macrothele).

huttoni, Cambridge. T.N.Z.I. vi. 200.

insignipes, Simon. Zool. Jahrb. Syst. xii. 420.

simoni, Hogg. P.Z.S. Igor, 269.

Cteniza, Latreille, I 825 .

antipodum, White. P.Z.S. 1849,3 (gen. dub.).

hexops, White. P.Z.S. 1849,3 (gen. dub.).

Family Dysderide.

Segestria, Latreille, I 804 .

suteri, Urquhart. T.N.Z.I. xxiv. 230.

Ö̈nops, Templeton, I 830 .

septem-cincta, Urquhart. T.N.Z.I. xxiii. 128.

\section{Family DraSSID瓜.}

Herpyllus, Urquhart, 1887 .

formicarius, Urquhart. T.N.Z.I. xix. 109.

Drassus, Walckenaer, I 805 .

pretiosus, Koch. Arach. Aust. 385 .

erebus, Koch. Arach. Aust. 387 .

scitulus, Urquhart. T.N.Z.I. xxv. 165.

Chiracanthium, Koch, 1839.

stratioticum, Koch. Arach. Aust. 408.

Clubiona, Latreille, I804.

cambridgei, Koch. Arach. Aust. 416.

peculiaris, Koch. Arach. Aust. 427.

viridicoma, Urquhart. T.N.Z.I. xxiv. 233.

chevronia, Urquhart. T.N.Z.I. xxiv. $23 \mathrm{I}$.

nitida, Urquhart. T.N.Z.I. xxv. I66. 
Uliodon, Koch, I875.

frenata, Koch. Arach. Aust. 440.

maritima, Cambridge. P.Z.S. I 883, 356 (Amaurobioides).

Family Agelenide.

Amaurobius, Koch, 1836 .

longinquus, Koch. Arach. Aust. $33^{6}$.

finschii, Koch. Arach. Aust. 339 .

martius, Simon. Zool. Jahrb. Syst. xii. 42 I (I 899).

chathamensis, Simon. Zool. Jahrb. Syst. xil. 432 (1899).

Badumna, Thorell, i89o.

sub-fasciata, Simon. Zool. Jahrb. Syst. xii. 422.

Desis, Walckenaer, I837.

marina, Hector. Cambridge, P.Z.S. ı 879, 687 ; Daindrigia dysderoides, White, P.Z.S. 1849,5 .

Tegenaria, Walckenaer, 1805 .

foliata, Koch. Arach. Aust. 356 .

arboricola, Urquhart. T.N.Z.I. xxiii. I 29.

livoris, Urquhart. T.N.Z.I. xxv. 167.

Cambridgea, Koch, I 872.

antipodiana, White. Simon, Zool. Jahrb. Syst. xii. 434;

C. fasciata, Koch, Cambridge, T.N.Z.I. vi. 202.

Amphicnecta, Simon, I 888.

decemmaculata, Simon.

Family THERIDHDÆ.

Argyrodes, Simon, I 864 .

lepida, Cambridge, P.Z.S. I 879, 688.

conus, Urquhart. 'T.N.Z.I. xvii. 40.

Ariamnes, Thorell, I870.

coniferus, Urquhart. T.N.Z.I. xix. 96.

triangulatus, Urquhart. T.N.Z.I. xix. 97.

attenuatus, Urquhart. T.N.Z.I. xix. 98.

flavo-notatus, Urquhart. T.N.Z.I. xxiii. 136.

Mimetus, Hentz, I 85 I.

mendicus, Cambridge. P.Z.S. 1879, 697.

sennio, Urquhart. T.N.Z.I. xxiii. 137.

atri-cinctus, Urquhart. T.N.Z.I. xxiv. 234.

Theridion, Walckenaer, I 805.

veruculatum, Urquhart. T.N.Z.I. xviii. I 88.

blatteus, Urquhart. T.N.Z.I. xviii. I 90.

calyciferum, Urquhart. T.N.Z.I. xviii. 192. cruciferum, Urquhart. T.N.Z.I. xviii. I93.

triloris, Urquhart. T.N.Z.I. xviii. 194. 
Theridion squalidæ, Urquhart. T.N.Z.I. xviii. 195. setiger, Urquhart. T.N.Z.I. xviii. 196. zantholabis, Urquhart. T.N.Z.I. xviii. 197. sericum, Urquhart. T.N.Z.I. xviii. 198 ; xxii. 258. melanozanthum, Urquhart. T.N.Z.I. xix. I02. tuberculatum, Urquhart. T.N.Z.I. xix. I 04. maculopes, Urquhart. T.N.Z.I. xix. 105. viridanum, Urquhart. T.N.Z.I. xix. 106. flabelliferum, Urquhart. T.N.Z.I. xix. 106. venustulum, Urquhart. T.N.Z.I. xix. 107. albo-guttatum, Urquhart. T.N.Z.I. xix. 108. nigro-folium, Urquhart. T.N.Z.I. xx. I I 2. helvolum, Urquhart. T.N.Z.I. xx. I I3. truncatum, Urquhart. T.N.Z.I. xx. I I 5. exornatum, Urquhart. T.N.Z.I. xx. II 5. brunnea-folium, Urquhart. T.N.Z.I. xxi. 142. niger-punctillum, Urquhart. T.N.Z.I. xxi. I 43. porphyreticum, Urquhart. T.N.Z.I. xxi. I 44. gracilipes, Urquhart. T.N.Z.I. xxi. I 45. zebrinia, Urquhart. T.N.Z.I. xxii. 256 . pusillulum, Urquhart. T.N.Z.I. xxii. 257 . pumilio, Urquhart. T.N.Z.I. xviii. I90. punica-punctatum, Urquhart. T.N.Z.I. xxiii. I 47. apiatum, Urquhart. T.N.Z.I. xxiii. I 48. literatum, Urquhart. T.N.Z.I. xxiii. I50. ampliatum, Urquhart. T.N.Z.I. xxiv. 237. albocinctum, Urquhart. T.N.Z.I. xxiv. 236 . argentatum, Urquhart. T.N.Z.I. xxiv. 235. gibbosum, Urquhart. T.N.Z.I. xxvi. 205.

Erycina, Urquhart, I89I. violacea, Urquhart. T.N.Z.I. xxiii. $\mathbf{1}^{2} 2$.

Latrodectus, Walckenaer, I 805. hasselti, Thorell. Koch, Arach. Aust. 276 ; L. katipo, Powell, T.N.Z.I. iii. 56 ; T.N.Z.I. xxviii. 755 ; Pickard, Cambridge, P.Z.S. I902, i. 255. var. atritus, Urquhart. T.N.Z.I. xxii. 259.

Lithyphantes, Thorell, I 869. lepidus, Cambridge. P.Z.S. $1879,690$.

Atkinsonia, Cambridge, I879. nana, Cambridge. P.Z.S. I879, 691 .

Phycosoma, Cambridge, I 879 . œcobioides, Cambridge. P.Z.S. $1879,692$.

Linyphia, Latreille, I804. subdola, Cambridge. P.Z.S. $1879,693$. 
Linyphia peramæna, Cambridge. P.Z.S. $1879,693$. melanopygia, Cambridge. P.Z.S. I879, 693. diloris, Urquhart. T.N.Z.I. xviii. I 84 . trispathulata, Urquhart. T.N.Z.I. xviii. I 86. blatifer, Urquhart. T.N.Z.I. xix. 99. rufocephala, Urquhart. T.N.Z.I. xx. Io9. lagenifera, Urquhart. T.N.Z.I. xx. I I I. purpura-punctata, Urquhart. T.N.Z.I. xxi. I34. nitidula, Urquhart. T.N.Z.I. xxi. 136. rufo-lineata, Urquhart. T.N.Z.I. xxi. I 37. nemoralis, Urquhart. T.N.Z.I. xxi. I 40. multicolor, Urquhart. T.N.Z.I. xxiii. I 40. cruenta, Urquhart. T.N.Z.I. xxxiii. I42. albiapiata, Urquhart, T.N.Z.I. xxxiii. I 43. pellos, Urquhart. T.N.Z.I. xxxiii. 146. mundenia, Urquhart. T.N.Z.I. xxvi. 207. decolora, Urquhart. T.N.Z.I. xxvi. 208. fucatinia, Urquhart. T.N.Z.I. xxvi. 209.

Tekella, Urquhart, I 895 . absidiata, Urquhart. T.N.Z.I. xxvi. 2 II.

Erigone, Savigny, r 8- (?). atriventer, Urquhart. T.N.Z.I. xix. 102.

Stegosoma, Cambridge, I879. quadratum, Cambridge. P.Z.S. 1879, 698. lacunosum, Urquhart. T.N.Z I. xxv. I69.

Cornicularia, Menge, I869. crinifrons, Urquhart. T.N.Z.I. xxiii. 155 .

Steatoda, Sundevall, 1833. nubilosa, Urquhart. T.N.Z.I. xxx. 168. exçussa, Urquhart. T.N.Z.I. xxx. I 7 I.

Habronestes, Koch, 1872 .

Family ENYOIDE. marinus, Goyen. T.N.Z.I. xxii. 269. celeripes, Urquhart. T.N.Z.I. xxiii. I 32. scitula, Urquhart. T.N.Z.I. xxiii. I35.

Huttonia, Cambridge, 1879. palpaminoides, Cambridge. P.Z.S. I $879,68_{5}$.

Family Tetragnathide.

Tetragnatha, Latreille, 1804.

typica, Urquhart. T.N.Z.I. xxii. $25 \mathrm{I}$. herbigrada, Urquhart. T.N.Z.I. xxii. 252. arborea, Urquhart. T.N.Z.I. xxxiii. 172. multi-punctata, Urquhart. T.N.Z.I. xxxiii. 176 . 
Tetragnatha flavida, Urquhart. T.N.Z.I. xxxiii. 177 . gulosa, Koch. Arach. Aust. I 76.

Orsinome, Thorell, I 890. australis, Simon. Zool. Jahrb. Syst. xii. 423 .

Deinagnatha, White, 1846 . daindridgi, White. A.M.N.H. I, xviii. 180.

Ischalea, Koch, I87 I. spinipes, Koch. Arach. Aust. 197.

Family EPEIRIDE.

Argiope, Saxigny, 18-(?). syrmatica, Koch. Arach. Aust. 2 I 3.

Epeira, Walckenaer, I805.

extuberata, Koch. Arach. Aust. 6I. crassa, Walck. Koch, Arach. Aust. 63 . undata, Koch. Arach. Aust. 73 . verrucosa, Walck. Koch, Arach. Aust, i 2. feredayi, Cambridge. Koch, Arach. Aust, I 22. brounii, Urquhart. T.N.Z.I. xvii. 32. attenuata, Urquhart. T.N.Z.I. xvii. 33 . corrugata, Urquhart. T.N.Z.I. xix. 72. pocillator, Urquhart. T.N.Z.I. xix. 74. oblitera, Urquhart. T.N.Z.I. xix. 77 . trituberculata, Urquhart. T.N.Z.I. xix. 78 , and $\mathrm{xx} . \mathbf{I} 20$. orientalis, Urquhart. T.N.Z.I. xix. 79, and Xx. I $2 \mathrm{I}$. bi-albimacula, Urquhart. T.N.Z.I. xix. 8r. saxitalis, Urquhart. T.N.Z.I. xix. 82. subcompta, Urquhart. T.N.Z.I. xix. 83 . viridata, Urquhart. T.N.Z.I. xix. 85 . discolora, Urquhart. T.N.Z.I. xix. 86. veruta, Urquhart. T.N.Z.I. xix. 87. var. veruina, Urquhart. T.N.Z.I. xix. 88. var. hastata, Urquhart. T.N.Z.I. xix. 89. var. lineola, Urquhart. T.N.Z.I. xix. 89. linea-acuta, Urquhart. T.N.Z.I. xix. 90. purpurea, Urquhart. T.N.Z.I. xix. 9I. viridicans, Urquhart. T.N.Z.I. xx. I I 6. mulleola, Urquhart, T.N.Z.I. xx. II8. helveo-guttata, Urquhart. T.N.Z.I. xx. II9. tuberculata, Urquhart. T.N.Z.I. xx. I 20. dumicola, Urquhart. T.N.Z.I. xxi. I 46. flavo-maculata, Urquhart. T.N.Z.I. xxii. 239. albo-stricta, Urquhart. T.N.Z.I. xxii. 240. guttata, Urquhart. T.N.Z.I. xxii. 242. ostri-brunnea, Urquhart. T.N.Z.I. xxii. 
Epeira albi-scuta, Urquhart. T.N.Z.I. xxii. 244. dubitabilis, Urquhart. T.N.Z.I. xxii. 246. tri-notata, Urquhart. T.N.Z.I. xxii. 247. var. olivinia, Urquhart. T.N.Z.I. xxii. 249. atri-apiata, Urquhart. T.N.Z.I. xxiii. I 56. acinta, Urquhart. T.N.Z.I. xxiii. I 58. nigro-hastula, Urquhart. T.N.Z.I. xxiii. 159. atri-hastula, Urquhart. T.N.Z.I. xxiii. I62. galbana, Urquhart. T.N.Z.I. xxiii. I63. venustula, Urquhart. T.N.Z.I. xxiii. 165. melania, Urquhart. T.N.Z.I. xxiii. I66. similaris, Urquhart. T.N.Z.I. xxiii. I68. lævigata, Urquhart. T.N.Z.I. xxiii. I 7 I. invisiblis, Urquhart. T.N.Z.I. xxiv. 239. sublutia, Urquhart. T.N.Z.I. xxiv. $24 \mathrm{I}$. simulata, Urquhart. T.N.Z.I. xxiv. 242. ventriosa, Urquhart. 'T.N.Z.I. xxiv. 243. leucisca, Urquhart. T.N.Z.I. xxiv. 245 . æstiva, Urquhart. T.N.Z.I. xxv. I 74 . decorosa, Urquhart. T.N.Z.I. xxvi. 2 I 2. powelli, Urquhart. T.N.Z.I. xxvi. 2 I 4. pironginia, Urquhart. T.N.Z.I. xxvi. 2 r 5. angusticlava, Urquhart. T.N.Z.I. xxv. I 73. viridana, Urquhart. T.N.Z.I. xxv. I75. munda, Urquhart. T.N.Z.I. xxv. 177 . var. inversa, Urquhart. T.N.Z.I. xxv. I 78. albolineata, Urquhart. T.N.Z.I. xxv. I 78 . blattea, Urquhart. T.N.Z.I. xxv. I 79.

Nephila, Leach, I8 5 . argentata, Urquhart. T.N.Z.I. xix. 92.

Arachnura, Vinson, 1863 . longicauda, Urquhart. T.N.Z.I. xvii. 39. nigritia, Urquhart. T.N.Z.I. xvii. 37. obtusa, Urquhart. T.N.Z.I. xvii. 37 . trilobata, Urquhart: T.N.Z.I. xvii. 37.

Celænia, Thorell, i 868.

\section{Family CeLæNIDÆ.}

atkinsoni, Cambridge. P.Z.S. I 879,699 (Thlaosoma). hectori, Cambridge. P.Z.S. I 879, 700 (Thlaosoma). olivacea, Urquhart. T.N.Z.I. xvii. 39, and xix. 95. penna, Urquhart. T.N.Z.I. xix. 94. tuberosa, Urquhart. T.N.Z.I. xix. I 49.

Episinus, Walckenaer, I 809 .

Family Episinide.

antipodianus, Cambridge. P.Z.S. I879, 70 I. 
Family Тноміside.

Stephanopis, Cambridge, I 869.

angulatus, Urquhart. T.N.Z.I. xvii. 42 , and xxii. 260.

angularis, Urquhart. T.N.Z.I. xvii. 43 .

Diæa, Thorell, i 869 .

albo-limbata, Koch. Arach. Aust. 588 .

Xysticus, Koch, I 835 .

albo-brunnea, Urquhart. T.N.Z.I. xxv. i 84.

Family PHILODROMIDÆ.

Philodromus, Walckenaer, i 805.

ambarus, Urquhart. T.N.Z.I. xvii. 43, and xix. I I 2.

sphæriodes, Urquhart. T.N.Z.I. xvii. 44 , and xix. I I I

ovatus, Urquhart. T.N.Z.I. xix. I I 3.

rubro-frontus, Urquhart. T.N.Z.I. xxiii. 179.

Hemiclæa. Thorell, 1870.

rogenhoferi, Koch. Arach. Aust. 637.

plautus, Urquhart. T.N.Z.I. xviii. I99, and xix. x10.

Lycosa, Latreille, I804.

Family Lycosid $F$.

sænica, Koch. Arach. Aust. 91 5.

hilaris, Koch. Arach. Aust. 920 and 979.

umbrata, Koch. Arach. Aust. 921.

virgata, Goyen. T.N.Z.I. xix. 201.

canescens, Goyen. T.N.Z.I. xix. 203.

taylori, Goyen. T.N.Z.I. xix. 206.

ærescens, Goyen. T.N.Z.I. xix. 206.

uliginosa, Goyen. T.N.Z.I. xx. 136 .

bellicosa, Goyen. T.N.Z.I. xx. I 38 .

proxima, Urquhart. T.N.Z.I. xviii. 201.

adumbrata, Urquhart. T.N.Z.I. xix. I I4.

arenaria, Urquhart. T.N.Z.I. xxiii. I82.

maura, Urquhart. T.N.Z.I. xxiv. 246.

urquharti, Simon. Hist. Nat. Araignées, and ed. ii. 30.

piratica, Clerck. Simon, Zool. Jahrb. 1 899, 427 (introduced?).

schauinslandi, Simon. Zool. Jahrb. 1899, 428.

tremula, Simon. Zool. Jahrb. I $899,429$.

Dolomedes, Latreille, 1804 .

imperiosus, Koch. Arach. Aust. 867.

lateralis, White. P.Z.S. $1849,4$.

sagittiger, White. P.Z.S. ז $849,4$.

minor, Koch. Arach. Aust. 864.

schauinslandi, Simon. Zool. Jahrb. Syst. xii. $43^{6}$. 
Pardosa, Koch, I 848 .

vicaria, Koch. Arach. Aust. 965.

Anoteropsis, Koch, I 878 .

flavescens, Koch. Arach. Aust. 97 I.

Cycloctenus, Koch, I879.

Family CTEnide.

fugax, Goyen. T.N.Z.I. xxii. 267.

flaviceps, Koch. C. lepidus, Urquhart. T.N.Z.I. xxii. $26 \mathrm{r}$. pulcher, Urquhart. T.N.Z.I. xxiii. 183 .

Oxyopes, Latreille, I 804 .

Family Oxyopide.

gregarius, Urquhart. T.N.Z.I. xvii. 5 I (Sphasus).

Sphasus, Walckenaer, I 805 .

gracilipes, White. P.Z.S. I 849,4 .

Læstrygones, Urquhart, 1892.

albiceres, Urquhart. T.N.Z.I. xxvi. 2 I 7 .

Salticus, Latreille, I 804.

Family AtT1DÆ.

appressus, Powell. T.N.Z.I. v. 28 I.

atratus, Powell. T.N.Z.I. v. 283 .

v-notatus, Powell. T.N.Z.I. v. 284 .

fumosus, Powell. T.N.Z.I. v. 284 .

mustilinus, Powell. T.N.Z.I. v. 285.

albo-barbatus, Powell. T.N.Z.I. v. 286.

Marpissa, Koch, I 847.

leucophæna, Urquhart. T.N.Z.I. xx. I2I.

arenaria, Urquhart. T.N.Z.I. xx. 123.

æri-hirta, Urquhart. T.N.Z.I. xx. I 24.

cineracea, Urquhart. T.N.Z.I. xxiii. I88.

armifera, Urquhart. T.N.Z.I. xxiv. 248.

nemoralis, Urquhart. T.N.Z.I. xxiv. 250.

Marptusa, Thorell, 1877 .

cinerea, Koch. Arach. Aust. I I 2.

leucocomus, Koch. Arach. Aust. 1096. marina, Goyen. T.N.Z.I. xxiv. 253.

Ballus, Koch, I 850 . compactus, Urquhart. T.N.Z.I. xvii. 50.

Plexippus, Koch, I 847 .

minax, Powell. T.N.Z.I. v. 282 (Salticus). herbigradus, Urquhart. T.N.Z.I. xxi. I 50. capillatus, Urquhart. T.N.Z.I. xxii. 265. silvarus, Urquhart. T.N.Z.I. xxiv. 252. 
Trite, Simon, $188_{5}$.

planiceps, Simon. Zool. Jahrb. Syst. xii. 430.

binotata, Simon. Zool. Jahrb. Syst. xii. 43 .

Triaenonyx, Sorensen, I 886.

obesus, Simon. Zool. Jahrb. Syst. xii. 43 I.

Attus, Walckenaer, I805.

abbreviatus, Walckenaer.

cookii, Walckenaer.

phrinoides, Walckenaer.

tabinus, Urquhart. T.N.Z.I. xvii. 46.

curvus, Urquhart. T.N.Z.I. xvii. 46.

furvus, Urquhart. T.N.Z.I. xvii. 47.

alpinus, Urquhart. T.N.Z.I. xvii. 48.

albo-palpis, Urquhart. T.N.Z.I. xvii. 49.

tenebricus, Urquhart. T.N.Z.I. xvii. $5 \mathrm{I}$.

auricomus, Urquhart. T.N.Z.I. xviii. 202.

zanthrofrontalis, Urquhart. T.N.Z.I. xviii. 203.

var. hirtus, Urquhart. T.N.Z.I. xviii. 204.

saxatilis, Urquhart. T.N.Z.I. xviii. 204.

aquilus, Urquhart. T.N.Z.I. xix. I 15.

bimaculosus, Urquhart. T.N.Z.I. xix. I 6.

subfuscus, Urquhart. T.N.Z.I. xix. I I 7 .

pullus, Urquhart. T.N.Z.I. xxii. $26_{3}$.

scindus, Urquhart. T.N.Z.I. xxii. 264.

montinus, Urquhart. T.N.Z.I. xxiii. I 84 .

monticola, Urquhart. T.N.Z.I. xxiii. I86.

valentulus, Urquhart. T.N.Z.I. xxiii. I 87.

ravus, Urquhart. T.N.Z.I. xxv. I 85 .

suffusus, Urquhart. T.N.Z.I. xxv. I86.

kirkii, Urquhart. T.N.Z.I. xxv. I 87.

tenebrosus, Urquhart. T.N.Z.I. xxv. I 88. adustus, Urquhart. T.N.Z.I. xxv. 189.

Order ACARINA.

Family IXODIDE.

Ixodes, Latreille, 1796.

eudyptidis, Maskell. T.N.Z.I. xvii. I9.

maskelli, Kirk. 'T.N.Z.I. xix. 65.

apteridis, Maskell. T.N.Z.I. xxix. 29I.

aptericola, Maskell. T.N.Z.I. xxix. 292.

intermedius, Neumann. Mem. Soc. Zool. France, r 899, I 32.

Alloptes, Canestrini, I 879.

mucronatus, Trouessart. Bull. Soc. Angers, r $899,58$.

Faculifer,

spinosus, Trouessart. Bull. Soc. Angers, I 899, I 7. 
Eupterolicus, Canestrini and Kramer, I 899.

favetti, Trouessart. Bull. Soc. Angers, 1899, 6.

Falciger, Trouessart, I 884 and I 885 .

spinosus, Trouessart. Bull. Soc. Ent. France, I895, 32 I.

Pterolichus, Robin, I 868.

favettei, Trouessart, Bull. Soc. Ent. France, $1898,290$.

Family HaLACARIDE.

Halacarus, Gosse, I 855.

parvus, Chilton. T.N.Z.I. xv. I 90.

truncipes, Chilton. T.N.Z.I. xv. I9I.

Family Hydrachnidæ.

Eylais, Latreille, I 796.

Koenike, Zool. Jahrb. xiii. 125 (1900).

Family GamasidÆ.

Uropoda, Latreille, r 806.

vegetans, De Geer. Maskell, T.N.Z.I. xxr. 201 ; Smith, T.N.Z.I. xxxiv. 199.

Leiosoma, Nicolet, I 855 .

longipilis, Moniez. Smith, T.N.Z.I. xxviii. 470.

Order Pedipalpida.

Family Phalanginde.

Dasylobus, Simon, 1879 .

australis, Simon. Zool. Jahrb. Syst. xii. 437 .

Phrynus, Latreille, I802.

Family Phrynide.

listeri, White. P.Z.S. I $849,6$.

cheliferoides, Colenso. T.N.Z.I. xv. I66.

Order PsEudosCoR PIONIDA.

Family Chernetide.

Chelifer, Geoffroy, 1764 .

pallipes, White. P.Z.S. 1849,6 .

Order Pycnogonida. Family NYMPHONIDE.

Sub-family NyMPHONINÆ.

Nymphon, Fabricius, 1837 . compactum, Hoek. Thomson, T.N.Z.I. xvi. 243 longicoxa, Hoek. Thomson, T.N.Z.I. xvi. 243. 
Sub-family CoLossendeinÆ.

Ammothea, Leach, I8I4.

dohrni, Thomson. T.N.Z.I. xvi. 243.

magnicipes, Thomson. T.N.Z.I. xvi. 245 .

Oorhynchus, Hoek, 188 I.

aucklandiæ, Hoek. Thomson, T.N.Z.I. xvi. 245.

Pallene, Johnston, I 837 .

Sub-family PaLleninæ.

novæ-zealandiæ, Thomson. T.N.Z.I. xvi. 246.

Phoxichilidium, Johnston, 1839.

obliquum, Thomson. T.N.Z.I. xvi. 247 .

Order TARDIGRADA.

This order is represented in New Zealand, but no species have as yet been described.

\section{CLASS, CRUSTACEA. \\ Sub-class Malacostraca. \\ Order DECAPODA. \\ Sub-order BRACHYURA. \\ Section MaIOIDEA. \\ Family INACHIDE.}

Stenorhynchus, Lamarck, I8 8 .

fissifrons, Haswell. P.L.S. N.S.W. iii. 409 and 432 .

Trichoplatus, A. Milne-Edwards, 1876 .

huttoni, A. Milne-Edwards. Miss. de l'Ile Campbell, $35^{2}$. = Halimus hectori, Miers. Cat. N.Z. Crust. 4 .

Halimus, Latreille, i 825 .

rubiginosus, Kirk. T.N.Z.I. xiii. 236.

Family Mande.

Paramithrax, Milne-Edwards, I 834 . peronii, Milne-Edwards. Cat. N.Z. Crust. 5. latreilli, Miers $=C$. barbicornis, Miers. Cat. N.Z. Crust. $6=$ C. cristatus, Filhol. Miss. de l'Ile Campbell, $35^{8}$. sternocostulatus, A. Milne-Edwards $=P$. gaimardii, Miers. Cat. N.Z. Crust. 6 ; Cat. Austral. Crust. I 3. minor, Filhol. Miss. de l'Ile Campbell, 356. longipes, G. M. Thomson. A.M.N.H. 7 , x. 36 I.

Leptomithrax, Miers, 1876 .

australis, Jacquinot and Lucas. Cat. N.Z. Crust. 7. longimanus, Miers. Cat. N.Z. Crust. 8. 
Acantophrys, A. Milne-Edwards, 1876.

filholi, A. Milne-Edwards. Miss. de l'Ile Campbell, 365 .

Micippa, Leach, I8I 7 .

spinosa, Stimpson. Cat. N.Z. Crust. 9.

Family Periceride.

Prionorhynchus, Jacquinot and Lucas, I 853.

edwardsii, Jacquinot and Lucas. Cat. N.Z. Crust II ; Miss. de l'Ile Campbell, $3^{67}$.

Family PARTHENOPIDE.

Eurynolambrus, Milne-Edwards and Lucas, I 84 I.

australis, Milne-Edwards and Lucas, Cat. N.Z. Crust. I 2.

Section Cancroidea.

Cancer, Linnæus, I 766 .

Family CanCRIDE.

novæ-zealandiæ, Jacquinot and Lucas. Cat. N.Z. Crust. I4.

Heterozius, A. Milne-Edwards, I 867.

rotundifrons, A. Milne-Edwards. Cat. N.Z. Crust. $\mathbf{5}$.

Xantho, Leach, I8I5.

spinotuberculata, Lock. T.N.Z.I. xi. 396.

Leptodius, A. Milne-Edwards, I 860.

nudipes, Dana. Cat. N.Z. Crust. I 7 ; Miss. de l'Ile Campbell, 374 .

eudorus, Herbst. Cat. N.Z. Crust. I 7 ; Miss. de l'Ile Campbell, 374.

Pilumnus, Leach, I8 8 .

vespertilio, Fabricius. Cat. N.Z. Crust. 19.

tomentosus, Latreille. Cat. N.Z. Crust. 20.

novæ-zealandiæ, Filhol. Miss. de l'Ile Campbell, 375.

spinosus, Filhol. Miss. de l'Ile Campbell, 377.

Pilumnopeus, A. Milne-Edwards, I867.

serratifrons, Kinahan. Cat. N.Z. Crust. 20 ; Miss. de l'Ile Campbell, 378 .

Ozius, A. Milne-Edwards, 1834 .

truncatus, A. Milne-Edwards. Cat. N.Z. Crust. 2 I.

Panopeus, A. Milne-Edwards, I 834 .

otagoensis, Filhol. Miss. de l'Ile Campbell, 379.

Ruppellioides, A. Milne-Edwards, 1867.

convexus, A. Milne-Edwards. Cat. N.Z. Crust. 23 ; Miss. de l'Ile Campbell, 38 I. 
Portunus, Fabricius, 1798 .

Family PORTunidæ:

pusillus, Leach. T.N.Z.I. xi. 402.

Neptunus, de Haan, 1833 .

sayi, A. Milne-Edwards. Cat. N.Z. Crust. 24.

pelagicus, Linnæus. Cat. N.Z. Crust. 25.

Thalamita, Latreille, r 829.

sima, A. Milne-Edwards. Cat. N.Z. Crust. 28.

Nectocarcinus, A. Milne-Edwards, 1860.

integrifrons, Latreille. Cat. N.Z. Crust. 30.

antarcticus, Jacquinot and Lucas. Cat. N.Z. Crust. 30.

Platyonichus, Latreille, $\mathrm{I} 8_{2} 5$.

bipustulatus, A. Milne-Edwards. Cat. N.Z. Crust. 32.

ocellatus, Herbst. T.N.Z.I. ix. 473; Miss. de l'Ile Campbell, 383 .

\section{Section OcyPoDIIDEA. \\ Family OCYPODIDÆ.}

Ommatocarcinus, White, I $85^{2}$.

huttoni, Filhol. Miss. de l'Ile Campbell, 384 .

macgillivrayi, White. Miss. de l'tle Campbell, $3_{5}{ }_{5}$; Challenger Reports, xvii. 247.

Macrophthalmus, Latreille, $x 829$.

hirtipes, Jacquinot and Lucas = Cleistostoma ? hirtipes. Voy. au Pole Sud. Crust. 68 ; A.M.N.H. 7, x. 462.

Hemiplax, Heller, r865.

hirtipes, Heller. Cat. N.Z. Crust. 34; Challenger Reports, xvii. $25 \mathrm{I}$.

Gelasimus, Latreille, $x 88 \circ$.

thomsoni, Kirk. T.N.Z.I. xiii. 237.

huttoni, Filhol. Miss. de l'Ile Campbell, 386.

Helœcius, Dana, $185^{2}$.

cordiformis, A. Milne-Edwards. Cat. N.Z. Crust. 35 .

Family Grapside.

Grapsus, Lamarck, I $80 \mathrm{I}$.

pictus, Latreille, Cat. N.Z. Crust. $3^{6}$; Miss. de l'Ile Campbell, 387 .

Leptograpsus, Milne-Edwards, I 853 .

variegatus, Fabricius. Cat. N.Z. Crust. 36 ; Challenger Reports, xvii. 257.

Heterograpsus, Lucas, 1849 .

sexdentatus, Milne-Edwards. Cat. N.Z. Crust. 37. 
Heterograpsus crenulatus, Milne-Edwards. Cat. N.Z. Crust. 38 .

maculatus, Milne-Edwards. Cat. N.Z. Crust. 39; Miss. de

l'Ile Campbell, 389 .

Nautilograpsus, A. Milne-Edwards, r837. (=Planes, Leach.) minutus, Linnæus. Cat. N.Z. Crust. 39; Challenger Reports, xvii. 254 .

Cyclograpsus, Milne-Edwards, i 837.

lavauxi, Milne-Edwards. Cat. N.Z. Crust. $4 \mathrm{I}$.

Chasmagnathus, de Haan, I 835 .

subquadratus, Dana. Cat. N.Z. Crust. 42.

lævis, Dana. Cat. N.Z. Crust. 42.

Helice, de Haan, 1835 .

crassa, Dana. Cat. N.Z. Crust. 43.

lucasi, A. Milne-Edwards. Cat. N.Z. Crust. 44; Miss. de l'Ile Campbell, 39I.

Sesarma, Say, i 8 r 7 .

pentagona, Hutton. T.N.Z.I. vii. 279.

Plagusia, Latreille, 1806.

chabrus, Linnæus. Cat. N.Z. Crust. 45; Miss. de l'Ile Campbell, 393.

Leiolophus, Miers, $\mathrm{r} 876$.

planissimus, Herbst. Cat. N.Z. Crust. 46 ; Miss. de l'Ile Campbell, 394 .

Family Pinnotheride.

Pinnotheres, Latreille, 1803. pisum, Linnæus. Cat. N.Z. Crust. 48. novæ-zealandiæ, Filhol. Miss. de l'Ile Campbell, 395.

Halicarcinus, White, $r 846$.

planatus, Fabricius. Cat. N.Z. Crust. 49; Challenger Reports, xvii. $28 \mathrm{I}$; P.Z.S. I 900, 524.

tridentatus, Jacquinot and Lucas. Miss. de l'Ile Campbell, 396.

huttoni, Filhol. Miss. de l'Ile Campbell, 398.

Hymenicus, Dana, 1852 .

varius, Dana. Cat. N.Z. Crust. 50.

pubescens, Dana. Cat. N.Z. Crust. 5 r.

depressus, Jacquinot and Lucas. Cat. N.Z. Crust. 5 I.

edwardsii, Filhol. Miss. de l'Ile Campbell, 400. cooki, Filhol. Miss. de l'Ile Campbell, $40 \mathrm{r}$. haasti, Filhol. Miss. de l'Ile Campbell, 402. marmoratus, Chilton. T.N.Z.I. xiv. I 72. 
Hymenosoma, Leach, 1825 .

lacustris, Chilton. T.N.Z.I. xv. 69; Fulton and Grant, Proc. R.S. Vict. xv. (New Series), 59.

Elamena, Milne-Edwards, 1837 .

quoyi, Milne-Edwards. Cat. N.Z. Crust. 52 ; Miss. de l'Ile Campbell, $4 \circ 3$.

whitei, Miers. Cat. N.Z. Crust. 52 ; Miss. de l'Ile Campbell, 403.

longirostris, Filhol. Miss. de l'Ile Campbell, 403. kirki, Filhol. Miss. de l'Ile Campbell, 405.

producta, Kirk. T.N.Z.I. xi. 395; Miss de l'Ile Campbell, 404 .

Section Leucosindea. Family LEUCOSIIDE.

Ebalia, Leach, I 8 I $7 . \quad$ (=Phlyxia, Bell.)

lævis, Bell. Cat. N.Z. Crust. 56 ; Challenger Reports, xvii. 306.

cheesemani, Filhol. Miss. de l'Ile Campbell, 407.

tumefacta, Kirk. T.N.Z.I. xi. 395.

tuberculosa, A. Milne-Edwards. Challenger Reports, xvii. 306.

Sub-order ANomura.

Group DROMIDEA.

Family Dromide.

Cryptodromia, Stimpson, I 858.

lateralis, Gray. T.N.Z.I. xxxi. I 70.

Group PAgURIDEA.

Family PAGURIDE.

Clibanarius, Dana, I $85 \mathrm{I}$.

cruentatus, Milne-Edwards. T.N.Z.I. xxxi. 172.

barbatus, Heller. T.N.Z.I. xxxi. I 72.

Eupagurus, Brandt, $185 \mathrm{I}$.

novæ-zealandiæ, Dana. T.N.Z.I. xxxi. I 73 .

kirkii, Filhol. T.N.Z.I. xxxi. 175.

cookii, Filhol. T.N.Z.I. xxxi. I 76 .

hectori, Filhol. T.N.Z.I. xxxi. I 77 .

lacertosus, Henderson. T.N.Z.I. xxxi. 178.

traversi, Filhol. T.N.Z.I. xxxi. I 79.

stewarti, Filhol. T.N.Z.I. xxxi. I 80.

rubricatus, Henderson. T.N.Z.I. xxxi. I 80.

spinulimanus, Miers. T.N.Z.I. xxxi. I 8 I.

edwardsi, Filhol. T.N.Z.I. xxxi. I 82.

campbelli, Filhol. T.N.Z.I. xxxi. I83.

thomsoni, Filhol. T.N.Z.I. xxxi. I 83 .

cristatus, Milne-Edwards. T.N.Z.I. xxxi. I 84. 
Aniculus, Dana, $185 \mathrm{I}$.

typicus, Dana. T.N.Z.I. xxxi. I 84 .

Stratiotes, Thomson, 1897 .

setosus, Filhol. T.N.Z.I. xxxi. 185.

Paguristes, Dana, $185 \mathrm{I}$.

pilosus, A. Milne-Edwarḍs. T.N.Z.I. xxxi. I87.

subpilosus, Henderson. Challenger Reports, xxvii. 77 ; T.N.Z.I. xxxi. I 87 .

Family Parapaguride.

Parapagurus, S. J. Smith, I $\$ 79$.

latimanus, Henderson. Challenger Reports, xxvii. 9I ; T.N.Z.I, xxxi. I 87.

Pagurodes, Henderson, r 888.

inarmatus, Henderson. Challenger Reports, xxvii. 94; T.N.Z.I. xxxi. 187 .

Genus incerte sedis.

Porcellanopagurus, Filhol, I $8 \delta_{5}$.

edwardsi, Filhol. T.N.Z.I. xxxi. I 87.

Group Galatheidea.

Family PORCELlanide.

Petrolisthes, Stimpson, I 858 .

elongatus, Milne-Edwards. T.N.Z.I. xxxi. I 89.

novæ-zealandiæ, Filhol. T.N.Z.I. xxxi. I 90.

Petrocheles, Miers, 1876 .

spinosus, Miers. T.N.Z.I. xxxi. I9I.

Porcellana, Lamarck, i 80 I.

rupicola, Stimpson. T.N.Z.I. xi. 396.

Family Galatheide.

Galathea, Fabricius, I793. pusilla, Henderson. T.N.Z.I. xxxi. I93.

Munida, Leach, r 820 . subrugosa, Henderson. Challenger Reports, xxvii. I 24 ;

T.N.Z.I. xxxi. I 94.

gracilis, Henderson. T.N.Z.I. xxxi. I96.

microphthalma, A. Milne-Edwards. T.N.Z.I. xxxi. 196.

Elasmonotus, A. Milne-Edwards, I88o. marginatus, Henderson. T.N.Z.I. xxxi. 196.

Uroptychus, Henderson, I 888.

spinimarginatus, Henderson. T.N.Z.I. xxxi. 196. politus, Henderson. T.N.Z.I. xxxi. I 96.

australis, Henderson. T.N.Z.I. xxxi. 197. 
Callianassa, Leach, I8 14 .

Sub-order Macrura.

Division TRICHOBRANCHIATA.

Group Aeerrantia.

Family Callianasside.

sp. indef, Kirk. T.N.Z.I. $40 \mathrm{I}$.

filholi, A. Milne-Edwards. Mission de l'Ile Campbell, 429.

Upogebia, Leach, $\mathbf{1} 8 \mathrm{I} 4=$ Gebia, Leach.

danai, Miers. Cat. N.Z. Crust. 70; G. hirtifrons, Kirk, T.N.Z.I. xi. 401.

Family AxIIDE.

Eiconaxius, Spence Bate, r 888.

kermadeci, Spence Bate. Challenger Reports, xxiv. 43.

parvus, Spence Bate. Challenger Reports, xxiv. 44.

Group Normalia.

Tribe Synaxidea.

Family SCYLLARIDÆ.

Ibacus, Leach, 1815 .

alticrenatus, Spence Bate. Challenger Reports, xxiv. 63 .

Family PaLINURIDE.

Jasus, T. J. Parker, $\mathbf{1} 88_{3}=$ Palinostylus, Spence Bate.

edwardsi, Hutton. T.N.Z.I. vii. 279 ; Parker, T.N.Z.I, xvi. 297 , and xix. 150.

lalandii, M. Edwards. Cat. N.Z. Crust. 74 ; Parker, T.N.Z.I. xvi. 297 , and xix. 150.

hugelii, Heller. Cat. Aust. Crust. I $72=$ Palinurus tumidus, Kirk, T.N.Z.I. xii. 3I 4 .

Tribe Astacidea.

Family ERYONTIDE.

Polycheles, Heller, I 862.

helleri, Spence Bate. Challenger Reports, xxiv. 138.

Pentacheles, Spence Bate, I 878 .

euthrix, Willemoes-Suhm. Challenger Reports, xxiv. 149.

Family NePHROPSIDÆ.

Nephrops, Leach, 18 I 6 .

thomsoni, Spence Bate. Challenger Reports, xxiv. 185.

Family Parastacide.

Paranephrops, White, I 842 . planifrons, White. Chilton, T.N.Z.I. xxxii. I4. zealandicus, White. Chilton, T.N.Z.I. xxxii. 15. setosus, Hutton. Chilton, T.N.Z.I. xxxii. I6. 


\section{Division Dendrobranchiata.}

Family PENÆIDÆ.

? Philonicus, Spence Bate, 1888.

? lucasii, Spence Bate. Ann. Mag. Nat. Hist. 5, viii. I85; Challenger Reports, xxiv. 277.

Haliporus, Spence Bate, I88r.

obliquirostris, Spence Bate. Challenger Reports, xxiv. 286.

Hemipenæus, Spence Bate, I88r.

semidentatus, Spence Bate. Challenger Reports, xxiv. 305.

Benthesicymus, Spence Bate, I88I.

brasiliensis, Spence Bate. Challenger Reports, xxiv. $33^{2}$.

iridescens, Spence Bate. Challenger Reports, xxiv. 335 .

altus, Spence Bate. Challenger Reports, xxiv. 336.

Family Sergestide.

Sergestes, Milne-Edwards, 1830.

kröyeri, Spence Bate. Challenger Reports, xxiv. 388.

? parvidens, Spence Bate. Challenger Reports, xxiv. 409.

Division Phyllobranchiata.

Tribe Crangonidei.

Family Crangonide.

Pontophilus, Leach, I 8 I 7 .

australis, Hutton. T.N.Z.I. xi. 23 I ; T.L.S. 2, viii. 434.

gracilis, Spence Bate. Challenger Reports, xxiv. 487 .

Tribe Polycarpidea.

Family NiKIDA.

Glyphocrangon, A. Milne-Edwards, i 884. regalis, Spence Bate. Challenger Reports, xxiv. 5 I 7 .

Family AlPHEID

Alpheus, Fabricius, 1778 .

socialis, Heller. Voy. Novara, Crust. pl. Io6; Cat. N Z.

Crust. 82 ; T.L.S. 2 , viii. 436.

novæ-zealandiæ, Miers. Cat. N.Z. Crust. 82.

halesii, Kirk. T.N.Z.I. xix. 194.

Betæus, Dana, i 852 .

æquimanus, Dana. Cat. N.Z. Crust. 83 ; T.L.S. 2 , viii. 438 .

Alope, White, I 848.

Family HipPolytide.

palpalis, White. Cat. N.Z. Crust. 84 ; T.L.S. 2, viii. 440.

Hippolyte, Leach, I8I5.

bifidirostris, Miers. Cat. N.Z. Crust. 8 I (Virbius); T.L.S. 2, viii. 443 . 
Merhippolyte, Spence Bate, I 888.

spinifrons, Milne-Edwards. Cat. N.Z. Crust. So (Hippolyte ,

T.L.S. 2, viii. 444 .

Nauticaris, Spence Bate, 1888 .

stewarti, G. M. Thomson. T.N.Z.I. xxi. 259 (Hippolyte); T.L.S. 2, viii. 445 .

Family Pandalide.

Plesionika, Spence Bate, I 888.

semilævis, Spence Bate. Challenger Reports, xxiv. 644.

Pandalus, Leach, I 8 I 7 .

magnoculus, Spence Bate. Challenger Reports, xxiv. 667.

Tribe MoNOCARPIDEA.

Family ATYID五.

Xiphocaris, v. Martens, I 872.

curvirostris, Heller. Cat. N.Z. Crust. 78 (Caridina); T.L.S.

2, viii. 447 .

Family PaLemonide.

Acanthephyra, A. Milne-Edwards, I88I.

sica, Spencer Bate. Challenger Reports, xxiv. 739.

brachytelsonis, Spencer Bate. Challenger Reports, xxiv.

753 .

Palæmon, Fabricius, I 798.

affinis, A. Milne-Edwards. Cat. N.Z. Crust. 85.

natator, A. Milne-Edwards. Cat. N.Z. Crust. 86.

Bithynis, Philippi, I860.

ornatus, Olivier. Cat. N.Z. Crust. 87.

Brachycarpus, Spence Bate, I 888.

audouini, Spence Bate. Challenger Reports, xxiv. 798.

Nematocarcinus, A. Milne-Edwards, I 884.

undulatipes, Spence Bate. Challenger Reports, xxiv. 8or .

gracilis, Spence Bate. Challenger Reports, xxiv. 8I5.

serratus, Spence Bate. Challenger Reports, xxiv. 8 I9.

hiatus, Spence Bate. Challenger Reports, xxiv. 82 I.

Family Stylodactylide.

Stylodactylus, A. Milne-Edwards, ${ }^{2} 88_{3}$.

discissipes, Spence Bate. Challenger Reports, xxiv. 85 I.

orientalis, Spence Bate. Challenger Reports, xxiv. 854 .

Order Schizopoda.

Family EUPHAUSIIDE.

Nyctiphanes, Sars, 1883 .

australis, Sars. Thomson, J.L.S. Zool. xxvii. 485 . 
Siriella, Dana, I 850 .

Family Mysıde.

denticulata (Thomson). J.L.S. Zool. xxvii. 482 .

Tenagomysis, Thomson, 1900.

novæ-zealandiæ, Thomson. J.L.S. Zool. xxvii. 484 .

Order Stomatopoda.

Squilla, Fabricius, I 793 .

Family SQUillidæ.

nepa, Latreille. Chilton, T.N.Z.I. xxiii. 60.

armata, A. Milne-Edwards. Chilton, T.N.Z.I. xxiii. 60.

Protosquilla, Brooks, 1886.

trispinosa, White. Chilton, T.N.Z.I. xxiii. 61.

Lysiosquilla, Latreille, 1825 .

spinosa, Wood-Mason. Chilton, T.N.Z.I. xxiii. 6I

Order Cumacea.

Family Cumidæ.

Cyclaspis, Sars, 1864 .

lævis, G. M. Thomson. J.L.S. Zool. xxiv. 264 .

Diastylis, Say, I 8 I 8 .

Family Diastylide.

neo-zealanica, G. M. Thomson. J.L.S. Zool. xxiv. 268.

Order Edriophthalmata.

Sub-order AMPHIPODA.

Tribe HyPERIDEA.

Vibilia, A. Milne-Edwards, 1830.

Family VIBILIDÆ.

propinqua, Stebbing. Challenger Reports, xxix. I279; Chilton, T.N.Z.I. xxiv. 261 .

Family Cystrsomide.

Cystisoma, Guérin-Méneville, 1842.

spinosum (Fabricius). Stebbing, Challenger Reports, xxix. I33I.

Phronima, Latreille, I803.

Family Phronimide.

novæ-zealandiæ, Powell. T.N.Z.I. vii. 294; Stebbing,

Challenger Reports, xxix. 1356.

Family HYPERIDE.

Euthemisto, Bovallius, I 887.

thomsoni, Stebbing (= Themisto antartica, Thomson); Challenger Reports, xxix. 1414 ; Chilton T.N.Z.I. xxiv. 263 . 
Family Phrosinid残.

Anchylomera, Milne-Edwards, 1830.

blossevillei, Milne-Edwards. Stebbing, Challenger Reports, xxix. 1433 .

Primno, Guérin-Méneville, I 836.

latreillei, Stebbing. Challenger Reports, xxix. 1445 .

Family Typhide.

Platyscelus, Spence Bate, I86r.

intermedius, G. M. Thomson. T.N.Z.I. xi. 244 ; Stebbing,

Challenger Reports, xxix. 1464 .

Family PRONOIDE.

Parapronoë, Claus, I879.

clausoides, Stebbing. Challenger Reports, xxix. 1529.

Family TRYPHanide.

Brachyscelus, Spence Bate, I86r.

crusculum, Spence Bate. Stebbing, Challenger Reports, 1547.

Family OXYcePhaLIDE.

Oxycephalus, Milne-Edwards, 1830.

edwardsii, G. M. Thomson. T.N.Z.I. xvi. 238 ; Stebbing, Challenger Reports, xxix. $15^{8} 5$.

Tribe Gammaridea.

Family ORCHESTIDE.

Orchestia, Leach, I 813 .

gammarellus, Pallas. Thomson, T.N.Z.I. xxxi. I99.

chiliensis, Milne-Edwards. Thomson, T.N.Z.I. xxxi. I99.

telluris, Spence Bate. Thomson, T.N.Z.I. xxxi. 200.

aucklandiæ, Spence Bate. Thomson, T.N.Z.I. xxxi. 20 I.

quoyana, A. Milne-Edwards. Thomson, T.N.Z.I. xxxi. 202. tumida, Thomson. T.N.Z.I. xxxi. 203.

sylvicola, Dana. Thomson, T.N.Z.I. xxxi. 203.

Hyale, Rathke, I837.

prevostii, Milne-Edwards. Thomson, T.N.Z.I. xxxi. 204.

pontica, Rathke. Thomson, T.N.Z.I. xxxi. 205.

lubbockiana, Spence Bate. Thomson, T.N.Z.I. xxxi. 206.

chiltoni, G. M. Thomson. T.N.Z.I. xxxi. 206.

Chiltonia, Stebbing, I 899.

mihiwaka, Chilton. Stebbing, T. Linn. Soc. Zool. vii. 408 ;

Hyalella mihizeraka, Thomson, T.N.Z.I. xxxi. 207.

Ceina, Della Valle, I 893 .

egregia, Chilton. Thomson, T.N.Z.I. xxxi. 207. 
Anonyx, Kröyer, 1838 .

Family Lysianasside.

exiguus, Stimpson.

G. M. Thomson, T.N.Z.I. xiv. 232.

Lysianax, Stebbing, 1888 (substitute for Lysianassa, MilneEdwards, pre-occupied).

kröyeri, Spence Bate. G. M. Thomson, T.N.Z.I. xi. 237.

Amaryllis, Haswell, I880.

macrophthalmus, Haswell. Thomson, A.M.N.H. 7, x. 463.

Andaniotes, Stebbing, I 897 .

corpulentus, Thomson. Stebbing, T. Linn. Soc. Zool. vii. 3 r.

Family AмPнILOCHIDÆ.

Amphilochus, Spence Bate, I862.

squamosus, Thomson. T.N.Z.I. xiii. $2 \times 4$.

Cyproidia, Haswell, r880.

otakensis, Chilton. A.M.N.H. 7, v. 24 r.

Tetradeion, Stebbing, I 899 .

crassa, Chilton. Stebbing, A.M.N.H. 7, iv. 207; Cyproidia

? crassa, Chilton, T.N.Z.I. xv. 80.

Family LeUCOTHOIDÆ.

Leucothoë, Leach, 1813 .

trailii, Thomson. T.N.Z.I. xiv. 234 .

tridens, Stebbing. Challenger Reports, xxix. 777 .

Seba, Spence Bate, r862.

saundersii, Stebbing. Chilton, T.N.Z.I. xxiv. 260.

Family PONTOPOREIIDE.

Phoxocephalus, Stebbing, I 888.

bassi, Stebbing. Thomson, A.M.N.H.

Parharpinia, Stebbing, 1899 .

villosus, Haswell. Stebbing, A.M.N.H. 7, iv. 207 ; Phoxus batei, G. M. Thomson, T.N.Z.I. xiv. 232.

Platyischnopus, Stebbing, I 888.

neo-zelanicus, Chilton. A.M.N.H. 6, xix. I.

Family EDICERIDE.

Ediceros, Kröyer, I 842. novæ-zealandiæ, Dana. Miers, Cat. N.Z. Crust. i 26.

Pleustes, Spence Bate, I 858.

Family Pleustide.

panoplus, Kröyer. Kirk, T.N.Z.I. xi. 402. 
Family IPHIMEDIDÆ

Iphimedia, Rathke, I 843 .

spinosa, G. M. Thomson. Panoptea spmo G. M. Thomson, T.N.Z.I. xiii. $2 \mathbf{r}_{3}$.

Family ATYLIDæ.

Halirages, Boeck, 1870 .

debilis, Thomson. Stebbing, Challenger Reports, xxix. 524 ;

Panoplea debilis, Thomson, T.N.Z.I. xiii. 2 I 3.

translucens, Chilton. Stebbing. Challenger Reports, xxix.

55I ; Panoptca translucens, Chilton, T.N.Z.I. xvi. 263.

Atylus, Leach, i 8 I 5 .

danai, Thomson. T.N.Z.I. xi. 238.

Tritaeta, Boeck, I 876 .

Family DexaminidÆ.

antarctica, Stebbing. Challenger Reports, xxix. 45I ; Polycheria obtusa, Thomson, T.N.Z.I. xiv. 233.

Paradexamine, Stebbing, I 899 .

pacifica, Thomson. Stebbing, A.M.N.H. 7, iv. 210 ; Dexamine pacifica, Thomson, T.N.Z.I. xi. 238.

levis, Thomson $=$ Amplithonotus levis, Thomson, T.N.Z.I. xiii. 215 .

\section{Family EUSIRIDÆ.}

Eusirus, Kröyer, I 845 .

longipes, Kröyer, Challenger Reports, xxix. 965.

Liljeborgia, Spence Bate, I862.

haswelli, Stebbing. Challenger Reports, xxix. 985 ; A.M.N.H. $7, x .463$.

Family CaLliopind.

Paracalliope, Stebbing, I 899.

fluviatilis, Thomson. Stebbing, A.M.N.H. 7, iv. 210 ; Calliope fluviatilis, Thomson, T.N.Z.I. xi. 240.

Paraleptamphopus, Stebbing, I 899 .

subterraneus, Chilton. Stebbing, A.M.N.H. 7, iv. 209;

Calliopius subterraneus, Chilton, T. Linn. Soc. Zool. vi. 234. cærulea, Thomson. Stebbing, A.M.N.H. 7, iv. 209, and

T.Z.S. xii. 206.

Calliopius, Liljeborg, I 865 . didactylus, Thomson. T.N.Z.I. xi. 240.

Pherusa, Leach, I 8 I 4.

novæ-zealandiæ, Thomson. T.N.Z.I. xi. 239. 
Stenothoë, Dana, I $85^{2}$.

Family STENothoide.

miersii, Haswell. Montaguana miersii, Chilton, T.N.Z.I. xv. 79 .

adhærens, Stebbing. Chilton, T.N.Z.I. xxiv. 259.

Family Ampeliscides.

Ampelisca, Kroyër, I 842 .

chiltoni, Stebbing. Challenger Reports, xxix. 1042.

acinaces, Stebbing. Challenger Reports, xxix. г 036 .

Bircenna, Chilton, I 884.

Family Phliadioæ.

fulva, Chilton. T.N.Z.I. xvi. 264.

Iphinotus, Stebbing, r 899 .

typica, Thomson, A.M.N.H. 7, x. 464 =chiltoni, Stebbing.

T. Linn. Soc. Zool. vii. 419.

Family GammaridÆ.

Phreatogammarus, Stebbing, I 899.

fragilis, Chilton. Stebbing, T. Linn. Soc. Zool. vii. 427 ;

Gammarus fragilis, Chilton, T. Linn. Soc. Zool. vi. 227.

Mæra, Leach, I 8 I 3 .

rubromaculata, Stebbing. Challenger Reports, xxix. Ioo8, spinosa, Chilton, T.N.Z.I. xv. 8I.

fasciculata, Thomson. T.N.Z.I. xiii. 2 I 8.

quadrimana, Spence Bate. Thomson, T.N.Z.I. xiv. 235. incerta, Chilton. T.N.Z.I. xv. 83 .

haswelli, Thomson. A.M.N.H. 6, xx. 449; Stebbing, A.M.N.H. 7 , iii.

Elasmopus, Costa, I 853 .

subcarinatus, Haswell. Stebbing, Challenger Reports, xxix. ror 9 ; Chilton, T.N.Z.I. xxiv. 26 I.

Melita, Leach, I8I3.

tenuicornis, Dana. Miers, Cat. N.Z. Crust. I 27.

Paracrangonyx, Stebbing, I 899 .

compactus, Chilton. Stebbing, T. Linn. Soc. Zool. vii. 424 ;

Crangonyx compactus, Chilton, T. Linn. Soc. Zool. vi. 220.

Aora, Kröyer, 1845 .

Family РнотіDж.

typica, Kröyer. Chilton, A.M.N.H. 5, xvi. 375 .

Gammaropsis, Liljeborg, I 855 .

typica = Paranenia typica, Chilton, T.N.Z.I. xvi. 259.

dentifera, Haswell. Parancenia dentifera, Chilton, T.N.Z.I.

xvi. 260. 
Gammaropsis longimana $=$ Paranania longimana, Chilton, T.N.Z.I. xvi. 26r.

thomsoni, Stebbing. Challenger Reports, xxix. 1103.

chiltoni $=$ Mera chiltoni, Thomson, A.M.N.H. 6, xx. 447 .

Podoceropsis, Boeck, 1860.

kermadeci, Stebbing. Challenger Reports, xxix. r 109.

\section{Family PODOCERIDÆ.}

Podocerus, Leach, I 8 I 4 .

validus, Dana. Stebbing, Challenger Reports, xxix. I I 35.

hoeki, Stebbing. Challenger Reports, xxix. I 136.

frequens, Chilton. T.N.Z.I. xv. 85 .

latipes, Chilton. T.N.Z.I. xvi. $25^{8}$ (name preoccupied).

longimanus, Haswell. Chilton, T.N.Z.I. xvi. 255.

cristatus, Thomson $=$ Cyrtophium cristatum, Thomson, T.N.Z.I. xiii. 2 I 9.

Harmonia, Haswell, 1880 (name preoccupied).

crassipes, Haswell. Chilton, T.N.Z.I. xv. 82.

Family COROPHIDÆ.

Corophium, Latreille, ISoo.

contractum, Stimpson. Thomson, T.N.Z.I. xiii. 220.

crassicorne, Bruzelius. Chilton, T.N.Z.I. xviii. I42.

Paracorophium, Stebbing, 1899.

excavatum, Thomson. Stebbing, A.M.N,H. 7, iii. $24 \mathrm{I}$ and $35^{\circ}$; Corophium excavatum, Thomson, T.N.Z.I. xvi. 236.

Family?

Haplocheira, Haswell, I 880.

barbimana, Stebbing. Challenger Reports, xxix. II 77 =Corophizm barbimanum, Thomson and Chilton, T.N.Z.I. xviii. I 4.3 .

Camacho, Stebbing, I 888.

bathyplous, Stebbing. Challenger Reports, xxix. I 79.

Caprella, Lamarck, I80I.

Tribe Caprellidea.

æquilibra, Say. Stebbing, Challenger Reports, xxix. I 254. linear is, Bate. Mayer, Capr. d. G. Neapel, $5^{8}$; C. lobata, Kirk, T.N.Z.I. xi. 393 .

Caprellinopsis, Stebbing, I888 (substitute for Caprellina, Thomson, preoccupied).

longicollet, Nicolet. Mayer, Capr. d. G. Neapel, 27. 
Cyamus, Latreille, $x 796$.

Family Cyamide.

ceti, Lamarck. Chilton, T.N.Z.I. xvi. $25^{2}$.

Sub-order ISOPODA.

Tribe Chelifera.

Apseudes, Leach, I8r4.

Family APSEUDIDE.

timaruvia, Chilton. T.N.Z.I. xv. 146.

latus, Chilton. T.N.Z.I. xvi. 249.

Family TANAIDE.

Tanais, A. Milne-Edwards, I829.

novæ-zealandiæ, G. M. Thomson. T.N.Z.I. xiii. 207.

Paratanais, Dana, 1852 .

tenuis, G. M. Thomson. T.N.Z.I. xiii. 207.

Tribe Flabellifera.

Anthura, Leach, r8 8 .

Family ANTHURIDE.

flagellata, Chilton. T.N.Z.I. xiv. I 72.

affinis, Chilton. T.N.Z.I. xv. 72 ; Stebbing, Hist. Crust. 332.

Paranthura, Bate and Westwood, I 866.

costana, Bate and Westwood. Thomson, T.N.Z.I. xiv. 230.

chiltoni, Beddard. Challenger Reports, xvii. 143 .

Cruregens, Chilton, I882.

fontanus, Chilton. T. Linn. Soc. Zool. vi. 2 Ix.

Meinertia, Stebbing, I 893 .

Family Сумотнопе.

imbricata, Fabricius. Stebbing, South African Crust. 58; Ceratothoa imbricata.

lineata, = Ceratothoa lineata, Miers. Cat. N.Z. Crust. I05. huttoni, = Ceratothoa huttoni, Filhol. Miss. de I'Ile Campbell, 447 .

novæ-zealandiæ, $=$ Ceratothoa novce-zealandic, Filhol, Miss. de l'Ile Campbell, 449.

Æga, Leach, I8r 5 .

novæ-zealandiæ, Dana. T.N.Z.I. xviii. I 53 .

maorum, Filhol. Miss. de l'Ile Campbell, $45^{2}$.

Pseudæga, G. M. Thomson, I882.

punctata, Thomson. T.N.Z.I. xvi. 234.

Livoneca, Leach, I8I8.

novæ-zealandiæ, Miers. Cat. N.Z. Crust. ıo6.

stewarti, Filhol. Miss. de l'Ile Campbell, $45^{\circ}$. 
Nerocila, Leach, J 8 I 8.

macleayii, Leach. Chilton, T.N.Z.I. xxiii. 68.

traillii, Filhol. Miss. de l'Ile Campbell, 45 I.

Cirolana, Leach, I 8I 8.

rossii, Miers. Cat. N.Z. Crust. rog.

cookii, Filhol. Miss. de l'Ile Campbell, 454.

hirtipes, A. Milne-Edwards. Filhol, Miss. de l'Ile Campbell, 455 .

Serolis, Leach, I8 8 .

Family SEROLide.

paradoxa, Fabricius. Beddard, Challenger Reports, xi. 23. scythei, Luitken. Beddard, Challenger Reports, xi. 40.

bromleyana, Willemoës-Suhm. Beddard. Challenger Reports, xi. 53 .

latifrons, White. Beddard, Challenger Reports, xi. 44.

Sphæroma, Latreille, iso2.

$$
\text { Family SPHÆROMIDж. }
$$

verrucauda, White. Miers, Cat. N.Z. Crust. I I I.

obtusa, Dana. Miers, Cat. N.Z. Crust. I I 2.

Exosphæroma, Stebbing, I 900.

gigas, Leach. Stebbing, P.Z.S. 1900, $55^{8}$; Spharoma gigas, Miers, Cat. N.Z. Crust. I Iо.

Cymodocella, Pfeffer, I887.

egregia, = Spharoma ? egregia, Chilton, T.N.Z.I. xxiv. 269.

Isocladus, Miers, i 876 .

armatus, A. Milne-Edwards. Miers, Cat. N.Z. Crust. 1 I 2.

spiniger, Dana. Miers, Cat. N.Z. Crust. I I3.

Cymodoce, Leach, i 8 I 4.

granulata, Miers. Cat. N.Z. Crust. I I 4.

convexa, Miers. Cat. N.Z. Crust. I I 4.

cordiforaminalis, Chilton. T.N.Z.I. xiv. 188.

bituberculata, Filhol. Miss. de l'Ile Campbell, 457.

huttoni, G. M. Thomson. Dynamene Inttoni, G. M. Thomson, T.N.Z.I. xi. 234 .

Scutuloidea, Chilton, r 882.

maculata, Chilton. T.N.Z.I. xv. 70.

Cassidina, A. Milne-Edwards, I 840.

neo-zelanica, G. M. Thomson. T.N.Z.I. xxi. 264 ; Stebbing,

P.Z.S. I 900,562 .

Amphoroidea, A. Milne-Edwards, i 840.

falcifer, G. M. Thomson. T.N.Z.I. xi. 233 .

Plakarthrium, Chilton, I882.

typicum, Chilton. T.N.Z.I. xv. 74 . 
Limnoria, Leach, I8r4.

Family LIMNORIIDE.

segnis, Chilton. T.N.Z.I. xv. 76.

Tribe VALV1Fera.

Family ARCTURIDæ.

Astacilla, Cordiner, I 795 .

tuberculatus, G. M. Thomson. Arturus tuberculatus, G. M. Thomson, T N.Z.I. xiii. 206.

Arcturus, Latreille, I804.

myops, Beddard. Challenger Reports, xvii. Ioo.

Idotea, Fabricius, 1798.

\section{Family IDOTE1DÆ.}

marina, Linnæus. Chilton, T.N.Z.I. xxii. I9 I.

festiva, Chilton. T.N.Z.I. xxii. I 92.

metallica, Bosc. Chilton, T.N.Z.I. xxii. 193.

lacustris, G. M. Thomson. Chilton, T.N.Z.I. xxiv. 263.

elongata, Miers. Chilton, T.N.Z.I. xxii. I98.

peronii, Milne-Edwards. Chilton, T.N.Z.I. xxii. r99.

stewarti, Filhol. Miss. de l'Ile Campbell, 492.

Paridotea, Stebbing, I 900.

ungulata, Pallas. Stebbing, South African Crust. 53; Idotea ungulata, Chilton, T.N.Z.I. xxii. 196.

Cleantis, Dana, I 849 .

tubicola, G. M. Thomson. Chilton, T.N.Z.I. xxir. 265 .

Iais, Bovallius, $x 886$.

Tribe Asellota.

Family AsELlide.

pubescens, Dana. Chilton, T.N.Z.I. xxiv. 266 ; Stebbing, P.Z.S. $1900,549$.

Iathrippa, Bovallius, 1886.

longicauda, Chilton. Janira longicauda, Chilton, T.N.Z.I. xvi. 250 , and xviii. 57 .

Stenetrium, Haswell, r88r.

fractum, Chilton. T.N.Z.1. xvi. 251, and xviii. 157.

Jæropsis, Koehler, r885.

neo-zelanica, Chilton. T.N.Z.I. xxiv. 267.

Munna, Kröyer, 1839.

neo-zelanica, Chilton. A.M.N.H. 6, ix. r.

Acanthomunna, Beddard, 1886.

proteus, Beddard. Challenger Reports, xvii. 47 . 
Munnopsis, M. Sars, I86I.

Family Munnopside.

gracilis, Beddard. Challenger Reports, xvii. $5 \mathrm{I}$.

Eurycope, Sars, 1864.

novæ-zealandiæ, Beddard. Challenger Reports, xvii. 6I.

Tribe Phreatoicidea.

Family Phreatoicide.

Phreatoicus, Chilton, I 882.

typicus, Chilton. T. Linn. Soc. Zool. vi. 196.

assimilis, Chilton. T. Linn. Soc. Zool. vi. I 86.

Tribe OnisCOIDEA.

Family LIGIIDE.

Ligia, Fabricius, 1798.

novæ-zealandiæ, Dana. Chilton, T. Linn. Soc. Zool viii. I07.

Family Trichoniscidæ.

Trichoniscus, Brandt, 1833 .

phormianus, Chilton. T. Linn. Soc. Zool. viii. I I 5 .

otakensis, Chilton. T. Linn. Soc. Zool. viii. I I 7.

thomsoni, Chilton. T. Linn. Soc. Zool. viii. I 8.

Haplophthalmus, Schöbl, I860.

helmsii, Chilton. T. Linn. Soc. Zool. viii. I I 9.

Tylos, Latreille, I 829 .

Family Tylide.

neo-zelanicus, Chilton. T. Linn. Soc. Zool. viii. I 20.

Scyphax, Dana, I 853.

Family ScyPhacide.

ornatus, Dana. Chilton, T. Linn. Soc. Zool. viii. I 23.

aucklandiæ, G. M. Thomson. Chilton, T. Linn. Soc. Zool. viii. 126.

Scyphoniscus, Chilton, I90I.

waitatensis, Chilton. T. Linn. Soc. Zool. viii. I 28.

Actæcia, Dana, I853.

euchroa, Dana. Chilton, T. Linn. Soc. Zool. viii. I 30.

opihensis, Chilton. T. Linn. Soc. Zool. viii. I 32.

Oniscus, Linnæus, 1767 .

Family ONISCIDE.

punctatus, G. M. Thomson. Chilton, T. Linn. Soc. Zool. viii. I 33 .

kenepurensis, Chilton. T. Linn. Soc. Zool. viii. I35.

cookii, Filhol. Chilton, T. Linn. Soc. Zool. viii. $3^{6}$. 
Philoscia, Latreille, I 804 .

pubescens, Dana. Chilton, T. Linn. Soc. Zool. viii. $\mathbf{r}_{3} 6$.

novæ-zealandiæ, Filhol. Chilton, T. Linn. Soc. Zool. viii. 138 .

Metoponorthus, Buddle-Lund, 1879 .

pruinosus, Brandt. Chilton, 'T. Linn. Soc. Zool. viii. I +1 .

\section{Family ARMadillide.}

Armadillo, Latreille, 1804 .

ambitiosus, Buddle-Lund. Chilton, T. Linn. Soc. Zool. viii. I 44 .

danæ, Heller. Chilton, T. Linn. Soc. Zool. viii. I4t.

speciosus, Dana. Chilton, T. Linn. Soc. Zool. viii. I 4 6.

rugulosus, Miers. Chilton, T. Linn. Soc. Zool. viii. I 47 .

monolinus, Dana. Chilton, T. Linn. Soc. Zool. viii. 147.

hamiltoni, Chilton. T. Linn. Soc. Zool. viii. I48.

macmahoni, Chilton. T. Linn. Soc. Zool. viii. 149.

spinosus, Dana. Chilton, T. Linn. Soc. Zool. viii. 150.

Nebalia, Leach, I 8 I 5 .

\section{Order Phyllocarida.}

longicornis, Thomson. T.N.Z.I. xiii. $22 \mathrm{I}$; Sayce, Victorian Naturalist, xviii. 15 I.

\section{Sub-class Entomostraca.}

Order BRANCHIOPODA.

Sub-order Cladocera.

Family DaPHNIDÆ.

Daphnia, Müller, I 785 .

thomsoni, G. O. Sars. Fresh-water Entom. of N.Z., Vidensk.-Selsk. Skr. $1894-5=D$. similis, Thomson, T.N.Z.I. xvi. 240.

Simocephalus, Schödeler. obtusatus, Thomson. Sars, Fresh-water Entom. of N.Z. Io; Daphnia obtusata, T.N.Z.I. xi. $26 \mathrm{I}$.

Ceriodaphnia, Dana, 1847. sublævis, Sars. Fresh-water Entom. of N.Z. I 4.

Chydorus, Leach, 18 I 9.

Family Lynceid minutus, Thomson. T.N.Z.I. xi. 262.

Alona, Baird, I 843 . eucostata, Sars. Fresh-water Entom. of N.Z. I 7. macrocopa, Sars. Fresh-water Entom. of N.Z. 20. 
Podon, Liljeborg, 1853 .

Family PoDontide.

polyphemoides, Leuckart. T.N.Z.I. xxvii. 22 I.

trisetosus, Krämer. T.N.Z.I. xxvii. 222.

Evadne, Lovén, I 835 .

aspinosa, Krämer. T.N.Z.I. xxvii. 222.

Family SIDIDE.

Penilia, Dana, I847.

pacifica, Krämer. T.N.Z.I. xxvii. 222.

Lepidurus, Leach, i 8 I 6 .

Order PhyLlopoda.

Family APODIDE.

viridis, Baird. Sayce, P.R.S. Vict. xv. n.s. $242=$ L. angasii. Sars, Description of some Australian Phyllopoda, Archiv. f. Math. og Natur. xvii. No. 2, 4,=L. kirkii, 'Thomson, T.N.Z.I. xi. $260,=$ compressus, Thomson. T.N.Z.I. xi. 260 .

Order Ostracoda.

Section PODOCOPA.

Family CYPRIDIDÆ.

Cypris, Müller, 1785 .

novæ-zealandiæ, Baird. Dieffenbach, New Zealand, ii. 268.

littoralis, Thomson. T.N.Z.I. xi. 253.

bennelong, King. Sars, Fresh-water Entom. of N.Z., Vidensk.-Selsk. Skr. I894, No. 5, 24.

sydneia, King. Sars, Fresh-water Entom. of N.Z. 27, $=$ C. ciliata, Thomson, T.N.Z.I. xi. 253 .

Cypridopsis, Brady, I868.

turgida, G. O. Sars. On Fresh-water Entom. from neighbourhood of Sydney, 62, =C. minna, King. Sars, Freshwater Entom. of N.Z. 30 .

viridis, Thomson $=$ Cypris viridis, Thomson, T.N.Z.I. xi. 253; Sars, Fresh-water Enton. of N.Z. 32.

Candonocypris, G. O. Sars, I 894.

candonoides, King. Sars, Fresh-water Entom. of N.Z. 35. assimilis, Sars. Fresh-water Entom. of N.Z. 36 .

Ilyodromus, G. G. Sars, I 894 .

stanleyanus, King. Sars, Fresh-water Entom. of N.Z. 39.

varrovillius, King. Sars, Fresh-water Entom. of N.Z. $4 \mathbf{I}$.

smaragdinus, Sars. Fresh-water Entom. of N.Z. 43.

substriatus, Sars. Fresh-water Entom. of N.Z. 45.

obtusus, Sars. Fresh-water Entom. of N.Z. 46. 
Paracypris, G. O. Sars, 1865. polita, Sars. Challenger Reports, i. 32.

Phlyctenophora, G. S. Brady, I880. zealandica, Brady. Challenger Reports, i. 33.

Cyprinotus, G. S. Brady, I885.

flavescens, Brady. T.Z.S. xiv. 440.

Aglaia, G. S. Brady, I 867.

clavata, Brady. Challenger Reports, i. 34 .

Macrocypris, G. S. Brady, I 868.

tumida, Brady. Challenger Reports, i. 43.

Bairdia, M'Coy, I 844 .

? ovata, Bosquet. Challenger Reports, i. 53.

Cythere, Müller, I 785 .

\section{Family CYTHERIDE.}

atra, Thomson. T.N.Z.I. xi. 254.

murrayana, Brady. Challenger Reports, i. 69.

rastromarginata, Brady. Challenger Reports, i. 83 .

arata, Brady. Challenger Reports, i. Io ı.

dasyderma, Brady. Challenger Reports, i. 105.

scutigera, Brady. Challenger Reports, i. rog.

brunnea, Brady. T.Z.S. xiv. 442.

innominata, Brady. T.Z.S. xiv. $443=C$. truncata,

Thomson, T.N.Z.I. xi. 254 .

truncula, Brady. T.Z.S. xiv. 444.

Krithe, Brady, Crosskey, and Robertson, I 874.

producta, Brady. Challenger Reports, i. I I4.

Loxoconcha, G. O. Sars, 1865 .

punctata, Thomson. T.N.Z.I. xi. 255.

Xestoleberis, G. O. Sars, 1865 .

luxata, Brady. T.Z.S. xiv. $44 \mathrm{I}$.

olivacea, Brady. T.Z.S. xiv. 442.

compressa, Brady. T.Z.S. xiv. 442.

Cytheropteron, G. O. Sars, I 865 .

wellingtoniense, Brady. Challenger Reports, i. I 36.

Trachyleberis, G. S. Brady, I 898 .

scabrocuneata, Brady. Challenger Reports, i. I03;

T.Z.S. xiv. 444.

Cytherideis, Jones, 1856 .

novæ-zealandiæ, Brady. T.Z.S. xiv. 446.

Sclerochilus, G. O. Sars, I 865 .

contortus, Norman. Challenger Reports, i. I47. 
Section Myodocopa.

Family CYPRIDINIDE.

Crossophorus, G. S. Brady, I880.

imperator, Brady. Challenger Reports, i. ${ }_{5} 8$.

Philomedes, Liljeborg, i 853 .

agilis, Thomson. T.N.Z.I. xi. 257 .

sculpta, Brady. T.Z.S. xiv. 434 .

flexilis, Brady. T.Z.S. xiv. 435 .

Asterope, Philippi, 1840 .

australis, Brady. T.Z.S. xiv. 43 $\mathrm{I}$.

quadrata, Brady. T.Z.S. xiv. 432 .

grisea, Brady. T.Z.S. xiv. 432.

Cyclasterope, G. S. Brady, 1897.

ovulum, Brady. T.Z.S. xiv. 432

tenera, Brady. T.Z.S. xiv. 433 .

zealandica, Baird. T.Z.S. xiv. $433=$ Cypredina zealanica,

Baird. P.Z.S. 1850,255 .

Family SARSIELLIDÆ

Sarsiella, Norman, 1869 .

hanseni, Brady. T.Z.S. xiv. $43^{8}$.

hispida, Brady. T.Z.S. xiv. 439 .

Section Platycopa.

Family Cytherellida.

Cytherella, Jones, 1849 .

polita, Brady. Challenger Reports, i. 72

pulchra, Brady. Challenger Reports, i. I 74

punctata, Brady. Challenger Reports, i. 174.

eburnea, Brady. T.Z.S. xiv. 447 .

Order Copepoda.

Section Gnathostoma.

Family Calanide.

Calanus, Leach, r816.

finmarchicus, Günner. T.Z.S. xv. 32 .

Paracalanus, Boeck, 1864.

parvus, Claus. 'T.N.Z.I. xxvii. 2 I 7 ; T.Z.S. xv. 32.

Clausocalanus, Giesbrecht, r 888.

arcuicornis, Dana. T.N.Z.I. xxvii. 217 ; T.Z.S. xv. $3^{2}$ furcatus, Brady. T.Z.S. xv. 32 .

Euchæta, Philippi, 1843 .

marina, Prestandrea. Pelagischen Copepoden des Golfes von Neapel, $245=E$. prestandrece, Philippi. 
Family Centropagire.

Centropages, Kröyer, I 849.

typicus, Kröyer, var. aucklandicus, Krämer. T.N.Z.I. xxvii. 2 I 7.

discaudatus, Brady. T.Z.S. xv. 35 . pectinatus, Brady. T.Z.S. xv. 36 .

Deguernea, Thomson, $1902=$ Guernea, Thomson, 1895 . antarctica, Thomson. T.N.Z.I. xxvii. 213.

Temora, Baird, 1850 .

turbinata, Dana. T.N.Z.I. xxvii. 218.

tenuicauda, Brady. T.Z.S. xv. 34 .

? stylifera, Dana $=T$. dubia, Lubbock.

Leuckartia, Claus, 1863.

n. sp. Krämer. T.N.Z.I. xxvii. 2 1 8.

Boeckella, Guerne and Richard = Boeckia, Thomson.

triarticulata, Thomson. T.N.Z.I. xv. 94.

Candace, Dana, 1846.

Family Candacide.

pectinata, Brady.

pachydactyla, Dana. U.S. Expl. Exp. Crust. I I 3.

Labidocera, Lubbock, I 853 .

Family PONTELLIDE.

cervi, Krämer. T.N.Z.I. xxvii. 218 ; T.Z.S. xv. 37.

simplex, Dana = Pontellina simplex, Dana. U.S. Expl. Exp.

Crust. I I 45.

Acartia, Dana, 1846 .

clausii, Giesbrecht. T.N.Z.I. xxvii. 2 I9.

ensifera, Brady. T.Z.S. xv. 33 .

Oithona, Baird, 1843 .

Family CyclopıdÆ.

nana, Giesbrecht. T.N.Z.I. xxvii. 2 I 9 .

spinifrons, Boeck. T.Z.S. xv. 38 .

Thorellia, Boeck, I864.

brunnea, Boeck, var. antartica, Thomson. T.N.Z.I. xv. 95 .

Cyclops, Müller, 1776 .

gigas, Claus. T.N.Z.I. xv. $9^{6 .}$

novæ-zealandiæ, Thomson. T.N.Z.I. xi. 258.

serrulatus, Fischer. T.N.Z.I. xv. 96.

chiltoni, Thomson. T.N.Z.I. xv. 97.

æquoreus, Fischer. T.N.Z.I. xv. 97.

ewarti, Brady. T.Z.S. xv. 38 . 
Ectinosoma, Boeck, I864.

Family HaRPaCticide.

australe, Brady. T.Z.S. xv. 39.

Euterpe, Claus, $\mathrm{x} 86_{3}$.

gracilis, Claus. T.Z.S. xv. 39 .

acutifrons, Dana. T.N.Z.I. xxvii. 219.

Amymome, Claus, 1863 .

clausii, Thomson. T.N.Z.I. xv. 98 ; T.Z.S. xv. 40.

Laophonte, Philippi, I 840.

australasica, Thomson. T.N.Z.I. xv. IOI.

serrata, Claus. T.Z.S. xv. 40.

meinerti, Brady. T.Z.S. xv. 40.

Diarthrodes, Thomson, 1882.

novæ-zealandiæ, Thomson. T.N.Z.I. xv. 99.

Merope, Thomson, I 882 .

hamata, Thomson. T.N.Z.I. xv. 100.

Dactylopus, Claus, 1863 .

tisboides, Claus. T.N.Z.I. xv. 102 ; T.Z.S. xv. $4 \mathrm{I}$.

hanseni, Brady. T.Z.S. xv. 4I.

Xouthous, Thomson, I 882 .

novæ-zealandiæ, Thomson. T.N.Z.I. xv. I03.

Flavia, Brady, I 899 .

crassicornis, Brady. T.Z.S. xv. 42.

Phroso, Brady, I 899.

gracilis, Brady. T.Z.S. xv. $4^{2}$.

Thalestris, Claus, 1863 .

forficula, Claus. T.N.Z.I. xv. I04; T.Z.S. xv. 43.

ciliata, Brady. T.Z.S. xv. 43 .

australis, Brady. T.Z.S. xv. 43 .

Harpacticus, Milne-Edwards, 1838 .

chelifer, Müller. T.N.Z.I. xv. 105.

glaber, Brady. T.Z.S. xv. 44.

Zaus, Goodsir, $\mathbf{1} 845$.

contractus, Thomson. T.N.ZI. xv. 106 ; xxvii. 2 I 3 .

Peltidium, Philippi, I 839 .

novæ-zealandiæ, Brady. T.Z.S. xv. 44 .

Porcellidium, Claus, 1860 .

fulvum, Thomson. T.N.Z.I. xv. 107 ; T.Z.S. xv. 46. interruptum, Thomson. T.N.Z.I. xv. 108. 
Scutellidium, Claus, $\mathrm{x} 866$.

tisboides, Claus. T.N.Z.I. xv. I 10.

plumosum, Brady. T.Z.S. xv. 45.

Idya, Philippi, 1843 .

furcata, Baird. T.N.Z.I. xv. Io8; T.Z.S. xv. 45.

Setella, Dana, 1846 .

gracilis, Dana = Miracia gracilis, Dana.

\section{Section P(ecilostoma.}

Oncæa, Philippi, i 843 .

$$
\text { Family CORYCÆIDÆ. }
$$

mediterranea, Claus. Pelagischen Copepoden des Goltes von Neapel, 59I = O. obtusa, Dana.

Oncæola, Krämer, I 895 .

specialis, Krämer. T.N.Z.I. xxvii. 220.

Corycæus, Dana, I 845 .

tenuis, Giesbrecht. T.N.Z.I. xxvii. 220.

aucklandicus, Krämer. T.N.Z.I. xxvii. 220.

robusta, Dana (?). T.Z.S. xv. 46.

Paurocope, Brady, I 899 .

robusta, Brady. T.Z.S. xv. 46 .

? Centromma, Brady, r 899.

thomsoni, Brady. T.Z.S. xv. 47.

Family SAPPHIRINIDA.

Sapphirina, Thomson, I 829 .

gemma, Dana.

Section Siphonostoma.

Entomolepis, Brady, 1899.

Family ENTOMOLEPIDA.

ovalis, Brady. T.Z.S. xv. 48 .

Family Artotrogide.

Conostoma, Thomson, I 882 .

elliptica, Thomson. T.N.Z.I. xv. x 12.

Artotrogus, Boeck, 1859.

boeckii, Brady. T.N.Z.I. xv. I I 3 .

ovatus, Thomson. T.N.Z.I. xv. $x_{3}$; T.Z.S. xv. 4 S.

brevicaudatus, Brady. T.Z.S. xv. 48.

Acontiophorus, Brady, I880.

scutatus, Brady and Robertson. T.N.Z.I. xv. I I4. 


\section{Family CaLigide.}

Lepeophtheirus, Nordmann, I $\delta_{32}$.
erecsoni, G. M. Thomson. T.N.Z.I. xxiii. 227.

Gloiopotes, Steenstrup and Lütken, I 860.

huttoni, G. M. Thomson (Lepeophtheirus). T.N.Z.I. xxii.

354 ; P. IV. Bassett-Smith, P.Z.S. ז899, $45^{8}$.

Caligus, Müller, I 785 .

productus, Dana. U.S. Expl. Exped. Crust. I 354 ; BassettSmith, P.Z.S. I $899,45^{2}$.

longicaudatus, Brady. T.Z.S. xv. 49.

Dinematura, Burmeister, 1837 .

ferox, Kröyer. P. W. Bassett-Smith, P.Z.S. I 899, 463; $=D$. carcharodonti, G. M. Thomson, T.N.Z.I. xxii. 360 . hamiltoni, G. M. Thomson. T.N.Z.I. xxii. 357 .

Echthrogaleus, Steenstrup and Lütken, I 860.

neo-zealanica, G. M. Thomson (Dinematura). T.N.Z.I. xxii. 359 .

affinis, Milne-Edwards. P.Z.S. I 899, 464; =E. braccatus, T.N.Z.I. xxii. $36 \mathrm{r}$.

Cecrops, Leach, I873.

latreillii, Leach. T.N.Z.I. xxii. 465 .

Gangliopus, Gerstaecker, I 854 .

? pyriformis, Gerstaecker. P.Z.S. ז 899,$465 ;=$ Nogagus curticaudatus $=$ Specilligus curticaudis, 'T.N.Z.I. xxii. 365 .

Pandarus, Leach, I 8 i 6.

dentatus, Milne-Edwards ( $\delta=$ Nogagus elongatus, Heller), T.N.Z.I. xxii. $35^{6}$ and 363 .

armatus, Heller ( $\delta=$ Nogagus latreillii, Milne-Edwards).

T.N.Z.Y. xxii. 363 and 364 .

affinis, V. Bened. $=P$. satyris, Dana $=P$. brevicaudatus

(? brevicaudis), Dana. T.N.Z.I. xxii. 365 .

Nogagus, Leach, I8r 9 .

validus, Dana. T.N.Z.I. xxii. $35^{6}$.

Family Dichelestinde.

Anthosoma, Leach, i 8 r 9 . crassum, Abgd. T.N.Z.I. xxii. 365 .

Lernanthropus, Blainville, 1832 . percis, G. M. Thomson. T.N.Z.I. xxii. 366 .

Family Philichthyide.

Philichthys, Steenstrup, I86I. xiphiæ, Steenstrup. T.N.Z.I. xxii. $3^{6} 7$. 
Penella, Edwards, I 840.

Family LERNÆIDÆ.

histiophori, G. M. Thomson. T.N.Z.I. xxii. 368.

Lernæa, Linnæus, 776 .

lotellæ, G. M. Thomson. T.N.Z.I. xxii. $3^{69}$.

Sphyrion, Cuvier, r 829 .

Family Chondracanthide.

lævigatum, Cuvier = Lesteira kröyeri, Thomson. P.Z.S. I 899, 489; T.N.Z.I. xxii. 370.

Chondracanthus, De la Roche, r 8 Ir.

lotellæ, G. M. Thomson. T.N.Z.I. xxii. 372.

chylomycteri, G. M. Thomson. T.N.Z.I. xxii. 37 I.

genypteri, G. M. Thomson. T.N.Z.I. xxii. 372 .

Family LERNÆOPODIDE.

Lernæopoda, Nordman, I 832.

galei, Kröyer $=L$. musteli, Thomson. P.Z.S. r899, 499; T.N.Z.I. xxii. 373 .

Brachiella, Latreille, r829.

parkeri, G. M. Thomson. T.N.Z.I. xxii. 374 .

Alepas, Rang, r829.

Order CiRRIPEDIA. Family LEPADIDE.

tubulosa, Quoy and Gaimard. Filhol, Miss. de l'Ile Campbell, 486.

Lepas, Linnæus, I 748.

hilli, Leach. Filhol, Miss. de l'Ile Campbell, 485 .

pectinata, Spengler. Hutton, T.N.Z.I. xi. 329.

australis, Darwin. Filhol, Miss. de l'Ile Campbell, 485 .

elongata, Quoy and Gaimard. Filhol, Miss. de l'Ile Campbell, 485 .

Pollicipes, Leach, I8r 7 .

sertus, Darwin. Filhol, Miss. de l'Ile Campbell, 486.

spinosus, Quoy and Gaimard. Filhol, Miss. de l'Ile Campbell, 486.

darwinii, Hutton. T.N.Z.I. xi. 329; Miss. de l'Ile Camp. bell, 486 .

Family BaLANIDE.

Coronula, Oken, i8r 5 . diadema, Lamarck. Filhol, Miss. de l'Ile Campbell, 489.

Tubicinella, Lamarck, 1802 .

trachealis, Shaw. Hutton, T.N.Z.I. xi. 330. 
Elminuus, King, r83o,

modestus, Darwin. Filhol, Miss. de l'Ile Campbell, 489.

sinuatus, Hutton. Filhol, Miss. de l'Ile Campbell, 489 .

plicatus, Gaimard. Filhol, Miss. de l'Ile Campbell, 489.

rugosus, Hutton. Filhol, Miss. de l'Ile Campbell, 489 .

Tetraclita, Schuhmacher, I8 I 7 .

purpurescens, Wood. Miss. de l'Ile Campbell, 488.

Balanus, Lister, 1685 .

decorus, Darwin. Filhol, Miss. de l'Ile Campbell, 486.

porcatus, Da Costa. Filhol, Miss. de l'Ile Campbell, 487 . vestitus, Darwin. Filhol, Miss. de l'Ile Campbell, 487 . campbelli, Filhol. Miss. de l'Ile Campbell, 487. amphitrite, Darwin. Filhol, Miss. de l'Ile Campbell, 486.

Chamæsipho, Darwin, I 854 .

columna, Spengler. Filhol, Miss. de l'Ile Campbell, 489 . 


\title{
PHYLUM, ANNULATA.
}

\author{
CLASS, POLYCHÆTA. \\ [For additional identifications of this Class, see Appendix.] \\ Order NEREIDIFORMIA. \\ Family Syllide.
}

Syllis, Savigny, Emend. Ehlers, 1864.

macrura, Schmarda. N.IV.Th. (2), 70 (Spharosyllis, Quatre-

fages, nec Claparède); Hutton, T.N.Z.I. xi. 323.

Family Hesionide.

Pseudosyllis, Quatrefages, I865.

incerta, Schmarda (Cirrosyllis $i$.). N.W.Th. (2), 79 ; Quatrefages, Hist. Nat. Annel. ii. Io6.

Family APHRODITID\&.

Sub-family Polynoina.

'Polynoe,' Savigny, i 809 .

aucklandica, Schmarda. N.W.Th. (2), I 58; Hutton, T.N.Z.I. xi. 318.

*comma, Thomson. T.N.Z.I. xxxiv. $24 \mathrm{I}$.

Lepidonotus, Leach, I8I9.

polychroma, Schmarda. N.W.Th. (2), I 53 ; Hutton, T.N.Z.I. xi. 31 9.

sinclairii, Baird. J.L.Soc. (Zool.) viii. I 84 ; Hutton, T.N.Z.I. xi. 319. [Probably identical with preceding.]

giganteus, Kirk. T.N.Z.I. xi. 400 ; Thomson, P.Z.S. I900, $974=$ Aphrodita squamosa, Quatrefages.

mirabilis, McIntosh (Macellicephala m.) Challenger Reports, xii. I 2 I.

Harmothoe, Kinberg, I 855 .

macrolepidota, Schmarda (Polynoe m.). N.IV.Th. (2), I 55 ; Hutton, T.N.Z.I. xi. 3 I 8.

* This species does not fit into any of the present genera of Polynoidre, though in some respects it resembles Halosydna, Kbg. '55. 
Harmothoe iphionoides, McIntosh (Eunoa i.). Challenger Reports, xii. 69.

kermadecensis, McIntosh (Lagisca? k.) Challenger Reports, xii. 93 .

magnipalpa, McIntosh (Polynoe m.). Challenger Reports, xii. I 18.

Sp. nov. innom. Benham MS.

A phrodita, Linnæus.

Sub-family HeRmionina.

talpa, Quatrefages. Hist. Nat. Annel. i. I96 ; Hutton, T.N.Z.I. xi. 318.

Sp. nov. innom. Benham MS.

Lætmonice, Kinberg, I 855 .

producta, Grube.

var. willemoesi, McIntosh. Challenger Reports, xii. 47.

Leanira, Kinberg, I 855 .

Sub-family Sigalionina.

lævis, McIntosh. Challenger Reports, xii. 156.

Sthenelais, Kinberg, 1855 .

semitecta, Ehlers MS.

Sub-family Polylepidia.

Psammolyce, Kinberg, I 855 .

antipoda, Schmarda (Pelogenia a.). N.W.Th. (2), I60; Hutton, T.N.Z.I. xi. 3 I 9.

Family Phyllodocide.

Phyllodoce, Savigny, i 809. sub-gen. Anaitis, Malmgren, 1865. ochracea, Ehlers MS.

Eulalia, Savigny, I809.

sub-gen. Eulalia.

microphylla, Schmarda. N. W. Th. (2), 86 ; Hutton,

T.N.Z.I. xi. $323,=E$. cæeca, Quatrefages.

novæ-zealandiæ, Grube. J. B. Schles. Gesellsch. 1vii. 2 I 0. Sp. nov. innom. Benham MS.

sub-gen. Eumida, Malmgren, I 865.

Sp. nov. innom. Benham MS.

sub-gen. Pterocirrus, Claparède.

Sp. nov. innom. Benham MS.

Nereis, Lamarck, i818.

Family LyCORIDÆ.

pacifica, Schmarda (Nereilepas p.). N.W.Th. (2), 107; Hutton, T.N.Z.I. xi. 32 I. (Not $N$. pacifica, Quatrefages.) 
Nereis neozelanica, Benham $=N$. robusta, Quatrefages. Hist. Nat. Annel. i. 544; Hutton, T.N.Z.I. xi. 32 I. vallata, Grube $=N$. latipalpa, Schmarda. Ehlers, Polych. Magellan. Chil. Str. I Io.

maghaelensis, Kinberg $=N$. eatoni, McIntosh. Ehlers, Polych. Magellan. Chil. Str. I04. australis, Schmarda (Heteronereis a.) N.IV.Th. (2), IоI; Hutton, T.N.Z.I. xi. 322.

3 spp. nov. innom. Benham MS.

\section{Family NEPHTHYDIDÆ.}

Nephthys, Cuvier, I8 77 .

macroura, Schmarda. N.W.Th. (2), $9 \mathrm{I}$; Hutton, T.N.Z.I. xi. $32 \mathrm{I}$.

dibranchis, Grube. McIntosh, Challenger Reports, xii. I6I. verrilli, McIntosh. Challenger Reports, xii. I63. $[?=d i$ branchis, Qr.-W. B. B.]

Sp. nov. innom. Benham MS.

Chlœia, Savigny, I809.

Family Amphinomidæ.

inermis, Quatrefages. Hist. Nat. Annel. i. 389 ; Hutton, T.N.Z.I. xi. 320.

spectabilis, Baird. J.L.S. x. 234 ; Hutton, T.N.Z.I. xi. 320.

Eunice, Cuvier, I8I 7 .

Family EuNicide.

gaimardi, Valenciennes MS. Quatrefages, Hist. Nat. Annel.

i. $32 \mathrm{I}$; Hutton, T.N.Z.I. xi. 320.

australis, Quatrefages. Hist. Nat. Annel. i. 321 .

aphroditois, Pallas. Benham MS.

leuconuchalis, Benham. 'N.Z. Gov. Rep. on Experim.

Trawling. I 900, 2 I.

Sp. nov. innom. Benham MS.

Diopatra, Audouin and Edwards, 1833 .

tenuisetis, McIntosh (Nothria t.). Challenger Reports, xii. 307 .

Sp. nov. innom. Benham MS.

Onuphis, Audouin and Edwards, 1833 .

tubicola, Müller.

var. longibranchiata, McIntosh (Hyalinacia l.). Challenger Reports, xii. 337 .

benthaliana, McIntosh (Hyalinocia b.). Challenger Reports, xii. 329.

minuta, McIntosh (Nothria m.). Challenger Reports, xii. 334. 
Nicidion, Kinberg, i 864 .

balfouriana, McIntosh. Challenger Reports, xii. $30 \mathrm{I}$.

Marphysa, Quatrefages, I 865.

corallina, Kinberg. Benham MS. Ehlers, Polych. Magellan.

Chil. Strand. I 34 (Chatham isl.).

depressa, Schmarda (Eunice d.). N.W.Th. (2), I 26.

Halla, Costa, i 844 .

2 spp. nov. innom. Benham MS.

Lumbriconereis, Blainville, I 828 .

sphærecephala, Schmarda (Notocirrus s.). N.W.Th. (2), I 6 ; Hutton, T.N.Z.I. xi. 320.

neozealania, McIntosh. Challenger Reports, xii. 248.

Arabella, Grube, I85 1 .

Sp. nov. innom. Benham MS.

Stauronereis, Verrill, I 900.

2 spp. nov. innom. Benham MIS.

Glycera, Savigny, i 820 .

Family GLyGERIDÆ.

ovigera, Schmarda. N.W.Th. (2), 95 ; Hutton, T.N.Z.I. xi. 322.

lamelliformis, McIntosh. Challenger Reports, xii. $3+7$. 5 spp. nov. innom. Benham MS.

Hemipodus, Quatrefages, I 865.

simplex, Grube. Ann. (Ersted. (fide Ehlers MS.).

Eone, Malmgren, I865.

Family Goniadide.

trifida, McIntosh. Challenger Reports, xii. $34 \mathrm{I}$.

Family ARICIIDÆ.

Gen. indet. Benham MS.

Order SPIONIFORMIIA.

Family AMMOCHARID \&.

Owenia, Delle Chiaje, I 842.

? fusiformis, Delle Chiaje. Benham MS.

Family SPIONIDÆ.

Gen. indet. Benham MS.

Order Terebelliformia.

Family CirRatulidæ.

Audouinia, Quatrefages, I 865.

anchylochætus, Schmarda (Cirratulus a.). N.W.Th. (2),

58 ; Hutton, T.N.Z.I. xi. 323 . 
Chætozone, Malmgren, 1865 .

pacifica, McIntosh. Challenger Reports, xii. 387 .

Timarete, Kinberg, I 865.

fecunda, Kinberg. Ann. nova. 254 ( fide Ehlers MS.).

Family TerebeLLIDÆ.

Thelepus, Leuckart, I 849 .

plagiostoma, Schmarda (Terebella p.). N.W.Th. (2), $4^{\mathrm{J}}$; Hutton, T.N.Z.I. xi. 324.

heterobranchia, Schmarda (Terebella h.). N.W.Th. (2),

42. [Probably synonymous with preceding.-W. B. B.]

Nicolea, Malmgren, r 865 .

Sp. nov. innom. Benham MS.

Amphitrite, Müller, I 77 I.

3 spp. nov. innom. Benham MS.

Lanice, Malmgren, 1865.

? conchilega, Pallas. Benham MS.

Leæna, Malmgren, 1865 .

neo-zealanica, McIntosh. Challenger Reports, xii. 460.

langerhansi, McIntosh. Challenger Reports, xii. 463 .

Lanassa, Malmgren, I 865 .

sarsi, McIntosh. Challenger Reports, xii. 436.

Terebella, Linnæus.

kermadecensis, McIntosh. Challenger Reports, xii. 446.

Terebellides, Sars, 1835 .

? stroemi, Sars. McIntosh, Challenger Reports, xii. 48 I.

Family AMPHARETIDE.

Melinna, Malmgren, I 865.

armandi, McIntosh. Challenger Reports, xii. 439.

Family AMPHICTENID.E.

Pectinaria, Lamarck, I $\delta$ I 2.

antipoda, Schmarda. M.IV.T. (2), 46 (fide Ehlers MS.).

Order Capitelliformia.

Family Capitellidæ.

Gen. indet. Benham MS.

Order SCOLECIFORMIA.

Fanily OPHELIIDÆ.

Thoracophelia, Ehlers, I 897.

Sp. nov. innom. Benham M.S. 
Travisia, Johnston, 1840 .

Sp. nov. innom. Benham MS.

Maldane, Grube, is6o.

Family Maldanide.

Sp. nov. innom. Benham MS.

Axiothea, Malmgren, 1865 . insecta, Ehlers MS.

Maldanella, McIntosh, $188_{5}$. neo-zealaniæ, McIntosh. Challenger Reports, xii. 398.

Family Telethuside.

Arenicola, Lamarck, i 8 I 8 .

assimilis, Ehlers.

var. affinis, Ashworth. Q.J.M.Sc. xlvi. 737.

Family SCALIBREgmide.

Scalibregma, Rathke, r 843 .

inflatum, Rathke. McIntosh, Challenger Reports, xii. 359.

Eumenia, Oersted, I 843 .

reticulata, McIntosh. Challenger Reports, xii. 360.

Flabelligera, Sars, I 829 .

Family Chloremide.

bicolor, Schmarda (Pherusa b.). N.W.Th. (2), 2 I ; Hutton, T.N.Z.I. xi. 324.

antarctica, Baird (Siphonostomum a.). J.L.S. xi. 95; Hutton, T.N.Z.I. xi. 324 .

Stylarioides, (D. Chiaje), Claparède, I 868.

? parmatus, Grube. Benham MS.

Sternaspis, Otto, I $82 \mathrm{I}$.

Family Sternaspide.

princeps, Selenka. Challenger Reports, xiii. 6.

Order SABElliformia.

Sabella, Savigny, i 809 .

Family SABELLIDE.

armata, Quatrefages. Hist. Nat. Annel. ii. 453; Hutton, T.N.Z.I. xi. 325 .

grandis, Baird. J.L.S. xiii. 160 ; Hutton, T.N.Z.I. xi. 325. ceratodaula, Schmarda. N.W.Th. (2), 33; Hutton, T.N.Z.I. xi. 325 .

Branchiomma, (Kölliker) Claparède, ı 868. suspiciens, Ehlers MS. 
Protula, Risso, I 826 .

* Family Serpulide.

Sp. nov. innom. Benham MS.

Apomatus, Philippi, I 844 .

elizabethæ, McIntosh. Challenger Reports, xii. 5 I 4 .

Pomatoceros, Philippi, 1844 .

strigiceps, Mörch. McIntosh, Challenger Reports, xii. 520. triqueter, Mörch.

Eupomatus, Philippi, I 844 .

boltoni, Baird. J.L.S. viii. I 2 ; Hutton, T.N.Z.I. xi. 326.

Serpula, Linnæus.

zeylandica, Baird. J.L.S. viii. 2 I ; Hutton, T.N.Z.I. xi. 325 . antarctica, Quatrefages. Hist. Nat. Annel. ii. 503 ; Hutton, T.N.Z.I. xi. 325 , suggests that it is synonymous with the preceding.

*Vermilia, Lamarck, i 8 I 8 .

cœrulea, Schmarda (Placostegus c.). N.W.Th. (2), 29.

carinifera, Gray in Dieffenbach's New Zealand, ii. 242;

Hutton, T.N.Z.I. xi. 326 , believes these to be synonymous. greyi, Quatrefages. Hist. Nat. Annel. ii. 5 Io; Hutton, T.N.Z.I. xi. 326.

mahoria, Quatrefages. Hist. Nat. Annel. ii. 520 ; Hutton, T.N.Z.I. xi. 326.

hombroni, Quatrefages. Hist. Nat. Annel. ii. 527 ; Hutton, T.N.Z.I. xi. 327 .

hystrix, Mörch. Quatrefages, Hist. Nat. Annel. ii. 534; Hutton, T.N.Z.I. xi. 327 .

Cymospira, Blainville, $\mathrm{I} 828$.

incompleta, Quatrefages. Hist. Nat. Annel. ii. 543 ; Hutton, T.N.Z.I. xi. 327 .

Spirorbis, Daudin, I 800 .

zelandica, Gray, in Dieffenbach's New Zealand, ii. 295; Hutton, T.N.Z.I. xi. 327 .

Order HerMelliformia.

Pallasia, Quatrefages, I 848 .

Family Heriellide.

quadricornis, Schmarda (Hermella q.). N.W.Th. (2), 25 ;

Hutton, T.N.Z.I. xi. 324 .

castelnaui, Grube. J. B. sches. Gesell. xlvii. 69.

Oncoscolex, Schmarda, I86I.

Incertæ sedis. [? OPHELIIDÆ.]

homochætus, Schmarda. N.W.Th. (2), 55.

* No attempt has been made to re-examine the Serpulids. 


\section{Class ECHIUROIDEA.}

Echiurus, Guérin (? I 83 I).

Family ECHIURIDÆ.

novæ-zealandiæ, Dendy. T.N.Z.I. xxx. 323 .

\section{Class OLIGOCHÆTA.}

[For additional species of this Class, see Appendix.]

Family Megascolecide.

Sub-family ACANTHODRILINE.

Maoridrilus, Michaelsen, I $899=-$ Acanthodrilus, Perrier, in part. uliginosus, Hatton, 1876 , T.N.Z.I. ix. $35 \mathrm{I}=A$ c. novazealandice, Beddard, 1885. Benham, T.N.Z.I. xxxiii. I22, pl. v.; Beddard, r895, Monogr. Oligoch. 534 .

dissimilis, Beddard. P.Z.S. 1 885, 813 , pl. 52, 53 .

rosæ, Beddard. P.Z.S. 1889,379 .

smithi, Beddard. P.Z.S. 1892. 675, pl. 46.

parkeri, Beddard. Monogr. I 895, 534 .

plumbeus, Beddard. Monogr. I $895,548$.

tetragonurus, Michaelsen, I 899. Zool. Jahrb. Syst. xii. 230.

Neodrilus, Beddard, i 887 .

monocystis, Beddard. Benham, Q.J.M.S. I 892 , xxxiii. $289, \mathrm{pl}$. I 5 .

Plagiochæta, Benham, I 892.

sylvestris, Hutton. Megascolex s., Hutton, T.N.Z.I. ix. $35^{2}$;

Benham, T.N.Z.I. г 898 , xxxv. $278 ;$ P. punctata, Benham, Q.J.M.S. xxxiii. 294.

lineata, Hutton. Megascolex l., Hutton, T.N.Z.I. ix. $35^{2}$; Benham, T.N.Z.I. xxxv. $28 \mathrm{I}$.

montana, Benham. T.N.Z.I. xxxv. 288.

ricardi, Benham. T.N.Z.I. xxxv. 286.

rossii, Benham. T.N.Z.I. xxxv. 284 .

[N.B.-Probably Baird's Megascolex antarcticus, I873, belongs to this genus.]

Notiodrilus, Michaelsen, I $899=$ Acanthodrilus, Perrier, in part. annectens, Beddard, is88. Q.J.M.S. xxix. 102, pl. I 2, I3; Beddard, Monogr. I 895,544 . paludosus, Beddard, I892. P.Z.S. 677.

macquariensis, Beddard, 1896 . Benham, T.N.Z.I. xxxiii. I $32, \mathrm{pl} .2$.

haplocystis, Benham, I 900 . T.N.Z.I. xxxiii. I 30 , pl. 2. aucklandicus, Benham, 1902 . T.N.Z.I. xxxv. 275.

Microscolex, Rosa, I887.

novæ-zealandiæ, Beddard, I 894 . Monogr. 463 . monticola, Beddard, i 895 . Monogr. 467. 
Rhododrilus, Beddard, I 899.

minutus, Beddard. P.Z.S. I889, 380 ; Monogr. 463.

huttoni, Benham, i 900 . Microscolex h., T.N.Z.I. xxxiii. 140 , pl. 4 .

Sub-family MegascolecinÆ.

Pontodrilus, Perrier, 1874 .

chathamianus, Michaelsen, i 899. Chathamensis, Benham, laps. cal., T.N.Z.I. I 900, xxxiii. I 36, pl. 3 .

Diporochæta, Beddard, I 890.

intermedia, Beddard. Q.J.M.S. xxx. 467, pl. 29 ; Monogr. 439.

chathamensis, Benham, I900. T.N.Z.I. xxx. I 34, pl. 3 .

Sub-family ОстоснжтілÆ.

Octochætus, Beddard, $\mathrm{I} 893=$ Acanthodrilus, Perrier, in part. levis, Hutton, I876. Penham, г898, T.N.Z.I. xxxi. I60. multiporus, Beddard, I885. P.Z.S. 8I 3, pl. 52, 53 ; Monogr. 526 ; Michaelsen, Tierr. 319. antarcticus, Beddard, I889. P.Z.S. 379; Monogr. 553. thomasi, Beddard, I 892 . P.Z.S. 671 ; Monogr. $55^{2}$. huttoni, Beddard, I893. P.Z.S. 674 ; Monogr. $55^{2}$.

Dinodrilus, Beddard, I 888 .

benhami, Beddard. Q.J.M.S. ı 888, xxix. ı०5, pl. І3 ; Monogr. 557 .

Family ENCHYTRÆIDÆ.

Fridericia, Michaelsen, 1889 .

galba, Hoffmeister, I 843 = antartica, Beddard, I 895 . Monogr. 349.

Henlea, Michaelsen, I889. ventriculosa, Udekem. Beddard, Monogr. $35^{\circ}$.

Family Haplotaxide.

Pelodrilus, Beddard, I $89 \mathrm{I}$. violaceus, Beddard, I891. A.M.N.H. ser. 6, vii. 91 ; Monog. I 92.

Haplotaxis, Hoffmeister, I 843 . smithi, Beddard, $1888=$ Phreoryctes s., Beddard. A.M.N.H. ser. 6, i. 393, pl. 23 ; Monogr. I9o.

Family PHREODRILIDÆ.

Phreodrilus, Beddard, I89I. subterraneus, Beddard. A.M.N.H. 6, vii. 92 ; Monogr. 275.

Tubifex, Lamarck, i 8 18. Family Tubificide.

tubifex, O. F. Müller, I $774=T$. rivulorum. Lamarck. Beddard, Monogr. 244 [? introduced]. 
Limnodrilus, Claparède, 1862.

novæ-zealandiæ, Beddard. Monogr. 247.

Sp. nov. innom. Beddard MS.

$$
\text { CLASS, MYZOSTOMIDA. }
$$

Myzostoma, Leuckart, I827.

tenuispinum, Graff. Challenger Reports, x. 68. willemœsii, Graff. Challenger Reports, x. 71 . murrayi, Graff. Challenger Reports, x. 74.

\section{CLASS, SIPUNCULACEA.}

Sipunculus, Lamarck, I 8 I 8 .

æneus, Baird. P.Z.S. 1868, 81 ; Hutton, T.N.Z.I. xi. 316.

? lutulentus, Hutton. T.N.Z.I. xii. 278.

Physcosoma, Selenka, I 897.

annulatum, Hutton (Phascolosoma a.). T.N.Z.I. xii. 278 .

Phascolosoma, Leuckart, i 862.

huttoni, Benham MS. ?= Sip. lutulentus, Hutton.

Aspidosiphon, Grube, 1867.

suteri, Benham MS.

\section{CLASS, HIRUDINEA.}

Order RhyNCHOBDELLIDA.

Family ICHTHYOBDELLIDE.

Branchellion, Savigny, I807.

rajæ, Parker. T.N.Z.I. xxiv. 7 I 4 .

Pontobdella, Leach, I 8 I 5 .

Sp. indet. Benham MS.

Glossiphonia, Johnson, 1816.

Family Glossiphonidæ.

novæ-zealandiæ, Dendy and Olliver. T.N.Z.I. xxxiii. 99.

Order GNathobdellida.

Hæmadipsa, Tennent.

Family HiRUdinidæ.

limbata, Grube. Moore, Proc. U.S. Mus. xxi. 543 (Geobdella limbata). [Probably attributed to N.Z. in error.] 


\title{
PHYLUM, ECHINODERMATA.
}

\author{
CLASS, HOLOTHURIOIDEA.
}

Order ACTiNOPODA.

Family AsPiDochirote.

Holothuria, Linnæus, 1758 .

robsoni, Hutton (Sp. dub.). Dendy, J.L.S. Zool. xxvi. 48 ; Ludwig, Hamburger Magalhaensische Sammelreise, Holothurien, 7 .

Stichopus, Brandt, I 835 .

mollis, Hutton. Dendy, J.L.S. Zool. xxvi. 46 ; Ludwig, Hamburger Magalhaensische Sammelreise, Holothurien, 7 .

Mesothuria, Oestergren, 1896 .

lactea, Théel = Holothuria lactea, Dendy, J.L.S. Zool. xxvi. 49. Oestergren, Zur Kenntniss der Subfamilie Synallectinæ unter den Aspidochiroten, Upsala, I 896. (Festskrift för Liljeborg.)

Enypniastes, Théel, I882.

Family Elasipode.

eximia, Théel. Challenger Reports, iv. 56.

Pannychia, Théel, I882.

moseleyi, Théel. Challenger Reports, iv. 88.

Family Dendrochirote.

Cucumaria, Blainville, 1830 .

alba, Hutton $=$ Colochirus alba, Dendy, J.L.S. Zool. xxvi. 35 . Ludwig, Hamburger Magalhaensische Sammelreise, Holothurien, 29.

ocnoides, Dendy $=$ Colochirus ocnoides, Dendy, J.L.S. Zool. xxvi. 36 ; Ludwigia ocnoides, Reiffen, Zeitschr. wiss. Zool. lxix. 598.

huttoni, Dendy. J.L.S. Zool. xxvi. 32 ; Ludwig, Hamburger Magalhaensische Sammelreise, Holothurien, 39.

thomsoni, Hutton (sp. dub.). Dendy, J.L.S. Zool. xxvi. 34 . turbinata, Hutton (sp. dub.). Dendy, J.L.S. Zool. xxvi. 34 . 
Phyllophorus, Grube, i 840 (Ludwig emend. i 887 ).

longidentis $($ Hutton $)=$ Thyonidium longidentis, Dendy, J.L.S. Zool. xxvi. 42. Ludwig, Hamburger Magalhaensische Sammelreise, Holothurien, 49.

Pseudopsolus, Ludwig, i 898 .

macquariensis, Dendy $=$ Psolus macquariensis, Dendy, J.L.S. Zool. xxvi. 4I. Ludwig, Hamburger Magalhaensische Sammelreise, Holothurien, 49.

Colochirus, Troschel, i 846 .

brevidentis, Hutton. Dendy, J.L.S. Zool. xxvi. to; Ludwig, Zool. Jahrb. Supp. iv. Heft 2, i $898,442$.

calcareus, Dendy (sp. dub.). J.L.S. Zool. xxvi. 38 ; Ludwig, Zool. Jahrb. Supp. iv. Heft 2, r 898, 442.

Family MolPadiIDE.

Caudina, Stimpson, i 853 . coriacea, Hutton. Dendy, J.L.S. Zool. xxvi. $28,45^{6}$.

Ankyroderma, Danielssen and Koren, i 879. marenzelleri, Théel. Challenger Reports, xiv. $4 \mathrm{I}$.

Trochostoma, Danielssen and Koren, I 877 .

albicans, var. glabrum, Théel. Challenger Reports, xir: 46. violaceum, Studer. Théel, Challenger Reports, xiv. 42.

Order PaRACTINOPODA. Family SyNAPTIDE.

Synapta, Eschscholtz, I829.

uncinata, Hutton. Dendy, J.L.S. Zool. xxri. 25.

inæqualis, Hutton (sp. dub.). Dendy, J.L.S. Zool, xxvi. 26.

Trochodota, Ludwig, I $89 \mathrm{I}$.

dunedinensis, Parker $=$ Chirodota dunedinensis, Dendy, J.L.S. Zool. xxvi. 26 ; Ludwig, Hamburger Magalhaensische Sammelreise, Holothurien, 87.

\section{CLASS, ECHINOIDEA.}

Order Spatangoidea.

Family Spatangide.

Pourtalesia, Agassiz, I869. laguncula, Agassiz. Farquhar, P.L.S.N.S.IV. xxiii. 322.

Echinocardium, Gray, i 825. australe, Gray. Farquhar, P.L.S.N.S.IW. xxiii. 322.

Brissiopsis, Agassiz, r840.

luzonica, Gray. Farquhar, P.L.S.N.S.IV. xxiii. $3^{22}$. 
Family Cassidulide.

Echinobrissus, Breyn, I 732.

recens, Milne-Edwards. Farquhar, P.L.S.N.S.IV. xxiii. 32 I.

Order Clypeastridea.

Arachnoides, Klein, I 734 .

Family SCUTELL1DÆ.

placenta, Linnæus. Farquhar, P.L.S.N.S.IV. xxiii. 321.

Goniocidaris, Des

Order Regularia.

Family CIDARIDE.

umbulacrum, Hutton. Farquhar, P.L.S.N.S.IV. xxiii. 3 I 6 .

Salenia, Gray, I825.

Family SALENide.

hastigera, Agassiz. Challenger Reports, iii. 54.

Family Diadematide.

Aspidodiadema, Agassiz, I 879.

tonsum, Agassiz. Challenger Reports, iii. 66.

Family ECHıNothURIDÆ.

Asthenosoma, Grube, I869. gracile, Agassiz. Farquhar, P.L.S.N.S.W. xxiii. 3 I 7 .

Phormosoma, Thomson, I 874 .

rigidum, Agassiz. Farquhar, P.L.S.N.S.W. xxiii. 3 I 7 .

Family Echinometridæ.

Echinometra, Rondel, I554.

lucunter, Leske. Agassiz, Challenger Reports, iii. I05.

Strongylocentrotus, Brandt, 1835 .

tuberculatus, Lamarck. Far(juhar, P.L.S.N.S.W. xxiii. 3 I 7.

Family ECHINIDE.

Temnopleurus, Agassiz, I84 I. reynaudi, Agassiz. Farquhar, P.L.S.N.S.IV. xxiii. 3 I 8 .

Trigonocidaris, Agassiz, I869. monolini, Agassiz. Challenger Reports, iii. I I .

Salmacis, Agassiz, i $84 \mathrm{I}$. globator, Agassiz. Farquhar, P.L.S.N.S.W. xxiii. 318.

Amblypneustes; Agassiz, I 84 I.

formosus, Valenciennes. Farquhar, P.L.S.N.S.IV. xxiii. 318. griseus, Blainville. Farquhar, P.L.S.N.S.IV. xxxiii. 319. 
Echinus, Rondel, I554.

angulosus, Klein. Farquhar, P.L.S.N.S.W. xxiii. 319.

magellanicus, Phillipi. Farquhar, P.L.S.N.S.W. xxiii. 320.

Evechinus, Verrill, i 846 .

chloroticus, Valenciennes. Farquhar, P.L.S.N.S.W. xxiii. 320.

Tripneustes, Agassiz, I84 I.

variegatus, Klein. (Kermadecs),

\section{CLASS, OPHIUROIDEA.}

\section{Order OpHIURIDA.}

Pectinura, Forbes, 1842 .

Family OPHIURIDE.

maculata, Verrill. Farquhar, P.L.S.N.S.W. xxiii. 306.

Ophiopeza, Pet.. I $8_{5}$ I.

cylindrica, Hutton. Farquhar, P.L.S.N.S.W. xxiii. 306. danbyi,

Ophioplocus, Lyman, i 861 .

huttoni, Farquhar. P.L.S.N.S.W. xxiv. 187.

Ophioglypha, Lyman, I86o.

rugosa, Lyman. Farquhar, P.L.S.N.S.IV. xxiii. 306.

ornata, Lyman. Challenger Reports, v. 6r.

Ophioceramis, Lyman, 1865.

clausa, Lyman. Challenger Reports, v. 26 .

obstricta, Lyman. Challenger Reports, v. 26.

Ophiocten, Lütken, I854.

hastatum, Lyman. Farquhar, P.L.S.N.S.W. xxiii. 307.

Ophiomusium, Lyman, I 869 .

lymani, Thomson. Farquhar, P.L.S.N.S.W. xxiii. 306.

scalare, Lyman. Challenger Reports, v. 95.

Ophiozona, Lyman, IS65.

stellata, Lyman. Farquhar, P.L.S.N.S.W. xxiii. 307 .

Ophiomastus, Lyman, 1878 .

tegulitius, Lyman. Farquhar, P.L.S.N.S.W. xxiii. $3 \circ 7$.

Ophiophyllum, Lyman, 1878 .

petilum, Lyman. Challenger Reports, v. Iо2.

Ophiactis, Lütken, I 856 .

flexuosa, Lyman. Challenger Reports, v. i 6.

cuspidata, Lyman. Challenger Reports, v. I I 7.

nama, Lyman. Challenger Reports, v. i I 7 .

nigrescens, Hutton. Farquhar, P.L.S.N.S.IV. xxiii. 307. 
Ophionereis, Lütken, I 859 .

schayeri, Muller and Troschel. Farquhar, P.L.S.N.S.W. xxxiii. 307 .

Amphiura, Forbes, 1842 .

argentea, Lyman. Challenger Reports, v. r 29.

lanceolata, Lyman. Farquhar, P.L.S.N.S.W. xxiii. 308.

canescens, Lyman. Challenger Reports, v. I 40.

rosea, Farquhar. P.L.S.N.S. IV. xxiii. 308 .

pusilla, Farquhar. P.L.S.N.S.IV. xxiii. 308.

elegans, Leach. Farquhar, P.L.S.N.S.IV. xxiii. 308.

parva, Hutton. Farquhar, P.L.S.N.S.W. xxiii. 308.

aster, Farquhar. T.N.Z.I. xxxiii. 250.

Ophiochiton, Lyman, I 878 .

lentus, Lyman. Challenger Reports, v. I77.

Ophiacantha, Müller and Troschel, i 842.

vepratica, Lyman. Challenger Reports, v. I 82.

cornuta, Lyman. Challenger Reports, v. I93.

Ophiomitra, Lyman, I 869 .

plicata, Lyman. Challenger Reports, v. 203.

Ophiopteris, Smith, I 877 .

antipodum, Smith. Farquhar, P.L.S.N.S.IV. xxiii. 308.

Ophiomyxa, Müller and Troschel, I 842.

australis, Lütken. Farquhar, P.L.S.N.S.IV. xxiii. 309.

Order EuRyalida.

Family ASTROPHY'TIDæ.

Astrochema, Örst. and Lutken, I 856 .

horridum, Lyman. Challenger Reports, v. 275.

salix, Lyman. Challenger Reports, v. 277.

Ophiocreas, Lyman, 1869.

constrictus, Farquhar. T.N.Z.I. xxxii. 405.

\section{CLASS, CRINOIDEA.}

Order Articulata.

Family Comatulide.

Antedon, De Fréminville, i 8 i I.

incerta, Carpenter. Challenger Reports, xxvi. 106.

breviradia, Carpenter. Challenger Reports, xxvi. i ro.

echinata, Carpenter. Challenger Reports, xxvi. I I 9.

basicurva, Carpenter. Challenger Reports, xxvi. I 20. incisa, Carpenter. Challenger Reports, xxvi. I 24.

alternata, Carpenter. Challenger Reports, xxvi. I 79 . inæqualis, Carpenter. Challenger Reports, xxvi. 244. 
Endiocrinus, Carpenter, I882.

semperi, Carpenter. Challenger Reports, xxvi. 82 .

Family Pentacrinide.

Metacrinus, Carpenter, I884.

varians, Carpenter. Challenger Reports, xi. 353.

wyvillei, Carpenter. Challenger Reports, xi. 358.

nodosus, Carpenter. Challenger Reports, xi. 364 .

Pentacrinus, Miller, I 82 I.

alternicirrus, Carpenter. Challenger Reports, xi. $32 \mathrm{I}$.

naresianus, Carpenter. Challenger Reports, xi. 324 .

\section{CLASS, ASTEROIDEA.}

Order Phanerozonia.

Family Astropectenide.

Astropecten, Linck, I 733 .

edwardsi, Verrill. Farquhar, P.L.S.N.S.IV. xxiii. 309.

polycanthus, Müller and Troschel. Farquhar, P.L.S.N.S.IV.

xxiii. $3 \circ 9$.

Psilaster, Sladen, 1885 .

acuminatus, Sladen. Challenger Reports, xxx. 225.

Family Pentagonasteride.

Astrogonium, Müller and Troschel, I 842.

pulchellum, Gray. Farquhar, P.L.S.N.S.W. xxiii. 310.

abnormale, Gray. Farquhar, P.L.S.N.S.W. xxiii. 3 Io.

Gnathaster, Sladen, i 889 .

miliaris, Gray. Farquhar, P.L.S.N.S.W. xxiii. 310.

rugosus, Hutton. Farquhar, P.L.S.N.S.W. xxiii. 3 I I.

Goniodon, Perrier.

dilatatus, Perrier. Farquhar, P.L.S.N.S.W. xxiii. 3 I I ; Loriol,

Notes pour servir à l'étude des Echinodermes, I 901,43 .

Family Gymasteridiæ.

Asterospis.

imperialis, Farquhar.

Family ASTERINIDÆ.

Asterina, Nardo, I834.

regularis, Verrill. Farquhar, P.L.S.N.S.W. xxiii. 3 r 2.

novæ-zealandiæ, Perrier. Farquhar, P.L.S.N.S.W. xxiii.

3 I 2.

Stegnaster, Sladen.

inflatus, Hutton. Farquhar, P.L.S.N.S.W. xxiii. $3^{\text {I } 2 .}$ 
Order Cryptozonia.

Family STICHASTERIDE.

Stichaster, Müller and Troschel, I 840.

australis, Verrill. Farquhar, P.L.S.N.S.W. xxiii. 31 I 3 . polyplax, Müller and Troschel. Farquhar, P.L.S.N.S.Wr. xxiii. 3 I 3 .

suteri, Loriol. Farquhar, P.L.S.N.S.W. xxiii. 3r 3 .

var. lævigatus, Hutton. Farquhar, P.L.S.N.S.W. xxii.. ${ }^{5} 313-$ insignis, Farquhar. P.L.S.N.S.IV. xxiii. 3 I 4.

Family LiNCKIIDF.

Ophidiaster, Agassiz, I 835 .

cylindricus. Sladen, Challenger Reports, xxx. 403.

Solaster, Forbes, I 839.

Family Solasteride.

torulatus, Sladen. Challenger Reports, xxx. 457.

Family EChINASTERIDÆ.

Cribrella, Agassiz, I 834 .

ornata, Perrier. Farquhar, P.L.S.N.S.W. xxiii. 3 r 4. compacta, Sladen. Farquhar, P.L.S.N.S.IV. xxiii. 3 I 4. sufflata, Sladen. Challenger Reports, xxx. 549.

lukinsii, Farquhar. T.N.Z.I. xxx. 190.

Echinaster, Gray, 1840 .

purpureus, Gray. Farquhar, P.L.S.N.S.W. xxiii. 3 I4.

Freyella, Perrier, I 885 .

Family BRISINGIDÆ.

polycnema, Sladen. Challenger Reports, xxx. 62 I.

Family ASTERIIDÆ.

Asterias, Linnæus, I 748 .

calamaria, Gray. Farquhar, P.L.S.N.S.W. xxiii. 315.

scabra, Hutton. Farquhar, P.L.S.N.S.W. xxiii. 3 I 5.

mollis, Hutton. Farquhar, P.L.S.N.S.W. xxiii. 3 I 6.

fragilis, Sladen. Farquhar, P.L.S.N.S.W. xxiii. $3 \mathbf{I} 6$. rodolphi, Perrier.

Uniofera, Gray, i 840 .

granifera, Lamarck. Farquhar, P.L.S.N.S.IV. xxiil. 316 . 


\title{
PHYLUM, MOLLUSCOIDA.
}

\author{
CLASS, BRACHIOPODA. \\ Order ARticulata. \\ Family Terebratulide.
}

Magellania,

lenticularis, Deshayes. Hutton, Man. N.Z. Moll. I 76 (Waldheimia).

Terebratella, D'Orbigny, i 847 .

sanguinea, Leach = cruenta, Dillwyn. Hutton, Man. N.Z. Moll. I 76 .

rubicunda, Solander. Hutton, Man. N.Z. Moll. 177.

Terebratulina, d'Orbigny, $\mathrm{I} 847$.

murrayi, Davidson. Challenger Reports, i. 39.

Family RHyNCHONELLIDÆ.

Rhynchonella, Fischer, i 809 .

nigricans, Sowerby. Hutton, Man. N.Z. Moll. i 7 S.

Pedicellinopsis, Hincks.

$$
\text { CIAAS, POLYZOA. }
$$

Sub-class ENDOPROCTA.

Order

Family

gracilis, Sars. Hilgendorf, T.N.Z.I. xxx. 2 I 8.

Sub-class Ectoprocta.

Order Phylactolæiata.

Family

Plumatella, Lamarck, i 8 i 6.

aplinii, MacGillivray (?). Hutton, Man. N.Z. Moll. I8So, I 79. repens, Allman. Hamilton, T.N.Z.I. xii. 302.

Order GyinolÆnata.

Sub-order C'Tenostomata.

Family HaLCYONELLIDE.

Flustrella, Ehrenberg, 1838 .

binderi, Harvey. Hutton, T.N.Z.I. xxiii. 107. 
Family Vesicularinide.

Amathia, Lamouroux, 1812.

swainsoni, Hutton. Cat. Marine Moll. N.Z. 9I (Beania).

Sub-order Cheilostomata.

Division Stolonata.

Family ÆTEIDÆ.

Etea, Lamouroux, 1812.

dilatata, Busk. Cat. Pol. Brit. Mus. i. 31 .

Family Eucrateade.

Hippothoa, Lamouroux, I 82 I.

flagellum, Manzoni. Hutton, T.N.Z.I. xxiii. זо6.

divaricata, Lamouroux. Hamilton, T.N.Z.I. xxx. 196.

Division RAdicellata.

Family Catenariade.

Catenicella, Blainville, i 830 .

ventricosa, Busk. Cat. Pol. Brit. Mus. i. 7 .

hastata, Busk. Cat. Pol. Brit. Mus. i. 7 (C. bicuspis, Gray).

cribraria, Busk. Cat. Pol. Brit. Mus. i. 9.

perforata, Busk. Cat. Pol. Brit. Mus. i. 9.

ringens, Busk. Cat. Pol. Brit. Mus. i. 10.

elegans, Busk. Cat. Pol. Brit. Mus. i. 10.

cornuta, Busk. Cat. Pol. Brit. Mus. i. I I.

carinata, Busk. Cat. Pol. Brit. Mus. i. I 2.

scutella, Hutton. T.N.Z.I. xxiii. I03.

alata, Wyville Thomson. Hamilton, T.N.Z.I. xxx. 199.

crystallina, Wyville Thomson. Nat. Hist. Rev. 1858.

Claviporella,

geminata, Wyvllle Thomson. MacGillivray, Cat. Vict. Pol. 12. aurita, MacGillivray. Cat. Vic. Pol. I2.

Family Cellulariade.

Cellularia, Pallas, 1760 .

cuspidata, Busk. Hutton, T.N.Z.I. xxiii. 103.

Menipea, Lamouroux, 18I2.

cirrata, Lamouroux. Busk, Cat. Pol. Brit. Mus. i. 2 I.

buskii, Wyville Thomson. Nat. Hist. Review, i 858 .

crystallina, Gray. In Dieffenbach's N.Z. ii. 293 (Emma). tricellata, Busk. Cat. Pol. Brit. Mus. i. 28 (Emma). patagonica, Busk. Cat. Pol. Brit. Mus. i. 22.

Scrupocellaria, Van Beneden, 1845.

scrupea, Busk. Cat. Pol. Brit. Mus. i. 24.

Caberea, Lamouroux, 1816.

texta, Lamarck. Hutton, T.N.Z.I. xxiii. 103. 
Caberea lyallii, Busk. Hutton, T.N.Z.I. xxiii. 103. rostrata, Busk. Hutton, T.N.Z.I. xxiii. 103. boryi, Audouin. Hamilton, T.N.Z.I. xxx. I94.

Bicellaria, Blainville, I 830 .

Family BicellariadÆ. tuba, Busk. Cat. Pol. Brit. Mus. i. 42.

Bugula, Oken, 1835 . neritina, Linnæus. Busk, Cat. Pol. Brit. Mus. i. 44. dentata, Lamouroux. Busk, Cat. Pol. Brit. Mus. i. 46. prismatica, Gray. Dieffenbach's N.Z. ii. 292. johnstonii, Gray. Dieffenbach's N.Z. ii. 292 (Halophila). avicularia, Linnæus. Hutton, T.N.Z.I. xxiii. ıо3.

\section{Family GEMeLLARIADE.}

Dimetopia, Busk, I 85 I.

spicata, Busk. Cat. Pol. Brit. Mus. i. 35 .

cornuta, Busk. Cat. Pol. Brit. Mus. i. 35 .

Family Farcininariadi.

Farciminaria, Busk, i $8_{52}$.

aculeata, Busk. Cat. Pol. Brit. Mus. i. 33 .

blainvillii, Lamouroux. Hutton, T.N.Z.I. xxiii. I04.

Family FLUSTR1De.

Flustra, Linnæus, I 745 .

episcopalis, Busk. Cat. Pol. Brit. Mus. i. 52 (Carbasea). indivisa, Busk. Cat. Pol. Brit. Mus. i. 53 (Carbasea). var. cyathyformis, MacGillivray. Trans. Phil. i. Vic. I 859,97 .

Carbasea, Gray, i 848 .

moselyi, Busk. Challenger Reports, x. 56 .

Diachoris, Busk, i $85 \mathrm{I}$.

elongata, Hincks. A.M.N.H. 5, xv. 244.

bilaminata, Hincks. A.M.N.H. 5, vii. 44 .

intermedia, Hincks. A.M.N.H. 5, viii. 74 .

magellanica, Busk. Cat. Pol. Brit. Mus. i. 54.

inermis, Busk. Cat. Pol. Brit. Mus. i. 54.

spinigera, MacGillivray. Cat. Vict. Pol. I 7 .

Family MEMBRANIPORIDE.

Membranipora, Blainville, 1830 .

membranacea, Linnæus. Busk, Cat. Pol. Brit. Mus. ii. 56.

pilosa, Linnæus. Busk, Cat. Pol. Brit. Mus. ii. 56 .

lineata, Linnæus. Busk, Cat. Pol. Brit. Mus. ii. 58 .

spinosa, Quoy and Gaimard. Hutton, T.N.Z.I. xxiii. $10_{4}$.

trifolium, IVood. Hutton, T.N.Z.I. xxiii. I04. 
Membranipora roborata, Hincks. Hutton, T.N.Z.I. xxiii. 104. solidula, Hincks. Hutton, T.N.Z.I. xxiii. 104.

valdemuniata, Hincks. Hutton, T.N.Z.I. xxiii. 104.

hians, Hincks. Hutton, T.N.Z.I. xxiii. I04.

pura, Hincks. Hutton, T.N.Z.I. xxiii. 104.

acuta, Hincks. Hutton, T.N.Z.I. xxiii. 104.

brunnea, Hutton. T.N.Z.I. xxiii. 104.

maorica, Stoliczka. Hutton, T.N.Z.I. xxiii. 104.

serrata, McGillivray. Hamilton, T.N.Z.I. xxx. 195 .

Family Microporide.

Micropora, Gray, I 848 .

lepida, Hincks. Hutton, T.N.Z.I. xxiii. ro5.

elongata, Hincks. Hutton, T.N.Z.I. xxiii. 105.

Steganoporella, Hincks, 1879 .

neozelanica, Busk. Q.J.M.S. n.s. i. I55 (Vincularia);

Harmer, Q.J.M.S. xliii. 259.

Family Salicornariade.

Cellaria, Ellis, 1754 .

malvinensis, Busk. Cat. Pol. Brit. Mus. i. I9.

setigera, Desmarest. Hutton, T.N.Z.I. xxiii. I03.

Family Tubucellariade.

Tubucellaria, d'Orbigny, I $85 \mathrm{I}$.

hirsuta, Lamouroux. Hutton, T.N.Z.I. xxiii. 103.

Calwellia, Thomson, 1859 .

Family ONCHOPORIDE.

bicornis, Wyville Thomson. Nat. Hist. Rev. ${ }_{1}{ }_{5} S$, i. 91.

Retepora, Lamarck, I80I.

Family RETEPORIDE.

fissa, McGillivray. IVaters, A.M.N.H. 6, iv. I 8. novæ-zealandiæ, Waters. J.L.S. xxv. 270.

monilifera, forma munita, Hincks. T.R.S.Vict. xx. 108. avicularis, McGillivray. Hamilton, T.N.Z.I. xxx. 196.

Cribrilina, Gray, 1848 .

Family CRIBRILINID瓜.

monoceros, Busk. Challenger Reports, x. I 33 .

figularis, Johnston. Hamilton, T.N.Z.I. xxx. 196.

radiata, Moll. Busk, Challenger Reports, x. I3 I.

Microporella, Hincks.

Family MiCROPORELLIDE.

ciliata, Pallas. Hutton, T.N.Z.I. xxiii. 105. 
Microporella decorata, var. angustipora, Hincks. A.M.N.H.

5, xv. 249 .

var. disjuncta, Hincks. A.M.N.H. 5, xv. 249.

yarraensis, Waters. Hutton, T.N.Z.I. xxiii. I05.

pellucida, Hutton. Cat. Marine Moll. N.Z. 97.

coscinophora, var. mucromata, McGillivray. Hamilton,

T.N.Z.I. xxx. 195.

Family EsCHARIDE.

Lepralia, Johnston, ${ }_{1} 838$.

lyallii, Busk. Cat. Pol. Brit. Mus. ii. 75 .

cincta, Hincks. A.M.N.H. 5, xv. 254 .

foraminigera, Hincks. A.M.N.H. 5, xi. sog.

poissonii, Audouin. Hincks, A.M.N.H. 5 , viii. 63 . rectilineata, Hincks. A.M.N.H. 5, xi. I Io.

flexuosa, Hutton. Cat. Marine Moll. N.Z. 99 (Eschara j.). pallasiana, Moll. Hincks, Brit. Mar. Pol. 297.

Chorizopora, Hincks. brongniartii, Audouin. Hamilton, T.N.Z.I. xxx. I95.

Porella, Gray, i 848 . malleolus, Hincks. A.M.N.H. 5, xiii. $36 \mathrm{I}$.

Smittia, Hincks, i 879 . napierii, Waters. A.M.N.H. 6, iv. I 7 . unispinosa, Waters. A.M.N.H. 6, iv. I 5. trispinosa, Johnston. Hincks, Brit. Marine Pol. 353. reticulata, McGillivray. A.M.N.H. I, ix. 467 .

Mucronella, Hincks, i 877 .

præstans, Hincks. Hutton, T.N.Z.I. xxiii. ro5.

bi-incisa, var. bicuspis, Hincks. A.M.N.H. 5, xi. I10.

diaphana, var. armata, Hincks. A.M.N.H. 5, ix. 98. tricuspis, Hincks. A.M.N.H. 5, viii. 66.

variolosa, Johnston. Busk, Cat. Pol. Brit. Mus. ii. 75 . peachii, var. octodentata, Hincks. Brit. Marine Pol. $3^{6} \mathrm{I}$.

Membraponella, nitida, Johnston. Busk, Cat. Pol. Brit. Mus. ii. 76 .

Monoporella, crustatina, Waters. Hutton, T.N.Z.I. xxiii. ro4. disjuncta, Manzoni. Hutton, T.N.Z.I. xxiii. I04.

Schizoporella, Hincks, 1877 .

hyalina, Linnæus. Hutton, T.N.Z.I. xxiii. 106. var. cornuta, Busk. Hutton, T.N.Z.I. xxiii. Io6. areolata, Busk. Cat. Pol. Brit. Mus. ii. 82. marsupifera, Busk. Challenger Reports, x. I65. 
Schizoporella biaperta, Michelin. Hincks, Brit. Marine Pol. 255.

biserialis, Hincks. A.M.N.H. 5. xv. $25^{\circ}$.

cinctipora, Hincks. A.M.N.H. 5, xi. rog.

var. personata, Waters. Q.J.Geol.S. xliii. I67.

cribrilifera, Hincks. A.M.N.H. 5, xv. 250.

spectabilis, Hincks. A.M.N.H. 6, vii. 292.

sinuosa, var. armata, Hincks. Brit. Marine Pol. 267.

conservata, Waters. Hincks, A.M.N.H. 5; x. 165.

auriculata, Hassall. A.M.N.H. i. ix. 4 I I.

Haswellia, Busk, I $88_{4}$.

auriculata, Busk. Challenger Reports, x. I 73.

Rhynchopora, Hincks, I 877.

bispinosa, Johnston. Hincks, Brit. Marine Pol. 385 .

Adeonellopsis,

Family Adfones.

parvipunctata, McGillivray. Hamilton, T.N.Z.I. xxx. I96.

Family Celleporidæ.

Cellepora, Gmelin, I 789 .

coronopus, Wood. Hutton, T.N.Z.I. xxiii. ז06.

bispinata, Busk. Cat. Pol. Brit. Mus. ii. 87.

mamillata, Busk. Cat. Pol. Brit. Mus. ii. 87 .

pustulata, Busk. Challenger Reports, x. 200.

ampliata, Hutton. Cat. Marine Moll. N.Z. 99.

agglutinans, Hutton. Cat. Marine Moll. N.Z. 99.

\section{Sub-order Cyclostoniata.}

Family CRISIAD※.

Crisia, Lamouroux, i8 12.

denticulata, var. patagonica, D'Orbigny. Busk, Cat. Pol. Brit. Mus. iii. S.

edwardsiana, D’Orbigny. Busk, Cat. Pol. Brit. Mus. iii. 5 .

\section{Family IDMONEIDE.}

Idmonea, Lamouroux, I82 I.

milneana, D'Orbigny. I. giebeliana, Stoliczka, Reise der Novara, i 15.

radians, Lamarck. Busk, Cat. Pol. Brit. Mus. iii. I I.

ramosa, D’Orbigny. Waters, Q.J.G.S. xliii. 339.

serpens, Linnæus. Hutton, T.N.Z.I. xxiii. I07.

Crisina,

hochstetteriana, Stoliczka. Reise der Novara, Palæ. I I 3

(Idmonea). 
Entalophora, Lamouroux, I82 I.

raripora, D’Orbigny. E. haastiana, Stoliczka, Reise der Novara, Palæ. I02.

intricaria, Busk. Cat. Pol. Brit. Mus. iii. 22.

purpurascens, Hutton. T.N.Z.I. ix. 361 (Pustulipora).

Cinctipora, Hutton, 1873 .

elegans, Cat. Marine Moll. N.Z. 103.

Hornera, Lamouroux, i 82 I.

foliacea, McGillivray. Hutton, T.N.Z.I. xxiii. Iо7.

striata, Stoliczka. Reise der Novara, Palæ. 107.

frondiculata, Lamouroux. Hamilton, T.N.Z.I. xxx. 197.

Family TUBUliPORIDE.

Tubulipora, Lamarck, is 86. biduplicata, Waters. Q.J.G.S. xliii. 343 . glomerata, Hutton. Cat. Marine Moll. N.Z. I०3.

Stromatopora, Blainville, is 8 o.

racemosa, Hutton. Cat. Marine Moll. N.Z. Iо3 (Alecto). major, Johnston. Hamilton, T.N.Z.I. xxx. I97.

\section{Family DiastopoRIDE.}

Diastopora, Lamouroux, I82 I.

patina, Lamarck. Hutton, T.N.Z.I. xxiii. 107.

perangulata, Waters. Q.J.G.S. xliii. 343 .

sarniensis, Norman. A.M.N.H. 3, xiii. 89.

suborbicularis, Hincks. Brit. Marine Pol. 464.

Family LICHENOPORIDÆ.

Lichenopora, Defrance, 1823 . holdsworthii, Busk. Hutton, T.N.Z.I. xxiii. 107. neozelanica, Busk. Cat. Pol. Brit. Mus. iii. 32 (Discoporella). clypeiformis, D'Orbigny. Hamilton, T.N.Z.I. xxx. 197. pristis, McGillivray. Cat. Vict. Pol. 336.

Defranchia, Bronn, I 835 .

dentata, Hutton. Cat. Marine Moll. N.Z. I99.

Family FrondiPORIDE.

Supercystis, D'Orbigny.

digitata, D'Orbigny. Busk, Cat. Pol. Brit. Mus. iii. 37 (Fasciculipora).

Family HeTEROPORIDE.

Heteropora, Blainville, I 830 . pelliculata, Waters. Hutton, T.N.Z.I. xxiii. 107. 


\section{PHYLUM, TROCHELMINTHES.}

\section{CLASS, ROTIFERA.}

Order RHizota.

Family FlosCULARIDE.

Floscularia, Oken, i 8 I 5 .

coronetta, Cubitt. Hudson and Gosse, The Rotifera, i. 49. ornata, Ehrenberg. Hudson and Gosse, The Rotifera, i. 50. ambigua, Hudson. Hudson and Gosse, The Rotifera, i. 53.

\section{Family MELICERTADE.}

Melicerta, Schrank, r 803 . ringens, Schrank. Hudson and Gosse, The Rotifera, i. 70.

Order Bdelloida.

Family Philodinade.

Philodina, Ehrenberg, I 830.

erythrophthalma, Ehrenberg. Hudson and Gosse, The Rotifera, i. 99.

roseola, Ehrenberg. Hudson and Gosse, The Rotifera, i. 99. megalotrocha, Ehrenberg. Hudson and Gosse, The Rotifera, i. Iо .

microps, Gosse. Hudson and Gosse, The Rotifera, Supplement, 8.

Rotifer, Cuvier, I 798.

vulgaris, Schrank. Hudson and Gosse, The Rotifera, i. 104. macrurus, Schrank. Hudson and Gosse, The Rotifera, i. I07.

Callidina, Ehrenberg, I 830 .

bihamata, Gosse. Hudson and Gosse, The Rotifera, i. I I I. quadridens, Hilgendorf. T.N.Z.I. xxxi. I I 3. venusta, Bryce.

Order Ploima.

Sub-order ILLORICATA.

Family HydatinadÆ.

Notops, Hudson, I 886.

minor, Rousselet. Quekett Jour. Jan. I $892=$ Postclausa circularis and minuta, Hilgendorf, T.N.Z.I. xxxi. I25-6. 
Hydatina, Ehrenberg, I 830 . senta, Ehrenberg. Hudson and Gosse, The Rotifera, ii. 9.

Family NotonnatadÆ.

Notommata, Ehrenberg, I830.

tripus, Ehrenberg. Hudson and Gosse, The Rotifera, ii. 22. pentophthalma, Hilgendorf. T.N.Z.I. xxxi. i 6.

Proales, Gosse, i 886.

decipiens, Ehrenberg. Hudson and Gosse, The Rotifera, ii. $3^{6}$.

Diglena, Ehrenberg, I 830 .

forcipata, Ehrenberg. Hudson and Gosse, The Rotifera, ii. $3^{6 .}$

Eosophora, Ehrenberg, I83o.

aurita, Ehrenberg. Hudson and Gosse, The Rotifera, ii. 47 .

Sub-order LORICATA.

Family RATTULIDE.

Mastigocerca, Ehrenberg, I 830 .

lophoessa, Gosse. Hudson and Gosse, The Rotifera, ii. 60 $=M$. rectocaudatus, Hilgendorf, T.N.Z.I. xxxi. I 20 .

Rattulus, Ehrenberg, i $8_{3}$ o.

tigris, Muller. Hudson and Gosse, The Rotifera, ii. 65.

cimolius, Gosse. Hudson and Gosse, The Rotifera, ii. 65. weberi, Jennings.

Cœlopus, Gosse, I 886.

tenuior, Gosse. Hudson and Gosse, The Rotifera, ii. 68 = Mastigocerca flectocaudatus, Hilgendorf, T.N.Z.I. xxxi. I 19. brachyurus, Gosse. Hudson and Gosse, The Rotifera, ii. 69 .

Family Dinocharide.

Dinocharis, Ehrenberg, I 830 .

inornata, Hilgendorf. T.N.Z.I. xxxi. I21.

Diaschiza, Gosse, i 886.

Family SALPINADE.

tenuior, Gosse. Hudson and Gosse, The Rotifera, ii. 8 I $=D$. taurocephalus, Hilgendorf, T.N.Z.I. xxxi. I 23 . semiaperta, Gosse. Hudson and Gosse, The Rotifera, ii. So. paeta, Gosse. Hudson and Gosse, The Rotifera, ii. 79. ventripes, Dixon-Nuttall.

Euchlanis, Ehrenberg, is 3 o.

Family Euchilanide.

dilatata, Ehrenberg. Hudson and Gosse, The Rotifera, ii. 90. 
Cathypna, Gosse, I 886.

hudsoni, Lord.

Monostyla, Ehrenberg, I 830 .

cornuta, Ehrenberg. Hudson and Gosse, The Rotifera, ii. 98. lunaris, Ehrenberg. Hudson and Gosse, The Rotifera, ii. 98.

Coluris, Ehrenberg, 1830 .

Family COLURID盾.

amblytelus or caudatus (or both), Ehrenberg. Hudson and Gosse, The Rotifera, ii. Io4.

Metopidia, Ehrenberg, I 830.

solidus, Gosse. Hudson and Gosse, The Rotifera, ii. Io6. acuminata, Ehrenberg. Hudson and Gosse, The Rotifera, ii. 107.

triptera, Ehrenberg. Hudson and Gosse, The Rotifera, ii. I08.

Pterodina, Ehrenberg, I 830.

Family Pterodinade.

patina, Ehrenberg. Hudson and Gosse, The Rotifera, ii. I I 2.

Family ANURÆADÆ.

Anuræa, Gosse, I 85 I, nec Ehrenberg.

hypelasma, Gosse. Hudson and Gosse, The Rotifera, ii. I 23.

Notholca, Gosse, 1886.

jugosa, Gosse. Hudson and Gosse, The Rotifera, Supplement, $5^{6}=N$. regularis, Hilgendorf, T.N.Z.I. xxxi. I 32 . 


\title{
PHYLUM, NEMATELMINTHES.
}

\author{
CLASS, NEMATOIDEA.*
}

Ascaris, Linnæus, I 746.

Family Ascaride.

macruri, v. Linstow. Challenger Reports, xxiii. 7, in Macruris rudis.

labiata, Rudolphi, in Conger vulgaris. ecaudata, Dujardin, in Conger vulgaris.

clavata, Rudolphi, in Conger vulgaris.

orthagorisci, Rudolphi, in Orthagoriscus mola.

incurva, Rudolphi, in Xiphias gladius.

bi-uncinata, Molin, in Zeus faber.

bramæ, v. Beneden, in Brama raii.

granulosa, Schneider, in Fregatta aquila.

spiculigera, Rudolphi, in Podiceps cristatus and in Phalacrocorax carbo.

microcephala, Rudolphi, in Herodias egretta.

aurita, v. Linstow, in Micranous leucocapillus.

conocephala, Krabbe, in Delphinus delphis.

apterycis, Chatin, in Apteryx sp.; Bull. Soc. Philom. 7, ix. 36 .

Heterakis, Dujardin, I 845 .

hians, Dujardin, in Conger vulgaris.

præcinctus, Dujardin, in Conger vulgaris.

congri, v. Beneden, in Conger vulgaris.

Ichthyonema, Diesing, I86o.

Family FiLARIDE.

congeri vulgaris, Molin, in Conger vulgaris.

Filaria, Müller, 1787 .

acuta, Diesing, in Podiceps cristatus.

recta, v. Linstow, in Podiceps cristatus.

squamata, v. Linstow, in Phalacrocorax carbo.

dubia, Leidy, in Diomedea exulans.

* This list of Parasites is for the most part abstracted from v. Linstow's 'Compendium d. Helminthologie,' I878, and Appendix, 1889, with a few recently described species from Zool. Record. It has been impossible to determine the synonyms, owing to absence of literature. 
Spirophera, Rudolphi, I8 I 9.

acanthocephalica, Molin, in Hydroprogne caspica.

campbelli, Chatin, in Notothenia filholi, Bull. Soc. Philom. 7 , ix. 37 .

Family TRICHOTRACHELID

Trichosoma, Rudolphi, i 8 i 9 .

carbonis, Rudolphi, in Phalacrocorax carbo.

convolutum, Fourment, in Ossiphraga gigantea.

Tropidocerca, Diesing, I 860.

certa, Leidy, in Diomedea exulans.

Family Strongylidæ.

Eustrongylus, Diesing, I 850.

papillosum, Rudolphi, in Phalacrocorax carbo.

tubifex, Diesing, in Podiceps cristatus.

Agamonema, Diesing, $185 \mathrm{I}$.

Family (?).

capsularia, Diesing, in Conger vulgaris.

zenis, Wedl, in Zeus faber.

fabri, Diesing, in Zeus faber.

carancum, Diesing, in Caraux trachurus.

campbelli, Chatin, Bull. Soc. Philom. 7 , ix. 37 , in various

fishes.

\section{CLASS, GORDIACEA.}

Family GORDIDE.

Gordius, Linnæus, I 767 .

diblastus, Oerley, Ann. Mag. N.H. 5, viii. $33 \mathrm{I}$.

pachydermus, Oerley, Ann. Mag. N.H. 5, viii. 33 I.

\section{CLASS, ACAN'THOCEPHALA.}

Family ECHINORHYNCHIDE.

Echinorhynchus, O. F. Müller, 1776.

urniger, Dujardin, in Zeus faber.

annulatus, Molin, in Lepidopus caudatus and in Conser vulgaris.

lamelliger, Diesing, in Naucrates ductor.

vasculosus, Rudolphi, in Brama raii.

acus, Rudolphi, in Conger vulgaris.

solitarius, Molin, in Conger vulgaris.

hystrix, Bremser, in Phalacrocorax rarbo.

striatus, Goeze, in Phalacrororax carbo.

longicollis, Villot, in Stepsilas interpres.

sphærocephalus, Bremser, in Larus dominicanus. 
Echinorhynchus capitatus, v. Linstow, in Pseudorca crassidens and in Globicephalus melas. porrigens, Rudolphi, in Balanoptera rostrata.

turbinella, Diesing, in Balcenoptera musculus and in Delphinus delphis.

\section{CLASS, CHÆTOGNATHA.}

Krohnia, Langerhans, I 880 .

Sp. innom., Parker. T.N.Z.I. xxviii. $75^{8}$ (? = Spadella hamata, Mœbius). 


\title{
PHYLUM, PLATYHELMINTHES.
}

\author{
CLASS, TURBELLARIA.
}

Order RHABDOCCELIDA.

Sub-order RHABDOCELA.

Family VORTICIDE.

Chonostomum, Schmarda, I 859.

crenulatum, Schmarda. N.W.Th. (1) 4 ; Hutton, T.N.Z.I.

xi. 3 I4.

Family Prorhynchidæ.

Prorhynchus, M. Schultze, $185 \mathrm{I}$.

putealis, Haswell. Q.J.M.S. xl. 63 I.

Order Polycladida.

Family LEPTOPLANIDE.

Leptoplana, Ehrenberg, i 83 I.

californica, Plehn. Zool. Jahrb. Syst. xii. 448.

? polysora, Schmarda. N.W.Th. (I), 25 (Centrostomum);

Hutton, T.N.Z.I. xi. $3^{1} 5$.

? brunnea, Cheeseman. T.N.Z.I. xv. 2 I 4.

Polycelis, Schmarda, 1859.

australis, Schmarda. N.W.Th. (I), 2 I ; Hutton, T.N.Z.I. xi. 315 .

Family PSEUdOCERIDE.

Thysanozoon, Grube, I 840.

brocchii, Risso. Lang, Die Polycladen, I $885,525=T$. cruciatum, Schmarda. Hutton, T.N.Z.I. xi. 3 I $5=T$. aucklandica, Cheeseman, T.N.Z.I. xv. 2 I 3.

var. huttoni, T. W. Kirk. T.N.Z.I. xiv. 267.

Family EURYLEPTIDA.

Prostheceræus, Schmarda, I 859.

herberti, T. W. Kirk (Eurylepta h.). T.N.Z.I. xiv. 267 .

Order TRICLADIA.

Tribe TERricola.

Family GEOPLANide.

Geoplana, Schultze, I 856 .

testacea, Hutton. T.N.Z.I. xii. 277 ; Graff, Mon. de Turbellarien, ii. 353 . 
Geoplana triangulata, Dendy. T.N.Z.I. xxvii. I 79.

var. australis, Dendy. T.N.Z.I. xxvii. I 80 ; xxviii. 2 I 1.

flavimarginata, Dendy. T.N.Z.I. xxix. 259.

purpurea, Dendy. T.N.Z.I. xxvii. 182 ; xxviii. 2 І 2.

garveyi, Dendy. T.N.Z.I. xxxiii. 234.

tenuis, Dendy. T.N.Z.I. xxvii. I 88.

traversii, Moseley. Q.J.M.S. n.s. xvii. 284.

challengeri, Graff. Mon. Turb. ii. 370.

moseleyi, Hutton. T.N.Z.I. xii. 277 ; Dendy, T.N.Z.I.

xxix. 261 .

graffii, Dendy. T.N.Z.I. xxvii. I 80 ; xxxiii. 224.

var. somersii, Dendy. T.N.Z.I. xxviii. 2 I 2.

var. nodosa, Dendy. T.N.Z.I. xxxiii. 227.

var. angusta, Dendy. T.N.Z.I. xxxiii. 228.

var. clintonensis, Dendy. T.N.Z.I. xxxiii. 228.

var. occidentalis, Dendy. T.N.Z.I. xxix. 260.

var. otiraensis, Dendy. T.N.Z.I. xxix. 261.

var. nigrescens, Dendy. T.N.Z.I. xxxiii. 230.

var. castanea, Dendy. T.N.Z.I. xxxiii. 225.

var. ocellata, Dendy. T.N.Z.I. xxxiii. 226.

var. wharekauriensis, Dendy. T.N.Z.I. xxxiii. 226.

var. dorsomarmorata, Dendy. T.N.Z.I. xxxiii. 228 .

iris, Dendy. T.N.Z.I. xxviii. 213.

sulphurea, Fletcher and Hamilton. Dendy, T.N.Z.I. xxvii. 183 .

alfordensis, Dendy. T.N.Z.I. xxviii. 212.

agricola, Dendy. T.N.Z.I. xxvii. I 84.

var. maori, Dendy. T.N.Z.I. xxxiii. 233.

inæqualistriata, Dendy. T.N.Z.I. xxvii. I 82.

fagicola, Dendy. T.N.Z.I. xxxiii. 233.

exulans, Dendy. T.N.Z.I. xxxiii. 233.

munda, Fletcher and Hamilton. T.N.Z.I. xxxiii. 236.

splendens, Dendy. T.N.Z.I. xxvii. I 83 .

laingii, Dendy. T.N.Z.I. xxvii. 185 .

spectabilis, Dendy. T.N.Z.I. xxvii. 185 .

hamiltoni, Dendy. T.N.Z.I. xxvii. I 86.

gelatinosa, Dendy. T.N.Z.I. xxvii. I 86.

quinquelineata, Fletcher and Hamilton. T.N.Z.I. xxviii.

$2 \mathrm{I} 2$.

jacksoniana, Dendy. T.N.Z.I. xxix. 262.

lateropunctata, Dendy. T.N.Z.I. xxxiii. $23^{2}$.

huttoni, Graff. Mon. Turb. ii. 352.

subquadrangulata, Dendy. T.N.Z.I. xxvii. I 87 .

mœbiusi, Graff. Mon. Turb. ii. 386.

meridionalis, Graff. Mon. Turb. ii. 377.

suteri, Dendy. T.N.Z.I. xxix. 263.

aucklandica, Dendy. T.N.Z.I. xxxiii. 235. 
Geoplana howesi, Dendy. T.N.Z.I. xxxiii. ${ }_{2} S$.

cooperi, Dendy. T.N.Z.I. xxxiii. 239.

latissima, Dendy. T.N.Z.I. xxviii. 2 I I.

cucullata, Dendy. T.N.Z.I. xxix. 259.

mariæ, Dendy. T.N.Z.I. xxvii. I84.

\section{CLASS, TEMNOCEPHALOIDEA.}

Order DactYlifera.

Family TEMNOCEPHALIDE.

Temnocephala, Blanchard, I 849.

novæ-zealandiæ, Haswell. Q.J.M.S. xxviii. 279 ; Macleay Mem. 1893, I38; Chilton, T.N.Z.I. xxi. 252 (on Paranephrops).

CLASS, TREMATODA.*

Order Heterocotylea.

Family TRISTOMIDÆ.

Tristomum, Cuvier, i 8 I 7 . coccineum, Cuvier, in Xiphias gladius. papillosum, Diesing, in Xiphias gladius. molæ, Blanchard, in Orthagoriscus mola. rudolphianum, Diesing, in Orthagoriscus mola. cephala, Risso, in Orthagoriscus mola. squali, Blanchard, in a shark (unnamed) from New Zealand.

Encotyllabe, Diesing, I 850 .

nordmanni, Diesing, in Brama raii.

Family Microcotylidæ.

Gastrocotyle, Van Beneden and Hesse, I863.

trachuri, Van Beneden and Hesse, in Caranx trachurus.

Order Aspidocotylea.

Family ASPIDOBOTHRIDÆ.

Macraspis, Olsson, i 868.

Sp. Benham MS. in Callorhynchus.

Order Malacotylea.

* This list of Parasites is for the most part abstracted from v. Linstow's 'Compendium d. Helminthologie,' 1878, and Appendix, 1889, and a few have been taken from the Zoological Record of more recent date. It has not been possible in all cases to determine the matter of synonymy, owing to the absence of literature. 
Family Distomidæ.

Distomum, Retzius, 1776.

insigne, Diesing, in Echinorhinus spinosus.

veliporum, Creplin, in Srymnus lichia and in Echinorhinus spinosus.

macrocotyle, Diesing, in Orthagoriscus mola. contortum, Rudolphi, in Orthagoriscus mola.

nigroflavum, Rudolphi, in Orthagoriscus mola.

foliatum, Linton, in Orthagoriscus mola.

calceolus, Molin, in Conger vulgaris.

rufoviride, Rudolphi, in Conger vulgaris and in Zeus faber.

simplex, Rudolphi, in Conger vulgaris.

varicum, Zeder, in Conger vulgaris.

echeneidis remoræ, Rudolphi, in Echeneis remora.

lageniforme, Linton, in Echeneis remora.

monticelii, Linton, Echeneis remora.

dendriticum, Rudolphi, in Xiplizas gladius.

clavatum, Rudolphi, in Xiphicas gladius.

polonii, Molin, in Caranx trachurus.

giardii, Stossich, in Naucrates ductor.

grandiporum, Rudolphi, in Conger vulgaris.

laticolle, Rudolphi, in Caraux trachurus.

cochleariforme, Rudolphi, in Fregatta aquila.

concavum, Creplin, in Podiceps cristatus.

echinatum, Zeder, in Podiceps cristatus and in Phalacrocorax carbo.

spinulosum, Rudolphi, in Podiceps cristatus.

goliath, v. Beneden, in Balcenoptera rostrata.

palliatum, Looss, in Delphinus delphis.

dephini, Poirier, in Delphinus delphis.

erinaceum, Poirier, in Delphinus delphis.

rochebruni, Poirier, in Delphinus delphis.

longissimum, Poirier, in Tursiops tursio.

Kœllikeria, Cobbold, r 859 .

filicolle, Rudolphi, in Brama raii and in Orthagoriscus mola.

Family Holostonidi:

Holostomum, Nitzsche, i 8 I9.

platycephalum, Dujardin, in Phalacrocorax carbo and in Podiceps cristatus.

variegatum, Dujardin, in Podiceps cristatus and in Phalacrocorax carbo.

squamosum, Villot, in Strepsilas interpres.

Hemistomum, Diesing, $185^{\circ}$.

trilobum, Rudolphi, in Phalacrocorax carbo. 
Hemistomum pileatum, Brandes, in Hydroprogne caspia. commutatum, Diesing, in Hydroprogne caspia.

Monostomum, Zeder, I800.

Family Monostonide.

filarinum, Diesing, in Orthagoriscus mola.

filum, Dujardin, in Brama raii.

echinostomum, Diesing, in Sula fusca.

petasatum, Deslongchamps, in Strepsilas interpres.

holostomioides, Meblis, in Podiceps cristatus.

pingue, Mehlis, in Podiceps cristatus.

Family Gasterostonide.

Gasterostomum, V. Siebold, $\mathbf{1} 848$.

armatum, Molin, in Conger vulgaris.

Family DidymozoonTidÆ.

Didymozoon, Taschenberg, I 879 .

exocœti, Parona and Perugia in Exocetus.

\section{CLASS, CESTOIDEA.}

Order Gyrocotylacea.

Family GYROCOTYLIDæ.

Gyrocotyle, Diesing, 1850 .

urna, Wagner, in Callorhynchus (see Spencer, T.R.S.V. i. I36).

$$
\begin{aligned}
& \text { Order Caryophyllacea. } \\
& \text { Family Caryophylleide. }
\end{aligned}
$$

Monobothrium, Diesing, I 850 .

punctatum, Molin, in Conger vulgaris [? a young form of Tetrabothrid].

Order Pseudophylitidea.

Family BOTHRIOCEPHALIDE.

Anchistrocephalus, Monticelli, I 890.

macrocephalus, Rudolphi, in Orthagoriscus mola (Bothriocephalus $m$.).

Fistulicola, Lühe, I 899 .

plicatus, Rudolphi, in Xiphias gladius (Bothriocephalus p.).

Bothriocephalus, Rudolphi, I 8 ro.

quadratus, v. Linstow, in Ogmorhinus leptonyx:

bramæ, Ariola, in Brama raii.

Schistocephalus, Creplin, I 829 .

nodosus, Bloch, in Podiceps cristatus. 
Ligula, Bloch, 1782 .

monogramma, Creplin, in Podiceps cristatus.

digramma, Creplin, in Podiceps cristatus.

Order Tetraphyllidea.

Family Tetrabothride.

Tetrabothrium (Rudolphi), Fuhrmann, I 899.

maculatum, Olssen, in Lamna cornubica.

macrocephalum, Rudolphi, in Podiceps cristatus.

auriculatum, v. Linstow, in Thalassaca glacialoides and Daption capensis.

erostris, Limsberg, in Catarhactes chrysocome.

Orygmatobothrium, Diesing, 1854 . dohrnii, Oerley, in Scymmus lichia.

Amphoterocotyle, Diesing, 1863 . elegans, Diesing, in Daption capensis.

Phyllobothrium, v. Beneden, I 849. delphini, Gervais, in Tursiops tursio.

Anthobothrium, v. Beneden, I 849 . cornucopia, v. Beneden, in Lamna cornubica.

Cylindrophorus, Diesing, 1863 .

typicus, Diesing, in Carcharodon rondeletii.

Dinobothrium, v. Beneden, I889.

septaria, v. Beneden, in Lamna cornubica.

Diplobothrium, v. Beneden, I889.

simile, v. Beneden, in Lamna cornubica.

\section{Order Tetrarhyncha. \\ Family TETRARHYNCHIDÆ.}

Tetrarhynchus, sensu lato, Vaullegard, I899.

bicolor, v. Nordmann, in Carch. rondeletii = Abothros carcharias, Welch.

erinaceus, v. Beneden, in Scymnus lichia.

infulatus, Diesing, in Zeus faber and in Conger vulgaris.

paleaceus, Rudolphi, in Conger vulgaris and in Caranx trachucrus.

crassiceps, Diesing, in Brama raii.

gracilis, Wagener, in Orthagoriscus mola. attenuatus, Rudolphi, in Xiphias gladius. tenuicaudatus, Leidy, in Echeneis remora. gigas, Vaullegard = reptans, Wagener = elongatus, Rudolphi, in Zeus faber and in Orthagoriscus mola. 


\section{Order Tetracotylea.}

Family TæNıDÆ.

Drepanidotænia, Raillet, 1892.

apterygis, Benham. Q.J.M.S. xliii. $8_{3}$, in Apteryx.

minuta, Benham. Q.J.M.S. xliii. $8_{3}$, in Apteryx.

Tænia, sensu lato, Linnæus, 1758.

scolecina, Ruclolphi, in Phalacrocorax carbo.

increscens, v. Linstow, in Hamatopus unicolor.

coronata, Krefft, in Himantopus leucocephalus.

rugosa, Krefft, in Himantopus leucocephalus.

cylindrica, Krefft, in Anas superciliosa.

pediformis, Krefft, in Anas superciliosa.

flavescens, Krefft, in Anas superciliosa and in Spatula rhynchota.

bairdi, Kreff, in Anas superciliosa.

tuberculata, Krefft, in $N$ yroca australis.

filum, Goeze, in Strepsilas interpres.

clavigera, Krabbe, in Strepsilas interpres.

retirostris, Krabbe, in Strepsilas interpres.

furcifera, Krabbe, in Podiceps cristatus.

capillaris, Rudolphi, in Podiceps cristatus.

pelecani aquilæ, Rudolphi, in Fregatta aquila.

sulciceps, Baird, in Diomedea exulans.

apterycis, Chatin, in Apteryx, Bull. Soc. Philom. (7), ix. $3^{6 .}$

[Is probably identical with one of the two sp. Drepanidotcenia, above.]

\section{CLASS, NEMERTINI.}

\section{Order Metanenertini.}

? Amphiporus, Ehrenberg, I $83 \mathrm{I}$, or ? Eunemertes, Vaillant, I 890 .

heterophthalma, Schmarda. N.IV.Th. (1), 41 ; Ommatoplea h., Hutton, T.N.Z.I. xi. 316.

? Amphiporus or ? Drepanophorus, Hubrecht, 1874.

novæ-zealandiæ, Quoy and Gaimard. Voy. Astrolabe, Zool. iv. 290 (Borlasia, N.Z.); Hutton, T.N.Z.I. xii. 277.

Family Prosorhochmide.

Geonemertes, Semper, $\mathrm{I}_{86}$. novæ-zealandiæ, Dendy. T.N.Z.I. xxvii. 192 ; xxviii. 214. 
Order Heteronemertini.

Eupolia, Hubrecht, I 887.

Family EUPOLIIDÆ.

giardii, McIntosh. Challenger Reports, xix. I I.

australis, Hubrecht. Challenger Reports, xix. I 3.

Cerebratulus, Renier, I 804 .

Family LineIde.

macrostomus, Schmarda (Meckelia m.). N.W.Th. (r), +2 ; Hutton, T.N.Z.I. xi. 3 I6.

macrorhochmus, Schmarda (Meckelia m.). N.W.Th. (ז), 43 ; Hutton, T.N.Z.I. xi. 3 I 6 .

parkeri, Hubrecht. Challenger Reports, xix. 43 .

angusticeps, Hubrecht. Challenger Reports, xix. 44.

macroren, Hubrecht. Challenger Reports, xix. 46. 


\section{PHYLUM, CCELENTERATA.}

\section{CLASS, CTENOPHORA.}

Order Saccata.

Family Pleurobrachidæ.

Eschscholthia, Lesson, I 843 .

dimidiata, Lesson. Ann. Sc. Nat. v. 254 ; Acalèphes, 102.

\section{CLASS, ACTINOZOA.}

Sub-class ZOANTHARIA.

Order ACTINIARIa.

Family Phyllactide.

Oulactis, H. Milne-Edwards, 1857 . plicatus, Hutton. T.N.Z.I. xi. $31 \mathrm{I}$.

Gregoria, Gosse, I860.

Family Sagartiade.

albocincta, Hutton. T.N.Z.I. xi. $3 \mathbf{I} 2$.

Sagartia, Gosse, $x 8_{5} 8$.

crocata, Hutton (Diactis). T.N.Z.I. xii. 275 .

Family ANTHEADÆ.

Paractis, H. Milne-Edwards, 1857. monilifera, Drayton. Hutton, T.N.Z.I. xii. 275 .

Anthea, Johnstone, 1838 .

olivacea, Hutton. T.N.Z.I. xii. $3^{\text {I } 2 .}$

Actinia, Gmelin, 1789 .

Family Actiniade.

striata, Quoy and Gaimard (gen. dub.). Hutton, T.N.Z.I. xii. 213 .

tenebrosa, Farquhar. J.L.S. xxvi. 535 .

thomsoni, Coughtrey. T.N.Z.I. vii. 280 (genus doubtful); Farquhar, J.L.S. xxvi. 527 . 
Phymactis, H. Milne-Edwards, I 857 . polydactyla, Hutton. T.N.Z.I. xi. 3 I 3 . inconspicua, Hutton. T.N.Z.I. xi. $3^{\mathrm{I}} 3$.

Peachia, Gosse, r 854 .

Family IlyanthIDÆ.

carnea, Hutton. T.N.Z.I. xii. 275.

Minyas, Cuvier, 18 I 7 .

Family MiNYADidæ.

viridula, Quoy and Gaimard. Hutton, T.N.Z.I. xi. 3 I 4.

Order MADREPORARIA.

Section Aporosa.

Sub-section TURBINOLIDA.

Family TURBINOLIDÆ.

Flabellum, Lesson, i 8-. rubrum, Quoy and Gaimard. Voy. Astrolabe, iv. I 88.

Conocyathus, d'Orbigny, i 849 .

zealandiæ, Duncan. P.Z.S. I 876, 43 I.

Cylicia, Edwards and Haime, I $85 \mathrm{I}$.

rubeola, Quoy and Gaimard. Voy. Astrolabe, iv. 197.

huttoni, Ten. Woods. P.L.S.N.S.IV. iii. 132.

vacua, Ten. Woods. P.L.S.N.S.W. iii. I 33 .

Placotrochus, Edwards and Haime, r 848.

pedicellatus, Ten. Woods. P.L.S.N.S.IV. iii. I 34 .

Polyphyllia, Quoy and Gaimard, I833.

pelvis, Quoy and Gaimard. Voy. Astrolabe, iv. I 85.

Caryophyllia, Lamarck, r8or.

maculata, Pourtales. Moseley, Challenger Reports, ii. I39.

lamellifera, Moseley. Challenger Reports, ii. I 40.

Order ANTIPITHARIA.

Family ANTIPATHIDE.

Aphanipathes, Brook, I889.

fruticosa, Gray. Brook, Challenger Reports, xxxii. I 24.

Taxipathes, Brook, I 889 .

recta, Brook (?). Challenger Reports, xxxii. I 56. [Specimen in Canterbury Museum.]

\section{Sub-class Alcyonaria.}

Order Alcyonacea.

Family CORNULARIIDÆ.

Anthopodium, Verrill, I872.

australe, Verrill. Bull. U.S. Mus. I876, 76 . 
Family Alcyonıdæ.

Lobularia, Lamarck, i 8 i 6.

aurantiaca, Lamarck. Quoy, Voy. Astrolabe, iv. 277.

Order Pennatulacea.

Virgularia, Lamarck, i 8 I 6.

Family VIRGULARIDÆ.

gracillima, Kölliker. Dendy, T.N.Z.I. xxix. $25^{6}$.

Protocaulon, Kölliker, I 880.

Family Protocaulide.

molle, Kölliker. Challenger Reports, i. 26.

Family Protoptilide.

Leptoptilum, Kölliker, I880.

gracile, Kölliker. Challenger Reports, i. 27.

Order Gorgonacea.

Family DASYGORGIDE.

Dasygorgia, Verrill, i $\$ 83$.

expansa, Wright and Studer. Challenger Reports, xxxi. I6. acanthella, Wright and Studer. Challenger Reports, xxxi. I 8. axillaris, Wright and Studer. Challenger Reports, xxxi. 20.

Calytrophora, Gray, I 866 .

Family PRIMNOIDÆ.

wyvillei, Wright and Studer. Challenger Reports, xxxi. $5^{2}$.

Stachyodes, Wright and Studer, I 889.

regularis, Wright and Studer. Challenger Reports, xxxi. 55.

Thouarella, Gray, i 870 .

moseleyi, Wright and Studer. Challenger Reports, xxxi. 6r.

Primnoella, Gray, 1857 .

australasiæ, Gray. Wright and Studer, Challenger Reports, xxxi. 88 .

Rhipidigorgia, Valenciennes, I 854 .

Family ScLerogorgiadæ.

cribum, Valenciennes. H. Milne-Edwards, Coralleairies, i. 175

arenata, Valenciennes. H. Milne-Edwards, Coralleairies,

i. 176 .

CLASS, SCYPHOZOA.

Order STAUROMEDUSÆ.

Family LUCERNARID瓜.

Craterolophus, Clark, $186_{3}$.

macrocystis, Lendenfeld (nomen nudum). P.L.S.N.S.W. ix. 165 . 
Order Peromeduse.

Pericrypta, Haeckel, I 879.

Family PERICOLPIDE.

campana, Haeckel. Lendenfeld, P.L.S.N.S.IV. ix. 167.

Family Periphyllide.

Periphylla, Steenstrup, I 837 .

mirabilis, Haeckel. Lendenfeld, P.L.S.N.S.IV. ix. I68.

Order Discomeduse.

Archirhiza, Haeckel, I880.

Family TOREUMIDE.

aurosa, Haeckel. Lendenfeld, P.L.S.N.S.W. ix. 282 and 425 .

Toreuma, Haeckel, I880.

theophila, Haeckel. Lendenfeld, P.L.S.N.S.W. ix. 283 and 426 .

Cannorhiza, Haeckel, I88o.

connexa, Haeckel. Lendenfeld, P.L.S.N.S.W. ix. 294 and 427 .

\section{CLASS, HYDROZOA.}

Order SipHONOPHORA.

Velella, Lamarck, i 8i 6.

Family VeLELLIDE.

cyanea, Lesson. Haeckel, Challenger Reports, xxviii. $\delta_{3}$.

Desmalia, Haeckel, i 888.

Family Desnophyide.

imbricata, Haeckel. Challenger Reports, xxviii. I69.

Diphyes, Cuvier, I8 7 .

Family DıPHyıdæ.

appendicularia, Eschscholtz. Hutton, T.N.Z.I. xii. 274.

Family Agalmidæ.

Stephanomia, Péron and Lesueur, i 807.

imbricata, Quoy and Gaimard. Voy. Astrolabe, iv. 7 I.

Physophora, Forskal, I 775 .

Family Discolabide.

australis, Quoy and Gaimard. Hutton, T.N.Z.I. xii. 274.

Family STEPHALIDE.

Stephonalia, Haeckel, i 888 .

bathyphysa, Haeckel. Challenger Reports, xxviii. 299. 
Physalia, Lamarck, i 818.

$$
\text { Family Physalide. }
$$

utriculus, Eschscholtz. Haeckel, Challenger Reports, xxviii. $35^{\mathrm{I}}$.

Order HYDROCORALLINA.

Stylaster, Gray, I 83 I.

$$
\text { Family STYLASTERIDÆ. }
$$

erubescens, Pourtales. Moseley, Challenger Reports, ii. 8I. gracilis, H. Milne-Edwards and Haime. Moseley, Challenger Reports, ii. 8 I.

lævis, Studer. Moseley, Challenger Reports, ii. SI.

sanguineus, Valenciennes. Lendenfeld, P.L.S.N.S.W. ix. 6 I 5 .

Allopora, Ehrenberg, I 834 .

profunda, Moseley. Challenger Reports, ii. 62.

Cryptohelia, H. Milne-Edwards and Haime.

pudica, H. Milne-Edwards and Haime. Moseley, Challenger Reports, ii. 7 I.

Conopora, Moseley, I88I.

tenuis, Moseley. Challenger Reports, ii. 82 .

Errina, Gray, I 835 .

[Two species are in the Canterbury Museum.]

Tubiclava, Allman, 1862 .

Order HYDROIDA.

Sub-order Gymnoblastea.

Family Clavide.

rubra, Farquhar. T.N.Z.I. xxvii. 209.

fruticosa, Allman. Hilgendorf, T.N.Z.I. xxx. $20 \mathrm{r}$.

Cordylophora, Allman, I 844 .

lacustrus (?), Hamilton. N.Z. Jour. Science, i. 4 I 9.

Coryne, Lamarck, i80 I.

Family CORYNIDE.

tenella, Farquhar. T.N.Z.I. xxvii. 208.

Family EUDENDRIDÆ.

Eudendrium, Ehrenberg, I 834 . novæ-zealandiæ, Marktanner-Turneretscher. Farquhar, T.N.Z.I. xxviii. 459 .

Family BougainvilLide.

Hemitheca, Hilgendorf, I 898. intermedia, Hilgendorf. T.N.Z.I. xxx. 202. 
Family Tubularidde.

Tubularia, Linnæus, 1756 .

attenuoides, Coughtrey. T.N.Z.I. viii. 302 ; Hilgendorf, T.N.Z.I. Xxx. 202.

Pelagohydra, Dendy, I 902.

mirabilis, Dendy. Q.J.M.S. xlvi. I.

\section{Sub-order Calyptoblastea.}

Family Campanularinde.

Eucopella, Lendenfeld, I 883 .

crenata, Hartlaub.

C. caliculata var. makrogona, Lendenfeld. Farquhar, T.N.Z.I. xxviii. 459.

Hypanthea, Allman, 1876 .

bilabiata, Coughtrey. Farquhar, T.N.Z.I. xxviii. 460 ; Hilgendorf, T.N.Z.I. xxx. 213.

asymmetrica, Hilgendorf. T.N.Z.I. xxx. 2 I 2.

Campanularia, Lamarck, i 8 i 6 .

carduella, Allman. Farquhar, T.N.Z.I. xxviii. 460.

fruticosa, Esper. Farquhar, T.N.Z.I. xxviii. 460.

Obelia, Lamarck, I82 I.

geniculata, Linnæus. Farquhar, T.N.Z.I. xxviii. 460.

australis, Lendenfeld. Farquhar, T.N.Z.I. xxviii. 460.

pygmæa, Coughtrey. Farquhar, T.N.Z.I. xxviii. 460.

nigrocaulus, Hilgendorf. T.N.Z.I. xxx. 203.

Hebella, Allman, i 888 .

scandens, Bale. Farquhar, T.N.Z.I. xxviii. 460.

Calycella,

parkeri, Hilgendorf. T.N.Z.I. xxx. 205.

Clytia, Lamarck, i 8 i 2.

elongata, Marktanner-Turneretscher (gen. dub.). Farquhar, T.N.Z.I. xxviii. $46 \mathrm{I}$.

johnstoni, Alder. Hartlaub, Zoologischen Jahrbüchern, xiv. 364 .

Eucope, Geg.

annulata, Lendenfeld. Farquhar, T.N.Z.I. xxviii. $46 \mathrm{I}$.

Thyroscyphus, Allman, I 877 .

tridentatus, Bale. Hartlaub, Zoologischen Jahrbiichern, xiv. 364 .

Lafoëa, Lamouroux, I82 I.

Family PerisiphonidDe.

cylindrica, Lendenfeld. Farquhar, T.N.Z.I. xxviii. 46r.

dumosa, Coughtrey (sp. dub.). Farquhar, T.N.Z.I. xxviii. 46 I. 
Cryptolaria, Busk, 1857 .

gracilis, Allman. Farquhar, T.N.Z.I. xxviii. $46 \mathrm{I}$.

Perisiphonia, Allman, 1888.

pectinata, Allman. Farquhar, T.N.Z.I. xxviii. 46I.

Halecium, Oken, 1815.

Family HaLECIIDE.

delicatula, Coughtrey. Farquhar, T.N.Z.I. xxviii. 46 I. parvulum, Bale. Farquhar, T.N.Z.I. xxviii. 46 I.

Family Sertularidde.

Sertularia, Linnæus, I 748 .

elongata, Lamouroux. Farquhar, T.N.Z.I. xxviii. 46 I.

bispinosa, Gray. Farquhar, T.N.Z.I. xxviii. 462.

crinis, Allman. Farquhar, T.N.Z.I. xxviii. 462.

trispinosa, Coughtrey. Farquhar, T.N.Z.I. xxviii. 462.

ramulosa, Coughtrey. Farquhar, T.N.Z.I. xxviii. 462.

operculata, Linnæus. Farquhar, T.N.Z.I. xxviji. 462.

minima, Thompson. Farquhar, T.N.Z.I. xxviii. 462.

unguiculata, Busk. Farquhar, T.N.Z.I. xxviii. 463.

simplex, Lendenfeld. Farquhar, T.N.Z.I. xxviii. 463 .

huttoni, Marktanner-Turneretscher. Farquhar, T.N.Z.I. xxviii. 463 .

unilateralis, Allman. Farquhar, T.N.Z.I. xxviii. $46_{3}$.

australis, Thompson. Lendenfeld, P.L.S.N.S.W. ix. 408.

Diphasia, Agassiz, 1862 .

symmetrica, Lendenfeld. P.L.S.N.S.W. ix. $4 \mathrm{I} 4$.

Sertularella, Gray, 1848 .

johnstoni, Gray. Farquhar, T.N.Z.I. xxviii. 463.

capillaris, Allman. Farquhar, T.N.Z.I. xxviii. $46_{3}$.

polyzonias, Linnæus. Farquhar, T.N.Z.I. xxviii. 463 .

robusta, Coughtrey. Farquhar, T.N.Z.I. xxviii. 464.

pygmæa, Bale. Farquhar, T.N.Z.I. xxviii. 464 .

ramosa, Thompson. Farquhar, T.N.Z.I. xxviii. 464.

exigua, Thompson. Farquhar, T.N.Z.I. xxviii. 464.

integra, Allman. Farquhar, T.N.Z.I. xxviii. 464.

muelleri, Kirchenpauer. Farquhar, T.N.Z.I. xxviii. 464 .

episcopus, Allman. Farquhar, T.N.Z.I. xxxviii. 464.

tenella, Alder. Hartlaub, Zoologischen Jahrbüchern, xiv. 370.

solidula, Bale. Hartlaub, Zoologischen Jahrbüchern, xiv. $37 \mathrm{I}$.

fusiformis, var. nana, Hartlaub. Zoologischen Jahrbüchern, xiv. 372 .

Thuiaria, Fleming, I 828 .

zelandica, Gray. Farquhar, T.N.Z.I. xxviii. 464 . monilifera, Hutton. Farquhar, T.N.Z.I. xxviii. 465. subarticulata, Coughtrey. Farquhar, T.N.Z.I. xxviii. 465 . quadridens, Bale. Farquhar, T.N.Z.I. xxviii. 465. 
Selaginopsis, Allman, ${ } S_{7} 6$.

novæ-zealandiæ, Thompson. Farquhar, T.N.Z.I. xxviii. 465 .

Desmoscyphus, Allman, i 876 .

buskii, Allman. Farquhar, T.N.Z.I. xxviii. 465.

Hydrallmania, Hincks, i 869 .

bicalicula, Coughtrey (Gen. dub.). Farquhar, T.N.Z.I. xxviii. 465 .

Family SyntheCIDÆ.

Synthecium, Allman, 1876 .

elegans, Allman. Farquhar, T.N.Z.I. xxviii. 465 .

ramosum, Allman. Farquhar, T.N.Z.I. xxviii. 465.

campylocarpum, Allman. Farquhar, T.N.Z.I. xxviii. 466.

Family Plumularidide.

Plumularia, Lamarck, i 8 i 5 .

setacea, Ellis. Farquhar, T.N.Z.I. xxviii. 466.

spinulosa, Bale. Farquhar, T.N.Z.I. xxviii. 466.

turgida, Bale. Farquhar, T.X.Z.I. xxviii. 466.

campanula, Busk. Farquhar, T.N.Z.I. xxviii. 466.

multinoda, Allman. Farquhar, T.N.Z.I. xxviii. 466.

simplex, Coughtrey (Gen. dub.). Farquhar, T.N.Z.I. xxviii. 466.

tripartita, Lendenfeld. P.L.S.N.S.IV. i.x. 477.

Antennularia, Lamarck, I8I6.

antennina, Linnæus. Farquhar, T.N.Z.I. xxviii. 466.

Family Aglaophenidid.

Aglaophenia, Lamarck, 1812.

incisa, Coughtrey. Farquhar, T.N.Z.I. xxviii. 467.

banksii, Gray. Farquhar, T.N.Z.I. xxviii. 467.

huttoni, Coughtrey. Farquhar, T.N.Z.I. xxviii. 467.

acanthocarpa, Allman. Farquhar, T.N.Z.I. xxviii. 467 .

laxa, Allman. Farquhar, T.N.Z.I. xxviii. 467.

formosa, Busk. Farquhar, T.N.Z.I. xxviii. 467.

filicula, Allman. Hilgendorf, T.N.Z.I. xxx. 215.

Idia, Lamarck, 18 I 6 .

Family IDIIDE.

pristis, Lamouroux. Farquhar, T.N.Z.I. xxviii. 467.

Sub-order ElEUTHEROBLASTEA.

Hydra, Linnæus, $175^{6}$.

Family HYDRIDA:

viridis, Linnæus. Farquhar, 'T.N.Z.I. xxviii. 468. 


\title{
PHYLUM, PORIFERA.
}

\author{
CLASS, CALCAREA.
}

Order HoMOCOELA.

Family LEUCOSOLENIDE.

Leucosolenia, Bowerbank, I862.

lucasis, Dendy. T.N.Z.I. xxvi. I 78. echinata, Kirk. T.N.Z.I. xxvi. I 77 .

cerebrum, Haeckel. T.N.Z.I. xxviii. 207.

challengeri, Polejaeff. T.N.Z.I. xxviii. 207.

clathrus, Schmidt. T.N.Z.I. xxviii. 206.

depressa, Dendy. T.N.Z.I. xxviii. 209. intermedia, Kirk. T.N.Z.I. xxviii. 208.

laxa, Kirk. T.N.Z.I. xxviii. 208.

proxima, Dendy. T.N.Z.I. xxviii. 207. rosea, Kirk. T.N.Z.I. xxviii. 209.

Order Heterocoela. Family LEUCASCIDE.

Leucascus, Dendy, I892.

simplex, Dendy. T.N.Z.I. xxx. 313.

Family Sycettide.

Sycon, Risso, 1827 .

dendyi, Kirk. T.N.Z.I. xxvii. 287. inconspicua, Lendenfeld. P.L.S.N.S.W. 1885 , 1093. ornatum, Kirk. T.N.Z.I. xxix. 3 I4. pedicellatum, Kirk. T.N.Z.I. xxix. 3 r 3 . 3 sp. innominata.

Lamontia, Kirk, r 894 .

Family Grantide.

zona, Kirk. T.N.Z.I. xxvii. 289.

Leucandria, Haeckel, I 872.

Species innominata.

Grantessa, Lendenfeld, I 885 . intus-articulata, Carter.

Vosmaeropsis, Dendy, r892. macera, Carter. 
CLASS, SIILICEA.

Sub-class Hexactinellida.

Family EuPLECTELLID

Walteria, Schulze, 1887 .

flemmingii, Schulze. Challenger Reports, xxi. 96.

Family RosSellide.

Euryplegma, Schulze, I 887 .

auriculare, Schulze. Challenger Reports, xxi. I 76.

Aulochone, Schulze, I 887.

cylindrica, Schulze. Challenger Reports, xxi. I68.

Family Hyalonematide.

Poliopogon, Wyville-Thomson, I 877 .

gigas, Schulze. Challenger Reports, xxi. 254.

Family FARREIDÆ.

Farrea, Bowerbank, r862. Challenger Reports, xxi. 277.

Family CoscinosPoride.

Chonelasma, Schulze, I887.

lamella, Schulze. Challenger Reports, xxi. $32 x$.

hamatum, Schulze. Challenger Reports, xxi. 323.

Sub-class Triaxonia. Order Hexaceratina.

Family Aplysyllide.

Dendrilla, Lendenfeld, I 883 .

rosea, Lendenfeld. Cat. Sponges in Aust. Mus. zS.

Sub-class Tetrazonia.

Order Chondrospongia.

Family TETHYDE.

Tethya, Lamarck, i 8 I 6 .

fissurata, Lendenfeld. Cat. Sponges in Aust. Mus. 48.

Family Stellettide.

Tethyopsis, Stewart, 1870 .

radiata, Marshall. Challenger Reports, xxv. I90.

Axinella, Schmidt, I 862 .

Family AxineLLidF..

sinclairi, Gray. T.N.Z.I. xxx. 3 I 7 .

Family SPONGILID

Spongilla, Lamarck, i 8 i 6.

lacustris, Johnston. N.Z. Jour. Sci. i. $3 \delta_{3}$ and 572 . 
Order CoRnacuspongia.

Family Desaiacidonide.

Cladorhiza, Sars, 1872 .
pentacrini, Dendy.
A.M.N.H. $5, \mathrm{xx} .279$.

Myxilla, Schmidt, i 862 .

frondosa, Ridley and Dendy. Challenger Reports, xx. I44.

Clathria, Schmidt, I 862.

macropora, Lendenfeld. Cat. Sponges in Aust. Mus. $22 \mathrm{I}$.

Rhaphidophlus, Ehlers, i 870.

anchoratum, var. lamellosus, Lendenfeld. Cat. Sponges in Aust. Mus. 220 (Echinonema).

Family HETERORRAPHIDE.

Stylotella, Lendenfeld, I 888 .

digitata, Lendenfeld. Cat. Sponges in Aust. Mus. I85.

Family SPONGEL1D瓜.

Sigmatella, Lendenfeld, I 888.

corticata, var. papillosa, Marshall. Lendenfeld, Man. Horny Sponges, 620.

Psammopemma, Marshall, i880.

crassum, Carter. Lendenfeld, Man. Horny Sponges, 638 .

Haastia, Lendenfeld, I889.

navicularis, Lendenfeld. Man. Horny Sponges, 628 .

Spongelia, Nardo, 1834 .

ramsayi, Lendenfeld. Cat. Sponges in Aust. Mus. 209.

varia, Gray. T.N.Z.I. xxx. 319.

elastica, Schulze, var. lobosa, Lendenfeld. A.M.N.H. 6, xix. I 24.

Chalina, Grant, I86r.

Family HOMORRAPHIDE.

ramosa, Gray. T.N.Z.I. xxx. $3 \mathrm{I} 8$.

Cacochalina, Schmidt, I 870 .

pandæa, Lendenfeld. Zool. Jahrb. 1887 , ii. 763 .

velinae, Lendenfeld. Zool. Jahrb. I887, ii. 762 .

truncatella, Lendenfeld. Zool. Jahrb. I 887 , ii. 763 .

typica, Lendenfeld. Zool. Jahrb. 1887, ii. 764 .

Cladochalina, Schmidt, ז 870.

dendroides, Lendenfeld. Zool. Jahrb. I 887 , ii. 769 . mammillata, Lendenfeld. Zool. Jahrb. 1887 , ii. 769 . tenuiraphis, Lendenfeld. Zool. Jahrb. 1887 , ii. 769 .

Pachychalina, Schmidt, I 868.

paucispina, Lendenfeld. Zool. Jahrb. I887, ii. 776 .

ramulosa, Lendenfeld. Zool. Jahrb. 1887 , ii. 777 . 
Pachychalina macrospina, Lendenfeld. Zool. Jahrb. I 887 , ii. 777 .

elegans, Lendenfeld. Zool. Jahrb. 1887 , ii. 777 .

rigida, Lendenfeld. Zool. Jahrb. 1887 , ii. 778 .

Ceraochalina, Lendenfeld, I $\$ 87$.

papillata, Lendenfeld. Zool. Jahrb. I 887 , ii. 780 .

microrhaphis, Lendenfeld. Zool. Jahrb. 1887 , ii. 783 .

multiformis, Lendenfeld. Zool. Jahrb. I 887 , ii. $78_{3}$.

reteplax, Lendenfeld. Zool. Jahrb. I 887 , ii. 785 .

extrema, Lendenfeld. Zool. Jahrb. i 887 , ii. 785 .

Antherochalina, Lendenfeld, 1887.

renicroides, Lendenfeld. Zool. Jahrb. 1887 , ii. 788 .

concentrica, Lendenfeld. A.M.N.H. 6, xix. I 24.

Placochalina, Lendenfeld, r 887.

arenosa, Lendenfeld. Zool. Jahrb. I887, ii. 79г.

Toxochalina, Ridley, I884.

folioides, Lendenfeld. Zool. Jahrb. I 887 , ii. 797.

Phylosiphonia, Lendenfeld, I887.

pumila, Lendenfeld. Zool. Jahrb. r 887 , ii. 799 .

Dactylochalina, Lendenfeld, I 885 .

arenosa, Carter. Zool. Jahrb. I887, ii. 8I I.

candelabrum, Lendenfeld. Zool. Jahrb. ii. SI 2.

Euchalinopsis, Lendenfeld, I 887.

oculata, Lendenfeld. Zool. Jahrb. I887, ii. 8 15.

minima, Lendenfeld. Zool. Jahrb. ז887, ii. 8 г6.

Euchalina, Lendenfeld, i 887.

paucispina, Lendenfeld. Zool. Jahrb. I887, ii. 8 I 8 .

Family SPONGIDE.

Phyllospongia, Ehlers, I872.

perforata, Hyatt. Lendenfeld, Man. Horny Sponges, I 72.

macropora, Lendenfeld. Man. Horny Sponges, I 73 .

papyracea, Esper. Man. Horny Sponges, I 87.

distans, Lendenfeld. Man. Horny Sponges, i 89.

arbuscula, Lendenfeld. Man. Horny Sponges, I 90.

foliascens, Pallas. Man. Horny Sponges, I 96.

spiralis, Lendenfeld. Man. Horny Sponges, 200.

Chalinopsilla, Lendenfeld, i 889 .

australis, var. reticulata, Lendenfeld. Cat. Sponges in Aust. Mus. 105.

arborea, var. macropora, Lendenfeld. Cat. Sponges in

Aust. Mus. i Io.

arborea, var. massa, Lendenfeld. Man. Horny Sponges, I 53. 
Euspongia, Bronn, I 859 .

irregularis, var. silicata, Lendenfeld. Cat. Sponges in Aust. Mus. 134.

irregularis, var. tenuis, Lendenfeld. Man. Horny Sponges, $25 \mathrm{I}$.

irregularis, var. villosa, Lendenfeld. Man. Horny Sponges, 252.

Hippospongia, Schulze, I879.

equina, var. elastica, Lendenfeld. Man. Horny Sponges, 307 .

canaliculata, var. microtuba, Lendenfeld. Man. Horny Sponges, 325 .

Thorecta, Lendenfeld, i 888.

exemplum, var. tertia, Lendenfeld. Cat. Sponges in Aust. Mus. I 45 .

squalidus, Lendenfeld. Man. Horny Sponges, 348.

meandrinus, Lendenfeld. Man. Horny Sponges, $35^{\circ}$.

byssoides, Lamarck. Man. Horny Sponges, 365 .

Aplysina, Nardo, 1833 .

ramosa, Lendenfeld. Man. Horny Sponges, 4 I I.

procumbens, Lendenfeld. Man. Horny Sponges, 4I 6. meandrina, Lendenfeld. Man. Horny Sponges, 4 I 8.

Stelospongia, Schmidt, I 870 .

serta, Lendenfeld. Man. Horny Sponges, 489. australis, var. conulata, Lendenfeld. Man. Horny Sponges, $5^{16}$. 


\title{
PHYLUM, PROTOZOA.
}

\author{
CLASS, INFUSORIA.
}

Order Tentaculifera.

Acineta, Ehrenberg, 1832 .

speciosa, Maskell. 'T.N.Z.I. xix. 60, and xx. г8.

simplex, Maskell. T.N.Z.I. xix. 6 I.

flos, Maskell. T.N.Z.I. xx. I 7 .

angularis, Maskell. T.N.Z.I. xx. I 7 .

tulipa, Maskell. 'T.N.Z.I. xx. i 8.

lasanicola, Maskell. T.N.Z.I. xx. i8.

Trichophyra, Claparède and Lachmann, i 859.

epistilidis, Claparède and Lachmann. Maskell, 'T.N.Z.I.

xx. 17 .

Sphærophyra, Claparède and Lachmann, i 859.

magna, Maupas. Maskell, T.N.Z.I. xix. 60.

Order Ciliata.

Sub-order Нуроткісна.

Family EUPLOTIDE.

Euplotes, Ehrenberg, I 832 .

patella, Ehrenberg. Maskell, T.N.Z.I. xix. 60.

charon, Müller. Maskell, T.N.Z.I. xx. i 7 .

Glaucoma, Ehrenberg, i $\delta_{3}$.

scintillaris, Ehrenberg. Maskell, T.N.Z.I. xix. 60.

Aspidisca, Ehrenberg, I 830 .

turrita, Claparède and Lachmann. Maskell, T.N.Z.I. xix. 60.

Family OXYTRICHIDÆ.

Stylonychia, Ehrenberg, I 830 . mytilus, Ehrenberg. Maskell, T.N.Z.I. xix. 60.

Histrio, Sterki.

acuminatus, Maskell. T.N.Z.I. xix. 59.

Opisthotricha, Saville-Kent, I882.

parallela, Engelmann. Maskell, T.N.Z.I. xx. I 7 .

Gastrostyla, Engelmann, i 86 I.

steinii, Engelmann. Maskell, T.N.Z.I. xix. 59. 
Uroleptus, Ehrenberg, I 832.

musculus, Müller. Maskell, T.N.Z.I. xix. 59.

Stichotricha, Pertz, I 852.

remex, Hudson. Maskell, T.N.Z.I. xix. 59.

Keroma, Müller, I 786 .

polyporum, Ehrenberg. Maskell, T.N.Z.I. xx. I6.

Psilotricha, Stein, I 859 .

acuminata, Stein. Maskell, T.N.Z.I. xix. 59.

Family Dysteriade.

Egyria, Claparède and Lachmann. astyla, Maskell. T.N.Z.I. xx. I 5. distyla, Maskell. T.N.Z.I. xx. I 5 .

Family Chlamydodontride.

Loxodes, Ehrenberg, I 830. rostrum, Ehrenberg. Maskell, T.N.Z.I. xix. 59.

Chilodon, Ehrenberg, I 834 . cucullulus, Müller. Maskell, T.N.Z.I. xix. 59.

Phascolodon, Stein. elongatus, Maskell. T.N.Z.I. xx. I4.

Family LitonotidÆ.

Litonotus, Wrzesmiowski, i 870 . fasciola, Ehrenberg. Maskell, T.N.Z.I. xix. 59.

Family ThUROPHORID..

Thurophora, Maskell, I888. lucens, Maskell. 'T.N.Z.I. xx. I6.

Sub-order PERITRICHA.

Family VORTICELLIDE.

Vorticella, Müller, I 773 .

annularis, Müller. Kirk, T.N.Z.I. xviii. 2 I 5. longifilum, Kent. Kirk, 'T.N.Z.I. xviii. 2 I6. campanula, Ehrenberg. Maskell, T.N.Z.I. xix. 58. cratera, Kent. Maskell, T.N.Z.I. xix. 58. citrina, Ehrenberg. Maskell, T.N.Z.I. xix. 58. zealandica, Kirk. T.N.Z.I. xviii. 2 I 6. elongata, De Fromentel. Maskell, T.N.Z.I. xix. 58. patellina, Müller. Maskell, T.N.Z.I. xix. 58. nebulifera, Ehrenberg. Maskell, T.N.Z.I. xix. 58. marina, Greeff. Kirk, T.N.Z.I. xviii. 2 I 5 . oblonga, Kirk. T.N.Z.I. xviii. 215. striata, Dujardin. Kirk, T.N.Z.I. xviii. 2 I 5. aperta, De Fromentel. Kirk, T.N.Z.I. xviii. 2 I 7 . 
Carchesium, Ehrenberg, i 838 . polypinum, Linnæus. Maskell, T.N.Z.I. xx. 13.

Zoöthamnium, Ehrenberg, i 838 . affine, Stein. Maskell, T.N.Z.I. xix. $5^{8}$. limpidum, Maskell. T.N.Z.I. xx. I3.

Epistylis, Ehrenberg, I830. leucoa, Ehrenberg. Maskell, T.N.Z.I. xix. $5^{8}$. anastatica, Linnæus. Maskell, T.N.Z.I. xx. I 3 .

Opercularia, Goldfuss, 1820 . parallela, Maskell. T.N.Z.I. xix. $5^{8}$. nutans, Ehrenberg. Maskell, T.N.Z.I. xx. I 3.

Vaginicola, Lamarck, i 8 I 6. crystallina, Ehrenberg. Maskell, T.N.Z.I. xix. 59.

Thuricola, Saville-Kent, $188 \mathrm{r}$. valvata, Wright. Maskell, T.N.Z.I. xx. I 3.

Cothurnia, Ehrenberg, I 832 . patellæ, Hutton. T.N.Z.I. xi. $33^{\circ}$. parallela, Maskell. T.N.Z.I. xx. I 3.

Pyxicola, Saville-Kent, 1882.

furcifer, Hutton. Jour. R. Micros. Soc. I 878 , p. 49 (Cothurnia).

Platycola, Saville-Kent, I 882.

decumbens, Ehrenberg. Maskell, T.N.Z.I. xx. I3.

Family UrCeolarime.

Licnophora, Claparède, 1867. setifera, Maskell. T.N.Z.I. xix. 57.

Family GYrocoride.

Gyrocoris, Stein, I 867 . oxyura, Stein. Maskell, T.N.Z.I. xix. 57.

Urocentrum, Nitzsch, i 8 I 7 .

turbo, Müller. Maskell, T.N.Z.I. xix. 57.

Halteria, Dujardin.

Family Halterinde.

grandinella, Müller. Maskell, T.N.Z.I. xix. 57.

Strombidion, Claparède and Lachmann, 1859. claparedii, Kent. Maskell, T.N.Z.I. xix. 57. intermedium, Maskell. T.N.Z.I. xx. I 2. 


\section{Sub-order Heterotricha.}

Family TinTINNODE.

Tintinnidium, Saville-Kent, I88I.
fluviatile, Stein.
Maskell, T.N.Z.I. xx. I I.

Family Stentoride.

Stentor, Oken, I $\mathrm{S}_{\mathrm{I}} 5$.

gracilis, Maskell. T.N.Z.I. xix. 56 . striatus, Maskell. T.N.Z.I. xix. 57.

attenuatus, Maskell. T.N.Z.I. xx. 20.

Family SPIROSTOMIDE.

Spirostomum, Ehrenberg, I 833 .

ambiguum, Ehrenberg. Maskell, T.N.Z.I. xix. $5^{6 .}$

Family Bursaridde.

Metopus, Claparède and Lachmann, r 858.

sigmoides, Müller. Maskell, T.N.Z.I. xix. 56.

Sub-order Holotricha.

Family PleURONEMIDA.

Pleuronema, Dujardin, 1836 . coronata, Kent. Maskell, T.N.Z.I. xix. 55. cyclidium, Maskell. T.N.Z.I. xix. 56.

Cyclidium, Müller, I 786.

glaucoma, Ehrenberg. Maskell, T.N.Z.I. xix. $5^{6}$.

Uronema, Dujardin.

marinum, Dujardin. Maskell, T.N.Z.I. xix. 56.

Colpidium, Stein.

Family Ophryoglenid £.

cucullus, Schrank. Maskell, T.N.Z.I. xix. 55.

Plagiopyla, Stein, IS60.

varians, Maskell. T.N.Z.I. xix. 55.

Family Trachelinde.

Amphileptus, Ehrenberg, I 830 .

anser, Ehrenberg. Maskell, T.N.Z.I. xix. 55.

irregularis, Maskell. T.N.Z.I. xx. 9.

rotundus, Maskell. T.N.Z.I. xx. 9 .

tracheloides, Maskell. T.N.Z.I. xx. 9.

Family 'Trachelocercide.

Trachelocera, Ehrenberg, I 834.

filiformis, Maskell. T.N.Z.I. xix. 54.

Chœnia, Quennerstedt.

crassa, Maskell. T.N.Z.I. xx. 8. 
Tillina, Gruber, I 879 .

Family Erchelyide.

enormis, Maskell. T.N.Z.I. xix. 53 . inequalis, Maskell. T.N.Z.I. xix. 54 .

Family COLEPIDÆ.

Coleps, Nitzsch, i8 8 .

hirtus, Ehrenberg. Maskell, T.N.Z.I. xix. 53.

Family Trachelophyllide.

Trachelophyllum, Claparède and Lachmann, I 859 .

apiculatum, Pertz. Maskell, 'T.N.Z.I. xix. 53.

Prorodon, Ehrenberg, I 834 .

Family Prorodontide.

sulcatus, Maskell. T.N.Z.I. xix. 53.

Nassula, Ehrenberg, I 834 . ambigua, Stein. Maskell, T.N.Z.I. xx. 8.

Family PARAMCECIDE.

Paramecium, Hill, I $85 \mathrm{I}$.

aurelia, Müller. Maskell, T.N.Z.I. xix. 53.

bursaria, Ehrenberg. Maskell, T.N.Z.I. xix. 53 .

\section{CLASS, MASTIGOPHORA.}

Order Choanoflagellata.

Family SALPINGGCIDA.

Salpingœca, Jas. Clark, i 866.

amphoridium, Clark. Maskell, T.N.Z.I. xix. 5 I. inquillata, Kent. Maskell, T.N.Z.I. xx. 6.

steinii, Kent. Maskell, T.N.Z.I. xx. 7 .

Monosiga, Saville-Kent.

Family Codonosigid

consociata, Kent. Maskell, T.N.Z.I. xix. $5 \mathbf{I}$.

breviceps, Kent. Maskell, T.N.Z.I. xx. 6.

Codosiga, Jas. Clark, i 866.

botrytis, Ehrenberg. Maskell, T.N.Z.I. xx. 6 .

Order Flagellata.

Sub-order Cilio-Flagellata.

Family PERIDINIIDE.

Gymnodinium, Stein, 1879

varians, Maskell. T.N.Z.I. xx. 7 .

Peridinium, Ehrenberg, ${ }_{1} 8_{32}$.

tabulatum, Ehrenberg. Maskell, T.N.Z.I. xix. $5^{2 .}$ 
Sub-order Eustomata.

Anisonema, Dujardin.

Family ANISONEMID.E.

grande, Ehrenherg. Vaskell, T.N.Z.I. xx. 7 . ovatum, Maskell. T.N.Z.I. xx. 7 .

Family Zygoselmid

Sterromonas, Saville-Kent, ISSo.

formicina, Kent. Maskell, T.N.Z.I. xix. 52.

Family Chrysomonadid.£.

Uvella, Bory, is 24 .

virescens, Ehrenberg. Maskell, 'T.N.Z.I. xix. 52.

Dinobryon, Ehrenberg, is 34 .

sertularia, Ehrenberg. Maskell, T.N.Z.I. xix. 52.

Family Euglenid.£.

Euglena, Ehrenberg, i $\mathrm{S}_{32}$.

viridis, Ehrenberg. Maskell, T.N.Z.I. xix. 52.

spirogyra, Ehrenberg. Maskell, T.N.Z.I. xix. 52. acus, Ehrenberg. Maskell, T.N.Z.I. xis. 52.

Amblyophis, Ehrenberg, I $S_{32}$.

viridis, Ehrenberg. Maskell, T.N.Z.I. xix. 52.

Phacus, Nitzsch, isi6.

triqueter, Ehrenberg. Maskell, T.N.Z.I. xix. 52.

Trachelomonas, Ehrenberg, $\mathrm{I}_{34}$.

volvocina, Ehrenberg. Naskell, T.N.Z.I. xix. 52.

armata, Ehrenberg. Maskell, T.N.Z.I. xix. 52.

crenulatocollis, Maskell. T.N.Z.I. xix. 52.

hispida, Pertz. Maskell, T.N.Z.I. xx. 7 .

teres, Maskell. T.N.Z.I. xx. 7 .

cylindrica, Ehrenberg. Maskell, T.N.Z.I. x. 7 .

Family ASTASIADE.

Astasia, Ehrenberg, is $S_{3}$.

trichophora, Ehrenberg. Maskell, T.N.Z.I. xix. 52.

Phialonema, Stein.

Family PaRAMONADID.£.

cyclostomum, Stein. Maskell, T.N.Z.I. xix. 52.

Sub-order Pantostomata.

Heteromita, Dujardin, IS 40.

Family Heteromitide.

lens, Miiller., Maskell, T.N.Z.I. xix. 5 I. 
Rhipidodendron, Stein.

Family SPONGOMONADIDE.

huxleyi, Kent. Maskell, T.N.Z.I. xix. 5 I.

splendidum, Stein. Maskell, T.N.Z.I. xx. 6.

Spongomonas, Siein.

discus, Stein. Maskell, T.N.Z.I. xix. 5 I.

sacculus, Kent. Maskell, T.N.Z.I. xix. 51.

Goniomonas, Stein.

Family Amphimonadide.

truncata, Fres. Maskell, T.N.Z.I. xix. 5 I.

elongata, Maskell. T.N.Z.I. xx. 5 .

Family BIKCECIDE.

Stylobryon, Fromentel, i 875.

petiolatum, Dujardin. Maskell, T.N.Z.I. xx. 5.

Dendromonas, Stein.

Family DENDROMONADIDE.

virgaria, Weisse. Maskell, T.N.Z.I. xix. 5 I.

producta, Maskell. T.N.Z.I. xx. 5 .

Anthophysis, Bory, 1822.

socialis, Fromentel. Maskell, T.N.Z.I. xix. $5^{\text {I. }}$

vegetans, Müller. Maskell, T.N.Z.I. xix. 5ı.

Cephalothamnium, Stein.

coronatum, Maskeil. T.N.Z.I. xx. 5 .

Family Cercomonadide.

Oikomonas, Saville-Kent, I880.

mutabilis, Kent. Maskell, T.N.Z.I. xix. 50.

termo, Clark. Maskell, T.N.Z.I. xx. 5.

Monas, Müller, I 773 .

Family Monadide.

fluida, Dujardin. Maskell, T.N.Z.I. xix 50.

attenuata, Dujardin. Maskell, T.N.Z.I. xix. 50.

irregularis, Pertz. Maskell, T.N.Z.I. xx. 4.

Scytomonas, Stein.

pusilla, Stein. Maskell, T.N.Z.I. xix. 50.

\section{CLASS, RHIZOPODA.}

Order ForaminifERA.

Family Miliolide.

Nubecularia, Defrance, 1825 .

lucifuga, Defrance. Haeusler, T.N.Z.I. xix. 197. 
Biloculina, d'Orbigny, 1826 .

irregularis, d'Orbigny. Challenger Reports, ix. I 40. sphæra, d'Orbigny. Challenger Reports, ix. I 4 I. ringens, Lamarck. Challenger Reports, ix. I42. comata, Brady. Challenger Reports, ix. I 44. depressa, d'Orbigny. Challenger Reports, ix. I 45. var. serrata, Brady. Challenger Reports, ix. 146. var. murrhyna, Schwager. Challenger Reports, ix. 146.

Spiroloculina, d'Orbigny, 1826 .

Species undetermined. Haeusler, T.N.Z.I. xix. 197.

Miliolina, Williamson, I 858 .

oblonga, Montagu. Challenger Reports, ix. I60. valvularis, Reuss. Challenger Reports, ix. I6 1 . venusta, Karrer. Challenger Reports, ix. 162. tricarinata, d'Orbigny. Challenger Reports, ix. I 65. secans, d'Orbigny. Haeusler, T.N.Z.I. xix. 198. circularis, Bornemann. Challenger Reports, ix. I69. labiosa, d'Orbigny. Haeusler, T.N.Z.I. xix. 198. bicornis, Walker and Jacob. Haeusler, T.N.Z.I. xix. I9S. seminulum, Linnæus. Challenger Reports, ix. 157. linnæana, d'Orbigny. Haeusler, T.N.Z.I. xix. 198.

Planispirina, Seguenza, I880.

celata, Costa. Challenger Reports, ix. 197.

sigmoidea, Brady. Challenger Reports, ix. 197.

Cornuspira, Schultze, I 854 .

foliacea, Philippi. Challenger Reports, ix. I 99.

Family AstrorhIZIDÆ.

Pelosina, Brady, I 879 . variabilis, Brady. Challenger Reports, ix. 235. rotundata, Brady. Challenger Reports, ix. 236. cylindrica, Brady. Challenger Reports, ix. 236.

Technitella, Norman, I 875 . melo, Norman. Challenger Reports, ix. 246.

Bathysiphon, Sars, I 87 I. filiformis, Sars. Challenger Reports, ix. 248.

Psammosphæra, Schulze, 1874 . fusca, Schulze. Challenger Reports, ix. 249.

Jaccullela, Brady, ı 879 . acuta, Brady. Challenger Reports, ix. 255.

Hyperammira, Brady, I 878 . friabilis, Brady. Challenger Reports, ix. 258. ramosa, Brady. Challenger Reports, ix. 26 I. 
Rhabdamina, Sars, I 868 .

abyssorum, Sars. Haeusier, T.N.Z.I. xix. 198.

discreta, Brady. Challenger Reports, ix. 268.

linearis, Brady. Challenger Reports, ix. 269.

cornuta, Brady. Challenger Reports, ix. 270.

Family LiTUOLIDA.

Reophax, Montfort, I 808 .

diffulgiformis, Brady. Challenger Reports, ix. 289.

scorpiurus, Montfort. Challenger Reports, ix. 291.

dentaliniformis, Brady. Challenger Reports, ix. 293.

nodulosa, Brady. Challenger Reports, ix. 294.

guttifera, Brady. Challenger Reports, ix. 295.

distans, Brady. Challenger Reports, ix. 296.

adunca, Brady. Challenger Reports, ix. 296.

Haplophragmium, Reuss, i 860.

agglutinans, d'Orbigny. Challenger Reports, ix. $30 \mathrm{r}$.

tenuimargo, Brady. Challenger Reports, ix. 303 .

foliaceum, Brady. Challenger Reports, ix. 30.4 .

emaciatum, Brady. Challenger Reports, ix. 305 .

latidorsatum, Bornemann. Challenger Reports, ix. 307.

scitulum, Brady. Challenger Reports, ix. 308.

glomeratum, Brady. Challenger Reports, ix. 309.

canariense, d'Orbigny. Haeusler, T.N.Z.I. xix. 198.

nanum, Brady. Challenger Reports, ix. 3 I I.

globigeriniforme, Parker and Jones. Challenger Reports, ix. 3 I 2 .

Placopsilina, d'Orbigny, I 850 .

bulla, Brady. Challenger Reports, ix. 3 r 5 .

Thurammina, Brady, I 879 .

papillata, Brady. Challenger Reports, ix. $32 \mathrm{I}$.

Hormosina, Brady, i 879 .

globulifera, Brady. Challenger Reports, ix. 326.

monile, Brady. Challenger Reports, ix. 328 .

normani, Brady. Challenger Reports, ix. 329.

Ammodiscus, Reuss, I86r.

incertus, d'Orbigny. Challenger Reports, ix. 330.

tenuis, Brady. Challenger Reports, ix. $33^{2}$.

charoides, Jones and Parker. Challenger Reports, ix. 334.

Trochammina, Jones and Parker, 1859.

squamata, Jones and Parker. Challenger Reports, is. 337.

trullissata, Brady. Challenger Reports, ix. 342.

galeata, Brady. Challenger Reports, ix. 344.

pauciloculata, Brady. Challenger Reports, ix. 344. 
Webbina, d'Orbigny, I 839 .

clavata, Jones and Parker. Challenger Reports, ix. 349 .

hemispherica, Jones and Parker. Challenger Reports, ix. $35^{\circ}$.

Cyclammina, Brady, 1876 .

cancellata, Brady. Challenger Reports, ix. $35 \mathrm{I}$.

orbicularis, Brady. Challenger Reports, ix. 353.

Textularia, Defrance, I824.

Family Textularide.

quadrilatera, Schwager. Challenger Reports, ix. 358 .

concava, Karrer. Challenger Reports, ix. 360.

sagittula, Defrance. Challenger Reports, ix. 36 I.

agglutinans, d'Orbigny. Challenger Reports, ix. $3^{6} 3$.

aspera, Brady. Challenger Reports, ix. 367.

Gaudryina, d'Orbigny, I 840 .

pupoides, d'Orbigny. Challenger Reports, ix. 378 .

siphonella, Reuss. Challenger Reports, ix. 382 .

Verneuilina, d'Orbigny, I 840.

pygmæa, Egger. Challenger Reports, ix. $38_{5}$.

propinqua, Brady. Challenger Reports, ix. $3^{87}$.

Valvulina, d'Orbigny, I 826 .

concinna, Jones and Parker. Haeusler, T.N.Z.I. xix. 198.

Clavulina, d'Orbigny, I 826 .

communis, d'Orbigny. Challenger Reports, ix. 394.

Bulimina, d'Orbigny, i 826 .

elegans, d'Orbigny. Challenger Reports, ix. 398.

pyrula, d'Orbigny. Challenger Reports, ix. 399.

ovata, d'Orbigny. Challenger Reports, ix. 400.

affinis, d'Orbigny. Challenger Reports, ix. 400.

aculeata, d'Orbigny. Challenger Reports, ix. 406. inflata, Seguenza. Challenger Reports, ix. 406.

buchiana, d'Orbigny. Challenger Reports, ix. 407.

Virgulina, d'Orbigny, I $\$ 26$.

schreibersiana, Czjzek. Challenger Reports, ix. 4 I 4 . subsquamosa, Egger. Challenger Reports, ix. 4I 5. subdepressa, Brady. Challenger Reports, ix. 4 I 6 .

Bolivina, d'Orbigny, I 839 .

punctata, d'Orbigny. Haeusler, T.N.Z.I. xix. 198.

textilarioides, Reuss. Challenger Reports, ix. 4I9.

Cassidulina, d'Orbigny, I 826 .

subglobosa, Brady. Challenger Reports, ix. 430.

Ehrenbergina, Reuss, i 849.

serrata, Reuss. Challenger Reports, ix. 434. 
Family ChilostomeLlidæ.

Chilostomella, Reuss, I 849 .

ovoidea, Reuss. Challenger Reports, ix. $43^{6 .}$

Family LAgenide.

Lagena, Williamson, r 848 .

globosa, Montagu. Challenger Reports, ix. $45^{2}$.

apiculata, Reuss. Haeusler, T.N.Z.I. xix. 198.

lævis, Montagu. Challenger Reports, ix. 455.

truncata, Brady. Challenger Reports, ix. 457.

hispida, Reuss. Challenger Reports, ix. 459.

striata, d'Orbigny. Challenger Reports, ix. 460.

acuticosta, Reuss. Challenger Reports, ix. 464 .

gracilis, Williamson. Challenger Reports, ix. 464 .

striato-punctata, Jones and Parker. Haeusler, T.N.Z.I.

xix. I 98 .

squamosa, Montagu. Challenger Reports, ix. 47 I.

hexagona, Williamson. Challenger Reports, ix. 472.

marginata, Walker and Boys. Challenger Reports, ix. ${ }_{47} 6$.

var. semi-marginata, Reuss. Challenger Reports, ix. 477. quadricostulata, Reuss. Challenger Reports, ix. 486. auriculata, Brady. Challenger Reports, ix. 487 .

Nodosaria, Lamarck, i8 6 .

lævigata, d'Orbigny. Challenger Reports, ix. 490.

radicula, Linnæus. Challenger Reports, ix. 495 .

simplex, Silvestri. Challenger Reports, ix. 496.

soluta, Reuss. Challenger Reports, ix. $5 \circ 3$.

communis, d'Orbigny. Challenger Reports, ix. 504.

obliqua, Linnæus. Challenger Reports, ix. 5 r 3.

Vaginulina, d'Orbigny, 1826 .

spinigera, Brady. Challenger Reports, ix. $53 \mathrm{I}$.

Cristellaria, Lamarck, I 8 г6.

compressa, d'Orbigny. Challenger Reports, ix. 538.

reniformis, d'Orbigny. Challenger Reports, ix. 539.

rotulata, Lamarck. Challenger Reports, ix. 547.

cultrata, Montfort. Challenger Reports, ix. $55^{\circ}$.

Polymorphina, d'Orbigny, I 826 .

angusta, Egger. Challenger Reports, ix. 563.

Uvigerina, d'Orbigny, I 826 .

tenuistriata, Reuss. Challenger Reports, ix. 574 . angulosa, Williamson. Challenger Reports, ix. 576 . aculeata, d'Orbigny. Challenger Reports, ix. 578 . asperula, Czjzek. Challenger Reports, ix. 578 . var. auberiana, d'Orbigny. Challenger Reports, ix. 579 . 
Family GLobigerinidæ.

Globigerina, d'Orbigny, I 826 .

bulloides, d'Orbigny. Challenger Reports. ix. 593.

var. triloba, Reuss. Challenger Reports, ix. 595.

dubia, Egger. Challenger Reports, ix. 595.

inflata, d'Orbigny. Challenger Reports, ix. 60I.

æquilateralis, Brady. Challenger Reports, ix. 605.

Orbulina, d'Orbigny, I 839.

universa, d'Orbigny. Challenger Reports, ix. 608.

Pullenia, Parker and Jones, I 862.

sphæroides, d'Orbigny. Challenger Reports, ix. 6I 5 .

quinqueloba, Reuss. Challenger Reports, ix. 6 I 7.

obliquiloculata, Parker and Jones. Challenger Reports, ix. 6 i 8 .

Sphæroidina, d'Orbigny, 1826 .

bulloides, d'Orbigny. Challenger Reports, ix. 620 .

dehiscens, Parker and Jones. Challenger Reports, ix. 62 I.

Family ROTALIDE.

Patellina, Williamson, I $85^{8}$. corrugata, Williamson. Haeusler, T.N.Z.I. xix. 198.

Discorbina, Parker and Jones, 1862.

turbo, d'Orbigny. Haeusler, T.N.Z.I. xix. I 99.

globularis, d'Orbigny. Haeusler, T.N.Z.I. xix. 199.

valvulata, d'Orbigny. Haeusler, T.N.Z.I. xix. I 99.

rosacea, d'Orbigny. Haeusler, T.N.Z.I. xix. I 99.

orbicularis, Terquem. Haeusler, T.N.Z.I. xix. I 99.

Truncatulina, d'Orbigny, i 826 .

lobatula, Walker and Jacob. Challenger Reports, ix. 660. muellerstorfi, Schwager. Challenger Reports, ix. 662.

akneriana, d'Orbigny. Challenger Reports, ix. 663.

tenera, Brady. Challenger Reports, ix. 665 .

pygmæa, Hantken. Challenger Reports, ix. 666.

Pulvinulina, Parker and Jones, 1862.

repanda, Fichtel and Moll. Haeusler, T.N.Z.I. xix. 199.

canariensis, d'Orbigny. Challenger Reports, ix. 692.

crassa, d'Orbigny. Challenger Reports, ix. 694.

micheliniana, d'Orbigny. Challenger Reports, ix. 694.

exigua, Brady. Challenger Reports, ix. 696.

pauperata, Parker and Jones. Challenger lieports, ix. 696.

elegans, d'Orbigny. Challenger Reports, ix. 699.

Rotalia, Lamarck, 1804.

beccarii, Linnæus. Haeusler, T.N.Z.I. xix. I 99.

orbicularis, d'Orbigny. Challenger Reports, ix. 706. 
Rotalia soldani, d'Orbigny. Challenger Reports, ix. 706. clathrata, Brady. Challenger Reports, ix. 709.

Family Nummulinide.

Nonionina, d'Orbigny, I826.

depressula, Walker and Jacob. Haeusler, T.N.Z.I. xix. 199. scapha, Fichtel and Moll. Haeusler, T.N.Z.I. xix. 199. asteriscus, Walker and Jacob. Haeusler, T.N.Z.I. xix. I99. umbilicatula, Montagu. Challenger Reports, ix. 726 . pompiloides, Fichtel and Moll. Challenger Reports, ix. 727.

Polystomella, Lamarck, 1822.

striatopunctata, Fichtel and Moll. Haeusler, T.N.Z.I. xix. 199.

crispa, Linnæus. Haeusler, T.N.Z.I. xix. I99.

macella, Fichtel and Moll. Haeusler, T.N.Z.I. xix. I99.

Order Radiolaria.

Sub-order SPUMELlaRia.

Series Collodaria.

Family Thalassicolide.

Thalassicolla, Huxley, $185 \mathrm{I}$.

australis, Haeckel. Challenger Reports, xviii. 20.

Collozoum, Haeckel, i 862.

inerme, Haeckel. Challenger Reports, xviii. 25.

Family SPHÆOZOIDÆ.

Rhaphidozoum, Haeckel, i 862.

australe, Haeckel. Challenger Reports, xviii. 48.

Series SPHÆRELLARIA.

Family COLLOSPHÆRIDE.

Siphonosphæra, Müller, I 858 .

fragilis, Haeckel. Challenger Reports, xviii. Io6.

Family DRUPPULIDE.

Stylatractus, Haeckel, I 887 .

disetaneus, Haeckel. Challenger Reports, xviii. 33 I.

Series ACANTHOMETRA.

Family AstroLoPHID E.

Actinelius, Haeckel, I 865.

protogenes, Haeckel. Challenger Reports, xviii. 730.

Sub-order ACANTHAR1A.

Series ACANTHOMETRA.

Family As'TROLONCHIDE.

Stauracantha, Haeckel, i 88 I. orthostaura, Haeckel. Challenger Reports, xviii. 762 . 
Pristacantha, Haeckel, r 887 .

polyodon, Haeckel. Challenger Reports, xviii. 766.

Series Acanthophracta.

Family DORATASPIDE.

Dodecaspis, Haeckel, r 887.

trizonia, Haeckel. Challenger Reports, xviii. 835 .

Sub-order NASSELlaria.

Series Plectellaria.

Family PlaAonide.

Hexaplagia, Haeckel, i 88 r.

australis, Haeckel. Challenger Reports, xviii. 9r6.

Lampromitra, Haeckel, r88I.

Series Cyrtellaria.

Family TRIPOCYRTIDÆ.

huxleyi, Haeckel. Challenger Reports, xviii. I 2 I 5.

Sub-order PhäOdARIA.

Series Pheocystina.

Family AulacanthidÆ.

Aulodendron, Haeckel, I887.

australe, Haeckel. Challenger Reports, xviii. ${ }_{5} 89$.

Series Pheogromia.

Castanidium, Haeckel, 1879.

Family Castanellide.

bromleyi, Haeckel. Challenger Reports, xviii. 1685.

Order LoBosa.

Family Am৫BidÆ.

Amceba, Ehrenberg, I83o.

Several undetermined species. 


\section{A D D I T I O NS.}

AVES.

Page 29.-Cyanorhamphus forbesi, Rothschild. P.Z.S. I893, 529.

" 32.-Himantopus leucocephalus, Gould. C.B.M. xxiv. 3r 7 ; Buller, ii. 2 I I.

"38.-Anchylochilus subarquatus, Guildenst. C.B.M. xxiv. 386 ; T.N.Z.I. xxxvi.

PISCES.

" 44.-Centrolophus britannicus, Gunther. Cat. Fishes B.M. ii. 402 ; T.N.Z.I. xxxvi.

" 49.-Genypterus microstomus, Regan. A.M.N.H. 7, xi. 599.

\section{MOLLUSCA.}

"63.-Ranfurlyea constanciæ, Suter. P.Mal.S. 1903.

"70.-Cavolina uncinata, Rang et Souleyet. Hist. Nat. Moll. Ptéropodes, 37 .

longirostris, Lesueur. Rang et Souloyet, Hist.

Nat. Moll. Ptéropodes, $4 \mathrm{I}$.

trispinosus, Lesueur. Rang et Souleyet, Hist.

Nat. Moll. Ptéropodes, 45.

\section{COLEOPTERA.}

" I 39.-Lissotes auriculatus, Broun. A.M.N.H. 7, xi. 6 I 5.

" I39.-Mitophyllus comognathus, Broun. A.M.N.H. 7, xi. 6 I 6.

” I42.-Mecodema costellatum, Broun. A.M.N.H. 7, xi. 45 I. intricatum, Broun. A.M.N.H. 7, xi. 45 I. nitidum, Broun. A.M.N.H. 7 , xi. $45^{2}$.

variolosum, Broun. A.M.N.H. 7, xi. 453 . 
Page 143.-Metaglymma rugiceps, Broun. A.M.N.H. 7, xi. 454. calcaratum, Broun. A.M.N.H. 7 , xi. 455 .

" I 43.-Dichrochile cordicolle, Broun. A.M.N.H. 7, xi. 455.

"I44.-Anchomenus walkeri, Broun. A.M.N.H. 7, xi. 456.

" I45.-Trichosternus walkeri, Broun. A.M.N.H. 7, xi. 602. akaroaensis, Broun. A.M.N.H. 7, xi. 603 . bucolicus, Broun. A.M.N.H. 7, xi. 604 .

" I 46.-Pterostichus kirkianus. A.M.N.H. 7, xi. 604 . memes, Broun. A.M.N.H. 7, xi. 605 . prasignis, Broun. A.M.N.H. 7, xi. 606. setiventris, Broun. A.M.N.H. 7, xi. 606 .

I47.-Allocinopus sculpticollis, Broun. 608.

A.M.N.H. 7 , xi.

I49. - Tarastethus simplex, Broun. A.M.N.H. 7, xi. 457. lævicollis, Broun. A.M.N.H. 7, xi. $45^{8}$.

I49.-Oöpterus latipennis. Broun. A.M.N.H. 7, xi. 609. probus, Broun. A.M.N.H. 7, xi. 6ro. parvulus, Broun. A.M.N.H. 7, xi. 6Iо.

149.-Bembidium actuarium, Broun. A.M.N.H. 7, xi. 6 I I.

I 5 I.-Scopodes viridis, Broun. A.M.N.H. 7, xi. 6r 2.

I 52.-Rygmodus nigripennis, Broun. A.M.N.H. 7, xi. 61 2 . I52.-Cytomissus glabratus. Broun. A.M.N.H. 7, xi. 6I 3 . I 52.-Zeadolopus spinipes, Broun. A.M.N.H. 7, xi. 6I4. I 7I.-Pycnomerus nitiventris, Broun. A.M.N.H. 7, xi. $6 r 7$.

I 7 I.-Bothrideres picipes, Broun. A.M.N.H. 7, xi. 618.

I84. - Trogophlæus maritimus, Broun. A.M.N.H. 7, xi. 6r 5. I 85.-Syrphetodes simplex, Broun. A.M.N.H. 7, xii. 69. I go.-Thelyphassa furcata, Broun. A.M.N.H. 7, xii. 70. I90.-Techmessa longicollis, Broun. A.M.N.H. 7, xii. 70.

I90.-Exocalopus antennalis, Broun. A.M.N.H. 7 , xii. 7 I.

I 96.-Hylobasius cognatus, Broun. A.M.N.H. 7, xii. 82. laticollis, Broun. A.M.N.H. 7, xii. 83 . gracilipes, Broun. A.M.N.H. 7, xii. 84 . genalis, Broun. A.M.N.H. 7 , xii. 84 . 
Page 199.-Cecyropa lineifera, Broun. A.M.N.H. 7 , xii. 72. striata, Broun. A.M.N.H. 7, xii. 73 .

", 202.-Brachylous albescens, Broun. A.M.N.H. 7, xii. 73.

cervalis, Broun. A.M.N.H. 7, xii. 74 .

"202.-Aphela pictipes, Broun. A.M.N.H. 7, xii. 75 .

,202.-Anagotus pallescens, Broun. A.M.N.H. 7, xii. 76.

" 202.-Sargon carinatus, Broun. A.M.N.H. 7, xii. 77.

"202.-Memes rufirostris, Broun. A.M.N.H. 7, xii. 79.

"202.-Xerostygnus binodulus, Broun. A.M.N.H. 7, xii. 80.

"209.-Stephanorhynchus pygmæus, Broun. A.M.N.H. 7 , xii. 80 .

"209.-Drototelus politus, Broun. A.M.N.H. 7, xii. 82.

" 209.-Eualema walkeri, Broun. A.M.N.H. 7, xii. 86.

\section{HEMIPTERA.}

" 222.-Henicocephalus maclachlani, Kirkaldy. Ent. Mag. xxxvii. 2 I 7 (I 901 ).

\section{MYRIAPODA.}

236.-Necrophlæsophagus spenceri, Pocock. A.M.N.H. 7, viii. 462 .

\section{ARACHNIDA.}

" 246.-Eylais schauinslandi, Koenike. Zool. Jahrb. Syst. xiii. 125 (I 90 I).

\section{PEDIPALPIDA.}

246.-Pantopsalis coronata, Pocock. A.M.N.H. 7, xi. 436.

trippi, Pocock. A.M.N.H. 7, xi. 437.

jenningsi, Pocock. A.M.N.H. 7, xi. 437 .

" 246.-Sorensenella bicornis, Pocock. A.M.N.H. 7, xi. 439. 
CRUSTACEA.

Page 250.-Pinnotheres schauinslandi, Lenz. Zool. Jahrb. Syst. xiv. 468 (I 90 I).

"251.-Eupagurus intermedius, Lenz. Zool. Jahrb. Syst. xiv. 446 (I 90 I).

OLIGOCHÆTA.

"283.-Notiodrilus aucklandicus, Benham. T.N.Z.I. xxxv. 275 .

\section{HIRUDINEA.}

"285.-Hirudo antipodum, Benham. T.N.Z.1. xxxvi.

\section{POLYZOA.}

"292.-Paludicella ehrenbergi, Van Beneden. T.N.Z.I. xxxv. 262.

\section{CELENTERATA.}

"3I9.-Eucopella crenata, Hartlaub. Zool. Jahrb. Syst, xiv. 364 (I 901$)$. 


\section{A P P E N D I X.}

\section{PHYLUM, ANNULATA.}

CLASS, POLYCHÆTA.

The following additional identifications were received from Ehlers since the list on $p p .276-282$ was printed:-

Family Syllide.

Syllis closterobranchia, Schmarda. N.W.Th. (2), 72, fide Ehlers MS.

Trypanoscyllis, Claparède, I 864. occipitalis, Ehlers M.S.

Family APHRODITIDE,

'Polynoe' australis, Schmarda. N.W.Th. (2), I 54, fide Ehlers MS.

Family Lycoride.

Nereis amblyodonta, Schmarda (Nereilepas a.). N.W.Th. (2), I06, fide Ehlers MS.

Family Eunicidæ.

Lumbriconereis brevicirrus, Schmarda (Notocirrus b.). N.W.Th. (2), I 7 , fide Ehlers MS.

Stauronereis australis, Haswell. P.L.S.N.S.W. x. 747 , fide Ehlers MS.

Spio, Lamarck, i 8 I 8.

Family SPIONIDÆ.

æqualis, Ehlers MS.

Polydora, Bosc, I802.

polybranchia, Haswell. P.L.S.N.S.W.x. 275, fide Ehlers MS. monilaris, Ehlers M.S

Family Terebellide.

Leprea, Malmgren, 1865 .

orotavæ, Langerhaus, fide Ehlers MS. 
Family Scalinregmide.

Eumenia analis, Ehlers MS.

Family Chlorænide.

Flabelligera semiannulata, Ehlers MS.

CLASS, OLIGOCH ETA.

The following additional Oligochata have been identified since pp. 283-285 were printed:-

Family Phreodrilide.

Phreodrilus, Beddard, i 891 . lacustris, Benham. Q.J.M.S. I903. mainensis, Benham. Q.J.M.S. 1903. beddardi, Benham. Q.J.M.S. I903.

Family Tubicifide.

Taupodrilus, Benham, I 903.

simplex, Benham. P.Z.S. 1903.

Limnodrilus vejdovskyanus, Benham. P.Z.S. I903. lucasi, Benham. P.Z.S. I 903 .

Family ENCHYTRÆIDE.

Euchytræus, Heule, I 837 . simulans, Benham. P.Z.S. I903.

Achæta, Vejsdovsky, I877.

maorica, Benham. P.Z.S. 1903

Family HaPLOTAXIDE.

Haplotaxis heterogyne, Benham. Q.J.M.S. I903.

Plutellus, Perrier, I 873 .

Family Megascolide.

lacustris, Benham. P.Z.S. I 903 .

Diporochæta aquațica, Benham. P.Z.S. I903. 


\section{LIST OF NATURALISED ANIMALS.}

This list includes those animals which have been introduced, either intentionally or unintentionally, by human agencies, and have become so well established that they may be considered as part of the fauna. Domesticated animals which have not gone wild are not included.

\section{MAMMALIA.}

Mustelus putorius, Linnæus. (Ferret.) Europe. erminea, Linnæus. (Stoat.) Europe. vulgaris, Gmelin. (Weasel.) Europe.

Erinaceus europeus, Linnæus. (Hedgehog.) Europe.

Mus decumanus, Pallas. (Brown rat.) Europe. rattus, Linnæus. (Black rat.) British islands. exulans, Peale. (Maori rat.) Polynesia. musculus, Linnæus. (Mouse.) Europe. Cervus elaphus, Linnæus. (Red deer.) Europe. Dama vulgaris, Gray. (Fallow deer.) Europe. Sus scrofa, Linnæus. (Pig.) Europe and North Africa. Halmaturus, sp. (Rock wallaby.) Australia. Trichosurus fuliginosus, Ogilby. (Sooty opossum.) Tasmania. vulpecula, Kerr. (Opossum.) Australia.

AVES.

Turdus musicus, Linnæus. (Song-thrush.) Europe. merula, Linnæus. (Blackbird.) Europe.

Accentor modularis, Linnæus. (Hedge-sparrow.) Europe.

Passer domesticus, Linnæus. (Sparrow.) Europe.

Ligurinus chloris, Linnæus. (Greenfinch.) Europe.

Fringilla cœlebs, Linnæus. (Chaffinch.) Europe.

Carduelis elegans, Stephens. (Goldfinch.) Europe.

Linota rufescens, Vieillart. (Red-poll.) Europe.

Emberiza citrinella, Linnæus. (Yellow-hammer.) Europe. cirlus, Linnæus. (Cirl-bunting.) Europe.

Sternus vulgaris, Linnæus. (Starling.) Europe.

Acridotheres tristis, Linnæus. (House mynah.) India 
Corvus frugilegus, Linnæus. (Rook.) Europe.

Gymnorhina leuconota, Gould. (Australian magpie.) Australia.

Alauda arvensis, Linnæus. (Skylark.) Europe.

Cygnus olor, Gmelin. (White swan.) Europe. atratus, Lathrades. (Black swan.) Australia.

Synœcus australis, Lathrades. (Swamp quail.) Australia.

Callipepla californica, Shaw. (Californian quail.) California.

Phasianus colchicus, Linnæus. (Pheasant.) England. torquatus, Gmelin. (Ring-necked pheasant.) China.

\section{AMPHIBIA.}

Hyla aurea, Lesson. (Green frog.) Australia.

\section{PISCES.}

Perca fluviatilis, Linnæus. (Perch.) Europe.

Salmo fario, Linnæus. (Trout.) Europe. iridens, Mitchell. (Rainbow trout.) California.

Carassius vulgaris, Nilsson. (Carp.) Europe. auratus, Linnæus. (Gold-fish.) China.

\section{MOLLUSCA.}

\section{Pulmonata.}

Limnæa stagnalis, Linnæus. Europe.

Testacella maugei, Férussac. (T. vagans, Hutton.) Europe. Limax agrestis, Linnæus. (Slug.) (L. molestus, Hutton.) Europe.

maximus, Linnæus. Europe.

variegatus, Drapanaud. Europe.

Amalia gagates, Drapanaud. Europe.

(=L. fuliginosa, Gould; L. antipoda, Pfeiffer); and M. emarginata, Hutton.

Hyalina cellaria, Mueller. ( $H$. corneo-fulva, Pfeiffer.) Europe.

alliaria, Mueller. Europe.

crystallina, Mueller. Europe.

Helix hortensis, Mueller. Europe.

aspera, Mueller. (Garden snail.) Europe.

Helicella caperata, Montagu. Europe.

Cochlostyla fulgetrum, Broderip. (B. antipodarum, Gray.) Philippines. 
Cochlostyla daphnis, Broderip. Philippines.

tricolor, Pfeiffer. Solomon Islands.

Vallonia excentrica, Sterki. Europe and North America.

Arion empiricorum, Férussac. Europe.

subfusca, Drapanand. (A. incommodus, Hutton.) Europe. hortensis, Férussac. Europe.

Cochlicopa lubrica, Mueller. Europe.

\section{HYMENOPTERA.}

\section{APIDE.}

Apis mellifica, Linnæus. (Honey-bee.) Asia (?).

ligustica, Spin. (Ligurian bee.) Italy.

Bombus terrestris, var. virginalis, Kirby. (Humble-bee.) Europe.

hortorum, Linnæus. Europe.

hortorum, var. harrisellus, Kirby. Europe.

FORMICIDE.

Prenolepis longicornis, Fabricius. Europe and Asia. T.N.Z.I. xxxiii. I 20.

\section{Parasitica.}

Lissopimpla semipunctata, Kirby. Australia (?).

Bassus lætatorius, Fabricius. (S. varipes, Smith; B. generosus, Cameron.) Europe.

Dacnusa, sp. Cameron, Manchester Memoirs, xlvi. p. 26.

Eulophus albitarsis, Ashmead. North America.

\section{TENTHREDINIDF.}

Eriocampa adumbrata, Klug. (Monostegia antipoda, Kirby.) (Slug-worm.) Europe.

\section{LEPIDOPTERA.}

CARAdRINida.

Utethesia pulchella, Linnæus. Europe.

Pyralidina.

Melliphora grisella, Fabricius. Europe.

Pyralis farinelis, Linnæus. Europe.

TORTRICINA.

Carpocapsa pomonella, Linnæus. (Codlin moth.) Europe. 
Tineina.

Trochilium tipuliforme, Cramer. (Currant moth.) Europe. Ecophora pseudospretella, Stainton. Europe.

Plutella cruciferarum, Zeller. Europe.

Chorentis bjerkandrella, Thunberg. Europe.

Bedellia somnulentella, Zeller. Europe.

Monopsis ferruginella, Hübner. Europe.

ethelella, Newman. Europe.

Tineola biselliella, Humboldt. Europe.

Trichophaga tapetiella, Linnæus. (Clothes moth.) Europe.

Tinea fuscipunctella, Hawthorn. Europe.

terranea, Butler. Europe.

Lita solanella, Boisduval. (Potato moth.) Africa (?).

\section{DIPTERA.}

SCHIZOPHORA.

Gasterophilus equi, Meigen. (Bot-fly.) Europe. hæmorrhoidalis, Linnæus. Europe.

CEstrus ovis, Linnæus. Europe.

Sarcophaga impatiens, Walker. Australia.

Lucilia cæsar, Linnæus. (Greenbottle.) Europe.

Calliphora erythrocephala, Meigen. (Bluebottle.) Europe.

Musca domestica, Linnæus. (House-fly.) Europe.

corvina, Fabricius. (fide Hilgendorf.) Europe.

Muscina stabulans, Meigen. Europe.

Stomoxys calcitrans, Fabricius. (Horse-fly.) Europe.

Homalomyia canicularis, Linnæus. Europe.

Tephrites tryoni, Froggart. Australia.

\section{Aschiza.}

Eristalis tenax, Linnæus. (Drone-fly.) Europe.

Nemocera.

Cecidomyia destructor, Say. (Hessian-fly.) N. America (?). Scatopse notata, Linnæus. Europe.

Psychoda phalænoides, Linnæus. Europe.

\section{Pupipara.}

Melophagus ovinus, Linnæus. Europe.

\section{APHANiptera.}

Pulex irritans, Linnæus. (Flea.) Europe (?). serraticeps, Gervasson. (Dog-flea.) Europe. avium, Taschb. (Pigeon-flea.) Europe. 


\section{COLEOPTERA.}

\section{LAMELLICORNIA.}

Onthophagus granulatus, Boh. Australia. posticus, Erichson. Australia.

Aphodius granarius, Linnæus. Europe.

Proctophanes sculptus, Hope. Australia.

Trox, sp. Reported by Dr. Swale.

\section{ADEPHAGA.}

Læmosthenes complanatus, Dejean. S. Europe, \&c. Rhytisternus puella, Chaudoir. New South Wales. Hypharpax australasiæ. Dejean. Australia. australis. Dejean. Australia. Agonochila binotata, White. Australia.

\section{POLYMORPHA.}

Hydrobius assimilis, Hope. Australia.

Paracymus nitidiusculus. Australia.

Cyclonotum marginale, Sharp. Australia.

Cercyon flavipes, Fabricius. Europe.

Homalota sordida, Marsham. Europe.

Quedius fulgidus, Fabricius. Europe.

Philonthus æneus, Rossi. Europe.

scybalarius. Europe.

affinis, Roth. Europe.

nigritulus, Gravenhorst. Europe.

Xantholinus punctulatus, Paykull. Europe.

Oxytelus rugosus. Australia.

Carcinops I4-striata, Stephens. Europe.

Platysoma bakewelli, Marseul. Australia.

Carpophilus hemipterus, Linnæus. Cosmopolitan. mutilatus, Erichson. Cosmopolitan.

Osmosita colon, Linnæus. Europe.

Tenebrioides mauritanicus, Linnæus. Cosmopolitan.

Silvanus surinamensis, Linnæus. Cosmopolitan. unidentatus, Fabricius. Europe.

Coccinella II-punctata, Linnæus. (Lady-bird.) Europe.

Novius cardinalis, Mulsart. Australia.

Monotoma picipes, Herbst. . Europe.

sub-4-foveolata, Watson. Europe.

spinicollis, Aubé. Europe.

Coniuomus nodifer, Westwood. Europe.

Metiorhynchus rufipennis, Fabricius. Tasmania.

Dermestes vulpinus, Fabricius. Cosmopolitan.

lardarius, Linnæus. Cosmopolitan. 
Anthrenus mussæorum, Linnæus. Cosmopolitan.

Lyctus brunneus, Stephens. Europe.

Ptinus fur, Linnæus. Cosmopolitan.

Anobium domesticum, Linnæus. (Wood-borer.) Europe.

paniceum, Linnæus. Cosmopolitan.

Necrobia ruficollis, Fabricius. Cosmopolitan.

rufipes, De Geer. Cosmopolitan.

Buprestis lauta, Leconte. North America.

Stigmodera gulielmi, White. Australia.

Lacon variabilis, Candêze.* Australis.

Monocrepidius exsul, Sharp. Australis.

Heteromera.

Tenebrio obscurus, Fabricius. Cosmopolitan. molitor, Linnæus. Cosmopolitan.

Gnathocerus cornutus, Fabricius. Europe.

Tribolium ferrugineum, Fabricius. Cosmopolitan.

Anthicus floralis, Linnæus. Europe.

Macerdes melanura, Schmidt. Europe.

\section{Phytophaga.}

Bruchus rufimanus, Boh. Europe.

Hylotrupes bajulus, Linneus. (T.N.Z.I. vii. 320.) Europe. Phoracantha recurva, Newman. (T.N.Z.I. vi. I54.) Australia. Callirhoe allaspa, Newman. Australia.

Tessaromma undatum, Newman. Australia.

RHYNCHOPHORA.

Doticus pestilens, Oliff. Australia.

Otiorhynchus sulcatus, Fabricius. Europe.

Gonypterus sp. Australia.

Calandra granaria, Linnæus. Cosmopolitan.

oryzæ, Linnæus. Cosmopolitan.

Anthronomus pomorum, Linnæus. Europe.

Sitones lineatus, Linnæus. Europe.

\section{HEMIPTERA.}

\section{Heteroptera.}

Sciocoris helferi, Fieber. Reported by Novara Expd. Europe. Nezara prasina, Linnæus(?). Europe.

* In T.N.Z.I. xxx, 156, I mentioned Lacon murinus as introduced into the Chatham Islands. This is a mistake, as the insect referred to is really Psorochroa granulata. 
Nabis lineatus, Dahlberg(?). Europe.

Cimex lectularius, Linnæus. (Bed-bug.) Europe.

\section{HOMOPTERA.}

Ricania australis, Walker. Australia.

Aspidiotus aurantii, Maskell. T.N.Z.I. xxvii. 2. Australia, \&c. budleiæ, Signoret. Europe. camelliæ, Signoret. Europe. epidendrii, Bouché. Europe. nerii, Bouché. Europe.

Diaspis boisduvalii, Signoret. Europe. rosæ, Sandberg. Europe.

Mytilaspis citricola, Packard. North America. pomorum, Bouché. Europe.

Chionaspis citri, Comstock. North America.

Leucanium hemisphæricum, Targioni-Tozzetti. Europe. hesperidum, Linnæus. Europe. hibernaculorum, Boisduval. Europe. maculatum, Signoret. Europe. mori, Signoret. Europe. nigrum, Nietner. Australia. oleæ, Bernard. Europe. ribis, Fitch. North America.

Pulvinaria camellicola, Signoret. Furope.

Dactylopius adonidum, Linnæus. Europe.

Siphonophora rosæ, Reaum. (Rose aphis.) Europe. granaria, Kirby. Europe.

Mysus cerasi, Fabricius. Europe.

Aphis brassicæ, Linnæus. Europe.

Chermes corticalis, Kaltenbach. Europe.

pini, Koch. (T.N.Z.I. xviii. I3.) Europe.

Schizoneura lanigera, Haus. (Woolly aphis.) Europe.

Phylloxera vastatrix, Planchon. Europe.

Rhizobius graminis, Thompson. Europe.

ANOpleura.

Pediculus capitis, Nitzsch. (Louse.) Cosmopolitan. vestimenti, Nitzsch. Cosmopolitan.

Hæmatopinus eurysternus, Nitzsch. Cosmopolitan.

\section{ORTHOPTERA.}

Gryllus servillei, Saussure. (Field-cricket.) Australia. Cœdicia olivacea, Brunner. Australia. Orthodera ministralis, Fabricius. (T.N.Z.I. xxix. 242) Mantis. Australia. 
Blatta germanica, Linnæus. (Cockroach.) Cosmopolitan. latipennis, Brunner. India (?).

Polyzosteria truncata, Brunner. Australia.

Periplaneta americana, Linnæus. Cosmopolitan.

Forficula auricularia, Linnæus. (Ear-wig.) Europe.

Chelisoches morio, Fabricius. Malay Archipelago.

\section{NEUROPTERA.}

Chrysopa perla, Schneider. Europe. (Fide Hilgendorf.) Trichodectes scalaris, Nitzsch. (Ox-louse.)

Thrips sp.

\section{THYSANOPTERA.}

\section{APTERA.}

Lepisma saccharina, Linnæus. (Silver-fish.) Cosmopolitan. Entomobrya multifasciata, Tullberg. Smith, T.N.Z.I. xxviii. 475 .

Achorutes armatus, Nicolet. Smith, T.N.Z.I. xxviii. 475 .

\section{ARACHNIDA.}

\section{Araneida.}

Theridion tepidariorum, Blackwall. (House-spider.) Europe? rufipes, Lucas. Simon, Zool. Jahr. 1899, 422. Europe and Asia.

Walckenæra cristata, Blackwall. Europe.

\section{ACARINA.}

Ixodes ricinus, Linnæus. (Sheep-tick.) Europe.

Tyroglyphus siro, Linnæus. (Cheese-mite.) Cosmopolitan. Glycyphagus domesticus, de Geer. (Common-mite.) Cosmopolitan.

Tetranychus telarius, Linnæus. (Red-spider.) Cosmopolitan. Morton, T.N.Z.I. vi. 380.

\section{CRUSTACEA.}

ISOPODA.

Porcellio scaber, Latreille. (Wood-louse.) Chilton, T.L.S. Zool. viii. I39. Cosmopolitan.

Armadillidium vulgare, Latreille. Chilton, T.L.S. Zool. viii. I49. Europe. 


\section{ANNULATA.}

\section{Oligocheta.}

Eiseniella tetraedrus, Savigny. Europe.

Eisenia fotida, Savigny. Europe. rosea, Savigny. Europe.

Helodrilus caliginosus, Savigny. Europe. rubidus, Savigny. Europe.

Otoclasium cyaneum, Savigny. Europe.

Lumbricus rubellus, Hoffmeister. (L. campestris, Hutton.) Europe.

castaneus, Savigny. Europe.

terrestris, Linnæus. T.N.Z.I. xxv. I I 7 ( fide Smith). Europe. Didymogaster sylvatica, Fletcher. New South Wales.

Eudrilus eugeniæ, Kinberg. Equatorial West Africa, but widely spread in the tropics.

Pontoscolex corethrurus, Fr. Müller. Central America, but widely spread in the tropics.

\section{PLATYHELMINTHES.}

Turbellaria。

Geoplana sanguinea, Moseley. T.N.Z.I. xxvii. I 78. Australia. cærulea, Moseley. T.N.Z.I. xxvii. I8I. Australia.

Placocephalus kewensis, Moseley. Cosmopolitan.

Platydesmus moseleyi, Fletcher and Hamilton. T.N.Z.I. xxvii. I 89. Australia. 



\section{INDEX TO THE GENERA.}

ABREUS, 173

Acalles, 2 I 2

Acallistus, I43

Acallopais, 214

Acanthephyra, 255

Acanthidositta, 29

Acanthochzera, 37

Acanthochites, 86

Acanthoclinus, 46

Acanthoderus, 234

Acanthodoris, 67

Acanthomunna, 264

Acanthopleura, 86

Acantophrys, 248

Acartia, 270

Accentor, 347

Achæta, 346

Achorutes, 354

Acineta, 327

Aclis, 80

Acmæa, 85

Acontiophorus, 272

Acrantus, 219

Acosmetus, I70

Acrocyrta, 194

Actæcia, 265

Actæon, 69

Actenonyx, I 50

Actidium, I68

Actinelius, 339

Actinia, 3I4

Actinopteryx, I68

Actizeta, 185

Adelium, 187

Adeonellopsis, 298

Adolopus, I53

Adoxophyes, I 6

Adula, 94

Ega, 262

Agiatilis, $3^{8}$

Egoenicthys, 45

Æolidiella, 68

Élis, 67

Agyria, 328

Aschna, 230

Aschrodomus, 60

Etea, 294

Etobatus, 53

Agacalles, 2 I2

Agalba, 154

Agamonema, 304

Agandecca, 225
Aganeuma, 208

Agapanthida, I92

Agastegnus, 216

Agathinus, 210

Aglaia, 268

Aglaja, 69

Aglaophenia, 32I

Aglophus, I 56

Agonochila, 150

Agonostomus, 46

Agræcia, 23 I

Agrilochilus, 217

Agromyza, 129

Agrostis, 106

Aka, 225

Akera, 69

Aldonus, 2 I I

Alema, 198

Aleochara, I8I

Alepas, 274

Alepisaurus, 50

Aleurodes, 225

Allaorus, 218

Allasterna, I99

Allocatops, I75

Allocharis, 198

Allocinopus, 342

Allodiscus, 63

Alloparnus, I 53, I62

Allopora, 318

Alloprocas, 207

Allopterus, 188

Alloptes, 245

Allotherma, ro2

Alogus, 147

Alona, 266

Alope, 254

Alopias, 54

Alpheus, 254

Alysia, 100

Amalia, 348

Amarosoma, I88

Amarotypus, I 4 I

Amathia, 294

Amarygmus, I 88

Amaryllis, $25^{8}$

Amaura, 8o

Amaurobius, 238

Ambeodontus, 192

Amblyophis, $33^{2}$

Amblypneustes, 288

Amblypone, 97
Amblyrhynchotus, 52

Amblytelus, IoI

Ameletus, 231

Ammodiscus, 335

Ammothea, 247

Ammotretis, 48

Amœba, $34^{\circ}$

Ampelisca, 260

Amphibola, 66

Amphileptus, 330

Amphilochus, 258

Amphinecta, 238

Amphipeplea, 66

Amphiplatys, I 57

Amphiporus, 312

Amphitrite, 280

Amphiura, 290

Amphixystis, I24

Amphoroidea, 263

Amphitretus, 57

Amphoterocotyle, 3 I I

Amplectopus, I 57

Amylopterus, 2 Io

Amymone, 27I

Anabarhynchus, I3I

Anacharis, 102

Anagotus, 203, 343

Anaitis, 89

Anarhynchus, 32

Anas, 36

Anchistrocephalus, 3 ro

Anchomenus, 143, 342

Anchylochilus, 34I

Ancilla, 74

Ancistropterus, 2Io

Anclylomera, 257

Andaniotes, 258

Aneuma, 208

Aneura, I34

Aneurus, 222

Anguilla, 52

Aniculus, 252

Anillus, 150

Anisolabis, 234

Anisomeristes, I74

Anisonema, 332

Anisoplaca, II 8

Anisops, 223

Anobium, r6r

Anomalomyia, I34

Anomia, 95

Anomyx, $25^{8}$ 
Anosia, 103, 125

Anoteropsis, 244

Anoura, 234

Antedon, 290

Antennularia, 321

Anthea, 3r4

Anthicus, 189, $35^{2}$

Anthobothrium, 3 II

Anthonomus, 352

Anthophysis, 333

Anthopodium, 315

Anthornis, 28

Anthorochalina, 325

Anthosoma, 273

Anthrenus, 352

Anthribus, 219

Anthura, 262

Anthus, 28

Antiporus, I5I

Anubis, 22 I

Anuraea, 302

Aora, 260

Apale, 162

Apatolestes, I32

Apeosima, r9r

Aphænogaster, 97

Aphanasium, 194

Aphanipathes, 315

Aphela 343

Aphelomera, 134

Aphilon, I98

Aphis, 353

Aphobetus, 99

Aphodius, 139, $35^{\text {I }}$

Aphrodita, 277

Aphrophora, 224

Aphytopus, I8I

Apion, 210

Apis, 349

Aplysina, 326

Apollo, 75

Apomatus, 282

Aporolobus, 201

Apsetta, 48

Apseudes, 262

Apsoma, I 3 I

Aptenodytes, 35

Apterina, I29

Apteryx, 37

Apthora, 186

Arabella, 279

Arachnoides, 288

Arachnura, 242

Aradus, 222

Aræocerus, 22I

Aræoscapus, 205

Arbanitis, 237

Archidoris, 67

Archirhiza, 3I7

Architeuthis, 58

Arctocephalus, 25

Arctoneura, I.33

Arcturus, 264

Ardetta, 3I
Arecocryptus, 217

Arecopais, 220

Arecophaga, 2 I8

Arenaria, $3 \mathbf{I}$

Arenicola, 28I

Argentina, 5I

Argiope, 24I

Argonauta, 57

Argosarcus, 234

Argutor, 147

Argyrodes, 238

Argyropelecus, 50

Argyrophegna, 103

Ariamnes, 238

Arion, 349

Armadililidium, 354

Armadillo, 266

Arnomus, 197

Arocatus, 222

Arripis, 4I

Arthopus, 187

Arthracanthus, 160

Artotrogus, 272

Artystoma, 187

Asaphodes, 108

Ascaris, $3 \circ 3$

Aschleta, I I9

Ascidia, $5^{6}$

Ascogaster, roo

Asilis, I6r

Asilus, I31

Askyroderma, 287

Asobara, I0o

Asphærites, 174

Aspidiotus, 225, 353

Aspidisca, 327

Aspidodiadema, 288

Aspidosiphon, 285

Assiminea, 77

Astacilla. 264

Astasia, 3,32

Asteia, I 29

Asterias, 292

Asterina, 29r

Asterope, 269

Asteropsis, 29I

Astetholea, I93

mstetholid + , 193

isthena, ro8

Asthenosoma, 288

Astralium, 8 I

Astrape, 53

Astrochema, 290

Astrogonium, 29I

Astropecten, 29I

Astyplus, 205

Asymphus, I 55

Atactodea, 91

Atagena, 67

Atalophebia, 23I

Atherina, 46

Athoracophorus, 59

Athysaurus, 224

Atkinsonia, 239
Atomatricha, I I9

Atopida, 158

Atricatus, I97

Attus, 245

Atylus, 259

Atypichthys, 4I

Auchenoceros, 49

Auchenopteru $=, 46$

Audouinia, 279

Aulochrone, 323

Aulodendron, $34^{\circ}$

Axinella, 323

Axothea, 28I

BACTRA, II6

Baculipalpus, Igo

Badumma, $23^{8}$

Bxeocera, I74

Bxeorhopalus, 217

Bæosilpha, 175

Bairdia, 268

Balæena, 25

Balæenoptera, 26

Balanus, 275

Balcus, I60

Bantiades, 205

Barbatia, 94

Barlerina, 78

Barnea, 88

Bassus, 244

Bathygadus, 49

Bathymetis, Ior

Bathypterois, 50

Bathysaurus, 50

Bathysyphon, 334

Batrachedra, I2I

Bedelia, r23, $35^{\circ}$

Bembidium, I49, 342

Benthesicymus, 254

Berardius, 26

Beris, 132

Berosus, I 52

Beryx, 43

Betæus, 254

Betarmon, I57

Betyla, 98

Bicellaria. 295

Bidessus, I 5 I

Biloculina, 334

Bircenna, 260

Bithynis, 255

Bitoma, 16y

Bittium, 77

Bityla, 106

Blabophanes, I 25

Blatta, 234, 354

Blediotrogus, 184

Blosyropus, 193

Boeckella, 270

Holitophagus, 186

Bolitophila, 133

Bolivina, $33^{6}$

Boltenia, $5^{6}$ 
Bombus, 349

Borborus, 129

Botaurus, 3 I

Bothrideres, I7I, 342

Bothriocephalus, 310

Botryllus, 55

Bovichthys, 43

Brachaspis, 233

Brachicarpus, 255

Brachidocrania, 134

Brachiella, $27 t$

Brachylous, 202, 343

Brachynopus, 174

Brachypeplus, 172

Brachypleura, 48

Brachysara, II9

Brachyscelus, 257

Bradypatæe, 204

Brama, 44

Branchellion, 285

Branchiomma, 28 I

Brevicornu, I34

Brexius, 210

Bristiopsis, 287

Brontopriscus, I67

Brounia, I57

Bruchus, 352

Brullea, I 43

Bryobates, 199

Bugula, 295

Bulla, 69

Bulimina, 336

Bullinula, 70

Buprestis, $35^{2}$

Butalis, I 2 I

Byrrhocryptus, I 57

Byrrhodes, I 57

Cabal.us, 30

Caberea, 294

Caccomolpus, I98

Cacochalina, 324

Cacodrotus, 193

Cacœecia, II 7

Cadulus, 86

Cacioperc 1, 4 I

Cænophanes, 208

Cafius, 183

Cafioquedus, I 82

Calandra, 352

Calanus, 269

Calathus, I 43

Calcager, 126

Calicotis, I2I

Caligus, 273

Callianassa, 253

Callidina, 300

Calliopius, 259

Calliostoma, 83

Callipepia, 348

Calliphora, 127, 350

Calliprason, I93

Callirhoë, 352
Calliteuthis, 57

Callochiton, 87

Callorhynchus, 53

Calodera, I8I

Calotermes, 230

Calwellia, 296

Calycella, 3 I9

Calyptrea, 79

Calytrophora, 3I6

Camacho, 26I

Cambridgia, $23^{8}$

Camiarus, I74

Campanularid, 3 I9

Camptocladius, I 37

Camptoscapus, 2 I7

Campylomyza, I35

Cancellaria, 7 I

Cancer, $2+8$

Candace, 270

Candonocypris, 267

Canno hiza, 317

Cantharidus, 82

Caphius, 183

Caprella, 26I

Caprellinop sis, 26r

Caprodon, 4 I

Capromimus, 45

Capsus, 223

Capua, I 6

Caranx, 44

Carbasea, 295

Carcharias, 53

Carcharinus, 53

Carcharodon, 54

Carchesium, 329

Cardiastethus, 223

Cardita, 92

Cardium, 89

Carduelis, $3+7$

Carinaria, 70

Carpocapsa, 349

Carpophilus, 35 I

Carasius, 348

Carsinops, 35 I

Carthæa, 64

Caryophyllia, 315

Casa, I33

Casarca, 36

Cassidina, 263

Cassidulina, 336

Castanidium, 340

Catarrhactes, 36

Catenicella, 29.4

Cateristis, I23

Cathartocryptus, 167

Cathypna, 302

Catopsolius, 175

Catoptes, 20I

Caudina, 287

Caulopsetta, 48

Cavolina, 70, 34I

Cecidomyia, I35, $35^{\circ}$

Cecrops, 273

Cecyropa, I99, 343
Ceina, 257

Celania, $2+2$

Celetotelus, 208

Cellaria, 296

Cellepora, 298

Cellularia, 294

Centriscops, 46

Centrolophus, 44, 34I

Centromma, 272

Centropages, 270

Cephalorhynchus, 26

Cephaloscillium, 54

Cephalothamnium, 333

Cerabilia, I 47

Ceraochalina, 325

Ceratognathus, I39

Ceratopogon, I37

Cerchneis, $3^{8}$

Cercyodes, I53

Cercyon, 35I

Cerebratulus, 3 I 3

Ceresium, I94

Ceriodaphnia, 266

Cerithiopsis, 77

Cermatia, 235

Cermatulus, 22I

Cerodolus, 188

Ceroplatus, I 33

Cerosomyia, 126

Cerozodia, I 37

Certhiparus, 28

Cervus, $3+7$

Cetonurus, 49

Cetorhinus, $5+$

Chærodes, I85

Chætosoma, I66

Chætozone, 280

Chalastria, I I I

Chalcococcyx, 29

Chalcodrya, 189

Chalina, 324

Chalinolobas, 25

Chalinopsilla, 325

Chalinura, 49

Chamæsephis, 205

Chamæsipho, 275

Chamostrea, 87

Chanos, 5 I

Charadrius, $3 \mathbf{I}$

Charopa, 59

Chasmagnathus, 250

Chauliodes, 229

Cheimarrichthys, 43

Chelifer, 246

Chelisoches, 354

Chermes, 353

Chilodactylus, 42

Chilodon, 328

Chilostomella, 337

Chiltonia, 257

Chimæra, 53

Chionaspis, 226353

Chione, 89

Chiracanthium, 237 
Chironemus, 42

Chironomus, 137

Chiton, 86

Chloia, 278

Chloroclystis, 107

Chlorophthalmus, 50

Chœenia, $33^{\circ}$

Choleva, I75

Chondracanthus, 274

Chonelasma, 323

Chorascus, I70

Choreutis, $35^{\circ}$

Chorinæus, IO2

Chorizopoda, 297

Chromodoris, 67

Chronostomum, 306

Chrosis, I55

Chrysopa, 354

Chrysophanes, ro3

Chydorus, 266

Cicindela, I4I

Ciconissins, I7I

Cilibe, 186

Cillenum, I 50

Cimex, 353

Cinctipora, 299

Circia, I22

Circostella, I23

Circus, 30

Cirolana, 263

Cirroteuthis, 57

Cirsonella, 83

Cis, 162

Cixius, 224

Cladochalina, 324

Cladorhiza, 324

Clambus, I73

Clathria, 324

Clathurella, $7 \mathrm{I}$

Clausocalanus, 269

Claviporella, 294

Clavulina, 336

Cleantis, 264

Clepricosma, II 4

Clibanaria, $25^{I}$

Clinocera, I30

Clitarchus, 233

Clitellaria, I 32

Clivina, I 42

Clubinona, 237

Clupea, 5I

Clypeorhynchus, 205

Clytia, 319

Clytus, I9.4

Coccinella, I64. $35^{\mathrm{I}}$

Cochlicopa, 349

Cochlodesma, 87

Cochlostyla, 348

Codosiga, 33 i

Cœdicia, 353

Cœlopa, I28

Cœlopus, 30I

Cœlostoma, 228

Cœlorhynchus, 49
Cœnocydnus, 222

Cœnosia, I27

Cogia, 26

Coleps, 331

Collozoum, 339

Colobracis, IOI

Coloburiscus, 23r

Colochirrus, 287

Cologrammus, 46

Colpidium, 330

Columbella, 72

Coluris, 302

Cominella, 73

Compsistis, I23

Cona, 225

Condylocardia, 92

Congermurzena, 52

Congiopodus, 42

Coninomus, 35I

Conocyathus, 3I 5

Conopomæpha, I23

Conopora, 318

Conostoma, 272

Conurus, 182

Copelatus, I5I

Coprostygnus, 184

Coptoma, 194

Corbula, 88

Cordylophora, 318

Cordylura, I 28

Corethra, 138

Coridodax, 47

Coriscium, I23

Corixa, 224

Cormocephalus, 236

Cornicularia, 240

Cornuspira, 334

Coronula, 274

Corophium, 26I

Corticaria, 164

Corvus, 348

Corycæus, 272

Corymbites, I54

Coryne, 318

Cosmocephalus, 236

Cosmodes, 106

Costleya, 140

Cotes, 189

Cothurnia, 329

Cottapistus, 42

Coturnix, 30

Coxelus, I69

Crambus, II2

Cranophorus, 165

Crapatalus, 42

Craspedosoma, 235

Crassatellites, 92

Cratena, 68

Craterolophus, 316

Crayracion, 52

Creadion, 27

Cremogenes, 120

Crepidogaster. 47

Crepidula, 79
Cribella, 292

Cribrilina, 296

Crisina, 298

Crissia, 298

Crissius, 212

Cristellaria, 337

Cristiceps, 46

Crocidophora, III

Crossophorus, 269

Cruregens, 262

Crymophilus, 38

Cryptamorpha, 167

Cryptodacne, I66

Cryptodromia, 251

Cryptohelia. 318

Cryptohynus, 155

Cryptolaria, 320

Cryptomera, IgI

Cryptomina, II2

Cryptophagus, I66

Cryptops, 236

Cryptus, IOI

Cteniza, 237

Ctenoneurus, 222

Ctenochiton, 226

Ctenognathus, I44

Ctenoplectron, I88

Ctenopseustes, II7

Cubiceps 44

Cucumaria, 286

Culeolus, 56

Culex, 138

Cuniopterus, 205

Curimus, 162

Cuspidaria, 87

Cyamiomactra, 90

Cyamium, 92

Cyamus, 262

Cyanorhainphus, 29, 34I

Cybium, 45

Cyclacalles, 2I4

Cyclammina, $33^{6}$

Cyclaspis, 256

Cyclasterope, 269

Cyclaxyra, I 73

Cyclidium, 330

Cyclocterus, 244

Cyclogaster, I32

Cyclograpsus, 250

Cycloneura, I34

Cyclonotum, I53 $35 \mathrm{I}$

Cyclops, 270

Cyclothorax, I49

Cylichna, 69

Cylicia, 315

Cylindrophorus 3 II

Cyliosoma, 234

Cyloma, 152

Cymodoce, 263

Cymodocella, 263

Cymodroma, 34

Cymolutes, 48

Cymospira, 282

Cyphanus, 157 
Cyphon, I 58

Cyphotelus, I59

Cypridopsis, 267

Cyprinotus, 268

Cypris, 267

Cyprobius, 157

Cyproidia, 258

Cystisoma, 256

Cystodytes, 55

Cythere, 268

Cythereides, 268

Cytherella, 269

Cytheropteron, 268

Cytilissus, 163

Cytomissus, 342

Cyttalia, 208

Cyttus, 45

DACNOPHYLLA, 206

Dacnusa, 349

Dactvlochalina, 325

Dactylopius, 227, 353

Dactylopus, 27 I

Dalatias, 54

Dalma, 178

Dalminissus, 178

Dama, 347

Dammarobius, Igo

Daphnella, 7 I

Daphnia, 266

Daption, 35

Dasybatis, 53

Dasycolletes, 96

Dasygorgia, 316

Dasylobus, 246

Dasypodia, I06

Dasytes, I6o

Dasyuris, Iog

Deana, II 5

Decadarchis, I24

Declana, I I I

Defranchia, 299

Degithina, Ior

Deguernea, 270

Deinacrida, 23 I

Deinagnatha, 241

Delatus, 54

Deleatidiun 1,23 I

Delphinapterus, 26

Delphinus, 27

Demetrida, I 50

Demiegretta, 3 I

Demtrius, 187

Dendrilla, 323

Dendroblax, I39

Dendrocygna, 39

Dendromonas, 333

Dendrophagus, 167

Dendrostygnus, 212

Dendrotrupes, 2 I9

Dentalium, 85

Dermestes, $35 \mathrm{I}$

Dermochelys, 40
Dermothrius, 2 I 4

Dermotrichus, 205

Desis, $23^{8}$

Desmalia, 3 I 7

Desmocyphus, 321

Diachasma, I00

Diachoris, 295

Diadema, I 25

Diaea, 243

Diagrypnodes, I 66

Diaprepocoris, 224

Diapria, 98

Diarthrocera, I64

Diarthrodes, 27 I

Diaschiza, 301

Diasemia, I 44

Diaspis, 226, 353

Diastemerus, I97

Diastopora, 299

Diastylis, $25^{6}$

Dichrochile, 143, $3+2$

Dichromodus, I Io

Dicœlotus, IOI

Dicotylichthys, 52

Dicranomyia, 136

Dictyotus, 221

Didymocantha, I9I

Didymogaster, 355

Didymozoon, 310

Diglena, 30 I

Diglimma, I43

Digrammus, 182

Dilophus, I 33

Dimetopia, 295

Dinematichthys, 49

Dinematrum, 273

Dinobothrium, 3 I I

Dinobryum, 332

Dinocharis, 301

Dinodrilus, 284

Diöedimorpha, 217

Diomedea, 35

Diopatra, 278

Diphasia, 320

Diphyes, 317

Diplobothrium, 3I I

Diplocrepis, 47

Diplodon, 91

Diplodonta, 92

Diplopseustis, II 5

Diplosis, I 35

Diporochæeta, 284, 346

Dipterina, I 17

Diptychophora, I I 2

Diretmus, 45

Discorbina, $33^{8}$

Discothyrea, 97

Distoma, 309

Divaricella, 92

Dodecaspis, $34^{\circ}$

Dorlonidia, IO3

Dolichernis. I 23

Dolichoglossus, 56

Dolichopezea, I35
Doliocenthus, 205

Doliolum, 56

Dolium, 75

Dolomedes, 243

Domina, I 29

Dorcatoma, I6I

Doriopsis, 67

Doris, 67

Doryctomorpha, 99

Dorydium, 224

Doryichthys, $5^{2}$

Dorytomus, 207

Dosinia, 89

Doticus, 352

Drassus, 237

Drasterius, 154

Drepanepteryx, 229

Drepanidotænia, 312

Drepanodes, I I I

Drepanura, 234

Drillia, 70

Drimostona, I 44

Drosophila, I29

Drototelus, 343

Drotus, 193

Dryandula, 124

Dryocera, I66

Dryopais, 204

Dryptops, I68

Ducoscolex, 282

Duymzria, 48

Dysnocryptus, 220

Ebalia, 25I

Ebmrida, 193

Echinaster, 292

Echinobrissus, 288

Echinocardium, 287

Echinometra, 288

Echinophthirus, 228

Echinorhinus, 54

Echinorhynchus, 304

Echinus, 289

Echiurus, 283

Echthrogaleus, 273

Ectatomma 97

Ectinosoma, 271

Ectopis, 2 I I

Edalus, 187

Ehrenbergina, 336

Eiconaxius, 253

Eiratus, 204

Eiseniella, 355

Eisenia, 355

Elachista, I2 I

Elamena, 25 I

Elasmonetta, 36

Elasmonotus, 252

Elasmopus, 260

Eledone, 57

Eleotris. 45

Elminius, 275

Elvira, 107 
Emarginula, 84

Emberiza, 347

Emesoderma, 223

Emmelichthys, $4 \mathrm{I}$

Empis, 130

Enarsus, 169

Encephalia, I8I

Endophthora, 124

Endrosis, I2I

Entalophord, 229

Entium, 217

Entomobrya, 354

Entomolepis, 272

Enypniaster, 286

Eone, 279

Eosophora, 301

Epalxiphora, I I6

Epheus, 193

Ephydra, I29

Epiblema, I 6

Epidosis, I35

Epiera, 24I

Eprerus, 173

Epiphthora, I 8

Epirranthis, rro

Episinus, 242

Epistranus, 170

Epistylus, 329

Epltimestes, 200

Epuræa, 172

Erana, 106

Erebiola, 103

Erechthris, 124

Erennetis, I24

Ericentrus, 46

Erigone, 240

Erinaceus 347

Eriocampa, 35r

Eriochiton, 227

Eriococcus, 227

Erirhinnus 203

Eristalis, 350

Errina, 318

Erycina, 239

Erymnetus, 204

Eschatotypa, I24

Eschscholthia, 3I4

Ethalia, 83

Etheophanus, 208

Etnalis, 220

Eualema, 343

Euchreta, 269

Euchalina, 325

Euchatinopsis, 325

Euckelus, 83

Euchœca, 108

Euchytræus, 346

Euclanis, $30 \mathrm{r}$

Eucolaspis, 197

Eucope, 319

Eucopelli, 319, 344

Eucossoinus, 2,6

Eucotyllabe, 308

Euctenopus, ro\%
Eudendrium, 3 I 8

Euderia, I62

Eudiocrinus, 29I

Eudoxochiton, 86

Eudyptula, 36

Eudrilus, 355

Euglena, 332

Euglyptus, I76

Eugoniscus, 220

Eugonomus, 208

Eulalia, 277

Eulechria, I 9

Eulinella, 74

Eulophus, 349

Eumede, 160

Eumenıa, 28I, 346

Eumetopias, 25

Eunice, 278

Euodontes, 218

Eupagurus, 25 I, 344

Eupelmus, 99

Euplectus, I79

Euplocamus, 67

Euplotes, 327

Eupolia, 313

Eupomatus, 282

Euprocas, 207

Eupines, 177

Eupterolicus, 246

Eiryceras, I 34

Eurychxena, 196

Eurycope, 265

Eurynolainurus, 248

Euryphegma, 323

Eurystomus, 37

Eurythecta, II7

Eurytoma, 99

Eusirus, 259

Eusoma, I40

Euspongia, 326

Eustrongylus, 304

Euterpe, 27I

Euthemisto, 256

Euthenarus, I 48

Euthria, 73

Euthyrhinus, 214

Eutora, I23

Eutornus, 218

Evadne, 267

Evechinus, 289

Evistius, 44

Exaireta, I32

Exeirarthra, I79

Exilis, 220

Exocalopus, I90, 342

Exocoetus, 49

Exœolus, 155

Exohad!nus, 185

Exomesistes, 2 I 8

Exoria, I 16

Exospherona, 263

Exsul, I27

Exydrus, 152

Eylais, 246, $3+3$
FACELINA, 68

Faculifer, 245

Falagria, $18 \mathrm{r}$

Falciger, 246

Farciminaria, 295

Farrea, 323

Fhogra, 100

Filaria, 303

Fiona, 68

Fiorinia, 226

Fissurella, 84

Fistulicola, 310

Flabelligera, 28r, 346

Flabellum, 315

Flammulina o2

Flavid, 27I

Floscularia, 300

Flustra, 295

Flustrella, 293

Forficula, 354

Fossarina, 83

Fraudator. I3I

Fregatta, 33, 38

Fr yella, 292

Fridericia, 284

Fringilla, 347

Fuligula, 37

Fusus, 73

Gadinia, 68

Galathea, 252

Galaxias, 5 I

Galeorhinus, $5+$

Galeus, 54

Gallinago, 32, 38

Gammaroparnops, 232

Gammaropsis, 260

Gangliopus, 273

Garrodia, 34

Gasterochisma, 45

Gasteropinilus, 350

Gasterostomum, 3 I0

Gasteruption, 99

Gastrobothrus, I77

Gastrocotyle, 308

Gastrophysus, 52

Gastrosarus, 193

Gastrostyla, 327

Gathocles, 169

Gaudrina, $33^{6}$

Gehyra, 39

Gelasimus, 249

Gelechia, I 18

Genotia, 70

Genyagnus, 42

Genypterus, 49, 34 I

Geochus, $2 \mathrm{O}_{3}$

Geonemertes, 312

Geophilus, 236

Geoplana, 306, 355

Geotomus, 22I

Geotria, 5.5

Ceramus, 154 
Geranomyia, I36

Gerontia, 64

Getacalles, 212

Gibbula, 82

Gilbertia, 40

Glaucoma, 327

Glaucopsis, 27

Glenentela, 170

Globiocephalus, 27

Globigerina, $33^{8}$

Gloiopotes, 273

Glossiphonia, 285

Glottis, $3^{8}$

Glycera 279

Glycymeris, 94

Glycyphagus, 354

Glyphipteryx, I22

Glyhocrangon, 254

Glyphoramphus, 217

Gnathaster, 29I

Gnathocerus, $35^{2}$

Gnomodes, I93

Gnophomyia, 136

Gobiomorphus, 45

Gobius, 46

Goniocidaris, 288

Goniodon, 29I

Goniomonas, 333

Gonophylla, I I I

Gonorhynchus, 5t

Gonostoma, $5^{\circ}$

Gonypteıus, $35^{2}$

Gordius, $30+$

Gorytes, 98

Gossyparia, 227

Gracillaria, I 23

Grammaroparnops, $23^{2}$

Grampus, 27

Grantessa, 322

Graspus, 249

Graucalus 37

Gregoria. 314

Gryllus, 349

Grynoma, I 72

Gundlachia, 66

Gygis, 33

Gymnobathrd, I I9

Gymnodinium, 33 I

Gymnophania I27

Gymnoplectron 232

Gymnorhina 348

Gynoplistia, I37

Gyrinu :, I5I

Gyrocoris, 329

Gyrocotyle, 3I0

Gyrophæena, I8I

\section{Haastia, 324}

Habronestes, 240

Habrophila, 124

Hadracalles, 212

Hremadipsa, 285

Hæmatopinus, 353
Hæmatopus, 3 I

Halacarus, $2+6$

Halargyreus, 48

Halcyon, 29

Halecium, 320

Halicarcinus, $25^{\circ}$

Halictus, 96

Halimus, 247

Haliotis, 83

Haliporus, 254

Halirages, 259

Halla, 279

Halmaturus, 317

Haloboena, 35

Halteria, 329

Halytes, 160

Haminea, 69

Hamotulus, 177

Haplocheira, 26I

Haplodactylus, 4I

Haplolobus, 202

Haplophragmium. 335

Haplophthalmus, 265

Haplotaxis. $28+3+6$

Harnologa, 117

Harmonis, 26r

Harmothoë 276

Harpacticus, 27 I

Harpalus, I. 78

Haswellia, 298

Hebella, 3 I9

Hednota, I I I

Helæcius, 2.49

Helicæ, $25^{\circ}$

Helicarion, 65

Helicella, $34^{8}$

Heliostribes, I 22

Heliothela, II 3

Heliothis, In6

Helix, $34^{8}$

Helle, I 31

Helodrilus, 355

Heloecius, 249

Helomyza, I28

Helophilus, I 30

Hemerocotes, 46

Hemiclava, 243

Hemideina, 231

Hemipenæus, 254

Hemiphaga, 30

Hemiplax, 29

Hemipodus, 279

Hemirhamphus, 49

Hemistomum, 309

Hemiteles, IOI

Hemitheca, 318

IJendecastricha, I 6

Henicocephalus, 343

Henicops, 235

Henlea, $28+$

Henops, I3I

Hepialus, 125

Heptatrema, 55

Herodias, $3 \mathbf{I}$
Herpyllus, 237

Hesperophanes, 192

Heteractites, $3^{8}$

Heteragus, 169

Heterakis, 303

Heteralocha, 27

Heteraomus, 203

Heterocrossa, II 7

Heterodactylus, I44

Heterograpsus, 249

Heteromita, $33^{2}$

Heteromyza, I28

Heteronyx, I 70

Heteropleuron, 55

Heteropora, 299

Heteropsis, 2 I 7

Heteropygia, 32

Heterostoma, 235

Heterostyles, 203

Heterozius, 2.48

Hexaplagia, $34^{\circ}$

Hexathele, 237

Hexatricha, I94

Hilara, I30

Himantopıs, 32, 34I

Hippocampus, 52

Hippolyte, 254

Hipponyx, 79

Hippospongia, 326

Hippothoa, 294

Hirudo, $34+$

Histiophorus, 43

Histrio, 327

Hochstetteria, 93

Hofmannia, 123

Holcaspis, $1_{4} 6$

Holoparainecus, I64

Holopsis, 165

Holostomunt, 309

Holothuria, 286

Holotrochus, I 84

Homalomyia, I27, 350

Homalota, I8I

Homepuræa, 172

Homodus, 20I

Homœodytes, I 5 I

Homœeosoma, I I I

Homoreda, 2 I I

Hoplocneme, 209

Hoplodactylus, 39

Hormosina, 335

Hornera, 299

Huberia, 97

Huttonia, 240

Huxelhydrus, I 5 I

Hyale, 257

Hyalina, $34^{8}$

Hybernia, II I

Hybolasius, 196

Hydatina, 301

Hydora, I53, I6 3

Hydra, 321

Hydrallmannia, $32 \mathrm{I}$

Hydriomena, IO7 
Hydrobiosis, 229

Hydrobius, $35 \mathrm{I}$

Hydrocena, 80

Hydroessa, 223

Hydrocheilodon, $3^{8}$

Hydroprogne, 32

Hydropsyche, 229

Hydrostygnus, I 52

Hydrus, to

Hygrochus, 201

Hyla, $34^{8}$

Hylastes, 2 r 9

Hylobasius, 196, 342

Hylobia, I89

Hymenia, I 13

Hymenicus, 250

Hymenolcemus, 37

Hymenosoma $25 \mathrm{I}$

Hypanthea, 319

Hypenodes, 106

Hyperammina, 334

Hyperosoma, I 83

Hypeuryntis, I 23

Hypharpax, I48, 35 I

Hypolycodes, 49

Hypotzenidia, 30

Hypotagea, 207

Hystricia, I 26

IAIS, 264

Iathrippa, 264

Ibacus, 253

Icerya, 228

Ichmalius, 2 ro

Ichneumon, I00

Ichneutica, Io5

Ichthybotus, 23I

Ichthyocampus, $5^{2}$

Ichthyoneura, 303

Icosidesmus, 235

Idia, 32 I

Idmonea, 298

Idotasia, 215

Idotea, 264

Idus, 217

Idya, 272

Ilyodromus, 267

Incentia, 2 I I

Incisidens, 4I

Inglisia, 226

Inocatops, 174

Inocatoptes, 202

Inopelonia, I99

Inophlzeus, 202

Inosomus, 2 I 8

Ipana, I I I

Iphimedia, 259

Iphinctus, 250

Ips, 173

Ischalea, $24 \mathrm{I}$

Ischnochiton, 87

Ischnoderus, 184
Ischyroplectron, $23^{2}$

Isidora, 66

Isochasta, I I 8

Isocladus, 263

Isocolon, 175

Isonomentis, I 18

Isoplectron, 232

Isurus, 54

Itamus, I 31

Ithris, I70

Ixodes, 245,350

J ACULELLA, 334

Jæropsis, 264

Jantbina, 80

Jasus, 253

Julus, 235

Junonia, Io3

KATHESOSTOMA, 43

Kellia, 9I

Keroma, 328

Kœllikeria, 309

Krithe, 268

Krohnia, 305

LABIDOCERA, 270

Lacon, $35^{2}$

Lamosthenes, 351

Læstrygones, 244

Lætmonice, 277

Lævilitorina, 79

Lafoëa, 319

Lagena, 337

Lagochilus, 78

Lagrioda, 188

Lamontia, 322

Lampadena, 50

Lampris, 44

Lamprocolletes, 96

Lampromitra, 340

Lamyctes, 235

Lanassa, 280

Lancetes, I $5 \mathrm{r}$

Lanice, 280

Laoma, 6I

Laophonte, 27 I

Larus, 33

Lasea, 92

Lasiorhynchus, 219

Lasius, 96

Lathicrossa, I I 9

Lathridius, 164

Latia, 66

Latris, 42

Latrodectus, 239

Lauxamia, 128

Leachia, 228
Leæna, 280

Leanira, 277

Lebia, I50

Lecanium, 227

Lecanochiton, 226

Lecanomerus, 148

Leda, 95

Leiolophus, 250

Leiopeplus, 188

Leioproctus, 96

Leiosoma, 246

Leis, $16_{5}$

Lenax, 173

Lepas, 274

Lepeophtheirus, 273

Leperina, I72

Lepidonotus, 276

Lepidopleurus, 87

Lepidoplois, 48

Lepidopus, 43

Lepidothynnus, 45

Lepidotrigla, 45

Lepidurus, 267

Lepisma, $35^{\circ}$

Lepralia, 297

Leprea, 345

Leptachrous, 192

Leptocephalus, 52

Leptoceras, 229

Leptoclinum, 55

Leptodius, 248

Leptograpsus, 249

Leptomeris, I Io

Leptomerocoris, 223

Leptomithrax, 247

Leptoperla, 23 I

Leptoplana, 306

Leptoptilum, 3 I6

Leptoscopus, 42

Leptotbyra, 8I

Leria, 128

Lernaeopoda, 274

Lernacea, 274

Lernanthropus, 273.

Lestes, 230

Lestremia, I35

Leucandria, 322

Leucania, 104

Leucanium, 353

Leucascus, $j 22$

Leuckartia, 270

Leuconopsis, 67

Leucosolenia, 322

Leucothoë, $25^{8}$

Leucotina, 70

Lianculus, I30

Licophora, 329

Lichenopora, 299

Ligia, 265

Ligula, 3 I I

Ligurinus, 343

Liljeborgia, 259

Lima, 93

Limax, 348 
Limnxa, 66,348

Limnerium, 102

Limnia. 128

Limnichus, I63

Limnobia, 136

Limnodrilus, 285,346

I.imnœcia, I2I

Limnophila, 137

Limnophora, 127

Limnoria, 264

Limonites, 32

Limopsis, 94

Limosa, $32,3^{8}$

Linota, 347

Linyphea, 239

Liochoria, I63

Liogramma, I9I

Liopelma, 40

Lippistes, 79

Lipura, 234

Lirus, 4I, 44

Lissonota, $\mathrm{IO}_{2}$

Lissopimpla, 349

Lissotes, I $_{3}^{8}, 34 \mathrm{I}$

Lissotrachelus, 23 I

Lita, 350

Lithobius, 235

Lithochar's, 183

Lithocia, 205

Lithophaga, 94

Lithostygnus, I70

Lithyphantes, 239

Litonotus, 328

Litorina, $7^{8}$

Livoneca, 262

Lobivanellus, 38

Lobularia, 316

Lomemus, I 56

Lonchæa, 129

Lophotes, 47

Lorelus, I86

Lotorium, 75

Loxoconcha, 263

Loxodes, 328

Lucilia, $35^{\circ}$

Lumbriconereis, 279, 345

Lumbricus, 355

Luperus, 198

Lycana, 103

Lycosa, 243

Lyctus, 352

Lygosoma, 39

Lymnichus, I63

Lyperobates, 20I

Lyperobius, 203

Lyperopais, 204

Lysiana, $25^{8}$

Lysiosquilla, 256

Lysiphragma, I2I

Lythria, Iog

MACERDEs, $35^{2}$

Macoma, 9 I
Macraspis, 308

Macratia, I9o

Macrocera, I33

Macrochlamys, 65

Macrocypris, 268

Macromastix, 136

Macropathus, 233

Macrophthalmus, 249

Macrorhinus, 25

Macroscytalus, 217

Macrurorus, 49

Macrurus, 49

Mactra, 90

Mæra, 260

Magellania, 293

Majaqueus, 34

Maldane, $28 \mathrm{I}$

Maldanella, 28I

Malletia, 95

Mallobathra, I24

Mangelia, 7 I

Maoriana, 237

Maoridrilus, 283

Maquartia, 126

Margareta, 222

Marginella, 74

Marginula, 67

Marphysa, 279

Marpissa, 244

Marptusa, 2.14

Marsenia, 80

Mastigocerca, 30 r

Mathesis, I60

Maurolichus, 50

Mecastrus, I 56

Mecistostylus, 2I5

Mecodema, I 42,34 I

Mecyna, I I 5

Medyla, 65

Megacolabus, 208

Megacraspedus, I I 8

Megadromus, I48

Megadyptes, 36

Megalestris, 33

Megalocerœa, 223

Megaptera, 26

Megatebenus, 84

Meinertia, 262

Melainpsalta, 224

Melanchra, I05

Melanopsis, 76

Melanostoma, I30

Melanus, I 54

Melicerta, 300

Melinna, 280

Melita, 26o

Melliphora, 349

Melophagus, $35^{\circ}$

Membranipora, 295

Membraponella, 297

Memes, 343

Mendosoma, 42

Meninius, 185

Menipea, 294
Meretrix, 89

Merganser, 37

Merhippolyte, 255

Merluccias, 49

Merope, 27 I

Mesagyrites, I75

Mesocolon, I75

Mesocyphon, $15^{8}$

Mesodesma, 90

Mesolamia, I95

Mesoleptus, 102

Mesopatrum, I85

Mesoplodon, 26

Mesoreda, 2 I I

Mesoscolopax, $3^{8}$

Mesoscolytus, 2 I9

Mesotenus, Ior

Mesothuria, 286

Mesoxenophasis, 2I6

Metablax, 157

Metacalles, 2 I4

Metacrias, IO4

Metacrinus, 29I

Metagerra, 222

Metaglymma, 142, 342

Meteorus, 99

Methemus, I6r

Metopidia, 302

Metoponchus, 183

Metoponorthus, 266

Metopus, 330

Metriorhynchus, $35 \mathrm{r}$

Miaster, I 35

Micippa, 248

Micranous, 33

Microdes, 107

Microlamia, I95

Micromus, 230

Micropora, 296

Microporella, 296

Micropus, 37

Microscolex, 283

Microsilpha, I74

Microtribus, 2I6

Microtyrus, 176

Migas, 236

Milesia, I 30

Milichia, I29

Miliolina, 334

Mimetas, $23^{8}$

Minyas, $3 \mathbf{I} 5$

Miotopus, 232

Miro, 28

Miselia, I0.4

Mitophyllus, I39, 34 I

Mitra, 73

Mitrastethus, 215

Mnesarchæa, I25

Modiolarca, 94

Modiolaria, 94

Mohua, 28

Mola, 53

Monacanthus, 52

Monas, 333 
Monilea, 83

Monobothiluın, 310

Monocrepidius, 156,352

Monodonta, $8 \mathrm{I}$

Monomiorium. 97

Monoporella 297

Monops s, $35^{\circ}$

Monosiga, 33I

Monostomum, 3 Io

Monostyla, 302

Monotoma, 35

Mordeıla, I9I

Mordellisterna, I9I

Morna. 223

Mucronella, 297

Mugil. 46

Munida, 252

Munna, $26+$

Munnopsis, 265

Muræena, 5 I

Murex, 7 I

Mus, 347

Musca, 350

Muscina, 350

Musotina, II

Mustelus, 347

Mycetophila, I34

Myctophum, 50

Mygopsocus, 230

Mylitta, 92

Myodora, 88

Myrnielton, 229

Mystacops 25

Mysiss, 353

Myrilaspis, 226, 353

Mytilus, 94

Myxilla, 324

Myzostoma, 285

NABIS, 223, 353

Nascis, 153

Nassa. 72

Nassula, 331

Natica, 80

Naucrates, 44

Naultinus, 39

Nausitora, 88

Nauticaris, 255

Nautilograpsus, 250

Navomorpha, I94

Nebalia, 266

Necrobia, $35^{2}$

Necrophilus, I74

Necrophløesophagus, 236 , 343

Nectocarcinus, 249

Neides, 222

Nematccarcinus, 255

Nematonurus, 49

Nemorea, I26

Neobalæna, 26

Neochanna, 5 I

Neocharis, I 5.3
Neodrilus, 283

Neolepton, 92

Neomycta, 207

Neonetus, 232

Neophrynichthys, 42

Neoscopelus, 50

Nephila, 242

Nephrops, 253

Nephthys, 278

Nepticula, I23

Neptunus, 249

Nertis, 278, $3+5$

Nerita, 8I

Nerocila, 263

Nervijuncta, I33

Nesarcha, I 15

Nesierax, 30

Nesolimnas, 30

- Nesonetta, 36

Nestor, 29

Nestrius, 206

Nettion, 36

Neuronectes,

Nezara, 221, $35^{2}$

Nicreana, I99

Nicidion, 279

Nicolea, 280

Ninox, 29

Nitidula, 172

Nodosaria, 337

Nogagus, 273

Nonionina, 339

Nonnotus, $2=0$

Norix, r70

Nosodendron, 162

Notacanthus, 47

Notacantolica, 229

Notarchus, 69

Noteriurda, I 16

Nothaldonus, 2 I I

Notholea, 302

Notiodrilus, 283, 344

Notiopate, 20I

Notommata, 30 I

Notophoryx, 3 I

Notops, 300

Notoreas, Iog

Notorhynchus, 54

Notornis, 3I

Notothenia, 43

Notoulus, I 68

Novitas, 217

Novius, $35^{1}$

Nubecularia, 333

Nucula, 95

Numenius, $3^{8}$

Nyctemera, $\mathrm{IO}_{4}$

Nycticorax, 3I

Nyctiphanes, 255

Nymphon, 246

Nymphostota, I I8

Nyroca, 36

Nyxetes, 2 Io

Nyxius, 222
OBELIA, 3 I9

Ocalea, I 8I

Oceanites, 34

Ochosternus, $I=5$

Ochthrodromus, 32

Ocydromus, 30

Occissor, 126

Ochina, I6

Ochrocydus I9I

Ochrbiphila, 129

Octochæetes, 284

Octopus, 57

Odax, 47

Odontomyia, I 32

Odontostoma, 74

Odontria, I 40

Eceticus, I 5

Echalia, 22I

Econesus, 228

Ecophora, I20, 350

Ediceros, 258

Emona, Ig2

Estralata, 34

Estrus, 350

Ogmorhinus, 25

Oikomonas, 333

Oithona, $27 \mathrm{C}$

Oliarius, 225

Olinga, 228

Omalissus, 184

Omalium, 184

Onedes, I 88

Ommatocarcinus, 249.

Omosita, I72

Omphalotropis, 78

Oncrea, 272

Oncreola, 272

Onchoscolex, 282

Oncidiella, $5^{8}$

Onichoteuthes, $5^{8}$

Onicigaster, 23I

Oniscus, 265

Onos, 48

Onosandrus, 232

Onsinome, 24I

Onthophagus, 35I

Onuphis, 278

Onysius, 189

Oonops, 237

Ooperipatus, 236

Oopterus, I48, $34^{2}$

Oorbynchus, 247

Opatrum, 185

Opercularia, 329

Ophiacantha, 290

Ophiactis, 289

Ophioceramis, 289 .

Ophiochiton, 290

Ophiocreas, 290

Ophidiaster, 292

Ophiocten, 289

Ophioglypha, 289

Ophiomitra, 290

Ophiomastus, 289, 
Ophiomusium, 289

Ophiomyxa, 290

Ophion, IOI

Ophionereis, 290

Ophiopeza, 289

Ophiophorus, $=89$

Ophiophyllum, 289

Ophiopterus, 290

Ophiozona, 289

Ophisurus, $5^{\mathrm{I}}$

Ophryops. 192

Opifex, I 36

Opisthotricha, 327

Opomyza, I29

Optonurus, 49

Orbulina, $33^{8}$

Orchestia 257

Orca, 27

Orectognathus, 97

Oreda, 2 I I

Oregus, I 43

Oreocharis, 209

Ornithomyia, 138

Ornithochiton, 86

Orocrambus, I I 3

Orophora, I 15

Oropterus, 2 Io

Orsinome, 24I

Orthenches, 122

Orthocladius, 137

Orthodera, 353

Orthosia, 104

Orygmatobothrium, 3II

Oscinis, 129

Ossifraga, 35

Ostenia, I 30

Ostrea, 93

Othius, I 83

Otiorhynchus, $35^{2}$

Otoclasium, 355

Otoconcha, 65

Oulactis, $3 \mathbf{I} 4$

Owenia. 279

Oxycephalus, 257

Oxyethra, 229

Oxylasma, I 56

Oxynotus, 54

Oxyopes, 244

Oxytelus, $35^{\mathrm{I}}$

Ozius, 248

PACHYCHALINA, 324

Pachycorinus, $18_{3}$

Pachycotes, 2 I9

Pachykellia, 92

Pachylopus, I73

Pachymorpha, 233

Pachyprypnus, 205

Pachyrhamma, 232

Pachyrhina, I 35

Pachytylus, 233

Pachyura, 210
Pactola, 209

Predaretus, 204

Prelocharis, 20 I

Pagrosomus, fI

Pagurites, 252

Pagurodes, 252

Palæemon, 255

Palæomicra, 125

Palaina, 78

Pallasia, 282

Pallene, 247

Palluricella, $34+4$

Pandalus, 255

Pandrus, 273

Pangrus, 221

Pangonia, I32

Paniscus, I32

Pannichia, 286

Panopea, 88

Panopeus, 248

Panspœus, I 56

Pantopsalis, 343

Pantosperma, I22

Paprides, 233

Parabrontes, I67

Paracalamus, 269

Paracalliope, 259

Paracorophium, 26 I

Paracrangonyx, 260

Paractis, 314

Paracymus, 35I

Paracypris, 268

Paradetis, 107

Paradexamine, 259

Paradoxa, I 34

Paraleptamphopus, 259

Paramecium, 33I

Paramecosoma, I66

Paramithrax, 247

Paranephrops, 253

Paranomocerus, 2 I 5

Paranthurd, 262

Parapagurus. 252

Paraperris, 43

Paraphylax, 185

Paraponyx, II 4

Parapronoe, 257

Paratanais, 262

Pardosa, 244

Paresuris, 222

Parharpinia, $25^{8}$

Paridotea, 264

Parinus, I5t

Parmipalpes, I7 8

Parmius, I 59

Parvicellula, I34

Paryphanta, 65

Passer, 347

Patella, 84

Patellina, $33^{8}$

Paurocope 272

Paupris, I 59

Paxillus, 78

Peachid, 3I5
Pecten, 93

Pectilabrus,

Pectinaria, 280

Pectinura, 289

Pedalopia, I 50

Pedicellinopsis, 293

Pediculus, 353

Pedilophorus, I6 3

Pelagodroma, 34

Pelagohydra, 3 I9

Pelecanoides, 35

Pelodrilas, 284

Pelogenia, 277

Peloniu "1, I 59

Pelosina, 334

Peltidium, 27 I

Peltorhamphus, 48

Peltophora, I I9

Penella, 274

Penillia, 267

Peniticus. 197

Pentacheles, 253,

Pentacrinus, 29I

Pentarthrum, 2 I 5

Perca, $34^{8}$

Percnodaimon, IO3

Peremptor, 126

Periatrium, I85

Pericoma, I 38

Pericoptis, I4I

Pericrypta, 317

Peridinium, 33I

Peripatoides, 236

Periphylla, 3 I 7

Periplaneta, 354

Perisponia, 320

Perrierina, 90

Petrocheles, $25^{2}$

Petrochelida, 37

Petroica, 28

Petrolisthes, 252

Phacus, 332

Phacussa. 6

Phaeophanus, 203

Phroosaces, I I8

Phæthon, 33

Phaganophana, I76

Phaiussa, 64

Phalacrocorax, 34

Phania, I27

Phanophilus, $\mathrm{I} 83$

Pharmacus, 233

Pharcolodon, 328

Pharcolosoma, 285

Phasianella, 8I

Phasianus, 348

Phaulacridium, 233

Phelerosus, I 52

Phemus, 206

Phenacharopa, 6I

Phenachohelis, 63

Pherusa, 259

Phialonema, 332

Philacta, 206 
Philiniscus, 229

Phlæenus, 224

Phlæopoda, I I9

Philhydrus, I 5 I

Philichthys, 273

Philine, 69

Philobrya, 93

Philodina, 300

Philodromus, 243

Philomelas, 269

Philonicus, 254

Philonthus, 35I

Philoscia, 266

Philothermus, I7 I

Phlœopola, I I9

Phlyctænodes, 194

Phlyctenophora, 268

Phœebetria, 35

Pholadidea, 88

Phoracantha, $35^{2}$

Phorbia, I 27

Phorinosoma, 288

Phorocera, 126

Phorostichus, 208

Phosichthys, 50

Phoxichilidium, 247

Phoxocephalus, $25^{8}$

Phoxoteles, 203

Phreatogammarus, 260

Phreatoicus, 265

Phreodrilus, 284, 346

Phrissogonus, 107

Phrixgnathus, 6I

Phronima, 256

Phronira, 206

Phroso, 27I

Phryganostola, 122

Phrynixus, 204

Phrynus, 246

Phycosoma, 239

Phycochus, I40

Phycosecis, I72

Phyllobothrium, 3II

Phyllodore, 277

Phyllodytes, 206

Phyllophorus, 287

Phyllospongia, 325

Phyllotreta, I99

Phlœephagosoma, 218

Phylosiphonia, 325

Phyloxera, 353

Phymactris, 3 I 5

Phymalophœe, I60

Phymata, 222

Physalia, 318

Physcosoma, 285

Physeter, 26

Physetica, I04

Physiculus, 48

Physolæethus, I43

Physophora, 317

Phytilea, I9o

Picrotus, 167

Pilacoslaspis, I97
Pilumnopeus, 248

Pilumnus, $24^{8}$

Pinna, 94

Pinnoctopus, 57

Pinnotheres, 250, 344

Pirates, 223

Pisania, 73

Pisidium, 9 I

Pison, 98

Placocephalus, 355

Placochalina, 325

Placopsilina, 335

Placotiochus, 3 I 5

Placostylus, 59

Placunanomia, 95

Plagiochæta, 283

Plagiogenion, 4I

Plagiophila, 330

Plagusia, 250

Plakathrium, 263

Planaxis, 76

Planchonia, 227

Planispirina, 334

Planorbis, 66

Platalea, $3^{8}$

Platipidia, 173

Platurus, 40

Platycola, 329

Platycoris, 22I

Platydesmus, 355

Platyischnopus, $25^{8}$

Platyonichus, 249

Platyomida, 200

Platyptilia, I 5

Platypus, 2 I9

Platyscelus, 257

Platysoma, I73, 351

Platyura, I33

Platyzosteria, 234

Plaxiphora, 86

Plectomorphus, 179

Plegadia, $3^{8}$

Pleioplectron, 232

Plesionika, 255

Pleuraltica, I99

Pleurobranchæa, 68

Pleurobranchus, 68

Pleuronema, 330

Pleurotoma, 70

Pleustes, $25^{8}$

Plexippus, 244

Plociomerus, 222

Plotnus, 206

Plotus, 39

Plumatella, 293

Plumularia, $32 \mathrm{I}$

Plusia, I06

Plutella, I23, 350

Plutellus, 346

Podicipes, 36

Podoceropsis, 26I

Podocerus, 26I

Podon,

Pœcilippe, I96
Paccilodiscus, I40

Pogonorhinus, 218

Pogonornis, 28

Poliaspis, 226

Poliopogon, 323

Pollicipes, 274

Polycarpa, 56

Polycelis, 306

Polycentropus, 229

Polycheles, 253

Polydesmus, 235

Polydora, 345

Polylobus, I8I

Polymorphina, 337

Polynoë, 276, 345

Polyphyllia, 3I5

Polyprion, 40

Polystomella, 339

Polysyncraton, 55

Polyzosterium, 234, 354

Pomatoceros, 282

Ponera, 97

Pontobdella, 285

Pontodrilus, 284

Pontophilus, 254

Pontoscolex, 355

Porcellana, 252

Porcellanopagurus, 252

Porcellidium, 27 I

Porcellio, 354

Pordo, 267

Porella, 297

Porina, 125

Porphyrio, 3 I

Porrhotheles, 237

Porroa, 277

Portunus, 249

Porzana, 31

Potamides, 77

Potaminus, 153,162

Potamopyrgus, 77

Pourtalesia, 287

Praolepra, 208

Prenolepis, 349

Priasilpha, I73

Priates, I73

Primoella, 3 I 6

Prinino, 257

Priocella, 34

Priofinus, 34

Prion, 35

Prionoplus, I9I

Prionorhynchus, 248

Pristacantha, 340

Pristancylus, I44

Proales, 301

Probolus, IOI

Procelsterna, 33

Procissio, I26

Proconus, 217

Proctophanes, $35^{I}$

Proctotrupes, 98

Prodelphinus, 27

Promanus, 172 
Promethichthys, 43

Prophila, 129

Prorhynchus, 306

Prorodon, 33I

Prosacantha, 99

Proscoporhinus, 220

Proselena, I r6

Prosaprognus, $\mathrm{I}_{44}$

Prosopis, 96

Prostheceræus, 306

Prosthemadera, 28

Protarphius, r7o

Protelater, 154

Proteodes, I 18

Proternia, I 15

Proterocossa, 121

Prothelymna, I 77

Protithona, I 16

Protocaulon, 316

Protolobus, 2OI

Protoparnus, I 53, I62

Protophormus, 200

Protosquilla, 256

Protosynæma, 122

Prototroctes, 50

Protula, 282

Psanımobia, 88

Psammopemma, 324

Psammosphæra, 334

Pselactus, 2r 8

Pselaphus, 177

Psephoboragus, I53

Psepholax, 2I I

Pseudagenia, 97

Pseudæga, 262

Pseudocalliprason, I92

Pseudococcus, 228

Pseudœeconesus, 228

Pseudogerygone, 28

Pseudolabrus, 47

Pseudonema, 228

Pseudopatrum, I85

Pseudopentaceros, 4 I

Pseudophycis, 48

Pseudopsolus, 287

Pseudorca, 27

Pseudoreda, 2 I I

Pseudosemnus, r92

Pseudosyllis, 276

Psilaster, 29I

Psilochoremus, 229

Psilocnzeia, 195

Psilodontria, I40

Psilomorpha, I93

Psilopus, I30

Psilotricha, 328

Psorochroa, I56

Psychoda, I38, 350

Ptenidium, 167

Pteraclis, 44

Pterodina, 302

Pterolichus, 246

Pteronalus, 99

Pterophorus, II 5
Pteropterix, 99

Pterostichus, 145, 342

Ptinella, r68

Ptinus, I6I, 352

Ptychodon, 6r

Ptychopterus, 193

Puffinus, 34

Pulex, I38, 350

Pullenia, $33^{8}$

Pulvinaria, 349

Pulvinulina, $33^{8}$

Purpura, $7 \mathbf{r}$

Pycnocentra, 228

Pycnomerodes, I7 I

Pycnomerus, I71, 342

Pygoscelis, 36

Pyralis, 349

Pyramidella, 74

Pyrgotis, I 16

Pyronota, 140

Pyrosoma, 56

Pyrrha, 63

Pyxicola, 329

Quenius, $r 82,35 \mathrm{r}$

RACHIDISCUS, 205

Ræta, 90

Raia, 53

Ranfurlyea, 34I

Rattulus, 301

Realia, 78

Recurvirostra, 32

Recyntus, 170

Regalicus, 47

Rembus, 143

Remora, 45

Reophax, 335

Resania, 90

Rethusus, 16

Retipora, 296

Retropinna, 5I

Reuda, 223

Rhabdamina, 335

Rhabdinosomus, 203

Rhamphidia, I36

Rhantus, $\mathrm{I}_{5} \mathrm{I}$

Rhaphidophlus,

Rhaphidozoom, 339

Rhaphiodophlus, 324

Rhapsa, 106

Rhenia, 65

Rhinanisus, 217

Rhinocola, 225

Rhinorhynchus, 2 ro

Rhinoscopelas, 50

Rhipidodendron, 333

Rhipidogorgia, 316

Rhipidura, 28

Rhipisterna, I9I

Rhizobius, 353

Rhizococcus, 227
Rhizonium, 170

Rhododrilus, 284

Rhogas, 99

Rhombocyttus, 45

Rhombosolex, 48

Rhopalimorpha, 22 I

Rhopalium, 98

Rhynchogonus, 2OI

Rhynchonella, 293

Rhynchophora, 298

Rhyneodes, 215

Rhynorhynchus, 210

Rhypha, I35

Rhypholopus, I 36

Rhyssa, 102

Rhyssodes, 167

Rhytida, 65

Rhytiodontus, I70

Rhytisternus, $147,35^{r}$

Rhyzonium, I70

Ricania, 353

Ripersia, 227

Risella, 79

Risellopsis, 79

Rissoia, 77

Rissoina, 78

Rotalia, 338

Rotifer, 300

Ruppeloides, 2.48

Rygmodus, 152, 342

Rystheus, 206

SABELLA, 28I

Sacina, 174

Sagartia, 3r4

Sagephora, I24

Sagola, 178

Sagolonus, 179

Salda, 223

Salenia, 288

Salius, 97

Salmacis, 288

Salmo, $34^{8}$

Salpa, 56

Salpingœca, 331

Salpingus, 189

Salticus, 244

Salltius, $x 66$

Samana, I Io

Saphobius, 139

Saphophagus, I67

Saphydrus, I 52

Sapphirina, 272

Saprinus, 173

Sapromyza, 128

Sarcophaga, 350

Sarda, 45

Sargon, 343

Saropogon, I 3 I

Sarsiella, 269

Saxicava, 88

Scalaria, 80

Scalibregma, 28r 
Scaphella, 74

Scaphisoma, 174

Scaphodius, 198

Scatopse, I33, 350

Sceliodes, II 5

Scelodolichus, 2I4

Sceloglaux, 29

Scenoploca, II2

Schauinslandica, Ioo

Schistocephalus, 310

Schizoglossa, 66

Schizoneura, 353

Schizoporella, 297

Schylus, 214

Sciacharis, I76

Sciara, I34

Scieropepla, I 18

Sciocoris, 352

Sciomya, 128

Sciophila, I33

Scissurella, 83

Sclerochilus, 268

Scolobates, 102

Scolopterus, 2 ro

Scoloptethus, 222

Scomber, 44

Scombresox, 49

Scoparia, II3

Scopodes, I50, $34^{2}$

Scoriodycta, I24

Scorpæena, 42

Scorpis, $4 \mathrm{I}$

Scraptogetus, Igo

Scrupocellaria, 294

Scutellidium, 272

Scutuloidea, 263

Scutum, 84

Scydmænus, I75

Scymmus, 165

Scyphax, 265

Scyphoniscus, 265

Scythrodes, I40

Seytomonas, 333

Seba, $25^{8}$

Sebastapristes, 42

Segestria, 237

Selaginopsis, 321

Selenopalpus, Igo

Selidosema, I Iо

Semicassis, 75

Semicosma, I 9

Semo, 225

Senoprosop1s, I3I

Sepia, $5^{8}$

Sepimentum, I27

Sepiola, $5^{8}$

Sepioteuthis, $5^{8}$

Serangodes, 235

Sergestes, 254

Sericoderus, 174

Sericospilus, I 40

Sericotrogus, 2 r 6

Seriola, 44

Seriolella, 44
Serolis, 263

Serpula, 282

Sertularella, 320

Sertularia, 320

Sesarma, 250

Sessinia, Igo

Sestra, I I I

Setella, 272

Setodes, 229

Sharpia, I9I

Sibinia, 210

Siculodes, I I 5

Sierola, 99

Sigaus, 233

Signuatella, 32.4

Silene, I 56

Silphotelus, 175

Silvanus, $35 \mathrm{I}$

Simachus, 208

Simæthis, I22

Simocephalus, 266

Simulium, I33

Siphonaria, 68

Siphonalia, 72

Siphonophora, 353

Siphonosphara, 339

Siphostoma, 52

Sipunculus, 285

Siriella, 256

Sitones, 352

Smittia, 297

Solarium, 79

Solaster, 292

Solegnathus, 52

Solenomya, 95

Solenophora, 227

Solenotellina, 89

Solidula, 69

Somatidia, 195

Somatochlora, 230

Sorensenella, 343

Soronia, 172

Sosgenes, 206

Spatula, 36

Sphærium, 9I

Sphæroidina, $33^{8}$

Sphærophysa, 327

Sphasus, 244

Sphenodon, 40

Spencecus, 28

Spheroma, 263

Sphindoteles, 16I

Sphinx, I I I

Sphyræna, 46

Sphryrna, 53

Sphyrion, 274

Spilomicrus, 98

Spilotrogia, I96

Spio, I 45

Spiroloculina, 334

Spirosphera, 304

Spirostomum, 330

Spirostreptus, 235

Spirorbis, 282
Spirotropis, 70

Spirula, $5^{8}$

Spongelia, 324

Spongilla, 323

Spongomonas, 333

Spondylus, 93

Spongiochiton, 86

Squalus, 54

Squilla, 256

Stachyodes, 316

Standella, 90

Staphilinus, I83

Startes, I78

Stathomopoda, 120

Stauracanthus, 339

Stauronereis, 279. 345

Steatoda, 2.40

Steenstrupia, $5^{8}$

Steganoporella, 296

Steg، sonia, 240

Stelospongi $\uparrow, 326$

stenellipsis, 195

Stenetrium, 264

Stenopotes, I93

Stenoperla, 230

Stenoptila, I 5

Stenorhynchus, 247

Stenosmylus, 229

Stenothoe, 260

Stentor, 330

Stephanomia, 317

Stephanopsis, 243

Stephanorhynchus, 209, 343

Stephonalia, $3^{17}$

Stercorarius, 33

Sterna, 32

Sternagasta, 29I

Sternaspis, 28I

Sternopteryx, 50

Sternus, 347

Steropus, I 47

Sterromonas, $33^{2}$

Stethaspes, 140

Sthenelais, 277

Stichaster, 292

Sternaulax, 173

Stichopus, 286

Stichotricha, $3^{28}$

Stigmatophora, 52

Stigmodera, $35^{2}$

Stilbocara, 218

Stilbopsis, 208

Stiliger, 68

Stolephorus, $5^{\text {I }}$

Stolotermes, 230

Stomoxys, 350

Stratiotes, 252

Strepsicrates, I I6

Stringops, 30

Stromatopora, 299

Strombidium, 329

Strongylocentratus, 288

Strongylosoma, 235

Strumigemys, 97 
Struthiolaria, 75

Stygeopetes, 199

Stygnohydrus, I 52

Stylarioides, 28I

Stylaster, 318

Stylatrachus, 339

Stylobryon, 333

Stylodactylus, 255

Stylonictia, 327

Stylotella, 324

Styphotelus, 205

Subemarginula, 84

Sula, 33, 39

Supercystis, 299

Surcula, 70

Suteria, 63

Sus, 347

Sycon, 322

Syllectus 148

Syllis, 276, 34 I

Sympedius, 212

Sympetrum, 230

Sympiestus, I49

Sympiezoscelus, 2 I 5

Synapta, 287

Syncalus, $\mathbf{x} 70$

Synœcus, 348

Synthecium, 321

Syntomactis, I $2 \mathrm{r}$

Syrphetodes, 185,342

Syrphus, I30

Tabanus, 132

Tachina, 126

Tachusa, I8I

Tachys, 150

Tachytes, 98

Tænia, 312

Tæniorhynchus, $1_{3} 8$

Talerax, I 54

Talitropsis, $23^{2}$

Tanais, 262

Tanyderus, 137

Tanypus, 137

Tanytarsus, $\mathbf{r} 37$

Taonius, 57

Tapes, 89

Tarastethus, I49, 342

Targarema, 222

Taron, 73

Tarphiominus, 168

Tatosoma, I07

Taupodrilus, 346

Tauroscopa, I I I

Taxipathes, $3 \mathbf{I} 5$

Techmessi, I90, 342

Techmessodes, I9I

Technitella, 334

Tegenaria, $23^{8}$

Tekella, 240

Tellina, 9 I

Telmatophilus, 166

Temnocephala, 308
Temnopleurus, 288

Temora, 270

Tenagodes, 76

Tenagomysis, 256

Tenebrio, 352

Tenebrioides, 35I

Tephrites, 350

Terebella, 280

Terebellides, 280

Terebra, 70

Terebratella, 293

Terebratulina, 293

Tessaromma, $35^{2}$

Testacella, $34^{8}$

Tetanocera, 128

Tethya, 323

Tethyopsis, 323

Tethys, 68

Tetrabothrius, 3 II

Tetraclita, 275

Tetradeion, 258

Tetragnatha, 240

Tetragoneura, I34

Tetranychus, 354

Tetrarhynchus, $3 \mathrm{rr}$

Tetrorea, 196

Textularia, 336

Thalamita, 249

Thalassicolla, 339

Thalassogeron, 35

Thalassohelix, 64

Thalestris, 271

Thallis, $\mathbf{1 6 6}$

Thamnosara, Irg

Thaumatodon, 6 I

'Thelepas, 280

Thelyphassa, I90, 342

Theoxena, I10

Therasia, 63

Theridion, 238, 354

Thersites, 43

Thinornis, 32

Thinosotia, II2

Thiotrichia, 118

Thoracophelia, 280

Thoramus, I 57

Thorecta, 326

Thorellia, 270

Thortus, I67

Thouarella, 316

Thracia, 87

Thrips, 354

Thuiaria, 320

Thurammina, 335

Thuricola, 329

Thurophora, 328

Thylacosceles, I2I

Thynasotia, II2

Thynnus, 44

Thyrocyphus, 3I9.

Thyrsites, 43

Thysanozoon, 306

Tigones, 200

'Tillina, 33
Timarete, 280

Tinea, 125, 350

Tinemyia, I37

Tineola, $35^{\circ}$

Titanomis, 124

Tintinnidium, 330

Tipula, 136

Todarodes, $5^{8}$

Tomicus, 219

Tornissus, 152

Tormus, I 52

Tornatellina, 59

Toreuma, $3^{17}$

Tornatina, 70

Torpedo, 53

Tortrix, II7

Torymus, 99

Toxochalina, 325

Trachelocera, 330

Trachelochismus, 47

Trachelomonas, $33^{2}$

Trachelophyllum, 331

Trachyichthys, 43

Trachurus, 44

Trachyleberis, 268

Trachypepla, II9

Trachypterus, 47

Trachyrhynchus, 49

Trachyroplectron, 232

Trachytetra, I99

Traversia, 29

Travesia, 281

Tralia, 66

Triæenyx, 245

Tribolium,

Trichocera, 136

Trichodectes,

Trichoniscus, 265

Trichophaga, 350

Trichophthicus, I27

Trichophyra, 327

Trichoplatus, 247

Trichopteryx, 167

Trichosia, 134

Trichosoma, 304

Trichosternus, I45, 342

Trichosurus, 347

Trichotropsis, 79

Triforis, 76

Trigla, 45

Trisoniza, 233

Trigonocidaris, 288

Trigonometopus, 128

Tringa, 32

Trioza, 225

Triphyllus, 165

Triplax, I66

Triplosaurus, 147

Tripneustes, 289

Tripterygion, 46

Tristomum, 308

Tritæeta, 259

Trite, 245

Trivia, 75 
Trochammina, 335

Trochilium, 350

Trochobola, 136

Trochodota. 287

Trochostoma, 287

Trochus, 8I

Trogoderma, I63

Trogophlæus, I84, 342

Trogosita, I7I

Trophon, 72

Tropidocera, 304

Trox, 35I

Truncatulina, 338

Trypanoscyllis, 345

Tryphera, 126

Tryphon, IO2

Tubicinella, 274

Tubiclava, 318

Tubifex, 284

Tubucellaria, 296

Tubularia, 3 Ig

Tubulipora, 299

Tularthrium, 167

Turbo, 8I

Turbonilla, 74

Turdus, 347

Turnagra, 27

Turritella, 76

Tursiops, 27

Tychanopais, 212

Tychanus, 2II

Tylos, 265

Tympanopalpus, I97

Typhæa, 165

Tyrogetus, I76

Tyroglyphus, 354

Tyrus, I76

Tysius, 208

ULIODON, 238

Uloma, I86

Ulomotypus, I86

Ulonotus, I68

Uniofera, 292

Upeneichtlyys, 4 I
Upogebia, 253

Uranotœnia, ${ }_{13} 8$

Urocentrum, 329

Urodynamis, 29

Uroleptus, 328

Uronema, 330

Uropetala, 230

Uropoda, 246

Uroptychus, 252

Utethesia, 349

Uvella, 332

Uvigerina, 337

VAGINICOI.A, 329

Vaginulina, 337

Vallonia. 349

Valvulina, 336

Vanessa, I03

Vanicella, I20

Velella, 3I7

Venericardia, 93

Venerupis, 90

Venusia, I08

Vermicularia, 76

Vermilia, 282

Verneuilina, 336

Veronatus, $15^{8}$

Veronicobius, I65

Vibilia, 256

Vidanus, I79

Virgularia, 3 I 6

Virgulina, 336

Vitiacus, 169

Volsella, 94

Vorticella, 328

Vosmaeropsis, 322

Votum, I92

Vulpicula, 74

WAKEFIELDIA, I 50

Walckenaera, 354

Walteria, 323

Webbina, $33^{6}$

Whitea, I79
XANTHAGRION, 230

Xanthia, ${ }^{0} 4$

Xantho, 248

Xantholinus, $183,35 \mathrm{I}$

Xanthorhoë, I08

Xenanthribus, 221

Xenicus, 29

Xenocnema, $2 I 8$

Xenogonus, I6I

Xenophora, 79

Xenoscelis, I72

Xerostygnus, 343

Xesta, 65

Xestoleberis, 268

Xiphias, 43

Xiphidium, 23I

Xiphocharis, 255

Xiphydria, I03

Xouthous, 27 I

Xuthodes, I93

Xylochus, 188

Xylostygnus, I40

Xyloteles, I94

Xysticus, 243

YPONOMEUTA, I22

ZABRONOTHUS, $\mathrm{I}_{4} 8$

Zapyrastra, I2I

Zaus, 27I

Zeacalles, 2 I4

Zeadolopus, 342

Zeaglophus, I56

Zeamordella, I9I

Zelandius, I79

Zenatia, 90

Zeopcecilus, $\mathbf{1} 45$

Zestocormus, IOI

Zeus, 45

Ziphius, 26

Zolodinus, I 86

Zolus, I48

Zoothamnium, 329

Zorion, I93

Zosterops, 28

Zygomyia, I34 

